AIII02 146064

NBS

PUBLICATIONS

NAT'L INST OF STANDARDS \& TECH R.IC.

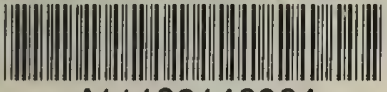

A11102146064

OC100 U573 V4:1;1966 C.2 NBS-PUB-C 1964

Aloming Tiransition

Prohativifitios

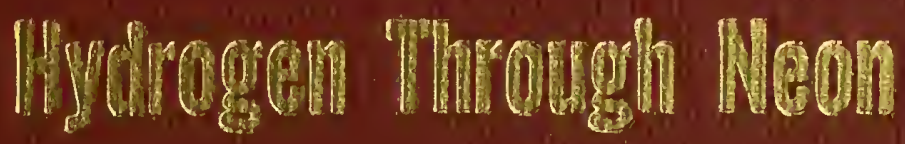

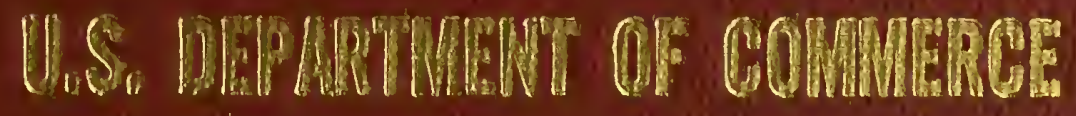

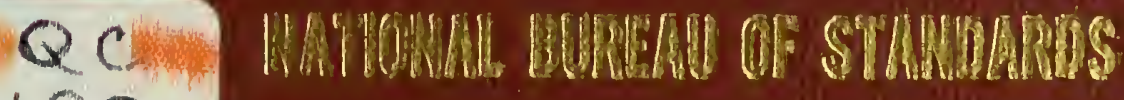

100

4573

No. 4

1966

( . . 2
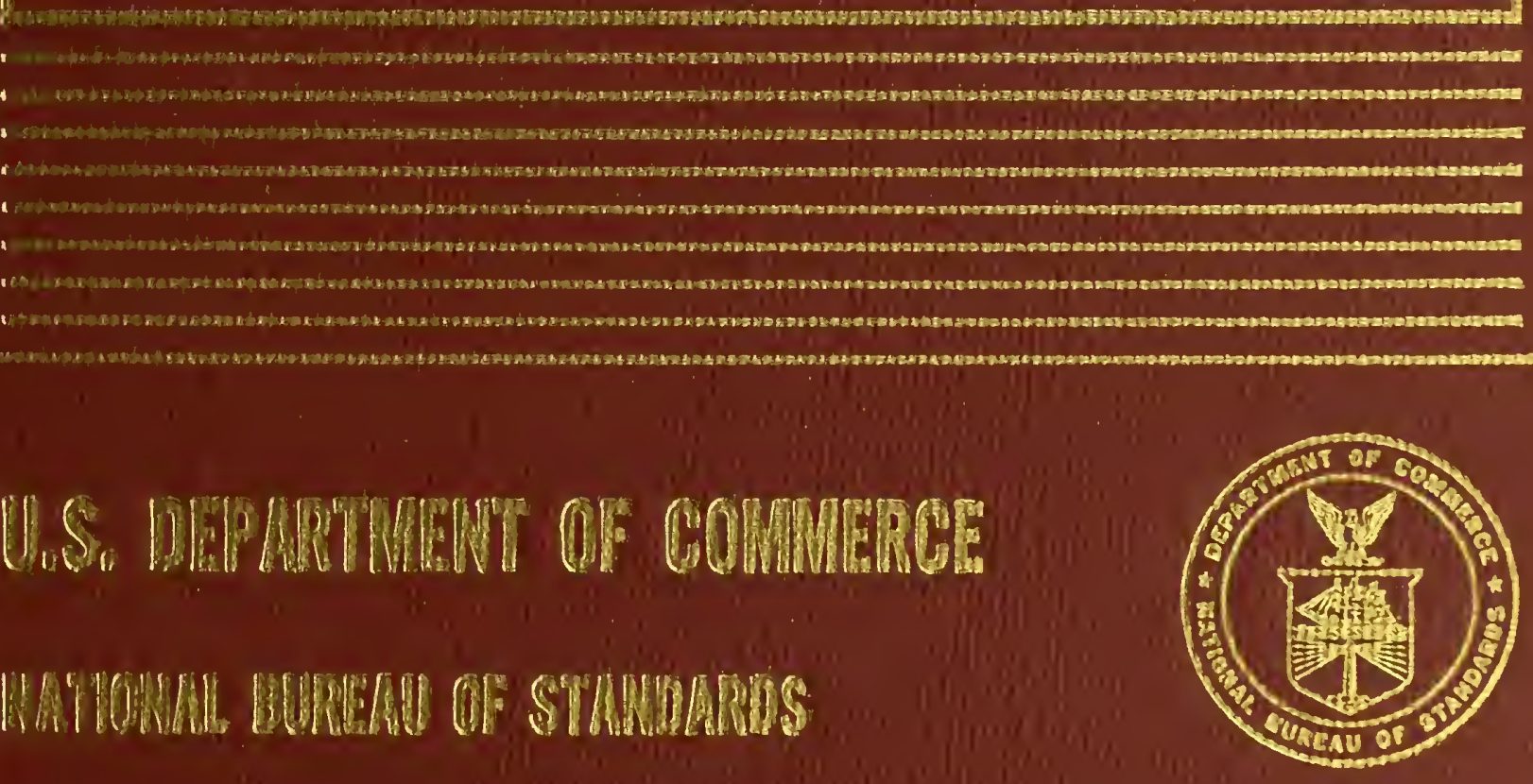

1

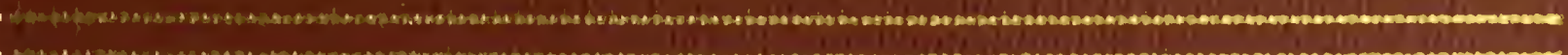

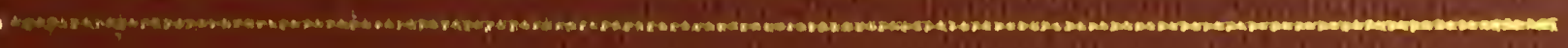







\title{
Atomic Transition Probabilities
}

\section{Volume I Hydrogen Through Neon}

A Critical Data Compilation

\author{
W. L. Wiese, M. W. Smith, and B. M. Glennon \\ Institute for Basic Standards \\ National Bureau of Standards \\ Washington, D.C.
}

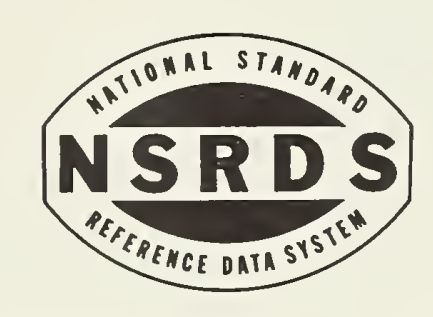

NSRDS-NBS 4-

National Standard Reference Data Series

National Bureau of Standards 4

(Category 3 - Atomic and Molecular Properties)

Issued May 20, 1966

For sale by the Supcrintendent of Documents, U.S. Government P'rinting Office

Washington, D.C., 20402 - Price $\$ 2.50$ 
National Bureau of Standards AUG $3 \quad 966$

133,190

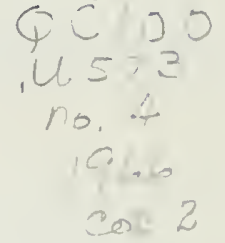

Library of Congress Catalog Card Number : 65-60078 


\section{National Standard Reference Data System}

The National Standard Reference Data System is a government-wide effort to give to the technical community of the United States optimum access to the quantitative data of physical science, critically evaluated and compiled for convenience. This program was established in 1963 by the President's Office of Science and Technology, acting upon the recommendation of the Federal Council for Science and Technology. The National Bureau of Standards has been assigned responsibility for administering the effort. The general objective of the System is to coordinate and integrate existing data evaluation and compilation activities into a systematic, comprehensive program, supplementing and expanding technical coverage when necessary, establishing and maintaining standards for the output of the participating groups, and providing mechanisms for the dissemination of the output as required.

The NSRDS is conducted as a decentralized operation of nation-wide scope with central coordination by NBS. It comprises a complex of data centers and other activities, carried on in government agencies, academic institutions, and nongovernmental laboratories. The independent operational status of existing crictical data projects is maintained and encouraged. Data centers that are components of the NSRDS produce compilations of critically evaluated data, critical reviews of the state of quantitative knowledge in specialized areas, and computations of useful functions derived from standard reference data.

For operational purposes, NSRDS compilation activities are organized into seven categories as listed below. The data publications of the NSRDS, which may consist of monographs, looseleaf sheets, computer tapes, or any other useful product, will be classified as belonging to one or another of these categories. An additional "General" category of NSRDS publications will include reports on detailed classification schemes, lists of compilations considered to be Standard Reference Data, status reports, and similar material. Thus, NSRDS publications will appear in the following eight categories:

Category
1
2
3
4
5
6
7
8

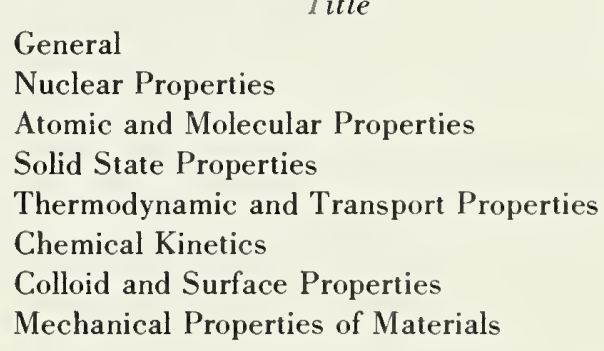

Within the National Bureau of Standards publication program a new series has been established, called the National Standard Reference Data Series. The present report, which is in Category 3 of the above list, is Volume $I$ of Number 4 of the new series and is designated NSRDS-NBS 4 Vol. I. 



\section{Contents}

General Introduction
A. INTRODUCTORY REMARKS
B. SCOPE OF THE TABLES
I

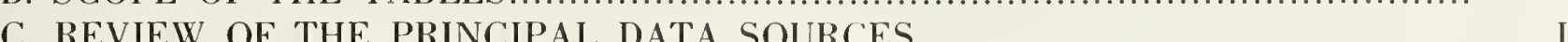
D. METHOD OF EVALUATION.
E. ARRANGEMENT AND EXPLANATION OF COLUMNS.
F. FUTURE PLANS AND ACKNOWLEDGEMENTS
REFERENCES
VIII
APPENDIX A
VIII
APPENDIX B
$\mathrm{XI}$
XI

List of Tables

Spectrum

Hydrogen $\mathrm{H} \mathrm{I}^{*}$.....

Helium He I. .

Lithium

Li I....

Li II. .

Beryllium Be

Be II

Be III. ...

Boron

B I.

B II

B III...

B IV

Carbon

C I

C II.............................

C III............................

C IV

$\mathrm{C}$

Nitrogen

$\mathrm{N}$ I.

$\mathrm{N}$ II...........................

N III.........................

$\mathrm{N}$ IV

$\mathrm{N}$ v

$\mathrm{N}$ VI.

Page
1
9
16
19
22
23
24
25
27
28
29
30
37
41
45
47
48
56
65
71
74
76

Page

$$
1
$$

$$
9
$$$$
16
$$

19

22

23

24

25

27

28

29

30

37

41

45

47

48

56

65

71

74

76

Spectrum
Oxygen
Fluorine
Neon

0

(O)

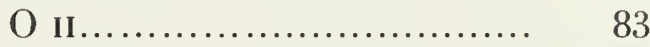

O III........................... 95

O IV............................ 103

O v

O vi.......................... 110

O VII......................... 112

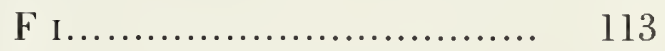

F II......................... 115

F III....................... 117

F IV........................ 119

F v ........................... 120

F vi........................ 122

F VII....................... 123

F VIII....................... 125

Ne I.......................... 126

Ne II.......................... 130

Ne III....................... 138

$\mathrm{Ne}$ IV......................... 142

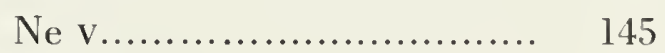

$\mathrm{Ne}$ VI......................... 148

$\mathrm{Ne}$ vil....................... 150

Ne vill...................... 151

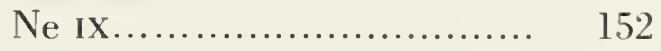

LIST OF RECENT ADDITIONAL MATERIAL 



\title{
ATOMIC TRANSITION PROBABILITIES*
}

\author{
(A critical data compilation)
}

Volume I

Elements Hydrogen through Neon

\author{
W. L. Wiese, M. W. Smith, and B. M. Glennon
}

\begin{abstract}
Atomic transition probabilities for about 4,000 spectral lines of the first ten elcments, based on all available literature sources, are critically compiled. The data are presented in separate tables for each element and stage of ionization. For each ion the transitions are arranged according to multiplets, supermultiplets, transition arrays, and increasing quantum numbers. Allowed and forbidden transitions are listed senarately. For each line the transition probability for spontaneous emission, the absorption oscillator strength, and the line strength are given along with the spectroscopic designation. the wavelength, the statistical weights, and the energy levels of the upper and lower states. In addition, the estimated accuracy and the source are indicated. In short introductions, which precede the tables for each ion, the main justifications for the choice of the adopted data and for the accuracy rating are discussed. A general introduction contains a critical review of the major data sources.
\end{abstract}

\section{A. INTRODUCTORY REMARKS}

After a long period of limited activity in atomic spectroscopy, the last half dozen years have brought rapid growth to this field. This has been sparked largely by urgent needs from areas in which basic atomic data are employed, namely plasma physics, astrophysics, and space research. As part of these developments, the pace and scope of determining atomic transition probabilities has greatly increased, so that the amount of accumulated material appears now to be sufficiently large to make a critical data compilation worthwhile and desirable. To realize this idea, a data center on atomic transition probabilities was established at the National Bureau of Standards in 1960. As a first step of the program, a search for the widely scattered literature references was undertaken. This phase of the work was essentially completed in 1962 with the publication of a "Bibliography on Atomic Transition Probabilities" (NBS Monograph 50) [1]. After that, only the monitoring of the current literature had to be kept up, and the emphasis of the work therefore could be shifted to the critical evaluation of the literature and the tabulation of the numerical data. Since the lightest ten elements have been of dominant interest, and on the other hand, the largest amount of data are available for them, it was decided to concentrate on these and publish their "best" values as the first part of a general compilation. pilation.

When the present tabulation was started, it was hoped that sufficient reliable material was available for a fairly comprehensive list, which would contain data for at least all the strong prominent transitions. This hope materialized only partially. A number of gaps and large discrepancies were found, and the theoretical and experimental efforts of several members of the Plasma Spectroscopy Section at NBS were needed to remedy the most critical

\footnotetext{
*This research is a part of project DEFENDER, sponsored by the Advanced Research Projects Agency, Department of Defense, through the Office of Naval Reacarcli.
}

situations. Furthermore, the Coulomb approximation of Bates and Damgaard [2] was extensively applied to obtain additional data. Although this unforeseen extra work delayed the publication of this compilation somewhat, we feel that we are now able to present a more useful and sub. stantial collection of data.

\section{B. SCOPE OF THE TABLES}

In the present compilation the "best" available transition probabilities ${ }^{1}$ of allowed (i.e., electric dipole) and forbidden (i.e., electric quadrupole and magnetic dipole) lines of the first ten elements, including their ions, are tabulated. The hydrogen-like ions are excluded; their transition probabilities may be obtained by scaling the hydrogen values according to the formulas given in table I. Furthermore. $f$-values or absorption coefficients for continua, i.e., boundfree transitions are not listed. As source material all the literature given in Ref. [1] plus later articles obtained from continuous scanning of the current literature are available.

It is our opinion that a tabulation of the present kind must contain fairly reliable values for at least all the stronger. characteristic lines of the various ions in order to be of general usefulness. We have tried to adhere to this goal from the start. More specifically, we have felt that for most atoms and ions at least the "prominent" half of the multiplets listed in the "Revised Multiplet Table" [3]. and the "Ultraviolet Multiplet Table" [4] should be included in the tabulation, and uncertainties should be smaller than 50 percent. A number of gaps in the data and inferior values were noticed at the start, and-as already mentioned-it has been largely due to the efforts of some

Hereafter, we sluall use the eqursalens terms "transition probability, onsillator strength wr fivalue. and line strength" on an interehangeable basis. The numerical relationships between these guan. ities are given in table 11 ! 
members of the Plasma Spectroscopy Section at NBS and the availability of the Coulomb approximation [2] that the most glaring defects could be eliminated. Although we still must compromise in some cases by including inferior material (marked in the accuracy column as " $E$ ") we feel that waiting for these improvements would unduly delay the publication of the table.

Aside from this objective of including at least all the stronger lines, we have listed all additional available material with uncertainties smaller than 50 percent. We have deviated from this scheme only in a few instances: In these cases we have excluded data for very highly excited transitions, because these transitions have never been observed (no experimental wavelengths are available) and are of little practical interest. However, we have listed this additional material by spectrum in table II.

Most final tabulations were undertaken during 1964. Thus the literature through 1963 and in some cases even later work could be included. However, a few 1963 articles, which have been found in abstracting journals, came to our attention too late. These are listed, together with other recent material, in the list of additions at the end of the tables.

\section{REVIEW OF THE DATA SOURCES}

The present status of our knowledge of atomic transition probabilities must be considered as being far from ideal. The available material leaves much to be desired in quality as well as quantity [5]. This becomes especially evident if comparison is made with the other most important quantity of a spectral line, its wavelength. The only transition probabilities known with an accuracy comparable to that for wavelengths are available for hydrogen and hydrogenlike ions and a few lines of helium. For all other elements more or less reliable values have been obtained from various experimental and theoretical approaches. While experimental work has provided, with very high accuracy, practically all the data for the wavelengths of lines, it could not accomplish nearly the same in the case of transition probabilities. The measurement techniques are quite complicated and laborious, and it has proved to be very difficult to obtain accuracies of 10 percent or better. On the other hand, advanced theoretical approximations have been quite successful for the light, relatively simple atomic systems, and large amounts of data have been obtained from their applications. But the theoretical methods have the shortcoming that they do not permit estimates of the size of the errors as do the experiments.

In view of this reliability problem it is very important to discuss in detail how the accuracy ratings for the tabulated values have been obtained. For this purpose, a brief discussion is given in the individual introductions for each ion. Furthermore, to provide a better background and understanding for these short explanations, we include the following discussion of those major experimental and theoretical methods from which the bulk of the material for the lightest ten elements has been obtained.

\section{Experimental Sources}

a. Measurements in Emission

Experimentally, the largest number of $f$-values has been obtained from measurements of the intensities of spectral lines which are emitted from plasmas under known conditions. With this method the first and second spectra of carbon, nitrogen, and oxygen, the third spectrum of oxygen,- and the first spectrum of neon have been studied. The plasma sources are various types of stabilized arcs, and, to a lesser extent, shock-tubes. In brief, the method [6] is as follows: The transition probability for spontaneous emission from upper state $k$ to lower state $i, A_{k i}$, is related to the total intensity $I_{k i}$ of a line of frequency $\nu_{i k}$ by

$$
I_{k i}=\frac{1}{4 \pi} A_{k i} h \nu_{i k} N_{k}
$$

where $h$ is Planck's constant and $N_{k}$ the population of state $k$. $A_{k i}$ may therefore be obtained from the measurement of $I_{k i}$ and the determination of $N_{k}$.

The experimental conditions are chosen so that the plasma is approximately in a state of local thermodynamic equilibrium (LTE), because $N_{k}$ is then a function of temperature and total density of the species only, and may be determined from the application of equilibrium and conservation equations and measurements of the temperature and electron density. The measurements have always been done spectroscopically from the determinations of the intensities of lines and continua of known transition probabilities and absorption coefficients, or by measuring line profiles and utilizing the results of line broadening theory in plasmas.

Checks for the existence of LTE have been made repeatedly. It appears to be always closely approximated, except in the high-temperature magnetically driven shocktubes where only partial LTE exists [7]. Also, the investigated lines have generally been checked for self-absorption. A demixing effect in arcs $[8,9]$ has introduced uncertainties into the results of some earlier arc experiments with gas mixtures, in which the mixture ratio was employed for the analysis. Since primarily the densities are affected, larger uncertainties in the absolute $f$-value scale are likely, but the relative $f$-values should be still quite accurate. However, this effect has been circumvented in most of the recent arc experiments used for this data compilation. Significant errors in emission experiments may arise from difficulties in determining the continuous background, from neglecting intensity contributions of the distant line wings [10], from uncertainties in the calibration of standard light sources, and from uncertainties in the high-density corrections in plasmas [11]. Applications of wall-stabilized arcs $[12,13]$ have given the most accurate results of all emission measurements.

The best absolute $f$-values obtained from emission experiments are estimated to be accurate within 15 percent, but for the bulk of the tabulated data errors between 20 to 50 percent must be expected. It should finally be noted that absorption measurements (only one is encountered in the case of $\mathrm{Ne} \mathrm{I}$ ) are quite analogous to the above mentioned emission experiments.

\section{b. Lifetime Determinations}

The direct measurement of lifetimes of excited atomic states has important applications for helium and neon. The method $[6,14]$ employed here consists essentially of exciting atoms by radiation or electron impact in short bursts and of observing the subsequent depopulation of excited levels by studying the time decay of the emitted radiation (delayed coincidence technique). The population $N_{k}$ of an excited state $k$ decays according to

$$
N_{k}=N_{o, k} \exp \left(-\gamma_{k} t\right)
$$

where $N_{o, k}$ is the population at time $t=0$ and $\gamma_{k}$ the decay 
constant. Thus, an exponential decay in the radiation is observed. The mean lifetime $\tau_{k}=\gamma_{k}^{-1}$ of the atomic state is related to the transition probability $A_{k i}$ for spontaneous emission by

$$
\tau_{i}^{-1}=\sum_{i} A_{k i}+Q
$$

neglecting absorption and induced emission. $Q$ denotes a term for collisional population and depopulation rates. In order to obtain $\sum_{i} A_{k i}$, one has to choose experimental conditions such that the collisional term $Q$ (as well as the less critical absorption and induced emission) becomes negligible. This condition is achieved at very low densities.

It is seen that from lifetime mcasurements generally the sum of all probabilities for transitions to lower levels $i$ is obtained, and individual transition probabilities may be obtained explicitly only in the following two cases: (a) The sum reduces to a single term, i.e., only transitions to the ground state are possible. This is, for example, the case for resonance lines. (b) The sum is dominated by one strong term (this is likely if it contains a transition of comparatively high frequency $\nu_{i k}\left(A_{k i}\right.$ is proportional to $\left.\nu_{i k}^{3}\right)$, or if all transitions but one are "forbidden", i.e., have very small transition probabilities). Furthermore, one may use lifetime experiments to normalize available relative transition probabilities to an absolute scale, if all relative probabilities contributing to the sum are known.

The lifetime method is, therefore, limited to only a few lines per spectrum, namely those originating from the lowest excited levels. But the results should be very accurate, with uncertainties less than 10 percent, since the method is simple and the instrumentation is by now well developed [14]. The major uncertainties arise from radiative cascading from higher levels, which repopulates the initial level, and from depopulation by collisions.

c. Measurement of $f$-values from the Anomalous Dispersion at the Edges of Spectral Lines

This method has found applications for lines of neutral lithium and neon. It is based on the following relation: In the neighborhood of a spectral line the index of refraction $n$ varies according to

$$
n-1=\frac{e^{2} N_{i} f_{i k}}{4 \pi m_{e} c^{2}} \frac{\lambda_{0}^{3}}{\lambda-\lambda_{0}}\left(1-\frac{N_{k} g_{i}}{N_{i} g_{k}}\right) \text {. }
$$

Here $g$ denotes the statistical weight; $\lambda$ the wavelength; $N_{i}$ the population of the lower state $i$; and $e, m_{e}, c$ are the usual natural constants. The experimental conditions are chosen such that the excited states are populated according to the Boltzmann formula, so that generally $N_{k}<<N_{i}$, and the term $N_{k} g_{i} / N_{i} g_{k}$ may be neglected. For the determination of $N_{i} f_{i k}$ the index of refraction $n$ at the wavelength distance $\lambda-\lambda_{0}$ from the center of the line, $\lambda_{0}$, has to be measured. This can be done most precisely with the "hook" method developed by Rozhdestvenskii [15] and recently reviewed by Penkin [16]. In this method the gas to be studied fills a tube, which is part (one arm) of a Jamin or Mach-Zehnder interferometer. 'The tube must be at an elevated temperature to achicve sufficient population of the excited levels. Light from the continuum source pelletrates the tube as well as an evacuated comparison tube of the same length, and the resulting interference fringes are sent into a spectrograph. On either side of an absorption line the interference fringes are characteristically bent due to the rapid change in the index of refraction. By introducing a thick glass plate into the compensating arm of the interferometer, a tilting of the fringes and the formation of the looks is accomplished. The measurement of the wavelength distance between the cxtrema then permits a precise determination of the index of refraction. In the three experiments encountered for this compilation, the absolute number densities for the lower states $N_{i}$ could not be determined, so that only relative $f$-values for lines originating from the same lower levels werc measured. Uncertainties in the relative values should not exceed 10 to 20 percent. In the original papers, absolute $f$-values were then obtained from applications of the Thomas-Kuhn-Reiche $f$-sum rule, but for this compilation we have normalized the relative values to diffcrent scales, which are based on other, more accurate material.

\section{Theoretical Sources}

\section{a. The Coulomb Approximation}

Under the assumption of Russell-Saunders (or LS.) coupling, which is generally very well fulfilled for the first ten elements, the line strength $S$ may be expressed as the product of three factors [2]

$$
S=\Im(\mathfrak{M}) \Subset(?) \sigma^{2}
$$

(The relations of $S$ with $A$ and $f$ are given in table III.) The first two factors in eq (5) represent the strength of the multiplet $\left(\Im\left(\mathfrak{O}_{i}\right)\right)$ and the fractional strength of the spectral line within the multiplet $(\Im(?))$. The numerical values for these may be obtained from tables by Goldberg [17], and White and Eliason [18], which have also been reproduced by Allen [19].

The difficult problem is the evaluation of the transition integral $\sigma$. Bates and Damgaard [2] showed that for most transitions the main contribution to the integral comes from a region in which the deviation of the potential of an atom or ion from its asymptotic Coulomb form is so small that it may be replaced by the latter. Since for the Coulomb potential the transition integral may be expressed analytically, it is possible to calculate $\sigma^{2}$ as a function of the observed term value and the azimuthal quantum number. Bates and Damgaard have thus compiled tables with numerical values of $\sigma^{2}$ for $s-p, p-d$, and $d-f$ transitions.?

The Coulomb approximation is restricted to transitions between levels having the same parent term. It gives the best results if the degree of cancellation in the transition integral is small, i.e., if $\sigma^{2}$ is not too close to zero, and if the upper and lower levels of the excited electron are in a shell which contains no other electrons. This is true for the moderately and highly excited levels. But even if the lower level is in a shell which contains other electrons, the results often agree fairly well with those obtained by other methods.

On the whole, the Coulomb approximation has given impressive results and has proved to be of great value. In most cases where comparisons are available-there are several hundred of them for the first ten elements-the results agree within 20-40 percent with those from advanced theoretical and experimental methods. We have therefore made extensive use of this approximation ${ }^{3}$ to smpplement

\footnotetext{
${ }^{2}$ The custumary spectrosecopice notations are neced thremghoue

We have heen fortunate in wheaining a computer program for the calculation of Bates. hangaded

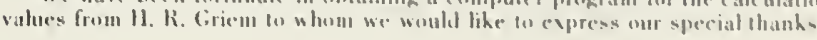


the available material. However, we have restricted ourselves to the medium-strong or stronger lines (as judged from the intensity data supplied in the multiplet tables) for which experimental wavelength and energy level data are available and for which the lower state is significantly above the ground state. If the need for $f$-values of other higher excited lines should arise, we strongly recommend the application of the Coulomb approximation.

On the basis of many comparisons, the uncertainties of the Bates-Damgaard values have been estimated as follows: For transitions between excited states in the spectra of neutral helium, lithium, beryllium, boron, and their isoelectronic sequences they do not exceed 25 percent and in favorable situations may be as low as 10 percent. For the more complex atoms among the first ten elements, namely carbon, nitrogen, oxygen, fluorine, and their equivalent ions, we have estimated the uncertainties to be within 50 percent for the moderately excited transitions including $3 s-3 p$ and within 25 percent for the medium and highly excited lines, i.e., transitions of the types $3 p-3 d, 3 d-4 f$, etc. A few of the tabulated values may be much more uncertain than the stated error limit because of cancellation in the transition integral which we did not check in each case.

It is worth noting that in many instances the results of the Coulomb approximation appear to be as good as those from other, more elaborate theoretical treatments, such as the self-consistent field approximation with exchange. This is primarily indicated from comparisons with the most advanced theoretical and experimental methods.

\section{b. Calculations Based on the Self-Consistent Field (SCF)} Approximation

This method has found, in varying degrees of refinement, widespread use for the calculation of $f$-values. It provides a set of wave functions for the atomic electrons which produce an approximately self-consistent electric field. The transition probabilities are then determined by integration over the radial parts of these wave functions. A short outline of the procedure, developed by Hartree, and extensively described by him [20], is given below:

It is assumed that the charge density distribution of the atomic sysiem is spherically symmetric, i.e., the potentials of the electrons depend only on their radial positions. Correlations between the electrons are at first neglected; all are supposed to move independently in the central field, experiencing only the averaged charge distribution of the other electrons and the nucleus. With these simplifications the motions of the individual electrons can be calculated by assuming trial wave functions for the others, and from the resulting wave functions the charge density distribution is computed and compared with the initial one obtained with the trial functions. If self-consistency is not achieved, the new, computed wave functions are used as trial functions, and the procedure is repeated until initial and final charge distributions are identical, i.e., the field is self-consistent.

This basic procedure was improved by Fock [21], who included exchange effects between the electrons, by Trefftz et al. [22], and Biermann and Lübeck [23] who in the special cases of $\mathrm{He} I$ and $\mathrm{C} I \mathrm{I}$ took into account other correlations between the electrons. More recently, large-scale computations were made possible after the introduction of elaborate computer programs by Roothaan and co-workers [24].

An assessment of the errors resulting from the various approximations in the calculations has not been feasible. But a number of comparisons with experimental results and with more accurate variational calculations, as well as con- sistency checks made by applying the dipole length and dipole velocity representations of the matrix element have shown that for simple atomic systems accurate transition probabilities with uncertainties smaller than 10 percent may be obtained when a refined procedure including exchange effects is applied. This is particularly true for He I and $\mathrm{Li} I$ and their isoelectronic sequences, for which the extensive calculations by Weiss [25], and Trefftz et al. [22] are available. The large-scale computations by Kelly [26] for lines of nitrogen and oxygen contain the exchange effects only in an approximate way (the exchange term is replaced by an averaged potential) and errors of about 20 percent must be expected for most of the moderately excited transitions as judged from many comparisons. In a few cases, the positive and negative contributions to the transition integral are almost equal to each other (the ratios are listed by Kelly); i.e., cancellations are encountered and a much lower accuracy must be expected. In these cases we have given preference to available experimental results.

For the breakdown of the transition integrals into multiplet and line values we have used the $L S$-coupling strengths [19] unless special results have been available, as for example for O II. We have generally avoided using SCF calculations if they were done without considering exchange effects, but have had to make an exception for some important lines of B I because no other comparable material is available.

Large uncertainties in the SCF calculations as well as other theoretical treatments are expected for transitions where configuration interaction becomes important. For the first ten elements these transitions are of the type $1 s^{2} 2 s^{m} 2 p^{n} \rightarrow 1 s^{2} 2 s^{m-1} 2 p^{n+1}$. Only a few attempts have yet been made to take configuration interaction into account. Very recently, Weiss [27] has undertaken limited calculations for some CI and Be I lines and Yutsis, Bolotin and co-workers have for some time employed a "many configuration approximation," [28] as they call it. The Russian group has greatly simplified its approach by including only one interacting term for the lower state, which is always the ground state ("double configuration approximation") and none for the upper state. In addition to this simplification, relatively crude wave functions have been employed, namely analytical one-electron wave functions or SCF functions without exchange. Unfortunately, practically all these transitions are in the far ultraviolet; only two experimental investigations by Boldt [29] and Labuhn [30], both done with a wall-stabilized arc, are available for a detailed comparison. From the experimental results one must judge that the success of the many-configuration method in its present form is only fair. Errors of factors of two or more must be expected. This seems to be also the case for Weiss' somewhat more elaborate treatment of configuration interaction (up to three interacting terms for the lower state). Thus, the transition probabilities for the $1 s^{2} 2 s^{m} 2 p^{n} \rightarrow 1 s^{2} 2 s^{m-1} 2 p^{n+1}$ transitions are among the least well known for the lightest ten elements, and further improvements for these lines are urgently needed.

\section{c. Quantum Mechanical Calculations of Forbidden Tran- sitions}

We have considered as forbidden lines all magnetic dipole and electric quadrupole lines. The extensive calculations by Garstang [31, 32, 33] and Naqvi [34], and-to a lesser extent - the papers by Seaton and Osterbrock [35], Yamanouchi and Horie [36], and Ufford and Gilmour [37], have been principal sources. All these calculations have as a common starting point the general expressions for the line 
strengths of forbidden lines in the $p^{2}, p^{3}$, and $p^{4}$ configurations, which were given algebraically and tabulated by Short. ley et al. [38], and later extended by Naqvi to the few transitions of the $s p, p$, and $p^{5}$ configurations.

The principal differences between the various calculations are the approaches chosen to determine the most important parameters:

(a) The "spin-orbit," and "spin-spin and spin-other-orbit" integrals, usually designated by $\zeta$ and $\eta$, have been determined either empirically or by using available wave functions. Garstang has compared the empirical and theoretical values for some ions - the latter obtained from SCF functions with exchange - and has found differences of up to 20 percent for $\zeta$ and up to 30 percent for $\eta$. When a choice is available, we have given preference to the empirical values.

(b) The term intervals. Here one has the choice between using exclusively experimental energy values or combining some of these with the results of the Slater theory [39] for inter-multiplet separations, that is, by employing the Slater parameters $F_{2}$. Differences between the two approaches arise mainly due to the effects of configuration interaction. These are neglected in all calculations and may cause deviations up to a factor of two. A study by Garstang [40] in 1956 led to the result that the exclusive use of observational material partially includes, at least in simple cases, the effects of configuration interaction, when the latter is otherwise not taken into account. Thus the work based on experimental term intervals has been adopted whenever available.

Naqvi used in his calculations essentially the second of the above-mentioned approaches. He compared empirically determined Slater parameters $F_{2}$ for the various term intervals with theoretically derived values, and selected the one experimental parameter which was in best agreement with theory. Then he employed this particular $F_{2}$ and the Slater theory for the determination of all other term intervals. In view of the above mentioned study by Garstang we have used from Naqvi's work only the transition probabilities based entirely on this initial parameter, i.e., based exclusively on observational material. Consequently, his data for the $p^{3}$ configuration have not been applied, with the exception of the ion F III, since in this case his work was the only available source. On the other hand, Naqvi's calculations for the simpler $s p$-configuration are all based on the empirical value for the one term interval there and should, therefore, take the effects of configuration interaction partially in to account.

(c) Transformation coefficients. The atoms and ions under consideration are most closely represented by the intermediate coupling scheme, but for the calculations of transition probabilities the actual wave functions are more conveniently expressed in terms of $L S$-coupling wave functions. The transformation coefficients were first derived by Shortley et al. [38] and were later refined by several others, in particular by Naqvi [34]. Thus, Naqvi's results have been adopted whenever the choice of the transformation coefficients became important and when he accounted for the effects of configuration interaction in the abovementioned manner. It is especially worth noting that by including the effects of spin-spin and spin-other-orbit interactions on the transformation coefficients of the $p^{4}$ configuration some results are improved by about 10 percent.

(d) The integral $s_{q}$ for electric quadrupole transitions. This depends principally on the quality of the employed wave functions. We have preferred calculations with SCF wave functions over those with hydrogenic functions or screening constants and, among SCF calculations, we have preferred those with exchange effects included over those without exchange. The improvement with SCF wave funetions is estimated to be of the order of 20 percent. In general, the electric quadrupole transitions are not as accurate as the magnetic dipole values for transitions of the same general type because of the additional uncertainty in the determination of $s_{q}$. This uncertainty should generally be in the neighborhood of 20 percent.

A good assessment of the uncertainties in the calculated values for forbidden lines is possible due to the fortunate circumstance that some forbidden lines of $\mathrm{O}_{\mathrm{I}}$ have been determined experimentally. These lines appear strongly in the aurora, which has been utilized as a "light source". The transition probabilities could be accurately determined via a measurement of the lifetimes of the upper atomic states. Extensive auroral observations by Omholt [41] gave for the ${ }^{1} \mathrm{D}-{ }^{1} \mathrm{~S}$ transition a transition probability of $1.43 \mathrm{sec}^{-1}$, while the best calculated value is $1.25 \mathrm{sec}^{-1}$. For another case, namely the lifetime of the ${ }^{1} \mathrm{D}$ state, the averaged experimental result is approximately $160 \mathrm{sec}$, while the theory gives $135 \mathrm{sec}$.

The theoretical transition probabilities involved in this comparison depend sensitively on the choice of some parameters, particularly $s_{q}$ and $\zeta$. The good agreement with the observations indicates that uncertainties no greater than 25 to 50 percent have to be generally expected.

For a number of magnetic dipole transitions, the uncertainties should be even smaller, since the results are almost independent of the choice of the parameters. In the $p^{2}$ and $p^{4}$ configurations these are the transitions ${ }^{3} \mathrm{P}_{2}-{ }^{3} \mathrm{P}_{1}$ and ${ }^{3} \mathrm{P}_{1}-{ }^{3} \mathrm{P}_{0}$, which have, near $L S$-coupling, the strengths of 2 and 2.5 respectively. In the $p^{3}$ configuration one encounters the transitions ${ }^{2} \mathrm{P}_{3 / 2}^{\circ}-{ }^{2} \mathrm{P}_{1 / 2}^{\circ}$ with a strength of 1.33 and the transition ${ }^{2} \mathrm{D}_{5 / 2}^{\circ}-{ }^{2} \mathrm{D}_{3 / 2}^{\circ}$ with a strength of 2.4 , again near $L S$. coupling. For all these lines the effects of configuration interaction and deviations from $L S$-coupling do not enter sensitively into the results. Thus, these transition probabilities should be considered accurate to within 10 percent, while all other magnetic dipole lines are uncertain within about 25 percent.

Analogously, the transition probabilities for a number of electric quadrupole lines depend essentially only on the quadrupole integral $s_{q}$. These are the transitions ${ }^{1} \mathrm{~S}_{0}-{ }^{1} \mathrm{D}_{2}$, ${ }^{3} \mathrm{P}_{2}-{ }^{3} \mathrm{P}_{1}$, and ${ }^{3} \mathrm{P}_{2}-{ }^{3} \mathrm{P}_{0}$ for the $p^{2}$ and $p^{4}$ configurations and ${ }^{2} \mathrm{D}_{5 / 2}^{\circ}-{ }^{2} \mathrm{P}_{3 / 2}^{\circ},{ }^{2} \mathrm{D}_{3 / 2}^{\circ}-{ }^{2} \mathrm{P}_{3 / 2}^{\circ},{ }^{2} \mathrm{D}_{5 / 2}^{\circ}-{ }^{2} \mathrm{P}_{1 / 2}^{\circ}$, and ${ }^{2} \mathrm{D}_{3 / 2}^{\circ}-{ }^{2} \mathrm{P}_{1 / 2}^{\circ}$ for the $p^{3}$ configuration. Within a given spectrum these should be the best available quadrupole lines and they have been estimated to be accurate within 25 percent, while the rest of the quadrupole transitions should be accurate within 50 percent. On the whole, electric quadrupole lines have been rated to be of lower accuracy than magnetic dipole lines, since the uncertainties in the quadrupole integral must be added to the other uncertainties already present for the magnetic dipole lines.

Further details on the calculations of forbidden line strengths may be found in the recent review article by Garstang [42].

\section{METHOD OF EVALUATION}

We shall now discuss the general steps in the evaluation of the data: The literature, as taken from the files of our data center, has first been screened for outdated and superseded material. The remaining articles have then been individually studied and the results collected in comparison 
tables. Additional values have been computed by employing the Coulomb approximation by Bates and Damgaard [2] whenever this has been considered necessary and useful. When large discrepancies or odd values have appeared in the comparison tables, we have searched for likely sources of numerical errors, and have also communicated in many instances with the respective authors.

The evaluation and final selection of the sets of best values depends so much on the particular material available for each ion that the main justifications for the selections has to be delegated to the individual introductions. Only a few general rules on the selection may be given now:

Thus, self-consistent field calculations with exchange effects have been regarded as superseding those not including these effects ${ }^{4}$; the Coulomb approximation is generally not employed when the transition is very far from being hydrogen-like, e.g., when the lower state is the ground state or when it contains two or more electrons of the same principal quantum number; experiments employing photoelectric techniques are preferred over similar experiments utilizing photographic detection; measurements with wallstabilized arc sources are considered superseding analogous measurements with fluid- or gas-stabilized arc sources because of the stability problems of the latter. For forbidden lines, the calculations based on empirical term intervals are preferred to those based on the Slater theory for intermultiplet separations, since a theoretical study [40] shows that the effects of configuration interaction, which are often important, are at least partially taken into account by the first approach.

When several methods of comparable quality are available, the results are averaged to obtain the best value. If one method appears clearly better than the others, only those results are employed.

The final step in the evaluation is the estimate of the uncertainties. At the present status of our knowledge, we find it impossible to assign specific numerical error limits to each transition. Instead we have introduced a classification scheme with several classes of accuracy, and assigned each transition probability to a certain class. We have used the following arbitrary notation:

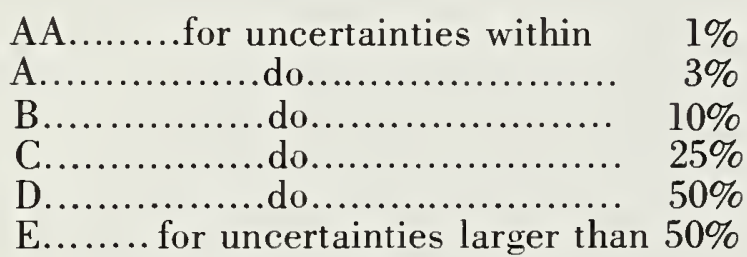

The word uncertainty is being used in the meaning "extent of possible error" or "possible deviation from the true value". We are aware that this is far from being a precise definition of error, but, considering the multitude of approaches to the error discussions in the various papers (or the lack of them), it seems impossible to find a better common denominator. Uncertainties of class " $A A$ ", i.e., values that are essentially exact, are found only in hydrogen and a few transitions of helium. Going to the other extreme, we have included class " $E$ " data, i.e., very uncertain values, only in those special cases, when for the most important and most characteristic lines of a spectrum no better data are available, so that otherwise these lines would

${ }^{4}$ Fortunately, most of the selected self-consistent field calculations include exchange effects in varying degrees of refinement. have to be omitted. Occasionally, we have made a further differentiation in the classification scheme by assigning plus or minus signs to some transitions. This serves to indicate that these lines are significantly better or worse than the average values, but do no quite belong into the next higher or lower class. They should be therefore the first or last choice among similar lines.

Since the theoretical treatments essentially do not permit error estimates per se, these have to be obtained from comparisons with experimental and other theoretical determinations or from general consistency checks, such as applications of $f$-sum rules, etc. A few rather audacious extrapolations have had to be undertaken, when no reliable comparison material was available. On the other hand, the errors given by the experimentalists are sometimes only indications of their precision, and no allowance is made for systematic errors. Therefore, we have generally been more conservative with our error estimates, and hope that we have arrived at a realistic and consistent error presentation.

\section{E. ARRANGEMENT AND EXPLANATION OF COLUMNS}

We have adopted the present arrangement of the tables after consulting with a number of physicists working in three fields from which -it is anticipated-most of the users of this compilation will come, i.e., spectroscopy, astrophysics, and plasma physics.

We feel that of the multitude of units in which transition probabilities are expressed, the adopted combination of the transition probability for spontaneous emission $A_{k i}\left(\right.$ in $\sec ^{-1}$ ), the absorption oscillator strength $f_{i k}$ (dimensionless), the $\log g f$ (a further discussion of the statistical weight $g$ is given in Appendix B) and the line strength $S$ (in atomic units) gives a very adequate representation. The other units are either not commonly used or, in case of $g f$ and $g A$, may be obtained by simply multiplying two columns of the table. The units that are only occasionally used are:

1. The transition probability of absorption $B_{i k}(i=$ lower, $k=$ upper state) which is related to $A_{h i}$ by

$$
B_{i k}=6.01 \lambda^{3} \frac{g_{i}}{g_{\imath}} A_{k i}
$$

( $\lambda$ is the wavelength in Angstrom units, and $g_{i}, g_{h}$ are the statistical weights, further discussed in Appendix B)

2. The transition probability of induced emission $B_{k i}$, which is related to $A_{k i}$ by

$$
B_{k i}=6.01 \lambda^{3} A_{k i}
$$

3. The emission oscillator strength $f_{k i}$, which is related to the absorption oscillator strength $f_{i k}$ by

$$
f_{k i}=-\frac{g_{i}}{g_{k}} f_{i k}
$$

In addition, some authors have introduced still other quantities, but these have not found general acceptance and will not be considered further.

The conversion factors between the tabulated quantities $A_{k i}, f_{i k}$, and $S$ are listed in table III, as reproduced from reference [1]. (For the case of hydrogen, we have employed the reduced mass and other appropriate constants in the conversion factors.) 
The general arrangement of the tables according to increasing atomic number and stage of ionization needs no further comments. The material for the individual ions is further subdivided into sections for allowed (electric dipole) and forbidden transitions. As forbidden transitions we have considered all magnetic dipole and electric quadrupole lines. Intercombination lines, although they are forbidden in the case of pure $L S$-coupling, are listed under allowed transitions, since they are electric dipole transitions.

The tabulations for each ion start out with listings of the ground state configuration and the ionization potential, both taken from ref. [43]. In all cases, where we have tabulated more than 20 allowed lines per ion, we have then assembled a "finding list," i.e., we have arranged the lines in order of increasing wavelength and indicated their position in the main tables - which are arranged according to spectroscopic notation - by listing their running numbers. The latter are given in front of the spectroscopic notation in the tables. These finding lists should, for many applications, permit one to find out quickly which lines are covered in the present tabulation.

Each table is then preceded by a short introduction containing in brief the major reasons which have led to the selection of the presented data and their classification in terms of accuracy. This is followed by a reference list of the selected articles.

It remains to discuss the columns of the main tables: The first part of the tables contains data connected with the identification of lines, i.e., spectroscopic notation, wavelength, and energy levels. All these data have been taken from the compilations by Mrs. Moore-Sitterly, i.e., the "Revised Multiplet Table" [3], the "Ultraviolet Multiplet Tables" [4], and "Atomic Energy Levels," Vol. I, and addenda in Vols. II and III, recent reports of the Triple Commission for Spectroscopy [44], and from newer material generously furnished by her. All designations, as usual, are written in terms of the absorption process, i.e., the lower (initial) state first. For the classification of the lines we have employed the standard spectroscopic notation for $L S$. coupling, with the exception of $\mathrm{Ne} I$, where we have used the $j l$-coupling notation, all in accordance with the above mentioned "Atomic Energy Levels" tables. In listing the transition arrays, we have presented only the electrons in the unfilled shells. Furthermore, to distinguish between the different supermultiplets, we have inserted the parent terms in the notation. If they are given only once, as is generally the case, then no change occurs from lower to upper state.

For all spectra with pronounced multiplet structure, i.e., for Be I through $\mathrm{Ne} I$ and their isoelectronic sequences, we have arranged the lines according to a configurational order: They are grouped to multiplets, supermultiplets, transition arrays, and increasing quantum numbers. Within the transition arrays, the multiplets are in order of increasing lower energy levels. Individual lines within the multiplets are listed whenever the total wavelength spread amounts to more than 0.01 percent. This arrangement is convenient for the application of $f$-sum rules ${ }^{5}$ and to the similar one used in the "Revised Multiplet Table" [3] and "Ultraviolet Multiplet Tables" [4]. At first we attempted to copy and extend these older arrangements, but this would have meant that many new multiplets had to be inserted. Therefore, we abandoned this plan. We have, however, made reference to

"It should he mentioned, that for allowed transitions the strengths $S$ of lines in a multiplet add up to the total multiplet strength (see also appendix B), and that for forbidden transitions the total
transition probability is obtained by adding the clectric quadrupole and magnetic dipole probalilities. the iwo multiplet lists by including the multiplet numbers in the present tabulation. The numbers are given in parentheses under the multiplet designation. The letters " $u v "$ are added if the numbers are from the "Ultraviolet Multiplet Tables."

For the He I isoelectronic sequence we have changed the arrangement slightly by listing the singlets and triplets separately.

For hydrogen we have made several changes in the arrangement to adapt the tables to the very divergent applications in theory and experiment: The tables are split into four separate parts: In table A we list the "average" transition probabilities, etc., for the transitions between lower state of principal quantum number $n_{i}$ and upper state $n_{k}$.

These are defined by the following relations:

$$
\begin{aligned}
& A_{n_{k}, n_{i}}^{*}=\sum_{l_{k}, l_{i}} \frac{2 l_{k}+1}{n_{k}^{2}} A_{(n l)_{k},(n l)_{i}} \\
& f_{n_{i}, n_{k}}^{*}=\sum_{l_{k}, l_{i}} \frac{2 l_{i}+1}{n_{i}^{2}} f_{(n l)_{i},(n l)_{k}} \\
& S_{n_{i}, n_{k}}^{*}=\sum_{l_{k}, l_{i}} S_{(n l)_{i},(n l)_{k} .}
\end{aligned}
$$

These "average" values are applicable to most problems in plasma spectroscopy and astrophysics. This is due to the circumstance that states with the same principal $(n)$, but different orbital ( $l$ ) quantum numbers fall practically together ("degeneracy"), so that only a single line is observed for all the possible combinations between states of different principal quantum numbers. The only assumption entering into the application of "average" values is that the atomic substates must be occupied according to their statistical weights [45]. The above assumption is fulfilled for any reasonably dense plasma, where the excited atoms undergo many collisions during their lifetimes.

In table $B$ the probabilities for transitions between the various sublevels $(n l)_{i}-(n l)_{k}$ are listed. This table should be useful primarily for theoretical applications. Tables $\mathrm{C}$ and $\mathrm{D}$, finally, contain the most important fine structure $\left[(n l j)_{i}-(n l j)_{k}\right]$ and hyperfine structure lines $\left[(n l j f)_{i}-\left(n l j f_{k}\right]\right.$ of hydrogen $(j=$ inner or total electronic angular momentum quantum number; $f=$ total atomic angular momentum quantum number). For these two special tables we have made a further change by presenting frequencies and energy differences rather than the usual wavelengths and energy levels.

In all other tables, the energy levels are given (in units of $\mathrm{cm}^{-1}$ ) relative to the ground state with $E_{0}=0$. We have limited the numerical values to six digits which should be more than sufficient for all applications. The same limitation was imposed on the wavelengths.

In a number of cases we have had to calculate wavelengths from energy level differences. These are given in brackets to distinguish them from the presumably more accurate observed material. However, hydrogen is an obvious exception. Also given in brackets are all energy levels which are not derived from the analysis of spectra, but are extrapolated or obtained from approximate wave function calculations. We have included in this category those energy levels derived from observational material that are shifted by an unknown amount indicated by an 
"x" or " $y$ " in ref. [43]. Those calculated or extrapolated values which we expect to be quite uncertain have been indicated, in addition to the brackets, by a question mark.

The averaged energy levels for a multiplet have been obtained by weighting the component levels according to their statistical weights, and the multiplet wavelength is calculated from the averaged energy levels, taking the refraction of air into account for wavelengths longer than 2000 $\AA$ [42]. These averaged values are indicated by italics.

The statistical weights $g$ have been included in this tabulation because of their importance in applications involving line intensities. They have been obtained from the inner quantum numbers $J$ listed in the "Atomic Energy Levels" by applying the relations given in Appendix B.

The second part of the table contains the data proper. The numerical values contain as many digits as are consistent with the estimated accuracy of the data. ${ }^{6}$

The numbers in the source column refer to the references listed after the individual introductions. If two or more references are listed, we have given each source equal weight in arriving at the averaged value. If the data for all lines of a multiplet are given, then these are either obtained from the relationships listed in Appendix B and from $L S$ coupling tables [17-19], which is indicated in the source column by " $l s "$, or they are obtained directly from the literature. In the latter case they are sometimes marked " $n$ ", if they are normalized to a basis which is different from the one chosen originally by the authors. Similarly, the multiplet values sometimes have been renormalized and have been marked " $n$ ".

For the forbidden lines, a few small changes in the arrangement have been made. First, we have indicated the type of transition, i.e., we have listed an " $m$ " for magnetic dipole and " $e$ " for electric quadrupole lines. Furthermore, the $\log g f$ and $f_{i k}$ columns are omitted, since these units are not used for forbidden lines. (The line strength $S$, which now has different atomic units (see table III), is also used infrequently). It should be noted that the total transition probabilities of forbidden lines are obtained by adding the magnetic dipole and electric quadrupole values.

We finally would like to mention that we have assembled and explained in Appendix A all abbreviations appearing in the tabulations.

\section{F. FUTURE PLANS AND ACKNOWLEDGEMENTS}

It is our plan to extend this critical compilation to all other elements and make revisions whenever necessary. The present tables should therefore be regarded as the first volume of a larger work spanning all elements. However, realization of this large project in a systematic fashion, say, in order of increasing atomic number, does not appear feasible at the present time, since there are relatively few reliable data available for many heavier elements, and essentially none for higher stages of ionization. As an interim solution, we probably shall attempt to assemble, in an irregular fashion, tables of best values for the spectra of those heavier elements and ions for which extensive and worthwhile data are presently at hand. These will be primarily the heavier noble gases, some of the wellknown metals, the alkalis, and the alkaline earths. Also, the third period of the periodic table appears to be promising.

\footnotetext{
${ }^{6}$ During the computations and conversions all digits were retained and finally rounded off. Thus
}

Finally, it is our wish and hope that this compilation may stimulate further work on the lightest ten elements, since many gaps and unreliable data are evident on closer inspection of the tables. The two areas that merit the highest attention are the higher ions and those lower excited transitions that are subject to the effects of configuration interaction.

It is our pleasure to acknowledge the help and collaboration of many workers in this field. In particular, we would like to thank P. S. Kelly and B. H. Armstrong for sending us preprints of their extensive SCF-calculations on nitrogen and oxygen, H. R. Griem for supplying us with a computer program to calculate Bates-Damgaard values; and R. H. Garstang and A. M. Naqvi for extensive discussions and some re-calculations of transition probabilities for forbidden lines.

We also express our sincere gratitude to the students who have worked during the past summers on the prelimiary aspects of this compilation: These are Mary DesJardins, Maureen Zagronic, Berry Cobb, Don Hall, and Paul Voigt.

We would finally like to thank several of our collegues at NBS; especially A.W. Weiss for many useful discussions and for carrying out many SCF-calculations when serious gaps in the data showed up; Mrs. C. E. Moore-Sitterly for generously furnishing us with new material on energy levels and wavelengths; and J. Z. Klose for undertaking a lifetime study for important excited states of $\mathrm{Ne}$ I.

It is also a pleasure to acknowledge the competent help of Miss Jean Bates and Mrs. Marilyn Duffany in typing and proofreading the manuscript.

\section{References}

[1] Glennon, B. M. and Wiese, W. L., National Bureau of Standards Monograph 50 (1962) (U.S. Government Printing Office, Washington, D.C.).

[2] Bates, D. R. and Damgaard, A., Phil. Trans. Roy. Soc. London A242, $101(1949)$

[3] Moore, C. E., "A Multiplet Table of Astrophysical Interest, Revised Edition," National Bureau of Standards Tech. Note 36 (1959) (U.S. Government Printing Office, Washington, D.C.).

[4] Moore, C. E., "An Ultraviolet Multiplet Table," National Bureau of Standards .Circular 488, Sec. 1 (1950) (U.S. Government Printing Office, Washington, D.C.)

[5] Wiese, W. L. in "Proceedings Xth Colloq. Spectrosc. Internat.," p. 37, (Spartan Books, Washington, D.C., 1963).

[6] Foster, E. W., Repts. Prog. in Physics, XXVII, 469 (1964).

[7] Berg, H. F., Eckerle, K. L. Burris, R. W., and Wiese, W. L.. Astrophys. J. 139, 751 (1964).

[8] Richter, J., Z. Astrophysik 53, 262 (1961).

[9] Frie, W, and Maecker, H., Z. Physik 162, 69.(1961)

[10] Wiese, W. L., Chapter VI in "Plasma Diagnostics," (edited by Huddlestone, R. H. and Leonard, S. L., Academic Press, New York, 1965).

[11] Griem, H. R., Phys. Rev. 128, 997 (1962).

[12] Shumaker, Jr., J. B., Rev. Sci. Instr. 32, 65 (1961).

[13] Maecker, H., Z. Naturforsch. 1 1, 457 (1956).

[14] Bennett, Jr., W. R., Kindlmann, P. J., and Mercer, G. N., Applied Optics, Supplement 2 of Chemical Lasers, 34 (1965).

[15] Rozhdestvenskii, D., Ann. Physik 39, 307 (1912).

[16] Penkin, N. P., J. Quant. Spectrosc. Radiat. Transfer 4, 41 (1964).

[17] Goldberg, L., Astrophys J. 82, I (1935) and 84, 11 (1936).

[18] White, H. E and Eliason, A. Y., Phys. Rev, 44, 753 (1933).

[19] Allen, C. W., "Astrophysical Quantities," 2d Ed. (The Athlone Press, London, 1963).

[20] Hartree, D. R.. "The Calculation of Atomic Structures," (John Wiley and Sons, New York, 1956).

[21] Fock, V., Z. Physik 61, 126 (1930).

[22] Trefftz, E., Schlüter, A., Dettmar, K. H., and Jörgens, K., Z. Astrophysik 44, 1 (1957).

[23] Biermann, L. and Lübeck. K., Z. Astrophysik 25, 325 (1948).

[24] Roothaan, C. C. J. and Bagus. P. S., in "Methods in Computational Physics". Vol. II p. 47 (Academic Press, New York. 1963).

[25] Weiss, A. W.. Astrophys. J. 138, 1262 (1963). 
26] Kelly, P. J Ouant. Spectrose. Radiat. Transfer 4, $117(1964)$.

[27] Weiss, $\Lambda$. W., private communication (1964).

[28] Yutsis, A. P., Zhur. Eksptl. i Troret. Fiz. 23, 129 (1952)

20) Boldt G, Z Naturforseh. $18 \mathrm{~A}, 1107$ (1963).

[30] Labuhn, F., to be pulslished in \%. Naturforsclı.

[31) Garstang, R. H., Monthly Notices Roy. Astron. Soc. 11 1, 115 (19.51).

32) Garstang, R. H., Astrophys. J. 115,500 (1952).

[33] Garstang, R. H., Monthly Notices Roy. Astron. Soc. 120, 201 (1960).

[34] Na(pvi, A. M., Thesis Harvard (1951).

[35] Seaton, M. J. and Osterbrock, D. E., Astrophys. J. 125, 66 (1957).

[36] Yamanouchi, T. and Horie, H., J. Phys. Soc. Jayan 7, 52 (1952).

[37] Ufford, C. W. and Gilmour, R. M., Astrophys. J. 11 1, 580 (1950).

[38] Shortley, G. H., Aller, L. H., Baker, J. G., and Menzel, D. H., Astro. phys. J. 93, 178 (1941).

[39] Slater, J. C., Phys. Rev. 34, 1293 (1929).
[40] Garstang, R. H. Proc: Cambridere P'hil Soc: 52, 107 (]9.56).

[41] Omboht, A., Geofys. Publikasjoner Norske Videnskaps. - Akad. Oclo, $21,1(1959)$.

[42] Garstang, R. H., Chapter I in "Alomie and Molecular Procesces", (codited by Bates, D. R., Acarlemic: J'ress, Now York, 1962).

[43] Moore, C. E., "Atomic Energy Levels", National Burreau of Standards Circular 467; Vol. I (1949); Vol. II (1952); Vol. III (1958) (U.S. Grov. ernment Printing Office, Washington, f).C.).

144] Reports of the Triple Commission for Spectroseopy, J. Opt. Soe. Am. $\mathbf{5 2}, 476(1962) ; \mathbf{5 2}, 583$ (1962); 53, 885 (1963).

[45] Bethe, H. A. and Salpeter, E. E. "Quantum Mechanirs of One-and Two-Electron Atoms", (Academic P'ress, New York, 1\%57).

[46] Coleinan, C. D., Bozman, W. R., and Meggers, W. F., National Bureau of Standards Monograph 3 (1960) (U.S. Government Printing Office, Washington, D.C.).

Table 1.

Relationships between the quantities for hydrogen, indicated by $\mathrm{H}$, and liydrogen-like ions of eharge $Z$ (the other symbols are explained in Sec. E).

$$
f_{Z}=f_{\mathrm{H}} \quad S_{\mathrm{Z}}=Z^{-2} S_{\mathrm{H}} \quad A_{\mathrm{Z}}=Z^{4} A_{\mathrm{H}}
$$

Table II.

Reference list of additional material which is considered to be quite reliable but not covered in the table because of its very limited use (the quantum numbers are given in their customary notation).

\begin{tabular}{|c|c|c|}
\hline Spectrum & Transitions & Author \\
\hline H I & $\begin{array}{l}2 \rightarrow n \text { for } 41 \leqslant n \leqslant 60 \\
n_{i} l_{i} \rightarrow n_{k} l_{k} \text { for } \\
5 \leqslant n_{i} \leqslant 20,6 \leqslant n_{k} \leqslant 20 \\
\text { and all possible } l \text {-values } \\
2 l \rightarrow n l \text { for } 2 l \leqslant n \leqslant 60\end{array}$ & $\begin{array}{l}\text { Green, L. C., Rush, P. P., and Chandler, } \\
\text { C. D., Astrophys. J. Suppl. Ser. 3, } 37 \\
\text { (1957). }\end{array}$ \\
\hline $\mathrm{H}_{\mathbf{I}}$ & $\begin{array}{l}l s \rightarrow n p \text { for } 7 \leqslant n \leqslant 50 \\
2 l \rightarrow n l \text { for } 7 \leqslant n \leqslant 50 \\
\text { and all possible } l \text {-values } \\
3 l \rightarrow n l \text { for } 7 \leqslant n \leqslant 50 \\
\text { and all possible } l \text {-values } \\
4 l \rightarrow n l \text { for } 7 \leqslant n \leqslant 50 \\
\text { and all possible } l \text {-values }\end{array}$ & $\begin{array}{l}\text { Harriman, J. M., Phys. Rev. 101, } 594 \text { (1956) } \\
\text { and Document No. 4705, American Docu- } \\
\text { mentation Institute Auxiliary Publica- } \\
\text { tions Project, Photoduplication Service, } \\
\text { Library of Congress, Washington, D. C. }\end{array}$ \\
\hline $\mathrm{N}_{\mathbf{I}}$ & $\begin{array}{l}s-p, p-d, \text { and } d-f \text { for } \\
2 \leqslant n \leqslant 11 ; \text { with } 1 s^{2} 2 s^{2} 2 p^{2} \\
\text { core } \\
s^{-} p, p-d, \text { and } d-f \text { for } \\
2 \leqslant n \leqslant 8 ; \text { with } 1 s^{2} 2 s 2 p^{3} \\
\text { and } 1 s^{2} 2 p^{4} \text { cores }\end{array}$ & $\begin{array}{l}\text { Kelly, P. S., J. Quant. Spectrosc. Radiat. } \\
\text { Transfer 4, 117-148 (1964). }\end{array}$ \\
\hline N II, O III & $\begin{array}{l}s-p, p-d, \text { and } d-f \text { for } \\
2 \leqslant n \leqslant 8 ; \text { witl } 1 s^{2} 2 s^{2} 2 p \\
\text { core }\end{array}$ & $\begin{array}{l}\text { Kelly, P. S., J. Quant. Spectrosc. Radiat. } \\
\text { Transfer 4, 117-148 (1964). }\end{array}$ \\
\hline $\mathrm{N}$ III, O IV & $\begin{array}{l}s-p, p-d, \text { and } d-f \text { for } \\
2 \leqslant n \leqslant 8 ; \text { with } 1 s^{2} 2 s^{2} \text { core }\end{array}$ & $\begin{array}{l}\text { Kelly, P. S., J. Quant. Spectrosc. Radiat. } \\
\text { Transfer 4, 117-148 (1964). }\end{array}$ \\
\hline $\mathrm{N}$ IV, $\mathrm{O} v$ & $\begin{array}{l}s^{-} p, p-d, \text { and } d-f \text { for } \\
2 \leqslant n \leqslant 8 ; \text { with } 1 s^{2} 2 s \text { core }\end{array}$ & $\begin{array}{l}\text { Kelly, P. S., J. Quant. Spectrosc. Radiat. } \\
\text { Transfer 4, 1 17-148 (1964). }\end{array}$ \\
\hline $\mathrm{N} v, \mathrm{O} v \mathrm{I}$ & $\begin{array}{l}s-p, p-d, \text { and } d-f \text { for } \\
2 \leqslant n \leqslant 8 ; \text { with } 1 s^{2} \text { core }\end{array}$ & $\begin{array}{l}\text { Kelly, P. S., J. Quant. Spectrosc. Radiat. } \\
\text { Transfer 4, 117-148 (1961). }\end{array}$ \\
\hline $\mathrm{O}_{\mathrm{I}}$ & $\begin{array}{l}s-p, p-d, \text { and } d-f \text { for } \\
2 \leqslant n \leqslant 8 ; \text { with } 1 s^{2} 2 s^{2} 2 p^{3} \text { core }\end{array}$ & $\begin{array}{l}\text { Kelly, P. S., J. Quant. Spectrosc. Radiat. } \\
\text { Transfer 4, 117-148(1964). }\end{array}$ \\
\hline O II & $\begin{array}{l}s-p, p-d, \text { and } d-f \text { for } \\
2 \leqslant n \leqslant 8 ; \text { with } l s^{2} 2 s^{2} 2 p^{2} \text { core }\end{array}$ & $\begin{array}{l}\text { Kelly, P. S., J. Quant. Spectrosc. Radiat. } \\
\text { Transfer 4, 117-148 (1964). }\end{array}$ \\
\hline
\end{tabular}


Table III.

Conversion factors. The factor in each box converts by multiplication the quantity above it into the one at its left.

\begin{tabular}{|c|c|c|c|}
\hline & $A_{k i}$ & $f_{i k}$ & $S$ \\
\hline \multirow{3}{*}{$A_{k \mathrm{i}}$} & \multirow{3}{*}{1} & \multirow{3}{*}{$\frac{6.670_{2} \times 10^{15}}{\lambda^{2}} \frac{g_{i}}{g_{k}}$} & $\begin{array}{l}E_{d} \\
\frac{2.026_{1} \times 10^{18}}{g_{k} \lambda^{3}}\end{array}$ \\
\hline & & & $\begin{array}{l}E_{q} \\
\frac{1.679_{8} \times 10^{18}}{g_{i} \lambda^{5}}\end{array}$ \\
\hline & & & $\begin{array}{l}M_{d} \\
\frac{2.697_{2} \times 10^{13}}{g_{k} \lambda^{3}}\end{array}$ \\
\hline \multirow{3}{*}{$f_{i k}$} & \multirow{3}{*}{$1.4992 \times 10^{-16} \lambda^{2} \frac{g_{k}}{g_{i}}$} & \multirow{3}{*}{1} & $\begin{array}{l}E_{d} \\
\frac{303.7_{5}}{g_{i} \lambda}\end{array}$ \\
\hline & & & $\begin{array}{l}E_{q} \\
\quad \frac{251.8}{g_{i} \lambda^{3}}\end{array}$ \\
\hline & & & $\begin{array}{l}M_{d} \\
\qquad \frac{4.043_{6} \times 10^{-3}}{g_{i} \lambda}\end{array}$ \\
\hline \multirow{3}{*}{$S$} & $\begin{array}{ll}E_{d} & 4.935_{6} \times 10^{-19} g_{k} \lambda^{3}\end{array}$ & $\begin{array}{cc}E_{d} & 3.292_{1} \times 10^{-3} g_{i} \lambda\end{array}$ & \multirow{3}{*}{1} \\
\hline & $\begin{array}{ll}E_{q} & 5.953 \times 10^{-19} g_{k} \lambda^{5}\end{array}$ & $\begin{array}{ll}E_{q} & 3.971 \times 10^{-3} g_{i} \lambda^{3}\end{array}$ & \\
\hline & $\begin{array}{ll}M_{d} & \\
& 3.707_{6} \times 10^{-14} g_{k} \lambda^{3}\end{array}$ & $247.3_{0} g_{i} \lambda$ & \\
\hline
\end{tabular}

The line strength is given in atomic units, which are: For electric dipole transitions (allowed-denoted by $E_{d}$ ):

$$
a_{0}^{2} e^{2}=6.459_{4} \times 10^{-36} \mathrm{~cm}^{2} \mathrm{esu}^{2}
$$

for electric quadrupole transitions (forbidden-denoted by $E_{q}$ ):

$$
a_{0}^{4} e^{2}=1.808_{8} \times 10^{-52} \mathrm{~cm}^{4} \mathrm{esu}^{2} ;
$$

for magnetic dipole transitions (forbidden-denoted by $M_{d}$ ):

$$
e^{2} h^{2} / 16 \pi^{2} m_{e}^{2} c^{2}=8.599 \times 10^{-41} \text { erg }^{2} \text { gauss }^{-2} .
$$

The transition probability is in units $\sec ^{-1}$, and the $f$-value is dimensionless. The wavelength $\lambda$ is given in Angstrom units, and $g_{i}$ and $g_{k}$ are the statistical weights of the lower and upper state, respectively. 


\section{APPENDIX A}

Key to abbreviations and symbols used in the tables

(A) Symbols for indication of accuracy:
AA ...... uncertainties within
$1 \%$
A...... do
$3 \%$
B...... do
$10 \%$
C...... do
$25 \%$
D...... do
$50 \%$
E. ...... uncertainties larger than
$50 \%$

(B) Abbreviations appearing in the source column of allowed transitions

$\iota s=L S$-coupling

$c a=$ Coulomb approximation

$n=$ normalized to a different scale

(C) Types of forbidden lines

$e=$ electric quadrupole line

$m=$ magnetic dipole line

(Total transition probabilities of forbidden lines are obtained by adding the $e$ - and $m$-values).

(D) Special symbols used in the wavelength and energy level columns.

Number in parentheses under multiplet notation refers to running number of ref. [3] (Revised Multiplet Table)

If letters " $u v$ " are added, reference is made to running number in ref. [4] (Ultraviolet Multiplet Table)

Numbers in italics indicate multiplet values, i.e., weighted averages of line values.

Numbers in square brackets approximate calculated or extrapolated values.

Question marks indicate rather uncertain values.

\section{APPENDIX B}

(A) Statistical Weights:

The statistical weights are related to the inner quantum number $J_{L}$ (in one-electron spectra $j$ ) of a level (initial and final states of a line) by

$$
g_{l}=2 J_{l}+1,
$$

and to the quantum numbers of a term (initial and final states of a multiplet) by

$$
g_{M}=(2 L+1)(2 S+1) .
$$

(The "multiplet" values $g_{M}$ may also be obtained by summing over all possible "line" values $g_{L} . \quad S$ is the resultant spin.) (B) Relations between the strengths of lines and the total multiplet strength:

1. Line strength $S$ :

$$
\begin{gathered}
S(i, k)=\sum_{J_{i}, J_{k}} S\left(J_{i}, J_{k}\right) \\
\text { or } S \text { (Multiplet) }=\sum S(\text { line })
\end{gathered}
$$

( $k$ denotes the upper and $i$ the lower term).

2. Absorption oscillator strength:

$$
f_{i k}^{\text {multiplet }}=\frac{1}{\bar{\lambda}_{i k} \sum_{J_{i}}\left(2 J_{i}+1\right)} \sum_{J_{k}, J_{i}}\left(2 J_{i}+1\right) \times \lambda\left(J_{i}, J_{k}\right) \times f\left(J_{i}, J_{k}\right)
$$

The mean wavelength for the multiplet $\bar{\lambda}_{i k}$ may be obtained from the weighted energy levels. Usually the wavelength differences for the lines within a multiplet are very small, so that the wavelength factors may be neglected.

3. Transition probabilities

$A_{k i}^{\text {multiplet }}=\frac{1}{\left(\bar{\lambda}_{i k}\right)^{3} \sum_{J_{k}}\left(2 J_{k}+1\right)} \sum_{J_{i}, J_{k}}\left(2 J_{k}+1\right) \times \lambda\left(J_{i}, J_{k}\right)^{3} \times A\left(J_{k}, J_{i}\right)$

Relative strengths $S\left(J_{i}, J_{k}\right)$ of the components of a multiplet are listed in refs. [17-19] for the case of $L S$-coupling. 


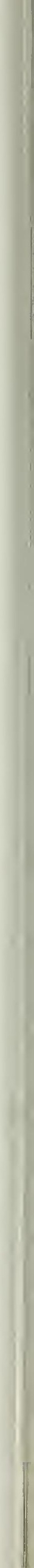




\section{HYDROGEN}

\section{H}

Ground State

Ionization Potential
$1 s{ }^{2} \mathrm{~S}_{1 / 2}$

$13.595 \mathrm{eV}=109678.758 \mathrm{~cm}^{-1}$

\section{Allowed Transitions}

For hydrogen a special tabular arrangement is used. In Table A the "average" transition probabilities for transitions between lower states of principal quantum number $(n)_{i}$ to upper states $(n)_{k}$ are listed. They are taken from extensive calculations by Green, Rush, and Chandler; Harriman; Herdan and Hughes; Karzas and Latter; and Menzel and Pekeris [1]. These values are applicable to most problems in plasma spectroscopy and astrophysics (see general introduction, Sec. E). Table B contains the probabilities for transitions between the various sublevels $(n l)_{i}$ $-(n l)_{k}$. This table should be useful primarily for theoretical applications. Both tables include only four significant figures since relativistic effects, which are of the order of $\alpha^{2}$, have been neglected in the calculations ( $\alpha$ is the fine structure constant). It should be noted that Green, Rush, and Chandler; and Harriman list more transitions, but these, not being of any practical importance, are omitted.

Table C contains the values for nine fine structure lines as calculated from the work of Wild [2]. The effect of the Lamb shift has been taken into account by using his equation (4a) to calculate the line strength and then by using the energy levels given in NBS Circular 467 (Atomic Energy Levels) for conversion into the other quantities.

The values for the transition between the two hyperfine structure components of the $1 s{ }^{2} \mathrm{~S}_{1 / 2}$ level are also taken from Wild [2] and are given in Table D. This magnetic dipole transition has a statistical weight of $2 f+1$, where $f$ is $j \pm 1 / 2$ for hydrogen.

The metastable $2 s{ }^{2} \mathrm{~S}_{1 / 2}$ level gives rise to transitions to the ground state only by means of twophoton emission. This process was studied in particular by Shapiro and Breit [3]. Their calculation of the transition probability for the $1 s^{2} \mathrm{~S}_{1 / 2}-2 s^{2} \mathrm{~S}_{1 / 2}$ transition gives a value of $8.23 \mathrm{sec}^{-1}$ with an estimated accuracy of better than 3 percent. The transition itself gives rise to a continuum; hence no $f$ or $S$ values are given.

Finally, it should be mentioned that in the conversion factors used for hydrogen the reduced mass and other appropriate constants are taken into account.

\section{References}

[1] Green, L. C., Rush, P. P., and Chandler, C. D., Astrophys. J. Suppl. Ser. 3, 37-50 (1957): Harriman, J. M., Phys. Rev. 101, 594-598 (1956) and Document No. 4705, American Documentation Institute Auxiliary Publications Project, Photoduplication Service, Library of Congress, Washington, D. C.; Herdan, R., and Hughes, T. P., Astrophys. J. 133, 294-298 (1961); Karzas, W. J., and Latter, R., Astrophys. J. Suppl. Ser. 6, 167-212 (1961); Menzel, D. H., and Pekeris. C. L., Monthly Notices Roy. Astron. Soc. 96, 77-111 (1935).

[2] Wild, J. P., Astrophys. J. 115, 206-221 (1952).

[3] Shapiro, J., and Breit, G., Phys. Rev. 113, 179-181 (1959). 
H-Table A. $(n)_{i}-(n)_{k}$ Transitions (Average Values).

\begin{tabular}{|c|c|c|c|c|c|c|c|c|c|c|c|}
\hline Transition & $\lambda(\AA)$ & $E_{i}\left(\mathrm{~cm}^{-1}\right)$ & $E_{k}\left(\mathrm{~cm}^{-1}\right)$ & $g_{i}$ & $g_{k}$ & $A_{k i}\left(\sec ^{-1}\right)$ & $f_{i k}$ & $S$ (at.u.) & $\log g f$ & $\begin{array}{l}\text { Accu- } \\
\text { racy }\end{array}$ & Source \\
\hline $\begin{array}{l}1-2\left(\mathrm{~L}_{\alpha}\right) \\
1-3\left(\mathrm{~L}_{\beta}\right) \\
1-4\left(\mathrm{~L}_{\gamma}\right) \\
1-5\left(\mathrm{~L}_{\delta}\right) \\
1-6\left(\mathrm{~L}_{\epsilon}\right)\end{array}$ & $\begin{array}{c}1215.67 \\
1025.72 \\
792.537 \\
949.743 \\
937.803\end{array}$ & $\begin{array}{l}0 \\
0 \\
0 \\
0 \\
0\end{array}$ & $\begin{array}{r}82259 \\
97492 \\
102824 \\
105292 \\
106632\end{array}$ & $\begin{array}{l}2 \\
2 \\
2 \\
2 \\
2\end{array}$ & $\begin{array}{r}8 \\
18 \\
32 \\
50 \\
72\end{array}$ & $\begin{array}{l}4.699 \times 10^{8} \\
5.575 \times 10^{7} \\
1.278 \times 10^{7} \\
4.125 \times 10^{6} \\
1.644 \times 10^{6}\end{array}$ & $\begin{array}{l}0.4162 \\
7.910 \times 10^{-2} \\
2.899 \times 10^{-2} \\
1.394 \times 10^{-2} \\
7.799 \times 10^{-3}\end{array}$ & $\begin{array}{l}3.330 \\
0.5339 \\
0.1855 \\
8.711 \times 10^{-2} \\
4.813 \times 10^{-2}\end{array}$ & $\begin{array}{l}-0.0797 \\
-0.8008 \\
-1.2367 \\
-1.5548 \\
-1.8069\end{array}$ & $\begin{array}{l}\text { AA } \\
\text { AA } \\
\text { AA } \\
\text { AA } \\
\text { AA }\end{array}$ & $\begin{array}{l}1 \\
1 \\
1 \\
1 \\
1\end{array}$ \\
\hline $\begin{array}{l}1-7 \\
1-8 \\
1-9 \\
1-10 \\
1-11\end{array}$ & $\begin{array}{l}930.748 \\
926.226 \\
923.150 \\
920.963 \\
919.352\end{array}$ & $\begin{array}{l}0 \\
0 \\
0 \\
0 \\
0\end{array}$ & $\begin{array}{l}107440 \\
107965 \\
108325 \\
108582 \\
108772\end{array}$ & $\begin{array}{l}2 \\
2 \\
2 \\
2 \\
2\end{array}$ & $\begin{array}{r}98 \\
128 \\
162 \\
200 \\
242\end{array}$ & $\begin{array}{l}7.568 \times 10^{5} \\
3.869 \times 10^{5} \\
2.143 \times 10^{5} \\
1.263 \times 10^{5} \\
7.834 \times 10^{4}\end{array}$ & $\begin{array}{l}4.814 \times 10^{-3} \\
3.183 \times 10^{-3} \\
2.216 \times 10^{-3} \\
1.605 \times 10^{-3} \\
1.201 \times 10^{-3}\end{array}$ & $\begin{array}{l}2.948 \times 10^{-2} \\
1.940 \times 10^{-2} \\
1.346 \times 10^{-2} \\
9.729 \times 10^{-3} \\
7.263 \times 10^{-3}\end{array}$ & $\begin{array}{l}-2.0165 \\
-2.1961 \\
-2.3534 \\
-2.4934 \\
-2.6196\end{array}$ & $\begin{array}{l}\text { AA } \\
\text { AA } \\
\text { AA } \\
\text { AA } \\
\text { AA }\end{array}$ & $\begin{array}{l}1 \\
1 \\
1 \\
1 \\
1\end{array}$ \\
\hline $\begin{array}{l}1-12 \\
1-13 \\
1-14 \\
1-15 \\
1-16\end{array}$ & $\begin{array}{l}918.129 \\
917.181 \\
916.429 \\
915.824 \\
915.329\end{array}$ & $\begin{array}{l}0 \\
0 \\
0 \\
0 \\
0\end{array}$ & $\begin{array}{l}108917 \\
109030 \\
109119 \\
109191 \\
109250\end{array}$ & $\begin{array}{l}2 \\
2 \\
2 \\
2 \\
2\end{array}$ & $\begin{array}{l}288 \\
338 \\
392 \\
450 \\
512\end{array}$ & $\begin{array}{l}5.066 \times 10^{4} \\
3.393 \times 10^{4} \\
2.341 \times 10^{4} \\
1.657 \times 10^{4} \\
1.200 \times 10^{4}\end{array}$ & $\begin{array}{l}9.214 \times 10^{-4} \\
7.227 \times 10^{-4} \\
5.774 \times 10^{-4} \\
4.686 \times 10^{-4} \\
3.856 \times 10^{-4}\end{array}$ & $\begin{array}{l}5.567 \times 10^{-3} \\
4.362 \times 10^{-3} \\
3.482 \times 10^{-3} \\
2.824 \times 10^{-3} \\
2.323 \times 10^{-3}\end{array}$ & $\begin{array}{l}-2.7345 \\
-2.8400 \\
-2.9375 \\
-3.0281 \\
-3.1129\end{array}$ & $\begin{array}{l}\text { AA } \\
\mathrm{AA} \\
\mathrm{AA} \\
\mathrm{AA} \\
\mathrm{AA}\end{array}$ & $\begin{array}{l}1 \\
1 \\
1 \\
1 \\
1\end{array}$ \\
\hline $\begin{array}{l}1-17 \\
1-18 \\
1-19 \\
1-20 \\
1-21\end{array}$ & $\begin{array}{l}914.919 \\
914.576 \\
914.286 \\
914.039 \\
913.826\end{array}$ & $\begin{array}{l}0 \\
0 \\
0 \\
0 \\
0\end{array}$ & $\begin{array}{l}109299 \\
109340 \\
109375 \\
109405 \\
109430\end{array}$ & $\begin{array}{l}2 \\
2 \\
2 \\
2 \\
2\end{array}$ & $\begin{array}{l}578 \\
648 \\
722 \\
800 \\
882\end{array}$ & $\begin{array}{l}8858 \\
6654 \\
5077 \\
3928 \\
3077\end{array}$ & $\begin{array}{l}3.211 \times 10^{-4} \\
2.702 \times 10^{-4} \\
2.296 \times 10^{-4} \\
1.967 \times 10^{-4} \\
1.698 \times 10^{-4}\end{array}$ & $\begin{array}{l}1.933 \times 10^{-3} \\
1.626 \times 10^{-3} \\
1.381 \times 10^{-3} \\
1.183 \times 10^{-3} \\
1.021 \times 10^{-3}\end{array}$ & $\begin{array}{l}-3.1924 \\
-3.2673 \\
-3.3381 \\
-3.4052 \\
-3.4691\end{array}$ & $\begin{array}{l}\mathrm{AA} \\
\mathrm{AA} \\
\mathrm{AA} \\
\mathrm{AA} \\
\mathrm{AA}\end{array}$ & $\begin{array}{l}1 \\
1 \\
1 \\
1 \\
1\end{array}$ \\
\hline $\begin{array}{l}1-22 \\
1-23 \\
1-24 \\
1-25 \\
1-26\end{array}$ & $\begin{array}{l}913.641 \\
913.480 \\
913.339 \\
913.215 \\
913.104\end{array}$ & $\begin{array}{l}0 \\
0 \\
0 \\
0 \\
0\end{array}$ & $\begin{array}{l}109452 \\
109471 \\
109488 \\
109503 \\
109517\end{array}$ & $\begin{array}{l}2 \\
2 \\
2 \\
2 \\
2\end{array}$ & $\begin{array}{r}968 \\
1058 \\
1152 \\
1250 \\
1352\end{array}$ & $\begin{array}{l}2438 \\
1952 \\
1578 \\
1286 \\
1057\end{array}$ & $\begin{array}{l}1.476 \times 10^{-4} \\
1.291 \times 10^{-4} \\
1.136 \times 10^{-4} \\
1.005 \times 10^{-4} \\
8.928 \times 10^{-5}\end{array}$ & $\begin{array}{l}8.874 \times 10^{-4} \\
7.761 \times 10^{-4} \\
6.827 \times 10^{-4} \\
6.037 \times 10^{-4} \\
5.364 \times 10^{-4}\end{array}$ & $\begin{array}{l}-3.5299 \\
-3.5880 \\
-3.6436 \\
-3.6970 \\
-3.7482\end{array}$ & $\begin{array}{l}\text { AA } \\
\mathrm{AA} \\
\mathrm{AA} \\
\mathrm{AA} \\
\mathrm{AA}\end{array}$ & $\begin{array}{l}1 \\
1 \\
1 \\
1 \\
1\end{array}$ \\
\hline $\begin{array}{l}1-27 \\
1-28 \\
1-29 \\
1-30 \\
1-31\end{array}$ & $\begin{array}{l}913.006 \\
912.918 \\
912.839 \\
912.768 \\
912.703\end{array}$ & $\begin{array}{l}0 \\
0 \\
0 \\
0 \\
0\end{array}$ & $\begin{array}{l}109528 \\
109539 \\
109548 \\
109557 \\
109565\end{array}$ & $\begin{array}{l}2 \\
2 \\
2 \\
2 \\
2\end{array}$ & $\begin{array}{l}1458 \\
1568 \\
1682 \\
1800 \\
1922\end{array}$ & $\begin{array}{l}875.3 \\
729.7 \\
612.2 \\
516.7 \\
438.6\end{array}$ & $\begin{array}{l}7.970 \times 10^{-5} \\
7.144 \times 10^{-5} \\
6.429 \times 10^{-5} \\
5.806 \times 10^{-5} \\
5.261 \times 10^{-5}\end{array}$ & $\begin{array}{l}4.788 \times 10^{-4} \\
4.292 \times 10^{-4} \\
3.862 \times 10^{-4} \\
3.487 \times 10^{-4} \\
3.160 \times 10^{-4}\end{array}$ & $\begin{array}{l}-3.7975 \\
-3.8450 \\
-3.8908 \\
-3.9351 \\
-3.9779\end{array}$ & $\begin{array}{l}\mathrm{AA} \\
\mathrm{AA} \\
\mathrm{AA} \\
\mathrm{AA} \\
\mathrm{AA}\end{array}$ & $\begin{array}{l}1 \\
1 \\
1 \\
1 \\
1\end{array}$ \\
\hline $\begin{array}{l}1-32 \\
1-33 \\
1-34 \\
1-35 \\
1-36\end{array}$ & $\begin{array}{l}912.645 \\
912.592 \\
912.543 \\
912.499 \\
912.458\end{array}$ & $\begin{array}{l}0 \\
0 \\
0 \\
0 \\
0\end{array}$ & $\begin{array}{l}109572 \\
109578 \\
109584 \\
109589 \\
109594\end{array}$ & $\begin{array}{l}2 \\
2 \\
2 \\
2 \\
2\end{array}$ & $\begin{array}{l}2048 \\
2178 \\
2312 \\
2450 \\
2592\end{array}$ & $\begin{array}{l}374.2 \\
320.8 \\
276.3 \\
239.0 \\
207.6\end{array}$ & $\begin{array}{l}4.782 \times 10^{-5} \\
4.360 \times 10^{-5} \\
3.986 \times 10^{-5} \\
3.653 \times 10^{-5} \\
3.357 \times 10^{-5}\end{array}$ & $\begin{array}{l}2.872 \times 10^{-4} \\
2.618 \times 10^{-4} \\
2.394 \times 10^{-4} \\
2.194 \times 10^{-4} \\
2.016 \times 10^{-4}\end{array}$ & $\begin{array}{l}-4.0193 \\
-4.0595 \\
-4.0985 \\
-4.1363 \\
-4.1730\end{array}$ & $\begin{array}{l}\text { AA } \\
\text { AA } \\
\text { AA } \\
\text { AA } \\
\text { AA }\end{array}$ & $\begin{array}{l}1 \\
1 \\
1 \\
1 \\
1\end{array}$ \\
\hline $\begin{array}{l}1-37 \\
1-38 \\
1-39 \\
1-40\end{array}$ & $\begin{array}{l}912.420 \\
912.385 \\
912.353 \\
912.324\end{array}$ & $\begin{array}{l}0 \\
0 \\
0 \\
0\end{array}$ & $\begin{array}{l}109599 \\
109603 \\
109607 \\
109610\end{array}$ & $\begin{array}{l}2 \\
2 \\
2 \\
2\end{array}$ & $\begin{array}{l}2738 \\
2888 \\
3042 \\
3200\end{array}$ & $\begin{array}{l}181.0 \\
158.4 \\
139.1 \\
122.6\end{array}$ & $\begin{array}{l}3.092 \times 10^{-5} \\
2.854 \times 10^{-5} \\
2.640 \times 10^{-5} \\
2.446 \times 10^{-5}\end{array}$ & $\begin{array}{l}1.856 \times 10^{-4} \\
1.713 \times 10^{-4} \\
1.585 \times 10^{-4} \\
1.469 \times 10^{-4}\end{array}$ & $\begin{array}{l}-4.2088 \\
-4.2436 \\
-4.2774 \\
-4.3105\end{array}$ & $\begin{array}{l}\text { AA } \\
\text { AA } \\
\text { AA } \\
\text { AA }\end{array}$ & $\begin{array}{l}1 \\
1 \\
1 \\
1\end{array}$ \\
\hline $\begin{array}{l}2-3\left(\mathrm{H}_{\alpha}\right) \\
2-4\left(\mathrm{H}_{\beta}\right) \\
2-5\left(\mathrm{H}_{\lambda}\right) \\
2-6\left(\mathrm{H}_{\delta}\right) \\
2-7\left(\mathrm{H}_{\epsilon}\right)\end{array}$ & $\begin{array}{l}6562.80 \\
4861.32 \\
4340.46 \\
4101.73 \\
3970.07\end{array}$ & $\begin{array}{l}82259 \\
82259 \\
82259 \\
82259 \\
82259\end{array}$ & $\begin{array}{r}97492 \\
102824 \\
105292 \\
106632 \\
107440\end{array}$ & $\begin{array}{l}8 \\
8 \\
8 \\
8 \\
8\end{array}$ & $\begin{array}{l}18 \\
32 \\
50 \\
72 \\
98\end{array}$ & $\begin{array}{l}4.410 \times 10^{7} \\
8.419 \times 10^{6} \\
2.530 \times 10^{6} \\
9.732 \times 10^{5} \\
4.389 \times 10^{5}\end{array}$ & $\begin{array}{l}0.6407 \\
0.1193 \\
4.467 \times 10^{-2} \\
2.209 \times 10^{-2} \\
1.270 \times 10^{-2}\end{array}$ & $\begin{array}{c}110.7 \\
15.27 \\
5.105 \\
2.386 \\
1.328\end{array}$ & $\begin{array}{r}0.7098 \\
-0.0202 \\
-0.4469 \\
-0.7527 \\
-0.9929\end{array}$ & $\begin{array}{l}\text { AA } \\
\text { AA } \\
\text { AA } \\
\text { AA } \\
\text { AA }\end{array}$ & $\begin{array}{l}1 \\
1 \\
1 \\
1 \\
1\end{array}$ \\
\hline $\begin{array}{l}2-8 \\
2-9 \\
2-10 \\
2-11 \\
2-12\end{array}$ & $\begin{array}{l}3889.05 \\
3835.38 \\
3797.90 \\
3770.63 \\
3750.15\end{array}$ & $\begin{array}{l}82259 \\
82259 \\
82259 \\
82259 \\
82259\end{array}$ & $\begin{array}{l}107965 \\
108325 \\
108582 \\
108772 \\
108917\end{array}$ & $\begin{array}{l}8 \\
8 \\
8 \\
8 \\
8\end{array}$ & $\begin{array}{l}128 \\
162 \\
200 \\
242 \\
288\end{array}$ & $\begin{array}{l}2.215 \times 10^{5} \\
1.216 \times 10^{5} \\
7.122 \times 10^{4} \\
4.397 \times 10^{4} \\
2.834 \times 10^{4}\end{array}$ & $\begin{array}{l}8.036 \times 10^{-3} \\
5.429 \times 10^{-3} \\
3.851 \times 10^{-3} \\
2.835 \times 10^{-3} \\
2.151 \times 10^{-3}\end{array}$ & $\begin{array}{l}0.8228 \\
0.5482 \\
0.3851 \\
0.2815 \\
0.2124\end{array}$ & $\begin{array}{l}-1.1919 \\
-1.3622 \\
-1.5114 \\
-1.6443 \\
-1.7643\end{array}$ & $\begin{array}{l}\text { AA } \\
\text { AA } \\
\text { AA } \\
\text { AA } \\
\text { AA }\end{array}$ & $\begin{array}{l}1 \\
1 \\
1 \\
1 \\
1\end{array}$ \\
\hline $\begin{array}{l}2-13 \\
2-14 \\
2-15 \\
2-16 \\
2-17\end{array}$ & $\begin{array}{l}3734.37 \\
3721.94 \\
3711.97 \\
3703.85 \\
3697.15\end{array}$ & $\begin{array}{l}82259 \\
82259 \\
82259 \\
82259 \\
82259\end{array}$ & $\begin{array}{l}109030 \\
109119 \\
109191 \\
109250 \\
109299\end{array}$ & $\begin{array}{l}8 \\
8 \\
8 \\
8 \\
8\end{array}$ & $\begin{array}{l}338 \\
392 \\
450 \\
512 \\
578\end{array}$ & $\begin{array}{l}1.893 \times 10^{4} \\
1.303 \times 10^{4} \\
9210 \\
6658 \\
4910\end{array}$ & $\begin{array}{l}1.672 \times 10^{-3} \\
1.326 \times 10^{-3} \\
1.070 \times 10^{-3} \\
8.764 \times 10^{-4} \\
7.270 \times 10^{-4}\end{array}$ & $\begin{array}{l}0.1644 \\
0.1300 \\
0.1046 \\
8.547 \times 10^{-2} \\
7.077 \times 10^{-2}\end{array}$ & $\begin{array}{l}-1.8737 \\
-1.9743 \\
-2.0674 \\
-2.1542 \\
-2.2354\end{array}$ & $\begin{array}{l}\text { AA } \\
\text { AA } \\
\text { AA } \\
\text { AA } \\
\text { AA }\end{array}$ & $\begin{array}{l}1 \\
1 \\
1 \\
1 \\
1\end{array}$ \\
\hline
\end{tabular}


H-Table A. $\quad(n)_{i}-(n)_{k}$ Transitions (Average Values)-Continued

\begin{tabular}{|c|c|c|c|c|c|c|c|c|c|c|c|}
\hline Transition & $\lambda(\AA)$ & $E_{i}\left(\mathrm{~cm}^{-1}\right)$ & $E_{k}\left(\mathrm{~cm}^{-1}\right)$ & $g_{i}$ & $g_{k}$ & $A_{k i}\left(\sec ^{-1}\right)$ & $f_{i k}$ & $S($ at.u.) & $\log g f$ & $\begin{array}{c}\text { Accu- } \\
\text { racy }\end{array}$ & Srource \\
\hline $\begin{array}{l}2-18 \\
2-19 \\
2-20 \\
2-21 \\
2-22\end{array}$ & $\begin{array}{l}3691.55 \\
3686.83 \\
3682.81 \\
3679.35 \\
3676.36\end{array}$ & $\begin{array}{l}82259 \\
82259 \\
82259 \\
82259 \\
82259\end{array}$ & $\begin{array}{l}109340 \\
109375 \\
109405 \\
109430 \\
109452\end{array}$ & $\begin{array}{l}8 \\
8 \\
8 \\
8 \\
8\end{array}$ & $\begin{array}{l}648 \\
722 \\
800 \\
882 \\
968\end{array}$ & $\begin{array}{l}3685 \\
2809 \\
2172 \\
1700 \\
1347\end{array}$ & $\begin{array}{l}6.099 \times 10^{-4} \\
5.167 \times 10^{-4} \\
4.416 \times 10^{-4} \\
3.805 \times 10^{-4} \\
3.302 \times 10^{-4}\end{array}$ & $\begin{array}{l}5.928 \times 10^{-2} \\
5.016 \times 10^{-2} \\
4.283 \times 10^{-2} \\
3.686 \times 10^{-2} \\
3.196 \times 10^{-2}\end{array}$ & $\begin{array}{l}-2.3117 \\
-2.3837 \\
-2.4518 \\
-2.5165 \\
-2.5781\end{array}$ & $\begin{array}{l}\text { AA } \\
\text { AA } \\
\text { AA } \\
\text { AA } \\
\text { AA }\end{array}$ & $\begin{array}{l}1 \\
1 \\
1 \\
1 \\
1\end{array}$ \\
\hline $\begin{array}{l}2-23 \\
2-24 \\
2-25 \\
2-26 \\
2-27\end{array}$ & $\begin{array}{l}3673.76 \\
3671.48 \\
3669.46 \\
3667.68 \\
3666.10\end{array}$ & $\begin{array}{l}82259 \\
82259 \\
82259 \\
82259 \\
82259\end{array}$ & $\begin{array}{l}109471 \\
109488 \\
109503 \\
109517 \\
109528\end{array}$ & $\begin{array}{l}8 \\
8 \\
8 \\
8 \\
8\end{array}$ & $\begin{array}{l}1058 \\
1152 \\
1250 \\
1352 \\
1458\end{array}$ & $\begin{array}{l}1078 \\
870.7 \\
709.6 \\
583.0 \\
482.6\end{array}$ & $\begin{array}{l}2.884 \times 10^{-4} \\
2.534 \times 10^{-4} \\
2.238 \times 10^{-4} \\
1.987 \times 10^{-4} \\
1.772 \times 10^{-4}\end{array}$ & $\begin{array}{l}2.790 \times 10^{-2} \\
2.449 \times 10^{-2} \\
2.163 \times 10^{-2} \\
1.919 \times 10^{-2} \\
1.711 \times 10^{-2}\end{array}$ & $\begin{array}{l}-2.6369 \\
-2.6931 \\
-2.7470 \\
-2.7987 \\
-2.8484\end{array}$ & $\begin{array}{l}\text { AA } \\
\text { AA } \\
\text { AA } \\
\text { AA } \\
\text { AA }\end{array}$ & $\begin{array}{l}1 \\
1 \\
1 \\
1 \\
1\end{array}$ \\
\hline $\begin{array}{l}2-28 \\
2-29 \\
2-30 \\
2-31 \\
2-32\end{array}$ & $\begin{array}{l}3664.68 \\
3663.40 \\
3662.26 \\
3661.22 \\
3660.28\end{array}$ & $\begin{array}{l}82259 \\
82259 \\
82259 \\
82259 \\
82259\end{array}$ & $\begin{array}{l}109539 \\
109548 \\
109557 \\
109565 \\
109572\end{array}$ & $\begin{array}{l}8 \\
8 \\
8 \\
8 \\
8\end{array}$ & $\begin{array}{l}1568 \\
1682 \\
1800 \\
1922 \\
2048\end{array}$ & $\begin{array}{l}402.2 \\
337.4 \\
284.7 \\
241.6 \\
206.1\end{array}$ & $\begin{array}{l}1.587 \times 10^{-4} \\
1.427 \times 10^{-4} \\
1.288 \times 10^{-4} \\
1.167 \times 10^{-4} \\
1.060 \times 10^{-4}\end{array}$ & $\begin{array}{l}1.532 \times 10^{-2} \\
1.377 \times 10^{-2} \\
1.242 \times 10^{-2} \\
1.125 \times 10^{-2} \\
1.021 \times 10^{-2}\end{array}$ & $\begin{array}{l}-2.8962 \\
-2.9424 \\
-2.9869 \\
-3.0300 \\
-3.0717\end{array}$ & $\begin{array}{l}\text { AA } \\
\text { AA } \\
\text { AA } \\
\text { AA } \\
\text { AA }\end{array}$ & $\begin{array}{l}1 \\
1 \\
1 \\
1 \\
1\end{array}$ \\
\hline $\begin{array}{l}2-33 \\
2-34 \\
2-35 \\
2-36 \\
2-37\end{array}$ & $\begin{array}{l}3659.42 \\
3658.64 \\
3657.92 \\
3657.27 \\
3656.66\end{array}$ & $\begin{array}{l}82259 \\
82259 \\
82259 \\
82259 \\
82259\end{array}$ & $\begin{array}{l}109578 \\
109584 \\
109589 \\
109594 \\
109599\end{array}$ & $\begin{array}{l}8 \\
8 \\
8 \\
8 \\
8\end{array}$ & $\begin{array}{l}2178 \\
2312 \\
2450 \\
2592 \\
2738\end{array}$ & $\begin{array}{c}176.7 \\
152.2 \\
131.6 \\
114.3 \\
99.66\end{array}$ & $\begin{array}{l}9.658 \times 10^{-5} \\
8.825 \times 10^{-5} \\
8.086 \times 10^{-5} \\
7.427 \times 10^{-5} \\
6.837 \times 10^{-5}\end{array}$ & $\begin{array}{l}9.305 \times 10^{-3} \\
8.501 \times 10^{-3} \\
7.788 \times 10^{-3} \\
7.152 \times 10^{-3} \\
6.583 \times 10^{-3}\end{array}$ & $\begin{array}{l}-3.1120 \\
-3.1512 \\
-3.1892 \\
-3.2261 \\
-3.2620\end{array}$ & $\begin{array}{l}\mathrm{AA} \\
\mathrm{AA} \\
\mathrm{AA} \\
\mathrm{AA} \\
\mathrm{AA}\end{array}$ & $\begin{array}{l}1 \\
1 \\
1 \\
1 \\
1\end{array}$ \\
\hline $\begin{array}{l}2-38 \\
2-39 \\
2-40\end{array}$ & $\begin{array}{l}3656.11 \\
3655.59 \\
3655.12\end{array}$ & $\begin{array}{l}82259 \\
82259 \\
82259\end{array}$ & $\begin{array}{l}109603 \\
109607 \\
109610\end{array}$ & $\begin{array}{l}8 \\
8 \\
8\end{array}$ & $\begin{array}{l}2888 \\
3042 \\
3200\end{array}$ & $\begin{array}{l}87.20 \\
76.57 \\
67.46\end{array}$ & $\begin{array}{l}6.309 \times 10^{-5} \\
5.834 \times 10^{-5} \\
5.405 \times 10^{-5}\end{array}$ & $\begin{array}{l}6.073 \times 10^{-3} \\
5.615 \times 10^{-3} \\
5.202 \times 10^{-3}\end{array}$ & $\begin{array}{l}-3.2969 \\
-3.3310 \\
-3.3641\end{array}$ & $\begin{array}{l}\mathrm{AA} \\
\mathrm{AA} \\
\mathrm{AA}\end{array}$ & $\begin{array}{l}1 \\
1 \\
1\end{array}$ \\
\hline $\begin{array}{l}3-4\left(\mathrm{P}_{\alpha}\right) \\
3-5\left(\mathrm{P}_{\beta}\right) \\
3-6\left(\mathrm{P}_{\gamma}\right) \\
3-7\left(\mathrm{P}_{\delta}\right) \\
3-8\left(\mathrm{P}_{\epsilon}\right)\end{array}$ & $\begin{array}{c}18751.0 \\
12818.1 \\
10938.1 \\
10049.4 \\
9545.98\end{array}$ & $\begin{array}{l}97492 \\
97492 \\
97492 \\
97492 \\
974.92\end{array}$ & $\begin{array}{l}102824 \\
105292 \\
106632 \\
107440 \\
107965\end{array}$ & $\begin{array}{l}18 \\
18 \\
18 \\
18 \\
18\end{array}$ & $\begin{array}{r}32 \\
50 \\
72 \\
98 \\
128\end{array}$ & $\begin{array}{l}8.986 \times 10^{6} \\
2.201 \times 10^{6} \\
7.783 \times 10^{5} \\
3.358 \times 10^{5} \\
1.651 \times 10^{5}\end{array}$ & $\begin{array}{l}0.8421 \\
0.1506 \\
5.584 \times 10^{-2} \\
2.768 \times 10^{-2} \\
1.604 \times 10^{-2}\end{array}$ & $\begin{array}{l}935.4 \\
114.3 \\
36.18 \\
16.48 \\
9.069\end{array}$ & $\begin{array}{r}1.1806 \\
0.4331 \\
0.0022 \\
-0.3025 \\
-0.5396\end{array}$ & $\begin{array}{l}\text { AA } \\
\mathrm{AA} \\
\mathrm{AA} \\
\mathrm{AA} \\
\mathrm{AA}\end{array}$ & $\begin{array}{l}1 \\
1 \\
1 \\
1 \\
1\end{array}$ \\
\hline $\begin{array}{l}3-9 \\
3-10 \\
3-11 \\
3-12 \\
3-13\end{array}$ & $\begin{array}{l}9229.02 \\
9014.91 \\
8862.79 \\
8750.47 \\
8665.02\end{array}$ & $\begin{array}{l}97492 \\
97492 \\
97492 \\
97492 \\
97492\end{array}$ & $\begin{array}{l}108325 \\
108582 \\
108772 \\
108917 \\
109030\end{array}$ & $\begin{array}{l}18 \\
18 \\
18 \\
18 \\
18\end{array}$ & $\begin{array}{l}162 \\
200 \\
242 \\
288 \\
338\end{array}$ & $\begin{array}{l}8.905 \times 10^{4} \\
5.156 \times 10^{4} \\
3.156 \times 10^{4} \\
2.021 \times 10^{4} \\
1.343 \times 10^{4}\end{array}$ & $\begin{array}{l}1.023 \times 10^{-2} \\
6.980 \times 10^{-3} \\
4.996 \times 10^{-3} \\
3.711 \times 10^{-3} \\
2.839 \times 10^{-3}\end{array}$ & $\begin{array}{l}5.595 \\
3.728 \\
2.623 \\
1.924 \\
1.457\end{array}$ & $\begin{array}{l}-0.7347 \\
-0.9009 \\
-1.0461 \\
-1.1752 \\
-1.2916\end{array}$ & $\begin{array}{l}\mathrm{AA} \\
\mathrm{AA} \\
\mathrm{AA} \\
\mathrm{AA} \\
\mathrm{AA}\end{array}$ & $\begin{array}{l}1 \\
1 \\
1 \\
1 \\
1\end{array}$ \\
\hline $\begin{array}{l}3-14 \\
3-15 \\
3-16 \\
3-17 \\
3-18 \\
3-19 \\
3-20\end{array}$ & $\begin{array}{l}8598.39 \\
8545.39 \\
8502.49 \\
8467.26 \\
8437.96 \\
8413.32 \\
8392.40\end{array}$ & $\begin{array}{l}97492 \\
97492 \\
97492 \\
97492 \\
97492 \\
97492 \\
97492\end{array}$ & $\begin{array}{l}109119 \\
109191 \\
109250 \\
109299 \\
109340 \\
109375 \\
109405\end{array}$ & $\begin{array}{l}18 \\
18 \\
18 \\
18 \\
18 \\
18 \\
18\end{array}$ & $\begin{array}{l}392 \\
450 \\
512 \\
578 \\
648 \\
722 \\
800\end{array}$ & $\begin{array}{l}9211 \\
6490 \\
4680 \\
3444 \\
2580 \\
1964 \\
1517\end{array}$ & $\begin{array}{l}2.224 \times 10^{-3} \\
1.776 \times 10^{-3} \\
1.443 \times 10^{-3} \\
1.188 \times 10^{-3} \\
9.916 \times 10^{-4} \\
8.361 \times 10^{-4} \\
7.118 \times 10^{-4}\end{array}$ & $\begin{array}{l}1.133 \\
0.8992 \\
0.7267 \\
0.5963 \\
0.4957 \\
0.4167 \\
0.3539\end{array}$ & $\begin{array}{l}-1.3977 \\
-1.4952 \\
-1.5855 \\
-1.6696 \\
-1.7484 \\
-1.8225 \\
-1.8924\end{array}$ & $\begin{array}{l}\text { AA } \\
\text { AA } \\
\text { AA } \\
\text { AA } \\
\text { AA } \\
\text { AA } \\
\text { AA }\end{array}$ & $\begin{array}{l}1 \\
1 \\
1 \\
1 \\
1 \\
1 \\
1\end{array}$ \\
\hline $\begin{array}{l}4-5 \\
4-6 \\
4-7 \\
4-8 \\
4-9\end{array}$ & $\begin{array}{l}40512.0 \\
26252.0 \\
21655.0 \\
19445.6 \\
18174.1\end{array}$ & $\begin{array}{l}102824 \\
102824 \\
102824 \\
102824 \\
102824\end{array}$ & $\begin{array}{l}105292 \\
106632 \\
107440 \\
107965 \\
108325\end{array}$ & $\begin{array}{l}32 \\
32 \\
32 \\
32 \\
32\end{array}$ & $\begin{array}{r}50 \\
72 \\
98 \\
128 \\
162\end{array}$ & $\begin{array}{l}2.699 \times 10^{6} \\
7.711 \times 10^{5} \\
3.041 \times 10^{5} \\
1.424 \times 10^{5} \\
7.459 \times 10^{4}\end{array}$ & $\begin{array}{l}1.038 \\
0.1793 \\
6.549 \times 10^{-2} \\
3.230 \times 10^{-2} \\
1.870 \times 10^{-2}\end{array}$ & $\begin{array}{l}4428 \\
495.6 \\
149.4 \\
66.14 \\
35.79\end{array}$ & $\begin{array}{r}1.5212 \\
0.7586 \\
0.3213 \\
0.0143 \\
-0.2230\end{array}$ & $\begin{array}{l}\text { AA } \\
\text { AA } \\
\text { AA } \\
\text { AA } \\
\text { AA }\end{array}$ & $\begin{array}{l}1 \\
1 \\
1 \\
1 \\
1\end{array}$ \\
\hline $\begin{array}{l}4-10 \\
4-11 \\
4-12 \\
4-13 \\
4-14\end{array}$ & $\begin{array}{l}17362.1 \\
16806.5 \\
16407.2 \\
16109.3 \\
15880.5\end{array}$ & $\begin{array}{l}102824 \\
102824 \\
102824 \\
102824 \\
102824\end{array}$ & $\begin{array}{l}108582 \\
108772 \\
108917 \\
109030 \\
109119\end{array}$ & $\begin{array}{l}32 \\
32 \\
32 \\
32 \\
32\end{array}$ & $\begin{array}{l}200 \\
242 \\
288 \\
338 \\
392\end{array}$ & $\begin{array}{l}4.235 \times 10^{4} \\
2.556 \times 10^{4} \\
1.620 \times 10^{4} \\
1.069 \times 10^{4} \\
7288\end{array}$ & $\begin{array}{l}1.196 \times 10^{-2} \\
8.187 \times 10^{-3} \\
5.886 \times 10^{-3} \\
4.393 \times 10^{-3} \\
3.375 \times 10^{-3}\end{array}$ & $\begin{array}{c}21.87 \\
14.49 \\
10.17 \\
7.452 \\
5.645\end{array}$ & $\begin{array}{l}-0.4171 \\
-0.5817 \\
-0.7250 \\
-0.8521 \\
-0.9665\end{array}$ & $\begin{array}{l}\text { AA } \\
\text { AA } \\
\text { AA } \\
\text { AA } \\
\text { AA }\end{array}$ & $\begin{array}{l}1 \\
1 \\
1 \\
1 \\
1\end{array}$ \\
\hline $\begin{array}{l}4-15 \\
4-16 \\
4-17 \\
4-18 \\
4-19 \\
4-20\end{array}$ & $\begin{array}{l}15700.7 \\
15556.5 \\
15438.9 \\
15341.8 \\
15260.6 \\
15191.8\end{array}$ & $\begin{array}{l}102824 \\
102824 \\
102824 \\
102824 \\
102824 \\
102824\end{array}$ & $\begin{array}{l}109191 \\
109250 \\
109299 \\
109340 \\
109375 \\
109405\end{array}$ & $\begin{array}{l}32 \\
32 \\
32 \\
32 \\
32 \\
32\end{array}$ & $\begin{array}{l}450 \\
512 \\
578 \\
648 \\
722 \\
800\end{array}$ & $\begin{array}{l}5110 \\
3671 \\
2693 \\
2013 \\
1529 \\
1178\end{array}$ & $\begin{array}{l}2.656 \times 10^{-3} \\
2.131 \times 10^{-3} \\
1.739 \times 10^{-3} \\
1.439 \times 10^{-3} \\
1.204 \times 10^{-3} \\
1.019 \times 10^{-3}\end{array}$ & $\begin{array}{l}4.392 \\
3.492 \\
2.827 \\
2.324 \\
1.936 \\
1.631\end{array}$ & $\begin{array}{l}-1.0706 \\
-1.1662 \\
-1.2547 \\
-1.3370 \\
-1.4141 \\
-1.4865\end{array}$ & $\begin{array}{l}\text { AA } \\
\text { AA } \\
A A \\
A A \\
A A \\
A A\end{array}$ & $\begin{array}{l}1 \\
1 \\
1 \\
1 \\
1 \\
1\end{array}$ \\
\hline
\end{tabular}


$\mathbf{H}$-Table A. $(n)_{i}-(n)_{k}$ Transitions (Average Values)-Continued

\begin{tabular}{|c|c|c|c|c|c|c|c|c|c|c|c|}
\hline Transition & $\lambda(\AA)$ & $E_{i}\left(\mathrm{~cm}^{-1}\right)$ & $E_{k}\left(\mathrm{~cm}^{-1}\right)$ & $g_{i}$ & $g_{k}$ & $A_{k i}\left(\sec ^{-1}\right)$ & $f_{i k}$ & $S$ (at.u.) & $\log g f$ & $\begin{array}{c}\text { Accu- } \\
\text { racy }\end{array}$ & Source \\
\hline $\begin{array}{l}5-6 \\
5-7 \\
5-8 \\
5-9 \\
5-10\end{array}$ & $\begin{array}{l}74578 \\
46525 \\
37395 \\
32961 \\
30384\end{array}$ & $\begin{array}{l}105292 \\
105292 \\
105292 \\
105292 \\
105292\end{array}$ & $\begin{array}{l}106632 \\
107440 \\
107965 \\
108325 \\
108582\end{array}$ & $\begin{array}{l}50 \\
50 \\
50 \\
50 \\
50\end{array}$ & $\begin{array}{r}72 \\
98 \\
128 \\
162 \\
200\end{array}$ & $\begin{array}{l}1.025 \times 10^{6} \\
3.253 \times 10^{5} \\
1.388 \times 10^{5} \\
6.908 \times 10^{4} \\
3.800 \times 10^{4}\end{array}$ & $\begin{array}{l}1.231 \\
0.2069 \\
7.448 \times 10^{-2} \\
3.645 \times 10^{-2} \\
2.104 \times 10^{-2}\end{array}$ & $\begin{array}{l}1.511 \times 10^{4} \\
1584 \\
458.3 \\
197.7 \\
105.2\end{array}$ & $\begin{array}{l}1.7893 \\
1.0147 \\
0.5710 \\
0.2607 \\
0.0219\end{array}$ & $\begin{array}{l}\text { AA } \\
\text { AA } \\
\text { AA } \\
\text { AA } \\
\text { AA }\end{array}$ & $\begin{array}{l}1 \\
1 \\
1 \\
1 \\
1\end{array}$ \\
\hline $\begin{array}{l}5-11 \\
5-12 \\
5-13 \\
5-14 \\
5-15\end{array}$ & $\begin{array}{l}28722 \\
27575 \\
26744 \\
26119 \\
25636\end{array}$ & $\begin{array}{l}105292 \\
105292 \\
105292 \\
105292 \\
105292\end{array}$ & $\begin{array}{l}108772 \\
108917 \\
109030 \\
109119 \\
109191\end{array}$ & $\begin{array}{l}50 \\
50 \\
50 \\
50 \\
50\end{array}$ & $\begin{array}{l}242 \\
288 \\
338 \\
392 \\
450\end{array}$ & $\begin{array}{l}2.246 \times 10^{4} \\
1.402 \times 10^{4} \\
9148 \\
6185 \\
4308\end{array}$ & $\begin{array}{l}1.344 \times 10^{-2} \\
9.209 \times 10^{-3} \\
6.631 \times 10^{-3} \\
4.959 \times 10^{-3} \\
3.821 \times 10^{-3}\end{array}$ & $\begin{array}{l}63.55 \\
41.79 \\
29.18 \\
21.32 \\
16.12\end{array}$ & $\begin{array}{l}-0.1725 \\
-0.3368 \\
-0.4794 \\
-0.6056 \\
-0.7189\end{array}$ & $\begin{array}{l}\text { AA } \\
\text { AA } \\
\text { AA } \\
\text { AA } \\
\text { AA }\end{array}$ & $\begin{array}{l}1 \\
1 \\
1 \\
1 \\
1\end{array}$ \\
\hline $\begin{array}{l}5-16 \\
5-17 \\
5-18 \\
5-19 \\
5-20\end{array}$ & $\begin{array}{l}25254 \\
24946 \\
24693 \\
24483 \\
24307\end{array}$ & $\begin{array}{l}105292 \\
105292 \\
105292 \\
105292 \\
105292\end{array}$ & $\begin{array}{l}109250 \\
109299 \\
109340 \\
109375 \\
109405\end{array}$ & $\begin{array}{l}50 \\
50 \\
50 \\
50 \\
50\end{array}$ & $\begin{array}{l}512 \\
578 \\
648 \\
722 \\
800\end{array}$ & $\begin{array}{l}3079 \\
2249 \\
1675 \\
1268 \\
975.1\end{array}$ & $\begin{array}{l}3.014 \times 10^{-3} \\
2.425 \times 10^{-3} \\
1.984 \times 10^{-3} \\
1.646 \times 10^{-3} \\
1.382 \times 10^{-3}\end{array}$ & $\begin{array}{c}12.53 \\
9.957 \\
8.062 \\
6.631 \\
5.528\end{array}$ & $\begin{array}{l}-0.8218 \\
-0.9162 \\
-1.0035 \\
-1.0846 \\
-1.1605\end{array}$ & $\begin{array}{l}\text { AA } \\
\text { AA } \\
\text { AA } \\
\text { AA } \\
\text { AA }\end{array}$ & $\begin{array}{l}1 \\
1 \\
1 \\
1 \\
1\end{array}$ \\
\hline $\begin{array}{l}6-7 \\
6-8 \\
6-9 \\
6-10 \\
6-11\end{array}$ & $\begin{array}{r}123680 \\
75005 \\
59066 \\
51273 \\
46712\end{array}$ & $\begin{array}{l}106632 \\
106632 \\
106632 \\
106632 \\
106632\end{array}$ & $\begin{array}{l}107440 \\
107965 \\
108325 \\
108582 \\
108772\end{array}$ & $\begin{array}{l}72 \\
72 \\
72 \\
72 \\
72\end{array}$ & $\begin{array}{r}98 \\
128 \\
162 \\
200 \\
242\end{array}$ & $\begin{array}{l}4.561 \times 10^{5} \\
1.561 \times 10^{5} \\
7.065 \times 10^{4} \\
3.688 \times 10^{4} \\
2.110 \times 10^{4}\end{array}$ & $\begin{array}{l}1.424 \\
0.2340 \\
8.315 \times 10^{-2} \\
4.038 \times 10^{-2} \\
2.320 \times 10^{-2}\end{array}$ & $\begin{array}{l}\quad 4.173 \times 10^{4} \\
4160 \\
1164 \\
490.6 \\
256.8\end{array}$ & $\begin{array}{l}2.0108 \\
1.2266 \\
0.7772 \\
0.4635 \\
0.2227\end{array}$ & $\begin{array}{l}\text { AA } \\
\text { AA } \\
\text { AA } \\
\text { AA } \\
\text { AA }\end{array}$ & $\begin{array}{l}1 \\
1 \\
1 \\
1 \\
1\end{array}$ \\
\hline $\begin{array}{l}6-12 \\
6-13 \\
6-14 \\
6-15 \\
6-16\end{array}$ & $\begin{array}{l}43753 \\
41697 \\
40198 \\
39065 \\
38184\end{array}$ & $\begin{array}{l}106632 \\
106632 \\
106632 \\
106632 \\
106632\end{array}$ & $\begin{array}{l}108917 \\
109030 \\
109119 \\
109191 \\
109250\end{array}$ & $\begin{array}{l}72 \\
72 \\
72 \\
72 \\
72\end{array}$ & $\begin{array}{l}288 \\
338 \\
392 \\
450 \\
512\end{array}$ & $\begin{array}{l}1.288 \times 10^{4} \\
8271 \\
5526 \\
3815 \\
2707\end{array}$ & $\begin{array}{l}1.479 \times 10^{-2} \\
1.012 \times 10^{-2} \\
7.289 \times 10^{-3} \\
5.455 \times 10^{-3} \\
4.207 \times 10^{-3}\end{array}$ & $\begin{array}{c}153.3 \\
100.0 \\
69.43 \\
50.50 \\
38.07\end{array}$ & $\begin{array}{r}0.0273 \\
-0.1374 \\
-0.2800 \\
-0.4059 \\
-0.5186\end{array}$ & $\begin{array}{l}\text { AA } \\
\text { AA } \\
\text { AA } \\
\text { AA } \\
\text { AA }\end{array}$ & $\begin{array}{l}1 \\
1 \\
1 \\
1 \\
1\end{array}$ \\
\hline $\begin{array}{l}6-17 \\
6-18 \\
6-19 \\
6-20\end{array}$ & $\begin{array}{l}37484 \\
36916 \\
36449 \\
36060\end{array}$ & $\begin{array}{l}106632 \\
106632 \\
106632 \\
106632\end{array}$ & $\begin{array}{l}109299 \\
109340 \\
109375 \\
109405\end{array}$ & $\begin{array}{l}72 \\
72 \\
72 \\
72\end{array}$ & $\begin{array}{l}578 \\
648 \\
722 \\
800\end{array}$ & $\begin{array}{l}1966 \\
1457 \\
1099 \\
842.4\end{array}$ & $\begin{array}{l}3.324 \times 10^{-3} \\
2.679 \times 10^{-3} \\
2.196 \times 10^{-3} \\
1.825 \times 10^{-3}\end{array}$ & $\begin{array}{l}29.53 \\
23.44 \\
18.96 \\
15.59\end{array}$ & $\begin{array}{l}-0.6209 \\
-0.7146 \\
-0.8011 \\
-0.8815\end{array}$ & $\begin{array}{l}\text { AA } \\
\text { AA } \\
\text { AA } \\
\text { AA }\end{array}$ & $\begin{array}{l}1 \\
1 \\
1 \\
1\end{array}$ \\
\hline $\begin{array}{l}7-8 \\
7-9 \\
7-10 \\
7-11 \\
7-12\end{array}$ & $\begin{array}{r}190570 \\
113060 \\
87577 \\
75061 \\
67701\end{array}$ & $\begin{array}{l}107440 \\
107440 \\
107440 \\
107440 \\
107440\end{array}$ & $\begin{array}{l}107965 \\
108325 \\
108582 \\
108772 \\
108917\end{array}$ & $\begin{array}{l}98 \\
98 \\
98 \\
98 \\
98\end{array}$ & $\begin{array}{l}128 \\
162 \\
200 \\
242 \\
288\end{array}$ & $\begin{array}{l}2.272 \times 10^{5} \\
8.237 \times 10^{4} \\
3.905 \times 10^{4} \\
2.117 \times 10^{4} \\
1.250 \times 10^{4}\end{array}$ & $\begin{array}{l}1.616 \\
0.2609 \\
9.163 \times 10^{-2} \\
4.416 \times 10^{-2} \\
2.525 \times 10^{-2}\end{array}$ & $\begin{array}{l}\quad 9.931 \times 10^{4} \\
9514 \\
2588 \\
1069 \\
551.3\end{array}$ & $\begin{array}{l}2.1996 \\
1.4077 \\
0.9533 \\
0.6363 \\
0.3935\end{array}$ & $\begin{array}{l}\text { AA } \\
\text { AA } \\
\text { AA } \\
\text { AA } \\
\text { AA }\end{array}$ & $\begin{array}{l}1 \\
1 \\
1 \\
1 \\
1\end{array}$ \\
\hline $\begin{array}{l}7-13 \\
7-14 \\
7-15 \\
7-16 \\
7-17\end{array}$ & $\begin{array}{l}62902 \\
59552 \\
57099 \\
55237 \\
53783\end{array}$ & $\begin{array}{l}107440 \\
107440 \\
107440 \\
107440 \\
107440\end{array}$ & $\begin{array}{l}109030 \\
109119 \\
109191 \\
109250 \\
109299\end{array}$ & $\begin{array}{l}98 \\
98 \\
98 \\
98 \\
98\end{array}$ & $\begin{array}{l}338 \\
392 \\
450 \\
512 \\
578\end{array}$ & $\begin{array}{l}7845 \\
5156 \\
3516 \\
2471 \\
1781\end{array}$ & $\begin{array}{l}1.605 \times 10^{-2} \\
1.097 \times 10^{-2} \\
7.891 \times 10^{-3} \\
5.905 \times 10^{-3} \\
4.556 \times 10^{-3}\end{array}$ & $\begin{array}{c}325.7 \\
210.6 \\
145.3 \\
105.2 \\
79.03\end{array}$ & $\begin{array}{r}0.1967 \\
0.0313 \\
-0.1116 \\
-0.2376 \\
-0.3502\end{array}$ & $\begin{array}{l}\text { AA } \\
\text { AA } \\
\text { AA } \\
\text { AA } \\
\text { AA }\end{array}$ & $\begin{array}{l}1 \\
1 \\
1 \\
1 \\
1\end{array}$ \\
\hline $\begin{array}{l}7-18 \\
7-19 \\
7-20\end{array}$ & $\begin{array}{l}52622.5 \\
51679 \\
50899\end{array}$ & $\begin{array}{l}107440 \\
107440 \\
107440\end{array}$ & $\begin{array}{l}109340 \\
109375 \\
109405\end{array}$ & $\begin{array}{l}98 \\
98 \\
98\end{array}$ & $\begin{array}{l}648 \\
722 \\
800\end{array}$ & $\begin{array}{l}1312 \\
984.9 \\
751.7\end{array}$ & $\begin{array}{l}3.602 \times 10^{-3} \\
2.905 \times 10^{-3} \\
2.383 \times 10^{-3}\end{array}$ & $\begin{array}{l}61.13 \\
48.43 \\
39.13\end{array}$ & $\begin{array}{l}-0.4522 \\
-0.5456 \\
-0.6316\end{array}$ & $\begin{array}{l}\text { AA } \\
\text { AA } \\
\text { AA }\end{array}$ & $\begin{array}{l}1 \\
1 \\
1\end{array}$ \\
\hline $\begin{array}{l}8-9 \\
8-10 \\
8-11 \\
8-12 \\
8-13\end{array}$ & $\begin{array}{r}277960 \\
162050 \\
123840 \\
105010 \\
93894\end{array}$ & $\begin{array}{l}107965 \\
107965 \\
107965 \\
107965 \\
107965\end{array}$ & $\begin{array}{l}108325 \\
108582 \\
108772 \\
108917 \\
109030\end{array}$ & $\begin{array}{l}128 \\
128 \\
128 \\
128 \\
128\end{array}$ & $\begin{array}{l}162 \\
200 \\
242 \\
288 \\
338\end{array}$ & $\begin{array}{l}1.233 \times 10^{5} \\
4.676 \times 10^{4} \\
2.301 \times 10^{4} \\
1.287 \times 10^{4} \\
7804\end{array}$ & $\begin{array}{l}1.807 \\
0.2876 \\
0.1000 \\
4.787 \times 10^{-2} \\
2.724 \times 10^{-2}\end{array}$ & $\begin{array}{l}2.116 \times 10^{5} \\
1.964 \times 10^{4} \\
5217 \\
2117 \\
1077\end{array}$ & $\begin{array}{l}2.3642 \\
1.5661 \\
1.1072 \\
0.7873 \\
0.5424\end{array}$ & $\begin{array}{l}\text { AA } \\
\text { AA } \\
\text { AA } \\
\text { AA } \\
\text { AA }\end{array}$ & $\begin{array}{l}1 \\
1 \\
1 \\
1 \\
1\end{array}$ \\
\hline $\begin{array}{l}8-14 \\
8-15 \\
8-16 \\
8-17 \\
8-18 \\
8-19 \\
8-20\end{array}$ & $\begin{array}{l}86621 \\
81527 \\
77782 \\
74930 \\
72696 \\
70908 \\
69448\end{array}$ & $\begin{array}{l}107965 \\
107965 \\
107965 \\
107965 \\
107965 \\
107965 \\
107965\end{array}$ & $\begin{array}{l}109119 \\
109191 \\
109250 \\
109299 \\
109340 \\
109375 \\
109405\end{array}$ & $\begin{array}{l}128 \\
128 \\
128 \\
128 \\
128 \\
128 \\
128\end{array}$ & $\begin{array}{l}392 \\
450 \\
512 \\
578 \\
648 \\
722 \\
800\end{array}$ & $\begin{array}{l}5010 \\
3359 \\
2331 \\
1664 \\
1216 \\
906.9 \\
688.6\end{array}$ & $\begin{array}{l}1.726 \times 10^{-2} \\
1.177 \times 10^{-2} \\
8.456 \times 10^{-3} \\
6.323 \times 10^{-3} \\
4.877 \times 10^{-3} \\
3.856 \times 10^{-3} \\
3.112 \times 10^{-3}\end{array}$ & $\begin{array}{c}629.8 \\
404.1 \\
277.1 \\
199.6 \\
149.4 \\
115.2 \\
91.04\end{array}$ & $\begin{array}{r}0.3442 \\
0.1778 \\
0.0344 \\
-0.0919 \\
-0.2046 \\
-0.3066 \\
-0.3998\end{array}$ & $\begin{array}{l}\text { AA } \\
\text { AA } \\
\text { AA } \\
\text { AA } \\
\text { AA } \\
\text { AA } \\
\text { AA }\end{array}$ & $\begin{array}{l}1 \\
1 \\
1 \\
1 \\
1 \\
1 \\
1\end{array}$ \\
\hline
\end{tabular}


H-Table A. $(n)_{i}-(n)_{k}$ Transitions (Average Values)-Continued

\begin{tabular}{|c|c|c|c|c|c|c|c|c|c|c|c|}
\hline Transition & $\lambda(\AA)$ & $E_{i}\left(\mathrm{~cm}^{-1}\right)$ & $E_{k}\left(\mathrm{~cm}^{-1}\right)$ & $g_{i}$ & $g_{k}$ & $A_{k i}\left(\sec ^{-1}\right)$ & $f_{i k}$ & S(at.u.) & $\log g f$ & $\begin{array}{l}\text { Accu- } \\
\text { racy }\end{array}$ & Source \\
\hline $9-10$ & 388590 & 108325 & 108582 & 162 & 200 & $7.151 \times 10^{4}$ & 1.999 & $4.141 \times 10^{5}$ & 2.5103 & $\mathrm{AA}$ & ] \\
\hline $9-11$ & 223340 & 108325 & 108772 & 162 & 242 & $2.813 \times 10^{4}$ & 0.3143 & $3.742 \times 10^{4}$ & 1.7068 & $\mathrm{AA}$ & 1 \\
\hline $9-12$ & 168760 & 108325 & 108917 & 162 & 288 & $1.427 \times 10^{4}$ & 0.1083 & 9746 & 1.2442 & $\mathrm{AA}$ & 1 \\
\hline $9-13$ & 141790 & 108325 & 109030 & 162 & 338 & 8192 & $5.152 \times 10^{-2}$ & 3895 & 0.9215 & $\mathrm{AA}$ & 1 \\
\hline $9-14$ & 125840 & 108325 & 109119 & 162 & 392 & 5080 & $2.918 \times 10^{-2}$ & 1958 & 0.6746 & $\mathrm{AA}$ & 1 \\
\hline $9-15$ & 115360 & 108325 & 109191 & 162 & 450 & 3325 & $1.843 \times 10^{-2}$ & 1134 & 0.4750 & $\mathrm{AA}$ & 1 \\
\hline $9-16$ & 108010 & 108325 & 109250 & 162 & 512 & 2268 & $1.254 \times 10^{-2}$ & 721.9 & 0.3077 & $\mathrm{AA}$ & l \\
\hline $9-17$ & 102580 & 108325 & 109299 & 162 & 578 & 1598 & $8.995 \times 10^{-3}$ & 492.0 & 0.1635 & $\mathrm{AA}$ & 1 \\
\hline $9-18$ & 98443 & 108325 & 109340 & 162 & 648 & 1156 & $6.719 \times 10^{-3}$ & 352.7 & 0.0368 & $\mathrm{AA}$ & 1 \\
\hline $9-19$ & 95191 & 108325 & 109375 & 162 & 722 & 855.5 & $5.180 \times 10^{-3}$ & 262.9 & -0.0762 & $\mathrm{AA}$ & 1 \\
\hline $9-20$ & 92579 & 108325 & 109405 & 162 & 800 & 645.2 & $4.094 \times 10^{-3}$ & 202.1 & -0.1783 & $\mathrm{AA}$ & 1 \\
\hline $10-11$ & 525200 & 108582 & 108772 & 200 & 242 & $4.377 \times 10^{4}$ & 2.190 & $7.571 \times 10^{5}$ & 2.6415 & $\mathrm{AA}$ & 1 \\
\hline $10-12$ & 298310 & 108582 & 108917 & 200 & 288 & $1.774 \times 10^{4}$ & 0.3408 & $6.692 \times 10^{4}$ & 1.8335 & $\mathrm{AA}$ & 1 \\
\hline $10-13$ & 223250 & 108852 & 109030 & 200 & 338 & 9231 & 0.1166 & $1.713 \times 10^{4}$ & 1.3676 & $\mathrm{AA}$ & 1 \\
\hline $10-14$ & 186100 & 108582 & 109119 & 200 & 392 & 5417 & $5.513 \times 10^{-2}$ & 6753 & 1.0424 & $\mathrm{AA}$ & 1 \\
\hline $10-15$ & 164070 & 108582 & 109191 & 200 & 450 & 3424 & $3.109 \times 10^{-2}$ & 3358 & 0.7937 & $\mathrm{AA}$ & 1 \\
\hline $10-16$ & 149580 & 108582 & 109250 & 200 & 512 & 2280 & $1.958 \times 10^{-2}$ & 1927 & 0.5928 & $\mathrm{AA}$ & 1 \\
\hline $10-17$ & 139380 & 108582 & 109299 & 200 & 578 & 1578 & $1.328 \times 10^{-2}$ & 1219 & 0.4243 & $\mathrm{AA}$ & 1 \\
\hline $10-18$ & 131840 & 108582 & 109340 & 200 & 648 & 1127 & $9.515 \times 10^{-3}$ & 825.8 & 0.2794 & $\mathrm{AA}$ & 1 \\
\hline $10-19$ & 126080 & 108582 & 109375 & 200 & 722 & 825.2 & $7.099 \times 10^{-3}$ & $\begin{array}{l}589.0 \\
589.2\end{array}$ & 0.1522 & $\mathrm{AA}$ & 1 \\
\hline $10-20$ & $12 \mathbf{1 5 3 0}$ & 108582 & 109405 & 200 & 800 & 617.3 & $5.468 \times 10^{-3}$ & 437.4 & 0.0389 & $\mathrm{AA}$ & 1 \\
\hline $11-12$ & 690500 & 108772 & 108917 & 242 & 288 & $2.799 \times 10^{4}$ & 2.381 & $\times 10^{6}$ & 2.7606 & AA & 1 \\
\hline $11-13$ & 388320 & 108772 & 109030 & 242 & 338 & $1.163 \times 10^{4}$ & 0.3673 & $1.136 \times 10^{5}$ & 1.9489 & $\mathrm{AA}$ & 1 \\
\hline $11-14$ & 288230 & 108772 & 109119 & 242 & 392 & 6186 & 0.1248 & $2.865 \times 10^{4}$ & 1.4800 & $\mathrm{AA}$ & 1 \\
\hline $11-15$ & 238620 & 108772 & 109191 & 242 & 450 & 3699 & $5.872 \times 10^{-2}$ & 1.11 & 1.1526 & $\mathrm{AA}$ & 1 \\
\hline $11-16$ & 209150 & 108772 & 109250 & 242 & 512 & 2377 & $3.298 \times 10^{-2}$ & 5495 & 0.9021 & $\mathrm{AA}$ & 1 \\
\hline $11-17$ & 189730 & 108772 & 109299 & 242 & 578 & 1606 & $2.070 \times 10^{-2}$ & 3129 & 0.6999 & $\mathrm{AA}$ & 1 \\
\hline $11-18$ & 176030 & 108772 & 109340 & 242 & 648 & 1127 & $1.402 \times 10^{-2}$ & 1965 & 0.5304 & $\mathrm{AA}$ & 1 \\
\hline $11-19$ & 165900 & 108772 & 109375 & 242 & 722 & 814.1 & $1.002 \times 10^{-2}$ & 1324 & 0.3848 & $\mathrm{AA}$ & 1 \\
\hline $11-20$ & 158120 & 108772 & 109405 & 242 & 800 & 602.6 & $7.468 \times 10^{-3}$ & 940.5 & 0.2570 & $\mathrm{AA}$ & 1 \\
\hline $12-13$ & 887300 & 108917 & 109030 & 288 & 338 & $1.857 \times 10^{4}$ & 2.572 & $2.163 \times 10^{6}$ & 2.8697 & $\mathrm{AA}$ & 1 \\
\hline & 494740 & 108917 & 109119 & 288 & 392 & 7884 & 0.3938 & 1.8 & 2.0547 & $\mathrm{AA}$ & 1 \\
\hline $12-15$ & 364610 & 108917 & 109191 & 288 & 450 & 4271 & 0.1330 & $4.596 \times 10^{4}$ & 1.5832 & $\mathrm{AA}$ & 1 \\
\hline $12-16$ & 300020 & 108917 & 109250 & 288 & 512 & 2596 & $6.228 \times 10^{-2}$ & $1.771 \times 10^{4}$ & 1.2538 & $\mathrm{AA}$ & 1 \\
\hline $12-17$ & 261610 & 108917 & 109299 & 288 & 578 & 1693 & $3.486 \times 10^{-2}$ & 8644 & 1.0017 & $\mathrm{AA}$ & 1 \\
\hline $12-18$ & 236260 & 108917 & 109340 & 288 & 648 & 1159 & $2.182 \times 1 \overline{0}^{-2}$ & 4886 & 0.7982 & $\mathrm{AA}$ & 1 \\
\hline $12-19$ & 218360 & 108917 & 109375 & 288 & 722 & 822.3 & $1.474 \times 10^{-2}$ & 3050 & 0.6278 & $\mathrm{AA}$ & 1 \\
\hline $12-20$ & 205090 & 108917 & 109405 & 288 & 800 & 600.5 & $1.052 \times 10^{-2}$ & 2045 & 0.4814 & $\mathrm{AA}$ & 1 \\
\hline $13-14$ & 1118000 & 109030 & 109119 & 338 & 392 & $1.271 \times 10^{4}$ & 2.76 & $34:$ & 2.9703 & $A A$ & 1 \\
\hline & 619000 & 109030 & 109191 & 338 & 450 & 5496 & 0.42 & & 2.1524 & $\mathrm{AA}$ & 1 \\
\hline $13-16$ & 453290 & 109030 & 109250 & 338 & 512 & 302 & 0.1412 & $7.119 \times 10^{4}$ & 1.6787 & $\mathrm{AA}$ & 1 \\
\hline 17 & 371000 & 109030 & 109299 & 338 & 578 & 186 & $6.584 \times 10^{-2}$ & $2.717 \times 10^{4}$ & 1.3474 & $\mathrm{AA}$ & 1 \\
\hline & 322000 & 109030 & 109340 & 338 & 648 & 1232 & $3.672 \times 10^{-2}$ & $1.316 \times 10^{4}$ & 1.0939 & $\mathrm{AA}$ & 1 \\
\hline $13-19$ & 289640 & 109030 & 109375 & 338 & 722 & 853.2 & $2.292 \times 10^{-2}$ & 7386 & 0.8892 & $\mathrm{AA}$ & 1 \\
\hline $13-20$ & 266740 & 109030 & 109405 & 338 & 800 & 611.9 & $1.545 \times 10^{-2}$ & 4584 & 0.7178 & $\mathrm{AA}$ & 1 \\
\hline $14-$ & 1386000 & 109119 & 109191 & 392 & 450 & $89:$ & $29:$ & $5284 \times 10^{6}$ & 3.0637 & $A A$ & 1 \\
\hline & 762300 & 109119 & 109250 & 39 & 512 & $399^{\circ}$ & $\begin{array}{l}2.954 \\
0.4467\end{array}$ & $4.393 \times 10^{5}$ & 2.2433 & $\mathrm{AA}$ & 1 \\
\hline $14-1$ & 555200 & 109119 & 109299 & 392 & 578 & 2192 & 0.1494 & $1.070 \times 10^{5}$ & 1.7675 & $\mathrm{AA}$ & 1 \\
\hline $14-18$ & 452220 & 109119 & 109340 & 392 & 648 & 1369 & $6.938 \times 10^{-2}$ & $4.048 \times 10^{4}$ & 1.4345 & $A A$ & l \\
\hline
\end{tabular}


H-Table A. $(n)_{i}-(n)_{k}$ Transitions (Average Values)-Continued

\begin{tabular}{|c|c|c|c|c|c|c|c|c|c|c|c|}
\hline Transition & $\lambda(\AA)$ & $E_{i}\left(\mathrm{~cm}^{-1}\right)$ & $E_{k}\left(\mathrm{~cm}^{-1}\right)$ & $g_{i}$ & $g_{k}$ & $A_{k i}\left(\sec ^{-1}\right)$ & $f_{i k}$ & $S$ (at.u.) & $\log g f$ & $\begin{array}{l}\text { Accu- } \\
\text { racy }\end{array}$ & Source \\
\hline $14-19$ & 390880 & 109119 & 109375 & 392 & 722 & 914.4 & $3.858 \times 10^{-2}$ & $1.946 \times 10^{4}$ & 1.1796 & $\mathrm{AA}$ & 1 \\
\hline $14-20$ & 350300 & 109119 & 109405 & 392 & 800 & 639.7 & $2.402 \times 10^{-2}$ & $1.086 \times 10^{4}$ & 0.9738 & $\mathrm{AA}$ & 1 \\
\hline $15-16$ & 1694000 & 109191 & 109250 & 450 & 512 & 6429 & 3.145 & $7.889 \times 10^{6}$ & 3.1509 & AA & 1 \\
\hline $15-17$ & 926100 & 109191 & 109299 & 450 & 578 & 2864 & 0.4731 & $6.489 \times 10^{5}$ & 2.3281 & $\mathrm{AA}$ & 1 \\
\hline $15-18$ & 671200 & 109191 & 109340 & 450 & 648 & 1620 & 0.1575 & $1.566 \times 10^{5}$ & 1.8505 & AA & 1 \\
\hline $15-19$ & 544400 & 109191 & 109375 & 450 & 722 & 1023 & $7.292 \times 10^{-2}$ & $5.879 \times 10^{4}$ & 1.5160 & $\mathrm{AA}$ & 1 \\
\hline $15-20$ & 468760 & 109191 & 109405 & 450 & 800 & 690.3 & $4.043 \times 10^{-2}$ & $2.807 \times 10^{4}$ & 1.2599 & AA & 1 \\
\hline $16-17$ & 2044000 & 109250 & 109299 & 512 & 578 & 4720 & 3.336 & $1.149 \times 10^{7}$ & 3.2325 & AA & 1 \\
\hline $16-18$ & 1112000 & 109250 & 109340 & 512 & 648 & 2130 & 0.4995 & $9.358 \times 10^{5}$ & 2.4078 & $\mathrm{AA}$ & 1 \\
\hline $16-19$ & 802300 & 109250 & 109375 & 512 & 722 & 1217 & 0.1657 & $2.240 \times 10^{5}$ & 1.9285 & $\mathrm{AA}$ & 1 \\
\hline $16-20$ & 648200 & 109250 & 109405 & 512 & 800 & 776.7 & $7.644 \times 10^{-2}$ & $8.349 \times 10^{4}$ & 1.5926 & $\mathrm{AA}$ & 1 \\
\hline $17-18$ & 2438000 & 109299 & 109340 & 578 & 648 & 3530 & 3.527 & $1.636 \times 10^{7}$ & 3.3094 & $\mathrm{AA}$ & 1 \\
\hline $17-19$ & 1321000 & 109299 & 109375 & 578 & 722 & 1610 & 0.5259 & $1.321 \times 10^{6}$ & 2.4828 & $\mathrm{AA}$ & 1 \\
\hline $17-20$ & 949200 & 109299 & 109405 & 578 & 800 & 929.6 & 0.1738 & $3.139 \times 10^{5}$ & 2.0020 & $\mathrm{AA}$ & 1 \\
\hline & 2882000 & 109340 & 109375 & 648 & 722 & 2680 & 3.718 & $2.285 \times 10^{7}$ & 3.3819 & $\mathrm{AA}$ & 1 \\
\hline $18-20$ & 1554000 & 109340 & 109405 & 648 & 800 & 1235 & 0.5523 & $1.831 \times 10^{6}$ & 2.5537 & $\mathrm{AA}$ & 1 \\
\hline $19-20$ & 3374000 & 109375 & 109405 & 722 & 800 & 2067 & 3.909 & $3.134 \times 10^{7}$ & 3.4506 & $\mathrm{AA}$ & 1 \\
\hline
\end{tabular}

$\mathbf{H}-$ Table B. $(n l)_{i}-\left(n l_{k}\right.$ Transitions

\begin{tabular}{|c|c|c|c|c|c|c|c|c|c|c|c|}
\hline Transition & $\lambda(\AA)$ & $E_{i}\left(\mathrm{~cm}^{-1}\right)$ & $E_{k}\left(\mathrm{~cm}^{-1}\right)$ & $g_{i}$ & $g_{k}$ & $A_{k i}\left(\sec ^{-1}\right)$ & $f_{i k}$ & $S$ (at.u.) & $\log g f$ & $\begin{array}{l}\text { Accu- } \\
\text { racy }\end{array}$ & Source \\
\hline $1 s-2 p$ & 1215.67 & 0 & 82259 & 2 & 6 & $6.265 \times 10^{8}$ & 0.4162 & 3.330 & -0.0797 & $\mathrm{AA}$ & 1 \\
\hline $1 s-3 p$ & 1025.72 & 0 & 97492 & 2 & 6 & $1.672 \times 10^{8}$ & $7.910 \times 10^{-2}$ & $\begin{array}{l}0.530 \\
0.5339\end{array}$ & -0.8008 & $\mathrm{AA}$ & 1 \\
\hline $1 s-4 p$ & 972.537 & 0 & 102824 & 2 & 6 & $6.818 \times 10^{7}$ & $2.899 \times 10^{-2}$ & 0.1855 & -1.2367 & $\mathrm{AA}$ & 1 \\
\hline $1 s-5 p$ & 949.743 & 0 & 105292 & 2 & 6 & $3.437 \times 10^{7}$ & $1.394 \times 10^{-2}$ & $8.711 \times 10^{-2}$ & -1.5548 & $\mathrm{AA}$ & 1 \\
\hline $1 s-6 p$ & 937.804 & 0 & 106632 & 2 & 6 & $1.973 \times 10^{7}$ & $7.800 \times 10^{-3}$ & $4.813 \times 10^{-2}$ & -1.8069 & $\mathrm{AA}$ & 1 \\
\hline $2 p-3 s$ & 6562.86 & 82259 & 97492 & 6 & 2 & $6.313 \times 10^{6}$ & $1.359 \times 10^{-2}$ & 1.761 & -1.0886 & $\mathrm{AA}$ & 1 \\
\hline & 4861.35 & 82259 & 102824 & 6 & 2 & $2.578 \times 10^{6}$ & $3.045 \times 10^{-3}$ & 0.2923 & -1.7383 & AA & 1 \\
\hline $2 p-5 s$ & 4340.48 & 82259 & 105292 & 6 & 2 & $1.289 \times 10^{6}$ & $1.213 \times 10^{-3}$ & 0.1040 & $\begin{array}{r}-2.1379 \\
\end{array}$ & $\mathrm{AA}$ & 1 \\
\hline $2 p-6 s$ & 4101.75 & 82259 & 106632 & 6 & 2 & $7.350 \times 10^{5}$ & $6.180 \times 10^{-4}$ & $5.006 \times 10^{-2}$ & -2.4309 & $\mathrm{AA}$ & 1 \\
\hline $2 s-3 p$ & 6562.74 & 82259 & 97492 & 2 & 6 & $2.245 \times 10^{7}$ & 0.4349 & 18.79 & -0.0606 & $\mathrm{AA}$ & 1 \\
\hline $2 s-4 p$ & 4861.29 & 82259 & 102824 & 2 & 6 & $9.668 \times 10^{6}$ & 0.1028 & 3.288 & -0.6871 & $\mathrm{AA}$ & 1 \\
\hline $2 s-5 p$ & 4340.44 & 82259 & 105292 & 2 & 6 & $4.948 \times 10^{6}$ & $4.193 \times 10^{-2}$ & $\begin{array}{l}-.200 \\
1.198\end{array}$ & -1.0764 & $\mathrm{AA}$ & 1 \\
\hline $2 s-6 p$ & 4101.71 & 82259 & 106632 & 2 & 6 & $2.858 \times 10^{6}$ & $2.163 \times 10^{-2}$ & 0.5840 & -1.3639 & $\mathrm{AA}$ & 1 \\
\hline $2 p-3 d$ & 6562.81 & 82259 & 97492 & 6 & 10 & $6.465 \times 10^{7}$ & 0.6958 & 90.17 & 0.6206 & $\mathrm{AA}$ & 1 \\
\hline $2 p-4 d$ & 4861.33 & 82259 & 102824 & 6 & 10 & $2.062 \times 10^{7}$ & 0.1218 & 11.69 & -0.1362 & $\mathrm{AA}$ & 1 \\
\hline $2 p-5 d$ & 4340.47 & 82259 & 105292 & 6 & 10 & $9.425 \times 10^{6}$ & $4.437 \times 10^{-2}$ & 3.803 & -0.5748 & $\mathrm{AA}$ & 1 \\
\hline $2 p-6 d$ & 4101.74 & 82259 & 106632 & 6 & 10 & $5.145 \times 10^{6}$ & $2.163 \times 10^{-2}$ & $\begin{array}{l}1.000 \\
1.752\end{array}$ & -0.8868 & AA & 1 \\
\hline
\end{tabular}


H-Table B. $(n l)_{i}-(n l)_{k}$ Transitions. - Continued

\begin{tabular}{|c|c|c|c|c|c|c|c|c|c|c|c|}
\hline Transition & $\lambda(\hat{A})$ & $E_{i}\left(\mathrm{~cm}^{-1}\right)$ & $E_{k}\left(\mathrm{~cm}^{-1}\right)$ & $g_{i}$ & $g_{k}$ & $A_{k i}\left(\sec ^{-1}\right)$ & $f_{i k}$ & S(at.u.) & $\log g f$ & $\begin{array}{c}\text { Accu- } \\
\text { racy }\end{array}$ & Source \\
\hline $3 s-4 p$ & 18750.8 & 97492 & 102824 & 2 & 6 & $3.065 \times 10^{6}$ & 0.4847 & 59.83 & -0.0135 & $A \mathrm{~A}$ & 1 \\
\hline $3 s-5 p$ & 12818.0 & 97492 & 105292 & 2 & 6 & $1.638 \times 10^{6}$ & 0.1210 & 10.21 & -0.6161 & $\mathrm{AA}$ & 1 \\
\hline $3 s-6 p$ & 10938.0 & 97492 & 106632 & 2 & 6 & $9.55] \times 10^{5}$ & $5.139 \times 10^{-2}$ & 3.700 & -0.9881 & $A A$ & 1 \\
\hline $3 p-4 s$ & 18751.1 & 97492 & 102824 & 6 & 2 & $1.835 \times 10^{6}$ & $3.225 \times 10^{-2}$ & 11.94 & -0.7133 & $\mathrm{AA}$ & 1 \\
\hline $3 p-5 s$ & 12818.1 & 97492 & 105292 & 6 & 2 & $9.046 \times 10^{5}$ & $7.428 \times 10^{-3}$ & 1.880 & -1.3510 & $\mathrm{AA}$ & 1 \\
\hline $3 p-6 s$ & 10938.1 & 97492 & 106632 & 6 & 2 & $5.071 \times 10^{5}$ & $3.032 \times 10^{-3}$ & 0.6550 & -1.7401 & $\mathrm{AA}$ & 1 \\
\hline $3 p-4 d$ & 18750.9 & 97492 & 102824 & 6 & 10 & $7.037 \times 10^{6}$ & 0.6183 & 228.9 & 0.5693 & $A \mathrm{~A}$ & 1 \\
\hline $3 p-5 d$ & 12818.0 & 97492 & 105292 & 6 & 10 & $3.391 \times 10^{6}$ & 0.1392 & 35.24 & -0.0781 & AA & 1 \\
\hline $3 p-6 d$ & 10938.1 & 97492 & 106632 & 6 & 10 & $1.878 \times 10^{6}$ & $5.614 \times 10^{-2}$ & 12.13 & -0.4726 & AA & I \\
\hline $3 d-4 p$ & 18751.2 & 97492 & 102824 & 10 & 6 & $3.475 \times 10^{5}$ & $1.099 \times 10^{-2}$ & 6.783 & -0.9589 & $\mathrm{AA}$ & 1 \\
\hline $3 d-5 p$ & 12818.2 & 97492 & 105292 & 10 & 6 & $1.495 \times 10^{5}$ & $2.210 \times 10^{-3}$ & 0.9324 & -1.6556 & AA & 1 \\
\hline $3 d-6 p$ & 10938.1 & 97492 & 106632 & 10 & 6 & $7.824 \times 10^{4}$ & $8.420 \times 10^{-4}$ & 0.3031 & -2.0747 & $\mathrm{AA}$ & 1 \\
\hline $3 d-4 f$ & 18751.1 & 97492 & 102824 & 10 & 14 & $1.379 \times 10^{7}$ & 1.018 & 628.0 & 1.0075 & $\mathrm{AA}$ & 1 \\
\hline $3 d-5 f$ & 12818.1 & 97492 & 105292 & 10 & 14 & $4.542 \times 10^{6}$ & 0.1566 & 66.08 & 0.1949 & AA & 1 \\
\hline $3 d-6 f$ & 10938.1 & 97492 & 106632 & 10 & 14 & $2.146 \times 10^{6}$ & $5.389 \times 10^{-2}$ & 19.40 & -0.2685 & AA & 1 \\
\hline $4 s-5 p$ & 40511 & 102824 & 105292 & 2 & 6 & $7.372 \times 10^{5}$ & 0.5442 & 145.1 & 0.0368 & AA & 1 \\
\hline $4 s-6 p$ & 26251 & 102824 & 106632 & 2 & 6 & $4.456 \times 10^{5}$ & 0.1381 & 23.87 & -0.5587 & AA & 1 \\
\hline $4 p-5 s$ & 40512 & 102824 & 105292 & 6 & 2 & $6.450 \times 10^{5}$ & $5.291 \times 10^{-2}$ & 42.33 & -0.4983 & AA & 1 \\
\hline $4 p-6 s$ & 26251 & 102824 & 106632 & 6 & 2 & $3.582 \times 10^{5}$ & $1.234 \times 10^{-2}$ & 6.396 & -1.1306 & AA & 1 \\
\hline $4 p-5 d$ & 40511 & 102824 & 105292 & 6 & 10 & $1.486 \times 10^{6}$ & 0.6093 & 487.4 & 0.5630 & $\mathrm{AA}$ & 1 \\
\hline $4 p-6 d$ & 26251 & 102824 & 106632 & 6 & 10 & $8.622 \times 10^{5}$ & 0.1485 & 76.96 & -0.0502 & $\mathrm{AA}$ & 1 \\
\hline $4 d-5 p$ & 40512 & 102824 & 105292 & 10 & 6 & $1.884 \times 10^{5}$ & $2.782 \times 10^{-2}$ & 37.10 & -0.5556 & AA & 1 \\
\hline $4 d-6 p$ & 26252 & 102824 & 106632 & 10 & 6 & $9.416 \times 10^{4}$ & $5.837 \times 10^{-3}$ & 5.044 & -1.2338 & $\mathrm{AA}$ & 1 \\
\hline $4 d-5 f$ & 40512 & 102824 & 105292 & 10 & 14 & $2.584 \times 10^{6}$ & 0.8903 & 1187 & 0.9495 & AA & 1 \\
\hline $4 d-6 f$ & 26252 & 102824 & 106632 & 10 & 14 & $1.287 \times 10^{6}$ & 0.1862 & 160.8 & 0.2699 & $\mathrm{AA}$ & 1 \\
\hline $4 f-5 d$ & 40512 & 102824 & 105292 & 14 & 10 & $5.047 \times 10^{4}$ & $8.871 \times 10^{-3}$ & 16.56 & -0.9059 & $\mathrm{AA}$ & 1 \\
\hline $4 f-6 d$ & 26252 & 102824 & 106632 & 14 & 10 & $2.145 \times 10^{4}$ & $1.583 \times 10^{-3}$ & 1.915 & -1.6544 & $\mathrm{AA}$ & 1 \\
\hline $4 f-5 g$ & 40512 & 102824 & 105292 & 14 & 18 & $4.254 \times 10^{6}$ & 1.346 & 2512 & 1.2751 & $\mathrm{AA}$ & 1 \\
\hline $4 f-6 g$ & 26252 & 102824 & 106632 & 14 & 18 & $1.373 \times 10^{6}$ & 0.1824 & 220.6 & 0.4070 & AA & 1 \\
\hline $5 s-6 p$ & 74577 & 105292 & 106632 & 2 & 6 & $2.430 \times 10^{5}$ & 0.6078 & 298.4 & 0.0848 & $A A$ & 1 \\
\hline $5 p-6 s$ & 74578 & 105292 & 106632 & 6 & 2 & $2.682 \times 10^{5}$ & $7.454 \times 10^{-2}$ & 109.8 & -0.3495 & Ad & 1 \\
\hline $5 p-6 d$ & 74578 & 105292 & 106632 & 6 & 10 & $4.495 \times 10^{5}$ & 0.6247 & 920.0 & 0.5738 & AA & 1 \\
\hline $5 d-6 p$ & 74579 & 105292 & 106632 & 10 & 6 & $9.593 \times 10^{4}$ & $4.800 \times 10^{-2}$ & 117.8 & -0.3188 & $A A$ & 1 \\
\hline $5 d-6 f$ & 74578 & 105292 & 106632 & 10 & 34 & $7.232 \times 10^{5}$ & 0.8443 & 2072 & 0.9265 & AA & 1 \\
\hline $5 f-6 d$ & 74579 & 105292 & 106632 & 14 & 10 & $3.908 \times 10^{4}$ & $2.328 \times 10^{-2}$ & 79.98 & -0.4870 & $\mathrm{AA}$ & 1 \\
\hline $5 f-6 g$ & 74578 & 105292 & 106632 & 14 & 18 & $1.106 \times 10^{6}$ & 1.185 & 4073 & 1.2200 & $\mathrm{AA}$ & 1 \\
\hline $5 g-6 f$ & 74579 & 105292 & 106632 & 18 & 14 & $1.137 \times 10^{4}$ & $7.376 \times 10^{-3}$ & 32.59 & -0.8769 & $\mathrm{AA}$ & 1 \\
\hline $5 g-6 h$ & 74578 & 105292 & 106632 & 18 & 22 & $1.645 \times 10^{6}$ & 1.676 & 7406 & 1.4796 & $A A$ & 1 \\
\hline
\end{tabular}


H-Table C. $\quad(n l j)_{i}-(n l j)_{k}$ Transitions (Fine Structure Lines)

\begin{tabular}{|c|c|c|c|c|c|c|c|c|c|c|}
\hline Transition & $\nu(\mathrm{Mc} / \mathrm{sec})$ & $\Delta E\left(\mathrm{~cm}^{-1}\right)$ & $g_{i}$ & $g_{k}$ & $A_{k i}\left(\sec ^{-1}\right)$ & $f_{i k}$ & $S$ (at.u.) & $\log g f$ & $\begin{array}{c}\text { Accu- } \\
\text { racy }\end{array}$ & Source \\
\hline $2 s^{2} \mathrm{~S}_{1 / 2}-2 p^{2} \mathrm{P}_{3 / 2}^{\circ}$ & 9884 & 0.3297 & 2 & 4 & $6.54 \times 10^{-7}$ & $1.80 \times 10^{-5}$ & 36.0 & -4.443 & A & 2 \\
\hline $3 s^{2} \mathrm{~S}_{1 / 2}-3 p^{2} \mathrm{P}_{3 / 2}^{\circ}$ & 2944 & 0.0982 & 2 & 4 & $1.04 \times 10^{-7}$ & $3.22 \times 10^{-5}$ & 216 & -4.191 & A & 2 \\
\hline $3 p^{2} \mathrm{P}_{1 / 2}^{\circ}-3 d^{2} \mathrm{D}_{3 / 2}$ & 3244 & 0.1082 & 2 & 4 & $8.67 \times 10^{-8}$ & $2.22 \times 10^{-5}$ & 135 & -4.353 & A & 2 \\
\hline $3 p^{2} \mathrm{P}^{\circ}{ }_{3 / 2}-3 d^{2} \mathrm{D}_{5 / 2}$ & 1082 & 0.0361 & 4 & 6 & $3.86 \times 10^{-9}$ & $6.67 \times 10^{-6}$ & 243 & -4.574 & A & 2 \\
\hline $4 s^{2} \mathrm{~S}_{1 / 2}-4 p^{2} \mathrm{P}_{3 / 2}^{\circ}$ & 1247 & 0.0416 & 2 & 4 & $2.63 \times 10^{-8}$ & $4.55 \times 10^{-5}$ & 720 & -4.041 & A & 2 \\
\hline $4 p^{2} \mathrm{P}^{\circ}{ }_{1 / 2}-4 d^{2} \mathrm{D}_{3 / 2}$ & 1367 & 0.0456 & 2 & 4 & $2.77 \times 10^{-8}$ & $3.99 \times 10^{-5}$ & 576 & -4.098 & A & 2 \\
\hline $4 p^{2} \mathrm{P}_{3 / 2}^{\circ}-4 d^{2} \mathrm{D}_{5 / 2}$ & 456 & 0.0152 & 4 & 6 & $1.23 \times 10^{-9}$ & $1.20 \times 10^{-5}$ & 1040 & -4.320 & A & 2 \\
\hline $4 d^{2} \mathrm{D}_{3 / 2}-4 f^{2} \mathrm{~F}_{5 / 2}^{\circ}$ & 456 & 0.0152 & 4 & 6 & $7.18 \times 10^{-10}$ & $6.98 \times 10^{-6}$ & 605 & -4.554 & A & 2 \\
\hline $4 d^{2} \mathrm{D}_{5 / 2}-4 f^{2} \mathrm{~F}^{\circ}{ }_{7 / 2}$ & 228 & 0.0076 & 6 & 8 & $9.61 \times 10^{-11}$ & $3.33 \times 10^{-6}$ & 864 & -4.700 & A & 2 \\
\hline
\end{tabular}

H-Table D. $(n l j f)_{i}-(n l j f)_{k}$ Transition (Hyperfine Structure Line, Magnetic Dipole Transition)

\begin{tabular}{c|c|c|c|c|c|c|c|c|c|c}
\hline \hline Transition & $\nu(\mathrm{Mc} / \mathrm{sec})$ & $\Delta E\left(\mathrm{~cm}^{-1}\right)$ & $g_{i}$ & $g_{k}$ & $A_{k i}\left(\mathrm{sec}^{-1}\right)$ & $f_{i k}$ & $S($ at.u. $)$ & $\log g f$ & $\begin{array}{c}\text { Accu- } \\
\text { racy }\end{array}$ & Source \\
\hline $1 s^{2} \mathrm{~S}_{1 / 2}(f=0 \rightarrow f=1)$ & 1420.4 & 0.04738 & 1 & 3 & $2.87 \times 10^{-15}$ & $5.75 \times 10^{-12}$ & 3.00 & -11.241 & $\mathrm{~A}$ & 2 \\
\hline
\end{tabular}


HELIUM

\section{He I}

Ground State

$1 s^{2}{ }^{1} \mathrm{~S}_{0}$

Ionization Potential

$24.580 \mathrm{eV}=198305 \mathrm{~cm}^{-1}$

Allowed Transitions

List of tabulated lines:

\begin{tabular}{|c|c|c|c|c|c|}
\hline Wavelength $[\AA]$ & No. & Wavelength $[\AA]$ & No. & Wavelength $[\AA]$ & No. \\
\hline $\begin{array}{l}506.200 \\
506.570 \\
507.058 \\
507.718 \\
508.643\end{array}$ & $\begin{array}{r}11 \\
10 \\
9 \\
8 \\
7\end{array}$ & $\begin{array}{l}4387.93 \\
4437.55 \\
4471.5 \\
4713.2 \\
4921.93\end{array}$ & $\begin{array}{l}29 \\
23 \\
86 \\
80 \\
28\end{array}$ & $\begin{array}{l}19543 \\
20425 \\
20581.3 \\
21120 \\
21132.0\end{array}$ & $\begin{array}{r}105 \\
113 \\
12 \\
97 \\
39\end{array}$ \\
\hline $\begin{array}{l}509.998 \\
512.098 \\
515.617 \\
522.213 \\
537.030\end{array}$ & $\begin{array}{l}6 \\
5 \\
4 \\
3 \\
2\end{array}$ & $\begin{array}{l}5015.68 \\
5047.74 \\
5875.7 \\
6678.15 \\
7065.19\end{array}$ & $\begin{array}{l}13 \\
22 \\
85 \\
27 \\
79\end{array}$ & $\begin{array}{l}21494 \\
21608 \\
21617 \\
21841 \\
22284\end{array}$ & $\begin{array}{r}116 \\
126 \\
67 \\
60 \\
57\end{array}$ \\
\hline $\begin{array}{l}584.334 \\
2677.1 \\
2696.1 \\
2723.2 \\
2763.8\end{array}$ & $\begin{array}{r}1 \\
78 \\
77 \\
76 \\
75\end{array}$ & $\begin{array}{l}7065.71 \\
7281.35 \\
8361.77 \\
9463.57 \\
9603.42\end{array}$ & $\begin{array}{l}79 \\
21 \\
96 \\
95 \\
38\end{array}$ & $\begin{array}{l}23063 \\
24727 \\
26113 \\
26185 \\
26198\end{array}$ & $\begin{array}{r}54 \\
120 \\
63 \\
125 \\
66\end{array}$ \\
\hline $\begin{array}{l}2829.07 \\
2945.10 \\
3187.74 \\
3231.27 \\
3258.28\end{array}$ & $\begin{array}{l}74 \\
73 \\
72 \\
20 \\
19\end{array}$ & $\begin{array}{l}9702.66 \\
10311 \\
10667.6 \\
10829.1 \\
10830.2\end{array}$ & $\begin{array}{r}100 \\
104 \\
99 \\
70 \\
70\end{array}$ & $\begin{array}{l}26248 \\
26251 \\
26531 \\
26671 \\
26881\end{array}$ & $\begin{array}{r}69 \\
128 \\
59 \\
122 \\
115\end{array}$ \\
\hline $\begin{array}{l}3296.77 \\
3354.55 \\
3447.59 \\
3554.4 \\
3562.95\end{array}$ & $\begin{array}{l}18 \\
17 \\
16 \\
92 \\
84\end{array}$ & $\begin{array}{l}10830.3 \\
10902.2 \\
10912.9 \\
10917.0 \\
10996.6\end{array}$ & $\begin{array}{r}70 \\
48 \\
110 \\
51 \\
107\end{array}$ & $\begin{array}{l}27600 \\
28542 \\
33299 \\
37026 \\
40053\end{array}$ & $\begin{array}{r}56 \\
112 \\
53 \\
119 \\
62\end{array}$ \\
\hline $\begin{array}{l}3587.3 \\
3613.64 \\
3634.2 \\
3652.0 \\
3705.0\end{array}$ & $\begin{array}{l}91 \\
15 \\
90 \\
83 \\
89\end{array}$ & $\begin{array}{l}11013.1 \\
11045.0 \\
11225.9 \\
11969.1 \\
12528\end{array}$ & $\begin{array}{r}37 \\
44 \\
41 \\
103 \\
94\end{array}$ & $\begin{array}{l}40365 \\
40396 \\
40536 \\
40550 \\
41216\end{array}$ & $\begin{array}{r}124 \\
65 \\
68 \\
127 \\
58\end{array}$ \\
\hline $\begin{array}{l}3819.6 \\
3833.55 \\
3867.5 \\
3871.79 \\
3888.65\end{array}$ & $\begin{array}{l}88 \\
34 \\
82 \\
33 \\
71\end{array}$ & $\begin{array}{l}12755.7 \\
12785 \\
12790.3 \\
12846 \\
12968.4\end{array}$ & $\begin{array}{r}47 \\
109 \\
50 \\
98 \\
43\end{array}$ & $\begin{array}{l}42430 \\
42497 \\
46053 \\
46936 \\
74351\end{array}$ & $\begin{array}{r}121 \\
93 \\
55 \\
114 \\
35\end{array}$ \\
\hline $\begin{array}{l}3926.53 \\
3935.91 \\
3964.73 \\
4009.27 \\
4023.97\end{array}$ & $\begin{array}{l}32 \\
26 \\
14 \\
31 \\
25\end{array}$ & $\begin{array}{l}12985 \\
13411.8 \\
15083.7 \\
17002 \\
18555.6\end{array}$ & $\begin{array}{r}106 \\
40 \\
36 \\
102 \\
46\end{array}$ & $\begin{array}{l}108800 \\
180950 \\
186200 \\
439440 \\
957600\end{array}$ & $\begin{array}{r}111 \\
52 \\
101 \\
118 \\
45\end{array}$ \\
\hline $\begin{array}{l}4026.2 \\
4120.8 \\
4143.76 \\
4168.97\end{array}$ & $\begin{array}{l}87 \\
81 \\
30 \\
24\end{array}$ & $\begin{array}{l}18686 \\
18696.9 \\
19063 \\
19089.4\end{array}$ & $\begin{array}{r}108 \\
49 \\
117 \\
42\end{array}$ & $\begin{array}{l}2.16 \times 10^{6} \\
1.39 \times 10^{7} \\
1.82 \times 10^{7}\end{array}$ & $\begin{array}{r}61 \\
123 \\
64\end{array}$ \\
\hline
\end{tabular}


Aside from hydrogen, extremely precise $f$-values exist only for a few lines of helium. These are the result of extensive variational calculations (up to 220 parameter wave functions) of Schiff and Pekeris, [1] which give an agreement of $1 \%$ or less between the dipole length, velocity, and acceleration forms of the transition matrix element. Similar calculations have been undertaken for a few other transitions by Weiss, [2] but are not quite as refined (up to 54 parameter wave functions) and the agreement between the different forms of the matrix element is not quite as good.

For transitions to higher excited states, recourse has to be taken to somewhat less elaborate theoretical approximations. The following work has been used: Calculations by Low and Stewart (unpublished, but results quoted by Dalgarno and Stewart [3]) with variational wave functions for the ground state and modified hydrogenic wave functions for the excited states; similar calculations by Körwien [6]; self-consistent field calculations, including exchange and correlation effects, by Trefftz, Schlüter, Dettmar, and Jörgens [4]; and applications of various sum rules to modify calculated values by Dalgarno and Lynn [5], and Dalgarno and Kingston [7]. Furthermore, the results of Goldberg [8], who employed screened hydrogenic wave functions, and Hylleraas [9], who employed variational wave functions have been used in a few instances. (Hylleraas' work has not been extensively used, since it probably contains quite a number of numerical errors.) Finally, for all other tabulated transitions, the Coulomb approximation (see general introduction) has been employed, and is expected to give reliable results for this simple atom (uncertainties within 10\%). It should be noted that all above-mentioned calculations are nonrelativistic.

For some important helium lines reliable experimental data [10-13] are also available. They all result from determinations of lifetimes of excited states and agree generally quite closely, often within $10 \%$, with the tabulated theoretical re:sults, which are considered more accurate in these cases.

\section{References}

[1] Schiff, B., and Pekeris, C. L., Phys. Rev. 134, A638-A640 (1964).

[2] Weiss, A. W., private communication (1962).

[3] Dalgarno, A., and Stewart, A. L., Proc. Phys. Soc. London A 76, 49-55 (1960).

[4] Trefftz, E., Schlüter, A., Dettmar, K. H., and Jörgens, K., Z. Astrophys. 44, 1-17 (1957).

[5] Dalgarno, A., and Lynn, N., Proc. Phys. Soc. London A 70, 802-808 (1957).

[6] Körwien, H., Z. Physik 91, 1-36 (1934).

[7] Dalgarno, A., and Kingston, A. E., Proc. Phys. Soc. London A 72, 1053-1060 (1958).

[8] Goldberg, L., Astrophys. J. 90, 414-428 (1939).

[9] Hylleraas, E. A., Z. Physik 106, 395-404 (1937).

[10] Heron, S., McWhirter, R. W. P., and Rhoderick, E. H., Proc. Roy. Soc. London A 234, 565-582 (1956).

[11] Osherovich, A. L., and Savich, J. G., Optika i Spektroskopiya 4, 715-718 (1958) (Translated in "Optical Transition Probabilities," Office of Technical Services, U.S. Department of Commerce, Washington, D.C.)

[12] Bennett, R. G., and Dalby, F. W., J. Chem. Phys. 31, 434-441 (1959).

[13] Kindlmann, P. J., and Bennett, W. R., Jr., Bull. Am. Phys. Soc. II 8, 87 (1963). 
He 1. Allowed Transitions

\begin{tabular}{|c|c|c|c|c|c|c|c|c|c|c|c|c|c|}
\hline No. & $\begin{array}{l}\text { Transition } \\
\text { Array }\end{array}$ & Multiplet & $\lambda(\AA)$ & $E_{i}\left(\mathrm{~cm}^{-1}\right)$ & $E_{k}\left(\mathrm{~cm}^{-1}\right)$ & $g_{i}$ & $g_{k}$ & $A_{k i}\left(10^{\mathrm{K}} \sec ^{-1}\right)$ & $f_{i k}$ & $S($ at.u.) & $\log g f$ & $\begin{array}{c}\text { Accu- } \\
\text { racy }\end{array}$ & Source \\
\hline 1 & $1 s^{2}-1 s 2 p$ & $\begin{array}{l}{ }^{1} \mathrm{~S}-{ }^{1} \mathrm{P}^{\circ} \\
(2 \mathrm{uv})\end{array}$ & 584.334 & 0 & 171135 & 1 & 3 & 17.99 & 0.2762 & 0.5313 & -0.5588 & $\mathrm{AA}$ & 1 \\
\hline 2 & $1 s^{2}-1 s 3 p$ & $\begin{array}{c}{ }^{1 S}-{ }^{1} \mathrm{P}^{\circ} \\
(3 \mathrm{uv})\end{array}$ & 537.030 & 0 & 186210 & 1 & 3 & 5.66 & 0.0734 & 0.1298 & -1.1343 & $A A$ & 1 \\
\hline 3 & $1 s^{2}-1 s 4 p$ & $\begin{array}{c}{ }^{1} \mathrm{~S}-{ }^{1} \mathrm{P}^{\circ} \\
(4 \mathrm{uv})\end{array}$ & 522.213 & 0 & 191493 & 1 & 3 & 2.46 & 0.0302 & 0.0519 & -1.520 & A & 2 \\
\hline 4 & $1 s^{2}-1 s 5 p$ & $\begin{array}{c}{ }^{1} \mathrm{~S}-{ }^{1} \mathrm{P}^{\circ} \\
(5 \mathrm{uv})\end{array}$ & 515.617 & 0 & 193943 & 1 & 3 & 1.28 & 0.0153 & 0.0260 & -1.815 & $\mathrm{~B}^{+}$ & 3 \\
\hline 5 & $1 s^{2}-1 s 6 p$ & $\begin{array}{c}{ }^{1} \mathrm{~S}-{ }^{1} \mathrm{P}^{\circ} \\
(6 \mathrm{uv})\end{array}$ & 512.098 & 0 & 195275 & 1 & 3 & 0.719 & 0.00848 & 0.0143 & -2.072 & $\mathrm{~B}^{+}$ & 3,4 \\
\hline 6 & $1 s^{2}-1 s 7 p$ & $\begin{array}{l}{ }^{1} \mathrm{~S}-{ }^{1} \mathrm{P}^{\circ} \\
(7 \mathrm{uv})\end{array}$ & 509.998 & 0 & 196079 & 1 & 3 & 0.507 & 0.00593 & 0.00995 & -2.227 & $\mathrm{~B}$ & 5,6 \\
\hline 7 & $1 s^{2}-1 s 8 p$ & $\begin{array}{l}{ }^{1} \mathrm{~S}-{ }^{1} \mathrm{P}^{\circ} \\
(8 \mathrm{uv})\end{array}$ & 508.643 & 0 & 196602 & 1 & 3 & 0.343 & 0.00399 & 0.00668 & -2.399 & B & 5,6 \\
\hline 8 & $1 s^{2}-1 s 9 p$ & $\begin{array}{c}{ }^{1} \mathrm{~S}-{ }^{1} \mathrm{P}^{\circ} \\
(9 \mathrm{uv})\end{array}$ & 507.718 & 0 & 196960 & 1 & 3 & 0.237 & 0.00275 & 0.00459 & -2.561 & B & 5,6 \\
\hline 9 & $1 s^{2}-1 s 10 p$ & $\begin{array}{l}{ }^{1} \mathrm{~S}-{ }^{1} \mathrm{P}^{\circ} \\
(10 \mathrm{uv})\end{array}$ & 507.058 & 0 & 197216 & 1 & 3 & 0.181 & 0.00209 & 0.00349 & -2.680 & B & 5,6 \\
\hline 10 & $1 s^{2}-1 s 11 p$ & ${ }^{1} \mathrm{~S}-{ }^{1} \mathrm{P}^{\circ}$ & 506.570 & 0 & 197406 & 1 & 3 & 0.130 & 0.00150 & 0.00250 & -2.824 & B & 6 \\
\hline 11 & $1 s^{2}-1 s 12 p$ & ${ }^{1} \mathrm{~S}-{ }^{1} \mathrm{P}^{\circ}$ & 506.200 & 0 & 197550 & 1 & 3 & 0.104 & 0.00119 & 0.00199 & -2.923 & B & 6 \\
\hline 12 & $1 s 2 s-1 s 2 p$ & ${ }^{1} \mathrm{~S}-{ }^{1} \mathrm{P}^{0}$ & 20581.3 & 166278 & 171135 & 1 & 3 & 0.01976 & 0.3764 & 25.50 & -0.4244 & $\mathrm{AA}$ & 1 \\
\hline 13 & $1 s 2 s-1 s 3 p$ & ${ }^{1} \mathrm{~S}-{ }^{1} \mathrm{P}^{\circ}$ & 5015.68 & 166278 & 186210 & 1 & 3 & 0.1338 & 0.1514 & 2.500 & -0.8199 & $\mathrm{AA}$ & 1 \\
\hline 14 & $1 s 2 s-1 s 4 p$ & ${ }^{1} \mathrm{~S}-{ }^{1} \mathrm{P}^{\circ}$ & 3964.73 & 166278 & 191493 & 1 & 3 & 0.0717 & 0.0507 & 0.662 & -1.295 & A & 2 \\
\hline 15 & $1 s 2 s-1 s 5 p$ & $\begin{array}{c}{ }^{1} \mathrm{~S}-{ }^{1} \mathrm{P}^{\circ} \\
(6)\end{array}$ & 3613.64 & 166278 & 193943 & 1 & 3 & 0.0376 & 0.0221 & 0.263 & -1.656 & B & 7 \\
\hline 16 & $1 s 2 s-1 s 6 p$ & $\begin{array}{c}{ }^{1} \mathrm{~S}-{ }^{1} \mathrm{P}^{0} \\
(7)\end{array}$ & 3447.59 & 166278 & 195275 & 1 & 3 & 0.0239 & 0.0128 & 0.145 & -1.894 & A & 4 \\
\hline 17 & $1 s 2 s-1 s 7 p$ & ${ }^{1 S}-{ }^{1} \mathrm{P}^{\circ}$ & 3354.55 & 166278 & 196079 & 1 & 3 & 0.0130 & 0.00660 & 0.0729 & -2.180 & B & 7 \\
\hline 18 & $1 s 2 s-1 s 8 p$ & $\begin{array}{c}{ }^{1} \mathrm{~S}-{ }^{1} \mathrm{P}^{\circ} \\
(9)\end{array}$ & 3296.77 & 166278 & 196602 & 1 & 3 & 0.00901 & 0.00440 & 0.0478 & -2.356 & B & 7 \\
\hline 19 & $1 s 2 s-1 s 9 p$ & ${ }^{1} \mathrm{~S}-{ }^{1} \mathrm{P}^{\circ}$ & 3258.28 & 166278 & 196960 & 1 & 3 & 0.00650 & 0.00310 & 0.0333 & -2.508 & B & $i$ \\
\hline 20 & $1 s 2 s-1 s 10 p$ & ${ }^{1} \mathrm{~S}-{ }^{1} \mathrm{P}^{\circ}$ & 3231.27 & 166278 & 197216 & 1 & 3 & 0.00490 & 0.00230 & 0.0245 & -2.638 & B & 7 \\
\hline 21 & $1 s 2 p-1 s 3 s$ & ${ }^{1} \mathrm{P}^{\circ}-{ }^{1} \mathrm{~S}$ & 7281.35 & 171135 & 184865 & 3 & 1 & 0.181 & 0.0480 & 3.45 & $-0.8+2$ & $A$ & 2 \\
\hline 22 & $1 s 2 p-1 s 4 s$ & $\begin{array}{c}{ }^{1} \mathrm{P}^{\circ}-{ }^{1 \mathrm{~S}} \\
(47)\end{array}$ & 5047.74 & 171135 & 190940 & 3 & 1 & 0.0655 & $0.0083 t$ & 0.416 & -1.602 & $A$ & + \\
\hline 23 & $1 s 2 p-1 s 5 s$ & $\begin{array}{c}{ }^{1} \mathrm{P}^{\circ}-{ }^{15} \mathrm{~S} \\
(50)\end{array}$ & 4437.55 & 171135 & 193663 & 3 & 1 & 0.0313 & 0.00308 & 0.135 & -2.034 & B & $c a$ \\
\hline 24 & $1 s 2 p-1 s 6 s$ & ${ }_{(52)}^{1} \mathrm{P}^{\circ}{ }^{1 \mathrm{~S}}$ & 4168.97 & 171135 & 195115 & 3 & 1 & 0.0176 & 0.00153 & 0.0630 & -2.338 & A & 4 \\
\hline
\end{tabular}


He I. Allowed Transitions-Continued

\begin{tabular}{|c|c|c|c|c|c|c|c|c|c|c|c|c|c|}
\hline No. & $\begin{array}{c}\text { Transition } \\
\text { Array }\end{array}$ & Multiplet & $\lambda(\AA ̊)$ & $E_{i}\left(\mathrm{~cm}^{-1}\right)$ & $E_{k}\left(\mathrm{~cm}^{-1}\right)$ & $g_{i}$ & $g_{k}$ & $A_{k i}\left(10^{8} \sec ^{-1}\right)$ & $f_{i k}$ & $S$ (at.u.) & $\log g f$ & $\begin{array}{l}\text { Accu- } \\
\text { racy }\end{array}$ & Source \\
\hline 25 & $1 s 2 p-1 s 7 s$ & ${ }^{1} \mathrm{P}^{\circ}-{ }^{15} \mathrm{~S}$ & 4023.97 & 171135 & 195979 & 3 & 1 & 0.0109 & $8.81 \times 10^{-4}$ & 0.0350 & -2.578 & $\mathrm{~B}^{+}$ & 4 \\
\hline 26 & $1 s 2 p-1 s 8 s$ & $\begin{array}{c}{ }^{1} \mathrm{P}^{\circ}-{ }^{1} \mathrm{~S} \\
(57)\end{array}$ & 3935.91 & 171135 & 196535 & 3 & 1 & 0.00718 & $5.56 \times 10^{-4}$ & 0.0216 & -2.778 & $\mathrm{~B}^{+}$ & 4 \\
\hline 27 & $1 s 2 p-1 s 3 d$ & $\begin{array}{c}{ }^{1} \mathrm{P}^{\circ}-{ }^{1} \mathrm{D} \\
(46)\end{array}$ & 6678.15 & 171135 & 186105 & 3 & 5 & 0.638 & 0.711 & 46.9 & 0.329 & $A$ & 2 \\
\hline 28 & $1 s 2 p-1 s 4 d$ & $\begin{array}{c}{ }^{1} \mathrm{P}^{\circ}-{ }^{1} \mathrm{D} \\
(48)\end{array}$ & 4921.93 & 171135 & 191447 & 3 & 5 & 0.202 & 0.122 & 5.95 & -0.435 & A & 4 \\
\hline 29 & $1 s 2 p-1 s 5 d$ & $\begin{array}{c}{ }^{1} \mathrm{P}^{\circ}-{ }^{1} \mathrm{D} \\
(51)\end{array}$ & 4387.93 & 171135 & 193918 & 3 & 5 & 0.0907 & 0.0436 & 1.89 & -0.883 & A & 4 \\
\hline 30 & $1 s 2 p-1 s 6 d$ & $\begin{array}{c}{ }^{1} \mathrm{P}^{\circ}-{ }^{1} \mathrm{D} \\
(53)\end{array}$ & 4143.76 & 171135 & 195261 & 3 & 5 & 0.0495 & 0.0213 & 0.870 & -1.195 & B & $c a$ \\
\hline 31 & $1 s 2 p-1 s 7 d$ & 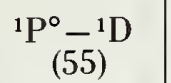 & 4009.27 & 171135 & 196070 & 3 & 5 & 0.0279 & 0.0112 & 0.444 & -1.473 & $\mathrm{C}^{+}$ & 8 \\
\hline 32 & $1 s 2 p-1 s 8 d$ & $\begin{array}{c}{ }^{1} \mathrm{P}^{\circ}-{ }^{1} \mathrm{D} \\
(58)\end{array}$ & 3926.53 & 171135 & 196596 & 3 & 5 & 0.0195 & 0.00750 & 0.291 & -1.648 & A & 4 \\
\hline 33 & $1 s 2 p-1 s 9 d$ & $\begin{array}{c}{ }^{1} \mathrm{P}^{\circ}-{ }^{1} \mathrm{D} \\
(60)\end{array}$ & 3871.79 & 171135 & 196956 & 3 & 5 & 0.0126 & 0.00471 & 0.180 & -1.850 & $\mathrm{C}^{+}$ & 8 \\
\hline 34 & $1 s 2 p-1 s 10 d$ & ${ }_{(62)}^{{ }^{1} P^{\circ}-{ }^{1} \mathrm{D}}$ & 3833.55 & 171135 & 197213 & 3 & 5 & 0.00971 & 0.00357 & 0.135 & -1.971 & A & 4 \\
\hline 35 & $1 s 3 s-1 s 3 p$ & ${ }^{1} \mathrm{~S}-{ }^{1} \mathrm{P}^{\circ}$ & [74351] & 184865 & 186210 & 1 & 3 & 0.00253 & 0.629 & 154 & -0.201 & A & 2 \\
\hline 36 & $1 s 3 s-1 s 4 p$ & ${ }^{1} \mathrm{~S}-{ }^{1} \mathrm{P}^{\circ}$ & 15083.7 & 184865 & 191493 & 1 & 3 & 0.0137 & 0.140 & 6.95 & -0.854 & B & 2 \\
\hline 37 & $1 s 3 s-1 s 5 p$ & $\begin{array}{c}{ }^{1} \mathrm{~S}-{ }^{1} \mathrm{P}^{\circ} \\
(70)\end{array}$ & 11013.1 & 184865 & 193943 & 1 & 3 & 0.00956 & 0.0521 & 1.89 & -1.283 & B & $c a$ \\
\hline 38 & $1 s 3 s-1 s 6 p$ & $\begin{array}{c}{ }^{1} \mathrm{~S}-{ }^{1} \mathrm{P}^{\circ} \\
(71)\end{array}$ & 9603.42 & 184865 & 195275 & 1 & 3 & 0.00564 & 0.0234 & 0.739 & -1.631 & B & 9 \\
\hline 39 & $1 s 3 p-1 s 4 s$ & ${ }^{1} \mathrm{P}^{\circ}-{ }^{1} \mathrm{~S}$ & 21132.0 & 186210 & 190940 & 3 & 1 & 0.0459 & 0.103 & 21.4 & -0.512 & B & $c a$ \\
\hline 40 & $1 s 3 p-1 s 5 s$ & ${ }^{1} \mathrm{P}^{\circ}-{ }^{1} \mathrm{~S}$ & [134.11.8] & 186210 & 193664 & 3 & 1 & 0.0202 & 0.0182 & 2.41 & -1.263 & B & $c a$ \\
\hline 41 & $1 s 3 p-1 s 6 s$ & $\begin{array}{c}{ }^{1} \mathrm{P}^{\circ}-{ }^{1} \mathrm{~S} \\
(87)\end{array}$ & 11225.9 & 186210 & 195115 & 3 & 1 & 0.0110 & 0.00690 & 0.765 & -1.684 & B & $c a$ \\
\hline 42 & $1 s 3 p-1 s 4 d$ & ${ }^{1} \mathrm{P}^{\circ}-{ }^{1} \mathrm{D}$ & 19089.4 & 186210 & 191447 & 3 & 5 & 0.0711 & 0.647 & 122 & 0.288 & B & $c a$ \\
\hline 43 & $1 s 3 p-1 s 5 d$ & ${ }^{1} \mathrm{P}^{\circ}-{ }^{1} \mathrm{D}$ & 12968.4 & 186210 & 193918 & 3 & 5 & 0.0331 & 0.139 & 17.8 & -0.380 & B & $c a$ \\
\hline 44 & $1 s 3 p-1 s 6 d$ & $\begin{array}{c}{ }^{1} \mathrm{P}^{\circ}-{ }^{1} \mathrm{D} \\
(88)\end{array}$ & 11045.0 & 186210 & 195261 & 3 & 5 & 0.0181 & 0.0553 & 6.03 & -0.780 & B & $c a$ \\
\hline 45 & $1 s 3 d-1 s 3 p$ & ${ }^{1} \mathrm{D}-{ }^{1} \mathrm{P}^{\circ}$ & [957600] & 186105 & 186210 & 5 & 3 & $1.68 \times 10^{-6}$ & 0.0139 & 219 & -1.158 & B & 2 \\
\hline 46 & $1 s 3 d-1 s 4 p$ & ${ }^{1} \mathrm{D}-{ }^{1} \mathrm{P}^{\circ}$ & 18555.6 & 186105 & 191447 & 5 & 3 & 0.00277 & 0.00858 & 2.62 & -1.368 & $\mathrm{C}^{+}$ & 2 \\
\hline 47 & $1 s 3 d-1 s 5 p$ & ${ }^{1} \mathrm{D}-{ }^{1} \mathrm{P}^{\circ}$ & 12755.7 & 186105 & 193943 & 5 & 3 & 0.00127 & 0.00186 & 0.390 & -2.032 & B & $c a$ \\
\hline 48 & $1 s 3 d-1 s 6 p$ & ${ }^{1} \mathrm{D}-{ }^{1} \mathrm{P}^{\circ}$ & 10902.2 & 186105 & 195275 & 5 & 3 & $9.23 \times 10^{-4}$ & $9.86 \times 10^{-4}$ & 0.177 & -2.307 & B & 9 \\
\hline 49 & $1 s 3 d-1 s 4 f$ & ${ }^{1} \mathrm{D}-{ }^{1} \mathrm{~F}^{\circ}$ & 18696.9 & 186105 & 191452 & 5 & 7 & 0.138 & 1.01 & 312 & 0.705 & B & $c a$ \\
\hline 50 & $1 s 3 d-1 s 5 f$ & ${ }^{1} \mathrm{D}-{ }^{1} \mathrm{~F}^{\circ}$ & 12790.3 & 186105 & 193921 & 5 & 7 & 0.0461 & 0.158 & 33.3 & -0.102 & B & $c a$ \\
\hline 51 & $1 s 3 d-1 s 6 f$ & $\begin{array}{c}{ }^{1} \mathrm{D}-{ }^{1} \mathrm{~F}^{\circ} \\
(84)\end{array}$ & 10917.0 & 186105 & 195263 & 5 & 7 & 0.0212 & 0.0529 & 9.51 & -0.577 & B & $c a$ \\
\hline
\end{tabular}


He I. Allowed Transitions-Continued

\begin{tabular}{|c|c|c|c|c|c|c|c|c|c|c|c|c|c|}
\hline No. & $\begin{array}{c}\text { Transition } \\
\text { Array }\end{array}$ & Multiplet & $\lambda(\AA)$ & $E_{i}\left(\mathrm{~cm}^{-1}\right)$ & $E_{k}\left(\mathrm{~cm}^{-1}\right)$ & $g_{i}$ & $g_{k}$ & $A_{k i}\left(10^{\mathrm{H}} \sec ^{-1}\right)$ & $f_{i k}$ & $S($ at.u.) & $\log g f$ & $\begin{array}{l}\text { Accu- } \\
\text { racy }\end{array}$ & Source \\
\hline 52 & $1 s 4 s-1 s 4 p$ & ${ }^{1} \mathrm{~S}-{ }^{1} \mathrm{P}^{\circ}$ & [180950] & 190940 & 191493 & 1 & 3 & $5.79 \times 10^{-4}$ & 0.853 & 508 & -0.069 & B & $c a$ \\
\hline 53 & $1 s 4 s-1 s 5 p$ & ${ }^{1} \mathrm{~S}-{ }^{1} \mathrm{P}^{\circ}$ & [33299] & 190940 & 193943 & 1 & 3 & 0.00302 & 0.151 & 16.5 & -0.822 & B & $c a$ \\
\hline 54 & $1 s 4 s-1 s 6 p$ & ${ }^{1} \mathrm{~S}-{ }^{1} \mathrm{P}^{\circ}$ & [23063] & 190940 & 195275 & 1 & 3 & 0.00250 & 0.0599 & 4.5 .5 & -1.222 & B & $c a$ \\
\hline 55 & $1 s 4 p-1 s 5 s$ & ${ }^{1} \mathrm{P}^{\circ}-{ }^{1} \mathrm{~S}$ & [46053] & 191493 & 193664 & 3 & 1 & 0.0150 & 0.159 & 72.2 & -0.322 & B & $c a$ \\
\hline 56 & $1 s 4 p-1 s 6 s$ & ${ }^{1} \mathrm{P}^{\circ}-{ }^{1} \mathrm{~S}$ & {$[27600]$} & 191493 & 195115 & 3 & l & 0.00721 & 0.0274 & 7.48 & -1.085 & B & $c a$ \\
\hline 57 & $1 s 4 p-1 s 7 s$ & ${ }^{1} \mathrm{P}^{\circ}-{ }^{1} \mathrm{~S}$ & [22284] & 191493 & 195979 & 3 & 1 & 0.00438 & 0.0109 & 2.39 & -1.487 & B & $c a$ \\
\hline 58 & $1 s 4 p-1 s 5 d$ & ${ }^{1} \mathrm{P}^{\circ}-{ }^{1} \mathrm{D}$ & [41216] & 191493 & 193918 & 3 & 5 & 0.0153 & 0.649 & 264 & 0.289 & B & $c a$ \\
\hline 59 & $1 s 4 p-1 s 6 d$ & ${ }^{1} \mathrm{P}^{\circ}-{ }^{1} \mathrm{D}$ & [26531] & 191493 & 195261 & 3 & 5 & 0.00861 & 0.152 & 39.7 & -0.342 & B & $c a$ \\
\hline 60 & $1 s 4 p-1 s 7 d$ & ${ }^{1} \mathrm{P}^{\circ}-{ }^{1} \mathrm{D}$ & [21841] & 191493 & 196070 & 3 & 5 & 0.00533 & 0.0635 & 13.7 & -0.720 & B & $c a$ \\
\hline 61 & $1 s 4 d-1 s 4 p$ & ${ }^{1} \mathrm{D}-{ }^{1} \mathrm{P}^{\circ}$ & {$\left[2.16 \times 10^{6}\right]$} & 191447 & 191493 & 5 & 3 & $5.70 \times 10^{-7}$ & 0.0240 & 856 & -0.920 & B & $c a$ \\
\hline 62 & $1 s 4 d-1 s 5 p$ & ${ }^{1} \mathrm{D}-{ }^{1} \mathrm{P}^{\circ}$ & [40053] & 191447 & 193943 & 5 & 3 & 0.00166 & 0.0240 & 15.8 & -0.922 & B & $c a$ \\
\hline 63 & $1 s 4 d-1 s 6 p$ & ${ }^{1} \mathrm{D}-{ }^{1} \mathrm{P}^{\circ}$ & [26113] & 191447 & 195275 & 5 & 3 & $7.85 \times 10^{-4}$ & 0.00482 & 2.07 & -1.618 & B & $c a$ \\
\hline 64 & $1 s 4 d-1 s 4 f$ & ${ }^{1} \mathrm{D}-{ }^{1} \mathrm{~F}^{\circ}$ & {$\left[1.82 \times 10^{7}\right]$} & 191447 & 191452 & 5 & 7 & $3.63 \times 10^{-10}$ & 0.00253 & 757 & -1.899 & B & $c a$ \\
\hline 65 & $1 s 4 d-1 s 5 f$ & ${ }^{1} \mathrm{D}-{ }^{1} \mathrm{~F}^{\circ}$ & [40396] & 191447 & 193921 & 5 & 7 & 0.0259 & 0.887 & 590 & 0.647 & B & $c a$ \\
\hline 66 & $1 s 4 d-1 s 6 f$ & ${ }^{1} \mathrm{D}-{ }^{1} \mathrm{~F}^{\circ}$ & [26198] & 191447 & 195263 & 5 & 7 & 0.0130 & 0.187 & 80.7 & -0.029 & B & $c a$ \\
\hline 67 & $1 s 4 d-1 s 7 f$ & ${ }^{1} \mathrm{D}-{ }^{1} \mathrm{~F}^{\circ}$ & [21617] & 191447 & 196071 & 5 & 7 & 0.00734 & 0.0719 & 25.6 & -0.444 & B & $c a$ \\
\hline 68 & $1 s 4 f-1 s 5 d$ & ${ }^{1} \mathrm{~F}^{\circ}-{ }^{1} \mathrm{D}$ & [40536] & 191452 & 193918 & 7 & 5 & $5.20 \times 10^{-4}$ & 0.00915 & 8.55 & -1.193 & B & $c a$ \\
\hline 69 & $1 s 4 f-1 s 6 d$ & ${ }^{1} \mathrm{~F}^{\circ}-{ }^{1} \mathrm{D}$ & [26248] & 191452 & 195261 & 7 & 5 & $2.49 \times 10^{-4}$ & 0.00184 & 1.11 & -1.891 & B & $c a$ \\
\hline 70 & $1 s 2 s-1 s 2 p$ & ${ }^{3} S-{ }^{3} \mathrm{P}^{\circ}$ & 10830 & 159856 & 169087 & 3 & 9 & 0.1022 & 0.5391 & 57.66 & 0.2088 & AA & 1 \\
\hline & & & 10830.3 & 159856 & 169087 & 3 & 5 & 0.1022 & 0.2994 & 32.03 & -0.0466 & AA & ls \\
\hline & & & $\begin{array}{l}10830.2 \\
10829.1\end{array}$ & $\begin{array}{l}159856 \\
159856\end{array}$ & $\begin{array}{l}169087 \\
169088\end{array}$ & $\begin{array}{l}3 \\
3\end{array}$ & $\begin{array}{l}3 \\
1\end{array}$ & $\begin{array}{l}0.1022 \\
0.1022\end{array}$ & $\begin{array}{l}0.1797 \\
0.05990\end{array}$ & $\begin{array}{c}19.22 \\
6.407\end{array}$ & $\begin{array}{l}-0.2684 \\
-0.7454\end{array}$ & $\begin{array}{l}\mathrm{AA} \\
\mathrm{AA}\end{array}$ & $\begin{array}{l}l s \\
l s\end{array}$ \\
\hline 71 & $1 s 2 s-1 s 3 p$ & $\begin{array}{c}{ }^{3} \mathrm{~S}-{ }^{3} \mathrm{P}^{\circ} \\
(2)\end{array}$ & 3888.65 & 159856 & 185565 & 3 & 9 & 0.09478 & 0.06446 & 2.476 & -0.7136 & $\mathrm{AA}$ & l \\
\hline 72 & $1 s 2 s-1 s 4 p$ & $\begin{array}{c}{ }^{3} \mathrm{~S}-{ }^{3} \mathrm{P}^{\circ} \\
(3)\end{array}$ & 3187.74 & 159856 & 191217 & 3 & 9 & 0.0505 & 0.0231 & 0.727 & -1.159 & B & 2 \\
\hline 73 & $1 s 2 s-1 s 5 p$ & $\begin{array}{c}{ }^{3} \mathrm{~S}-{ }^{3} \mathrm{P}^{\circ} \\
(11 \mathrm{uv})\end{array}$ & 2945.10 & 159856 & 193801 & 3 & 9 & 0.0293 & 0.0114 & 0.332 & -1.465 & B & 7 \\
\hline 74 & $1 s 2 s-1 s 6 p$ & $\begin{array}{c}{ }^{3} \mathrm{~S}-{ }^{3} \mathrm{P}^{\circ} \\
(12 \mathrm{uv})\end{array}$ & 2829.07 & 159856 & 195193 & 3 & 9 & 0.0169 & 0.00608 & 0.170 & -1.739 & B & 7 \\
\hline 75 & $1 s 2 s-1 s 7 p$ & ${ }^{3} \mathrm{~S}-{ }^{3} \mathrm{P}^{\circ}$ & 2763.8 & 159856 & 196027 & 3 & 9 & 0.0111 & 0.00381 & 0.104 & -1.942 & B & 7 \\
\hline 76 & $1 s 2 s-1 s 8 p$ & ${ }^{3} \mathrm{~S}-{ }^{3} \mathrm{P}^{\circ}$ & 2723.2 & 159856 & 196567 & 3 & 9 & 0.00780 & 0.00260 & 0.0700 & -2.108 & B & 7 \\
\hline 77 & $1 s 2 s-1 s 9 p$ & ${ }^{3} \mathrm{~S}-{ }^{3} \mathrm{P}^{0}$ & 2696.1 & 159856 & 196935 & 3 & 9 & 0.00550 & 0.00180 & 0.0479 & -2.268 & B & 7 \\
\hline 78 & $1 s 2 s-1 s 10 p$ & ${ }^{3} \mathrm{~S}-{ }^{3} \mathrm{P}^{\circ}$ & 2677.1 & 159856 & 197198 & 3 & 9 & 0.00404 & 0.00130 & 0.0344 & -2.409 & B & 7 \\
\hline \multirow[t]{3}{*}{79} & $1 s 2 p-1 s 3 s$ & ${ }^{3} \mathrm{P}^{\circ}-{ }^{3} \mathrm{~S}$ & 7065.3 & 169087 & 183237 & 9 & 3 & 0.278 & 0.0693 & 14.5 & -0.205 & A & 2 \\
\hline & & & $\begin{array}{l}7065.19 \\
7065.19\end{array}$ & $\begin{array}{l}169087 \\
169087\end{array}$ & $\begin{array}{l}183237 \\
183237\end{array}$ & $\begin{array}{l}5 \\
3\end{array}$ & $\begin{array}{l}3 \\
3\end{array}$ & 0.154 & $\begin{array}{l}0.0693 \\
0.0692\end{array}$ & $\begin{array}{r}8.06 \\
+83\end{array}$ & $\begin{array}{l}-0.460 \\
-0.683\end{array}$ & $A$ & ls \\
\hline & & & 7065.71 & 169088 & 183237 & l & 3 & 0.0308 & 0.0692 & 1.61 & -1.160 & A & is \\
\hline
\end{tabular}


He I. Allowed Transitions-Continued

\begin{tabular}{|c|c|c|c|c|c|c|c|c|c|c|c|c|c|}
\hline No. & $\begin{array}{c}\text { Transition } \\
\text { Array }\end{array}$ & Multiplet & $\lambda(\AA)$ & $E_{i}\left(\mathrm{~cm}^{-1}\right)$ & $E_{k}\left(\mathrm{~cm}^{-1}\right)$ & $g_{i}$ & $g_{k}$ & $A_{k i}\left(10^{8} \sec ^{-1}\right)$ & $f_{i k}$ & $S$ (at.u.) & $\log g f$ & $\begin{array}{c}\text { Accu- } \\
\text { racy }\end{array}$ & Source \\
\hline 80 & $1 s 2 p-1 s 4 s$ & $\begin{array}{c}{ }^{3} \mathrm{P}^{\circ}-{ }^{3} \mathrm{~S} \\
(12)\end{array}$ & 4713.2 & 169087 & 190298 & 9 & 3 & 0.106 & 0.0118 & 1.65 & -0.973 & B & 4 \\
\hline 81 & $1 s 2 p-1 s 5 s$ & $\begin{array}{c}{ }^{3} \mathrm{P}^{\circ}-{ }^{3} \mathrm{~S} \\
(16)\end{array}$ & 4120.8 & 169087 & 193347 & 9 & 3 & 0.0430 & 0.00365 & 0.446 & -1.483 & B & $c a$ \\
\hline 82 & $1 s 2 p-1 s 6 s$ & $\begin{array}{c}{ }^{3} \mathrm{P}^{\circ}-{ }^{3} \mathrm{~S} \\
(20)\end{array}$ & 3867.5 & 169087 & 194936 & 9 & 3 & 0.0236 & 0.00176 & 0.202 & -1.800 & B & $c a$ \\
\hline 83 & $1 s 2 p-1 s 8 s$ & $\begin{array}{c}{ }^{3} \mathrm{P}^{\circ}-{ }^{3} \mathrm{~S} \\
(27)\end{array}$ & 3652.0 & 169087 & 196461 & 9 & 3 & 0.0108 & $7.21 \times 10^{-4}$ & 0.0780 & -2.188 & B & 4 \\
\hline 84 & $1 s 2 p-1 s 10 s$ & $\begin{array}{c}{ }^{3} \mathrm{P}^{\circ}-{ }^{3} \mathrm{~S} \\
(33)\end{array}$ & 3562.95 & 169087 & 197145 & 9 & 3 & 0.00543 & $3.45 \times 10^{-4}$ & 0.0364 & -2.508 & B & 4 \\
\hline 85 & $1 s 2 p-1 s 3 d$ & $\begin{array}{c}{ }^{3} \mathrm{P}^{\circ}-{ }^{3} \mathrm{D} \\
(11)\end{array}$ & 5875.7 & 169087 & 186102 & 9 & 15 & 0.706 & 0.609 & 106 & 0.739 & A & 2 \\
\hline 86 & $1 s 2 p-1 s 4 d$ & $\begin{array}{c}{ }^{3} \mathrm{P}^{\circ}-{ }^{3} \mathrm{D} \\
(14)\end{array}$ & 4471.5 & 169087 & 191445 & 9 & 15 & 0.251 & 0.125 & 16.6 & 0.052 & A & 4 \\
\hline 87 & $1 s 2 p-1 s 5 d$ & $\begin{array}{c}{ }^{3} \mathrm{P}^{\circ}-{ }^{3} \mathrm{D} \\
(18)\end{array}$ & 4026.2 & 169087 & 193917 & 9 & 15 & 0.117 & 0.0474 & 5.66 & -0.370 & A & 4 \\
\hline 88 & $1 s 2 p-1 s 6 d$ & $\begin{array}{c}{ }^{3} \mathrm{P}^{\circ}-{ }^{3} \mathrm{D} \\
(22)\end{array}$ & 3819.6 & 169087 & 195260 & 9 & 15 & 0.0589 & 0.0215 & 2.43 & -0.714 & B & $c a$ \\
\hline 89 & $1 s 2 p-1 s 7 d$ & $\begin{array}{c}{ }^{3} \mathrm{P}^{\circ}-{ }^{3} \mathrm{D} \\
(25)\end{array}$ & 3705.0 & 169087 & 196070 & 9 & 15 & 0.0444 & 0.0152 & 1.67 & -0.864 & $\mathrm{C}^{+}$ & 8 \\
\hline 90 & $1 s 2 p-1 s 8 d$ & $\begin{array}{c}{ }^{3} \mathrm{P}^{\circ}-{ }^{3} \mathrm{D} \\
(28)\end{array}$ & 3634.2 & 169087 & 196595 & 9 & 15 & 0.0261 & 0.00862 & 0.928 & -1.110 & A & 4 \\
\hline 91 & $1 s 2 p-1 s 9 d$ & $\begin{array}{c}{ }^{3} \mathrm{P}^{\circ}-{ }^{3} \mathrm{D} \\
(31)\end{array}$ & 3587.3 & 169087 & 196955 & 9 & 15 & 0.0205 & 0.00660 & 0.702 & -1.226 & $\mathrm{C}^{+}$ & 8 \\
\hline 92 & $1 s 2 p-1 s 10 d$ & $\begin{array}{c}{ }^{3} \mathrm{P}^{\circ}-{ }^{3} \mathrm{D} \\
(34)\end{array}$ & 3554.4 & 169087 & 197213 & 9 & 15 & 0.0131 & 0.00414 & 0.436 & -1.429 & A & 4 \\
\hline 93 & $1 s 3 s-1 s 3 p$ & ${ }^{3} \mathrm{~S}-{ }^{3} \mathrm{P}^{\circ}$ & [42947] & 183237 & 185565 & 3 & 9 & 0.0108 & 0.896 & 380 & 0.429 & A & 2 \\
\hline 94 & $1 s 3 s-1 s 4 p$ & ${ }^{3} \mathrm{~S}-{ }^{3} \mathrm{P}^{\circ}$ & 12528 & 183237 & 191217 & 3 & 9 & 0.00608 & 0.0429 & 5.31 & -0.890 & B & 2 \\
\hline 95 & $1 s 3 s-1 s 5 p$ & $\begin{array}{c}{ }^{3} \mathrm{~S}-{ }^{3} \mathrm{P}^{\circ} \\
(67)\end{array}$ & 9463.57 & 183237 & 193801 & 3 & 9 & 0.00608 & 0.0245 & 2.29 & -1.134 & B & $c a$ \\
\hline 96 & $1 s 3 s-1 s 6 p$ & 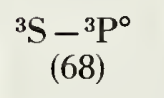 & 8361.77 & 183237 & 195193 & 3 & 9 & $7.16 \times 10^{-4}$ & 0.00225 & 0.186 & -2.170 & B & 9 \\
\hline 97 & $1 s 3 p-1 s 4 s$ & ${ }^{3} \mathrm{P}^{\circ}-{ }^{3} \mathrm{~S}$ & 21120 & 185565 & 190298 & 9 & 3 & 0.0652 & 0.145 & 91.0 & 0.117 & B & $c a$ \\
\hline 98 & $1 s 3 p-1 s 5 s$ & ${ }^{3} \mathrm{P}^{\circ}-{ }^{3} \mathrm{~S}$ & 12846 & 185565 & 193347 & 9 & 3 & 0.0269 & 0.0222 & 8.45 & -0.699 & B & $c a$ \\
\hline 99 & $1 s 3 p-1 s 6 s$ & $\begin{array}{c}{ }^{3} \mathrm{P}^{\circ}-{ }^{3} \mathrm{~S} \\
(73)\end{array}$ & 10667.6 & 185565 & 194936 & 9 & 3 & 0.0142 & 0.00810 & 2.56 & -1.137 & B & $c a$ \\
\hline 100 & $1 s 3 p-1 s 7 s$ & $\begin{array}{c}{ }^{3} \mathrm{P}^{\circ}-{ }^{3} \mathrm{~S} \\
(75)\end{array}$ & 9702.66 & 185565 & 195868 & 9 & 3 & 0.00858 & 0.00404 & 1.16 & -1.440 & B & $c a$ \\
\hline 101 & $1 s 3 p-1 s 3 d$ & ${ }^{3} \mathrm{P}^{\circ}-{ }^{3} \mathrm{D}$ & [186200] & 185565 & 186102 & 9 & 15 & $1.28 \times 10^{-4}$ & 0.111 & 613 & 0.000 & A & 2 \\
\hline 102 & $1 s 3 p-1 s 4 d$ & ${ }^{3} \mathrm{P}^{\circ}-{ }^{3} \mathrm{D}$ & 17002 & 185565 & 191445 & 9 & 15 & 0.0668 & 0.482 & 243 & 0.638 & B & $c a$ \\
\hline 103 & $1 s 3 p-1 s 5 d$ & $\begin{array}{c}{ }^{3} P^{\circ}-{ }^{3} \mathrm{D} \\
(72)\end{array}$ & 11969.1 & 185565 & 193917 & 9 & 15 & 0.0343 & 0.123 & 43.5 & 0.043 & B & $c a$ \\
\hline
\end{tabular}


He I. Allowed Transitions-Continued

\begin{tabular}{|c|c|c|c|c|c|c|c|c|c|c|c|c|c|}
\hline No. & $\begin{array}{c}\text { Transition } \\
\text { Array }\end{array}$ & Multiplet & $\lambda(\AA)$ & $E_{i}\left(\mathrm{~cm}^{-1}\right)$ & $E_{k}\left(c^{\prime} m^{-1}\right)$ & $g_{i}$ & $g_{k}$ & $A_{k i}\left(10^{k} \sec ^{-1}\right)$ & $f_{i k}$ & $S($ at.u.) & $\log g f$ & $\mid \begin{array}{l}\text { Accu- } \\
\text { racy }\end{array}$ & Sourere \\
\hline 104 & $1 s 3 p-1 s 6 d$ & $\begin{array}{c}{ }^{3} \mathrm{P}^{\circ}-{ }^{3} \mathrm{D} \\
\quad(74)\end{array}$ & 10311 & 185565 & 195260 & 9 & 15 & 0.0197 & 0.0524 & 16.0 & -0.327 & B & $c a$ \\
\hline 105 & $1 s 3 d-1 s 4 p$ & ${ }^{3} \mathrm{D}-{ }^{3} \mathrm{P}^{\circ}$ & 19543 & 186102 & 191217 & 15 & 9 & 0.00597 & 0.0205 & 19.8 & -0.512 & $\mathrm{C}^{+}$ & 2 \\
\hline 106 & $1 s 3 d-1 s 5 p$ & ${ }^{3} \mathrm{D}-{ }^{3} \mathrm{P}^{\circ}$ & 12985 & 186102 & 193801 & 15 & 9 & 0.00274 & 0.00415 & 2.66 & -1.206 & B & $c a$ \\
\hline 107 & $1 s 3 d-1 s 6 p$ & $\begin{array}{c}{ }^{3} \mathrm{D}-{ }^{3} \mathrm{P}^{\circ} \\
(78)\end{array}$ & 10996.6 & 186102 & 195193 & 15 & 9 & $5.67 \times 10^{-4}$ & $6.17 \times 10^{-4}$ & 0.335 & -2.034 & B & 9 \\
\hline 108 & $1 s 3 d-1 s 4 f$ & ${ }^{3} \mathrm{D}-{ }^{3} \mathrm{~F}^{\circ}$ & 18686 & 186102 & 191452 & 15 & 21 & 0.139 & 1.02 & 937 & 1.183 & B & $c a$ \\
\hline 109 & $1 s 3 d-1 s 5 f$ & ${ }^{3} \mathrm{D}-{ }^{3} \mathrm{~F}^{\circ}$ & 12785 & 186102 & 193921 & 15 & 21 & 0.0462 & 0.158 & 100 & 0.376 & B & $c a$ \\
\hline 110 & $1 s 3 d-1 s 6 f$ & $\begin{array}{c}{ }^{3} \mathrm{D}-{ }^{3} \mathrm{~F}^{\circ} \\
(79)\end{array}$ & 10912.9 & 186102 & 195263 & 15 & 21 & 0.0212 & 0.0531 & 28.6 & -0.099 & B & $c a$ \\
\hline 111 & $1 s 4 s-1 s 4 p$ & ${ }^{3} \mathrm{~S}-{ }^{3} \mathrm{P}^{\circ}$ & [108800] & 190298 & 191217 & 3 & 9 & 0.00227 & 1.21 & 1300 & 0.560 & B & $c a$ \\
\hline 112 & $1 s 4 s-1 s 5 p$ & ${ }^{3} \mathrm{~S}-{ }^{3} \mathrm{P}^{\circ}$ & [28542] & 190298 & 193801 & 3 & 9 & 0.00128 & 0.0468 & 13.2 & -0.852 & B & $c a$ \\
\hline 113 & $1 s 4 s-1 s 6 p$ & ${ }^{3} \mathrm{~S}-{ }^{3} \mathrm{P}^{\circ}$ & [20425] & 190298 & 195193 & 3 & 9 & 0.00147 & 0.0276 & 5.57 & -1.082 & B & $c a$ \\
\hline 114 & $1 s 4 p-1 s 5 s$ & ${ }^{3} \mathrm{P}^{0}-{ }^{3} \mathrm{~S}$ & [46936] & 191217 & 193347 & 9 & 3 & 0.0202 & 0.223 & 310 & 0.302 & B & $c a$ \\
\hline 115 & $1 s 4 p-1 s 6 s$ & ${ }^{3} \mathrm{P}^{0}-{ }^{3} \mathrm{~S}$ & [26881] & 191217 & 194936 & 9 & 3 & 0.00925 & 0.0334 & 26.6 & -0.522 & B & $c a$ \\
\hline 116 & $1 s 4 p-1 s 7 s$ & ${ }^{3} \mathrm{P}^{\circ}-{ }^{3} \mathrm{~S}$ & [21494] & 191217 & 195868 & 9 & 3 & 0.00543 & 0.0125 & 7.99 & -0.947 & B & $c a$ \\
\hline 117 & $1 s 4 p-1 s 8 s$ & ${ }^{3} \mathrm{P}^{\circ}-{ }^{3} \mathrm{~S}$ & [19063] & 191217 & 196461 & 9 & 3 & 0.00340 & 0.00618 & 3.49 & -1.255 & B & $c a$ \\
\hline 118 & $1 s 4 p-1 s 4 d$ & ${ }^{3} \mathrm{P}^{\circ}-{ }^{3} \mathrm{D}$ & [439440] & 191217 & 191445 & 9 & 15 & $4.15 \times 10^{-5}$ & 0.200 & 2610 & 0.256 & B & $c a$ \\
\hline 119 & $1 s 4 p-1 s 5 d$ & ${ }^{3} \mathrm{P}^{\circ}-{ }^{3} \mathrm{D}$ & [37026] & 191217 & 193917 & 9 & 15 & 0.0129 & 0.442 & 485 & 0.600 & B & $c a$ \\
\hline 120 & $1 s 4 p-1 s 6 d$ & ${ }^{3} \mathrm{P}^{\circ}-{ }^{3} \mathrm{D}$ & [24727] & 191217 & 195260 & 9 & 15 & 0.00795 & 0.121 & 89.0 & 0.039 & B & $c a$ \\
\hline 121 & $1 s 4 d-1 s 5 p$ & ${ }^{3} \mathrm{D}-{ }^{3} \mathrm{P}^{\circ}$ & [42430] & 191445 & 193801 & 15 & 9 & 0.00333 & 0.0539 & 113 & -0.092 & B & $c a$ \\
\hline 122 & $1 s 4 d-1 s 6 p$ & ${ }^{3} \mathrm{D}-{ }^{3} \mathrm{P}^{\circ}$ & [26671] & 191445 & 195193 & 15 & 9 & 0.00160 & 0.0102 & 13.5 & -0.813 & B & $c a$ \\
\hline 123 & $1 s 4 d-1 s 4 f$ & ${ }^{3} \mathrm{D}-{ }^{3} \mathrm{~F}^{\circ}$ & {$\left[1.39 \times 10^{7}\right]$} & 191445 & 191452 & 15 & 21 & $8.15 \times 10^{-10}$ & 0.00331 & 2270 & -1.305 & B & $c a$ \\
\hline 124 & $1 s 4 d-1 s 5 f$ & ${ }^{3} \mathrm{D}-{ }^{3} \mathrm{~F}^{\circ}$ & [40365] & 191445 & 193921 & 15 & 21 & 0.0260 & 0.888 & 1770 & 1.125 & B & $c a$ \\
\hline 125 & $1 s 4 d-1 s 6 f$ & ${ }^{3} \mathrm{D}-{ }^{3} \mathrm{~F}^{\circ}$ & [26185] & 191445 & 195263 & 15 & 21 & 0.0130 & 0.187 & 242 & 0.448 & B & $c a$ \\
\hline 126 & $1 s 4 d-1 s 7 f$ & ${ }^{3} \mathrm{D}-{ }^{3} \mathrm{~F}^{\circ}$ & [21608] & 191445 & 196071 & 15 & 21 & 0.00734 & 0.0720 & 76.8 & 0.033 & B & $c a$ \\
\hline 127 & $1 s 4 f-1 s 5 d$ & ${ }^{3} \mathrm{~F}^{\circ}-{ }^{3} \mathrm{D}$ & [40550] & 191452 & 193917 & 21 & 15 & $5.25 \times 10^{-4}$ & 0.00924 & 25.9 & -0.712 & B & $c a$ \\
\hline 128 & $1 s 4 f-1 s 6 d$ & ${ }^{3} \mathrm{~F}^{\circ}-{ }^{3} \mathrm{D}$ & [26251] & 191452 & 195260 & 21 & 15 & $2.51 \times 10^{-4}$ & 0.00185 & 3.36 & -1.410 & B & $c a$ \\
\hline
\end{tabular}




\section{LITHIUM}

\section{Li I}

Ground State

$1 s^{2} 2 s^{2} \mathrm{~S}_{1 / 2}$

Ionization Potential

$5.390 \mathrm{eV}=43487.19 \mathrm{~cm}^{-1}$

Allowed Transitions

List of tabulated lines:

\begin{tabular}{|c|c|c|c|c|c|}
\hline Wavelength $[\AA]]$ & No. & Wavelength $[\AA]$ & No. & Wavelength $[\AA]$ & No. \\
\hline 2333.94 & 12 & 10510.6 & 28 & 28417 & 38 \\
\hline 2340.16 & 11 & 10792.1 & 21 & 38081 & 40 \\
\hline 2348.22 & 10 & 10919.1 & 33 & 40294 & 45 \\
\hline 2358.92 & 9 & 11032.1 & 24 & 40562 & 47 \\
\hline 2373.55 & 8 & 12237.7 & 27 & 41791 & 42 \\
\hline 2394.36 & 7 & 12793.3 & 32 & 47804 & 50 \\
\hline 2425.41 & 6 & 12928.9 & 30 & 54633 & 37 \\
\hline 2475.06 & 5 & 13557.8 & 23 & 68592 & 34 \\
\hline 2562.31 & 4 & 17546.1 & 26 & 70319 & 53 \\
\hline 2741.19 & 3 & 18586.8 & 36 & 74379 & 56 \\
\hline 3232.63 & 2 & 18703.1 & 31 & 75010 & \\
\hline 3985.5 & 16 & 19274.8 & 29 & 77145 & 54 \\
\hline 4132.6 & 19 & 24464.7 & 22 & 102850 & 51 \\
\hline 4273.1 & 15 & 24971 & 35 & 139610 & 49 \\
\hline 4602.9 & 18 & 25197 & 41 & 279490 & 25 \\
\hline 4971.7 & 14 & 26201 & 46 & 650000 & 39 \\
\hline 6103.6 & 17 & 26260 & 48 & 1261000 & 52 \\
\hline 6707.8 & 1 & 26536 & 43 & $1.04 \times 10^{7}$ & 55 \\
\hline 8126.4 & 13 & 26877.8 & 20 & $1.47 \times 10^{7}$ & 44 \\
\hline
\end{tabular}

The transition probabilities for the $2 s-2 p, 2 p-3 s, 2 p-3 d, 3 s-3 p$, and $3 p-3 d$ transitions are taken from the dipole length calculations of Weiss [1]. The values for the $2 s-n p(n=3 \ldots 13)$ transitions are selected from the anomalous dispersion measurements of Filippov [2] normalized to Weiss' $f$-value for the resonance transition $2 s-2 p$. For this series, preference is given to Filippov's experimental values since the theoretical methods show strong cancellation effects in the transition integral. Uncertainties of not more than $10 \%$ are indicated by the very good agreement of the selected material with other determinations.

\section{References}

[1] Weiss, A. W., Astrophys. J. 138, 1262-1276 (1963).

[2] Filippov, A. N., Z. Physik 69, 526-547 (1931); Zhur. Eksptl. i Teoret. Fiz. 2, 24-41 (1932) (translated in "Optical Transition Probabilities," Office of Technical Services, U.S. Department of Commerce, Washington, D.C.). 
Li I. Allowed Transitions

\begin{tabular}{|c|c|c|c|c|c|c|c|c|c|c|c|c|c|}
\hline No. & $\begin{array}{c}\text { Transition } \\
\text { Array }\end{array}$ & Multiplet & $\lambda(\AA)$ & $E_{i}\left(\mathrm{~cm}^{-1}\right)$ & $E_{k}\left(\mathrm{~cm}^{-1}\right)$ & $g_{i}$ & $g_{k}$ & $A_{k i}\left(\mathrm{sec}^{-1}\right)$ & $f_{i k}$ & $S$ (at.u.) & $\log g f$ & Accu- & Source \\
\hline 1 & $2 s-2 p$ & $\begin{array}{c}{ }^{2} \mathrm{~S}-{ }^{2} \mathrm{P}^{\circ} \\
(1)\end{array}$ & 6707.8 & 0.00 & 14903.9 & 2 & 6 & $3.72 \times 10^{7}$ & 0.753 & 33.3 & 0.178 & A & l \\
\hline 2 & $2 s-3 p$ & $\begin{array}{c}{ }^{2} \mathrm{~S}-{ }^{2} \mathrm{P}^{\circ} \\
(2)\end{array}$ & 3232.63 & 0.00 & 30925.4 & 2 & 6 & $1.17 \times 10^{6}$ & 0.00552 & 0.117 & -1.957 & B & 2 \\
\hline 3 & $2 s-4 p$ & $\begin{array}{c}{ }^{2} \mathrm{~S}-{ }^{2} \mathrm{P}^{\circ} \\
(1 \mathrm{uv})\end{array}$ & 2741.19 & 0.00 & 36469.6 & 2 & 6 & $1.42 \times 10^{6}$ & 0.00480 & 0.0866 & -2.018 & B & 2 \\
\hline 4 & $2 s-5 p$ & $\begin{array}{c}{ }^{2} \mathrm{~S}-{ }^{2} \mathrm{P}^{\circ} \\
(2 \mathrm{uv})\end{array}$ & 2562.31 & 0.00 & 39015.6 & 2 & 6 & $1.07 \times 10^{6}$ & 0.00316 & 0.0533 & -2.199 & B & 2 \\
\hline 5 & $2 s-6 p$ & $\mid \begin{array}{c}{ }^{2} S-{ }^{2} P^{\circ} \\
(3 \text { uv })\end{array}$ & 2475.06 & 0.00 & 40390.8 & 2 & 6 & $6.97 \times 10^{5}$ & 0.00192 & 0.0313 & -2.416 & B & 2 \\
\hline 6 & $2 s-7 p$ & $\begin{array}{c}{ }^{2} \mathrm{~S}-{ }^{2} \mathrm{P}^{\circ} \\
(4 \mathrm{uv})\end{array}$ & 2425.41 & 0.00 & 41217.4 & 2 & 6 & $4.84 \times 10^{5}$ & 0.00128 & 0.0204 & -2.592 & B & 2 \\
\hline 7 & $2 s-8 p$ & $\mid \begin{array}{c}{ }^{2} \mathrm{~S}-{ }^{2} \mathrm{P}^{0} \\
(5 \mathrm{uv})\end{array}$ & 2394.36 & 0.00 & 41751.6 & 2 & 6 & $3.55 \times 10^{5}$ & $9.16 \times 10^{-4}$ & 0.0144 & -2.737 & B & 2 \\
\hline 8 & $2 s-9 p$ & ${ }^{2} \mathrm{~S}-{ }^{2} \mathrm{P}^{\circ}$ & 2373.55 & 0.00 & 42118.3 & 2 & 6 & $2.68 \times 10^{5}$ & $6.79 \times 10^{-4}$ & 0.0106 & -2.867 & B & 2 \\
\hline 9 & $2 s-10 p$ & ${ }^{2} \mathrm{~S}-{ }^{2} \mathrm{P}^{\circ}$ & 2358.92 & 0.00 & 42379.2 & 2 & 6 & $2.09 \times 10^{5}$ & $5.22 \times 10^{-4}$ & 0.00811 & -2.981 & B & 2 \\
\hline 10 & $2 s-11 p$ & ${ }^{2} \mathrm{~S}-{ }^{2} \mathrm{P}^{\circ}$ & 2348.22 & 0.00 & 42569.1 & 2 & 6 & $1.65 \times 10^{5}$ & $4.08 \times 10^{-4}$ & 0.00631 & -3.088 & B & 2 \\
\hline 11 & $2 s-12 p$ & ${ }^{2} \mathrm{~S}-{ }^{2} \mathrm{P}^{\circ}$ & 2340.16 & 0.00 & 42719.1 & 2 & 6 & $1.34 \times 10^{5}$ & $3.29 \times 10^{-4}$ & 0.00507 & -3.182 & B & 2 \\
\hline 12 & $2 s-13 p$ & ${ }^{2} \mathrm{~S}-{ }^{2} \mathrm{P}^{\circ}$ & 2333.94 & 0.00 & 42832.9 & 2 & 6 & $1.09 \times 10^{5}$ & $2.67 \times 10^{-4}$ & 0.00410 & -3.273 & B & 2 \\
\hline 13 & $2 p-3 s$ & $\begin{array}{c}{ }^{2} \mathrm{P}^{\circ}-{ }^{-2} \mathrm{~S} \\
(3)\end{array}$ & 8126.4 & 14903.9 & 27206.1 & 6 & 2 & $3.49 \times 10^{7}$ & 0.115 & 18.5 & -0.160 & $\mathrm{~B}+$ & 1 \\
\hline 14 & $2 p-4 s$ & ${ }^{2} \mathrm{P}^{\circ}-{ }^{2} \mathrm{~S}$ & 4971.7 & 14903.9 & 35012.1 & 6 & 2 & $1.01 \times 10^{7}$ & 0.0125 & 1.23 & -1.126 & B & $c a$ \\
\hline 15 & $2 p-5 s$ & ${ }^{2} \mathrm{P}^{\circ}-{ }^{2} \mathrm{~S}$ & 4273.1 & 14903.9 & 38299.5 & 6 & 2 & $4.60 \times 10^{6}$ & 0.00420 & 0.355 & -1.599 & B & $c a$ \\
\hline 16 & $2 p-6 s$ & ${ }^{2} \mathrm{P}^{\circ}-{ }^{2} \mathrm{~S}$ & 3985.5 & 14903.9 & 39987.6 & 6 & 2 & $2.50 \times 10^{6}$ & 0.00198 & 0.156 & -1.924 & B & $c a$ \\
\hline 17 & $2 p-3 d$ & $\mid \begin{array}{c}2 \mathrm{P}^{\circ}-{ }^{2} \mathrm{D} \\
(4)\end{array}$ & 6103.6 & 14903.9 & 31283.1 & 6 & 10 & $7.16 \times 10^{7}$ & 0.667 & 80.4 & 0.602 & $\mathrm{~B}+$ & 1 \\
\hline 18 & $2 p-4 d$ & $\mid \begin{array}{c}2 \mathrm{P}^{\circ}-{ }^{-2} \mathrm{D} \\
(6)\end{array}$ & 4602.9 & 14903.9 & 36623.4 & 6 & 10 & $2.30 \times 10^{7}$ & 0.122 & 11.1 & -0.137 & B & $c a$ \\
\hline 19 & $2 p-5 d$ & ${ }^{2} \mathrm{P}^{\circ}-{ }^{2} \mathrm{D}$ & 4132.6 & 14903.9 & 39094.9 & 6 & 10 & $1.06 \times 10^{7}$ & 0.0453 & 3.70 & -0.566 & B & $c a$ \\
\hline 20 & $3 s-3 p$ & ${ }^{2} \mathrm{~S}-{ }^{2} \mathrm{P}^{\circ}$ & 26877.8 & 27206.1 & 30925.4 & 2 & 6 & $3.77 \times 10^{6}$ & 1.23 & 217 & 0.389 & $\mathrm{~B}+$ & 1 \\
\hline 21 & $3 s-4 p$ & ${ }^{2} \mathrm{~S}-{ }^{2} \mathrm{P}^{\circ}$ & [10792.1] & 27206.1 & 36469.6 & 2 & 6 & $3.69 \times 10^{3}$ & $1.93 \times 10^{-4}$ & 0.0137 & -3.413 & B & $c a$ \\
\hline 22 & $3 p-4 s$ & ${ }^{2} \mathrm{P}$ & 24464.7 & 30925.4 & 35012.1 & 6 & 2 & $7.46 \times 10^{6}$ & 0.22 & 108 & 0.127 & B & $a$ \\
\hline 23 & $3 p-5 s$ & ${ }^{2} \mathrm{P}^{\circ}-{ }^{2} \mathrm{~S}$ & 13557.8 & 30925.4 & 38299.5 & 6 & 2 & $2.76 \times 10^{6}$ & 0.0254 & 6.80 & -0.817 & B & $c a$ \\
\hline 24 & $3 p-6 s$ & ${ }^{2} \mathrm{P}$ & 1 & 25.4 & 8.6 & 6 & 2 & 1.4 & 74 & 1.90 & -1.280 & B & $c a$ \\
\hline 25 & $3 p-3 d$ & ${ }^{2} \mathrm{P}^{0}-{ }^{2} \mathrm{D}$ & [279490] & 30925.4 & 31283.1 & 6 & 10 & $3.81 \times 10^{3}$ & 0.0743 & 410 & -0.351 & B & 1 \\
\hline 26 & $3 p-4 d$ & ${ }^{2} \mathrm{P}^{\circ}-{ }^{2} \mathrm{D}$ & 17546.1 & 30925.4 & 36623.4 & 6 & 10 & $6.85 \times 10^{6}$ & 0.527 & 183 & 0.500 & B & $c a$ \\
\hline 27 & $3 p-5 d$ & ${ }^{2} \mathrm{P}^{\circ}-{ }^{2} \mathrm{D}$ & 12237.7 & 30925.4 & 39094.9 & 6 & 10 & $3.41 \times 10^{6}$ & 0.128 & 30.9 & -0.116 & B & $c a$ \\
\hline 28 & $3 p-6 d$ & ${ }^{2} \mathrm{P}^{\circ}-{ }^{2} \mathrm{D}$ & 10510.6 & 30925.4 & 40437.3 & 6 & 10 & $1.94 \times 10^{6}$ & 0.0534 & 11.1 & -0.494 & B & $c a$ \\
\hline
\end{tabular}


Li I. Allowed Transitions-Continued

\begin{tabular}{|c|c|c|c|c|c|c|c|c|c|c|c|c|c|}
\hline No. & $\begin{array}{c}\text { Transition } \\
\text { Array }\end{array}$ & Multiplet & $\lambda(\AA)$ & $E_{i}\left(\mathrm{~cm}^{-1}\right)$ & $E_{k}\left(\mathrm{~cm}^{-1}\right)$ & $g_{i}$ & $g_{k}$ & $A_{k i}\left(\sec ^{-1}\right)$ & $f_{i k}$ & $S$ (at.u.) & $\log g f$ & $\begin{array}{r}\text { Accu- } \\
\text { racy }\end{array}$ & Source \\
\hline 29 & $3 d-4 p$ & ${ }^{2} \mathrm{D}-{ }^{2} \mathrm{P}^{\circ}$ & 19274.8 & 31283.1 & 36469.6 & 10 & 6 & $5.52 \times 10^{5}$ & 0.0184 & 11.7 & -0.734 & B & $c a$ \\
\hline 30 & $3 d-5 p$ & ${ }^{2} \mathrm{D}-{ }^{2} \mathrm{P}^{\circ}$ & [12928.9] & 31283.1 & 39015.6 & 10 & 6 & $2.31 \times 10^{5}$ & 0.00348 & 1.48 & -1.459 & B & $c a$ \\
\hline 31 & $3 d-4 f$ & ${ }^{2} \mathrm{D}-{ }^{2} \mathrm{~F}^{\circ}$ & 18703.1 & 31283.1 & 36630.2 & 10 & 14 & $1.38 \times 10^{7}$ & 1.01 & 625 & 1.006 & B & $c a$ \\
\hline 32 & $3 d-5 f$ & ${ }^{2} \mathrm{D}-{ }^{2} \mathrm{~F}^{\circ}$ & 12793.3 & 31283.1 & 39104.5 & 10 & 14 & $4.63 \times 10^{6}$ & 0.159 & 67.0 & 0.201 & B & $c a$ \\
\hline 33 & $3 d-6 f$ & ${ }^{2} \mathrm{D}-{ }^{2} \mathrm{~F}^{\circ}$ & [10919.1] & 31283.1 & [40439.0] & 10 & 14 & $2.11 \times 10^{6}$ & 0.0529 & 19.0 & -0.277 & B & $c a$ \\
\hline 34 & $4 s-4 p$ & ${ }^{2} \mathrm{~S}-{ }^{2} \mathrm{P}^{\circ}$ & [68592] & 35012.1 & 36469.6 & 2 & 6 & $7.72 \times 10^{5}$ & 1.63 & 737 & 0.514 & B & $c a$ \\
\hline 35 & $4 s-5 p$ & ${ }^{2} \mathrm{~S}-{ }^{2} \mathrm{P}^{\circ}$ & [24971] & 35012.1 & 39015.6 & 2 & 6 & $2.07 \times 10^{3}$ & $5.81 \times 10^{-4}$ & 0.0955 & -2.935 & B & $c a$ \\
\hline 36 & $4 s-6 p$ & ${ }^{2} \mathrm{~S}-{ }^{2} \mathrm{P}^{\circ}$ & [18586.8] & 35012.1 & 40390.8 & 2 & 6 & $8.38 \times 10^{3}$ & 0.00130 & 0.159 & -2.585 & B & $c a$ \\
\hline 37 & $4 p-5 s$ & ${ }^{2} \mathrm{P}^{\circ}-{ }^{2} \mathrm{~S}$ & [54633] & 36469.6 & 38299.5 & 6 & 2 & $2.25 \times 10^{6}$ & 0.335 & 362 & 0.303 & B & $c a$ \\
\hline 38 & $4 p-6 s$ & ${ }^{2} \mathrm{P}^{\circ}-{ }^{2} \mathrm{~S}$ & [28417] & 36469.6 & 39987.6 & 6 & 2 & $9.22 \times 10^{5}$ & 0.0372 & 20.9 & -0.651 & B & $c a$ \\
\hline 39 & $4 p-4 d$ & ${ }^{2} \mathrm{P}^{\circ}-{ }^{2} \mathrm{D}$ & [650000] & 36469.6 & 36623.4 & 6 & 10 & $1.28 \times 10^{3}$ & 0.135 & 1740 & -0.091 & B & $c a$ \\
\hline 40 & $4 p-5 d$ & ${ }^{2} \mathrm{P}^{\circ}-{ }^{2} \mathrm{D}$ & [38081] & 36469.6 & 39094.9 & 6 & 10 & $1.36 \times 10^{6}$ & 0.494 & 372 & 0.472 & B & $c a$ \\
\hline 41 & $4 p-6 d$ & ${ }^{2} \mathrm{P}^{\circ}-{ }^{2} \mathrm{D}$ & [25197] & 36469.6 & 40437.3 & 6 & 10 & $8.19 \times 10^{5}$ & 0.130 & 64.6 & -0.108 & B & $c a$ \\
\hline 42 & $4 d-5 p$ & ${ }^{2} \mathrm{D}-{ }^{2} \mathrm{P}^{\circ}$ & [41791] & 36623.4 & 39015.6 & 10 & 6 & $2.86 \times 10^{5}$ & 0.0450 & 61.9 & -0.347 & $\mathrm{~B}$ & $c a$ \\
\hline 43 & $4 d-6 p$ & ${ }^{2} \mathrm{D}-{ }^{2} \mathrm{P}^{\circ}$ & [26536] & 36623.4 & 40390.8 & 10 & 6 & $1.39 \times 10^{5}$ & 0.00879 & 7.67 & -1.056 & B & $c a$ \\
\hline 44 & $4 d-4 f$ & ${ }^{2} \mathrm{D}-{ }^{2} \mathrm{~F}^{\circ}$ & {$\left[1.47 \times 10^{7}\right]$} & 36623.4 & 36630.2 & 10 & 14 & $6.90 \times 10^{-2}$ & 0.00313 & 1520 & -1.504 & B & $c a$ \\
\hline 45 & $4 d-5 f$ & ${ }^{2} \mathrm{D}-{ }^{2} \mathrm{~F}^{\circ}$ & [40294] & 36623.4 & 39104.5 & 10 & 14 & $2.58 \times 10^{6}$ & 0.878 & 1160 & 0.944 & B & $c a$ \\
\hline 46 & $4 d-6 f$ & ${ }^{2} \mathrm{D}-{ }^{2} \mathrm{~F}^{\circ}$ & [26201] & 36623.4 & [40439.0] & 10 & 14 & $1.30 \times 10^{6}$ & 0.187 & 161 & 0.272 & B & $c a$ \\
\hline 47 & $4 f-5 d$ & ${ }^{2} \mathrm{~F}^{\circ}-{ }^{2} \mathrm{D}$ & [40562] & 36630.2 & 39094.9 & 14 & 10 & $5.23 \times 10^{4}$ & 0.00922 & 17.2 & -0.889 & B & $c a$ \\
\hline 48 & $4 f-6 d$ & ${ }^{2} \mathrm{~F}^{\circ}-{ }^{2} \mathrm{D}$ & [26260] & 36630.2 & 40437.3 & 14 & 10 & $2.50 \times 10^{4}$ & 0.00185 & 2.24 & -1.587 & B & $c a$ \\
\hline 49 & $5 s-5 p$ & ${ }^{2} \mathrm{~S}-{ }^{2} \mathrm{P}^{\circ}$ & [139610] & 38299.5 & 39015.6 & 2 & 6 & $2.33 \times 10^{5}$ & 2.05 & 1880 & 0.612 & B & $c a$ \\
\hline 50 & $5 s-6 p$ & ${ }^{2} \mathrm{~S}-{ }^{2} \mathrm{P}^{\circ}$ & [47804] & 38299.5 & 40390.8 & 2 & 6 & $1.72 \times 10^{3}$ & 0.00177 & 0.557 & -2.451 & B & $c a$ \\
\hline 51 & $5 p-6 s$ & ${ }^{2} \mathrm{P}^{\circ}-{ }^{2} \mathrm{~S}$ & [102850] & 39015.6 & 39987.6 & 6 & 2 & $8.48 \times 10^{5}$ & 0.448 & 911 & 0.430 & B & $c a$ \\
\hline 52 & $5 p-5 d$ & ${ }^{2} \mathrm{P}^{\circ}-{ }^{2} \mathrm{D}$ & [1261000] & 39015.6 & 39094.9 & 6 & 10 & $4.78 \times 10^{2}$ & 0.190 & 4730 & 0.057 & B & $c a$ \\
\hline 53 & $5 p-6 d$ & ${ }^{2} \mathrm{P}^{\circ}-{ }^{2} \mathrm{D}$ & [70319] & 39015.6 & 40437.3 & 6 & 10 & $3.98 \times 10^{5}$ & 0.491 & 683 & 0.470 & B & $c a$ \\
\hline 54 & $5 d-6 p$ & ${ }^{2} \mathrm{D}-{ }^{2} \mathrm{P}^{0}$ & [77145] & 39094.9 & 40390.8 & 10 & 6 & $1.42 \times 10^{5}$ & 0.0758 & 192 & -0.121 & B & $c a$ \\
\hline 55 & $5 d-5 f$ & ${ }^{2} \mathrm{D}-{ }^{2} \mathrm{~F}^{\circ}$ & {$\left[1.04 \times 10^{7}\right]$} & 39094.9 & 39104.5 & 10 & 14 & 0.696 & 0.0159 & 5440 & -0.800 & B & $c a$ \\
\hline 56 & $5 d-6 f$ & ${ }^{2} \mathrm{D}-{ }^{2} \mathrm{~F}^{\circ}$ & [74379] & 39094.9 & [40439.0] & 10 & 14 & $7.22 \times 10^{5}$ & 0.838 & 2050 & 0.924 & B & $c a$ \\
\hline 57 & $5 f-6 d$ & ${ }^{2} \mathrm{~F}^{\circ}-{ }^{2} \mathrm{D}$ & [75010] & 39104.5 & 40437.3 & 14 & 10 & $4.19 \times 10^{4}$ & 0.0252 & 87.3 & -0.452 & B & $c a$ \\
\hline
\end{tabular}




\section{Li II}

Ground State

$1 s^{2} \mathrm{i}_{0}$

Ionization Potential

$75.619 \mathrm{eV}=610079 \mathrm{~cm}^{-1}$

Allowed Transitions

List of tabulated lines:

\begin{tabular}{l|r||c|c|c|c}
\hline \hline Wavelength $[\AA]$ & No. & Wavelength $[\AA]$ & No. & Wavelength $[\AA]$ & No. \\
\hline & & & & & \\
178.015 & 2 & 1653.2 & 31 & 3306.5 & 17 \\
199.282 & 1 & 1681.8 & 10 & 3684.1 & 39 \\
861.36 & 30 & 1755.5 & 6 & 4156.3 & 14 \\
944.72 & 29 & 2605.1 & 47 & 4325.7 & 45 \\
972.30 & 34 & 2657.3 & 43 & 4637.8 & 23 \\
1018.0 & 37 & 2674.43 & 40 & 4671.8 & 50 \\
1031.9 & 33 & 2728.4 & 52 & 4678.4 & 24 \\
1044.3 & 9 & 2730.7 & 26 & 4787.5 & 19 \\
1093.2 & 5 & 2767.1 & 21 & 4840.8 & 48 \\
1102.6 & 12 & 2790.5 & 18 & 4881.3 & \\
1109.0 & 8 & 2952.5 & 15 & 5038.7 & 16 \\
1132.0 & 36 & 3029.1 & 46 & 5484.8 & 27 \\
1166.7 & 32 & 3155.4 & 42 & 9562.2 & 3 \\
1198.3 & 28 & 3195.8 & 51 & 21091 & 38 \\
1237.4 & 11 & 3199.4 & 25 & 33661 & 13 \\
1253.5 & 7 & 3235.7 & 49 & 57324 & 44 \\
1420.7 & 4 & 3250.1 & 20 & 211360 & 22 \\
1493.2 & 35 & & & & \\
\hline
\end{tabular}

The values are selected from Weiss' calculations [1] or, when not available, from the Coulomb approximation. The transition probabilities determined by Weiss are the result of extensive nonrelativistic variational calculations. Values have been determined in both the dipole length and dipole velocity approximations and agree to within $1 \%$, except for the $3 p^{1} \mathrm{P}^{\circ}-3 d^{1} \mathrm{D}$ transition where agreement is not as good. The average of the two approximations is adopted [1].

\section{Reference}

[1] Weiss, A. W., private communication (1964). 
Li II. Allowed Transitions

\begin{tabular}{|c|c|c|c|c|c|c|c|c|c|c|c|c|c|}
\hline No. & $\begin{array}{c}\text { Transition } \\
\text { Array }\end{array}$ & Multiplet & $\lambda(\AA)$ & $E_{i}\left(\mathrm{~cm}^{-1}\right)$ & $E_{k}\left(\mathrm{~cm}^{-1}\right)$ & $g_{i}$ & $g_{k}$ & $A_{k i}\left(\sec ^{-1}\right)$ & $f_{i k}$ & $S$ (at.u.) & $\log g f$ & $\begin{array}{r}\text { Accu } \\
\text { racy }\end{array}$ & Source \\
\hline 1 & $1 s^{2}-1 s 2 p$ & $\begin{array}{l}{ }^{1} S-{ }^{-1} \mathrm{P}^{\circ} \\
(1 \mathrm{uv})\end{array}$ & 199.282 & 0 & 501816 & 1 & 3 & 256 & 0.457 & 0.300 & -0.340 & A & 1 \\
\hline 2 & $1 s^{2}-1 s 3 p$ & $\begin{array}{c}{ }^{1 S}-{ }^{1} \mathrm{P}^{\circ} \\
(2 \mathrm{uv})\end{array}$ & 178.015 & 0 & 561749 & 1 & 3 & \begin{tabular}{|l|}
77.9 \\
\end{tabular} & 0.111 & 0.0651 & -0.955 & A & 1 \\
\hline 3 & $1 s 2 s-1 s 2 p$ & ${ }^{1} \mathrm{~S}-{ }^{1} \mathrm{P}^{\circ}$ & [9562.2] & 491361 & 501816 & 1 & 3 & 0.0518 & 0.213 & 6.71 & -0.672 & A & 1 \\
\hline 4 & $1 s 2 s-1 s 3 p$ & ${ }^{1} \mathrm{~S}-{ }^{1} \mathrm{P}^{\circ}$ & [1420.7] & 491361 & 561749 & 1 & 3 & 2.82 & 0.256 & 1.20 & -0.592 & A & 1 \\
\hline 5 & $1 s 2 s-1 s 4 p$ & ${ }^{1} \mathrm{~S}-{ }^{1} \mathrm{P}^{0}$ & [1093.2] & 491361 & 582832 & 1 & 3 & 1.32 & 0.0712 & 0.256 & -1.147 & B & $c a$ \\
\hline 6 & $1 s 2 p-1 s 3 s$ & ${ }^{1} \mathrm{P}^{0}-{ }^{1} \mathrm{~S}$ & [1755.5] & 501816 & 558779 & 3 & 1 & 2.04 & 0.0314 & 0.544 & -1.026 & B & $c a$ \\
\hline 7 & $1 s 2 p-1 s 4 s$ & ${ }^{1} \mathrm{P}^{0}-{ }^{1} \mathrm{~S}$ & [1253.5] & 501816 & 581590 & 3 & 1 & 0.776 & 0.00609 & 0.0754 & -1.738 & B & $c a$ \\
\hline 8 & $1 s 2 p-1 s 5 s$ & ${ }^{1} \mathrm{P}^{0}-{ }^{1} \mathrm{~S}$ & [1109.0] & 501816 & 591984 & 3 & 1 & 0.382 & 0.00235 & 0.0257 & -2.152 & B & $c a$ \\
\hline 9 & $1 s 2 p-1 s 6 s$ & ${ }^{1} \mathrm{P}^{\circ}-{ }^{1} \mathrm{~S}$ & [1044.3] & 501816 & 597574 & 3 & 1 & 0.214 & 0.00117 & 0.0120 & -2.456 & B & $c a$ \\
\hline 10 & $1 s 2 p-1 s 3 d$ & ${ }^{1} \mathrm{P}^{\circ}-{ }^{1} \mathrm{D}$ & [1681.8] & 501816 & 561276 & 3 & 5 & 10.1 & 0.714 & 11.9 & 0.331 & A & 1 \\
\hline 11 & $1 s 2 p-1 s 4 d$ & ${ }^{1} \mathrm{P}^{\circ}-{ }^{1} \mathrm{D}$ & [1237.4] & 501816 & 582631 & 3 & 5 & 3.10 & 0.119 & 1.45 & -0.448 & B & $c a$ \\
\hline 12 & $1 s 2 p-1 s 5 d$ & ${ }^{1} \mathrm{P}^{\circ}-{ }^{1} \mathrm{D}$ & [1102.6] & 501816 & 592508 & 3 & 5 & 1.37 & 0.0415 & 0.452 & -0.905 & B & $c a$ \\
\hline 13 & $1 s 3 s-1 s 3 p$ & ${ }^{1} \mathrm{~S}-{ }^{1} \mathrm{P}^{0}$ & [33661] & 558779 & 561749 & 1 & 3 & 0.00710 & 0.362 & 40.1 & -0.442 & B & $c a$ \\
\hline 14 & $1 s 3 s-1 s 4 p$ & $\begin{array}{c}{ }^{1} \mathrm{~S}-{ }^{1} \mathrm{P}^{\circ} \\
(3)\end{array}$ & 4156.3 & 558779 & 582832 & 1 & 3 & 0.343 & 0.267 & 3.65 & -0.574 & B & $c a$ \\
\hline 15 & $1 s 3 s-1 s 5 p$ & ${ }^{1} \mathrm{~S}-{ }^{1} \mathrm{P}^{\circ}$ & [2952.5] & 558779 & 592639 & 1 & 3 & 0.202 & 0.0791 & 0.769 & -1.102 & B & $c a$ \\
\hline 16 & $1 s 3 p-1 s 4 s$ & ${ }^{1} \mathrm{P}^{\circ}-{ }^{1} \mathrm{~S}$ & [5038.7] & 561749 & 581590 & 3 & 1 & 0.541 & 0.0687 & 3.42 & -0.686 & B & $c a$ \\
\hline 17 & $1 s 3 p-1 s 5 s$ & ${ }^{1} \mathrm{P}^{\circ}-{ }^{-1} \mathrm{~S}$ & [3306.5] & 561749 & 591984 & 3 & 1 & 0.252 & 0.0138 & 0.449 & -1.384 & B & $c a$ \\
\hline 18 & $1 s 3 p-1 s 6 s$ & ${ }^{1} \mathrm{P}^{\circ}-{ }^{-1} \mathrm{~S}$ & [2790.5] & 561749 & 597574 & 3 & 1 & 0.139 & 0.00542 & 0.149 & -1.789 & B & $c a$ \\
\hline 19 & $1 s 3 p-1 s 4 d$ & ${ }^{1} \mathrm{P}^{\circ}-{ }^{1} \mathrm{D}$ & [4787.5] & 561749 & 582631 & 3 & 5 & 1.14 & 0.654 & 30.9 & 0.293 & B & $c a$ \\
\hline 20 & $1 s 3 p-1 s 5 d$ & ${ }^{1} \mathrm{P}^{\circ}-{ }^{1} \mathrm{D}$ & [3250.1] & 561749 & 592508 & 3 & 5 & 0.528 & 0.139 & 4.48 & -0.379 & B & $c a$ \\
\hline 21 & $1 s 3 p-1 s 6 d$ & ${ }^{1} \mathrm{P}^{\circ}-{ }^{1} \mathrm{D}$ & [2767.1] & 561749 & 597877 & 3 & 5 & 0.289 & 0.0552 & 1.51 & -0.781 & B & $c a$ \\
\hline 22 & $1 s 3 d-1 s 3 p$ & ${ }^{1} \mathrm{D}-{ }^{1} \mathrm{P}^{0}$ & [211360] & 561276 & 561749 & 5 & 3 & $3.98 \times 10^{-5}$ & 0.0160 & 55.7 & -1.097 & B & 1 \\
\hline 23 & $1 s 3 d-1 s 4 p$ & ${ }^{1} \mathrm{D}-{ }^{1} \mathrm{P}^{0}$ & [4637.8] & 561276 & 582832 & 5 & 3 & 0.0471 & 0.00911 & 0.695 & -1.342 & B & $c a$ \\
\hline 24 & $1 s 3 d-1 s 4 f$ & ${ }^{1} \mathrm{D}-{ }^{1} \mathrm{~F}^{\circ}$ & [4678.4] & 561276 & 582645 & 5 & 7 & 2.21 & 1.02 & 78.2 & 0.706 & B & $c a$ \\
\hline 25 & $1 s 3 d-1 s 5 f$ & ${ }^{1} \mathrm{D}-{ }^{1} \mathrm{~F}^{\circ}$ & [3199.4] & 561276 & 592523 & 5 & 7 & 0.736 & 0.158 & 8.33 & -0.102 & B & $c a$ \\
\hline 26 & $1 s 3 d-1 s 6 f$ & ${ }^{1} \mathrm{D}-{ }^{1} \mathrm{~F}^{0}$ & [2730.7] & 561276 & 597886 & 5 & 7 & 0.338 & 0.0528 & 2.38 & -0.578 & B & $c a$ \\
\hline 27 & $1 s 2 s-1 s 2 p$ & $\begin{array}{c}{ }^{3} S-{ }^{3} \mathrm{P}^{\circ} \\
(1)\end{array}$ & 5484.8 & 476046 & 494273 & 3 & 9 & 0.228 & 0.308 & 16.7 & -0.034 & A & 1 \\
\hline 28 & $1 s 2 s-1 s 3 p$ & ${ }^{3} \mathrm{~S}-{ }^{3} \mathrm{P}^{\circ}$ & [1198.3] & 476046 & 559501 & 3 & 9 & 2.88 & 0.186 & 2.20 & -0.253 & A & 1 \\
\hline 29 & $1 s 2 s-1 s 4 p$ & ${ }^{3} \mathrm{~S}-{ }^{3} \mathrm{P}^{0}$ & [944.72] & 476046 & 581897 & 3 & 9 & 1.39 & 0.0558 & 0.521 & -0.776 & B & $c a$ \\
\hline 30 & $1 s 2 s-1 s 5 p$ & ${ }^{3} \mathrm{~S}-{ }^{3} \mathrm{P}^{0}$ & [861.36] & 476046 & 592141 & 3 & 9 & 0.722 & 0.0241 & 0.205 & -1.141 & B & $c a$ \\
\hline 31 & $1 s 2 p-1 s 3 s$ & ${ }^{3} \mathrm{P}^{\circ}-{ }^{3} \mathrm{~S}$ & [1653.2] & 494273 & 554761 & 9 & 3 & 2.85 & 0.0390 & 1.91 & -0.455 & B & $c a$ \\
\hline 32 & $1 s 2 p-1 s 4 s$ & ${ }^{3} \mathrm{P}^{\circ}-{ }^{3} \mathrm{~S}$ & [1166.7] & 494273 & 579982 & 9 & 3 & 1.02 & 0.00697 & 0.241 & -1.202 & B & $c a$ \\
\hline
\end{tabular}


Li II. Allowed Transitions-Continued

\begin{tabular}{|c|c|c|c|c|c|c|c|c|c|c|c|c|c|}
\hline No. & $\begin{array}{c}\text { Transition } \\
\text { Array }\end{array}$ & Multiplet & $\lambda(\AA)$ & $E_{i}\left(\mathrm{~cm}^{-1}\right)$ & $E_{k}\left(\mathrm{~cm}^{-1}\right)$ & $g_{i}$ & $g_{k}$ & $A_{k i}\left(\sec ^{-1}\right)$ & $f_{i k}$ & $S$ (at.u.) & $\log g f$ & $\begin{array}{l}\text { Accu- } \\
\text { racy }\end{array}$ & Source \\
\hline 33 & $1 s 2 p-1 s 5 s$ & ${ }^{3} \mathrm{P}^{\circ} \mathrm{L}^{\prime} \mathrm{S}$ & [1031.9] & 494273 & 591184 & 9 & 3 & 0.490 & 0.00261 & 0.0797 & -1.630 & B & $c a$ \\
\hline 34 & $1 s 2 p-1 s 6 s$ & ${ }^{3} \mathrm{P}^{\circ}-{ }^{3} \mathrm{~S}$ & [972.30] & 494273 & 597122 & 9 & 3 & 0.270 & 0.00128 & 0.0368 & -1.939 & B & $c a$ \\
\hline 35 & $1 s 2 p-1 s 3 d$ & ${ }^{3} \mathrm{P}^{\circ}-{ }^{3} \mathrm{D}$ & [1493.2] & 494273 & 561245 & 9 & 15 & 11.2 & 0.625 & 27.7 & 0.750 & A & 1 \\
\hline 36 & $1 s 2 p-1 s 4 d$ & ${ }^{3} \mathrm{P}^{\circ}-{ }^{3} \mathrm{D}$ & [1132.0] & 494273 & 582612 & 9 & 15 & 3.80 & 0.122 & 4.08 & 0.039 & B & $c a$ \\
\hline 37 & $1 s 2 p-1 s 5 d$ & ${ }^{3} \mathrm{P}^{\circ}-{ }^{3} \mathrm{D}$ & [1018.0] & 494273 & 592505 & 9 & 15 & 1.78 & 0.0461 & 1.39 & -0.382 & B & $c a$ \\
\hline 38 & $1 s 3 s-1 s 3 p$ & ${ }^{3} \mathrm{~S}-{ }^{3} \mathrm{P}^{\circ}$ & [21091] & 554761 & 559501 & 3 & 9 & 0.0254 & 0.509 & 106 & 0.184 & B & $c a$ \\
\hline 39 & $1 s 3 s-1 s 4 p$ & $\begin{array}{c}{ }^{3} S-{ }^{3} \mathrm{P}^{\circ} \\
(2)\end{array}$ & 3684.1 & 554761 & 581897 & 3 & 9 & 0.309 & 0.189 & 6.86 & -0.248 & B & $c a$ \\
\hline 40 & $1 s 3 s-1 s 5 p$ & $\begin{array}{l}{ }^{3} \mathrm{~S}-{ }^{3} \mathrm{P}^{\circ} \\
(4 u v)\end{array}$ & 2674.43 & 554761 & 592141 & 3 & 9 & 0.192 & 0.0617 & 1.63 & -0.733 & B & $c a$ \\
\hline 41 & $1 s 3 p-1 s 4 s$ & $\begin{array}{c}{ }^{3} \mathrm{P}^{0}-{ }^{3} \mathrm{~S} \\
(4)\end{array}$ & 4881.3 & 559501 & 579982 & 9 & 3 & 0.714 & 0.0850 & 12.3 & -0.116 & B & $c a$ \\
\hline 42 & $1 s 3 p-1 s 5 s$ & ${ }^{3} \mathrm{P}^{\circ}-{ }^{-3} \mathrm{~S}$ & [3155.4] & 559501 & 591184 & 9 & 3 & 0.318 & 0.0158 & 1.48 & -0.846 & B & $c a$ \\
\hline 43 & $1 s 3 p-1 s 6 s$ & ${ }^{3} \mathrm{P}^{0}-{ }^{3} \mathrm{~S}$ & [2657.3] & 559501 & 597122 & 9 & 3 & 0.172 & 0.00606 & 0.477 & -1.263 & B & $c a$ \\
\hline 44 & $1 s 3 p-1 s 3 d$ & ${ }^{3} \mathrm{P}^{\circ}-{ }^{3} \mathrm{D}$ & [57324] & 559501 & 561245 & 9 & 15 & 0.00110 & 0.0904 & 154 & -0.090 & A & 1 \\
\hline 45 & $1 s 3 p-1 s 4 d$ & $\begin{array}{c}{ }^{3} \mathrm{P}^{\circ}-{ }^{3} \mathrm{D} \\
(5)\end{array}$ & 4325.7 & 559501 & 582612 & 9 & 15 & 1.09 & 0.508 & 65.1 & 0.660 & B & $c a$ \\
\hline 46 & $1 s 3 p-1 s 5 d$ & ${ }^{3} \mathrm{P}^{\circ}-{ }^{3} \mathrm{D}$ & [3029.1] & 559501 & 592505 & 9 & 15 & 0.549 & 0.126 & 11.3 & 0.054 & B & $c a$ \\
\hline 47 & $1 s 3 p-1 s 6 d$ & ${ }^{3} \mathrm{P}^{\circ}-{ }^{3} \mathrm{D}$ & [2605.1] & 559501 & 597876 & 9 & 15 & 0.312 & 0.0530 & 4.09 & -0.322 & B & $c a$ \\
\hline 48 & $1 s 3 d-1 s 4 p$ & ${ }^{3} \mathrm{D}-{ }^{3} \mathrm{P}^{\circ}$ & [4840.8] & 561245 & 581897 & 15 & 9 & 0.0941 & 0.0198 & 4.74 & -0.527 & B & $c a$ \\
\hline 49 & $1 s 3 d-1 s 5 p$ & ${ }^{3} \mathrm{D}-{ }^{3} \mathrm{P}^{\circ}$ & [3235.7] & 561245 & 592141 & 15 & 9 & 0.0391 & 0.00369 & 0.589 & -1.257 & B & $c a$ \\
\hline 50 & $1 s 3 d-1 s 4 f$ & ${ }^{3} \mathrm{D}-{ }^{3} \mathrm{~F}^{\circ}$ & [4671.8] & 561245 & 582644 & 15 & 21 & 2.21 & 1.01 & 234 & 1.182 & B & $c a$ \\
\hline 51 & $1 s 3 d-1 s 5 f$ & ${ }^{3} \mathrm{D}-{ }^{3} \mathrm{~F}^{\circ}$ & [3195.8] & 561245 & 592527 & 15 & 21 & 0.739 & 0.158 & 25.0 & 0.376 & B & $c a$ \\
\hline 52 & $1 s 3 d-1 s 6 f$ & ${ }^{3} \mathrm{D}-{ }^{3} \mathrm{~F}^{\circ}$ & [2728.4] & 561245 & 597886 & 15 & 21 & 0.339 & 0.0530 & 7.14 & -0.100 & B & $c a$ \\
\hline
\end{tabular}




\section{BERYLLIUM}

\section{Be I}

Ground State

$1 s^{2} 2 s^{2}{ }^{1} \mathrm{~S}_{0}$

Ionization Potential

$9.320 \mathrm{eV}=75192.29 \mathrm{~cm}^{-1}$

\section{Allowed Transitions}

The results taken from Weiss' self-consistent field calculations [1] are estimated to be accurate to 10-25 percent because of the good agreement between the dipole length and velocity approximations and because of the inclusion of configuration interaction. The average of the two approximations is adopted [1]. For the resonance line the adopted value is within 10 percent of the result of calculations of Bolotin and Yutsis [2], who include configuration interaction in a more limited way. The Coulomb approximation, employed for the other transitions, is not considered to be very reliable here since the lower state has the same principal quantum number as the ground state.

\section{References}

[1] Weiss, A. W., private communication (1964).

[2] Bolotin, A. B., and Yutsis, A. P., Zhur. Eksptl. i Teoret. Fiz. 24, 537-543 (1953) (Translated in "Optical Transition Probabilities," Office of Technical Services, U.S. Department of Commerce, Washington, D.C.).

\section{Be I. Allowed Transitions}

\begin{tabular}{|c|c|c|c|c|c|c|c|c|c|c|c|c|c|}
\hline No. & $\begin{array}{c}\text { Transition } \\
\text { Array }\end{array}$ & Multiplet & $\lambda(\AA)$ & $E_{i}\left(\mathrm{~cm}^{-1}\right)$ & $E_{k}\left(\mathrm{~cm}^{-1}\right)$ & $g_{i}$ & $g_{k}$ & $A_{k i}\left(10^{8} \mathrm{sec}^{-1}\right)$ & $f_{i k}$ & $S$ (at.u.) & $\log g f$ & $\begin{array}{c}\text { Accu- } \\
\text { racy }\end{array}$ & Source \\
\hline 1 & $\begin{array}{l}2 s^{2}- \\
\quad 2 s 2 p\left({ }^{2} \mathrm{~S}\right) 2 p\end{array}$ & $\begin{array}{r}{ }^{1} S-{ }^{1} \mathrm{P}^{\circ} \\
(1 \mathrm{uv})\end{array}$ & 2348.61 & 0.0 & 42565.3 & 1 & 3 & 5.47 & 1.36 & 10.5 & 0.133 & $\mathrm{C}+$ & 1 \\
\hline 2 & $2 s 2 p-2 p^{2}$ & $\begin{array}{r}{ }^{3} \mathrm{P}^{\circ}-{ }^{3} \mathrm{P} \\
(2 \mathrm{uv})\end{array}$ & 2650.6 & 21980.1 & 59695.8 & 9 & 9 & 4.42 & 0.466 & 36.6 & 0.623 & $\mathrm{C}+$ & 1 \\
\hline 3 & & ${ }^{1} \mathrm{P}^{\circ}-{ }^{1} \mathrm{~S}$ & {$[3455.2]$} & 42565.3 & 71498.9 & 3 & 1 & 2.21 & 0.132 & 4.50 & -0.403 & C & 1 \\
\hline 4 & $\begin{array}{l}2 s 2 p- \\
\quad 2 s\left({ }^{2} \mathrm{~S}\right) 3 s\end{array}$ & $\frac{{ }^{3} \mathrm{P}^{\circ}-{ }^{3 \mathrm{~S}}}{(1)}$ & 3321.2 & 21980.1 & 52080.9 & 9 & 3 & 0.62 & 0.034 & 3.4 & -0.51 & $\mathrm{D}$ & $c a$ \\
\hline 5 & & $\begin{array}{c}{ }^{1} \mathrm{P}^{\circ}-{ }^{1} \mathrm{~S} \\
(2)\end{array}$ & 8254.10 & 42565.3 & 54677.2 & 3 & 1 & 0.38 & 0.13 & 11 & -0.41 & $\mathrm{D}$ & $c a$ \\
\hline 6 & $\begin{array}{l}2 s 2 p- \\
\quad 2 s\left({ }^{2} \mathrm{~S}\right) 3 d\end{array}$ & $\begin{array}{r}{ }^{3} \mathrm{P}^{\circ}-{ }^{3} \mathrm{D} \\
(3 \mathrm{uv})\end{array}$ & 2494.6 & 21980.1 & 62053.6 & 9 & 15 & 1.0 & 0.16 & 12 & 0.15 & D & $c a$ \\
\hline 7 & & ${ }^{1} \mathrm{P}^{\circ}-{ }^{1} \mathrm{D}$ & 4572.67 & 42565.3 & 64428.2 & 3 & 5 & 0.37 & 0.19 & 8.6 & -0.24 & $\mathrm{D}$ & $c a$ \\
\hline 8 & $\begin{array}{l}2 s 2 p- \\
\quad 2 s\left({ }^{2} \mathrm{~S}\right) 4 s\end{array}$ & ${ }^{1} \mathrm{P}^{\circ}-{ }^{1} \mathrm{~S}$ & 4407.91 & 42565.3 & 65245.4 & 3 & 1 & 0.090 & 0.0087 & 0.38 & -1.58 & D & $c a$ \\
\hline 9 & $\begin{array}{l}2 s 2 p- \\
2 s\left({ }^{2} \mathrm{~S}\right) 4 d\end{array}$ & ${ }^{1} \mathrm{P}^{\circ}-{ }^{1} \mathrm{D}$ & 3813.40 & 42565.3 & 68781.2 & 3 & 5 & 0.23 & 0.084 & 3.2 & -0.60 & D & $c a$ \\
\hline 10 & $\begin{array}{l}2 s 2 p- \\
\quad 2 s\left({ }^{2} \mathrm{~S}\right) 5 s\end{array}$ & $\begin{array}{c}{ }^{1} \mathrm{P}^{\circ}-{ }^{1} \mathrm{~S} \\
(6)\end{array}$ & 3736.28 & 42565.3 & 69322.3 & 3 & 1 & 0.038 & 0.0027 & 0.099 & -2.10 & D & $c a$ \\
\hline 11 & $\begin{array}{l}2 s 2 p- \\
\quad 2 s\left({ }^{2} \mathrm{~S}\right) 5 d\end{array}$ & $\frac{{ }^{1} \mathrm{P}^{\circ}-{ }^{1} \mathrm{D}}{(7)}$ & 3515.54 & 42565.3 & 71002.3 & 3 & 5 & 0.13 & 0.041 & 1.4 & -0.91 & $\mathrm{D}$ & $c a$ \\
\hline
\end{tabular}




\section{Forbidden Transitions}

Naqvi's calculations [1] are the only available source. The results for the ${ }^{3} \mathrm{P}^{\circ}-{ }^{3} \mathrm{P}^{\circ}$ transitions are essentially independent of the choice of the interaction parameters. For the ${ }^{3} \mathrm{P}^{\circ}-{ }^{1} \mathrm{P}^{\circ}$ transitions, Naqvi uses empirical term intervals, i.e., the effects of configuration interaction should be partially included.

\section{Reference}

[1] Naqvi, A. M., Thesis Harvard (1951).

Be I. Forbidden Transitions

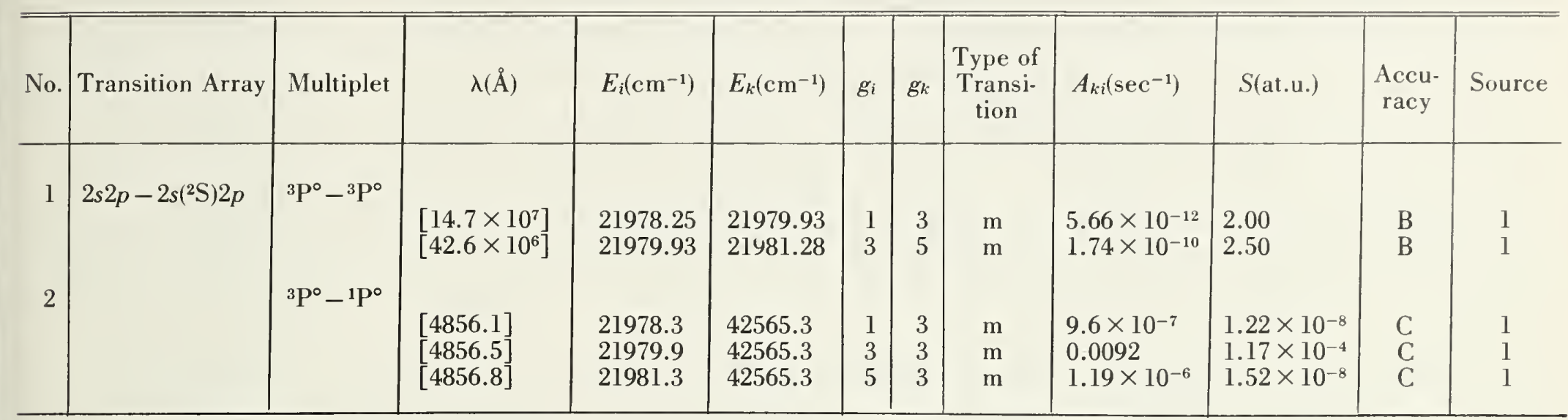

Be II

Ground State

Ionization Potential
$1 s^{2} 2 s^{2} \mathrm{~S}_{1 / 2}$

$18.206 \mathrm{eV}=146881.7 \mathrm{~cm}^{-1}$

\section{Allowed Transitions}

The values taken from Weiss' calculations [1] are estimated to be accurate to within 10 percent because of the very close agreement between his dipole length and dipole velocity approximations, except for the case of the $3 p-3 d$ transition where somewhat larger divergencies occur. The values calculated with the dipole length approximation are adopted.

\section{Reference}

[1] Weiss, A. W., Astrophys. J. 138, 1262-1276 (1963). 
Be II. Allowed Transitions

\begin{tabular}{|c|c|c|c|c|c|c|c|c|c|c|c|c|c|}
\hline No. & $\begin{array}{c}\text { Transition } \\
\text { Array }\end{array}$ & Multiplet & $\lambda(\AA)$ & $E_{i}\left(\mathrm{~cm}^{-1}\right)$ & $E_{k}\left(\mathrm{~cm}^{-1}\right)$ & $g_{i}$ & $g_{k}$ & $A_{k i}\left(10^{x} \sec ^{-1}\right)$ & $f_{i k}$ & $S$ (at.u.) & $\log g f$ & $\begin{array}{c}\text { Accu- } \\
\text { racy }\end{array}$ & Source \\
\hline 1 & $2 s-2 p$ & ${ }^{2} \mathrm{~S}-{ }^{2} \mathrm{P}^{\circ}$ & 3130.6 & 0.0 & 31933.2 & 2 & 6 & 1.15 & 0.505 & 10.4 & 0.004 & A & 1 \\
\hline 2 & $2 s-3 p$ & $\begin{array}{r}{ }^{2} \mathrm{~S}-{ }^{2} \mathrm{P}^{\circ} \\
(1 \mathrm{uv})\end{array}$ & 1036.27 & 0.0 & 96497.6 & 2 & 6 & 1.66 & 0.0804 & 0.549 & -0.794 & B & 1 \\
\hline 3 & $2 p-3 s$ & $\begin{array}{r}{ }^{2} \mathrm{P}^{\circ}-{ }^{2} \mathrm{~S} \\
\quad(3 \text { uv })\end{array}$ & 1776.2 & 31933.2 & 88231.2 & 6 & 2 & 4.22 & 0.0665 & 2.33 & -0.399 & $\mathrm{~B}+$ & 1 \\
\hline 4 & $2 p-3 d$ & $\begin{array}{r}{ }^{2} \mathrm{P}^{\circ}-{ }^{2} \mathrm{D} \\
(4 \mathrm{uv})\end{array}$ & 1512.4 & 31933.2 & 98053.2 & 6 & 10 & 11.4 & 0.652 & 19.5 & 0.592 & $\mathrm{~B}+$ & 1 \\
\hline 5 & $3 s-3 p$ & ${ }^{2} \mathrm{~S}-{ }^{2} \mathrm{P}^{\circ}$ & 12094 & 88231.2 & 96497.6 & 2 & 6 & 0.128 & 0.839 & 66.8 & 0.225 & B & 1 \\
\hline 6 & $3 s-4 p$ & $\begin{array}{c}{ }^{2} \mathrm{~S}-{ }^{2} \mathrm{P}^{\circ} \\
(2)\end{array}$ & 3274.64 & 88231.2 & 118760 & 2 & 6 & 0.143 & 0.0691 & 1.49 & -0.860 & B & $c a$ \\
\hline 7 & $3 p-4 s$ & $\begin{array}{c}{ }^{2} \mathrm{P}^{\circ}-{ }^{2} \mathrm{~S} \\
(3)\end{array}$ & 5270.7 & 96497.6 & 115465 & 6 & 2 & 0.969 & 0.134 & 14.0 & -0.093 & B & $c a$ \\
\hline 8 & $3 p-5 s$ & $\begin{array}{c}{ }^{2} \mathrm{P}^{\circ}-{ }^{2} \mathrm{~S} \\
(5)\end{array}$ & 3247.7 & 96497.6 & 127336 & 6 & 2 & 0.410 & 0.0216 & 1.38 & -0.888 & B & $c a$ \\
\hline 9 & $3 p-3 d$ & ${ }^{2} \mathrm{P}^{\circ}-{ }^{2} \mathrm{D}$ & 64266 & 96497.6 & 98053.2 & 6 & 10 & $7.83 \times 10^{-4}$ & 0.0808 & 103 & -0.314 & B & 1 \\
\hline 10 & $3 p-4 d$ & $\begin{array}{c}{ }^{2} \mathrm{P}^{\circ}-{ }^{2} \mathrm{D} \\
(4)\end{array}$ & 4360.9 & 96497.6 & 119422 & 6 & 10 & 1.09 & 0.519 & 44.7 & 0.493 & B & $\mathrm{ca}$ \\
\hline 11 & $3 d-4 f$ & $\begin{array}{c}{ }^{2} \mathrm{D}-{ }_{(6)}^{2} \mathrm{~F}^{\circ} \\
\text { (6) }\end{array}$ & 4673.46 & 98053.2 & 119445 & 10 & 14 & 2.21 & 1.01 & 156 & 1.006 & B & $c a$ \\
\hline
\end{tabular}

\section{BeIII}

Ground State

Ionization Potential
$1 s^{2} \mathrm{~S}_{0}$

$153.850 \mathrm{eV}=1241255 \mathrm{~cm}^{-1}$

\section{Allowed Transitions}

The results of extensive non-relativistic variational calculations by Weiss [1] are chosen. Values have been calculated in both the dipole length and dipole velocity approximations and agree to within 1 percent, except for the $3 p^{1} \mathrm{P}^{\circ}-3 d^{1} \mathrm{D}$ transition where agreement is not as good. The average of the two approximations is adopted [1].

\section{Reference}

[1] Weiss, A. W., private communication (1964). 
Be III. Allowed Transitions

\begin{tabular}{|c|c|c|c|c|c|c|c|c|c|c|c|c|c|}
\hline No. & $\begin{array}{c}\text { Transition } \\
\text { Array }\end{array}$ & Multiplet & $\lambda(\AA)$ & $E_{i}\left(\mathrm{~cm}^{-1}\right)$ & $E_{k}\left(\mathrm{~cm}^{-1}\right)$ & $g_{i}$ & $g_{k}$ & $A_{k i}\left(10^{H} \sec ^{-1}\right)$ & $f_{i k}$ & S(at.u.) & $\log g f$ & Accu- & Source \\
\hline 1 & $1 s^{2}-1 s 2 p$ & ${ }^{1} S_{-1}^{-1} P^{0}$ & [100.25] & 0 & 997466 & 1 & 3 & 1220 & 0.552 & 0.182 & -0.258 & A & 1 \\
\hline 2 & $1 s^{2}-1 s 3 p$ & ${ }^{1} \mathrm{~S}-{ }^{-1} \mathrm{P}^{\circ}$ & [88.314] & 0 & 1132323 & 1 & 3 & 362 & 0.127 & 0.0369 & -0.896 & A & 1 \\
\hline 3 & $1 s 2 s-1 s 2 p$ & ${ }^{1} \mathrm{~S}-{ }^{1} \mathrm{P}^{\circ}$ & [6141.2] & 981187 & 997466 & 1 & 3 & 0.0877 & 0.149 & 3.02 & -0.827 & A & 1 \\
\hline 4 & $1 s 2 s-1 s 3 p$ & ${ }^{1} \mathrm{~S}-{ }^{1} \mathrm{P}^{\circ}$ & [398.19] & 981187 & 1132323 & 1 & 3 & 42.8 & 0.305 & 0.400 & -0.516 & A & 1 \\
\hline 5 & $1 s 2 p-1 s 3 d$ & ${ }^{1} \mathrm{P}^{\circ}-{ }^{1} \mathrm{D}$ & [746.70] & 997466 & [1131389] & 3 & 5 & 51.0 & 0.711 & 5.24 & 0.329 & A & 1 \\
\hline 6 & $1 s 3 d-1 s 3 p$ & ${ }^{1} \mathrm{D}-{ }^{1} \mathrm{P}^{\circ}$ & [107000]? & [1131389] & 1132323 & 5 & 3 & $1.32 \times 10^{-4}$ & 0.0136 & 24.0 & -1.168 & $\mathrm{C}+$ & 1 \\
\hline 7 & $1 s 2 s-1 s 2 p$ & ${ }^{3} \mathrm{~S}-{ }^{3} \mathrm{P}^{\circ}$ & [3721.8] & [956496] & [983357] & 3 & 9 & 0.342 & 0.213 & 7.83 & -0.195 & A & 1 \\
\hline 8 & $1 s 2 s-1 s 3 p$ & ${ }^{3} \mathrm{~S}-{ }^{3} \mathrm{P}^{\circ}$ & [583.01] & [956496] & [1128020] & 3 & 9 & 16.5 & 0.252 & 1.45 & -0.122 & A & 1 \\
\hline 9 & $1 s 2 p-1 s 3 d$ & ${ }^{3} \mathrm{P}^{0}-{ }^{3} \mathrm{D}$ & [675.66] & [983357] & [1131360] & 9 & 15 & 56.1 & 0.640 & 12.8 & 0.760 & A & 1 \\
\hline 10 & $1 s 3 p-1 s 3 d$ & ${ }^{3} \mathrm{P}^{\circ}-{ }^{3} \mathrm{D}$ & [29930]? & [1128020] & [1131360] & 9 & 15 & 0.00318 & 0.0712 & 63.1 & -0.193 & A & 1 \\
\hline
\end{tabular}

\section{BORON}

\section{B I}

Ground State

Ionization Potential

$$
1 s^{2} 2 s^{2} 2 p{ }^{2} \mathrm{P}_{1 / 2}^{\circ}
$$

$8.296 \mathrm{eV}=66930 \mathrm{~cm}^{-1}$

\section{Allowed Transitions}

The values for the $2 s^{2} 2 p-2 s 2 p^{2}\left({ }^{2} \mathrm{P}^{\circ}-{ }^{2} \mathrm{D},{ }^{2} \mathrm{~S},{ }^{2} \mathrm{P}\right)$ transitions are taken from the calculations of Bolotin and Yutsis [1], who employ analytical one-electron wave functions. Self-consistent field calculations of Tsiunaitis and Yutsis [2] have been adopted for the $2 p^{2} \mathrm{P}^{\circ}-3 s^{2} \mathrm{~S}$ transition. Since both determinations take into account the important effects of configuration interaction only in a limited way, large uncertainties are expected.

\section{References}

[1] Bolotin, A. B., and Yutsis, A. P., Zhur. Eksptl. i Teoret. Fiz. 24, 537-543 (1953) (Translated in "Optical Transition Probabilities," Office of Technical Services, U.S. Department of Commerce, Washington, D.C.).

[2] Tsiunaitis, G. K., and Yutsis, A. P., Soviet Phys.-JETP 1, 358-363 (1955). 
B I. Allowed Transitions

\begin{tabular}{|c|c|c|c|c|c|c|c|c|c|c|c|c|c|}
\hline No. & $\begin{array}{l}\text { Transition } \\
\text { Array }\end{array}$ & Multiplet & $\lambda(\AA)$ & $E_{i}\left(\mathrm{~cm}^{-1}\right)$ & $E_{k}\left(\mathrm{~cm}^{-1}\right)$ & $g_{i}$ & $g_{k}$ & $A_{k i}\left(10^{8} \sec ^{-1}\right)$ & $f_{i k}$ & $S$ (at.u.) & $\log g f$ & $\begin{array}{c}\text { Accu- } \\
\text { racy }\end{array}$ & Source \\
\hline \multirow[t]{4}{*}{1} & $2 s^{2} 2 p-2 s 2 p^{2}$ & $\begin{array}{c}2 \mathrm{P}^{\circ}-{ }^{2} \mathrm{D} \\
(2 \mathrm{uv})\end{array}$ & 2089.3 & 10 & 47857 & 6 & 10 & 2.2 & 0.24 & 9.7 & 0.15 & $\mathrm{E}$ & 1 \\
\hline & & & 2089.57 & 15 & 47857 & 4 & 6 & 2.1 & 0.21 & 5.8 & -0.07 & $\mathrm{E}$ & ls \\
\hline & & & 2088.84 & 0 & 47857 & 2 & 4 & 1.8 & 0.23 & 3.2 & -0.33 & $\mathrm{E}$ & ls \\
\hline & & & [2089.6] & 15 & 47857 & 4 & 4 & 0.36 & 0.024 & 0.65 & -1.02 & $\mathrm{E}$ & ls \\
\hline \multirow[t]{3}{*}{2} & & ${ }^{2} \mathrm{P}^{\circ}-{ }^{2} \mathrm{~S}$ & 1573.5 & 10 & 63561 & 6 & 2 & 13 & 0.16 & 5.1 & -0.01 & $\mathrm{E}$ & 1 \\
\hline & & & {$[1573.7]$} & 15 & 63561 & 4 & 2 & 8.8 & 0.16 & 3.4 & -0.18 & $\mathrm{E}$ & ls \\
\hline & & & [1573.3] & 0 & 63561 & 2 & 2 & 4.4 & 0.16 & 1.7 & -0.48 & $\mathrm{E}$ & ls \\
\hline \multirow[t]{5}{*}{3} & & ${ }^{2} \mathrm{P}^{\circ}-{ }^{2} \mathrm{P}$ & 1378.7 & 10 & 72543 & 6 & 6 & 23 & 0.66 & 18 & 0.60 & $\mathrm{E}$ & 1 \\
\hline & & & [1378.7] & 15 & 72547 & 4 & 4 & 19 & 0.55 & 10 & 0.34 & $\mathrm{E}$ & ls \\
\hline & & & [1378.6] & 0 & 72535 & 2 & 2 & 15 & 0.44 & 4.0 & -0.05 & $\mathrm{E}$ & ls \\
\hline & & & [1378.9] & 15 & 72535 & 4 & 2 & 7.7 & 0.11 & 2.0 & -0.36 & $\mathrm{E}$ & ls \\
\hline & & & [1378.4] & 0 & 72547 & 2 & 4 & 3.9 & 0.22 & 2.0 & -0.36 & $\mathrm{E}$ & $l s$ \\
\hline \multirow[t]{3}{*}{4} & $2 p-\left({ }^{1} \mathrm{~S}\right) 3 s$ & $\begin{array}{c}{ }^{2} \mathrm{P}^{\circ}-{ }^{2} \mathrm{~S} \\
(1 \mathrm{uv})\end{array}$ & 2497.4 & 10 & 40040 & 6 & 2 & 3.6 & 0.11 & 5.5 & -0.17 & D & 2 \\
\hline & & & 2497.72 & 15 & 40040 & 4 & 2 & 2.4 & 0.11 & 3.7 & -0.35 & $\mathrm{D}$ & ls \\
\hline & & & 2496.77 & 0 & 40040 & 2 & 2 & 1.2 & 0.11 & 1.8 & -0.66 & $\mathrm{D}$ & is \\
\hline \multirow[t]{3}{*}{5} & $3 s-\left({ }^{1} \mathrm{~S}\right) 3 p$ & ${ }^{2} \mathrm{~S}-{ }^{2} \mathrm{P}^{\circ}$ & 11661 & 40040 & 48613 & 2 & 6 & 0.174 & 1.07 & 82 & 0.330 & C & $c a$ \\
\hline & & & 11660 & 40040 & 48613 & 2 & 4 & 0.174 & 0.71 & 54 & 0.152 & C & ls \\
\hline & & & 11662 & 40040 & 48612 & 2 & 2 & 0.174 & 0.355 & 27.2 & -0.149 & $\mathrm{C}$ & ls \\
\hline \multirow[t]{4}{*}{6} & $3 p-\left({ }^{1} \mathrm{~S}\right) 3 d$ & ${ }^{2} \mathrm{P}^{\circ}-{ }^{2} \mathrm{D}$ & 16243 & 48613 & 54768 & 6 & 10 & 0.138 & 0.90 & 291 & 0.73 & C & $c a$ \\
\hline & & & 16245 & 48613 & 54768 & 4 & 6 & 0.138 & 0.82 & 175 & 0.51 & C & $l s$ \\
\hline & & & 16240 & 48612 & 54768 & 2 & 4 & 0.115 & 0.91 & 97 & 0.259 & $\mathrm{C}$ & ls \\
\hline & & & 16245 & 48613 & 54768 & 4 & 4 & 0.0230 & 0.091 & 19.4 & -0.440 & $\mathrm{C}$ & ls \\
\hline \multirow[t]{3}{*}{7} & $3 p-\left({ }^{1} \mathrm{~S}\right) 4 s$ & ${ }^{2} \mathrm{P}^{\circ}-{ }^{2} \mathrm{~S}$ & 15628 & 48613 & 55010 & 6 & 2 & 0.154 & 0.188 & 58 & 0.052 & C & $c a$ \\
\hline & & & 15629 & 48613 & 55010 & 4 & 2 & 0.103 & 0.188 & 38.8 & -0.123 & C & ls \\
\hline & & & 15625 & 48612 & 55010 & 2 & 2 & 0.051 & 0.188 & 19.4 & -0.424 & C & $l s$ \\
\hline \multirow[t]{3}{*}{8} & $3 p-\left({ }^{1} \mathrm{~S}\right) 5 s$ & ${ }^{2} \mathrm{P}^{\circ}-{ }^{2} \mathrm{~S}$ & 8668.1 & 48613 & 60146 & 6 & 2 & 0.0486 & 0.0182 & 3.12 & -0.96 & $\mathrm{C}$ & $c a$ \\
\hline & & & 8668.6 & 48613 & 60146 & 4 & 2 & 0.0324 & 0.0182 & 2.08 & -1.137 & C & $l s$ \\
\hline & & & 8667.2 & 48612 & 60146 & 2 & 2 & 0.0162 & 0.0182 & 1.04 & -1.438 & $\mathrm{C}$ & ls \\
\hline
\end{tabular}

\section{Forbidden Transitions}

Naqvi's calculation [1] of the one possible transition in the ground state configuration $2 p$ is the only available source. The line strength should be quite accurate, since it does not sensitively depend on the choice of the interaction parameters.

Reference

[1] Naqvi, A. M., Thesis Harvard (1951).

B I. Forbidden Transitions

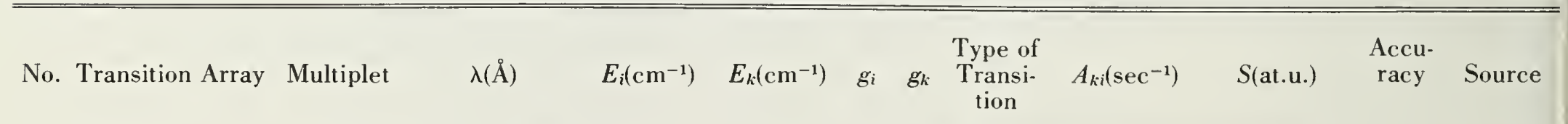

$1 \quad 2 p-2 p \quad{ }^{2} \mathrm{P}^{\circ}-{ }^{2} \mathrm{P}^{\circ} \quad\left[66.0 \times 10^{5}\right]$

$\begin{array}{llllll}0 & 15.15 & 2 & 4 & \mathrm{~m} & 3.15 \times 10^{-8}\end{array}$

1.33

C 
Ground State

Ionization Potential

$$
1 s^{2} 2 s^{2}{ }^{1} \mathrm{~S}_{0}
$$

$25.149 \mathrm{eV}=202895 \mathrm{~cm}^{-1}$

\section{Allowed Transitions}

Except for the $2 s 3 d^{3} \mathrm{D}-2 s 4 f^{3} \mathrm{~F}^{\circ}$ transition, for which a Coulomb approximation is employed, the values are taken from Weiss' self-consistent field calculations [1]. 'The length and velocity approximations disagree noticeably - for the $2 s 2 p^{1} \mathrm{P}^{\circ}-2 p^{2}{ }^{1} \mathrm{D}$ transition by as much as a factor of three. The average of the two approximations is adopted [1]. Accuracies within 50 percent are indicated by the following comparison: Weiss [1] has undertaken refined calculations, including configuration interaction, for the same transitions in $\mathrm{Be} \mathrm{I}$-the first member of this isoelectronic sequence-in addition to calculations of the type done for this ion. In all cases the agreement with the average of the dipole length and velocity approximations is close.

\section{Reference}

[1] Weiss, A. W., private communication (1964).

\section{B II. Allowed Transitions}

\begin{tabular}{|c|c|c|c|c|c|c|c|c|c|c|c|c|c|}
\hline No. & $\begin{array}{c}\text { Transition } \\
\text { Array }\end{array}$ & Multiplet & $\lambda(\AA)$ & $E_{i}\left(\mathrm{~cm}^{-1}\right)$ & $E_{k}\left(\mathrm{~cm}^{-1}\right)$ & $g_{i}$ & $g_{k}$ & $A_{k i}\left(10^{8} \sec ^{-1}\right)$ & $f_{i k}$ & $S($ at.u. $)$ & $\log g f$ & $\begin{array}{l}\text { Accu- } \\
\text { racy }\end{array}$ & Source \\
\hline 1 & $\begin{array}{l}2 s^{2}- \\
2 s\left({ }^{2} \mathrm{~S}\right) 2 p\end{array}$ & $\begin{array}{r}{ }^{1 S}-{ }^{1} \mathrm{P}^{\circ} \\
(1 \mathrm{uv})\end{array}$ & 1362.46 & 0.0 & 73396.7 & 1 & 3 & 13 & 1.1 & 4.9 & 0.04 & D & 1 \\
\hline \multirow[t]{7}{*}{2} & $2 s 2 p-2 p^{2}$ & $\begin{array}{r}{ }^{3} \mathrm{P}^{\circ}-{ }^{3} \mathrm{P} \\
(3 \mathrm{uv})\end{array}$ & {$[1624.0]$} & [37350.9] & [98925.5] & 9 & 9 & 8.4 & 0.33 & 16 & 0.48 & $\mathrm{D}$ & 1 \\
\hline & & & 1623.99 & {$[37356.4]$} & [98932.7] & 5 & 5 & 6.3 & 0.25 & 6.7 & 0.10 & $\mathrm{D}$ & ls \\
\hline & & & 1623.99 & {$[37340.0]$} & [98918.7] & 3 & 3 & 2.0 & 0.081 & 1.3 & -0.61 & D & ls \\
\hline & & & 1624.37 & {$[37356.4]$} & [98918.7] & 5 & 3 & 3.5 & 0.082 & 2.2 & -0.39 & D & ls \\
\hline & & & |[1624.2] & [37340.0] & [98910.3] & 3 & 1 & 8.5 & 0.11 & 1.8 & -0.47 & D & ls \\
\hline & & & 1623.57 & [37340.0] & [98932.7] & 3 & 5 & 2.1 & 0.14 & 2.2 & -0.39 & D & ls \\
\hline & & & [1623.8] & [37333.6] & [98918.7] & 1 & 3 & 2.8 & 0.34 & 1.8 & -0.47 & D & ls \\
\hline 3 & & ${ }^{1} \mathrm{P}^{\circ}-{ }^{1} \mathrm{D}$ & 3451.41 & 73396.7 & 102362 & 3 & 5 & 2.2 & 0.65 & 22 & 0.29 & $\mathrm{E}$ & 1 \\
\hline 4 & & ${ }^{1} \mathrm{P}^{0}-{ }^{1} \mathrm{~S}$ & [1842.8] & 73396.7 & 127662 & 3 & 1 & 6.8 & 0.12 & 2.1 & -0.46 & D & 1 \\
\hline 5 & $\begin{array}{l}2 s 3 d- \\
2 s\left({ }^{2} \mathrm{~S}\right) 4 f\end{array}$ & $\begin{array}{c}{ }^{3} \mathrm{D}-{ }^{3} \mathrm{~F}^{\circ} \\
(2)\end{array}$ & 4121.95 & [150649] & [174903] & 15 & 21 & 2.55 & 0.91 & 185 & 1.135 & C & $c a$ \\
\hline
\end{tabular}

\section{Forbidden Transitions}

Naqvi's calculations [1] are the only available source. The results for the ${ }^{3} \mathrm{P}^{\circ}-{ }^{3} \mathrm{P}^{\circ}$ transitions are essentially independent of the choice of the interaction parameters. For the ${ }^{3} \mathrm{P}^{\circ}-{ }^{1} \mathrm{P}^{\circ} \operatorname{transi-}$ tions, Naqvi uses empirical term intervals, i.e., the effects of configuration interaction should be partially included.

\section{Reference}

[1] Naqvi, A. M., Thesis Harvard (1951). 
B II. Forbidden Transitions

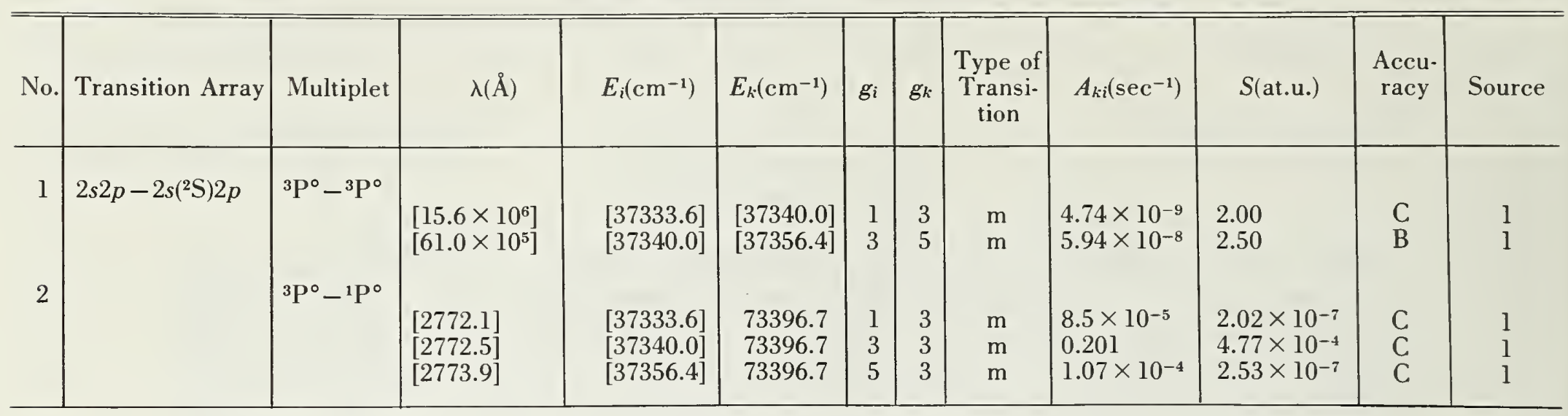

\section{BIII}

Ground State

Ionization Potential
$1 s^{2} 2 s^{2} \mathrm{~S}_{1 / 2}$

$$
37.920 \mathrm{eV}=305931.1 \mathrm{~cm}^{-1}
$$

\section{Allowed Transitions}

The values taken from Weiss' calculations [1] are estimated to be accurate to within 10 percent because of the very close agreement between his dipole length and dipole velocity approximations, except for the case of the $3 p-3 d$ transition where somewhat larger divergencies occur. The values calculated with the dipole length approximation are adopted.

\section{Reference}

[1] Weiss, A. W., Astrophys. J. 138, 1262-1276 (1963).

\section{B III. Allowed Transitions}

\begin{tabular}{|c|c|c|c|c|c|c|c|c|c|c|c|c|c|}
\hline No. & $\begin{array}{c}\text { Transition } \\
\text { Array }\end{array}$ & Multiplet & $\lambda(\AA)$ & $E_{i}\left(\mathrm{~cm}^{-1}\right)$ & $E_{k}\left(\mathrm{~cm}^{-1}\right)$ & $g_{i}$ & $g_{k}$ & $A_{k i}\left(10^{8} \sec ^{-1}\right)$ & $f_{i k}$ & $S($ at.u. $)$ & $\log g f$ & $\begin{array}{l}\text { Accu- } \\
\text { racy }\end{array}$ & Source \\
\hline 1 & $2 s-2 p$ & ${ }^{2} \mathrm{~S}-{ }^{2} \mathrm{P}^{\circ}$ & 2066.3 & 0.0 & 48381.2 & 2 & 6 & 1.91 & 0.366 & 4.98 & -0.136 & A & 1 \\
\hline 2 & $2 s-3 p$ & ${ }^{2} \mathrm{~S}-{ }^{2} \mathrm{P}^{\circ}$ & 518.25 & 0.0 & 192956 & 2 & 6 & 12.5 & 0.151 & 0.515 & -0.520 & B & 1 \\
\hline 3 & $2 p-3 s$ & ${ }^{2} \mathrm{P}^{\circ}-{ }^{2} \mathrm{~S}$ & 758.60 & 48381.2 & 180202 & 6 & 2 & 16.3 & 0.0470 & 0.704 & -0.550 & $B+$ & 1 \\
\hline 4 & $2 p-3 d$ & ${ }^{2} \mathrm{P}^{\circ}-{ }^{2} \mathrm{D}$ & 677.09 & 48381.2 & 196071 & 6 & 10 & 56.8 & 0.651 & 8.71 & 0.592 & $B+$ & 1 \\
\hline 5 & $3 s-3 p$ & ${ }^{2} \mathrm{~S}-{ }^{2} \mathrm{P}^{\circ}$ & 7838.5 & 180202 & 192956 & 2 & 6 & 0.222 & 0.614 & 31.7 & 0.089 & B & 1 \\
\hline 6 & $3 p-3 d$ & ${ }^{2} \mathrm{P}^{\circ}-{ }^{2} \mathrm{D}$ & 32094 & 192956 & 196071 & 6 & 10 & 0.00279 & 0.0719 & 45.6 & -0.365 & B & 1 \\
\hline 7 & $4 p-5 d$ & ${ }^{2} \mathrm{P}^{\circ}-_{(1)}^{2} \mathrm{D}$ & 4243.60 & 242832 & 266390 & 6 & 10 & 1.11 & 0.501 & 42.0 & 0.478 & B & $c a$ \\
\hline 8 & $4 d-5 f$ & $\begin{array}{c}{ }^{2} \mathrm{D}-{ }^{2} \mathrm{~F}^{\circ} \\
(2)\end{array}$ & 4487.46 & 244139 & 266417 & 10 & 14 & 2.10 & 0.887 & 131 & 0.948 & $\mathrm{~B}$ & $\mathrm{ca}$ \\
\hline
\end{tabular}




\section{B IV}

Ground State

$1 s^{2}{ }^{1} \mathrm{~S}_{0}$

Ionization Potential

$259.298 \mathrm{eV}=2091960 \mathrm{~cm}^{-1}$

\section{Allowed Transitions}

The results of extensive non-relativistic variational calculations by Weiss [1] are chosen. Values have been calculated in both the dipole length and dipole velocity approximations and agree to within I percent, except for the $3 p^{1} \mathrm{P}^{\circ}-3 d^{1} \mathrm{D}$ transition, where agreement is not as good. The average of the two approximations is adopted [1].

Reference

[1] Weiss, A. W., private communication (1964).

\section{B IV. Allowed Transitions}

\begin{tabular}{|c|c|c|c|c|c|c|c|c|c|c|c|c|c|}
\hline No. & $\begin{array}{c}\text { Transition } \\
\text { Array }\end{array}$ & Multiplet & $\lambda(\AA)$ & $E_{i}\left(\mathrm{~cm}^{-1}\right)$ & $E_{k}\left(\mathrm{~cm}^{-1}\right)$ & $g_{i}$ & $g_{k}$ & $A_{\text {hi }}\left(10^{8} \sec ^{-1}\right)$ & $f_{i k}$ & S(at.u.) & $\log g f$ & $\begin{array}{l}\text { Accu- } \\
\text { racy }\end{array}$ & Source \\
\hline 1 & $1 s^{2}-1 s 2 p$ & ${ }^{1} \mathrm{~S}-{ }^{1} \mathrm{P}^{0}$ & [60.313] & 0 & 1658020 & 1 & 3 & 3720 & 0.609 & 0.121 & -0.215 & A & 1 \\
\hline 2 & $1 s^{2}-1 s 3 p$ & ${ }^{1} \mathrm{~S}-{ }^{1} \mathrm{P}^{\circ}$ & [52.682] & 0 & 1898180 & 1 & 3 & 1080 & 0.135 & 0.0234 & -0.870 & A & 1 \\
\hline 3 & $1 s 2 s-1 s 2 p$ & ${ }^{1} \mathrm{~S}-{ }^{1} \mathrm{P}^{0}$ & [4499.4] & [1635801] & 1658020 & 1 & 3 & 0.125 & 0.114 & 1.69 & -0.943 & A & 1 \\
\hline 4 & $1 s 2 s-1 s 3 p$ & ${ }^{1} \mathrm{~S}-{ }^{1} \mathrm{P}^{0}$ & [381.13] & [1635801] & 1898180 & 1 & 3 & 51.0 & 0.333 & 0.418 & -0.478 & A & 1 \\
\hline 5 & $1 s 2 p-1 s 3 d$ & ${ }^{1} \mathrm{P}^{\circ}-{ }^{1} \mathrm{D}$ & [418.83] & 1658020 & [1896780] & 3 & 5 & 162 & 0.709 & 2.93 & 0.328 & A & 1 \\
\hline 6 & $1 s 3 d-1 s 3 p$ & ${ }^{1} \mathrm{D}-{ }^{1} \mathrm{P}^{\circ}$ & [71410]? & [1896780] & 1898180 & 5 & 3 & $2.62 \times 10^{-4}$ & 0.0120 & 14.1 & -1.222 & $\mathrm{C}+$ & 1 \\
\hline 7 & $1 s 2 s-1 s 2 p$ & ${ }^{3} \mathrm{~S}-{ }^{3} \mathrm{P}^{0}$ & 2823.4 & 1601505 & 1636913 & 3 & 9 & 0.455 & 0.163 & 4.55 & -0.311 & A & 1 \\
\hline 8 & $1 s 2 s-1 s 3 p$ & ${ }^{3} \mathrm{~S}-{ }^{3} \mathrm{P}^{\circ}$ & [344.19] & 1601505 & [1892046] & 3 & 9 & 54.6 & 0.291 & 0.989 & -0.059 & A & 1 \\
\hline 9 & $1 s 2 p-1 s 3 d$ & ${ }^{3} \mathrm{P}^{\circ}-{ }^{3} \mathrm{D}$ & [385.05] & 1636913 & [1896618] & 9 & 15 & 175 & 0.650 & 7.42 & 0.767 & A & 1 \\
\hline 10 & $1 s 3 p-1 s 3 d$ & ${ }^{3} \mathrm{P}^{\circ}-{ }^{3} \mathrm{D}$ & [21870]? & [1892046] & [1896618] & 9 & 15 & 0.00484 & 0.0578 & 37.5 & -0.284 & A & 1 \\
\hline
\end{tabular}




\section{Allowed Transitions}

List of tabulated lines:

\begin{tabular}{|c|c|c|c|c|c|}
\hline Wavelength $[\AA]]$ & No. & Wavelength [ $]$ & No. & Wavelength $[\AA]$ & No. \\
\hline $\begin{array}{c}945.193 \\
945.336 \\
945.566 \\
1260.75 \\
1260.9\end{array}$ & $\begin{array}{r}4 \\
4 \\
4 \\
13 \\
13\end{array}$ & $\begin{array}{l}1657.00 \\
1657.37 \\
1657.89 \\
1658.11 \\
1751.9\end{array}$ & $\begin{array}{r}5 \\
5 \\
5 \\
5 \\
20\end{array}$ & $\begin{array}{l}9078.32 \\
9088.57 \\
9094.89 \\
9111.85 \\
9603.09\end{array}$ & $\begin{array}{l}29 \\
29 \\
29 \\
29 \\
28\end{array}$ \\
\hline $\begin{array}{l}1260.96 \\
1261.12 \\
1261.4 \\
1261.56 \\
1274.13\end{array}$ & $\begin{array}{l}13 \\
13 \\
13 \\
13 \\
12\end{array}$ & $\begin{array}{l}1764 \\
1765 \\
1930.93 \\
2478.56 \\
2582.90\end{array}$ & $\begin{array}{c}24 \\
19 \\
6 \\
8 \\
7\end{array}$ & $\begin{array}{c}9620.86 \\
9658.49 \\
10124 \\
10548.0 \\
10683.1\end{array}$ & $\begin{array}{l}28 \\
28 \\
42 \\
48 \\
27\end{array}$ \\
\hline $\begin{array}{l}1277.15 \\
1277.27 \\
1277.4 \\
1277.62 \\
1277.77\end{array}$ & $\begin{array}{l}11 \\
11 \\
11 \\
11 \\
11\end{array}$ & $\begin{array}{l}2902.1 \\
2903.1 \\
2904.9 \\
2964.85 \\
2967.22\end{array}$ & $\begin{array}{r}26 \\
26 \\
26 \\
1 \\
1\end{array}$ & $\begin{array}{l}10685.3 \\
10691.2 \\
10707.3 \\
10729.5 \\
10754.0\end{array}$ & $\begin{array}{l}27 \\
27 \\
27 \\
27 \\
27\end{array}$ \\
\hline $\begin{array}{l}1277.8 \\
1279.25 \\
1279.90 \\
1280.15 \\
1280.34\end{array}$ & $\begin{array}{l}11 \\
10 \\
21 \\
21 \\
21\end{array}$ & $\begin{array}{l}4268.99 \\
4371.33 \\
4762.41 \\
4766.62 \\
4770.00\end{array}$ & $\begin{array}{l}39 \\
38 \\
34 \\
34 \\
34\end{array}$ & $\begin{array}{l}11330.3 \\
11330.4 \\
11602.9 \\
11609.9 \\
11619.0\end{array}$ & $\begin{array}{l}30 \\
41 \\
44 \\
44 \\
44\end{array}$ \\
\hline $\begin{array}{l}1280.65 \\
1280.89 \\
1328.82 \\
1329.10 \\
1329.58\end{array}$ & $\begin{array}{r}21 \\
21 \\
3 \\
3 \\
3\end{array}$ & $\begin{array}{l}4771.72 \\
4775.87 \\
4812.84 \\
4817.33 \\
4826.73\end{array}$ & $\begin{array}{l}34 \\
34 \\
33 \\
33 \\
33\end{array}$ & $\begin{array}{l}11631.6 \\
11638.6 \\
11653 \\
11656.0 \\
11667.1\end{array}$ & $\begin{array}{l}44 \\
44 \\
45 \\
45 \\
45\end{array}$ \\
\hline $\begin{array}{l}1364.14 \\
1431.60 \\
1432.12 \\
1432.54 \\
1459.05\end{array}$ & $\begin{array}{r}25 \\
9 \\
9 \\
9 \\
18\end{array}$ & $\begin{array}{l}4932.00 \\
5039.05 \\
5041.66 \\
5044.0 \\
5049.6\end{array}$ & $\begin{array}{l}37 \\
32 \\
32 \\
32 \\
32\end{array}$ & $\begin{array}{l}11667.1 \\
11677.0 \\
11747.5 \\
11754.0 \\
11778.0\end{array}$ & $\begin{array}{l}44 \\
44 \\
43 \\
43 \\
43\end{array}$ \\
\hline $\begin{array}{l}1463.33 \\
1467.45 \\
1469 \\
1470.20 \\
1472\end{array}$ & $\begin{array}{l}17 \\
23 \\
16 \\
15 \\
22\end{array}$ & $\begin{array}{l}5052.12 \\
5054.5 \\
5380.24 \\
5793.51 \\
5794.46\end{array}$ & $\begin{array}{l}36 \\
32 \\
35 \\
40 \\
40\end{array}$ & $\begin{array}{l}11801.8 \\
11820 \\
11824 \\
11849.3 \\
11863.0\end{array}$ & $\begin{array}{l}43 \\
49 \\
43 \\
49 \\
49\end{array}$ \\
\hline $\begin{array}{l}1481.77 \\
1560.31 \\
1560.70 \\
1561.29 \\
1561.3\end{array}$ & $\begin{array}{r}14 \\
2 \\
2 \\
2 \\
2\end{array}$ & $\begin{array}{l}5794.8 \\
5800.33 \\
5801.17 \\
5805.76 \\
6587.75\end{array}$ & $\begin{array}{l}40 \\
40 \\
40 \\
40 \\
50\end{array}$ & $\begin{array}{l}11880.4 \\
11894.9 \\
12551.0 \\
12565.0 \\
12582.3\end{array}$ & $\begin{array}{l}49 \\
49 \\
46 \\
46 \\
46\end{array}$ \\
\hline $\begin{array}{l}1561.40 \\
1656.26 \\
1656.92\end{array}$ & $\begin{array}{l}2 \\
5 \\
5\end{array}$ & $\begin{array}{l}8335.19 \\
9061.48 \\
9062.53\end{array}$ & $\begin{array}{l}31 \\
29 \\
29\end{array}$ & $\begin{array}{l}12602.6 \\
12614.8 \\
16890\end{array}$ & $\begin{array}{l}46 \\
46 \\
47\end{array}$ \\
\hline
\end{tabular}


The largest part of the data is from emission measurements with stabilized arc sources $[2,5$, $6,7]$. In the vacuum ultraviolet, recent measurements by Boldt [2] are given preference over theoretical determinations by Weiss [4] and Bolotin et al. [3], with which the experiment is in marked disagreement (factors of 2-3). At present there are strong indications that the principal source of the disagreement is the relatively crude theoretical treatment of configuration interaction, which is very critical for these transitions. Thus, the theoretical results $[3,4]$ have been employed only for a few strong transitions for which no experimental data are available. But it should be noted that some experimental values may be quite unreliable due to uncertainties in the identification of the lines.

For the higher excited transitions in the visible several experimental investigations are available. Richter's work with a carbon dioxide plasma [6] is regarded as the most advanced one and his results are principally used. However, from an analysis of his method it appears very likely that the absolute scale is shifted due to a demixing effect in the arc source (see general introduction). This is further substantiated by a disagreement with the Coulomb approximation by an almost constant factor. Since the Coulomb approximation has given very reliable results for $3 p-3 d$ transitions of atoms of analogous structure, Richter's absolute values are renormalized to give the best agreement with the Coulomb approximation for these latter type transitions. The normalization factor of 1.30 is then applied to all of his other transitions. The work of Maecker [5] and Foster [7], which is used for a few transitions not covered by Richter, is expected to be also subject to demixing effects of the same order of magnitude or higher, since these authors have used more complicated gas mixtures. Thus, their results have been also fitted to the scale established by the Coulomb approximation. Normalization factors of 2.50 and 1.40 are used, respectively.

Finally, two intercombination lines are taken from a recent paper by Garstang [1], who performed intermediate coupling calculations and normalized the values to a scale obtained from the Coulomb approximation.

\section{References}

[1] Garstang, R. H., The Observatory 82, 50-51 (1962).

[2] Boldt, G., Z. Naturforsch. 18a, 1107-1116 (1963).

[3] Bolotin, A. B., Levinson, I. B., and Levin, L. I., Soviet Phys. -JETP 2, 391-395 (1956).

[4] Weiss, A. W., private communication (1964).

[5] Maecker, H., Z. Physik 135, 13-22 (1953).

[6] Richter, J., Z. Physik 151, 114-123 (1958).

[7] Foster, E. W., Proc. Phys. Soc. London A 80, 882-893 (1962).

\section{I. Allowed Transitions}

\begin{tabular}{|c|c|c|c|c|c|c|c|c|c|c|c|c|c|}
\hline No. & $\begin{array}{c}\text { Transition } \\
\text { Array }\end{array}$ & Multiplet & $\lambda(\AA)$ & $E_{i}\left(\mathrm{~cm}^{-1}\right)$ & $E_{k}\left(\mathrm{~cm}^{-1}\right)$ & $g_{i}$ & $g_{k}$ & $A_{k i}\left(10^{8} \sec ^{-1}\right)$ & $f_{i k}$ & $S($ at.u.) & $\log g f$ & $\begin{array}{l}\text { Accu- } \\
\text { racy }\end{array}$ & Source \\
\hline \multirow[t]{3}{*}{1} & $\begin{array}{c}2 s^{2} 2 p^{2}- \\
2 s 2 p^{3}\end{array}$ & $\begin{array}{c}{ }^{3} \mathrm{P}-{ }^{5} \mathrm{~S}^{\circ} \\
(1 \mathrm{uv})\end{array}$ & & & & & & & & & & & \\
\hline & & & 2967.22 & 44 & 33735 & 5 & 5 & $2.4 \times 10^{-7}$ & $3.2 \times 10^{-8}$ & $1.6 \times 10^{-6}$ & -6.80 & E & 1 \\
\hline & & & 2964.85 & 16 & 33735 & 3 & 5 & $8.2 \times 10^{-8}$ & $1.8 \times 10^{-8}$ & $5.3 \times 10^{-7}$ & -7.27 & $\mathrm{E}$ & 1 \\
\hline \multirow[t]{7}{*}{2} & & $\begin{array}{c}{ }^{3} \mathrm{P}-{ }^{3} \mathrm{D}^{\circ} \\
(3 \mathrm{uv})\end{array}$ & & & 64091 & 9 & 15 & 1.5 & 0.091 & 4.2 & -0.09 & $\mathrm{D}$ & 2 \\
\hline & & & 1561.40 & 44 & 64089 & 5 & 7 & 1.5 & 0.078 & 2.0 & -0.41 & D & ls \\
\hline & & . & 1560.70 & 16 & 64093 & 3 & 5 & 1.1 & 0.065 & 1.0 & -0.71 & D & is \\
\hline & & & 1560.31 & 0 & 64092 & 1 & 3 & 0.84 & 0.091 & 0.47 & -1.04 & D & ls \\
\hline & & & 1561.29 & 44 & 64093 & 5 & 5 & 0.37 & 0.014 & 0.35 & -1.67 & $\mathrm{D}$ & ls \\
\hline & & & 1560.70 & 16 & 64092 & 3 & 3 & 0.62 & 0.023 & 0.35 & -1.67 & D & ls \\
\hline & & & [1561.3] & 44 & 64092 & 5 & 3 & 0.041 & $8.9 \times 10^{-4}$ & 0.023 & -2.35 & D & Is \\
\hline
\end{tabular}


CiI. Allowed Transitions-Continued

\begin{tabular}{|c|c|c|c|c|c|c|c|c|c|c|c|c|c|}
\hline No. & $\begin{array}{c}\text { Transition } \\
\text { Array }\end{array}$ & Multiplet & $\lambda(\AA)$ & $E_{i}\left(\mathrm{~cm}^{-1}\right)$ & $E_{k}\left(\mathrm{~cm}^{-1}\right)$ & $g_{i}$ & $g_{k}$ & $A_{k i}\left(10^{8} \mathrm{sec}^{-1}\right)$ & $f_{i k}$ & $S$ (at.u.) & $\log g f$ & $\begin{array}{l}\text { Accu- } \\
\text { racy }\end{array}$ & Sour \\
\hline \multirow[t]{6}{*}{3} & & $\begin{array}{c}{ }^{3} \mathrm{P}-{ }^{3} \mathrm{P}^{\circ} \\
(4, \mathrm{uv})\end{array}$ & & 30 & 75256 & 9 & 9 & 1.4 & 0.038 & 1.5 & -0.47 & D & \\
\hline & & & 1329.58 & 44 & 75256 & 5 & 5 & 1.1 & 0.029 & 0.63 & -0.84 & D & $l s$ \\
\hline & & & 1329.10 & 16 & 75256 & 3 & 3 & 0.37 & 0.0099 & 0.13 & -1.53 & D & ls \\
\hline & & & 1329.58 & 44 & 75256 & 5 & 3 & 0.60 & 0.0096 & 0.21 & -1.32 & D & ls \\
\hline & & & $\begin{array}{l}1329.10 \\
1329.10\end{array}$ & $\begin{array}{l}16 \\
16\end{array}$ & $\begin{array}{l}75256 \\
75256\end{array}$ & $\begin{array}{l}3 \\
3\end{array}$ & $\begin{array}{l}1 \\
5\end{array}$ & $\begin{array}{l}1.5 \\
0.36\end{array}$ & $\begin{array}{l}0.013 \\
0.016\end{array}$ & $\begin{array}{l}0.17 \\
0.21\end{array}$ & $\begin{array}{l}-1.41 \\
-1.32\end{array}$ & $\begin{array}{l}\mathrm{D} \\
\mathrm{D}\end{array}$ & ls \\
\hline & & & 1328.82 & 0 & 75256 & 1 & 3 & 0.49 & 0.039 & 0.17 & -1.41 & D & ls \\
\hline \multirow[t]{4}{*}{4} & & $\begin{array}{l}{ }^{3} \mathrm{P}-{ }^{3} \mathrm{~S}^{\circ} \\
(31 \mathrm{uv})\end{array}$ & 945.44 & 30 & 105801 & 9 & 3 & 61 & 0.27 & 7.6 & 0.39 & $\mathrm{E}$ & 3 \\
\hline & & & 945.566 & 44 & 105801 & 5 & 3 & 34 & 0.27 & 4.2 & 0.13 & E & ls \\
\hline & & & 945.336 & 16 & 105801 & 3 & 3 & 20 & 0.27 & 2.5 & -0.10 & $\mathrm{E}$ & ls \\
\hline & & & 94.193 & & 105801 & 1 & 3 & 6.7 & 0.27 & 0.84 & -0.57 & $\mathrm{E}$ & ls \\
\hline \multirow[t]{5}{*}{5} & $\begin{array}{l}2 p^{2}- \\
2 p\left({ }^{2} \mathrm{P}^{\circ}\right) 3 s\end{array}$ & $\begin{array}{l}{ }^{3} \mathrm{P}-{ }^{3} \mathrm{P}^{\circ} \\
(2 \mathrm{uv})\end{array}$ & 1657.2 & 30 & 60374 & 9 & 9 & 4.1 & 0.17 & 8.3 & 0.18 & D & 2 \\
\hline & & & $\begin{array}{l}1657.00 \\
1657.37\end{array}$ & $\begin{array}{l}44 \\
16\end{array}$ & $\begin{array}{l}60394 \\
60353\end{array}$ & $\begin{array}{l}5 \\
3\end{array}$ & $\begin{array}{l}5 \\
3\end{array}$ & 3.1 & 0.13 & $\begin{array}{l}3.5 \\
0.69\end{array}$ & $\begin{array}{l}-0.19 \\
-0.90\end{array}$ & D & ls \\
\hline & & & 1658.11 & 44 & 60353 & 5 & 3 & 1.8 & $\begin{array}{l}.0 .042 \\
0.044\end{array}$ & $\begin{array}{l}.09 \\
1.2\end{array}$ & $\begin{array}{l}-0.90 \\
-0.66\end{array}$ & $\begin{array}{l}\text { D } \\
\text { D }\end{array}$ & $\begin{array}{ll}l s \\
l s\end{array}$ \\
\hline & & & 1657.89 & 16 & 60334 & 3 & 1 & 4.1 & 0.056 & 0.92 & -0.77 & D & ls \\
\hline & & & $\begin{array}{l}1656.26 \\
1656.92\end{array}$ & $\begin{array}{r}16 \\
0\end{array}$ & $\begin{array}{l}60394 \\
60353\end{array}$ & $\begin{array}{l}3 \\
1\end{array}$ & $\begin{array}{l}5 \\
3\end{array}$ & $\begin{array}{l}1.1 \\
1.4\end{array}$ & $\begin{array}{l}0.073 \\
0.17\end{array}$ & $\begin{array}{l}1.2 \\
0.92\end{array}$ & $\begin{array}{l}-0.66 \\
-0.77\end{array}$ & $\begin{array}{l}\mathrm{D} \\
\mathrm{D}\end{array}$ & $l s$ \\
\hline \multirow[t]{4}{*}{6} & & ${ }^{1} \mathrm{D}-{ }^{1} \mathrm{P}^{\circ}$ & 1930.93 & 10194 & 61982 & 5 & 3 & 2.4 & 0.082 & 2.6 & -0.39 & E & 4 \\
\hline & & (33 uv) & & & & & & & & & & & \\
\hline & & $\begin{array}{l}{ }^{1} \mathrm{~S}-{ }^{3} \mathrm{P}^{0} \\
(60 \mathrm{uv})\end{array}$ & & & & & & & & & & & \\
\hline & & & 2582.90 & 21648 & 60353 & 1 & 3 & $8.6 \times 10^{-5}$ & $2.6 \times 10^{-5}$ & $2.2 \times 10^{-4}$ & -4.59 & D & $5 n$ \\
\hline 8 & & $\frac{{ }^{1} S-{ }^{1} \mathrm{P}^{\circ}}{(61 \mathrm{uv})}$ & 2478.56 & 21648 & 61982 & 1 & 3 & 0.34 & 0.094 & 0.77 & -1.03 & E & 4 \\
\hline \multirow[t]{3}{*}{9} & $\begin{array}{l}2 s 2 p^{3}- \\
2 s 2 p^{2}(4 \mathrm{P}) 3 s\end{array}$ & $\begin{array}{r}{ }^{5} \mathrm{~S}^{\circ}-{ }^{5} \mathrm{P} \\
(65 \text { uv) }\end{array}$ & 1431.9 & 33735 & 103570 & 5 & 15 & 1.4 & 0.13 & 3.1 & -0.19 & D & 2 \\
\hline & & & 1431.60 & 33735 & 103587 & 5 & 7 & 1.5 & 0.064 & 1.5 & -0.49 & D & 2 \\
\hline & & & $\begin{array}{l}1432.12 \\
143254\end{array}$ & 33735 & 103563 & 5 & 5 & 1. & 0.043 & & -0.67 & D & 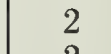 \\
\hline & & & 1432.54 & 33735 & 103542 & 5 & 3 & 1.3 & 0.024 & 0.57 & -0.92 & D & 2 \\
\hline \multirow[t]{2}{*}{10} & $\begin{array}{l}2 p^{2}- \\
2 p\left({ }^{2} \mathrm{P}^{\circ}\right) 3 d\end{array}$ & 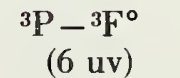 & & & & & & & & & & & \\
\hline & & & 1279.25 & 44 & 78216 & 5 & 7 & 0.11 & 0.0038 & 0.080 & -1.72 & D & 2 \\
\hline \multirow[t]{6}{*}{11} & & $\begin{array}{c}{ }^{3} \mathrm{P}-{ }^{3} \mathrm{D}^{\circ} \\
(7 \mathrm{uv})\end{array}$ & 1277.5 & 30 & 78310 & 9 & 15 & 1.6 & 0.063 & 2.4 & -0.24 & D & 2 \\
\hline & & & 1277.62 & 44 & 78316 & 5 & 7 & 1.5 & 0.052 & 1.1 & -0.58 & D & $l s$ \\
\hline & & & 1277.27 & 16 & 78307 & 3 & 5 & 1.2 & 0.048 & 0.60 & -0.85 & D & ls \\
\hline & & & $\begin{array}{l}1277.15 \\
1277.77\end{array}$ & $\begin{array}{r}0 \\
44\end{array}$ & $\begin{array}{l}78301 \\
78307\end{array}$ & $\begin{array}{l}1 \\
5\end{array}$ & $\begin{array}{l}3 \\
5\end{array}$ & $\begin{array}{l}0.88 \\
0.39\end{array}$ & $\begin{array}{l}0.064 \\
0.0095\end{array}$ & $\begin{array}{l}0.27 \\
0.20\end{array}$ & $\begin{array}{l}-1.19 \\
-1.32\end{array}$ & $\begin{array}{l}\mathrm{D} \\
\mathrm{D}\end{array}$ & $\begin{array}{l}\text { ls } \\
\text { ls }\end{array}$ \\
\hline & & & [1277.4] & 16 & 78301 & 3 & 3 & & & 0.20 & -1.32 & D & ls \\
\hline & & & [1277. & 4.4 & 78301 & 5 & 3 & 0.042 & $6.2 \times 10^{-4}$ & 0.013 & -2.51 & D & ls \\
\hline \multirow[t]{2}{*}{12} & & $\begin{array}{r}{ }^{3} \mathrm{P}-{ }^{1} \mathrm{~F}^{\circ} \\
(8 \mathrm{uv})\end{array}$ & & & & & & & & & & & \\
\hline & & & 1274.13 & 44 & 78531 & 5 & 7 & 0.0068 & $2.3 \times 10^{-4}$ & 0.0048 & -2.94 & D & 2 \\
\hline \multirow[t]{6}{*}{13} & & $\begin{array}{r}{ }^{3} \mathrm{P}-{ }^{3} \mathrm{P}^{\circ} \\
\text { (9 } \mathrm{uv} \text { ) }\end{array}$ & 1261.3 & 30 & 79315 & 9 & 9 & 1.2 & 0.029 & 1.1 & -0.58 & D & 2 \\
\hline & & & 1261.56 & 44. & 79311 & 5 & 5 & 0.93 & 0.022 & 0.46 & -0.96 & D & $l s$ \\
\hline & & & 1260.96 & 16 & 79319 & 3 & 3 & 0.31 & 0.0074 & 0.092 & -1.65 & D & ls \\
\hline & & & [1261.4] & 44 & $\begin{array}{l}79319 \\
70292\end{array}$ & 5 & 3 & 0.50 & 0.0072 & 0.15 & -1.44 & D & ls \\
\hline & & & $\begin{array}{l}{[1260.9]} \\
1261.12\end{array}$ & $\begin{array}{l}16 \\
16\end{array}$ & 79323 & $\begin{array}{l}3 \\
3\end{array}$ & $\begin{array}{l}1 \\
5\end{array}$ & $\begin{array}{l}1.2 \\
0.3\end{array}$ & $\begin{array}{l}0.0096 \\
0.012\end{array}$ & $\begin{array}{l}0.12 \\
0.15\end{array}$ & $\begin{array}{l}-1.54 \\
-1.44\end{array}$ & $\begin{array}{l}\mathrm{D} \\
\mathrm{D}\end{array}$ & ls \\
\hline & & & 1260.75 & 0 & 79319 & 1 & 3 & 0.40 & 0.029 & 0.12 & -1.54 & D & $s$ \\
\hline
\end{tabular}


C. I. Allowed Transitions-Continued

\begin{tabular}{|c|c|c|c|c|c|c|c|c|c|c|c|c|c|}
\hline No. & $\begin{array}{c}\text { Transition } \\
\text { Array }\end{array}$ & Multiplet & $\lambda(\AA)$ & $E_{i}\left(\mathrm{~cm}^{-1}\right)$ & $E_{k}\left(\mathrm{~cm}^{-1}\right)$ & $g_{i}$ & $g_{k}$ & $A_{k i}\left(10^{*} \sec ^{-1}\right)$ & $f_{i k}$ & $S($ at.u. $)$ & $\log g f$ & $\begin{array}{l}\text { Accu- } \\
\text { racy }\end{array}$ & Source \\
\hline 14 & & $\begin{array}{r}{ }^{1} \mathrm{D}-{ }^{1} \mathrm{D}^{\circ} \\
(34 \text { uv })\end{array}$ & 1481.77 & 10194 & 77681 & 5 & 5 & 0.33 & 0.011 & 0.27 & -1.26 & D & 2 \\
\hline 15 & & $\begin{array}{r}{ }^{1} \mathrm{D}-{ }^{3} \mathrm{~F}^{\circ} \\
(35 \mathrm{uv})\end{array}$ & 1470.20 & 10194 & 78216 & 5 & 7 & 0.0088 & $4.0 \times 10^{-4}$ & 0.0097 & -2.70 & D & 2 \\
\hline 16 & & ${ }^{1} \mathrm{D}-{ }^{3} \mathrm{D}^{\circ}$ & 1469 & 10194 & 78301 & 5 & 3 & 0.019 & $3.6 \times 10^{-4}$ & 0.0087 & -2.74 & D & 2 \\
\hline 17 & & $\begin{array}{l}{ }^{1} \mathrm{D}-{ }^{-1} \mathrm{~F}^{\circ} \\
(37 \mathrm{uv})\end{array}$ & 1463.33 & 10194 & 78531 & 5 & 7 & 2.1 & 0.093 & 2.2 & -0.33 & D & 2 \\
\hline 18 & & $\begin{array}{r}{ }^{1} \mathrm{D}-{ }^{1} \mathrm{P}^{\circ} \\
(38 \mathrm{uv})\end{array}$ & 1459.05 & 10194 & 78728 & 5 & 3 & 0.37 & 0.0070 & 0.17 & -1.46 & D & 2 \\
\hline 19 & & ${ }^{1} S-{ }^{3} D^{\circ}$ & 1765 & 21648 & 78301 & 1 & 3 & 0.0071 & 0.0010 & 0.0058 & -3.00 & D & 2 \\
\hline 20 & & 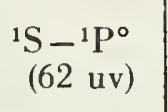 & 1751.9 & 21648 & 78728 & 1 & 3 & 0.87 & 0.12 & 0.69 & -0.92 & D & 2 \\
\hline 21 & 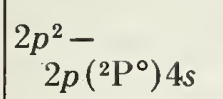 & $\begin{array}{c}{ }^{3} \mathrm{P}-{ }^{3} \mathrm{P}^{\circ} \\
(5 \text { uv })\end{array}$ & 1280.4 & 30 & 78133 & 9 & 9 & 0.82 & 0.020 & 0.76 & -0.74 & D & 2 \\
\hline & & & $\begin{array}{l}1280.34 \\
1280.34 \\
1280.89 \\
1280.65 \\
1279.90 \\
1280.15\end{array}$ & $\begin{array}{r}44 \\
16 \\
44 \\
16 \\
16 \\
0\end{array}$ & $\begin{array}{l}78148 \\
78117 \\
78117 \\
78105 \\
78148 \\
78117\end{array}$ & $\begin{array}{l}5 \\
3 \\
5 \\
3 \\
3 \\
1\end{array}$ & $\begin{array}{l}5 \\
3 \\
3 \\
1 \\
5 \\
3\end{array}$ & $\begin{array}{l}0.62 \\
0.20 \\
0.35 \\
0.81 \\
0.21 \\
0.27\end{array}$ & $\begin{array}{l}0.015 \\
0.0050 \\
0.0052 \\
0.0066 \\
0.0087 \\
0.020\end{array}$ & $\begin{array}{l}0.32 \\
0.063 \\
0.11 \\
0.084 \\
0.11 \\
0.084\end{array}$ & $\begin{array}{l}-1.12 \\
-1.83 \\
-1.58 \\
-1.70 \\
-1.58 \\
-1.70\end{array}$ & $\begin{array}{l}\mathrm{D} \\
\mathrm{D} \\
\mathrm{D} \\
\mathrm{D} \\
\mathrm{D} \\
\mathrm{D}\end{array}$ & $\begin{array}{l}l s \\
l s \\
l s \\
l s \\
l s \\
l s\end{array}$ \\
\hline 22 & & ${ }^{1} \mathrm{D}-{ }^{3} \mathrm{P}^{\circ}$ & 1472 & 10194 & 78117 & 5 & 3 & 0.0051 & $1.0 \times 10^{-4}$ & 0.0024 & -3.30 & D & 2 \\
\hline 23 & & $\begin{array}{l}{ }^{1} \mathrm{D}-{ }^{1} \mathrm{P}^{\circ} \\
(36 \mathrm{uv})\end{array}$ & 1467.45 & 10194 & 78338 & 5 & 3 & 0.46 & 0.0089 & 0.21 & -1.35 & D & 2 \\
\hline 24 & & ${ }^{1} \mathrm{~S}-{ }^{1} \mathrm{P}^{\circ}$ & 1764 & 21648 & 78338 & 1 & 3 & 0.022 & 0.0031 & 0.018 & -2.51 & D & 2 \\
\hline 25 & $\begin{array}{l}2 p^{2}- \\
2 p\left({ }^{2} \mathrm{P}^{\circ}\right) 4 d\end{array}$ & $\begin{array}{r}{ }^{1} \mathrm{D}-{ }^{1} \mathrm{D}^{\circ} \\
(39 \mathrm{uv})\end{array}$ & 1364.14 & 10194 & 83500 & 5 & 5 & 0.047 & 0.0013 & 0.029 & -2.19 & $\mathrm{D}$ & 2 \\
\hline 26 & $\begin{array}{c}2 s^{2} 2 p 3 p- \\
2 s 2 p^{3}\end{array}$ & ${ }^{3} \mathrm{P}-{ }^{3} \mathrm{~S}^{\circ}$ & 2903.9 & 71375 & 105801 & 9 & 3 & 0.044 & 0.0019 & 0.16 & -1.78 & $\mathrm{D}$ & $5 n$ \\
\hline & & & $\begin{array}{l}{[2904.9]} \\
{[2903.1]} \\
{[2902.1]}\end{array}$ & $\begin{array}{l}71386 \\
71365 \\
71353\end{array}$ & $\begin{array}{l}105801 \\
105801 \\
105801\end{array}$ & $\begin{array}{l}5 \\
3 \\
1\end{array}$ & $\begin{array}{l}3 \\
3 \\
3\end{array}$ & $\begin{array}{l}0.022 \\
0.017 \\
0.0066\end{array}$ & $\begin{array}{l}0.0017 \\
0.0021 \\
0.0025\end{array}$ & $\begin{array}{l}0.079 \\
0.061 \\
0.024\end{array}$ & $\begin{array}{l}-2.08 \\
-2.20 \\
-2.60\end{array}$ & $\begin{array}{l}\text { D } \\
\text { D } \\
\text { D }\end{array}$ & $\begin{array}{l}5 n \\
5 n \\
5 n\end{array}$ \\
\hline 27 & $\begin{array}{l}2 p 3 s- \\
\left.2 p{ }^{2} \mathrm{P}^{\circ}\right) 3 p\end{array}$ & $\begin{array}{c}{ }^{3} \mathrm{P}^{\circ}-{ }^{3} \mathrm{D} \\
\text { (1) }\end{array}$ & 10695 & 60374 & 69722 & 9 & 15 & 0.18 & 0.50 & 160 & 0.66 & D & $6 n$ \\
\hline & & & $\begin{array}{l}10691.2 \\
10683.1 \\
10685.3 \\
10729.5 \\
10707.3 \\
10754.0\end{array}$ & $\begin{array}{l}60394 \\
60353 \\
60334 \\
60394 \\
60353 \\
60394\end{array}$ & $\begin{array}{l}69744 \\
69711 \\
69690 \\
69711 \\
69690 \\
69690\end{array}$ & $\begin{array}{l}5 \\
3 \\
1 \\
5 \\
3 \\
5\end{array}$ & $\begin{array}{l}7 \\
5 \\
3 \\
5 \\
3 \\
3\end{array}$ & $\begin{array}{l}0.18 \\
0.13 \\
0.10 \\
0.043 \\
0.072 \\
0.0048\end{array}$ & $\begin{array}{l}0.43 \\
0.38 \\
0.51 \\
0.074 \\
0.12 \\
0.0050\end{array}$ & $\begin{array}{c}75 \\
40 \\
18 \\
13 \\
13 \\
0.89\end{array}$ & $\begin{array}{r}0.33 \\
0.06 \\
-0.29 \\
-0.43 \\
-0.43 \\
-1.60\end{array}$ & $\begin{array}{l}\mathrm{D} \\
\mathrm{D} \\
\mathrm{D} \\
\mathrm{D} \\
\mathrm{D} \\
\mathrm{D}\end{array}$ & $\begin{array}{l}\text { ls } \\
\text { ls } \\
\text { ls } \\
\text { ls } \\
\text { ls } \\
\text { ls }\end{array}$ \\
\hline 28 & & $\begin{array}{c}{ }^{3} \mathrm{P}^{\circ}-{ }^{3} \mathrm{~S} \\
(2)\end{array}$ & \begin{tabular}{|l}
9640.6 \\
9658.49 \\
9620.86 \\
9603.09
\end{tabular} & $\begin{array}{l}60374 \\
60394 \\
60353 \\
60334\end{array}$ & $\begin{array}{l}70744 \\
70744 \\
70744 \\
70744\end{array}$ & $\begin{array}{l}9 \\
5 \\
3 \\
1\end{array}$ & \begin{tabular}{l|l}
3 & \\
3 & \\
3 & \\
3 &
\end{tabular} & $\begin{array}{l}0.22 \\
0.12 \\
0.074 \\
0.024\end{array}$ & $\begin{array}{l}0.10 \\
0.10 \\
0.10 \\
0.10\end{array}$ & $\begin{array}{c}29 \\
16 \\
9.7 \\
3.2\end{array}$ & $\begin{array}{l}-0.04 \\
-0.30 \\
-0.51 \\
-0.99\end{array}$ & $\begin{array}{l}\mathrm{D} \\
\mathrm{D} \\
\mathrm{D} \\
\mathrm{D}\end{array}$ & $\begin{array}{l}6 n \\
\text { ls } \\
\text { ls } \\
\text { ls }\end{array}$ \\
\hline
\end{tabular}


C I. Allowed Transitions-Continued

\begin{tabular}{|c|c|c|c|c|c|c|c|c|c|c|c|c|c|}
\hline No & $\begin{array}{l}\text { Transition } \\
\text { Array }\end{array}$ & Multiplet & $\lambda(\AA)$ & $E_{i}\left(\mathrm{~cm}^{-1}\right)$ & $E_{k}\left(\mathrm{~cm}^{-1}\right)$ & $g_{i}$ & $g_{k}$ & $A_{k i}\left(10^{8} \mathrm{sec}^{-1}\right)$ & $f_{i k}$ & $S$ (at.u.) & $\log g f$ & $\begin{array}{c}\text { Accu- } \\
\text { racy }\end{array}$ & Source \\
\hline \multirow[t]{6}{*}{29} & & ${ }^{3} \mathrm{P}^{0}-{ }^{3} \mathrm{P}$ & 9087.6 & 60374 & 71375 & 9 & 9 & 0.25 & 0.31 & 83 & 0.44 & D & $6 n$ \\
\hline & & & 9094.89 & 60394 & 71386 & 5 & 5 & 0.19 & 0.23 & 35 & 0.07 & D & ls \\
\hline & & & 9078.32 & 60353 & 71365 & 3 & 3 & 0.062 & 0.077 & 6.9 & -0.64 & D & ls \\
\hline & & & $\begin{array}{l}9111.85 \\
0088.57\end{array}$ & 60394 & $\begin{array}{l}71365 \\
71353\end{array}$ & $\begin{array}{l}5 \\
3\end{array}$ & 3 & 0.11 & 0.080 & 12 & $\begin{array}{l}-0.40 \\
-0.51\end{array}$ & D & ls \\
\hline & & & 9061.48 & 60353 & 71386 & $\begin{array}{l}3 \\
3\end{array}$ & $\begin{array}{l}1 \\
5\end{array}$ & $\begin{array}{l}0.25 \\
0.065\end{array}$ & $\begin{array}{l}0.10 \\
0.13\end{array}$ & $\begin{array}{l}9.2 \\
12\end{array}$ & $\begin{array}{l}0.01 \\
-0.40\end{array}$ & $\begin{array}{l}\mathrm{D} \\
\mathrm{D}\end{array}$ & $\begin{array}{l}l s \\
l s\end{array}$ \\
\hline & & & 9062.53 & 60334 & 71365 & 1 & 3 & 0.083 & 0.31 & 9.2 & -0.51 & D & ls \\
\hline 30 & & $\begin{array}{c}{ }^{1} \mathrm{P}^{\circ}-{ }^{1} \mathrm{D} \\
(9)\end{array}$ & 11330.3 & 61982 & 72611 & 3 & 5 & 0.13 & 0.42 & 47 & 0.10 & D & $6 n$ \\
\hline 31 & & $\begin{array}{c}{ }^{1} \mathrm{P}^{\circ}-{ }^{1} \mathrm{~S} \\
(10)\end{array}$ & 8335.19 & 61982 & 73976 & 3 & 1 & 0.32 & 0.11 & 9.1 & -0.48 & D & $6 n$ \\
\hline \multirow[t]{6}{*}{32} & $\begin{array}{l}2 p 3 s- \\
2 p\left({ }^{2} \mathrm{P}^{\circ}\right) 4 p\end{array}$ & $\begin{array}{c}{ }^{3} \mathrm{P}^{\circ}-{ }^{3} \mathrm{D} \\
(4)\end{array}$ & 5041.7 & 60374 & 80203 & 9 & 15 & 0.0039 & 0.0025 & 0.37 & -1.65 & D & $6 n$ \\
\hline & & & 5041.66 & 60394 & 80223 & 5 & 7 & 0.0038 & 0.0020 & 0.17 & $\begin{array}{l}-1.99 \\
-0.96\end{array}$ & D & ls \\
\hline & & & 5039.05 & 60353 & 80192 & 3 & 5 & 0.0029 & 0.0018 & 0.092 & -2.26 & D & ls \\
\hline & & & $\begin{array}{r}5039.05 \\
{[5049.6]}\end{array}$ & 60334 & 80173 & 1 & 3 & 0.0022 & 0.0025 & 0.041 & $\begin{array}{l}-2.61 \\
-2.73\end{array}$ & D & ls \\
\hline & & & {$[5044.0]$} & $\begin{array}{l}00394 \\
60353\end{array}$ & $\begin{array}{l}80192 \\
80173\end{array}$ & $\begin{array}{l}5 \\
3\end{array}$ & $\begin{array}{r}5 \\
3\end{array}$ & $\begin{array}{l}9.8 \times 10^{-4} \\
0.0016\end{array}$ & $\begin{array}{l}3.1 \times 10^{-4} \\
6.2 \times 10^{-4}\end{array}$ & $\begin{array}{l}0.031 \\
0.031\end{array}$ & -2.73 & $\begin{array}{l}\text { D } \\
\text { D }\end{array}$ & $\begin{array}{l}l s \\
l s\end{array}$ \\
\hline & & & [5054.5] & 60394 & 80173 & 5 & 3 & $1.1 \times 10^{-4}$ & $2.5 \times 10^{-5}$ & 0.0021 & -3.90 & D & ls \\
\hline \multirow[t]{4}{*}{33} & & ${ }^{3} \mathrm{P}^{\circ}-{ }^{3} \mathrm{~S}$ & 4822.1 & 60374 & 81106 & 9 & 3 & 0.0084 & $9.8 \times 10^{-4}$ & 0.14 & -2.05 & D & $7 n$ \\
\hline & & & 4826.73 & 60394 & 81106 & 5 & 3 & 0.0047 & $9.8 \times 10^{-4}$ & 0.078 & -2.31 & D & ls \\
\hline & & & 4817.33 & 60353 & 81106 & 3 & $\begin{array}{r}3 \\
2\end{array}$ & 0.0028 & $9.9 \times 10^{-4}$ & 0.047 & $\begin{array}{l}-2.53 \\
-209\end{array}$ & D & ls \\
\hline & & & 4812.84 & 60334 & 81106 & 1 & 3 & $9.7 \times 10^{-4}$ & 0.0010 & 0.016 & -2.99 & D & \\
\hline \multirow[t]{6}{*}{34} & & $\begin{array}{c}{ }^{3} \mathrm{P}^{\circ}-{ }^{3} \mathrm{P} \\
(6)\end{array}$ & 4769.7 & 60374 & 81334 & 9 & 9 & 0.016 & 0.0053 & 0.75 & -1.32 & D & $6 n$ \\
\hline & & & 4771.72 & 60394 & 81344 & 5 & 5 & 0.012 & 0.0039 & 0.31 & -1.70 & D & ls \\
\hline & & & $\begin{array}{l}4766.62 \\
9775 \circ 7\end{array}$ & 60353 & 81326 & 3 & 3 & 0.0039 & 0.0013 & 0.063 & -2.40 & D & ls \\
\hline & & & $\begin{array}{l}4775.87 \\
4770.00\end{array}$ & $\begin{array}{l}60394 \\
60353\end{array}$ & $\begin{array}{l}81326 \\
81312\end{array}$ & $\begin{array}{l}5 \\
3\end{array}$ & $\begin{array}{l}3 \\
1\end{array}$ & $\begin{array}{l}0.0062 \\
0.015\end{array}$ & $\begin{array}{l}0.0013 \\
0.0018\end{array}$ & $\begin{array}{l}0.10 \\
0.083\end{array}$ & $\begin{array}{l}-2.20 \\
-2.28\end{array}$ & D & ls \\
\hline & & & 4762.41 & 60353 & 81344 & 3 & 5 & 0.0038 & 0.0021 & $\begin{array}{l}0.10 \\
0\end{array}$ & -2.20 & D & ls \\
\hline & & & 4762.41 & 60334 & 81326 & 1 & 3 & 0.0052 & 0.0053 & 0.083 & -2.28 & D & ls \\
\hline 35 & & $\begin{array}{l}{ }^{1} \mathrm{P}^{\circ}-{ }^{1} \mathrm{P} \\
\quad(11)\end{array}$ & 5380.24 & 61982 & 80564 & 3 & 3 & 0.016 & 0.0070 & 0.37 & -1.68 & D & $6 n$ \\
\hline 36 & & $\begin{array}{c}{ }^{1} \mathrm{P}^{\circ}-{ }^{1} \mathrm{D} \\
(12)\end{array}$ & 5052.12 & 61982 & 81770 & 3 & 5 & 0.017 & 0.011 & 0.54 & -1.49 & D & $6 n$ \\
\hline 37 & & $\begin{array}{c}{ }^{1} \mathrm{P}^{0}-1 \mathrm{~S} \\
(13)\end{array}$ & 4932.00 & 61982 & 82252 & 3 & 1 & 0.046 & 0.0055 & 0.27 & -1.78 & D & $6 n$ \\
\hline 38 & $\stackrel{2 p 3 s-}{2 p\left({ }^{-} \mathrm{P}^{\circ}\right) 5 p}$ & $\begin{array}{c}{ }^{1} \mathrm{P}^{\circ}-{ }^{1} \mathrm{P} \\
\quad(14)\end{array}$ & 4371.33 & 61982 & 84852 & 3 & 3 & 0.0097 & 0.0028 & 0.12 & -2.08 & $\mathrm{D}-$ & $c a$ \\
\hline 39 & & $\begin{array}{c}{ }^{1} \mathrm{P}^{\circ}-{ }^{1} \mathrm{D} \\
(16)\end{array}$ & 4268.99 & 61982 & 85400 & 3 & 5 & 0.0032 & 0.0015 & 0.062 & -2.36 & D & $7 n$ \\
\hline \multirow[t]{5}{*}{40} & $\begin{array}{l}2 s 2 p^{3}- \\
2 s^{2} 2 p\left({ }^{2} \mathrm{P}^{\circ}\right) 4 p\end{array}$ & $\begin{array}{c}{ }^{3} \mathrm{D}^{\circ}-{ }^{3} \mathrm{P} \\
(18)\end{array}$ & 5797.8 & 64091 & 81334 & 15 & 9 & 0.0039 & 0.0012 & 0.34 & -1.75 & D & $6 n$ \\
\hline & & & $\begin{array}{l}5793.51 \\
5801.17\end{array}$ & $\begin{array}{l}64089 \\
64093\end{array}$ & $\begin{array}{l}81344 \\
81326\end{array}$ & $\begin{array}{l}7 \\
5\end{array}$ & $\begin{array}{l}5 \\
3\end{array}$ & $\begin{array}{l}0.0033 \\
0.0029\end{array}$ & $\begin{array}{l}0.0012 \\
8.9 \times 10^{-4}\end{array}$ & $\begin{array}{l}0.16 \\
0.085\end{array}$ & $\begin{array}{l}-2.08 \\
-2.35\end{array}$ & D & ls \\
\hline & & & 5805.76 & 64092 & 81312 & 3 & 1 & 0.0039 & $6.6 \times 10^{-4}$ & $\begin{array}{l}0.085 \\
0.038\end{array}$ & $\begin{array}{l}-2.35 \\
-2.70\end{array}$ & $\begin{array}{l}\text { D } \\
\text { D }\end{array}$ & ls \\
\hline & & & 5794.46 & $\begin{array}{l}64093 \\
6400 ?\end{array}$ & $\begin{array}{l}81344 \\
81396\end{array}$ & 5 & $\begin{array}{l}5 \\
3\end{array}$ & $5.8 \times 10^{-4}$ & $2.9 \times 10^{-4}$ & 0.028 & -2.83 & D & ls \\
\hline & & & $\begin{array}{r}5800.23 \\
{[5794.8]}\end{array}$ & $\begin{array}{l}64092 \\
64092\end{array}$ & $\begin{array}{l}81326 \\
81344\end{array}$ & $\begin{array}{l}3 \\
3\end{array}$ & $\begin{array}{l}3 \\
5\end{array}$ & $\begin{array}{l}9.7 \times 10^{-4} \\
4.0 \times 10^{-5}\end{array}$ & $\begin{array}{l}4.9 \times 10^{-4} \\
3.3 \times 10^{-5}\end{array}$ & $\begin{array}{l}0.028 \\
0.0019\end{array}$ & $\begin{array}{l}-2.83 \\
-4.00\end{array}$ & $\begin{array}{l}\mathrm{D} \\
\mathrm{D}\end{array}$ & $\begin{array}{ll}l s \\
l s\end{array}$ \\
\hline 41 & $\begin{array}{l}2 p 3 p- \\
2 p\left({ }^{2} \mathrm{P}^{\circ}\right) 3 d\end{array}$ & $\begin{array}{c}{ }^{1} \mathrm{P}-{ }^{1} \mathrm{D}^{\circ} \\
(19)\end{array}$ & 11330.4 & 68858 & 77681 & 3 & 5 & 0.198 & 0.63 & 71 & 0.280 & C & $6 n, c a$ \\
\hline
\end{tabular}


C I. Allowed Transitions-Continued

\begin{tabular}{|c|c|c|c|c|c|c|c|c|c|c|c|c|c|}
\hline No. & $\begin{array}{c}\text { Transition } \\
\text { Array }\end{array}$ & Multiplet & $\lambda(\AA)$ & $E_{i}\left(\mathrm{~cm}^{-1}\right)$ & $E_{k}\left(\mathrm{~cm}^{-1}\right)$ & $g_{i}$ & $g_{k}$ & $A_{k i}\left(10^{k} \sec ^{-1}\right)$ & $f_{i k}$ & $S$ (at.u.) & $\log g f$ & $\begin{array}{l}\text { Accu- } \\
\text { racy }\end{array}$ & Source \\
\hline 42 & & ${ }^{1} \mathrm{P}-{ }^{1} \mathrm{P}^{\circ}$ & 10124 & 68858 & 78728 & 3 & 3 & 0.171 & 0.262 & 26.2 & -0.105 & $\mathrm{C}$ & $6 n, c a$ \\
\hline \multirow[t]{7}{*}{43} & & ${ }^{3} \mathrm{D}-{ }^{3} \mathrm{~F}^{\circ}$ & 11755 & 69722 & 78227 & 15 & 21 & 0.242 & 0.70 & 407 & 1.022 & C & $6 n, c a$ \\
\hline & & & 11754.0 & 69744 & 78250 & 7 & 9 & 0.241 & 0.64 & 174 & 0.65 & C & ls \\
\hline & & & 11754.0 & 69711 & 78216 & 5 & 7 & 0.216 & 0.63 & 121 & 0.495 & $\mathrm{C}$ & Is \\
\hline & & & 11747.5 & 69690 & 78199 & 3 & 5 & 0.202 & 0.70 & 81 & 0.321 & C & ls \\
\hline & & & 11801.8 & 69744 & 78216 & 7 & 7 & 0.0266 & 0.056 & 15.1 & -0.411 & C & ls \\
\hline & & & [11778] & 69711 & 78199 & 5 & 5 & 0.0375 & 0.078 & 15.1 & -0.410 & C & Is \\
\hline & & & [11824] & 69744 & 78199 & 7 & 5 & 0.00104 & 0.00156 & 0.426 & -1.96 & C & ls \\
\hline \multirow[t]{7}{*}{44} & & $\begin{array}{c}{ }^{3} \mathrm{D}-{ }^{3} \mathrm{D}^{\circ} \\
(25)\end{array}$ & 11641 & 69722 & 78310 & 15 & 15 & 0.065 & 0.132 & 76 & 0.297 & C & $6 n, c a$ \\
\hline & & & 11667.1 & 69744 & 78316 & 7 & 7 & 0.057 & 0.117 & 31.5 & -0.086 & $\mathrm{C}$ & $l s$ \\
\hline & & & 11631.6 & 69711 & 78307 & 5 & 5 & 0.0453 & 0.092 & 17.6 & -0.338 & C & ls \\
\hline & & & 11609.9 & 69690 & 78301 & 3 & 3 & 0.0492 & 0.099 & 11.4 & -0.53 & C & $l s$ \\
\hline & & & 11677.0 & 69744 & 78307 & 7 & 5 & 0.0101 & 0.0147 & 3.95 & -0.99 & C & ls \\
\hline & & & $\begin{array}{l}11638.6 \\
11619.0\end{array}$ & $\begin{array}{l}69711 \\
69711\end{array}$ & $\begin{array}{l}78301 \\
78316\end{array}$ & $\begin{array}{l}5 \\
5\end{array}$ & $\begin{array}{l}3 \\
7\end{array}$ & $\begin{array}{l}0.0163 \\
0.0073\end{array}$ & $\begin{array}{l}0.0198 \\
0.0207\end{array}$ & $\begin{array}{l}3.80 \\
3.95\end{array}$ & $\begin{array}{l}-1.004 \\
-0.99\end{array}$ & C & Is \\
\hline & & & 11602.9 & 69690 & 78307 & 3 & 5 & 0.0099 & 0.0332 & 3.80 & -1.002 & C & ls \\
\hline \multirow[t]{4}{*}{45} & & ${ }^{3} \mathrm{~S}-{ }^{3} \mathrm{P}^{0}$ & 11664 & 70744 & 79315 & 3 & 9 & 0.157 & 0.96 & 111 & 0.461 & C & $6 n, c a$ \\
\hline & & & 11667.1 & 70744 & 79311 & 3 & 5 & 0.158 & 0.54 & 62 & 0.208 & $\mathrm{C}$ & ls \\
\hline & & & 11656.0 & 70744 & 79319 & 3 & 3 & 0.158 & 0.321 & 37.0 & -0.016 & C & ls \\
\hline & & & [11653] & 70744 & 79323 & 3 & 1 & 0.157 & 0.107 & 12.3 & -0.494 & $\mathrm{C}$ & ls \\
\hline \multirow[t]{7}{*}{46} & & ${ }^{3} \mathrm{P}-{ }^{3} \mathrm{P}^{\circ}$ & 12591 & 71375 & 79315 & 9 & 9 & 0.105 & 0.249 & 93 & 0.351 & $\mathrm{C}$ & $6 n, c a$ \\
\hline & & & 12614.8 & 71386 & 79311 & 5 & 5 & 0.078 & 0.186 & 38.7 & -0.031 & C & ls \\
\hline & & & 12565.0 & 71365 & 79319 & 3 & 3 & 0.0262 & 0.062 & 7.7 & -0.73 & $\mathrm{C}$ & ls \\
\hline & & & 12602.6 & 71386 & 79319 & 5 & 3 & 0.0435 & 0.062 & 12.9 & -0.51 & C & ls \\
\hline & & & 12565.0 & 71365 & 79323 & 3 & 1 & 0.105 & 0.083 & 10.3 & -0.60 & C & ls \\
\hline & & & 12582.3 & 71365 & 79311 & 3 & 5 & 0.0262 & 0.104 & 12.9 & -0.51 & C & ls \\
\hline & & & 12551.0 & 71353 & 79319 & 1 & 3 & 0.0352 & 0.249 & 10.3 & -0.60 & C & ls \\
\hline 47 & & ${ }^{1} \mathrm{D}-{ }^{1} \mathrm{~F}^{\circ}$ & 16890 & 72611 & 78531 & 5 & 7 & 0.123 & 0.74 & 205 & 0.57 & $\mathrm{C}$ & $6 n, c a$ \\
\hline 48 & $\begin{array}{l}2 p 3 p- \\
\quad 2 p\left({ }^{2} \mathrm{P}^{\circ}\right) 4 s\end{array}$ & 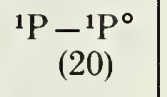 & 10548.0 & 68858 & 78338 & 3 & 3 & 0.010 & 0.017 & 1.8 & -1.29 & D & $6 n$ \\
\hline \multirow[t]{6}{*}{49} & & ${ }^{3} \mathrm{D}-{ }^{3} \mathrm{P}^{\circ}$ & & 69722 & 78133 & 15 & 9 & 0.11 & 0.14 & 85 & 0.34 & $\mathrm{D}$ & $6 n$ \\
\hline & & & 11894.9 & 69744 & 78148 & 7 & 5 & 0.096 & 0.15 & 40 & 0.01 & $\mathrm{D}$ & $l s$ \\
\hline & & & 11894.9 & 69711 & 78117 & 5 & 3 & 0.084 & 0.11 & 21 & -0.27 & D & $l s$ \\
\hline & & & 11880.4 & 69690 & 78105 & 3 & 1 & 0.11 & 0.080 & 9.4 & -0.62 & D & $l s$ \\
\hline & & & $\begin{array}{l}11849.3 \\
11863.0\end{array}$ & $\begin{array}{l}69711 \\
69690\end{array}$ & $\begin{array}{l}78148 \\
78117\end{array}$ & $\begin{array}{l}5 \\
3\end{array}$ & $\begin{array}{l}5 \\
3\end{array}$ & $\begin{array}{l}0.017 \\
0.029\end{array}$ & $\begin{array}{l}0.036 \\
0.061\end{array}$ & $\begin{array}{l}7.1 \\
7.1\end{array}$ & $\begin{array}{l}-0.74 \\
-0.74\end{array}$ & D & ls \\
\hline & & & [11820] & 69690 & 78148 & 3 & 5 & 0.0012 & 0.0040 & 0.47 & -1.92 & D & ls \\
\hline 50 & $\begin{array}{l}2 p 3 p- \\
\quad 2 p\left({ }^{2} \mathrm{P}^{\circ}\right) 4 d\end{array}$ & ${ }^{1} \mathrm{P}-{ }^{1} \mathrm{P}^{0}$ & 6587.75 & 68858 & 84032 & 3 & 3 & 0.024 & 0.015 & 1.0 & -1.34 & D & $c a$ \\
\hline
\end{tabular}




\section{Forbidden Transitions}

The adopted values are selected from calculations by Garstang [1], Naqvi [2], and Yamanouchi and Horie [3], which are very similar in character. All electric quadrupole values are taken from Garstang [1], since his estimate of $s_{q}$ appears to be the most advanced one. For most magnetic dipole lines, values could be taken from Garstang [1], as well as from Naqvi [2], and Yamanouchi and Horie [3], who all arrive at identical results. Only for the ${ }^{3} \mathrm{P}-{ }^{-1} \mathrm{~S}$ transition, for which configuration interaction is important, a difference occurs. In this case the results of Garstang [1], and Yamanouchi and Horie [3] are selected, since they represent, according to a later study by Garstang [4], the best available approximation (see also the general introduction).

\section{References}

[1] Garstang, R. H., Monthly Notices Roy. Astron. Soc. 111 , 115-124 (1951).

[2] Naqvi, A. M,. Thesis Harvard (1951).

[3] Yamanouchi, T. and Horie, H., J. Phys. Soc. Japan 7, 52-56 (1952).

[4] Garstang, R. H., Proc. Cambridge Phil. Soc. 52, 107-113 (1956).

CI. Forbidden Transitions

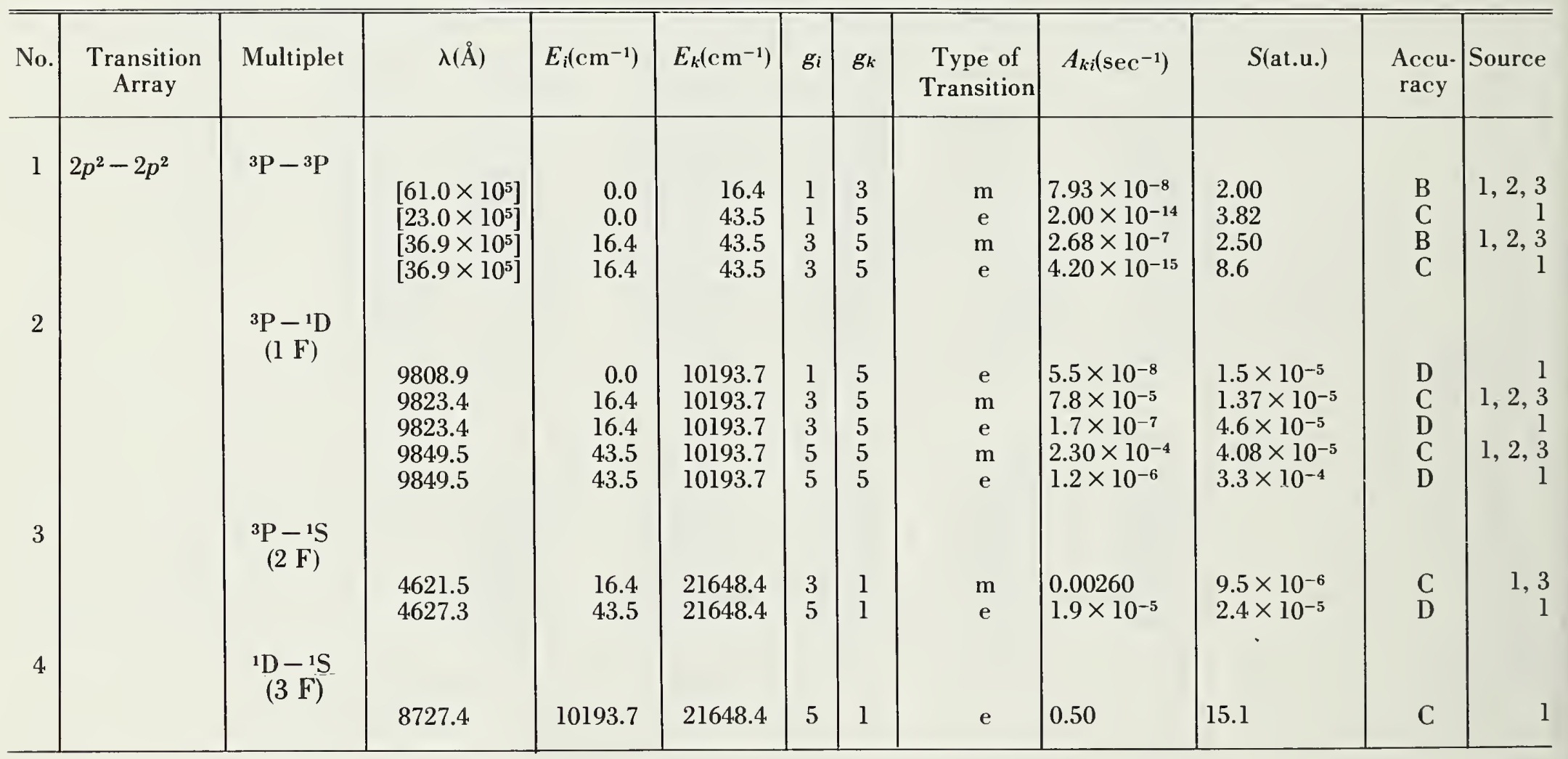




\section{Allowed Transitions}

List of tabulated lines:

\begin{tabular}{l|r||c|c||c|c}
\hline \hline Wavelength $[\AA]$ & No. & Wavelength $[\AA]$ & No. & Wavelength $[\AA]$ & No. \\
\hline & & & & & \\
687.059 & 10 & 3360.9 & 27 & 4413.2 & 24 \\
687.35 & 10 & 3361.09 & 27 & 4618.9 & 26 \\
687.355 & 10 & 3361.75 & 27 & 4628.1 & 26 \\
858.092 & 9 & 3581.80 & 18 & 5132.96 & 14 \\
858.559 & 9 & 3584.98 & 18 & 5133.29 & 14 \\
903.624 & 3 & 3585.83 & 18 & 5137.26 & 14 \\
903.962 & 3 & 3587.68 & 18 & 5139.21 & 14 \\
904.142 & 3 & 3588.92 & 18 & 5143.49 & 14 \\
904.480 & 3 & 3589.67 & 18 & 5145.16 & 14 \\
1009.85 & 4 & 3590.87 & 18 & 5151.08 & 14 \\
1010.07 & 4 & 3876.05 & & & 13 \\
1010.37 & 4 & 3876.19 & 22 & 5640.50 & 13 \\
1036.34 & 2 & 3876.41 & 22 & 5648.08 & 13 \\
1037.02 & 2 & 3876.67 & 22 & 5662.51 & 20 \\
1065.88 & 6 & 3878.22 & 22 & 5889.97 & 20 \\
1065.9 & 6 & 3879.60 & 22 & 5891.65 & 20 \\
1066.12 & 6 & 3880.59 & 22 & 6578.03 & 11 \\
1323.9 & 5 & 3881.2 & 22 & 6582.85 & 11 \\
1334.53 & 1 & 3883.8 & 22 & 6779.74 & 12 \\
1335.66 & 1 & 3918.98 & 17 & 6780.27 & \\
1335.71 & 1 & 3920.68 & 17 & 6783.75 & 12 \\
2173.8 & 15 & 4074.53 & 23 & 6787.09 & 12 \\
2174.1 & 15 & 4076.00 & 23 & 6791.30 & 12 \\
2509.11 & 7 & 4267.02 & 21 & 6798.04 & 12 \\
2511.71 & 7 & 4267.2 & 21 & 6800.50 & \\
2512.03 & 7 & 4267.27 & 21 & 6812.19 & 12 \\
2746.50 & 19 & 4371.59 & 25 & 7231.12 & 16 \\
2747.3 & 19 & 4372.49 & 25 & 7236.19 & 16 \\
2747.31 & 19 & 4374.28 & 25 & 7236.2 & 29 \\
2836.71 & 8 & 441.20 & 24 & 18895 & \\
2837.60 & 8 & 4411.52 & 24 & 18916 & \\
2992.6 & 28 & & & & \\
\hline & & & & & \\
\hline
\end{tabular}

Self-consistent field calculations by Weiss [1], and Biermann and Lübeck [3], and a high current arc experiment by Maecker [2] are utilized for the tabulation. The results for the lower and moderately excited transitions should be quite uncertain because in the calculations the strong effects of configuration interaction are essentially neglected, and the experimental work is subject to large systematic uncertainties.

\section{References}

[1] Weiss, A. W., private communication (1964).

[2] Maecker, H., Z. Physik 135, 13-22 (1953).

[3] Biermann, L. and Lübeck, K., Z. Astrophys. 25, 325-339 (1048). 
CII. Allowed Transitions

\begin{tabular}{|c|c|c|c|c|c|c|c|c|c|c|c|c|c|}
\hline No. & $\begin{array}{c}\text { Transition } \\
\text { Array }\end{array}$ & Multiplet & $\lambda(\AA)$ & $E_{i}\left(\mathrm{~cm}^{-1}\right)$ & $E_{k}\left(\mathrm{~cm}^{-1}\right)$ & $g_{i}$ & $g_{k}$ & $A_{k i}\left(10^{8} \sec ^{-1}\right)$ & $f_{i k}$ & $S$ (at.u.) & $\log g f$ & $\begin{array}{l}\text { Accu- } \\
\text { racy }\end{array}$ & Source \\
\hline \multirow[t]{4}{*}{1} & \multirow[t]{4}{*}{$2 s^{2} 2 p-2 s 2 p^{2}$} & \multirow{4}{*}{$\begin{array}{c}{ }^{2} \mathrm{P}^{\circ}-{ }^{2} \mathrm{D} \\
(1 \mathrm{uv})\end{array}$} & 1335.3 & 43 & 74932 & 6 & 10 & 6.0 & 0.27 & 7.0 & 0.20 & $\mathrm{E}$ & \\
\hline & & & 1335.71 & 64 & 74931 & 4 & 6 & 6.0 & 0.24 & 4.2 & -0.02 & $\mathrm{E}$ & \\
\hline & & & & 0 & 74933 & 2 & 4 & 40 & & 2.3 & -0.28 & $\mathrm{E}$ & \\
\hline & & & 1335.66 & 64 & 74.933 & 4 & 4 & 1.0 & 0.027 & 0.47 & -0.97 & $\mathrm{E}$ & \\
\hline \multirow[t]{3}{*}{2} & & \multirow{3}{*}{$\begin{array}{c}{ }^{2} \mathrm{P}^{\circ}-{ }^{2} \mathrm{~S} \\
(2 \mathrm{uv})\end{array}$} & 1036.8 & 43 & 96494 & 6 & 2 & 11 & 0.059 & 1.2 & -0.45 & $\mathrm{E}$ & \\
\hline & & & 1037.02 & 64 & 96494 & 4 & 2 & 7.3 & 0.059 & 0.80 & -0.63 & $\mathrm{E}$ & \\
\hline & & & 1036.34 & 0 & 96494 & 2 & 2 & 3.6 & 0.059 & 0.40 & -0.93 & E & \\
\hline \multirow[t]{5}{*}{3} & & \multirow{5}{*}{$\begin{array}{c}{ }^{2} \mathrm{P}^{\circ}-{ }^{2} \mathrm{P} \\
(3 \mathrm{uv})\end{array}$} & 904.09 & 43 & 110652 & 6 & 6 & 42 & 0.52 & 9.2 & 0.49 & $\mathrm{E}$ & \\
\hline & & & 904.142 & 64 & 110666 & 4 & 4 & 35 & 0.43 & 5.1 & 0.23 & E & \\
\hline & & & 903.962 & 0 & 110625 & 2 & 2 & 27 & 0.34 & 2.0 & -0.17 & $\mathrm{E}$ & \\
\hline & & & 904.480 & 64 & 110625 & 4 & 2 & 14 & 0.084 & 1. & -0.47 & $\mathrm{E}$ & \\
\hline & & & 903.624 & 0 & 110666 & 2 & 4 & 6.9 & 0.17 & 1.0 & -0.47 & $\mathrm{E}$ & \\
\hline \multirow[t]{4}{*}{4} & \multirow[t]{4}{*}{$2 s 2 p^{2}-2 p^{3}$} & \multirow{4}{*}{$\begin{array}{l}{ }^{4} \mathrm{P}-{ }^{4} \mathrm{~S}^{\circ} \\
(7 \mathrm{uv})\end{array}$} & 1010.2 & 43033 & 142024 & 12 & 4 & 32 & 0.16 & 6.5 & 0.29 & $\mathrm{E}$ & \\
\hline & & & \begin{tabular}{|l}
1010.37 \\
\end{tabular} & 43051 & 142024 & 6 & 4 & 16 & 0.17 & 3.3 & 0.00 & $\mathrm{E}$ & \\
\hline & & & 1010.07 & 43022 & 142024 & 4 & 4 & 11 & 0. & 2.2 & -0.18 & $\mathrm{E}$ & \\
\hline & & & 1009.85 & 43000 & 142024 & 2 & 4 & 5.4 & 0.17 & 1.1 & -0.48 & $\mathrm{E}$ & \\
\hline 5 & & $\begin{array}{l}{ }^{2} \mathrm{D}-{ }^{2} \mathrm{D}^{\circ} \\
(11 \mathrm{uv})\end{array}$ & 1323.9 & 74932 & 150465 & 10 & 10 & 8.7 & 0.23 & 10 & 0.36 & $\mathrm{E}$ & \\
\hline \multirow[t]{4}{*}{6} & & \multirow{4}{*}{$\begin{array}{l}{ }^{2} \mathrm{D}-{ }^{2} \mathrm{P}^{\circ} \\
(12 \mathrm{uv})\end{array}$} & 1066.0 & 74932 & 168744 & 10 & 6 & 8.1 & 0.083 & 2.9 & -0.08 & $\mathrm{E}$ & \\
\hline & & & 1065.88 & 74931 & 168750 & 6 & 4 & 7.1 & 0.081 & 1.7 & -0.31 & $\mathrm{E}$ & \\
\hline & & & $\begin{array}{l}1066.12 \\
1065.12\end{array}$ & 74933 & 168732 & 4 & 2 & 8 & 0.0 & 0. & -0.56 & $\mathrm{E}$ & \\
\hline & & & [1065.9] & 74933 & 168750 & 4 & 4 & 0.79 & 0.014 & 0.19 & -1.27 & $\mathrm{E}$ & \\
\hline \multirow[t]{4}{*}{7} & & \multirow{4}{*}{$\begin{array}{l}{ }^{2} \mathrm{P}-{ }^{2} \mathrm{D}^{\circ} \\
(14 \mathrm{uv})\end{array}$} & 2511.0 & 110652 & 150465 & 6 & 10 & 0.97 & 0.15 & 7.6 & -0.04 & $\mathrm{E}$ & \\
\hline & & & 2512.03 & 110666 & 150463 & 4 & 6 & 0.96 & 0.14 & 4.5 & -0.26 & $\mathrm{E}$ & \\
\hline & & & 2509.11 & 110 & 150468 & 2 & 4 & 08 & & 2. & -0.50 & $\mathrm{E}$ & \\
\hline & & & 25 & 110666 & 150468 & 4 & 4 & 0. & 0.016 & 0.52 & -1.20 & $\mathrm{E}$ & \\
\hline \multirow[t]{3}{*}{8} & \multirow{3}{*}{$\begin{array}{l}2 s 2 p^{2}- \\
2 s^{2}\left({ }^{1} \mathrm{~S}\right) 3 p\end{array}$} & \multirow{3}{*}{$\begin{array}{l}{ }^{2} \mathrm{~S}-{ }^{2} \mathrm{P}^{\circ} \\
(13 \mathrm{uv})\end{array}$} & 2837.0 & 96494 & 131732 & 2 & 6 & 0.35 & 0.13 & 2.4 & -0.59 & $\mathrm{E}$ & \\
\hline & & & 2836.71 & 964.94 & 131736 & 2 & 4 & 0.36 & 0.086 & 1.6 & -0.77 & E & \\
\hline & & & 2837.60 & 96494 & 131725 & 2 & 2 & 0.35 & 0.043 & 0.80 & -1.07 & $\mathrm{E}$ & \\
\hline \multirow[t]{3}{*}{9} & \multirow[t]{3}{*}{$2 p-\left({ }^{1} \mathrm{~S}\right) 3 s$} & \multirow{3}{*}{$\begin{array}{l}{ }^{2} \mathrm{P}^{\circ}-{ }^{2} \mathrm{~S} \\
(4 \mathrm{uv})\end{array}$} & 858.41 & 43 & 116538 & 6 & 2 & 12 & 0.046 & 0.78 & -0.56 & $\mathrm{D}$ & \\
\hline & & & 858.559 & 64 & 116538 & 4 & 2 & 8.3 & 0.046 & 0.52 & -0.74 & D & \\
\hline & & & 858.092 & 0 & 116538 & 2 & 2 & 4.2 & 0.046 & 0.26 & -1.04 & D & \\
\hline 10 & $2 p-\left({ }^{\mathrm{I}} \mathrm{S}\right) 3 d$ & ${ }^{2} \mathrm{P}^{\circ}-{ }^{2} \mathrm{D}$ & 687.25 & 43 & 145551 & 6 & 10 & 22 & 0.26 & 3.5 & 0.19 & $\mathrm{D}$ & \\
\hline & & & 687.355 & 64 & 145551 & 4 & 6 & 22 & 0.2 & 2.1 & -0.03 & $\mathrm{D}$ & \\
\hline & & & 607 & 0 & 145550 & 2 & 4 & 19 & 0 & 1. & -0.28 & D & \\
\hline & & & {$[687$} & 64 & 145 & 4 & 4 & 3.6 & 0.025 & 0.23 & -0.99 & D & \\
\hline 11 & $3 s-\left({ }^{1} \mathrm{~S}\right) 3 p$ & ${ }^{2} \mathrm{~S}-{ }^{2} \mathrm{P}^{\circ}$ & 6579.7 & 116538 & 131732 & 2 & 6 & 0.480 & 0.93 & 40.5 & 0.272 & C & 1,3 \\
\hline & & & 6578.03 & 116538 & 131736 & 2 & 4 & 0.480 & 0.6 & 27.0 & 0.096 & C & \\
\hline & & & 6582.85 & 116538 & 131725 & 2 & 2 & 0.479 & 0.311 & 13.5 & -0.206 & $\mathrm{C}$ & \\
\hline 12 & $2 s 2 p 3 s-$ & ${ }^{4} \mathrm{P}^{\circ}-{ }^{4} \mathrm{D}$ & 6785.6 & 167007 & 181740 & 12 & 20 & 0.369 & 0.424 & 114 & 0.71 & C & $c a$ \\
\hline & & & 6783.75 & 167033 & 181770 & 6 & 8 & 0.370 & 0.3 & 45.6 & 0.310 & C & \\
\hline & & & & & 181734 & 4 & 6 & & & 23. & & $\mathrm{C}$ & \\
\hline & & & & & 181709 & 2 & 4 & 0.1 & 0.2 & 9.5 & -0.373 & $\mathrm{C}$ & \\
\hline & & & 68 & 167033 & 181734 & 6 & 6 & 0.110 & 0.076 & 10.2 & -0.341 & $\mathrm{C}$ & \\
\hline
\end{tabular}


C.II. Allowed Transitions-Continued

\begin{tabular}{|c|c|c|c|c|c|c|c|c|c|c|c|c|c|}
\hline $\mathrm{No}$ & $\begin{array}{c}\text { Transition } \\
\text { Array }\end{array}$ & Multiplet & $\lambda(\AA)$ & $E_{i}\left(\mathrm{~cm}^{-1}\right)$ & $E_{k}\left(\mathrm{~cm}^{-1}\right)$ & $g_{i}$ & $g_{k}$ & $A_{k i}\left(10^{4} \sec ^{-1}\right)$ & $f_{i k}$ & $S$ (at.u.) & $\log g f$ & $\begin{array}{l}\text { Accu- } \\
\text { racy }\end{array}$ & Source \\
\hline & & & 6791.30 & 166988 & 181709 & 4 & 4 & 0.195 & 0.135 & 12.1 & -0.268 & $\mathrm{C}$ & ls \\
\hline & & & 6787.09 & 166965 & 181695 & 2 & 2 & 0.307 & 0.212 & 9.5 & -0.373 & C & ls \\
\hline & & & 6812.19 & 167033 & 181709 & 6 & 4 & 0.0183 & 0.0085 & 1.14 & -1.292 & C & Is \\
\hline & & & 6798.04 & 166988 & 181695 & 4 & 2 & 0.061 & 0.0211 & 1.89 & -1.074 & C & Is \\
\hline \multirow[t]{3}{*}{13} & & ${ }^{4} \mathrm{P}^{\circ}-{ }^{4} \mathrm{~S}$ & 5653.9 & 167007 & 184689 & 12 & 4 & 0.65 & 0.104 & 23.2 & 0.096 & C & $c a$ \\
\hline & & & 5662.51 & 167033 & 184689 & 6 & 4 & 0.325 & 0.104 & 11.6 & -0.205 & C & ls \\
\hline & & & $\begin{array}{l}5648.08 \\
5640.50\end{array}$ & $\begin{array}{l}166988 \\
166965\end{array}$ & $\begin{array}{l}184689 \\
184689\end{array}$ & $\begin{array}{l}4 \\
2\end{array}$ & $\begin{array}{l}4 \\
4\end{array}$ & $\begin{array}{l}0.217 \\
0.109\end{array}$ & $\begin{array}{l}0.104 \\
0.104\end{array}$ & $\begin{array}{l}7.7 \\
3.86\end{array}$ & $\begin{array}{l}-0.381 \\
-0.68\end{array}$ & $\begin{array}{l}\mathrm{C} \\
\mathrm{C}\end{array}$ & $\begin{array}{l}\text { ls } \\
\text { ls }\end{array}$ \\
\hline \multirow[t]{7}{*}{14} & & ${ }^{4} \mathrm{P}^{\circ}-{ }^{4} \mathrm{P}$ & 5141.8 & 167007 & 186450 & 12 & 12 & 0.86 & 0.342 & 69 & 0.61 & $\mathrm{C}$ & $c a$ \\
\hline & & & 5145.16 & 167033 & 186464 & 6 & 6 & 0.60 & 0.239 & 24.3 & 0.157 & $\mathrm{C}$ & ls \\
\hline & & & 5139.21 & 166988 & 186441 & 4 & 4 & 0.115 & 0.0456 & 3.09 & -0.74 & C & $l s$ \\
\hline & & & 5137.26 & 166965 & 186425 & 2 & 2 & 0.144 & 0.057 & 1.93 & -0.94 & $\mathrm{C}$ & ls \\
\hline & & & 5151.08 & 167033 & 186441 & 6 & 4 & 0.385 & 0.102 & 10.4 & -0.213 & C & ls \\
\hline & & & $\begin{array}{l}5143.49 \\
5133.29\end{array}$ & $\begin{array}{l}166988 \\
166988\end{array}$ & $\begin{array}{l}186425 \\
186464\end{array}$ & $\begin{array}{l}4 \\
4\end{array}$ & $\begin{array}{l}2 \\
6\end{array}$ & $\begin{array}{l}0.72 \\
0.260\end{array}$ & $\begin{array}{l}0.142 \\
0.154\end{array}$ & $\begin{array}{r}9.6 \\
10.4\end{array}$ & $\begin{array}{l}-0.246 \\
-0.210\end{array}$ & $\begin{array}{l}\mathrm{C} \\
\mathrm{C}\end{array}$ & $\begin{array}{l}l s \\
\text { ls }\end{array}$ \\
\hline & & & 5132.96 & 166965 & 186441 & 2 & 4 & 0.361 & 0.285 & 9.6 & -0.244 & $\mathrm{C}$ & ls \\
\hline \multirow[t]{3}{*}{15} & $3 s-\left({ }^{+} \mathrm{S}\right) 4 p$ & ${ }^{2} \mathrm{~S}-{ }^{2} \mathrm{P}^{\circ}$ & 2173.9 & 116538 & 162523 & 2 & 6 & 0.253 & 0.054 & 0.77 & -0.97 & C & 3 \\
\hline & & & [2173.8] & 116538 & 162525 & 2 & 4 & 0.251 & 0.0356 & 0.51 & -1.147 & C & ls \\
\hline & & & {$[2174.1]$} & 116538 & 162519 & 2 & 2 & 0.252 & 0.0179 & & -1.44 & & \\
\hline \multirow[t]{4}{*}{16} & $3 p-\left({ }^{1} \mathrm{~S}\right) 3 d$ & ${ }^{2} \mathrm{P}^{\circ}-{ }^{2} \mathrm{D}$ & 7234.4 & 131732 & 145551 & 6 & 10 & 0.450 & 0.59 & 84 & 0.55 & C & 1 \\
\hline & & & 7236.19 & 131736 & 145551 & 4 & 6 & 0.446 & 0.52 & 50 & 0.322 & C & ls \\
\hline & & & $\begin{array}{r}7231.12 \\
\end{array}$ & 131725 & 145550 & 2 & 4 & 0.375 & 0.59 & 28.0 & 0.071 & $\mathrm{C}$ & ls \\
\hline & & & {$[1,200.2]$} & 151750 & 145550 & & 4 & 0.075 & 0.059 & 5.6 & -0.63 & C & Is \\
\hline \multirow[t]{2}{*}{17} & $3 p-\left({ }^{1} \mathrm{~S}\right) 4 s$ & ${ }^{2} \mathrm{P}^{\circ}-{ }^{2} \mathrm{~S}$ & 3920.2 & 131732 & 157234 & 6 & 2 & 1.87 & 0.143 & 11.1 & -0.066 & C & 3 \\
\hline & & & $\begin{array}{l}3920.68 \\
391898\end{array}$ & 131736 & 157234 & 4 & 2 & 1.24 & 0.143 & $\begin{array}{l}7.4 \\
370\end{array}$ & -0.242 & $\mathrm{C}$ & Is \\
\hline \multirow{9}{*}{18} & & & & & & & & & & & & & \\
\hline & $\begin{array}{l}2 s 2 p 3 p- \\
\quad 2 s 2 p\left({ }^{3} \mathrm{P}^{\circ}\right) 4 s\end{array}$ & $\begin{array}{c}{ }^{4} \mathrm{D}-{ }^{4} \mathrm{P}^{\circ} \\
(23)\end{array}$ & 3589.3 & 181740 & 209593 & 20 & 12 & 0.92 & 0.107 & 25.3 & 0.330 & $\mathrm{C}$ & $c a$ \\
\hline & & & 3589.67 & 181770 & 209620 & 8 & 6 & 0.74 & 0.107 & 10.1 & -0.068 & C & Is \\
\hline & & & 3590.87 & 181734 & 209574 & 6 & 4 & 0.5 & $0.0^{7}$ & 5.3 & -0.347 & C & Is \\
\hline & & & $\begin{array}{l}3590.87 \\
3584.98\end{array}$ & $\begin{array}{l}181709 \\
181734\end{array}$ & $\begin{array}{l}209550 \\
209620\end{array}$ & 6 & $\begin{array}{l}2 \\
6\end{array}$ & $\begin{array}{l}0.465 \\
0.166\end{array}$ & $\begin{array}{l}0.0449 \\
0.0319\end{array}$ & $\begin{array}{l}2.12 \\
2.26\end{array}$ & $\begin{array}{l}-0.75 \\
-0.72\end{array}$ & C & Is \\
\hline & & & 3587.68 & 181709 & 209574 & 4 & 4 & 0.295 & 0.057 & 2.69 & -0.64 & $\mathrm{C}$ & $l s$ \\
\hline & & & 3588.92 & 181695 & 209550 & 2 & 2 & 0.466 & 0.090 & 2.1 & -0.74 & C & ls \\
\hline & & & 3581.80 & 181709 & 209620 & 4 & 6 & 0.0184 & 0.0053 & 0.250 & -1.67 & C & ls \\
\hline & & & 3585.83 & 181695 & 209574 & 2 & 4 & 0.0426 & 0.0178 & 0.420 & -1.450 & C & ls \\
\hline \multirow[t]{3}{*}{19} & $3 p-\left({ }^{1} \mathrm{~S}\right) 4 d$ & $\begin{array}{l}{ }^{2} \mathrm{P}^{\circ}-{ }^{2} \mathrm{D} \\
(15 \text { uv) }\end{array}$ & 2747.0 & 131732 & 168124 & 6 & 10 & 0.466 & 0.088 & 4.77 & -0.278 & C & $c a$ \\
\hline & & & $\begin{array}{l}2747.31 \\
274650\end{array}$ & $\begin{array}{l}131736 \\
131795\end{array}$ & $\begin{array}{l}168124 \\
168124\end{array}$ & 4 & 6 & 0.466 & 0.079 & 2.86 & -0.50 & C & ls \\
\hline & & & $\begin{array}{r}2746.50 \\
{[2747.3]}\end{array}$ & $\begin{array}{l}131725 \\
131736\end{array}$ & $\begin{array}{l}168124 \\
168124\end{array}$ & $\begin{array}{l}2 \\
4\end{array}$ & $\begin{array}{l}4 \\
4\end{array}$ & $\begin{array}{l}0.389 \\
0.078\end{array}$ & $\begin{array}{l}0.088 \\
0.0088\end{array}$ & $\begin{array}{l}1.59 \\
0.318\end{array}$ & $\begin{array}{l}-0.75 \\
-1.454\end{array}$ & $\begin{array}{l}\mathrm{C} \\
\mathrm{C}\end{array}$ & $\begin{array}{l}l s \\
l s\end{array}$ \\
\hline \multirow[t]{4}{*}{20} & $3 d-\left({ }^{1} \mathrm{~S}\right) 4 p$ & ${ }^{2} \mathrm{D}-{ }_{(5)}^{2} \mathrm{P}^{\circ}$ & 5890.4 & 145551 & 162523 & 10 & 6 & 0.337 & 0.105 & 20.4 & 0.022 & C & $c a$ \\
\hline & & & 5889.97 & 145551 & 162525 & 6 & 4 & 0.302 & 0.105 & 12.2 & -0.201 & C & $l s$ \\
\hline & & & 5891.65 & 145550 & 162519 & 4 & 2 & 0.337 & 0.088 & 6.8 & -0.455 & C & ls \\
\hline & & & [5889.4] & 145550 & 162525 & 4 & 4 & 0.0337 & 0.0175 & 1.36 & -1.154 & $\mathrm{C}$ & ls \\
\hline \multirow[t]{3}{*}{21} & $3 d-\left({ }^{1} \mathrm{~S}\right) 4 f$ & $\begin{array}{c}{ }^{2} \mathrm{D}-{ }^{2} \mathrm{~F}^{\circ} \\
(6)\end{array}$ & 4267.2 & 145551 & 168979 & 10 & 14 & 2.46 & 0.94 & 132 & 0.97 & $\mathrm{C}$ & $c a$ \\
\hline & & & 4267.27 & 145551 & 168979 & 6 & 8 & 2.44 & 0.89 & 75 & 0.73 & C & $l s$ \\
\hline & & & $\begin{array}{r}4267.02 \\
{[4267.2]}\end{array}$ & $\begin{array}{l}145550 \\
145551\end{array}$ & $\begin{array}{l}168979 \\
168979\end{array}$ & 6 & 6 & 0.164 & 0.0447 & 3.77 & -0.57 & $\mathrm{C}$ & $l s$ \\
\hline
\end{tabular}


C II. Allowed Transitions-Continued

\begin{tabular}{|c|c|c|c|c|c|c|c|c|c|c|c|c|c|}
\hline No & $\begin{array}{l}\text { Transition } \\
\text { Array }\end{array}$ & Multiplet & $\lambda(\AA)$ & $E_{i}\left(\mathrm{~cm}^{-1}\right)$ & $E_{k}\left(\mathrm{~cm}^{-1}\right)$ & $g_{i}$ & $g_{k}$ & $A_{k i}\left(10^{8} \mathrm{sec}^{-1}\right)$ & $f_{i k}$ & $S$ (at.u.) & $\log g f$ & $\begin{array}{l}\text { Accu- } \\
\text { racy }\end{array}$ & Source \\
\hline \multirow[t]{9}{*}{22} & $2 s 2 p 3 d-$ & ${ }^{4} \mathrm{~F}^{\circ}-{ }^{4} \mathrm{G}$ & 3876.7 & 195786 & 221574 & 28 & 36 & 2.66 & 0.77 & 275 & 1.333 & $\mathrm{C}$ & $c a$ \\
\hline & & & 3876.19 & 195812 & 221604 & 10 & 12 & 2.67 & 0.72 & 92 & 0.86 & C & ls \\
\hline & & & 3876.41 & 195785 & 221575 & 8 & 10 & 2.43 & 0.69 & 70 & 0.74 & $\mathrm{C}$ & ls \\
\hline & & & 3876.67 & 195765 & 221553 & 6 & 8 & 2.30 & 0.69 & 53 & 0.62 & C & ls \\
\hline & & & 3876.05 & 195751 & 221543 & 4 & 6 & 2.28 & 0.77 & 39.3 & 0.489 & $\mathrm{C}$ & ls \\
\hline & & & $\begin{array}{l}3880.59 \\
2070\end{array}$ & 195812 & $\begin{array}{l}221575 \\
791553\end{array}$ & $\begin{array}{ll}10 \\
8\end{array}$ & 10 & 0.218 & 0.0493 & 6.3 & -0.307 & $\mathrm{C}$ & ls \\
\hline & & & 3878.22 & 195765 & 221543 & $\begin{array}{l}0 \\
6\end{array}$ & $\begin{array}{l}8 \\
6\end{array}$ & $\begin{array}{l}0.360 \\
0.365\end{array}$ & $\begin{array}{l}0.081 \\
0.082\end{array}$ & $\begin{array}{l}8.3 \\
6.3\end{array}$ & $\begin{array}{l}-0.187 \\
-0.307\end{array}$ & $\begin{array}{l}\mathrm{C} \\
\mathrm{C}\end{array}$ & $\begin{array}{l}l s \\
l s\end{array}$ \\
\hline & & & [3883.8] & 195812 & 221553 & 10 & 8 & 0.0080 & 0.00145 & 0.186 & -1.84 & $\mathrm{C}$ & ls \\
\hline & & & [3881.2] & 195785 & 221543 & 8 & 6 & 0.0132 & 0.00224 & 0.229 & -1.75 & C & $l s$ \\
\hline \multirow[t]{3}{*}{23} & & ${ }^{4} \mathrm{D}^{\circ}-{ }^{4} \mathrm{~F}$ & & & & & & & & & & & \\
\hline & & & 4076.00 & 196581 & 221107 & 8 & 10 & 2.28 & 0.71 & 76 & 0.75 & C & $c a, l s$ \\
\hline & & & 4074.53 & 196571 & 221106 & 6 & 8 & 1.96 & 0.65 & 52 & 0.59 & C & $c a, l s$ \\
\hline \multirow[t]{4}{*}{24} & & ${ }^{2} \mathrm{D}^{\circ}-{ }^{2} \mathrm{~F}$ & 4411.4 & 198433 & 221095 & 10 & 14 & 2.11 & 0.86 & 125 & 0.93 & C & $c a$ \\
\hline & & & 4411.52 & 198437 & 221099 & 6 & 8 & 2.09 & 0.81 & 71 & 0.69 & $\mathrm{C}$ & ls \\
\hline & & & 4411.20 & 198426 & 221090 & 4 & 6 & 1.97 & 0.86 & 50 & 0.54 & $\mathrm{C}$ & $l s$ \\
\hline & & & [4413.2] & 198437 & 221090 & 6 & 6 & 0.140 & 0.0410 & 3.57 & -0.61 & C & ls \\
\hline \multirow[t]{4}{*}{25} & & $\left.{ }^{4} \mathrm{P}^{\circ}-{ }^{4} \mathrm{~T}\right) \mathrm{D}$ & & & & & & & & & & & \\
\hline & & & 4374.28 & $19884 \overline{2}$ & 221697 & 6 & 8 & 1.99 & 0.76 & 66 & 0.66 & C & $c a, l s$ \\
\hline & & & 4372.49 & 198864 & 221727 & 4 & 6 & 1.40 & 0.6 & 34.5 & 0.380 & $\mathrm{C}$ & $c a, l s$ \\
\hline & & & $40 \pi 1.09$ & $1988 / 8$ & 221746 & 2 & 4 & 0.83 & 0.475 & & & c & $c a$, ts \\
\hline \multirow[t]{4}{*}{26} & & ${ }^{2} \mathrm{~F}^{\circ}-{ }^{2} \mathrm{G}$ & 4619.1 & 199966 & 221609 & 14 & 18 & 2.24 & 0.92 & 196 & 1.110 & $\mathrm{C}$ & $c a$ \\
\hline & & & [4618.9] & 199984 & 221628 & 8 & 10 & 2.24 & 0.90 & 109 & 0.86 & $\mathrm{C}$ & $l s$ \\
\hline & & & [4618.9] & 199941 & 221585 & 6 & 8 & 2.16 & 0.92 & 84 & 0.74 & $\mathrm{C}$ & ls \\
\hline & & & [4628.1] & 199984 & 221585 & 8 & 8 & 0.079 & 0.0255 & 3.11 & -0.69 & C & ls \\
\hline \multirow[t]{3}{*}{27} & $3 d-\left({ }^{1} \mathrm{~S}\right) 5 p$ & ${ }^{2} \mathrm{D}-{ }_{(7)}^{-2} \mathrm{P}^{\circ}$ & 3361.3 & 145551 & 175293 & 10 & 6 & 0.121 & 0.0123 & 1.36 & -0.91 & $\mathrm{C}$ & $c a$ \\
\hline & & & 3361.09 & 145551 & 175295 & 6 & 4 & 0.109 & 0.0124 & 0.82 & -1.130 & $\mathrm{C}$ & ls \\
\hline & & & $\begin{array}{r}3361.75 \\
{[3360.9]}\end{array}$ & $\begin{array}{l}145550 \\
145550\end{array}$ & $\begin{array}{l}175288 \\
175295\end{array}$ & $\begin{array}{l}4 \\
4\end{array}$ & $\begin{array}{l}2 \\
4\end{array}$ & $\begin{array}{l}0.121 \\
0.0121\end{array}$ & $\begin{array}{l}0.0102 \\
0.00206\end{array}$ & $\begin{array}{l}0.453 \\
0.091\end{array}$ & $\begin{array}{l}-1.388 \\
-2.085\end{array}$ & $\begin{array}{l}\mathrm{C} \\
\mathrm{C}\end{array}$ & $\begin{array}{l}l s \\
l s\end{array}$ \\
\hline 28 & $3 d-\left({ }^{1} \mathrm{~S}\right) 5 f$ & $\begin{array}{c}{ }^{2} \mathrm{D}-{ }^{2} \mathrm{~F}^{\circ} \\
\end{array}$ & 2992.6 & 145551 & 178956 & 10 & 14 & 0.90 & 0.169 & 16.6 & 0.227 & $\mathrm{C}$ & $c a$ \\
\hline \multirow[t]{2}{*}{29} & $4 s-\left({ }^{1} \mathrm{~S}\right) 4 p$ & ${ }^{2} \mathrm{~S}-{ }^{2} \mathrm{P}^{\circ}$ & 18902 & 157234 & 162523 & 2 & 6 & 0.074 & 1.18 & 147 & 0.373 & $\mathrm{C}$ & 3 \\
\hline & & & $\begin{array}{l}{[18895]} \\
{[18916]}\end{array}$ & $\begin{array}{l}157234 \\
157234\end{array}$ & $\begin{array}{l}162525 \\
162519\end{array}$ & 2 & $\begin{array}{l}4 \\
2\end{array}$ & 0.074 & $\begin{array}{l}0.79 \\
0.393\end{array}$ & $\begin{array}{l}98 \\
490\end{array}$ & $\begin{array}{r}0.197 \\
-0104\end{array}$ & $\mathrm{C}$ & ls \\
\hline
\end{tabular}

\section{Forbidden Transitions}

Naqvi's calculation [1] of the one possible transition in the ground state configuration $2 p$ is the only available source. The line strength should be quite accurate, since it does not sensitively depend on the choice of the interaction parameters.

[1] Naqvi, A. M., Thesis Harvard (1951).

\section{Reference}

C.II. Forbidden Transitions

\begin{tabular}{|c|c|c|c|c|c|c|c|c|c|c|c|c|}
\hline No. & $\begin{array}{c}\text { Transition } \\
\text { Array }\end{array}$ & Multiplet & $\lambda(\AA)$ & $E_{i}\left(\mathrm{~cm}^{-1}\right)$ & $E_{k}\left(\mathrm{~cm}^{-1}\right)$ & $g_{i}$ & $g_{k}$ & $\begin{array}{l}\text { Type of } \\
\text { Transition }\end{array}$ & $A_{k i}\left(\sec ^{-1}\right)$ & $S($ at.u.) & $\begin{array}{l}\text { Accu- } \\
\text { racy }\end{array}$ & Source \\
\hline 1 & $2 p-2 p$ & ${ }^{2} \mathrm{P}^{\circ}-{ }^{2} \mathrm{P}^{\circ}$ & {$\left[15.6 \times 10^{5}\right]$} & 0 & 64 & 2 & 4 & $\mathbf{m}$ & $2.36 \times 10^{-6}$ & 1.33 & C & 1 \\
\hline
\end{tabular}




\section{III}

Ground State

$1 s^{2} 2 s^{2}{ }^{1} \mathrm{~S}_{0}$

Ionization Potential

$47.864 \mathrm{eV}=386213.9 \mathrm{~cm}^{-1}$

\section{Allowed Transitions}

List of tabulated lines:

\begin{tabular}{l|r||c|c||c|c}
\hline \hline Wavelength $[\AA]$ & No. & Wavelength $[\AA]$ & No. & Wavelength $[\AA]$ & No. \\
\hline 310.171 & 3 & 3609.96 & 23 & 4673.91 & 15 \\
371.694 & 11 & 3609.61 & 23 & 5244.5 & 14 \\
371.72 & 11 & 3703.52 & 18 & 5249.6 & 26 \\
371.747 & 11 & 3883.80 & 27 & 5253.55 & 14 \\
371.784 & 11 & 3885.99 & 27 & 5272.56 & 14 \\
371.80 & 11 & 3886.2 & 27 & 5696.0 & 17 \\
386.203 & 2 & 3889.18 & 27 & 5857.9 & 20 \\
459.462 & 9 & 3889.7 & 27 & 5862.8 & 20 \\
459.521 & 9 & 3889.8 & 27 & 5871.6 & 20 \\
459.633 & 9 & 4056.06 & 28 & 5871.8 & \\
459.64 & 9 & 4122.05 & 24 & 5880.4 & 20 \\
538.075 & 7 & 4325.70 & 16 & 5894.1 & 20 \\
538.150 & 7 & 4379.97 & 25 & 6727.1 & 13 \\
538.312 & 7 & 4383.24 & 25 & 6730.7 & 13 \\
574.279 & 10 & 4388.24 & 25 & 6742.1 & 13 \\
690.526 & 8 & 4516.02 & 22 & 6744.2 & 13 \\
977.026 & 1 & 4516.93 & 22 & 672.2 & 13 \\
1174.92 & 4 & 4647.40 & 12 & 673.7 & 19 \\
1175.25 & 4 & 4650.16 & 12 & 6851.2 & \\
1175.57 & 4 & 4650.9 & 15 & 6853.1 & 19 \\
1175.70 & 4 & 4651.35 & 12 & 6857.3 & 19 \\
1175.97 & 4 & 4651.8 & 15 & 6862.9 & 19 \\
1176.35 & 4 & 4659.0 & 15 & 6869.1 & 19 \\
1247.37 & 6 & 4663.53 & 15 & 6871.7 & \\
2296.89 & 5 & 4665.90 & 15 & 6881.4 & \\
3170.16 & 21 & & & & \\
& & & & & \\
\hline
\end{tabular}

Values for the $2 s^{2}-2 s 2 p$ and $2 s 2 p-2 p^{2}$ transition arrays are taken from the self-consistent field calculations of Weiss [1]. These calculations do not include the important effects of configuration interaction; hence fairly large uncertainties must be expected. The average of the dipole length and velocity approximations is adopted [I]. Accuracies within 50 percent are indicated by the following comparison: Weiss [1] has undertaken refined calculations, including configuration interaction, for the same transitions in Be I-the first member of this isoelectronic sequence-in addition to calculations of the type done for this ion. In all cases the agreement with the average of the dipole length and velocity approximations is close.

\section{Reference}

[1] Weiss, A. W., private communication (1964). 
C III. Allowed Transitions

\begin{tabular}{|c|c|c|c|c|c|c|c|c|c|c|c|c|c|}
\hline No. & $\begin{array}{l}\text { Transition } \\
\text { Array }\end{array}$ & Multiplet & $\lambda(\AA)$ & $E_{i}\left(\mathrm{~cm}^{-1}\right)$ & $E_{k}\left(\mathrm{~cm}^{-1}\right)$ & $g_{i}$ & $g_{k}$ & $A_{k i}\left(10^{8} \sec ^{-1}\right)$ & $f_{i k}$ & $S($ at.u. $)$ & $\log g f$ & $\begin{array}{c}\text { Accu- } \\
\text { racy }\end{array}$ & Source \\
\hline 1 & $2 s^{2}-2 s\left({ }^{2} \mathrm{~S}\right) 2 p$ & ${ }^{1} \mathrm{~S}-{ }^{1} \mathrm{P}^{\circ}$ & 977.026 & 0.0 & 102351 & 1 & 3 & 19 & 0.81 & 2.6 & -0.09 & D & 1 \\
\hline 2 & $2 s^{2}-2 s\left({ }^{2} \mathrm{~S}\right) 3 p$ & 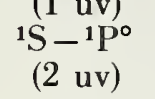 & 386.203 & 0.0 & 258931 & 1 & 3 & 38 & 0.26 & 0.32 & -0.59 & D & $c a$ \\
\hline 3 & $2 s^{2}-2 s\left({ }^{2} \mathrm{~S}\right) 4 p$ & $\begin{array}{c}{ }^{1} \mathrm{~S}-{ }^{1} \mathrm{P}^{\circ} \\
(3 \mathrm{uv})\end{array}$ & 310.171 & 0.0 & 322403 & 1 & 3 & 3.7 & 0.016 & 0.017 & -1.79 & D & $c a$ \\
\hline \multirow[t]{5}{*}{4} & \multirow[t]{8}{*}{$2 s 2 p-2 p^{2}$} & \multirow[t]{5}{*}{$\begin{array}{c}{ }^{3} \mathrm{P}^{\circ}-{ }^{3} \mathrm{P} \\
(4 \mathrm{uv})\end{array}$} & 1175.7 & 52419 & 137478 & 9 & 9 & 13 & 0.26 & 9.1 & 0.37 & D & 1 \\
\hline & & & $\begin{array}{l}1175.70 \\
1175.57\end{array}$ & $\begin{array}{l}52447 \\
52390\end{array}$ & $\begin{array}{l}137502 \\
137455\end{array}$ & $\begin{array}{l}5 \\
3\end{array}$ & $\begin{array}{l}5 \\
3\end{array}$ & 9.4 & 0.20 & 3.8 & -0.01 & D & $l s$ \\
\hline & & & $\begin{array}{l}117.5 / \\
1176.35\end{array}$ & $\begin{array}{l}52390 \\
52447\end{array}$ & 137455 & $\begin{array}{l}3 \\
5\end{array}$ & $\begin{array}{l}3 \\
3\end{array}$ & $\begin{array}{l}3.1 \\
5.2\end{array}$ & $\begin{array}{l}0.065 \\
0.065\end{array}$ & $\begin{array}{l}0.76 \\
1.3\end{array}$ & $\begin{array}{l}-0.71 \\
-0.49\end{array}$ & D & ls \\
\hline & & & 1175.97 & 52390 & 137426 & 3 & 1 & 13 & 0.087 & 1.0 & -0.58 & D & ls \\
\hline & & & $\begin{array}{l}1174.92 \\
1175.25\end{array}$ & $\begin{array}{l}52390 \\
59267\end{array}$ & 137502 & 3 & 5 & 3.1 & 0.11 & 1.3 & -0.49 & D & ls \\
\hline 5 & & & & & & & & & & & & & \\
\hline 5 & & $\begin{array}{l}{ }^{1} \mathrm{P}^{0}-{ }^{1} \mathrm{D} \\
(8 \mathrm{uv})\end{array}$ & 2296.89 & 102351 & 145875 & 3 & 5 & 3.6 & 0.47 & 11 & 0.15 & D & 1 \\
\hline 6 & & $\begin{array}{l}{ }^{1} \mathrm{P}^{0}-{ }^{1} \mathrm{~S} \\
(9 \mathrm{uv})\end{array}$ & 1247.37 & 102351 & 182520 & 3 & 1 & 12 & 0.090 & 1.1 & -0.57 & D & 1 \\
\hline \multirow[t]{3}{*}{7} & \multirow[t]{4}{*}{$2 s 2 p-2 s\left({ }^{2} \mathrm{~S}\right) 3 s$} & \multirow[t]{3}{*}{$\begin{array}{c}{ }^{3} \mathrm{P}^{\circ}-{ }^{3} \mathrm{~S} \\
(5 \text { uv })\end{array}$} & 538.23 & 52419 & 238212 & 9 & 3 & 21 & 0.031 & 0.49 & -0.55 & D & $c a$ \\
\hline & & & 538.312 & 52447 & 238212 & 5 & 3 & 12 & 0.031 & 0.27 & -0.81 & D & $l s$ \\
\hline & & & $\begin{array}{l}538.150 \\
538.075\end{array}$ & $\begin{array}{l}52390 \\
52367\end{array}$ & $\begin{array}{l}238212 \\
238212\end{array}$ & $\begin{array}{l}3 \\
1\end{array}$ & $\begin{array}{l}3 \\
3\end{array}$ & $\begin{array}{l}7.1 \\
2.3\end{array}$ & $\begin{array}{l}0.031 \\
0.031\end{array}$ & $\begin{array}{l}0.16 \\
0.054\end{array}$ & $\begin{array}{l}-1.04 \\
-1.51\end{array}$ & $\begin{array}{l}\mathrm{D} \\
\mathrm{D}\end{array}$ & ls \\
\hline 8 & & 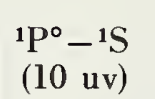 & 690.526 & 102351 & 247170 & 3 & 1 & 22 & 0.053 & 0.36 & -0.80 & D & $c a$ \\
\hline \multirow[t]{5}{*}{9} & \multirow[t]{6}{*}{$2 s 2 p-2 s\left({ }^{2} \mathrm{~S}\right) 3 d$} & \multirow[t]{5}{*}{$\begin{array}{c}{ }^{3 \mathrm{P}^{\circ}}-{ }^{3} \mathrm{D} \\
(6 \mathrm{uv})\end{array}$} & 459.57 & 52419 & 270013 & 9 & 15 & 79 & 0.42 & 5.7 & 0.57 & D & $\mathrm{ca}$ \\
\hline & & & $\begin{array}{l}459.633 \\
459591\end{array}$ & $\begin{array}{l}52447 \\
52300\end{array}$ & 270015 & 5 & $\begin{array}{l}7 \\
5\end{array}$ & $\begin{array}{l}79 \\
50\end{array}$ & 0.35 & 2.7 & $\begin{array}{r}0.24 \\
-\end{array}$ & D & ls \\
\hline & & & 459.462 & 52367 & 270009 & 1 & 3 & $\begin{array}{l}59 \\
44\end{array}$ & $\begin{array}{l}0.3 \\
0.4\end{array}$ & $\begin{array}{l}1.4 \\
0.63\end{array}$ & $\begin{array}{l}-0.03 \\
-0.38\end{array}$ & $\begin{array}{l}\mathrm{D} \\
\mathrm{D}\end{array}$ & ls \\
\hline & & & 459.633 & 52447 & 270011 & 5 & 5 & 20 & 0.063 & 0.47 & -0.51 & $\mathrm{D}$ & ls \\
\hline & & & $\begin{array}{r}459.521 \\
{[459.64]}\end{array}$ & $\begin{array}{l}52390 \\
52447\end{array}$ & $\begin{array}{l}270009 \\
270009\end{array}$ & $\begin{array}{l}3 \\
5\end{array}$ & $\begin{array}{l}3 \\
3\end{array}$ & $\begin{array}{c}33 \\
2.2\end{array}$ & $\begin{array}{l}0.10 \\
0.0042\end{array}$ & $\begin{array}{l}0.47 \\
0.032\end{array}$ & $\begin{array}{l}-0.50 \\
-1.68\end{array}$ & $\begin{array}{l}\text { D } \\
\text { D }\end{array}$ & $\begin{array}{l}l s \\
l s\end{array}$ \\
\hline 10 & & 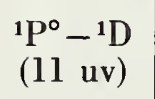 & 574.279 & 102351 & 276483 & 3 & 5 & 63 & 0.52 & 2.9 & 0.19 & D & $c a$ \\
\hline \multirow[t]{5}{*}{11} & \multirow[t]{5}{*}{$\begin{array}{l}2 s 2 p- \\
\quad 2 s\left({ }^{2} \mathrm{~S}\right) 4 d\end{array}$} & \multirow[t]{5}{*}{$\begin{array}{c}{ }^{3} \mathrm{P}^{\circ}-{ }^{3} \mathrm{D} \\
(7 \mathrm{uv})\end{array}$} & 371.73 & 52419 & 321435 & 9 & 15 & 34 & 0.12 & 1.3 & 0.03 & D & $c a$ \\
\hline & & & $\begin{array}{l}371.747 \\
371.694\end{array}$ & $\begin{array}{l}52447 \\
52390\end{array}$ & $\begin{array}{l}321450 \\
321427\end{array}$ & $\begin{array}{l}5 \\
3\end{array}$ & $\begin{array}{l}7 \\
5\end{array}$ & $\begin{array}{l}34 \\
26\end{array}$ & $\begin{array}{l}0.10 \\
0.089\end{array}$ & $\begin{array}{l}0.61 \\
0.33\end{array}$ & $\begin{array}{l}-0.30 \\
-0.57\end{array}$ & D & ls \\
\hline & & & 371.694 & 52367 & 321411 & 1 & 3 & 19 & 0.12 & 0.1 & -0.92 & $\begin{array}{l}\mathrm{D} \\
\mathrm{D}\end{array}$ & $\begin{array}{l}\text { ls } \\
l s\end{array}$ \\
\hline & & & 371.784 & 52447 & 321427 & 5 & 5 & 8.6 & 0.018 & 0.11 & -1.05 & D & ls \\
\hline & & & {$\left[\begin{array}{l}371.72] \\
{[371.80]}\end{array}\right]$} & $\begin{array}{l}52390 \\
52447\end{array}$ & $\begin{array}{l}321411 \\
321411\end{array}$ & $\begin{array}{l}3 \\
5\end{array}$ & $\begin{array}{l}3 \\
3\end{array}$ & $\begin{array}{l}14 \\
0.96\end{array}$ & 0.030 & $\begin{array}{l}0.11^{\circ} \\
0.0073\end{array}$ & $\begin{array}{l}-1.05 \\
-2.23\end{array}$ & $\begin{array}{l}\text { D } \\
\text { D }\end{array}$ & ls \\
\hline \multirow[t]{4}{*}{12} & \multirow{4}{*}{$\begin{array}{l}2 s 3 s- \\
2 s\left({ }^{2} \mathrm{~S}\right) 3 p\end{array}$} & \multirow{4}{*}{ 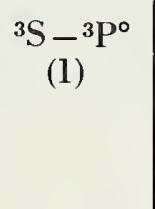 } & 4648.8 & 238212 & 259718 & 3 & 9 & 0.78 & 0.76 & 34.9 & 0.358 & $\mathrm{C}$ & $c a$ \\
\hline & & & 4647.40 & 238212 & 259724 & 3 & 5 & 0.78 & 0.42 & 19. & 0. & $\mathrm{C}$ & ls \\
\hline & & & 4650.16 & 238212 & 259711 & 3 & 3 & 0.78 & 0.253 & 11.6 & -0.121 & $\mathrm{C}$ & ls \\
\hline & & & 4651.35 & 238212 & 259706 & 3 & 1 & 0.78 & 0.084 & 3.88 & -0.60 & C & ls \\
\hline \multirow[t]{6}{*}{13} & \multirow[t]{6}{*}{$\begin{array}{l}2 p 3 s- \\
\quad 2 p\left({ }^{2} \mathrm{P}^{\circ}\right) 3 p\end{array}$} & \multirow{6}{*}{${ }^{3 \mathrm{P}^{\circ}-{ }^{3} \mathrm{D}}$} & 6740.8 & 308283 & 323114 & 9 & 15 & 0.267 & 0.303 & 61 & 0.436 & $\mathrm{C}$ & $c a$ \\
\hline & & & 6744.2 & 308317 & 323140 & 5 & 7 & 0.266 & 0.254 & 28.2 & 0.104 & C & ls \\
\hline & & & $\begin{array}{l}6730.7 \\
6727.1\end{array}$ & $\begin{array}{l}308248 \\
308215\end{array}$ & $\begin{array}{l}323101 \\
323076\end{array}$ & $\begin{array}{l}3 \\
1\end{array}$ & $\begin{array}{l}5 \\
3\end{array}$ & $\begin{array}{l}0.201 \\
0.149\end{array}$ & 0.2 & $\begin{array}{r}15.1 \\
6.7\end{array}$ & $\begin{array}{l}-0.167 \\
-0.52\end{array}$ & C & ls \\
\hline & & & [6762.2] & 308317 & 323101 & 5 & 5 & 0.066 & 0.0453 & 5.0 & -0.65 & C & $\begin{array}{l}\text { ls } \\
l s\end{array}$ \\
\hline & & & [6742.1] & 308248 & 323076 & 3 & 3 & 0.111 & 0.07 & 5.0 & -0.64 & $\mathrm{C}$ & ls \\
\hline & & & [6773.7] & 308317 & 323076 & 5 & 3 & 0.0073 & 0.00301 & 0.336 & -1.82 & $\mathrm{C}$ & is \\
\hline
\end{tabular}


CIII. Allowed Transitions-Continued

\begin{tabular}{|c|c|c|c|c|c|c|c|c|c|c|c|c|c|}
\hline No. & $\begin{array}{c}\text { Transition } \\
\text { Array }\end{array}$ & Multiplet & $\lambda(\AA)$ & $E_{i}\left(\mathrm{~cm}^{-1}\right)$ & $E_{k}\left(\mathrm{~cm}^{-1}\right)$ & $g_{i}$ & $g_{k}$ & $A_{k i}\left(10^{k} \sec ^{-1}\right)$ & $f_{i k}$ & $S($ at.u.) & $\log k f$ & $\begin{array}{c}\text { Accu- } \\
\text { racy }\end{array}$ & Sisurce \\
\hline \multirow[t]{4}{*}{14} & & ${ }^{3 \mathrm{P}^{\circ}}-^{-3} \mathrm{~S}$ & 5263.1 & 308283 & 327277 & 9 & 3 & 0.58 & 0.080 & 12.5 & -0.142 & C & \\
\hline & & & 5272.56 & 308317 & 327277 & 5 & 3 & 0.320 & 0.080 & 7.0 & -0.398 & C & \\
\hline & & & $\begin{array}{l}5253.55 \\
5244.5\end{array}$ & $\begin{array}{l}308248 \\
308215\end{array}$ & $\begin{array}{l}327277 \\
327277\end{array}$ & 3 & 3 & $\begin{array}{l}0.194 \\
0.065\end{array}$ & 0.080 & $\begin{array}{l}4.17 \\
1.20\end{array}$ & $\begin{array}{l}-0.62 \\
-1094\end{array}$ & $\mathrm{C}$ & \\
\hline & & & & & & & 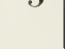 & & & & -1.094 & & \\
\hline \multirow[t]{6}{*}{15} & & ${ }^{3} \mathrm{P}^{\circ}-{ }^{3} \mathrm{P}$ & 4662.7 & 308283 & 329724 & 9 & 9 & 0.84 & 0.273 & 37.7 & 0.390 & C & \\
\hline & & & 4665.90 & 308317 & 329743 & 5 & 5 & 0.63 & 0.204 & 15.7 & 0.010 & C & \\
\hline & & & $\begin{array}{r}{[4659.0]} \\
4673.91\end{array}$ & $\begin{array}{l}308248 \\
308317\end{array}$ & $\begin{array}{l}329706 \\
320706\end{array}$ & $\begin{array}{l}3 \\
5\end{array}$ & $\begin{array}{l}3 \\
3\end{array}$ & 0.210 & 0.068 & 3.14 & $\begin{array}{l}-0.69 \\
-0.468\end{array}$ & C & \\
\hline & & & 4663.53 & 308248 & $\begin{array}{l}329706 \\
329685\end{array}$ & 3 & 1 & $\begin{array}{l}0.347 \\
0.84\end{array}$ & $\begin{array}{l}0.068 \\
0.091\end{array}$ & $\begin{array}{l}5.2 \\
4.19\end{array}$ & $\begin{array}{l}-0.468 \\
-0.56\end{array}$ & $\begin{array}{l}\mathrm{C} \\
\mathrm{C}\end{array}$ & \\
\hline & & & [4650.9] & 308248 & 329743 & 3 & 5 & 0.211 & 0.114 & 5.2 & -0.466 & C & \\
\hline & & & [4651.8] & 308215 & 329706 & 1 & 3 & 0.281 & 0.274 & 4.19 & -0.56 & C & \\
\hline 16 & & $\begin{array}{c}{ }^{1} \mathrm{P}^{0}-{ }^{-1} \mathrm{D} \\
(7)\end{array}$ & 4325.70 & 310005 & 333116 & 3 & 5 & 1.08 & 0.50 & 21.6 & 0.181 & C & \\
\hline 17 & $\begin{array}{l}2 s 3 p- \\
\quad 2 s\left({ }^{2} \mathrm{~S}\right) 3 d\end{array}$ & $\begin{array}{c}{ }^{1} \mathrm{P}^{\circ}-{ }^{1} \mathrm{D} \\
(2)\end{array}$ & 5696.0 & 258931 & 276483 & 3 & 5 & 0.50 & 0.407 & 22.9 & 0.087 & C & \\
\hline 18 & $\begin{array}{l}2 p 3 p- \\
\quad 2 p\left({ }^{2} \mathrm{P}^{\circ}\right) 3 d\end{array}$ & $\begin{array}{c}{ }^{1} \mathrm{P}-{ }^{1} \mathrm{P}^{\circ} \\
(12)\end{array}$ & 3703.52 & 319719 & 346713 & 3 & 3 & 0.320 & 0.066 & 2.41 & -0.70 & C & \\
\hline \multirow[t]{7}{*}{19} & & $\begin{array}{c}{ }^{3} \mathrm{D}-{ }^{3} \mathrm{D}^{\circ} \\
(19)\end{array}$ & 6865.8 & 323114 & 337675 & 15 & 15 & 0.066 & 0.0469 & 15.9 & -0.153 & $\mathrm{C}$ & $c a$ \\
\hline & & & 6871.7 & 323140 & 337688 & 7 & 7 & 0.059 & 0.0416 & 6.6 & -0.54 & C & $l$ \\
\hline & & & 6862.9 & 323101 & 337668 & 5 & 5 & & 0.0327 & 3. & -0.79 & C & \\
\hline & & & 6857.3 & 323076 & & 3 & 3 & & 0 & 2. & -0.98 & C & 10 \\
\hline & & & {$[6881.4]$} & 323140 & $\begin{array}{l}337 \\
327\end{array}$ & 7 & 5 & & 0.00 & 0.83 & $\begin{array}{l}-1.438 \\
-1450\end{array}$ & $\mathrm{C}$ & $1 \mathrm{~s}$ \\
\hline & & & {$[6853.1]$} & $\begin{array}{l}323101 \\
323101\end{array}$ & $\begin{array}{l}337655 \\
337688\end{array}$ & $\begin{array}{l}5 \\
5\end{array}$ & $\begin{array}{l}3 \\
7\end{array}$ & $\begin{array}{l}0.0100 \\
0.0074\end{array}$ & $\begin{array}{l}0.0070 \\
0.0073\end{array}$ & 0.83 & $\begin{array}{l}-1.450 \\
-1.436\end{array}$ & C & is \\
\hline & & & [6851.2] & 323076 & 337668 & 3 & 5 & 0.0100 & 0.0117 & 0.80 & -1.453 & $\mathrm{C}$ & \\
\hline \multirow[t]{6}{*}{20} & & ${ }^{3} \mathrm{D}-{ }^{3} \mathrm{P}^{\circ}$ & 5880.4 & 323114 & 340115 & 15 & 9 & 0.0124 & 0.00385 & 1.12 & -1.238 & $\mathrm{C}$ & $c a$ \\
\hline & & & 5894.1 & 323140 & 340101 & 7 & 5 & 0.0104 & 0.00385 & 0.52 & -1.57 & $\mathrm{C}$ & \\
\hline & & & 5871.6 & 323101 & 28 & 5 & 3 & & 0.0 & 0. & -1.84 & $\mathrm{C}$ & l. \\
\hline & & & $\begin{array}{c}5857.9 \\
{[58804]}\end{array}$ & 323076 & 340142 & 3 & $\begin{array}{l}1 \\
5\end{array}$ & 0.0126 & $\begin{array}{l}0.00216 \\
06 \times 10^{-4}\end{array}$ & 0.1 & $\begin{array}{l}-2.188 \\
-2.317\end{array}$ & C & ls \\
\hline & & & [5860.4] & $\begin{array}{l}323101 \\
323076\end{array}$ & $\begin{array}{l}340101 \\
340128\end{array}$ & $\begin{array}{l}5 \\
3\end{array}$ & $\begin{array}{l}3 \\
3\end{array}$ & $\begin{array}{l}0.00186 \\
0.00313\end{array}$ & $\begin{array}{l}9.6 \times 10^{-4} \\
0.00161\end{array}$ & $\begin{array}{l}0.093 \\
0.093\end{array}$ & $\begin{array}{l}-2.317 \\
-2.315\end{array}$ & $\begin{array}{l}\mathrm{C} \\
\mathrm{C}\end{array}$ & $\begin{array}{l}\text { ls } \\
\text { ls }\end{array}$ \\
\hline & & & [5871.8] & 323076 & 340101 & 3 & 5 & $1.25 \times 10^{-4}$ & $1.07 \times 10^{-4}$ & 0.0062 & -3.492 & $\mathrm{C}$ & is \\
\hline 21 & $\begin{array}{l}2 s 4 s- \\
\quad 2 s\left({ }^{2} S\right) 5 p\end{array}$ & $\stackrel{{ }^{1} \mathrm{~S}-{ }^{1} \mathrm{P}^{\circ}}{(8)}$ & 3170.16 & 311721 & 343256 & 1 & 3 & 0.325 & 0.147 & 1.53 & -0.83 & $\mathrm{C}$ & $a$ \\
\hline \multirow[t]{4}{*}{22} & ${ }_{\left.2 s s^{2} S\right) 5 s}^{2 s 4 p-}$ & 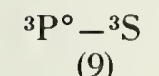 & 4516.5 & 317798 & 339933 & 9 & 3 & 1.66 & 0.169 & 22.6 & 0.182 & $\mathrm{C}$ & $c c$ \\
\hline & & & 4516.93 & 317800 & 339933 & 5 & 3 & 0.9 & 0.169 & 12.6 & -0.072 & C & \\
\hline & & & 4516.02 & 317795 & 339933 & 3 & 3 & & & 7. & -0.295 & C & ls \\
\hline & & & 4516.02 & 317795 & 339933 & 1 & 3 & 0.184 & 0.169 & 2.51 & -0.77 & $\mathrm{C}$ & ls \\
\hline \multirow[t]{6}{*}{23} & $\begin{array}{l}2 s 4 p- \\
2 s\left(^{2} \mathrm{~S}\right) 5 d\end{array}$ & $\begin{array}{c}{ }^{3} \mathrm{P}^{0}-{ }^{3} \mathrm{D} \\
(10)\end{array}$ & 3609.3 & 317798 & 345496 & 9 & 15 & 0.95 & 0.308 & 32.9 & 0.442 & C & $\mathrm{ca}$ \\
\hline & & & 3609.61 & 317800 & 345496 & 5 & 7 & 0.9 & 0.259 & 15.4 & 0.113 & C & $l s$ \\
\hline & & & & 317795 & & 3 & 5 & 0. & 0.2 & 8. & -0.160 & C & ls \\
\hline & & & 3608.96 & 317795 & 345496 & 1 & 3 & 0.5 & 0.308 & 3.6 & -0.51 & C & ls \\
\hline & & & $\begin{array}{l}3609.61 \\
3608.96\end{array}$ & $\begin{array}{l}317800 \\
317795\end{array}$ & $\begin{array}{l}345496 \\
345496\end{array}$ & $\begin{array}{l}5 \\
3\end{array}$ & $\begin{array}{l}5 \\
3\end{array}$ & $\begin{array}{l}0.236 \\
0.394\end{array}$ & $\begin{array}{l}0.0461 \\
0.077\end{array}$ & $\begin{array}{l}2.74 \\
2.74\end{array}$ & $\begin{array}{l}-0.64 \\
-0.64\end{array}$ & $\begin{array}{l}\mathrm{C} \\
\mathrm{C}\end{array}$ & $\begin{array}{l}l s \\
l s\end{array}$ \\
\hline & & & 3608.96 & 317795 & 345496 & 3 & 5 & 0.0158 & 0.0051 & 0.183 & -1.81 & C & ls \\
\hline 24 & & $\mid \begin{array}{c}{ }^{1} \mathrm{P}^{\circ}-{ }^{1} \mathrm{D} \\
(17)\end{array}$ & 4122.05 & 3224.03 & 346656 & 3 & 5 & 1.04 & 0.443 & 18.0 & 0.124 & C & $c a$ \\
\hline
\end{tabular}


C III. Allowed Transitions - Continued

\begin{tabular}{|c|c|c|c|c|c|c|c|c|c|c|c|c|c|}
\hline No. & $\begin{array}{c}\text { Transition } \\
\text { Array }\end{array}$ & Multiplet & $\lambda(\AA)$ & $E_{i}\left(\mathrm{~cm}^{-1}\right)$ & $E_{k}\left(\mathrm{~cm}^{-1}\right)$ & $g_{i}$ & $g_{k}$ & $A_{k i}\left(10^{8} \sec ^{-1}\right)$ & $f_{i k}$ & $S$ (at.u.) & $\log g f$ & $\begin{array}{l}\text { Accu- } \\
\text { racy }\end{array}$ & Source \\
\hline \multirow[t]{6}{*}{25} & \multirow{7}{*}{$\begin{array}{l}2 s 4 d- \\
\quad 2 s\left({ }^{2} \mathrm{~S}\right) 5 p\end{array}$} & \multirow{6}{*}{${ }^{3 \mathrm{D}}-{ }^{3} \mathrm{P}^{\circ}$} & 4385.1 & 321435 & 344233 & 15 & 9 & 0.267 & 0.0463 & 10.0 & -0.160 & C & $c a$ \\
\hline & & & 4388.24 & 321450 & 344233 & 7 & 5 & 0.224 & 0.0462 & 4.67 & -0.491 & C & ls \\
\hline & & & 4383.24 & 321427 & 344233 & 5 & 3 & 0.200 & 0.0346 & 2.50 & -0.76 & C & ls \\
\hline & & & 4379.97 & 321411 & 344233 & 3 & 1 & 0.268 & 0.0257 & 1.11 & -1.114 & C & ls \\
\hline & & & 4383.24 & 321427 & 344233 & 5 & 5 & 0.0401 & 0.0115 & $\begin{array}{l}0.83 \\
0.83\end{array}$ & $\begin{array}{l}-1.239 \\
-1.238\end{array}$ & C & ls \\
\hline & & & $\begin{array}{l}4379.97 \\
4379.97\end{array}$ & $\begin{array}{l}321411 \\
321411\end{array}$ & $\begin{array}{l}344233 \\
344233\end{array}$ & $\begin{array}{l}3 \\
3\end{array}$ & $\begin{array}{l}3 \\
5\end{array}$ & $\begin{array}{l}0.067 \\
0.00268\end{array}$ & $\begin{array}{l}0.0193 \\
0.00129\end{array}$ & $\begin{array}{l}0.83 \\
0.056\end{array}$ & $\begin{array}{l}-1.238 \\
-2.414\end{array}$ & $\begin{array}{l}\mathrm{C} \\
\mathrm{C}\end{array}$ & $\begin{array}{l}l s \\
l s\end{array}$ \\
\hline 26 & & $\begin{array}{c}{ }^{1} \mathrm{D}-{ }^{1} \mathbf{P}^{\circ} \\
(23)\end{array}$ & 5249.6 & 324212 & 343256 & 5 & 3 & 0.52 & 0.128 & 11.1 & -0.194 & C & $c a$ \\
\hline \multirow[t]{5}{*}{27} & \multirow[t]{6}{*}{$\begin{array}{l}2 s 4 d- \\
2 s\left({ }^{2} \mathrm{~S}\right) 5 f\end{array}$} & \multirow[t]{5}{*}{$\begin{array}{c}{ }^{3} \mathrm{D}-{ }^{3} \mathrm{~F}^{\circ} \\
(15)\end{array}$} & 3887.1 & 321435 & 347154 & 15 & 21 & 1.81 & 0.58 & 110 & 0.93 & C & $c a$ \\
\hline & & & $\begin{array}{l}3889.18 \\
200500\end{array}$ & 321450 & 347155 & 7 & 9 & 1.81 & 0.53 & 47.2 & 0.57 & C & ls \\
\hline & & & $\begin{array}{l}3885.99 \\
3883.80\end{array}$ & $\begin{array}{l}321427 \\
321411\end{array}$ & $\begin{array}{l}347153 \\
347151\end{array}$ & $\begin{array}{l}5 \\
3\end{array}$ & $\begin{array}{l}7 \\
5\end{array}$ & $\begin{array}{l}1.61 \\
1.52\end{array}$ & $\begin{array}{l}0.51 \\
0.57\end{array}$ & $\begin{array}{l}32.6 \\
22.0\end{array}$ & $\begin{array}{l}0.406 \\
0.236\end{array}$ & $\begin{array}{l}\mathrm{C} \\
\mathrm{C}\end{array}$ & $\begin{array}{l}l s \\
l s\end{array}$ \\
\hline & & & [3889.7] & 321450 & 347153 & 7 & 7 & 0.201 & 0.0456 & 4.09 & -0.496 & $\mathrm{C}$ & ls \\
\hline & & & [3886.2] & $\begin{array}{l}321427 \\
321450\end{array}$ & $\begin{array}{l}347151 \\
347151\end{array}$ & $\begin{array}{l}5 \\
7\end{array}$ & $\begin{array}{l}5 \\
5\end{array}$ & $\begin{array}{l}0.282 \\
0.0079\end{array}$ & $\begin{array}{l}0.064 \\
0.00128\end{array}$ & $\begin{array}{l}4.09 \\
0.115\end{array}$ & $\begin{array}{l}-0.495 \\
-2.047\end{array}$ & $\begin{array}{l}\mathrm{C} \\
\mathrm{C}\end{array}$ & $\begin{array}{l}l s \\
l s\end{array}$ \\
\hline 28 & & ${ }^{1} \mathrm{D}-{ }^{1} \mathrm{~F}^{\circ}$ & 4056.06 & 324212 & 348860 & 5 & 7 & 1.45 & 0.50 & 33.4 & 0.398 & C & $c a$ \\
\hline
\end{tabular}

\section{Forbidden Transitions}

Naqvi's calculations [1] are the only available source. The results for the ${ }^{3} \mathrm{P}^{\circ}-3 \mathrm{P}^{\circ}$ transitions are essentially independent of the choice of the interaction parameters. For the ${ }^{3} \mathrm{P}^{\circ}-{ }^{1} \mathrm{P}^{\circ}$ transitions, Naqvi uses empirical term intervals, i.e., the effects of configuration interaction should be partially included.

Reference

[1] Naqvi, A. M., Thesis Harvard (1951).

C III. Forbidden Transitions

\begin{tabular}{|c|c|c|c|c|c|c|c|c|c|c|c|c|}
\hline No. & $\begin{array}{c}\text { Transition } \\
\text { Array }\end{array}$ & Multiplet & $\lambda(\AA)$ & $E_{l}\left(\mathrm{~cm}^{-1}\right)$ & $E_{k}\left(\mathrm{~cm}^{-1}\right)$ & $g_{i}$ & $g_{k}$ & $\begin{array}{l}\text { Type of } \\
\text { Transi- } \\
\text { tion }\end{array}$ & $A_{k i}\left(\sec ^{-1}\right)$ & $S$ (at.u.) & $\begin{array}{c}\text { Accu- } \\
\text { racy }\end{array}$ & Source \\
\hline 1 & $2 s 2 p-2 s\left({ }^{2} \mathrm{~S}\right) 2 p$ & ${ }^{3} \mathrm{P}^{\circ}-{ }^{3} \mathrm{P}^{\circ}$ & $\begin{array}{l}{\left[43.5 \times 10^{5}\right]} \\
{\left[17.6 \times 10^{5}\right]}\end{array}$ & $\begin{array}{l}52366.7 \\
52389.7\end{array}$ & $\begin{array}{l}52389.7 \\
52446.5\end{array}$ & $\begin{array}{l}1 \\
3\end{array}$ & $\begin{array}{l}3 \\
5\end{array}$ & $\begin{array}{l}\mathrm{m} \\
\mathrm{m}\end{array}$ & $\begin{array}{l}2.18 \times 10^{-7} \\
2.47 \times 10^{-6}\end{array}$ & $\begin{array}{l}2.00 \\
2.50\end{array}$ & $\begin{array}{l}\text { B } \\
\text { B }\end{array}$ & 1 \\
\hline 2 & & ${ }^{3} \mathrm{P}^{\circ}-{ }^{1} \mathrm{P}^{0}$ & $\begin{array}{l}{[2000.0]} \\
{[2000.9]} \\
{[2003.2]}\end{array}$ & $\begin{array}{l}52366.7 \\
52389.7 \\
52446.5\end{array}$ & $\begin{array}{l}102351 \\
102351 \\
102351\end{array}$ & $\begin{array}{l}1 \\
3 \\
5\end{array}$ & $\begin{array}{l}3 \\
3 \\
3\end{array}$ & $\begin{array}{l}\mathrm{m} \\
\mathrm{m} \\
\mathrm{m}\end{array}$ & $\begin{array}{l}0.00142 \\
1.35 \\
0.00179\end{array}$ & $\begin{array}{l}1.26 \times 10^{-6} \\
0.00120 \\
1.60 \times 10^{-6}\end{array}$ & $\begin{array}{l}\mathrm{C} \\
\mathrm{C} \\
\mathrm{C}\end{array}$ & $\begin{array}{l}1 \\
1 \\
1\end{array}$ \\
\hline
\end{tabular}




\section{CIV}

Ground State

Ionization Potential
$1 s^{2} 2 s^{2} \mathrm{~S}_{1 / 2}$

$64.476 \mathrm{eV}=520177.8 \mathrm{~cm}^{-1}$

\section{Allowed Transitions}

List of tabulated lines:

\begin{tabular}{l|l||c|c||c|c}
\hline \hline Wavelength $[\AA]$ & No. & Wavelength $[\AA]$ & No. & Wavelength $[\AA]$ & No. \\
\hline & & 384.19 & 6 & 4647 & \\
222.791 & 4 & 49.525 & 5 & 4665 & 20 \\
244.907 & 3 & 419.714 & 5 & 4737 & 22 \\
259.471 & 9 & 1548.20 & 1 & 4789 & 18 \\
259.542 & 8 & 1550.77 & 1 & 5021 & 16 \\
289.143 & 8 & 2524.40 & 14 & 5023 & 16 \\
289.230 & 7 & 2595.14 & 13 & 5801.51 & 10 \\
296.857 & 7 & 2697.73 & 12 & 5812.14 & 10 \\
296.951 & 2698.70 & 12 & 6592 & 21 \\
312.418 & 2 & 3936 & 15 & 20694 & 11 \\
312.455 & 6 & 4440.4 & 17 & 20780 & 11 \\
384.032 & 6 & 4441.8 & 17 & 20828 & 11 \\
384.178 & & & & \\
\hline
\end{tabular}

The values taken from Weiss' calculations [1] are estimated to be accurate to within 10 percent because of the very close agreement between his dipole length and dipole velocity approximations. The values calculated with the length approximation are adopted.

\section{Reference}

[1] Weiss, A. W., Astrophys. J. 138, 1262-1276 (1963).

CIV. Allowed Transitions

\begin{tabular}{|c|c|c|c|c|c|c|c|c|c|c|c|c|c|}
\hline No. & $\begin{array}{c}\text { Transition } \\
\text { Array }\end{array}$ & Multiplet & $\lambda(\AA)$ & $E_{i}\left(\mathrm{~cm}^{-1}\right)$ & $E_{k}\left(\mathrm{~cm}^{-1}\right)$ & $g_{i}$ & $g_{k}$ & $A_{k i}\left(10^{8} \sec ^{-1}\right)$ & $f_{i k}$ & $S$ (at.u.) & $\log g f$ & $\begin{array}{c}\text { Accu- } \\
\text { racy }\end{array}$ & Source \\
\hline \multirow[t]{2}{*}{1} & $2 s-2 p$ & $\begin{array}{l}{ }^{2} \mathrm{~S}-{ }^{2} \mathrm{P}^{\circ} \\
(1 u v)\end{array}$ & 1549.1 & 0.0 & 64555 & 2 & 6 & 2.65 & 0.286 & 2.91 & -0.243 & A & 1 \\
\hline & & & $\begin{array}{l}1548.20 \\
1550.77\end{array}$ & $\begin{array}{l}0.0 \\
0.0\end{array}$ & $\begin{array}{l}64591 \\
64484\end{array}$ & $\begin{array}{l}2 \\
2\end{array}$ & $\begin{array}{l}4 \\
2\end{array}$ & $\begin{array}{l}2.65 \\
2.63\end{array}$ & $\begin{array}{l}0.190 \\
0.0950\end{array}$ & $\begin{array}{l}1.94 \\
0.970\end{array}$ & $\begin{array}{l}-0.420 \\
-0.721\end{array}$ & $\begin{array}{l}\mathrm{A} \\
\mathrm{A}\end{array}$ & $\begin{array}{l}\text { ls } \\
\text { ls }\end{array}$ \\
\hline \multirow[t]{2}{*}{2} & $2 s-3 p$ & $\begin{array}{l}{ }^{2} \mathrm{~S}-{ }^{2} \mathrm{P}^{\circ} \\
(2 \mathrm{uv})\end{array}$ & 312.43 & 0.0 & 320070 & 2 & 6 & 45.6 & 0.200 & 0.412 & -0.397 & $\mathrm{~B}+$ & 1 \\
\hline & & & $\begin{array}{l}312.418 \\
312.455\end{array}$ & $\begin{array}{l}0.0 \\
0.0\end{array}$ & $\begin{array}{l}320080 \\
320049\end{array}$ & $\begin{array}{l}2 \\
2\end{array}$ & $\begin{array}{l}4 \\
2\end{array}$ & $\begin{array}{l}45.7 \\
45.5\end{array}$ & $\begin{array}{l}0.134 \\
0.0666\end{array}$ & $\begin{array}{l}0.275 \\
0.137\end{array}$ & $\begin{array}{l}-0.573 \\
-0.876\end{array}$ & $\begin{array}{l}\mathrm{B}+ \\
\mathrm{B}+\end{array}$ & $\begin{array}{l}\text { ls } \\
\text { ls }\end{array}$ \\
\hline 3 & $2 s-4 p$ & $\begin{array}{c}{ }^{2} \mathrm{~S}-{ }^{2} \mathrm{P}^{\circ} \\
(3 \mathrm{uv})\end{array}$ & 244.907 & 0.0 & 408318 & 2 & 6 & 22.1 & 0.0597 & 0.0963 & -0.923 & B & $c a$ \\
\hline 4 & $2 s-5 p$ & $\begin{array}{c}{ }^{2} \mathrm{~S}-{ }^{2} \mathrm{P}^{\circ} \\
(4 \mathrm{uv})\end{array}$ & 222.791 & 0.0 & 448859 & 2 & 6 & 11.8 & 0.0263 & 0.0385 & -1.280 & B & $c a$ \\
\hline \multirow[t]{2}{*}{5} & $2 p-3 s$ & $\begin{array}{c}{ }^{2} \mathrm{P}^{\circ}-{ }^{2} \mathrm{~S} \\
(6 \mathrm{uv})\end{array}$ & 419.65 & 64555 & 302848 & 6 & 2 & 42.7 & 0.0376 & 0.312 & -0.647 & $B+$ & 1 \\
\hline & & & $\begin{array}{l}419.714 \\
419.525\end{array}$ & $\begin{array}{l}64591 \\
64484\end{array}$ & $\begin{array}{l}302848 \\
302848\end{array}$ & $\begin{array}{l}4 \\
2\end{array}$ & $\begin{array}{l}2 \\
2\end{array}$ & $\begin{array}{l}28.5 \\
14.3\end{array}$ & $\begin{array}{l}0.0376 \\
0.0376\end{array}$ & $\begin{array}{l}0.208 \\
0.104\end{array}$ & $\begin{array}{l}-0.822 \\
-1.123\end{array}$ & $\begin{array}{l}B+ \\
B+\end{array}$ & $\begin{array}{l}l s \\
l s\end{array}$ \\
\hline \multirow[t]{2}{*}{6} & $2 p-3 d$ & $\begin{array}{c}{ }^{2} \mathrm{P}^{\circ}-{ }^{2} \mathrm{D} \\
(7 \mathrm{uv})\end{array}$ & 384.12 & 64555 & 324887 & 6 & 10 & 177 & 0.654 & 4.96 & 0.594 & $B+$ & 1 \\
\hline & & & $\begin{array}{r}384.178 \\
384.032 \\
{[384.19]}\end{array}$ & $\begin{array}{l}64591 \\
64484 \\
64591\end{array}$ & $\begin{array}{l}324891 \\
324880 \\
324880\end{array}$ & $\begin{array}{l}4 \\
2 \\
4\end{array}$ & $\begin{array}{l}6 \\
4 \\
4\end{array}$ & \begin{tabular}{|c}
177 \\
148 \\
29.6
\end{tabular} & $\begin{array}{l}0.589 \\
0.653 \\
0.0654\end{array}$ & $\begin{array}{l}2.98 \\
1.65 \\
0.331\end{array}$ & $\begin{array}{r}0.372 \\
0.116 \\
-0.582\end{array}$ & $\begin{array}{l}B+ \\
B+ \\
B+\end{array}$ & $\begin{array}{l}\text { ls } \\
\text { ls } \\
\text { ls }\end{array}$ \\
\hline
\end{tabular}


CIV. Allowed Transitions-Continued

\begin{tabular}{|c|c|c|c|c|c|c|c|c|c|c|c|c|c|}
\hline No. & $\begin{array}{c}\text { Transition } \\
\text { Array }\end{array}$ & Multiplet & $\lambda(\AA)$ & $E_{i}\left(\mathrm{~cm}^{-1}\right)$ & $E_{k}\left(\mathrm{~cm}^{-1}\right)$ & $g_{i}$ & $g_{k}$ & $A_{k i}\left(10^{8} \sec ^{-1}\right)$ & $f_{i k}$ & $S($ at.u.) & $\log g f$ & $\begin{array}{c}\text { Accu- } \\
\text { racy }\end{array}$ & Source \\
\hline \multirow[t]{3}{*}{7} & $2 p-4 s$ & ${ }^{2} \mathrm{P}^{\circ}-{ }^{2} \mathrm{~S}$ & 296.92 & 64555 & 401347 & 6 & 2 & 17.5 & 0.00772 & 0.0453 & -1.334 & B & $c a$ \\
\hline & & & 296.951 & 64591 & 401347 & 4 & 2 & 11.7 & 0.00772 & 0.0302 & -1.510 & B & ls \\
\hline & & & 296.857 & 64484 & 401347 & 2 & 2 & 5.85 & 0.00773 & 0.0151 & -1.811 & B & $l s$ \\
\hline \multirow[t]{4}{*}{8} & $2 p-4 d$ & $\begin{array}{r}{ }^{2} \mathrm{P}^{\circ}-{ }^{2} \mathrm{D} \\
(9 \text { uv) }\end{array}$ & 289.20 & 64555 & 410336 & 6 & 10 & 58.1 & 0.121 & 0.694 & -0.137 & B & $c a$ \\
\hline & & & 289.230 & 64591 & 410338 & 4 & 6 & 58.2 & 0.109 & 0.417 & -0.359 & B & ls \\
\hline & & & 289.143 & 64484 & 410334 & 2 & 4 & 48.5 & 0.122 & 0.231 & -0.614 & B & ls \\
\hline & & & [289.23] & 64591 & 410334 & 4 & 4 & 9.69 & 0.0122 & 0.0463 & -1.313 & B & ls \\
\hline \multirow[t]{4}{*}{9} & $2 p-5 d$ & $\begin{array}{c}{ }^{2} \mathrm{P}^{\circ}-{ }^{2} \mathrm{D} \\
(10 \mathrm{uv})\end{array}$ & 259.52 & 64555 & 449887 & 6 & 10 & 22.8 & 0.0383 & 0.196 & -0.639 & B & $c a$ \\
\hline & & & 259.542 & 64591 & 449887 & 4 & 6 & 22.8 & 0.0345 & 0.118 & -0.860 & B & ls \\
\hline & & & 259.471 & 64484 & 449887 & 2 & 4 & 18.9 & 0.0382 & 0.0653 & -1.117 & B & ls \\
\hline & & & 259.542 & 64591 & 449887 & 4 & 4 & 3.80 & 0.00383 & 0.0131 & -1.814 & B & ls \\
\hline \multirow[t]{3}{*}{10} & $3 s-3 p$ & ${ }^{2} \mathrm{~S}-{ }^{2} \mathrm{P}^{\circ}$ & 5804.9 & 302848 & 320070 & 2 & 6 & 0.318 & 0.481 & 18.4 & -0.017 & B & 1 \\
\hline & & & 5801.51 & 302848 & 320080 & 2 & 4 & 0.319 & 0.322 & 12.3 & -0.191 & B & ls \\
\hline & & & 5812.14 & 302848 & 320049 & 2 & 2 & 0.316 & 0.160 & 6.13 & -0.494 & B & ls \\
\hline \multirow[t]{4}{*}{11} & $3 p-3 d$ & ${ }^{2} \mathrm{P}^{\circ}-{ }^{2} \mathrm{D}$ & 20754 & 320070 & 324887 & 6 & 10 & 0.00579 & 0.0623 & 25.5 & -0.427 & B & 1 \\
\hline & & & [20780] & 320080 & 324891 & 4 & 6 & 0.00576 & 0.0559 & 15.3 & -0.650 & B & $l s$ \\
\hline & & & [20694] & 320049 & 324880 & 2 & 4 & 0.00486 & 0.0624 & 8.50 & -0.904 & B & ls \\
\hline & & & [20828] & 320080 & 324880 & 4 & 4 & $9.53 \times 10^{-4}$ & 0.00620 & 1.70 & -1.606 & B & ls \\
\hline \multirow[t]{3}{*}{12} & $4 p-5 s$ & $\begin{array}{r}{ }^{2} \mathrm{P}^{\circ}-{ }^{2} \mathrm{~S} \\
(12 \mathrm{uv})\end{array}$ & 2698.4 & 408318 & 445366 & 6 & 2 & 4.09 & 0.149 & 7.94 & -0.049 & B & $c a$ \\
\hline & & & 2698.70 & 408322 & 445366 & 4 & 2 & 2.73 & 0.149 & 5.29 & -0.225 & B & ls \\
\hline & & & 2697.73 & 408309 & 445366 & 2 & 2 & 1.37 & 0.149 & 2.65 & -0.525 & B & ls \\
\hline 13 & $4 d-5 p$ & $\begin{array}{r}{ }^{2} \mathrm{D}-{ }^{2} \mathrm{P}^{\circ} \\
(13 \mathrm{uv})\end{array}$ & 2595.14 & 410336 & 448859 & 10 & 6 & 0.746 & 0.0452 & 3.86 & -0.345 & B & $c a$ \\
\hline 14 & $4 d-5 f$ & $\begin{array}{r}{ }^{2} \mathrm{D}-{ }^{2} \mathrm{~F}^{\circ} \\
(14 \mathrm{uv})\end{array}$ & 2524.40 & 410336 & 449938 & 10 & 14 & 7.44 & 0.995 & 82.7 & 0.999 & B & $c a$ \\
\hline 15 & $5 s-6 p$ & $\begin{array}{c}{ }^{2} \mathrm{~S}-{ }^{2} \mathrm{P}^{\circ} \\
(2)\end{array}$ & 3936 & 445366 & 470763 & 2 & 6 & 0.340 & 0.237 & 6.14 & -0.325 & B & $c a$ \\
\hline \multirow[t]{3}{*}{16} & $5 p-6 s$ & $\begin{array}{c}{ }^{2} \mathrm{P}^{\circ}-{ }^{2} \mathrm{~S} \\
(3)\end{array}$ & 5022.2 & 448859 & 468765 & 6 & 2 & 1.40 & 0.176 & 17.5 & 0.024 & B & $c a$ \\
\hline & & & 5023 & 448861 & 468765 & 4 & 2 & 0.935 & 0.177 & 11.7 & -0.150 & B & ls \\
\hline & & & 5021 & 448854 & 468765 & 2 & 2 & 0.467 & 0.176 & 5.83 & -0.453 & B & ls \\
\hline \multirow[t]{3}{*}{17} & $5 p-6 d$ & ${ }^{2} \mathrm{P}^{\circ}-{ }^{2} \mathrm{D}$ & 4441.8 & 448859 & 471368 & 6 & 10 & 1.05 & 0.516 & 45.3 & 0.491 & B & $c a$ \\
\hline & & & [4441.8] & 448861 & 471368 & 4 & 6 & 1.05 & 0.465 & 27.2 & 0.270 & B & $l s$ \\
\hline & & & $\begin{array}{l}{[4440.4]} \\
{[4441.8]}\end{array}$ & $\begin{array}{l}448854 \\
448861\end{array}$ & $\begin{array}{l}471368 \\
471368\end{array}$ & $\begin{array}{l}2 \\
4\end{array}$ & $\begin{array}{l}4 \\
4\end{array}$ & $\begin{array}{l}0.874 \\
0.175\end{array}$ & $\begin{array}{l}0.516 \\
0.0516\end{array}$ & $\begin{array}{c}15.1 \\
3.02\end{array}$ & $\begin{array}{r}0.014 \\
-0.685\end{array}$ & $\begin{array}{l}\mathrm{B} \\
\mathrm{B}\end{array}$ & $\begin{array}{l}l s \\
l s\end{array}$ \\
\hline 18 & $5 d-6 p$ & ${ }^{2} \mathrm{D}-{ }^{2} \mathrm{P}^{\circ}$ & 4789 & 449887 & 470763 & 10 & 6 & 0.341 & 0.0704 & 11.1 & -0.152 & B & $c a$ \\
\hline 19 & $5 d-6 f$ & $\begin{array}{c}{ }^{2} \mathrm{D}-{ }^{2} \mathrm{~F}^{\circ} \\
(6)\end{array}$ & 4647 & 449887 & 471403 & 10 & 14 & 1.85 & 0.837 & 128 & 0.923 & B & $c a$ \\
\hline 20 & $5 f-6 d$ & ${ }^{2} \mathrm{~F}^{\circ}-{ }^{2} \mathrm{D}$ & 4665 & 449938 & 471368 & 14 & 10 & 0.103 & 0.0241 & 5.18 & -0.472 & B & $c a$ \\
\hline 21 & $6 s-7 p$ & ${ }^{2 \mathrm{~S}-{ }^{2} \mathrm{P}^{\circ}} \frac{}{(10)}$ & 6592 & 468765 & 483931 & 2 & 6 & 0.132 & 0.258 & 11.2 & -0.288 & B & $c a$ \\
\hline 22 & $6 d-8 p$ & $\begin{array}{c}{ }^{2} \mathrm{D}-{ }^{2} \mathrm{P}^{\circ} \\
(12)\end{array}$ & 4737 & 471368 & 492473 & 10 & 6 & 0.104 & 0.0210 & 3.27 & -0.678 & B & $c a$ \\
\hline
\end{tabular}




\section{C v}

Ground State

$$
1 s^{2}{ }^{1} \mathrm{~S}_{0}
$$

Ionization Potential

$391.986 \mathrm{eV}=3162450 \mathrm{~cm}^{-1}$

\section{Allowed Transitions}

The results of extensive non-relativistic variational calculations by Weiss [1] are chosen. Values have been calculated in both the dipole length and dipole velocity approximations and agree to within 1 percent, except for the $3 p^{1} \mathrm{P}^{\circ}-3 d^{1} \mathrm{D}$ transition where agreement is not as good. The average of the two approximations is adopted [1].

\section{Reference}

[1] Weiss, A. W., private communication (1964).

C v. Allowed Transitions

\begin{tabular}{|c|c|c|c|c|c|c|c|c|c|c|c|c|c|}
\hline No. & $\begin{array}{c}\text { Transition } \\
\text { Array }\end{array}$ & Multiplet & $\lambda(\AA)$ & $E_{i}\left(\mathrm{~cm}^{-1}\right)$ & $E_{k}\left(\mathrm{~cm}^{-1}\right)$ & $g_{i}$ & $g_{k}$ & $A_{k i}\left(10^{8} \sec ^{-1}\right)$ & $f_{i k}$ & $S($ at.u.) & $\log g f$ & $\begin{array}{c}\text { Accu- } \\
\text { racy }\end{array}$ & Source \\
\hline 1 & $1 s^{2}-1 s 2 p$ & ${ }^{1} \mathrm{~S}-{ }^{1} \mathrm{P}^{\circ}$ & {$[40.270]$} & 0 & 2483240 & 1 & 3 & 8870 & 0.647 & 0.0858 & -0.189 & A & 1 \\
\hline 2 & $1 s^{2}-1 s 3 p$ & ${ }^{1} \mathrm{~S}-{ }^{1} \mathrm{P}^{\circ}$ & [34.973] & 0 & 2859350 & 1 & 3 & 2550 & 0.141 & 0.0162 & -0.852 & A & 1 \\
\hline 3 & $1 s 2 s-1 s 2 p$ & ${ }^{1} \mathrm{~S}-{ }^{1} \mathrm{P}^{\circ}$ & [3540.8] & {$[2455006]$} & 2483240 & 1 & 3 & 0.165 & 0.0931 & 1.09 & -1.031 & A & 1 \\
\hline 4 & $1 s 2 s-1 s 3 p$ & ${ }^{1} \mathrm{~S}-{ }^{1} \mathrm{P}^{\circ}$ & [247.31] & {$[2455006]$} & 2859350 & 1 & 3 & 128 & 0.351 & 0.286 & -0.455 & A & 1 \\
\hline 5 & $1 s 2 p-1 s 3 d$ & ${ }^{1} \mathrm{P}^{\circ}-{ }^{1} \mathrm{D}$ & {$[267.21]$} & 2483240 & [2857480] & 3 & 5 & 396 & 0.707 & 1.87 & 0.327 & A & 1 \\
\hline 6 & $1 s 3 d-1 s 3 p$ & ${ }^{1} \mathrm{D}-{ }^{1} \mathrm{P}^{\circ}$ & [53460]? & {$[2857480]$} & 2859350 & 5 & 3 & $3.97 \times 10^{-4}$ & 0.0102 & 8.98 & -1.292 & $\mathrm{C}+$ & 1 \\
\hline 7 & $1 s 2 s-1 s 2 p$ & ${ }^{3} \mathrm{~S}-{ }^{3} \mathrm{P}^{\circ}$ & 2273.9 & 2411244 & 2455207 & 3 & 9 & 0.565 & 0.132 & 2.95 & -0.404 & A & 1 \\
\hline 8 & $1 s 2 s-1 s 3 p$ & ${ }^{3} \mathrm{~S}-{ }^{3} \mathrm{P}^{\circ}$ & {$[227.37]$} & 2411244 & [2851060] & 3 & 9 & 136 & 0.316 & 0.710 & -0.023 & A & 1 \\
\hline 9 & $1 s 2 p-1 s 3 d$ & ${ }^{3} \mathrm{P}^{\circ}-{ }^{3} \mathrm{D}$ & 248.71 & 2455207 & 2857286 & 9 & 15 & 425 & 0.657 & 4.84 & 0.772 & A & 1 \\
\hline 10 & $1 s 3 p-1 s 3 d$ & ${ }^{3} \mathrm{P}^{\circ}-{ }^{3} \mathrm{D}$ & [16057]? & [2851060] & 2857286 & 9 & 15 & 0.00753 & 0.0485 & 23.1 & -0.360 & A & 1 \\
\hline
\end{tabular}


Allowed Transitions

List of tabulated lines:

\begin{tabular}{|c|c|c|c|c|c|}
\hline Wavelength $[\AA ̊]$ & No. & Wavelength $[\AA]$ & No. & Wavelength $[\AA]]$ & No. \\
\hline $\begin{array}{l}1100.7 \\
1134.17 \\
1134.42 \\
1134.98 \\
1163.87\end{array}$ & $\begin{array}{r}19 \\
1 \\
1 \\
1 \\
10\end{array}$ & $\begin{array}{l}4222.12 \\
4223.04 \\
4224.74 \\
4230.35 \\
4253.28\end{array}$ & $\begin{array}{l}29 \\
29 \\
29 \\
29 \\
28\end{array}$ & $\begin{array}{l}6622.54 \\
6626.8 \\
6636.94 \\
6644.96 \\
6646.51\end{array}$ & $\begin{array}{l}47 \\
47 \\
47 \\
47 \\
47\end{array}$ \\
\hline $\begin{array}{l}1163.88 \\
1164.31 \\
1167.45 \\
1168.42 \\
1168.54\end{array}$ & $\begin{array}{r}10 \\
10 \\
9 \\
9 \\
9\end{array}$ & $\begin{array}{l}4254.7 \\
4258.7 \\
4261.2 \\
4263.2 \\
4264.7\end{array}$ & $\begin{array}{l}28 \\
28 \\
28 \\
28 \\
28\end{array}$ & $\begin{array}{l}6653.46 \\
6656.51 \\
6926.90 \\
6945.22 \\
6951.7\end{array}$ & $\begin{array}{l}47 \\
47 \\
48 \\
48 \\
48\end{array}$ \\
\hline $\begin{array}{l}1169.69 \\
1176.4 \\
1176.6 \\
1177.7 \\
1199.55\end{array}$ & $\begin{array}{r}8 \\
14 \\
14 \\
14 \\
2\end{array}$ & $\begin{array}{l}4269.8 \\
4384.4 \\
4391.3 \\
4914.90 \\
4935.03\end{array}$ & $\begin{array}{l}28 \\
43 \\
43 \\
31 \\
31\end{array}$ & $\begin{array}{l}6960.4 \\
6973.0 \\
6979.10 \\
6981.8 \\
7423.64\end{array}$ & $\begin{array}{l}48 \\
48 \\
48 \\
48 \\
23\end{array}$ \\
\hline $\begin{array}{l}1200.22 \\
1200.71 \\
1231.7 \\
1243.17 \\
1243.30\end{array}$ & $\begin{array}{r}2 \\
2 \\
20 \\
5 \\
5\end{array}$ & $\begin{array}{l}5170.0 \\
5181.5 \\
5186.9 \\
5197.8 \\
5201.8\end{array}$ & $\begin{array}{l}18 \\
18 \\
18 \\
49 \\
49\end{array}$ & $\begin{array}{l}7442.30 \\
7468.31 \\
8184.85 \\
8188.01 \\
8200.36\end{array}$ & $\begin{array}{l}23 \\
23 \\
22 \\
22 \\
22\end{array}$ \\
\hline $\begin{array}{l}1243.31 \\
1310.54 \\
1310.97 \\
1316.29 \\
1319.04\end{array}$ & $\begin{array}{r}5 \\
13 \\
13 \\
12 \\
11\end{array}$ & $\begin{array}{l}5281.18 \\
5292.9 \\
5293.5 \\
5305.0 \\
5309.2\end{array}$ & $\begin{array}{l}17 \\
17 \\
17 \\
17 \\
17\end{array}$ & $\begin{array}{l}8210.71 \\
8216.32 \\
8223.12 \\
8242.37 \\
8567.74\end{array}$ & $\begin{array}{l}22 \\
22 \\
22 \\
22 \\
25\end{array}$ \\
\hline $\begin{array}{l}1319.72 \\
1326.63 \\
1327.96 \\
1411.94 \\
1492.62\end{array}$ & $\begin{array}{r}11 \\
15 \\
15 \\
6 \\
3\end{array}$ & $\begin{array}{l}5310.6 \\
5314.8 \\
5328.70 \\
5344.4 \\
5354.7\end{array}$ & $\begin{array}{l}17 \\
17 \\
16 \\
16 \\
16\end{array}$ & $\begin{array}{l}8590.01 \\
8629.24 \\
8655.87 \\
8680.27 \\
8683.40\end{array}$ & $\begin{array}{l}25 \\
25 \\
25 \\
21 \\
21\end{array}$ \\
\hline $\begin{array}{l}1492.67 \\
1494.67 \\
1742.72 \\
1742.73 \\
1745.25\end{array}$ & $\begin{array}{l}3 \\
3 \\
4 \\
4 \\
4\end{array}$ & $\begin{array}{l}5356.77 \\
5367.1 \\
5372.5 \\
5372.66 \\
5378.3\end{array}$ & $\begin{array}{l}16 \\
16 \\
16 \\
16 \\
16\end{array}$ & $\begin{array}{l}8686.16 \\
8703.26 \\
8711.71 \\
8718.84 \\
8728.91\end{array}$ & $\begin{array}{l}21 \\
21 \\
21 \\
21 \\
21\end{array}$ \\
\hline $\begin{array}{l}1745.26 \\
4099.95 \\
4109.96 \\
4113.97 \\
4137.63\end{array}$ & $\begin{array}{r}4 \\
26 \\
26 \\
26 \\
30\end{array}$ & $\begin{array}{l}5401.45 \\
5411.88 \\
5816.48 \\
5829.53 \\
5834.7\end{array}$ & $\begin{array}{l}32 \\
32 \\
50 \\
50 \\
50\end{array}$ & $\begin{array}{l}8747.36 \\
9028.92 \\
9045.88 \\
9049.47 \\
9049.89\end{array}$ & $\begin{array}{l}21 \\
33 \\
27 \\
27 \\
27\end{array}$ \\
\hline $\begin{array}{l}4143.42 \\
4151.46 \\
4214.73 \\
4215.92 \\
4218.87\end{array}$ & $\begin{array}{l}30 \\
30 \\
29 \\
29 \\
29\end{array}$ & $\begin{array}{l}5841.01 \\
5850.1 \\
5854.16 \\
5856.3 \\
6606.3\end{array}$ & $\begin{array}{l}50 \\
50 \\
50 \\
50 \\
47\end{array}$ & $\begin{array}{l}9060.72 \\
9386.81 \\
9392.79 \\
9460.68 \\
9776.90\end{array}$ & $\begin{array}{l}33 \\
24 \\
24 \\
24 \\
35\end{array}$ \\
\hline
\end{tabular}


List of tabulated lines-Continued

\begin{tabular}{|c|c|c|c|c|c|}
\hline Wavelength $[\AA]$ & No. & Wavelength $[\AA]$ & No. & Wavelength $[\AA]$ & No. \\
\hline $\begin{array}{l}9786.79 \\
9788.30 \\
9798.57 \\
9810.02 \\
9814.03\end{array}$ & $\begin{array}{l}35 \\
35 \\
35 \\
35 \\
35\end{array}$ & $\begin{array}{l}10563.3 \\
10591.9 \\
10596 \\
10597.0 \\
10623.2\end{array}$ & $\begin{array}{l}39 \\
44 \\
44 \\
44 \\
38\end{array}$ & $\begin{array}{l}11628.0 \\
11656.0 \\
11964 \\
11997.9 \\
12074.1\end{array}$ & $\begin{array}{r}7 \\
7 \\
42 \\
42 \\
42\end{array}$ \\
\hline $\begin{array}{c}9822.75 \\
9834.62 \\
9863.33 \\
9872.16 \\
10105.1\end{array}$ & $\begin{array}{l}35 \\
35 \\
35 \\
35 \\
34\end{array}$ & $\begin{array}{l}10644.0 \\
10653.0 \\
10675 \\
10693.2 \\
10713.6\end{array}$ & $\begin{array}{l}38 \\
38 \\
38 \\
37 \\
38\end{array}$ & $\begin{array}{l}12107.4 \\
12128.6 \\
12186.9 \\
12203.4 \\
12232.9\end{array}$ & $\begin{array}{l}42 \\
46 \\
46 \\
46 \\
46\end{array}$ \\
\hline $\begin{array}{l}10108.9 \\
10112.5 \\
10114.6 \\
10128.3 \\
10147.3\end{array}$ & $\begin{array}{l}34 \\
34 \\
34 \\
34 \\
34\end{array}$ & $\begin{array}{l}10718.0 \\
10730.5 \\
10757.9 \\
10775.0 \\
10879.2\end{array}$ & $\begin{array}{l}38 \\
37 \\
38 \\
37 \\
36\end{array}$ & $\begin{array}{l}12280 \\
12288.0 \\
12291 \\
12307 \\
12330\end{array}$ & $\begin{array}{l}46 \\
46 \\
40 \\
46 \\
40\end{array}$ \\
\hline $\begin{array}{l}10164.8 \\
10166.8 \\
10200.0 \\
10500.3 \\
10507.0\end{array}$ & $\begin{array}{l}34 \\
34 \\
34 \\
39 \\
39\end{array}$ & $\begin{array}{l}10884.6 \\
11180.1 \\
11227.1 \\
11237.6 \\
11266.2\end{array}$ & $\begin{array}{l}36 \\
45 \\
45 \\
45 \\
45\end{array}$ & $\begin{array}{l}12384 \\
12461.2 \\
12467.8 \\
12582.3\end{array}$ & $\begin{array}{l}40 \\
41 \\
41 \\
41\end{array}$ \\
\hline $\begin{array}{l}10513.4 \\
10520.6 \\
10533.8 \\
10539.6 \\
10549.6\end{array}$ & $\begin{array}{l}39 \\
39 \\
39 \\
39 \\
39\end{array}$ & $\begin{array}{l}11291.7 \\
11294.2 \\
11313.9 \\
11323.3 \\
11564.8\end{array}$ & $\begin{array}{r}45 \\
45 \\
45 \\
45 \\
7\end{array}$ & & \\
\hline
\end{tabular}

The numerical values for the $2 s^{2} 2 p^{3}-2 s 2 p^{4}, 2 p^{3}-2 p^{2} 3 s$, and $2 p^{3}-2 p^{2} 3 d$ transitions are taken exclusively from measurements with a wall-stabilized high current arc by Labuhn [1], since the available theoretical treatments for these transitions must be considered quite unreliable because of the strong effects of configuration interaction. However, even the experimental data should be considered with caution since they suffer, for example, from uncertainties in the identification of the lines.

The data for most higher excited transitions are taken from a stabilized-arc experiment by Richter [2], the Coulomb approximation, and approximate self-consistent field calculations by Kelly [6]. For about half of the $3 p-3 d$ transitions the numbers agree within an impressive 10 percent, and the results have been averaged. For most $3 s-3 p$ and $3 s-4 p$ transitions, strong cancellations in the calculations render the theoretical results unreliable. In these cases experimental results are exclusively used whenever available. The above material is supplemented by a few numbers obtained from a shock tube investigation by Doherty [5], a wall-stabilized arc study by Shumaker and Yokley [4], and work with a less refined arc source by Motschmann [3]. Motschmann's absolute values appear to be shifted by a constant factor due to a demixing effect in the arc and have been renormalized by applying a factor of 1.5 , which has given the best fit with Richter's data.

\section{References}

[1] Labuhn, F., to be published in Z. Naturforsch.

[2] Richter, J., Z. Astrophys. 5 1, 177-186 (1961).

[3] Motschmann, H., Z. Physik 143, 77-92 (1955).

[4] Shumaker, Jr., J. P., and Yokley, C. R., Applied Optics 3, 83-87 (1964).

[5] Doherty, L. R., Thesis Michigan (1961).

[6] Kelly, P. S., J. Quant. Spectrosc. Radiat. Transfer 4, 117-148 (1964). 
N I. Allowed Transitions

\begin{tabular}{|c|c|c|c|c|c|c|c|c|c|c|c|c|c|}
\hline No. & $\begin{array}{c}\text { Transition } \\
\text { Array }\end{array}$ & Multiplet & $\lambda(\AA)$ & $E_{i}\left(\mathrm{~cm}^{-1}\right)$ & $E_{k}\left(\mathrm{~cm}^{-1}\right)$ & $g_{i}$ & $g_{k}$ & $A_{k i}\left(10^{8} \sec ^{-1}\right)$ & $f_{i k}$ & $S$ (at.u.) & $\log g f$ & $\begin{array}{c}\text { Accu- } \\
\text { racy }\end{array}$ & Source \\
\hline \multirow[t]{4}{*}{1} & $2 s^{2} 2 p^{3}-$ & ${ }^{4} \mathrm{~S}^{\circ}-{ }^{4} \mathrm{P}$ & 1134.6 & 0.0 & 88135 & 4 & 12 & 2.3 & 0.13 & 2.0 & -0.27 & $\mathrm{D}-$ & 1 \\
\hline & & & 1134.98 & 0.0 & 88110 & 4 & 6 & 2.2 & 0.064 & 0.95 & -0.59 & $\mathrm{D}-$ & \\
\hline & & & 1134.42 & 0.0 & 88153 & 4 & 4 & 2.5 & 0.048 & 0.72 & -0.71 & $\mathrm{D}-$ & $1, l s$ \\
\hline & & & 1134.17 & 0.0 & 88173 & 4 & 2 & 2.5 & 0.024 & 0.36 & -1.02 & $\mathrm{D}-$ & $1, l s$ \\
\hline \multirow[t]{4}{*}{2} & $2 p^{3}-$ & ${ }^{4} \mathrm{~S}^{\circ}-{ }^{4} \mathrm{P}$ & 1199.9 & 0.0 & 83336 & 4 & 12 & 5.4 & 0.35 & 5.5 & 0.15 & $\mathrm{D}-$ & 1 \\
\hline & & & 1199.55 & 0.0 & 83366 & 4 & 6 & 5.5 & 0.18 & 2.8 & -0.15 & $\mathrm{D}-$ & 1 \\
\hline & & & 1200.22 & 0.0 & 83319 & 4 & 4 & 5.3 & 0.11 & 1.8 & -0.34 & $\mathrm{D}-$ & 1 \\
\hline & & & 1200.71 & 0.0 & 83286 & 4 & 2 & 5.5 & 0.059 & 0.94 & -0.62 & $\mathrm{D}-$ & 1 \\
\hline \multirow[t]{4}{*}{3} & & $\begin{array}{c}{ }^{2} \mathrm{D}^{\circ}-{ }^{2} \mathrm{P} \\
\text { (4 uv) }\end{array}$ & 1493.3 & 19228 & 86193 & 10 & 6 & 5.5 & 0.11 & 5.4 & 0.04 & D & 1 \\
\hline & & & 1492.62 & 19224 & 86221 & 6 & 4 & 5.3 & 0.12 & 3.5 & -0.15 & D & $1, l s$ \\
\hline & & & 1494.67 & 19233 & 86138 & 4 & 2 & 5.0 & 0.084 & 1.6 & -0.48 & D & 1 \\
\hline & & & 1492.67 & 19233 & 86221 & 4 & 4 & 0.58 & 0.019 & 0.38 & -1.11 & D & $1, l s$ \\
\hline \multirow[t]{5}{*}{4} & & $2 \mathbf{P}^{\circ}-{ }^{2} \mathbf{P}$ & 1743.6 & 28840 & 86193 & 6 & 6 & 2.0 & 0.091 & 3.1 & -0.26 & D & 1 \\
\hline & & & 1742.73 & 28840 & 86221 & 4 & 4 & 1.8 & 0.082 & 1.9 & -0.48 & D & $1, l s$ \\
\hline & & & 1745.25 & 28840 & 86138 & 2 & 2 & 1.3 & 0.059 & 0.68 & -0.93 & $\mathrm{D}$ & $1, l s$ \\
\hline & & & 1745.26 & 28840 & 86138 & 4 & 2 & 0.65 & 0.015 & 0.34 & -1.23 & D & $1, l_{s}$ \\
\hline & & & 1742.72 & 28840 & 86221 & 2 & 4 & 0.35 & 0.032 & 0.37 & -1.20 & D & $1, l s$ \\
\hline \multirow[t]{5}{*}{5} & $2 p^{3}-$ & $\begin{array}{c}{ }^{2} \mathrm{D}^{\circ}-{ }^{2} \mathrm{D} \\
(5 \mathrm{uv})\end{array}$ & 1243.3 & 19228 & 99663 & 10 & 10 & 4.6 & 0.11 & 4.4 & 0.03 & $\mathrm{D}$ & 1 \\
\hline & & & 1243.17 & 19224 & 99663 & 6 & 6 & 4.3 & 0.10 & 2.4 & -0.22 & D & $l s$ \\
\hline & & & 1243.30 & 19233 & 99663 & 4 & 4 & 4.3 & 0.10 & 1.6 & -0.40 & $\mathrm{D}$ & ls \\
\hline & & & 1243.17 & 19224 & 99663 & 6 & 4 & 0.45 & 0.0070 & 0.17 & -1.38 & D & $l s$ \\
\hline & & & 1243.31 & 19233 & 99663 & 4 & 6 & 0.30 & 0.010 & 0.17 & -1.38 & $\mathrm{D}$ & $l s$ \\
\hline 6 & & $\begin{array}{c}{ }^{2} \mathrm{P}^{\circ}-{ }^{2} \mathrm{D} \\
(10 \mathrm{uv})\end{array}$ & 1411.94 & 28840 & 99663 & 6 & 10 & 0.52 & 0.026 & 0.72 & -0.81 & $\mathrm{D}$ & 1 \\
\hline \multirow[t]{4}{*}{7} & $\begin{array}{l}2 s 2 p^{4}- \\
2 s^{2} 2 p^{2}\left({ }^{3} \mathrm{P}\right) 3 p\end{array}$ & ${ }^{4} \mathrm{P}-{ }^{4} \mathrm{~S}^{\circ}$ & 11602 & 88135 & 96752 & 12 & 4 & 0.0405 & 0.0273 & 12.5 & -0.485 & C & 2 \\
\hline & & & 11564.8 & 88110 & 96752 & 6 & 4 & 0.0210 & 0.0280 & 6.4 & -0.77 & C & $2, l s$ \\
\hline & & & 11628.0 & 88153 & 96752 & 4 & 4 & 0.0130 & 0.0264 & 4.04 & -0.98 & $\mathrm{C}$ & $2, l s$ \\
\hline & & & 11656.0 & 88173 & 96752 & 2 & 4 & 0.0066 & 0.0267 & 2.05 & -1.272 & $\mathrm{C}$ & $2, l s$ \\
\hline \multirow[t]{2}{*}{8} & $\begin{array}{l}2 p^{3}- \\
2 p^{2}\left({ }^{3} \mathrm{P}\right) 3 d\end{array}$ & ${ }^{2} \mathrm{D}^{\circ}-{ }^{4} \mathrm{~F}$ & & & & & & & & & & & \\
\hline & & & 1169.69 & 19224 & 104718 & 6 & 8 & 0.030 & $8.2 \times 10^{-4}$ & 0.019 & -2.31 & D & 1 \\
\hline \multirow[t]{4}{*}{9} & & ${ }^{2} \mathrm{D}^{\circ}-{ }^{2} \mathrm{~F}$ & 1167.9 & 19228 & 104852 & 10 & 14 & 1.2 & 0.034 & 1.3 & -0.46 & D & 1 \\
\hline & & & 1167.45 & 19224 & 104883 & 6 & 8 & 1.1 & 0.030 & 0.69 & -0.75 & D & 1 \\
\hline & & & 1168.54 & 19233 & 104811 & 4 & 6 & 1.3 & 0.040 & 0.61 & -0.80 & D & $1, l s$ \\
\hline & & & 1168.42 & 19224 & 104811 & 6 & 6 & 0.095 & 0.0019 & 0.045 & -1.93 & D & $1, l s$ \\
\hline \multirow[t]{4}{*}{10} & & $\mid{ }^{2} \mathrm{D}^{\circ}-{ }^{2} \mathrm{D}$ & 1164.0 & 19228 & 105135 & 10 & 10 & 0.47 & 0.0095 & 0.37 & -1.02 & D & 1 \\
\hline & & & 1163.88 & 19224 & 105144 & 6 & 6 & 0.43 & 0.0087 & 0.20 & -1.28 & $\mathrm{D}$ & $l s$ \\
\hline & & & 1164.31 & 19233 & 105121 & 4 & 4 & 0.43 & 0.0087 & 0.13 & -1.46 & D & ls \\
\hline & & & $\begin{array}{l}1164.31 \\
1163.87\end{array}$ & $\begin{array}{l}19224 \\
19233\end{array}$ & $\begin{array}{l}105121 \\
105144\end{array}$ & $\begin{array}{l}6 \\
4\end{array}$ & $\begin{array}{l}4 \\
6\end{array}$ & $\begin{array}{l}0.048 \\
0.032\end{array}$ & $\begin{array}{l}6.5 \times 10^{-4} \\
9.7 \times 10^{-4}\end{array}$ & $\begin{array}{l}0.015 \\
0.015\end{array}$ & $\begin{array}{l}-2.41 \\
-2.41\end{array}$ & $\begin{array}{l}\mathrm{D} \\
\mathrm{D}\end{array}$ & $\begin{array}{l}l s \\
l s\end{array}$ \\
\hline \multirow[t]{4}{*}{11} & & ${ }^{2} \mathbf{P}^{\circ}-{ }^{2} \mathbf{P}$ & 1319.5 & 28840 & 104628 & 6 & 6 & 1.3 & 0.034 & 0.88 & -0.69 & $\mathrm{D}$ & 1 \\
\hline & & 112 & 1319.72 & 28840 & 104615 & 4 & 4 & 1.1 & 0.029 & 0.50 & -0.94 & D & $1, l s$ \\
\hline & & & 1319.04 & 28840 & 104655 & 2 & 2 & 0.85 & 0.022 & 0.19 & -1.35 & D & $1, l s$ \\
\hline & & & $\begin{array}{l}1319.04 \\
1319.72\end{array}$ & $\begin{array}{l}28840 \\
28840\end{array}$ & $\begin{array}{l}104655 \\
104615\end{array}$ & $\begin{array}{l}4 \\
2\end{array}$ & $\begin{array}{l}2 \\
4\end{array}$ & $\begin{array}{l}0.42 \\
0.22\end{array}$ & $\begin{array}{l}0.0055 \\
0.011\end{array}$ & $\begin{array}{l}0.095 \\
0.10\end{array}$ & $\begin{array}{l}-1.66 \\
-1.64\end{array}$ & $\begin{array}{l}\mathrm{D} \\
\mathrm{D}\end{array}$ & $\begin{array}{l}1, l s \\
1, l s\end{array}$ \\
\hline
\end{tabular}


N I. Allowed Transitions-Continued

\begin{tabular}{|c|c|c|c|c|c|c|c|c|c|c|c|c|c|}
\hline No & $\begin{array}{l}\text { Transition } \\
\text { Array }\end{array}$ & Multiplet & $\lambda(\AA)$ & $E_{i}\left(\mathrm{~cm}^{-1}\right)$ & $E_{k}\left(\mathrm{~cm}^{-1}\right)$ & $g_{i}$ & $g_{k}$ & $A_{k i}\left(10^{k} \sec ^{-1}\right)$ & $f_{i k}$ & $S($ at.u.) & $\log g f$ & $\begin{array}{c}\text { Accu- } \\
\text { racy }\end{array}$ & Source \\
\hline 12 & & ${ }^{2} \mathrm{P}^{\circ}-{ }^{2} \mathrm{~F}$ & 1316.29 & 28840 & 104811 & 4 & 6 & 0.025 & $9.6 \times 10^{-4}$ & 0.017 & -2.42 & D & 1 \\
\hline \multirow[t]{4}{*}{13} & & $\begin{array}{c}2 \mathrm{P}^{\circ}-{ }^{2} \mathrm{D} \\
(13 \mathrm{uv})\end{array}$ & 1310.7 & 28840 & 105135 & 6 & 10 & 1.3 & 0.056 & 1.4 & -0.48 & D & 1 \\
\hline & & & 1310.54 & 28840 & 105144 & 4 & 6 & 1.3 & 0.050 & 0.87 & -0.70 & D & ls \\
\hline & & & 1310.97 & 28840 & 105121 & 2 & 4 & 1.1 & 0.057 & 0.49 & -0.95 & $\mathrm{D}$ & ls \\
\hline & & & 1310.97 & 28840 & 105121 & 4 & 4 & 0.23 & 0.0059 & 0.10 & -1.63 & D & ls \\
\hline \multirow[t]{4}{*}{14} & $2 p^{3}-$ & ${ }^{2} \mathrm{D}^{\circ}-{ }^{2} \mathrm{P}$ & 1176.9 & 19228 & 104199 & 10 & 6 & 1.1 & 0.014 & 0.53 & -0.86 & D & 1 \\
\hline & & & [1176.4] & 19224 & 104227 & 6 & 4 & 0.95 & 0.013 & 0.31 & -1.10 & D & $1, l s$ \\
\hline & & & {$[1177.7]$} & 19233 & 104142 & 4 & 2 & 1.3 & 0.014 & 0.21 & -1.27 & $\mathrm{D}$ & 1 \\
\hline & & & {$[1176.6]$} & 19233 & 104227 & 4 & 4 & 0.11 & 0.0023 & 0.035 & -2.04 & D & 1, ls \\
\hline \multirow[t]{5}{*}{15} & & $\begin{array}{l}{ }^{2} \mathrm{P}^{\circ}-{ }^{2} \mathrm{P} \\
(11 \mathrm{uv})\end{array}$ & 1.327 .0 & 28840 & 104199 & 6 & 6 & 0.20 & 0.0053 & 0.14 & -1.50 & D & 1 \\
\hline & & & 1326.63 & 28840 & 104227 & 4 & 4 & 0.15 & 0.0040 & 0.069 & -1.80 & D & 1, ls \\
\hline & & & 1327.96 & 28840 & 104142 & 2 & 2 & 0.17 & 0.0045 & 0.039 & -2.05 & D & 1, ls \\
\hline & & & 1327.96 & 28840 & 104142 & 4 & 2 & 0.085 & 0.0011 & 0.020 & -2.35 & D & 1, ls \\
\hline & & & 1326.63 & 28840 & 104227 & 2 & 4 & 0.030 & 0.0016 & 0.014 & -2.50 & D & $1, l s$ \\
\hline \multirow[t]{8}{*}{16} & $\begin{array}{l}2 s 2 p^{4}- \\
\quad 2 s^{2} 2 p^{2}\left({ }^{3} \mathrm{P}\right) 4 p\end{array}$ & $\begin{array}{c}{ }^{4} \mathrm{P}-{ }^{4} \mathrm{D}^{0} \\
(13)\end{array}$ & 5349.0 & 88135 & 106825 & 12 & 20 & 0.00252 & 0.00180 & 0.380 & -1.67 & $\mathrm{C}$ & 2 \\
\hline & & & 5328.70 & 88110 & 106871 & 6 & 8 & 0.00254 & 0.00144 & 0.152 & -2.062 & $\mathrm{C}$ & $3 n, l s$ \\
\hline & & & 5356.77 & 88153 & 106816 & 4 & 6 & 0.00189 & 0.00122 & 0.086 & -2.312 & C & $3 n, l s$ \\
\hline & & & 5372.66 & 88173 & 106780 & 2 & 4 & 0.00107 & $9.3 \times 10^{-4}$ & 0.0328 & -2.73 & $\mathrm{C}$ & $3 n, l s$ \\
\hline & & & {$[5344.4]$} & 88110 & 106816 & 6 & 6 & $6.2 \times 10^{-4}$ & $2.64 \times 10^{-4}$ & 0.0279 & -2.80 & C & $3 n, l s$ \\
\hline & & & [5367.1] & $\begin{array}{l}88153 \\
88173\end{array}$ & $\begin{array}{l}106780 \\
106761\end{array}$ & $\begin{array}{l}4 \\
2\end{array}$ & $\begin{array}{l}4 \\
2\end{array}$ & $\begin{array}{l}0.00118 \\
0.00210\end{array}$ & $\begin{array}{l}5.1 \times 10^{-4} \\
9.1 \times 10^{-4}\end{array}$ & $\begin{array}{l}0.0361 \\
0.0323\end{array}$ & $\begin{array}{l}-2.69 \\
-2.74\end{array}$ & $\stackrel{\mathrm{C}}{\mathrm{C}}$ & $3 n, l s$ \\
\hline & & & [5354.7] & 88110 & 106780 & 6 & 4 & $1.35 \times 10^{-4}$ & $3.88 \times 10^{-5}$ & 0.00410 & -3.63 & $\mathrm{C}$ & $3 n, l s$ \\
\hline & & & {$[5372.5]$} & 88153 & 106761 & 4 & 2 & $4.31 \times 10^{-4}$ & $9.3 \times 10^{-5}$ & 0.0066 & -3.428 & $\mathrm{C}$ & $3 n, l s$ \\
\hline \multirow[t]{7}{*}{17} & & ${ }^{4} \mathrm{P}-{ }^{4} \mathrm{P}^{\circ}$ & 5294.9 & 88135 & 107016 & 12 & 12 & 0.00373 & 0.00157 & 0.328 & -1.73 & $\mathrm{C}$ & 2 \\
\hline & & & 5281.18 & 88110 & 107039 & 6 & 6 & 0.00282 & 0.00118 & 0.123 & -2.150 & C & $3 n, l s$ \\
\hline & & & [5305.0] & 88153 & 106998 & 4 & 4 & $5.3 \times 10^{-4}$ & $2.23 \times 10^{-4}$ & 0.0156 & -3.049 & C & $3 n, l s$ \\
\hline & & & [5314.8] & 88173 & 106983 & 2 & 2 & $6.9 \times 10^{-4}$ & $2.91 \times 10^{-4}$ & 0.0102 & -3.234 & C & $3 n, l s$ \\
\hline & & & [5292.9] & 88110 & 106998 & 6 & 4 & 0.00167 & $4.68 \times 10^{-4}$ & 0.0489 & -2.55 & C & $3 n, l s$ \\
\hline & & & {$[5309.2]$} & 88153 & 106983 & 4 & 2 & 0.00273 & $5.8 \times 10^{-4}$ & 0.0403 & -2.64 & C & $3 n$, is \\
\hline & & & $\begin{array}{l}{[5293.5]} \\
{[5310.6]}\end{array}$ & $\begin{array}{l}88153 \\
88173\end{array}$ & $\begin{array}{l}107039 \\
106998\end{array}$ & $\begin{array}{l}4 \\
2\end{array}$ & $\begin{array}{l}6 \\
4\end{array}$ & $\begin{array}{l}0.00113 \\
0.00137\end{array}$ & $\begin{array}{l}7.1 \times 10^{-4} \\
0.00116\end{array}$ & $\begin{array}{l}0.0495 \\
0.0404\end{array}$ & $\begin{array}{l}-2.55 \\
-2.64\end{array}$ & $\stackrel{\mathrm{C}}{\mathrm{C}}$ & $\begin{array}{l}3 n, l s \\
3 n, l s\end{array}$ \\
\hline \multirow[t]{4}{*}{18} & & ${ }^{4} \mathrm{P}-{ }^{4} \mathrm{~S}^{\circ}$ & 5176.7 & 88135 & 107447 & 12 & 4 & 0.00427 & $5.7 \times 10^{-4}$ & 0.117 & -2.163 & C & 2 \\
\hline & & & {$[5170.0]$} & 88110 & 107447 & 6 & 4 & 0.00209 & $5.6 \times 10^{-4}$ & 0.057 & -2.475 & C & $3 n, l s$ \\
\hline & & & {$[5181.5]$} & 88153 & 107447 & 4 & 4 & 0.00144 & $5.8 \times 10^{-4}$ & 0.0395 & -2.64 & $\mathrm{C}$ & $3 n, l s$ \\
\hline & & & [5186.9] & 88173 & 107447 & 2 & 4 & $7.3 \times 10^{-4}$ & $5.9 \times 10^{-4}$ & 0.0201 & -2.93 & C & $3 n, l s$ \\
\hline \multirow[t]{4}{*}{19} & $\begin{array}{l}2 p^{3}- \\
2 p^{2}\left({ }^{3} \mathrm{P}\right) 5 s\end{array}$ & ${ }^{2} \mathrm{D}^{\circ}-{ }^{2} \mathrm{P}$ & 1100.7 & 19228 & 110082 & 10 & 6 & 0.33 & 0.0036 & 0.13 & -1.44 & $\mathrm{D}-$ & 1 \\
\hline & & ${ }^{2} \mathrm{P}^{\circ}-{ }^{2} \mathrm{P}$ & & & & & & & & & & & \\
\hline & & & [1231.7] & 28840 & 110029 & 2 & 2 & 0.022 & $5.0 \times 10^{-4}$ & 0.0041 & -2.99 & D & $1, l s$ \\
\hline & & & [1231.7] & 28840 & 110029 & 4 & 2 & 0.011 & $1.3 \times 10^{-4}$ & 0.0020 & -3.30 & D & 1, ls \\
\hline \multirow[t]{9}{*}{21} & $\begin{array}{l}2 p^{2} 3 s- \\
2 p^{2}\left({ }^{3} \mathrm{P}\right) 3 p\end{array}$ & ${ }^{4} \mathrm{P}-{ }^{4} \mathrm{D}^{\circ}$ & 8691.6 & 83337 & 94839 & 12 & 20 & 0.190 & 0.358 & 123 & 0.63 & C & 2 \\
\hline & & & 8680.27 & 83366 & 94883 & 6 & 8 & 0.191 & 0.287 & 49.2 & 0.236 & C & $2, l s$ \\
\hline & & & 8683.40 & 83319 & 94832 & 4 & 6 & 0.133 & 0.226 & 25.8 & -0.045 & C & $2, l s$ \\
\hline & & & 8686.16 & 83286 & 94795 & 2 & 4 & 0.079 & 0.178 & 10.2 & -0.448 & C & $2, l s$ \\
\hline & & & 8718.84 & 83366 & 94832 & 6 & 6 & 0.054 & 0.062 & 10.6 & -0.433 & C & $2, l s$ \\
\hline & & & 8711.71 & 83319 & 94795 & 4 & 4 & 0.101 & 0.115 & 13.2 & -0.337 & C & $2, l s$ \\
\hline & & & 8703.26 & 83286 & 94772 & 2 & 2 & 0.171 & 0.194 & 11.1 & -0.412 & C & 2. $l s$ \\
\hline & & & 8747.36 & 83366 & 94795 & 6 & 4 & 0.0079 & 0.0061 & 1.05 & -1.438 & C & $2, l s$ \\
\hline & & & 8728.91 & 83319 & 94772 & 4 & 2 & 0.0300 & 10.0171 & 1.97 & -1.164 & C & $2, l s$ \\
\hline
\end{tabular}


N I. Allowed Transitions-Continued

\begin{tabular}{|c|c|c|c|c|c|c|c|c|c|c|c|c|c|}
\hline No. & $\begin{array}{c}\text { Transition } \\
\text { Array }\end{array}$ & Multiplet & $\lambda(\AA)$ & $E_{i}\left(\mathrm{~cm}^{-1}\right)$ & $E_{k}\left(\mathrm{~cm}^{-1}\right)$ & $g_{i}$ & $g_{k}$ & $A_{k i}\left(10^{8} \mathrm{sec}^{-1}\right)$ & $f_{i k}$ & S(at.u.) & $\log g f$ & $\begin{array}{l}\text { Accu- } \\
\text { racy }\end{array}$ & Source \\
\hline \multirow[t]{8}{*}{22} & & ${ }^{4} \mathrm{P}-{ }^{4} \mathrm{P}^{\circ}$ & 8211.8 & 83337 & 95511 & 12 & 12 & 0.228 & 0.231 & 75 & 0.443 & C & 2,5 \\
\hline & & & 8216.32 & 83366 & 95533 & 6 & 6 & 0.160 & 0.162 & 26.3 & -0.012 & C & $2 n, l s$ \\
\hline & & & 8210.71 & 83319 & 95495 & 4 & 4 & 0.0363 & 0.0367 & 3.97 & -0.83 & $\mathrm{C}$ & $2 n, l s$ \\
\hline & & & 8200.36 & 83286 & 95477 & 2 & 2 & 0.0364 & 0.0367 & 1.98 & -1.135 & $\mathrm{C}$ & $2 n, l s$ \\
\hline & & & 8242.37 & 83366 & 95495 & 6 & 4 & 0.102 & 0.069 & 11.3 & -0.381 & C & $2 n, l s$ \\
\hline & & & 8223.12 & 83319 & 95477 & 4 & 2 & 0.202 & 0.103 & 11.1 & -0.387 & C & $2 n, l s$ \\
\hline & & & 8184.85 & 83319 & 95533 & 4 & 6 & 0.063 & 0.096 & 10.3 & -0.418 & $\mathrm{C}$ & $2 n, l s$ \\
\hline & & & 8188.01 & 83286 & 95495 & 2 & 4 & 0.092 & 0.185 & 10.0 & -0.431 & $\mathrm{C}$ & $2 n, l s$ \\
\hline \multirow[t]{4}{*}{23} & & ${ }^{4} \mathrm{P}-{ }^{4} \mathrm{~S}^{\circ}$ & 7452.2 & 83337 & 96752 & 12 & 4 & 0.318 & 0.088 & 26.0 & 0.025 & $\mathrm{C}$ & 2,5 \\
\hline & & & 7468.31 & 83366 & 96752 & 6 & 4 & 0.161 & 0.089 & 13.2 & -0.270 & C & $2 n, l s$ \\
\hline & & & 7442.30 & 83319 & 96752 & 4 & 4 & 0.106 & 0.088 & 8.6 & -0.454 & $\mathrm{C}$ & $2 n, l s$ \\
\hline & & & 7423.64 & 83286 & 96752 & 2 & 4 & 0.052 & 0.086 & 4.21 & -0.76 & C & $2 n, l s$ \\
\hline \multirow[t]{4}{*}{24} & & ${ }^{2} \mathrm{P}-{ }^{2} \mathrm{D}^{\circ}$ & 9395.3 & 86193 & 96834 & 6 & 10 & 0.217 & 0.478 & 89 & 0.458 & C & 2 \\
\hline & & & 9392.79 & 86221 & 96864 & 4 & 6 & 0.218 & 0.432 & 53 & 0.237 & $\mathrm{C}$ & $2, l s$ \\
\hline & & & 9386.81 & 86138 & 96788 & 2 & 4 & 0.183 & 0.482 & 29.8 & -0.016 & $\widetilde{C}$ & $2, l s$ \\
\hline & & & 9460.68 & 86221 & 96788 & 4 & 4 & 0.0334 & 0.0449 & 5.6 & -0.75 & $\mathrm{C}$ & $2, l s$ \\
\hline \multirow[t]{5}{*}{25} & & ${ }^{2} \mathrm{P}-{ }^{2} \mathrm{P}^{\circ}$ & 8617.5 & 86193 & 97794 & 6 & 6 & 0.286 & 0.318 & 54 & 0.281 & C & 2 \\
\hline & & & 8629.24 & 86221 & 97806 & 4 & 4 & 0.238 & 0.266 & 30.2 & 0.027 & C & $2, l s$ \\
\hline & & & 8590.01 & 86138 & 97770 & 2 & 2 & 0.190 & 0.210 & 11.9 & -0.376 & $\bar{C}$ & $2, l s$ \\
\hline & & & 8655.87 & 86221 & 97770 & 4 & 2 & 0.099 & 0.056 & 6.3 & -0.65 & C & $2, l s$ \\
\hline & & & 8567.74 & 86138 & 97806 & 2 & 4 & 0.0458 & 0.101 & 5.7 & -0.70 & C & $2, l s$ \\
\hline \multirow[t]{4}{*}{26} & $2 p^{2}\left({ }^{3} \mathrm{P}\right) 3 s-$ & ${ }^{2} \mathrm{P}-{ }^{2} \mathrm{D}^{\circ}$ & 4106.8 & 86193 & 110536 & 6 & 10 & 0.041 & 0.017 & 1.4 & -0.98 & D & $3 n$ \\
\hline & & & 4109.96 & 86221 & 110546 & 4 & 6 & 0.040 & 0.015 & 0.83 & -1.21 & D & $l s$ \\
\hline & & & 4099.95 & 86138 & 110522 & 2 & 4 & 0.034 & 0.017 & 0.46 & -1.47 & $\mathrm{D}$ & ls \\
\hline & & & 4113.97 & 86221 & 110522 & 4 & 4 & 0.0068 & 0.0017 & 0.093 & -2.16 & $\mathrm{D}$ & ls \\
\hline \multirow[t]{4}{*}{27} & $2 p^{2} 3 s^{\prime}-$ & ${ }^{2} \mathrm{D}-{ }^{2} \mathrm{~F}^{\circ}$ & 9047.6 & 99663 & 110713 & 10 & 14 & 0.272 & 0.467 & 139 & 0.669 & B & $2,6, c a$ \\
\hline & & & 9045.88 & 99663 & 110715 & 6 & 8 & 0.269 & 0.439 & 78.5 & 0.421 & B & $2 n, l s$ \\
\hline & & & 9049.89 & 99663 & 110711 & 4 & 6 & 0.258 & 0.475 & 56.6 & 0.279 & B & $2 n, l s$ \\
\hline & & & 9049.47 & 99663 & 110711 & 6 & 6 & 0.0180 & 0.0222 & 3.96 & -0.876 & B & $2 n, l s$ \\
\hline \multirow[t]{9}{*}{28} & $2 p^{2} 3 s-$ & ${ }^{4} \mathrm{P}-{ }^{4} \mathrm{D}^{\circ}$ & 4256.3 & 83337 & 106825 & 12 & 20 & 0.020 & 0.0089 & 1.5 & -0.97 & $\mathrm{E}$ & 6 \\
\hline & & & 4253.28 & 83366 & 106871 & 6 & 8 & 0.020 & 0.0071 & 0.60 & -1.37 & $\mathrm{E}$ & ls \\
\hline & & & 4254.7 & 83319 & 106816 & 4 & 6 & 0.014 & 0.0057 & 0.32 & -1.64 & $\mathrm{E}$ & $l s$ \\
\hline & & & 4254.7 & 83286 & 106780 & 2 & 4 & 0.0079 & 0.0043 & 0.12 & -2.07 & $\mathrm{E}$ & ls \\
\hline & & & [4263.2] & 83366 & 106816 & 6 & 6 & 0.0061 & 0.0017 & 0.14 & -2.00 & $\mathrm{E}$ & $l s$ \\
\hline & & & [4261.2] & 83319 & 106780 & 4 & 4 & 0.010 & 0.0029 & 0.16 & -1.94 & $\mathrm{E}$ & $l s$ \\
\hline & & & [4258.7] & 83286 & 106761 & 2 & 2 & 0.016 & 0.0043 & 0.12 & -2.07 & $\mathrm{E}$ & $l s$ \\
\hline & & & {$[4269.8]$} & 83366 & 106780 & 6 & 4 & $9.8 \times 10^{-4}$ & $1.8 \times 10^{-4}$ & 0.015 & -2.97 & $\mathrm{E}$ & $l s$ \\
\hline & & & {$[4264.7]$} & 83319 & 106761 & 4 & 2 & 0.0033 & $4.5 \times 10^{-4}$ & 0.025 & -2.75 & $\mathrm{E}$ & $l_{s}$ \\
\hline \multirow[t]{7}{*}{29} & & ${ }^{4} \mathrm{P}-{ }^{4} \mathrm{P}^{\circ}$ & 4222.0 & 83337 & 107016 & 12 & 12 & 0.073 & 0.020 & 3.3 & -0.63 & D & $3 n$ \\
\hline & & & 4223.04 & 83366 & 107039 & 6 & 6 & 0.051 & 0.014 & 1.1 & -1.09 & D & $l s$ \\
\hline & & & 4222.12 & 83319 & 106998 & 4 & 4 & 0.0098 & 0.0026 & 0.15 & -1.98 & D & $l s$ \\
\hline & & & 4218.87 & 83286 & 106983 & 2 & 2 & 0.012 & 0.0033 & 0.091 & -2.18 & D & $l s$ \\
\hline & & & 4230.35 & 83366 & 106998 & 6 & 4 & 0.033 & 0.0059 & 0.49 & -1.45 & D & $l s$ \\
\hline & & & $\begin{array}{l}4224.74 \\
4214.73\end{array}$ & $\begin{array}{l}83319 \\
83319\end{array}$ & $\begin{array}{l}106983 \\
107039\end{array}$ & 4 & 2 & 0.061 & 0.0082 & 0.45 & -1.49 & D & ls \\
\hline & & & 4215.92 & 83286 & 106998 & 2 & 4 & 0.031 & 0.016 & $\begin{array}{l}0.49 \\
0.45\end{array}$ & $\begin{array}{l}-1.45 \\
-1.49\end{array}$ & D & ls \\
\hline \multirow[t]{4}{*}{30} & & ${ }^{4} \mathrm{P}-{ }^{4} \mathrm{~S}^{\circ}$ & 4146.3 & 83337 & 107447 & 12 & 4 & 0.025 & 0.0021 & 0.35 & -1.59 & D & $3 n$ \\
\hline & & & 4151.46 & 83366 & 107447 & 6 & 4 & 0.013 & 0.0023 & 0.19 & -1.87 & D & $3 n, l s$ \\
\hline & & & 4143.42 & 83319 & 107447 & 4 & 4 & 0.0078 & 0.0020 & 0.11 & -2.09 & D & $3 n, l s$ \\
\hline & & & 4137.63 & 83286 & 107447 & 2 & 4 & 0.0039 & 0.0020 & 0.055 & -2.40 & D & $3 n, l s$ \\
\hline
\end{tabular}


N I. Allowed Transitions-Continued

\begin{tabular}{|c|c|c|c|c|c|c|c|c|c|c|c|c|c|}
\hline No. & $\begin{array}{c}\text { Transition } \\
\text { Array }\end{array}$ & Multiplet & $\lambda(\AA)$ & $E_{i}\left(\mathrm{~cm}^{-1}\right)$ & $E_{k}\left(\mathrm{~cm}^{-1}\right)$ & $g_{i}$ & $g_{k}$ & $A_{k i}\left(10^{*} \sec ^{-1}\right)$ & $f_{i k}$ & $S($ at.u.) & $\log g f$ & $\begin{array}{l}\text { Accu- } \\
\text { racy }\end{array}$ & Source \\
\hline \multirow[t]{2}{*}{31} & & ${ }^{2} \mathrm{P}-{ }^{2} \mathrm{~S}^{\circ}$ & 4928.2 & 8619.3 & 106479 & 6 & 2 & 0.0234 & 0.00285 & 0.277 & -1.768 & B & 2 \\
\hline & & & $\begin{array}{l}4935.03 \\
4914.90\end{array}$ & $\begin{array}{l}86221 \\
86138\end{array}$ & $\begin{array}{l}106479 \\
106479\end{array}$ & $\begin{array}{l}4 \\
2\end{array}$ & $\begin{array}{l}2 \\
2\end{array}$ & \begin{tabular}{|l|}
0.0158 \\
0.00759
\end{tabular} & $\begin{array}{l}0.00289 \\
0.00275\end{array}$ & $\begin{array}{l}0.188 \\
0.0889\end{array}$ & $\begin{array}{l}-1.937 \\
-2.260\end{array}$ & $\begin{array}{l}\mathrm{B} \\
\mathrm{B}\end{array}$ & $\begin{array}{l}4 n, l s \\
4 n, l s\end{array}$ \\
\hline \multirow[t]{2}{*}{32} & $2 p^{2}\left({ }^{3} \mathrm{P}\right) 3 p-$ & ${ }^{2} \mathrm{P}^{0}-{ }^{2} \mathrm{~S}$ & 5408.4 & 97794 & 116279 & 6 & 2 & 0.0111 & 0.00162 & 0.173 & -2.013 & C & \\
\hline & & & $\begin{array}{l}5411.88 \\
5401.45\end{array}$ & $\begin{array}{l}97806 \\
97770\end{array}$ & $\begin{array}{l}116279 \\
116279\end{array}$ & $\begin{array}{l}4 \\
2\end{array}$ & $\begin{array}{l}2 \\
2\end{array}$ & \begin{tabular}{|l}
0.0075 \\
0.00369
\end{tabular} & $\begin{array}{l}0.00164 \\
0.00161\end{array}$ & $\begin{array}{l}0.117 \\
0.057\end{array}$ & $\begin{array}{l}-2.183 \\
-2.491\end{array}$ & $\begin{array}{l}\mathrm{C} \\
\mathrm{C}\end{array}$ & $\begin{array}{l}2, l s \\
2, l s\end{array}$ \\
\hline \multirow[t]{2}{*}{33} & $\begin{array}{l}2 p^{2} 3 p- \\
2 p^{2}\left({ }^{3} \mathrm{P}\right) 3 d\end{array}$ & $\begin{array}{c}{ }^{2} \mathrm{~S}^{\circ}-{ }^{2} \mathrm{P} \\
(15)\end{array}$ & 9050.4 & 93582 & 104628 & 2 & 6 & 0.256 & 0.945 & 56.3 & 0.276 & B & 2,6 , \\
\hline & & & $\begin{array}{l}9060.72 \\
9028.92\end{array}$ & $\begin{array}{l}93582 \\
93582\end{array}$ & $\begin{array}{l}104615 \\
104655\end{array}$ & $\begin{array}{l}2 \\
2\end{array}$ & $\begin{array}{l}4 \\
2\end{array}$ & $\begin{array}{l}0.257 \\
0.255\end{array}$ & $\begin{array}{l}0.634 \\
0.311\end{array}$ & $\begin{array}{l}37.8 \\
18.5\end{array}$ & $\begin{array}{r}0.103 \\
-0.206\end{array}$ & $\begin{array}{l}\mathrm{B} \\
\mathrm{B}\end{array}$ & $\begin{array}{l}2 n, l s \\
2 n, l s\end{array}$ \\
\hline \multirow[t]{8}{*}{34} & & $\begin{array}{c}{ }^{4} \mathrm{D}^{\circ}-{ }^{4} \mathrm{~F} \\
(18)\end{array}$ & 10117 & 94839 & 104721 & 20 & 28 & 0.373 & 0.802 & 534 & 1.205 & B & $2,6, c a$ \\
\hline & & & 10114.6 & 94883 & 104767 & 8 & 10 & 0.374 & 0.717 & 191 & 0.759 & B & $l$ \\
\hline & & & $\begin{array}{l}10112.5 \\
101089\end{array}$ & $\begin{array}{l}94832 \\
94795\end{array}$ & $\begin{array}{l}104718 \\
104684\end{array}$ & $\begin{array}{l}6 \\
4\end{array}$ & $\begin{array}{l}8 \\
6\end{array}$ & $\begin{array}{l}0.321 \\
0.281\end{array}$ & $\begin{array}{l}0.656 \\
0.646\end{array}$ & 131 & $\begin{array}{l}0.595 \\
0.412\end{array}$ & $\mathrm{~B}$ & $l$. \\
\hline & & & 10108.9 & 94772 & $\begin{array}{l}104084 \\
104665\end{array}$ & $\begin{array}{l}4 \\
2\end{array}$ & $\begin{array}{l}0 \\
4\end{array}$ & $\begin{array}{l}.281 \\
0.262\end{array}$ & 0.803 & $\begin{array}{l}80.0 \\
53.4\end{array}$ & 0.206 & $\begin{array}{l}\mathrm{B} \\
\mathrm{B}\end{array}$ & \\
\hline & & & 10164.8 & 94883 & 104718 & 8 & 8 & 0.0523 & 0.0811 & 21.7 & -0.188 & B & \\
\hline & & & 47.3 & 94832 & 104684 & 6 & 6 & 0.0898 & 0.139 & 27.8 & -0.080 & B & \\
\hline & & & 10128.3 & 94795 & 104665 & 4 & 4 & 0.104 & 0.160 & 21.4 & -0.193 & B & \\
\hline & & & $\begin{array}{l}10200.0 \\
10166.8\end{array}$ & $\begin{array}{l}94883 \\
94832\end{array}$ & $\begin{array}{l}104684 \\
104665\end{array}$ & $\begin{array}{l}8 \\
6\end{array}$ & $\begin{array}{l}6 \\
4\end{array}$ & $\begin{array}{l}0.00353 \\
0.00737\end{array}$ & $\begin{array}{l}0.00413 \\
0.00762\end{array}$ & $\begin{array}{l}1.11 \\
1.53\end{array}$ & $\begin{array}{l}-1.481 \\
-1.340\end{array}$ & $\begin{array}{l}\mathrm{B} \\
\mathrm{B}\end{array}$ & \\
\hline \multirow[t]{10}{*}{35} & & $\begin{array}{c}{ }^{4} \mathrm{D}^{\circ}-{ }^{4} \mathrm{D} \\
(19)\end{array}$ & 9829.2 & 94839 & 105010 & 20 & 20 & 0.0992 & 0.144 & 93.0 & 0.458 & B & $2,6, c$ \\
\hline & & & 9863.33 & 94883 & 105020 & 8 & 8 & 0.101 & 0.147 & 38.1 & 0.069 & B & $2 n$ \\
\hline & & & 9822.75 & 94832 & 105011 & 6 & 6 & 0.0 & 33 & 15.2 & -0.328 & B & \\
\hline & & & 9798.57 & 94795 & 104998 & 4 & 4 & 0.0 & 0 & 6.42 & -0.701 & B & \\
\hline & & & 9788.30 & 94772 & 104987 & 2 & 2 & 0.0 & 0 & 2. & -1.050 & B & $2 n, l s$ \\
\hline & & & $\begin{array}{l}9872.16 \\
9834.62\end{array}$ & $\begin{array}{l}94883 \\
94832\end{array}$ & $\begin{array}{l}105011 \\
104998\end{array}$ & 8 & $\begin{array}{l}6 \\
4\end{array}$ & 0.0222 & 0.0243 & $\begin{array}{l}6.33 \\
8.65\end{array}$ & $\begin{array}{l}-0.711 \\
-0.573\end{array}$ & B & $2 n$, \\
\hline & & & $\begin{array}{l}4.62 \\
0.02\end{array}$ & $\begin{array}{l}94832 \\
94795\end{array}$ & $\begin{array}{l}104998 \\
104987\end{array}$ & $\begin{array}{l}6 \\
4\end{array}$ & $\begin{array}{l}4 \\
2\end{array}$ & $\begin{array}{l}0.0461 \\
0.0415\end{array}$ & ${ }_{00}^{15}$ & $\begin{array}{l}8 . \\
3.2\end{array}$ & $\begin{array}{l}-0.513 \\
-0.921\end{array}$ & $\begin{array}{l}\text { B } \\
\text { B }\end{array}$ & $2 n$ \\
\hline & & & 9814.03 & 94832 & 105020 & 6 & 8 & 0.0118 & 0.0227 & 4.41 & -0.865 & B & $2 n$ \\
\hline & & & $\begin{array}{l}9786.79 \\
0776.00\end{array}$ & 94795 & 105011 & 4 & 6 & 0.0144 & 0.0311 & 4.01 & -0.905 & B & $2 n$, \\
\hline & & & 9776.90 & 94.772 & 104998 & 2 & 4 & 0.0177 & 0.0506 & 3.26 & -0.995 & B & \\
\hline \multirow[t]{2}{*}{36} & & ${ }^{4} \mathrm{P}^{\circ}-{ }^{4} \mathrm{~F}$ & & & 104 & 6 & 8 & & 35 & & -170 & $C$ & \\
\hline & & & 10879.2 & 95495 & 104684 & 4 & 6 & 0.00160 & 0.00426 & 0.61 & -1.77 & C & \\
\hline \multirow[t]{4}{*}{37} & & ${ }^{4} \mathrm{P}^{\circ}-{ }^{2} \mathrm{~F}$ & & & & & & & & & & & \\
\hline & & & 10693.2 & 95533 & 104883 & 6 & 8 & 0.00402 & 0.0092 & 1.94 & -1.259 & C & \\
\hline & & & & & 104811 & 4 & 6 & & & & -0.75 & C & \\
\hline & & & 10775.0 & 95533 & 104811 & 6 & 6 & 0.00421 & 0.0073 & 1.56 & -1.357 & C & \\
\hline \multirow[t]{7}{*}{38} & & ${ }^{4} \mathrm{P}^{\circ}-{ }^{4} \mathrm{P}$ & 10708 & 95511 & 104847 & 12 & 12 & 0.126 & 0.216 & 91.5 & 0.414 & B & $2,6, c a$ \\
\hline & & & 10757.9 & 95533 & 104825 & 6 & 6 & 0.0868 & & 32. & -0.044 & B & \\
\hline & & & & 95 & & 4 & 4 & & & 4.06 & -0 & B & \\
\hline & & & & 95477 & 104886 & 2 & 2 & 0.0215 & 0 & 2.54 & -1.139 & B & \\
\hline & & & & 95533 & 104860 & 6 & 4 & 0.0564 & 0.0647 & 13. & -0.411 & B & \\
\hline & & & 10644.0 & $\begin{array}{l}95495 \\
05405\end{array}$ & 104886 & 4 & 2 & 0.107 & 0.0906 & 12. & -0.441 & B & \\
\hline & & & $\begin{array}{l}10713.6 \\
10653.0\end{array}$ & $\begin{array}{l}95495 \\
95477\end{array}$ & $\begin{array}{l}104825 \\
104860\end{array}$ & $\begin{array}{l}4 \\
2\end{array}$ & $\begin{array}{l}6 \\
4\end{array}$ & $\begin{array}{l}0.0376 \\
0.0532\end{array}$ & $\begin{array}{l}0.0971 \\
0.181\end{array}$ & $\begin{array}{l}13.7 \\
12.7\end{array}$ & $\begin{array}{l}-0.411 \\
-0.441\end{array}$ & $\begin{array}{l}\text { B } \\
\text { B }\end{array}$ & \\
\hline \multirow[t]{5}{*}{39} & & ${ }^{4} \mathrm{P}^{\circ}-{ }^{4} \mathrm{D}$ & 10525 & 95511 & 105010 & 12 & 20 & 0.248 & 0.688 & 286 & 0.917 & B & $2,6, c a$ \\
\hline & & & 10539.6 & 95533 & 105020 & 6 & 8 & 0.24 & 0.538 & & 0.509 & B & \\
\hline & & & 10507.0 & 95495 & 105011 & 4 & 6 & 0.132 & 0.3 & 45.2 & 0.116 & B & \\
\hline & & & 10500.3 & 95477 & 104998 & $\begin{array}{l}2 \\
6\end{array}$ & 4 & 0.0652 & 0.2 & 14. & -0.366 & B & \\
\hline & & & 10549.6 & 95533 & 105011 & & 6 & 0.126 & 0.210 & 43.8 & 0.101 & B & \\
\hline
\end{tabular}


N I. Allowed Transitions - Continued

\begin{tabular}{|c|c|c|c|c|c|c|c|c|c|c|c|c|c|}
\hline No. & $\begin{array}{l}\text { Transition } \\
\text { Array }\end{array}$ & Multiplet & $\lambda(\AA)$ & $E_{i}\left(\mathrm{~cm}^{-1}\right)$ & $E_{k}\left(\mathrm{~cm}^{-1}\right)$ & $g_{i}$ & $g_{k}$ & $A_{k i}\left(10^{8} \sec ^{-1}\right)$ & $f_{i k}$ & $S$ (at.u.) & $\log g f$ & $\begin{array}{c}\text { Accu- } \\
\text { racy }\end{array}$ & Source \\
\hline \multirow[t]{3}{*}{39} & (con.) & & 10520.6 & 95495 & 104998 & 4 & 4 & 0.162 & 0.269 & 37.2 & 0.031 & B & $2 n$ \\
\hline & & & $\begin{array}{l}10513.4 \\
10563.3\end{array}$ & 95477 & 104987 & 2 & 2 & 0.174 & 0.289 & 20.0 & -0.238 & ${ }_{B}^{B}$ & $2 n$ \\
\hline & & & 10533.8 & 95495 & 104987 & 4 & 2 & 0.0405 & 0.0337 & $\begin{array}{l}0.00 \\
4.67\end{array}$ & $\begin{array}{l}-0.000 \\
-0.871\end{array}$ & $\begin{array}{l}\mathrm{D} \\
\mathrm{B}\end{array}$ & $2 n, l s$ \\
\hline \multirow[t]{4}{*}{40} & & ${ }^{4} \mathrm{~S}^{\circ}-{ }^{4} \mathrm{P}$ & 12350 & 96752 & 104847 . & 4 & 12 & 0.124 & 0.85 & 138 & 0.53 & C & $6, c a$ \\
\hline & & & [12384] & 96752 & 104825 & 4 & 6 & 0.123 & 0.423 & 69 & 0.229 & C & $l s$ \\
\hline & & & [12330] & 96752 & 104860 & 4 & 4 & 0.124 & 0.283 & 46.0 & 0.054 & C. & ls \\
\hline & & & & & & & & & & & & & \\
\hline \multirow{3}{*}{41} & & $\begin{array}{c}{ }^{2} \mathrm{D}^{\circ}-{ }^{2} \mathrm{~F} \\
(36)\end{array}$ & 12469 & 96834 & 104852 & 10 & 14 & 0.216 & 0.71 & 290 & 0.85 & C & $6, c a$ \\
\hline & & & 12467.8 & $\begin{array}{l}96864 \\
06788\end{array}$ & 104883 & 6 & 8 & 0.217 & 0.67 & 166 & 0.61 & C & $l s$ \\
\hline & & & $\begin{array}{l}12461.2 \\
12582.3\end{array}$ & $\begin{array}{l}96788 \\
96864\end{array}$ & $\begin{array}{l}104811 \\
104811\end{array}$ & $\begin{array}{l}4 \\
6\end{array}$ & $\begin{array}{l}6 \\
6\end{array}$ & $\begin{array}{l}0.202 \\
0.0141\end{array}$ & $\begin{array}{l}0.71 \\
0.0334\end{array}$ & $\begin{array}{c}116 \\
8.3\end{array}$ & $\begin{array}{l}0.451 \\
-0.70\end{array}$ & $\begin{array}{l}\mathrm{C} \\
\mathrm{C}\end{array}$ & $\begin{array}{ll}l s \\
l s\end{array}$ \\
\hline \multirow[t]{4}{*}{42} & & ${ }^{2} \mathrm{D}^{\circ}-{ }^{2} \mathrm{D}$ & 12043 & 96834 & 105135 & 10 & 10 & 0.060 & 0.130 & 52 & 0.114 & C & $6, c a$ \\
\hline & & & 12074.1 & 96864 & 105144 & 6 & 6 & 0.055 & 0.121 & 28.8 & -0.140 & C & ls \\
\hline & & & $\begin{array}{l}11997.9 \\
12107.4\end{array}$ & $\begin{array}{l}96788 \\
96864\end{array}$ & $\begin{array}{l}105121 \\
105121\end{array}$ & $\begin{array}{l}4 \\
6\end{array}$ & $\begin{array}{l}4 \\
4\end{array}$ & $\begin{array}{l}0.054 \\
0.0059\end{array}$ & $\begin{array}{l}0.117 \\
0.0086\end{array}$ & $\begin{array}{l}18.5 \\
2.06\end{array}$ & $\begin{array}{l}-0.329 \\
-1.287\end{array}$ & $\begin{array}{l}\mathrm{C} \\
\mathrm{C}\end{array}$ & $\begin{array}{l}l s \\
l s\end{array}$ \\
\hline & & & [11964] & 96788 & 105144 & 4 & 6 & 0.00406 & 0.0131 & 2.06 & -1.282 & C & ls \\
\hline \multirow[t]{3}{*}{43} & $2 p^{2\left({ }^{(3} \mathrm{P}\right) 3 p-}-$ & ${ }^{2} \mathrm{P}^{\circ}-{ }^{2} \mathrm{~S}$ & 4389.1 & 97794 & 120572 & 6 & 2 & 0.0153 & 0.00148 & 0.128 & -2.053 & C & 2 \\
\hline & & & [4391.3] & 97806 & 120572 & 4 & 2 & 0.0102 & 0.00147 & 0.085 & -2.231 & $\mathrm{C}$ & $2, l s$ \\
\hline & & & {$[4384.4]$} & 97610 & 120562 & 2 & 2 & 0.0052 & 0.00151 & 0.0435 & -2.52 & $\mathrm{C}$ & \\
\hline \multirow[t]{3}{*}{44} & $2 p^{2} 3 p^{\prime}-$ & ${ }^{2} \mathrm{~F}^{\circ}-{ }^{2} \mathrm{G}$ & 10595 & 110713 & 120149 & 14 & 18 & 0.338 & 0.731 & 357 & 1.010 & B & $2,6, c a$ \\
\hline & & & 10597.0 & 110715 & 120149 & 8 & 10 & 0.337 & 0.709 & 198 & 0.754 & B & ls \\
\hline & & & $\begin{array}{l}10591.9 \\
{[10596]}\end{array}$ & $\begin{array}{l}110711 \\
110715\end{array}$ & $\begin{array}{l}120150 \\
120150\end{array}$ & $\begin{array}{l}6 \\
8\end{array}$ & $\begin{array}{l}8 \\
8\end{array}$ & $\begin{array}{l}0.326 \\
0.0121\end{array}$ & $\begin{array}{l}0.731 \\
0.0204\end{array}$ & $\begin{array}{c}153 \\
5.68\end{array}$ & $\begin{array}{r}0.642 \\
-0.788\end{array}$ & $\begin{array}{l}B \\
\mathrm{P}\end{array}$ & ls \\
\hline \multirow[t]{8}{*}{45} & $2 p^{2} 3 p-$ & ${ }^{4} \mathrm{D}^{\circ}-{ }^{4} \mathrm{P}$ & 11290 & 94839 & 103694 & 20 & 12 & 0.147 & 0.168 & 125 & 0.527 & $C+$ & 26 \\
\hline & $4 s$ & $(17$ & 7 & & & & & & & & & . & $2,0, c a$ \\
\hline & & & $\begin{array}{l}11291.6 \\
11313.9\end{array}$ & $\begin{array}{l}\begin{array}{l}94883 \\
94832\end{array} \\
9\end{array}$ & $\begin{array}{l}103737 \\
103668\end{array}$ & $\begin{array}{l}8 \\
6\end{array}$ & $\begin{array}{l}6 \\
4\end{array}$ & $\mid \begin{array}{l}0.117 \\
0.0920\end{array}$ & $\begin{array}{l}0.168 \\
0.118\end{array}$ & $\begin{array}{l}50.0 \\
26.3\end{array}$ & $\begin{array}{r}0.129 \\
-0151\end{array}$ & $\mathrm{C}+$ & $l s$ \\
\hline & & & 11323.2 & 947 & 103618 & 4 & 2 & 0.0726 & 0.0697 & 10 & $\begin{array}{l}-0.151 \\
-0.554\end{array}$ & $\begin{array}{l}\mathrm{C}+ \\
\mathrm{C}+\end{array}$ & $l s$ \\
\hline & & & 11227.1 & 94832 & 103737 & 6 & 6 & & & 11 & -0.515 & $\mathrm{C}+$ & $l s$ \\
\hline & & & $\begin{array}{l}11266.2 \\
11294.2\end{array}$ & $\begin{array}{l}94795 \\
94772\end{array}$ & 103668 & 4 & 4 & $\begin{array}{l}0.475 \\
0.0731\end{array}$ & 0.0 & 13. & $\begin{array}{l}-0.442 \\
-0.553\end{array}$ & $\mathrm{C}+$ & $l s$ \\
\hline & & & 11180.1 & 94795 & $\begin{array}{l}103618 \\
103737\end{array}$ & 4 & 6 & $\begin{array}{l}0.0631 \\
0.00302\end{array}$ & $\begin{array}{l}0.140 \\
0.00849\end{array}$ & $\begin{array}{c}10.4 \\
1.25\end{array}$ & $\begin{array}{l}-1.469 \\
-1.00\end{array}$ & $\mathrm{C}+$ & $l s$ \\
\hline & & & 11237.6 & 94772 & 103668 & 2 & 4 & 0.00746 & 0.0282 & 2.09 & -1.248 & $\mathrm{C}+$ & $l s$ \\
\hline \multirow[t]{7}{*}{46} & & ${ }^{4} \mathrm{P}^{\circ}-{ }^{4}{ }^{4} \mathrm{P}$ & 12217 & 95511 & 103694 & 12 & 12 & 0.076 & 0.171 & 83 & 0.312 & C & 6, $c a$ \\
\hline & & & 12186.9 & 955 & 103737 & 6 & 6 & 0.05 & 0.1 & 28 & -0.146 & C. & ls \\
\hline & & & 12232.9 & 95495 & 103668 & 4 & 4 & 0.0 & & 3.64 & -1.044 & C. & $l s$ \\
\hline & & & $\begin{array}{l}{[12280]} \\
12288.0\end{array}$ & $\begin{array}{l}95477 \\
95533\end{array}$ & $\begin{array}{l}103618 \\
103668\end{array}$ & $\begin{array}{l}2 \\
6\end{array}$ & $\begin{array}{l}2 \\
4\end{array}$ & \begin{tabular}{|l}
0.0125 \\
0.0336
\end{tabular} & $\begin{array}{l}0.0282 \\
0.051\end{array}$ & $\begin{array}{l}2.28 \\
12.3\end{array}$ & $\begin{array}{l}-1.249 \\
-0.52\end{array}$ & $\begin{array}{l}\mathrm{C} \\
\mathrm{C}\end{array}$ & 5 \\
\hline & & & [12307] & 954 & 103618 & 4 & 2 & 0.06 & 0.0 & 11 & -0.55 & C & ls \\
\hline & & & $\begin{array}{l}12128.6 \\
122034\end{array}$ & $\begin{array}{l}95495 \\
05477\end{array}$ & 103737 & 4 & 6 & 0.0233 & & 12. & -0 & C. & ls \\
\hline & & & $12<0.4$ & 95468 & 103668 & 2 & & 0.0318 & 0.142 & 11.4 & -0.55 & & $s$ \\
\hline \multirow[t]{7}{*}{47} & $\begin{array}{l}2 p^{2} 3 p- \\
\quad 2 p^{2\left({ }^{3} \mathrm{P}\right) 5 s}\end{array}$ & $\begin{array}{l}{ }^{4} \mathrm{D}^{\circ}-{ }^{4} \mathrm{P} \\
(20)\end{array}$ & 6644.0 & 94839 & 109886 & 20 & 12 & 0.0389 & 0.0154 & 6.8 & -0.51 & C & $6, c a$ \\
\hline & & & 6644.96 & 94883 & 109928 & 8 & 6 & 0.0311 & 0.0154 & 2.70 & -0.91 & C & ls \\
\hline & & & $\begin{array}{l}6653.46 \\
6656.51\end{array}$ & $\begin{array}{l}94832 \\
94795\end{array}$ & $\begin{array}{l}109858 \\
109814\end{array}$ & $\begin{array}{l}6 \\
4\end{array}$ & $\begin{array}{l}4 \\
2\end{array}$ & $\begin{array}{l}0.0244 \\
0.0193\end{array}$ & & $\begin{array}{l}1.4 \\
0.5\end{array}$ & $\begin{array}{l}-1.188 \\
-1.59\end{array}$ & $\begin{array}{l}\mathrm{C} \\
\mathrm{C}\end{array}$ & is \\
\hline & & & 6622.54 & 94832 & 109928 & 6 & 6 & 0.007 & 0.0 & 0. & -1.55 & $\mathrm{C}$ & ( \\
\hline & & & 6636.94 & 94795 & 109858 & 4 & 4 & 0.0125 & 0.0083 & 0.7 & -1.481 & C & 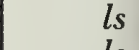 \\
\hline & & & 6646.51 & 94772 & 109814 & 2 & 2 & 0.0194 & 0.0128 & 0.5 & -1.59 & C. & /s \\
\hline & & & {$[6606.3]$} & 94772 & $\begin{array}{l}109928 \\
109858\end{array}$ & $\begin{array}{l}4 \\
2\end{array}$ & $\begin{array}{l}6 \\
4\end{array}$ & $\begin{array}{l}7.9 \times 10^{-4} \\
0.00197\end{array}$ & $\begin{array}{l}7.8 \times 10^{-} \\
0.00259\end{array}$ & $\begin{array}{l}0.068 \\
0.113\end{array}$ & $\begin{array}{l}-2.51 \\
-2.286\end{array}$ & $\begin{array}{l}\mathrm{C} \\
\mathrm{C}\end{array}$ & $l s$ \\
\hline
\end{tabular}


N I. Allowed Transitions-Continued

\begin{tabular}{|c|c|c|c|c|c|c|c|c|c|c|c|c|c|}
\hline No. & $\begin{array}{c}\text { Transition } \\
\text { Array }\end{array}$ & Multiplet & $\lambda(\AA)$ & $E_{i}\left(\mathrm{~cm}^{-1}\right)$ & $E_{k}\left(\mathrm{~cm}^{-1}\right)$ & $g_{i}$ & $g_{k}$ & $A_{k i}\left(10^{x} \sec ^{-1}\right)$ & $f_{i k}$ & $S($ at.u.) & $\log g f$ & $\begin{array}{l}\text { Accu- } \\
\text { racy }\end{array}$ & Source \\
\hline \multirow[t]{8}{*}{48} & & $\begin{array}{c}{ }^{4} \mathrm{P}^{\circ}-{ }^{4} \mathrm{P} \\
(29)\end{array}$ & 6954.6 & 95511 & 109886 & 12 & 12 & 0.0212 & 0.0154 & 4.22 & -0.73 & C & $6, c a$ \\
\hline & & & 6945.22 & 95533 & 109928 & 6 & 6 & 0.0149 & 0.0108 & 1.48 & -1.189 & C & $l s$ \\
\hline & & & [6960.4] & 95495 & 109858 & 4 & 4 & 0.00281 & 0.00204 & 0.187 & -2.088 & $\mathrm{C}$ & ls \\
\hline & & & [6973.0] & 95477 & 109814 & 2 & 2 & 0.00350 & 0.00255 & 0.117 & -2.293 & C & ls \\
\hline & & & 6979.10 & 95533 & 109858 & 6 & 4 & 0.0094 & 0.00459 & 0.63 & -1.56 & $\mathrm{C}$ & ls \\
\hline & & & [6981.8] & 95495 & 109814 & 4 & 2 & 0.0174 & 0.0064 & 0.59 & -1.59 & $\mathrm{C}$ & ls \\
\hline & & & 6926.90 & 95495 & 109928 & 4 & 6 & 0.0064 & 0.0069 & 0.63 & -1.56 & C & ls \\
\hline & & & [0951.7] & 95477 & 109858 & 2 & 4 & 0.0088 & 0.0128 & 0.59 & -1.59 & C & ls \\
\hline \multirow[t]{3}{*}{49} & $\begin{array}{l}2 p^{2} 3 p- \\
\quad 2 p^{2}\left({ }^{3} \mathrm{P}\right) 5 d\end{array}$ & ${ }^{2} \mathrm{~S}^{\circ}-{ }^{2} \mathrm{P}$ & 5200.5 & 93582 & 112806 & 2 & 6 & 0.023 & 0.028 & 0.96 & -1.25 & D & $3 n$ \\
\hline & & & [5201.8] & 93582 & 112801 & 2 & 4 & 0.023 & 0.019 & 0.64 & -1.43 & D & $l s$ \\
\hline & & & [5197.8] & 93582 & 112816 & 2 & 2 & 0.023 & 0.0094 & 0.32 & -1.73 & D & ls \\
\hline \multirow[t]{7}{*}{50} & $\begin{array}{l}2 p^{2} 3 p- \\
2 p^{2}\left({ }^{3} \mathrm{P}\right) 6 s\end{array}$ & $\begin{array}{c}{ }^{4} \mathrm{P}^{\circ}-{ }^{4} \mathrm{P} \\
(32)\end{array}$ & 5836.4 & 95511 & 112640 & 12 & 12 & 0.0092 & 0.00468 & 1.08 & -1.250 & C & $6, c a$ \\
\hline & & & 5829.53 & 95533 & 112683 & 6 & 6 & 0.0064 & 0.00328 & 0.378 & -1.71 & $\mathrm{C}$ & $l s$ \\
\hline & & & 5841.01 & 95495 & 112611 & 4 & 4 & 0.00122 & $6.2 \times 10^{-4}$ & 0.0480 & -2.60 & C & ls \\
\hline & & & $\begin{array}{r}{[5850.1]} \\
5854.16\end{array}$ & 95511 & $\begin{array}{l}112566 \\
112611\end{array}$ & $\begin{array}{l}2 \\
6\end{array}$ & $\begin{array}{l}2 \\
4\end{array}$ & $\begin{array}{l}0.00152 \\
0.00409\end{array}$ & $\begin{array}{l}7.8 \times 10^{-4} \\
0.00140\end{array}$ & $\begin{array}{l}0.0300 \\
0.162\end{array}$ & $\begin{array}{l}-2.81 \\
-2075\end{array}$ & C & ls \\
\hline & & & [5856.3] & 95495 & 112566 & 4 & 2 & 0.0076 & 0.00195 & 0.150 & -2.109 & $\mathrm{C}$ & $l \mathrm{~s}$ \\
\hline & & & 5816.48 & 95495 & 112683 & 4 & 6 & 0.00278 & 0.00212 & 0.162 & -2.073 & C & ls \\
\hline & & & [5834.7] & 95477 & 112611 & 2 & 4 & 0.00383 & 0.00390 & 0.150 & -2.107 & C & ls \\
\hline
\end{tabular}

\section{Forbidden Transitions}

For this ion we have performed the numerical calculations by utilizing Ufford and Gilmour's [1] values for the parameters $\zeta$ and $\eta$ and empirical term intervals, and by employing the general expressions of Shortley, Aller, Baker, and Menzel [2] for the line strengths in the $p^{3}$ configuration (see also general introduction). For the electric quadrupole lines we have employed Garstang's [3] estimate of the quadrupole integral $s_{q}$. For the ${ }^{2} \mathrm{D}^{\circ}-{ }^{2} \mathrm{D}^{\circ}$ transition a difference between the transition probability quoted by Garstang [4] and the tabulated value will be noticed. This is due to a revised experimental value for the term interval as given by Bowen [5].

\section{References}

[1] Ufford, C. W., and Gilmour, R. M., Astrophys. J. 111, 580-581 (1950).

[2] Shortley, G. H., Aller, L. H., Baker, J. G., and Menzel, D. H., Astrophys. J. 93, 178-184 (1941).

[3] Garstang, R. H., Astrophys. J. 115, 506-508 (1952).

[4] Garstang, R. H., "The Airglow and the Aurora," p. 324 (ed. Armstrong and Dalgarno, Pergamon Press, New York, 1956).

[5] Bowen, I. S., Astrophys. J. 121, 306 (1955).

N 1. Forbidden Transitions

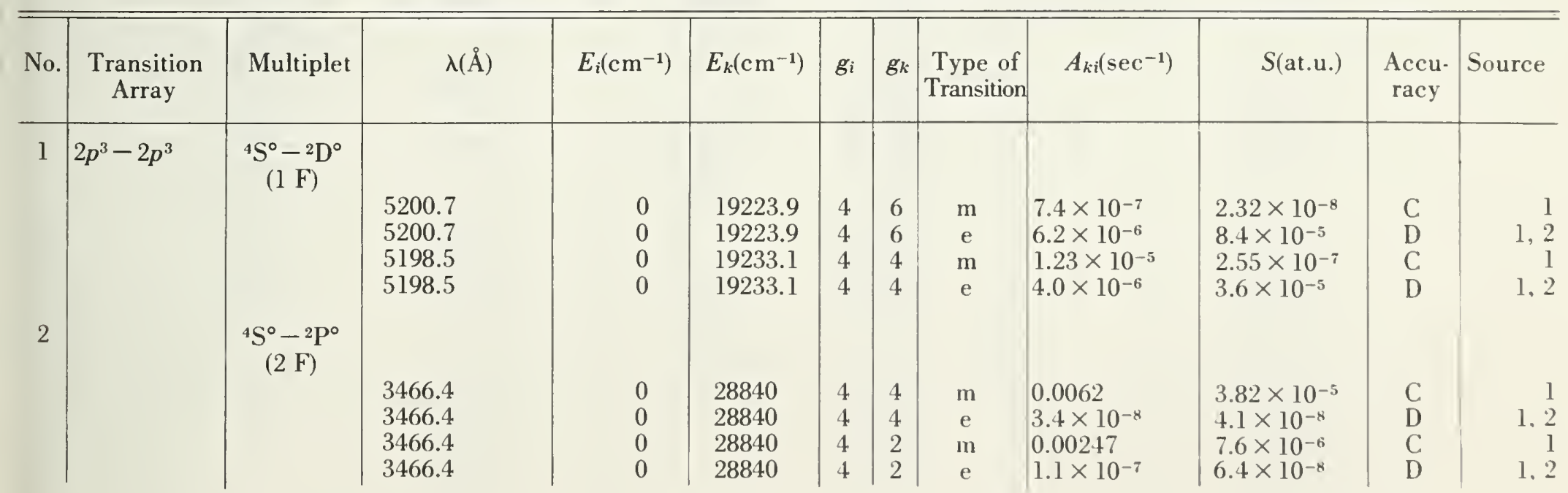


N I. Forbidden Transitions-Continued

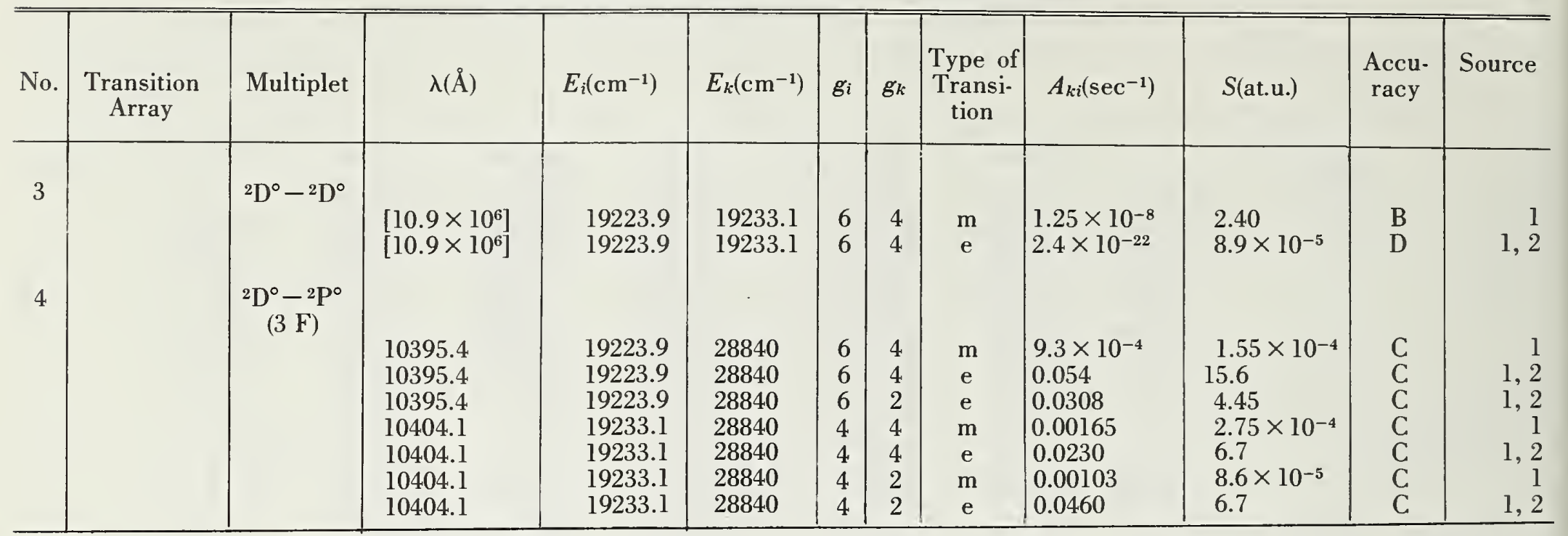

\section{N II}

Ground State

Ionization Potential
$1 s^{2} 2 s^{2} 2 p^{2}{ }^{3} \mathrm{P}_{0}$

$29.593 \mathrm{eV}=238751.1 \mathrm{~cm}^{-1}$

Allowed Transitions

List of tabulated lines:

\begin{tabular}{l|r||c|c||c|c}
\hline \hline Wavelength $[\AA]$ & No. & Wavelength $[\AA]$ & No. & Wavelength $[\AA]$ & No. \\
\hline & & & & & \\
529.343 & 10 & 745.836 & 6 & 2493.94 & 47 \\
529.405 & 10 & 746.976 & 8 & 2496.88 & 47 \\
529.481 & 10 & 775.957 & 4 & 2520.27 & 46 \\
529.627 & 10 & 915.603 & 2 & 2520.85 & 46 \\
529.713 & 10 & 915.955 & 2 & 2522.27 & 46 \\
529.860 & 10 & 916.004 & 2 & 2522.46 & 46 \\
533.504 & 9 & 916.700 & 2 & 2524.49 & 46 \\
533.51 & 9 & 1083.98 & 1 & 2526.17 & 46 \\
533.577 & 9 & 1084.57 & 1 & 2709.82 & 49 \\
533.644 & 9 & 1085.5 & 1 & 2799.20 & 48 \\
& & & & \\
533.726 & 9 & 1085.54 & 1 & 3006.86 & 38 \\
533.809 & 9 & 1085.70 & 1 & 3023.80 & 50 \\
572.07 & 13 & 188.82 & 24 & 3311.4 & 39 \\
574.650 & 12 & 2206.10 & 44 & 3318.14 & 39 \\
582.150 & 11 & 2316.46 & 45 & 3324.58 & 39 \\
635.180 & 14 & 2316.65 & 45 & 3328.79 & 39 \\
644.621 & 3 & 231.61 & 45 & 3330.30 & 39 \\
644.825 & 3 & 2319.94 & 45 & 3331.32 & 39 \\
645.167 & 3 & 2321.65 & 45 & 3437.16 & 20 \\
660.280 & 5 & 2325.16 & 45 & 3593.60 & 40 \\
671.014 & 7 & 2461.30 & 51 & 3609.09 & 40 \\
671.391 & 7 & 2488.12 & 47 & 3615.88 & 40 \\
671.629 & 7 & 2488.75 & 47 & 3829.80 & 41 \\
671.770 & 7 & 2490.37 & 47 & 3838.39 & 41 \\
671.999 & 7 & 2493.16 & 47 & 3842.20 & 41 \\
& & & & &
\end{tabular}


List of tabulated lines-Continued

\begin{tabular}{|c|c|c|c|c|c|}
\hline Wavelength $[\AA]$ & No. & Wavelength $[\hat{\Lambda}]$ & No. & Wavelength $[\AA]$ & No. \\
\hline $\begin{array}{l}3847.38 \\
3855.08 \\
3856.07 \\
3919.01 \\
3995.00\end{array}$ & $\begin{array}{l}41 \\
41 \\
41 \\
26 \\
19\end{array}$ & $\begin{array}{l}5012.03 \\
5016.39 \\
5023.11 \\
5025.67 \\
5040.76\end{array}$ & $\begin{array}{l}22 \\
27 \\
22 \\
27 \\
27\end{array}$ & $\begin{array}{l}5941.67 \\
5952.39 \\
5954.28 \\
5960.93 \\
6114.6\end{array}$ & $\begin{array}{l}31 \\
31 \\
60 \\
31 \\
52\end{array}$ \\
\hline $\begin{array}{l}4026.08 \\
4040.9 \\
4124.08 \\
4133.67 \\
4145.76\end{array}$ & $\begin{array}{l}62 \\
61 \\
23 \\
23 \\
23\end{array}$ & $\begin{array}{l}5045.10 \\
5104.45 \\
5168.24 \\
5170.08 \\
5171.30\end{array}$ & $\begin{array}{l}16 \\
43 \\
37 \\
37 \\
37\end{array}$ & $\begin{array}{l}6136.89 \\
6150.76 \\
6167.82 \\
6170.16 \\
6173.40\end{array}$ & $\begin{array}{l}52 \\
52 \\
52 \\
52 \\
52\end{array}$ \\
\hline $\begin{array}{l}4176.16 \\
4227.75 \\
4239.4 \\
4417.9 \\
4427.97\end{array}$ & $\begin{array}{l}63 \\
42 \\
64 \\
65 \\
65\end{array}$ & $\begin{array}{l}5171.46 \\
5172.32 \\
5173.37 \\
5174.46 \\
5175.89\end{array}$ & $\begin{array}{l}37 \\
35 \\
35 \\
37 \\
35\end{array}$ & $\begin{array}{l}6242.52 \\
6284.30 \\
6318.80 \\
6328.39 \\
6340.57\end{array}$ & $\begin{array}{l}59 \\
34 \\
55 \\
55 \\
55\end{array}$ \\
\hline $\begin{array}{l}4431.82 \\
4432.74 \\
4433.48 \\
4441.99 \\
4447.03\end{array}$ & $\begin{array}{l}65 \\
65 \\
65 \\
65 \\
25\end{array}$ & $\begin{array}{l}5176.56 \\
5177.06 \\
5179.50 \\
5179.50 \\
5180.34\end{array}$ & $\begin{array}{l}37 \\
35 \\
35 \\
37 \\
35\end{array}$ & $\begin{array}{l}6346.86 \\
6356.55 \\
6357.57 \\
6482.07 \\
6491.79\end{array}$ & $\begin{array}{l}55 \\
55 \\
55 \\
18 \\
54\end{array}$ \\
\hline $\begin{array}{l}4459.96 \\
4465.54 \\
4475.8 \\
4477.74 \\
4488.15\end{array}$ & $\begin{array}{l}29 \\
29 \\
29 \\
29 \\
29\end{array}$ & $\begin{array}{l}5183.21 \\
5184.96 \\
5186.17 \\
5190.42 \\
5191.97\end{array}$ & $\begin{array}{l}37 \\
35 \\
37 \\
35 \\
35\end{array}$ & $\begin{array}{l}6504.61 \\
6522.39 \\
6532.55 \\
6544.16 \\
6545.53\end{array}$ & $\begin{array}{l}54 \\
54 \\
54 \\
54 \\
54\end{array}$ \\
\hline $\begin{array}{l}4507.56 \\
4530.40 \\
4552.54 \\
4601.48 \\
4607.16\end{array}$ & $\begin{array}{l}29 \\
67 \\
66 \\
17 \\
17\end{array}$ & $\begin{array}{l}5199.50 \\
5313.43 \\
5320.96 \\
5327.45 \\
5338.66\end{array}$ & $\begin{array}{l}35 \\
36 \\
36 \\
36 \\
36\end{array}$ & $\begin{array}{l}6554.47 \\
6610.58 \\
6629.80 \\
6809.99 \\
6834.09\end{array}$ & $\begin{array}{l}54 \\
33 \\
53 \\
58 \\
58\end{array}$ \\
\hline $\begin{array}{l}4613.87 \\
4621.39 \\
4630.54 \\
4643.09 \\
4677.93\end{array}$ & $\begin{array}{l}17 \\
17 \\
17 \\
17 \\
68\end{array}$ & $\begin{array}{l}5340.20 \\
5351.21 \\
5452.12 \\
5454.26 \\
5462.62\end{array}$ & $\begin{array}{l}36 \\
36 \\
32 \\
32 \\
32\end{array}$ & $\begin{array}{l}6847.24 \\
6941.75 \\
6966.81 \\
6975.64 \\
7003.0\end{array}$ & $\begin{array}{l}58 \\
57 \\
57 \\
57 \\
57\end{array}$ \\
\hline $\begin{array}{l}4774.22 \\
4779.71 \\
4781.17 \\
4788.13 \\
4793.66\end{array}$ & $\begin{array}{l}28 \\
28 \\
28 \\
28 \\
28\end{array}$ & $\begin{array}{l}5478.13 \\
5480.10 \\
5495.70 \\
5526.26 \\
5530.27\end{array}$ & $\begin{array}{l}32 \\
32 \\
32 \\
21 \\
21\end{array}$ & $\begin{array}{l}7013.98 \\
7014.73 \\
7138.87 \\
7188.20 \\
7214.6\end{array}$ & $\begin{array}{l}57 \\
57 \\
56 \\
56 \\
56\end{array}$ \\
\hline $\begin{array}{l}4803.27 \\
4810.29 \\
4987.38 \\
4991.22 \\
4994.36\end{array}$ & $\begin{array}{l}28 \\
28 \\
30 \\
22 \\
22\end{array}$ & $\begin{array}{l}5535.39 \\
5540.16 \\
5543.49 \\
5551.95 \\
5552.54\end{array}$ & $\begin{array}{l}21 \\
21 \\
21 \\
21 \\
21\end{array}$ & $\begin{array}{l}7215.06 \\
7241.8 \\
7256.53\end{array}$ & $\begin{array}{l}56 \\
56 \\
56\end{array}$ \\
\hline $\begin{array}{l}4994.36 \\
4997.23 \\
5001.13 \\
5001.47 \\
5002.69\end{array}$ & $\begin{array}{l}30 \\
22 \\
27 \\
27 \\
16\end{array}$ & $\begin{array}{l}5565.30 \\
5666.64 \\
5676.02 \\
5679.56 \\
5686.21\end{array}$ & $\begin{array}{l}21 \\
15 \\
15 \\
15 \\
15\end{array}$ & & \\
\hline $\begin{array}{l}5005.14 \\
5005.14 \\
5007.32 \\
5010.62 \\
5011.24\end{array}$ & $\begin{array}{l}22 \\
27 \\
30 \\
16 \\
22 \\
\end{array}$ & $\begin{array}{l}5710.76 \\
5730.67 \\
5927.82 \\
5931.79 \\
5940.25\end{array}$ & $\begin{array}{l}15 \\
15 \\
31 \\
31 \\
31\end{array}$ & & \\
\hline
\end{tabular}


Data for the vacuum ultraviolet region of the spectrum are available from calculations of Bolotin et al. [1] and Kelly [2]. Bolotin et al. employ a "double configuration" approximation, i.e., they include to a first approximation the effects of configuration interaction which are expected to be drastic for these transitions. Kelly's calculations, in which these effects are neglected, are only used for some vacuum uv transitions for which configuration interaction is expected to be less pronounced. Nevertheless, his results may be quite uncertain. For higher excited lines, mainly in the visible, experimental work with a high current stabilized arc by Mastrup and Wiese [3] compares very well with approximate self-consistent field calculations by Kelly [4] and the Coulomb approximation. All these methods have been equally weighted in arriving at averaged values. However, the Coulomb approximation shows strong cancellation effects for $3 s-4 p$ and $3 p-4 d$ transitions and is not used there. Kelly's calculations, which seem to be less affected as judged from his fairly high ratios between the positive and negative contributions to the transition integrals, are exclusively used in these cases. For some multiplets with high azimuthal quantum numbers in the $3 d-4 f$ array (D-F and F-G) only multiplet values and no line values are listed, since intensity measurements by Eriksson [5] indicate considerable deviations from LS coupling and a transition to pair coupling.

\section{References}

[1] Bolotin, A. B., Levinson, I. B., and Levin, L. I., Soviet Phys. -JETP 2, 391-395 (1956).

[2] Kelly, P. S., Astrophys. J. 140, 1247-1268 (1964).

[3] Mastrup, F., and Wiese, W., Z. Astrophys. 44, 259-279 (1958).

[4] Kelly, P. S., J. Quant. Spectrosc. Radiat. Transfer 4, 117-148 (1964).

[5] Eriksson, K. B. S., Arkiv Fysik 13, 303-329 (1958).

NII. Allowed Transitions

\begin{tabular}{|c|c|c|c|c|c|c|c|c|c|c|c|c|c|}
\hline No. & $\begin{array}{c}\text { Transition } \\
\text { Array }\end{array}$ & Multiplet & $\lambda(\AA)$ & $E_{i}\left(\mathrm{~cm}^{-1}\right)$ & $E_{k}\left(\mathrm{~cm}^{-1}\right)$ & $g_{i}$ & $g_{k}$ & $A_{k i}\left(10^{8} \sec ^{-1}\right)$ & $f_{i k}$ & $S($ at.u.) & $\log g f$ & $\begin{array}{l}\text { Accu- } \\
\text { racy }\end{array}$ & Source \\
\hline \multirow[t]{6}{*}{1} & $2 s^{2} 2 p^{2}-2 s 2 p^{3}$ & $\begin{array}{c}{ }^{3} \mathrm{P}-{ }^{3} \mathrm{D}^{\circ} \\
\text { (luv) }\end{array}$ & 1085.1 & 89.3 & 92245 & 9 & 15 & 5.7 & 0.17 & 5.4 & 0.18 & $\mathrm{E}$ & 1 \\
\hline & & & 1085.70 & 131.3 & 92238 & 5 & 7 & 5.7 & 0.14 & 2.5 & -0.15 & $\mathrm{E}$ & ls \\
\hline & & & 1084.57 & 49.1 & 92251 & & 5 & 4.3 & 0.13 & 1.4 & -0.42 & $\mathrm{E}$ & ls \\
\hline & & & \begin{tabular}{|l|l|l|}
1083.98 \\
1085
\end{tabular} & $\begin{array}{r}0.0 \\
\end{array}$ & 92253 & $\begin{array}{l}1 \\
5\end{array}$ & $\begin{array}{l}3 \\
5 \\
\end{array}$ & 3.2 & 0.17 & 0.60 & -0.77 & $\mathrm{E}$ & ls \\
\hline & & & $\begin{array}{l}1085.54 \\
1084.57\end{array}$ & $\begin{array}{r}31.3 \\
49.1\end{array}$ & $\begin{array}{l}92251 \\
92253\end{array}$ & $\begin{array}{l}5 \\
3\end{array}$ & $\begin{array}{l}5 \\
3\end{array}$ & $\begin{array}{l}1.4 \\
2.4\end{array}$ & $\begin{array}{l}0.025 \\
0.042\end{array}$ & $\begin{array}{l}0.45 \\
0.45\end{array}$ & $\begin{array}{l}-0.90 \\
-0.90\end{array}$ & $\begin{array}{l}\mathrm{E} \\
\mathrm{E}\end{array}$ & $\begin{array}{l}l s \\
l s\end{array}$ \\
\hline & & & [1085.5] & 131.3 & 92253 & 5 & 3 & 0.16 & 0.0017 & 0.030 & -2.07 & $\mathrm{E}$ & ls \\
\hline \multirow[t]{6}{*}{2} & & $\begin{array}{c}{ }^{3} \mathrm{P}-{ }^{3} \mathrm{P}^{\circ} \\
(2 \mathrm{uv})\end{array}$ & 916.34 & 89.3 & 109219 & 9 & 9 & 18 & 0.22 & 6.0 & 0.30 & E & $\begin{array}{lll}1 & \end{array}$ \\
\hline & & & 916.700 & 131.3 & 109218 & 5 & 5 & 13 & 0.17 & 2.5 & -0.08 & $\mathrm{E}$ & $l s$ \\
\hline & & & 916.004 & 49.1 & $\begin{array}{l}109218 \\
189218\end{array}$ & 3 & $\begin{array}{l}3 \\
3 \\
2\end{array}$ & $\begin{array}{l}4.4 \\
7.2\end{array}$ & 0.055 & 0.50 & -0.78 & $\mathrm{E}$ & ls \\
\hline & & & $\begin{array}{l}910.700 \\
915.955\end{array}$ & $\begin{array}{r}131.3 \\
49.1\end{array}$ & $\begin{array}{l}109218 \\
109225\end{array}$ & $\begin{array}{l}5 \\
3\end{array}$ & $\begin{array}{l}3 \\
1\end{array}$ & 183 & $\begin{array}{l}0.055 \\
0.074\end{array}$ & $\begin{array}{l}0.84 \\
0.67\end{array}$ & $\begin{array}{l}-0.56 \\
-0.65\end{array}$ & $\mathrm{E}$ & ls \\
\hline & & & 916.004 & 49.1 & 109218 & 3 & 5 & 4.4 & 0.093 & 0.84 & -0.56 & $\mathrm{E}$ & ls \\
\hline & & & 915.603 & 0.0 & 109218 & 1 & 3 & 5.9 & 0.22 & 0.67 & -0.65 & $\mathrm{E}$ & ls \\
\hline \multirow[t]{4}{*}{3} & & $\begin{array}{c}{ }^{3} \mathrm{P}-{ }^{-3} \mathrm{~S}^{\circ} \\
(4 \mathrm{uv})\end{array}$ & 644.99 & 89.3 & 155130 & 9 & 3 & 110 & 0.23 & 4.4 & 0.32 & E & 1 \\
\hline & & & 645.167 & 131.3 & 155130 & 5 & 3 & 62 & 0.23 & 2.4 & 0.06 & E & ls \\
\hline & & & 644.825 & 49.1 & 155130 & 3 & $\begin{array}{l}3 \\
3\end{array}$ & 37 & 0.23 & 1.5 & -0.16 & E & $l s$ \\
\hline & & & 644.621 & 0.0 & 155130 & 1 & 3 & 12 & 0.23 & 0.49 & -0.64 & $\mathrm{E}$ & ls \\
\hline 4 & & $\begin{array}{c}{ }^{1} \mathrm{D}-{ }^{-} \mathrm{D}^{\circ} \\
(7 \mathbf{u v})\end{array}$ & 775.957 & 15316 & 144189 & 5 & 5 & 49 & 0.45 & 5.7 & 0.35 & $\mathrm{E}$ & 1 \\
\hline 5 & & $\begin{array}{l}{ }^{1} \mathrm{D}-{ }^{-1} \mathrm{P}^{\circ} \\
(9 \mathrm{uv})\end{array}$ & 660.280 & 15316 & 166766 & 5 & 3 & 77 & 0.30 & 3.3 & 0.18 & $\mathrm{E}$ & 1 \\
\hline 6 & & $\begin{array}{c}{ }^{1} S-{ }^{-1} \mathrm{P}^{\circ} \\
(12 \mathrm{uv})\end{array}$ & 745.836 & 32687 & 166766 & 1 & 3 & 16 & 0.40 & 0.98 & -0.40 & $\mathrm{E}$ & 1 \\
\hline
\end{tabular}


N II. Allowed Transitions-Continued

\begin{tabular}{|c|c|c|c|c|c|c|c|c|c|c|c|c|c|}
\hline No. & $\begin{array}{c}\text { Transition } \\
\text { Array }\end{array}$ & Multiplet & $\lambda(\AA)$ & $E_{i}\left(\mathrm{~cm}^{-1}\right)$ & $E_{k}\left(\mathrm{~cm}^{-1}\right)$ & $g_{i}$ & $g_{k}$ & $A_{\text {hid }}\left(10^{\kappa} \sec ^{-1}\right)$ & $f_{i k}$ & S(at.u.) & $\log g f$ & $\begin{array}{c}\text { Accu- } \\
\text { racy }\end{array}$ & Source \\
\hline \multirow[t]{7}{*}{7} & $\begin{array}{l}2 p^{2}- \\
2 p\left({ }^{2} \mathrm{P}^{\circ}\right) 3 s\end{array}$ & $\begin{array}{c}{ }^{3} \mathbf{P}-{ }^{3} \mathbf{P}^{\circ} \\
(3 u v)\end{array}$ & 671.48 & 89.3 & 149013 & 9 & 9 & 13 & 0.089 & 1.8 & -0.10 & $\mathrm{E}$ & 2 \\
\hline & & & 671.391 & 131.3 & 149077 & 5 & 5 & 9.9 & 0.067 & 0.74 & -0.48 & $\mathrm{E}$ & ls \\
\hline & & & 671.629 & 49.1 & 148941 & 3 & 3 & 3.3 & 0.022 & 0.15 & -1.18 & $\mathrm{E}$ & ls \\
\hline & & & 671.999 & 131.3 & 148941 & 5 & 3 & 5.5 & 0.022 & 0.25 & -0.95 & $\mathrm{E}$ & ls \\
\hline & & & 671.770 & 49.1 & 148909 & 3 & 1 & 13 & 0.030 & 0.20 & -1.05 & $\mathrm{E}$ & ls \\
\hline & & & 671.014 & 49.1 & 149077 & 3 & 5 & 3.3 & 0.037 & 0.25 & -0.95 & $\mathrm{E}$ & ls \\
\hline & & & 671.391 & 0.0 & 14894.1 & 1 & 3 & 4.4 & 0.89 & 0.20 & -1.05 & $\mathrm{E}$ & ls \\
\hline 8 & & $\begin{array}{c}{ }^{1} \mathrm{D}-{ }^{1} \mathrm{P}^{\circ} \\
(8 \mathrm{uv})\end{array}$ & 746.976 & 15316 & 149189 & 5 & 3 & 20 & 0.10 & 1.2 & -0.30 & $\mathrm{E}$ & 2 \\
\hline \multirow[t]{7}{*}{9} & $\begin{array}{l}2 p^{2}- \\
2 p\left({ }^{2} \mathrm{P}^{\circ}\right) 3 d\end{array}$ & $\begin{array}{c}{ }^{3} \mathrm{P}-{ }^{3} \mathrm{D}^{\circ} \\
(5 \mathrm{uv})\end{array}$ & 533.67 & 89.3 & 187472 & 9 & 15 & 36 & 0.26 & 4.1 & 0.37 & $\mathrm{D}-$ & 2 \\
\hline & & & 533.726 & 131.3 & 187493 & 5 & 7 & 36 & 0.22 & 1.9 & 0.04 & $\mathrm{D}-$ & ls \\
\hline & & & 533.577 & 49.1 & 187462 & 3 & 5 & 27 & 0.19 & 1.0 & -0.23 & $\mathrm{D}-$ & ls \\
\hline & & & 533.504 & 0.0 & 187438 & 1 & 3 & 20 & 0.26 & 0.45 & -0.59 & $\mathrm{D}-$ & ls \\
\hline & & & 533.809 & 131.3 & 187462 & 5 & 5 & 9.1 & 0.039 & 0.34 & -0.71 & $\mathrm{D}-$ & ls \\
\hline & & & 533.644 & 49.1 & 187438 & 3 & 3 & 15 & 0.065 & 0.34 & -0.71 & D- & ls \\
\hline & & & [533.51] & 0.0 & 187438 & 5 & 3 & 1.0 & 0.0026 & 0.023 & -1.89 & $\mathrm{D}-$ & $l s$ \\
\hline \multirow[t]{7}{*}{10} & & $\begin{array}{c}{ }^{3} \mathrm{P}-{ }^{3} \mathrm{P}^{\circ} \\
\text { (6uv) }\end{array}$ & 529.68 & 89.3 & 188884 & 9 & 9 & 20 & 0.082 & 1.3 & -0.13 & $\mathrm{D}-$ & 2 \\
\hline & & & 529.860 & 131.3 & 188858 & 5 & 5 & 15 & 0.062 & 0.54 & -0.51 & $\mathrm{D}-$ & $l s$ \\
\hline & & & 529.481 & 49.1 & 188910 & 3 & 3 & 4.9 & 0.020 & 0.11 & -1.21 & $\mathrm{D}-$ & ls \\
\hline & & & 529.713 & 131.3 & 188910 & 5 & 3 & 8.1 & 0.021 & 0.18 & -0.99 & $\mathrm{D}-$ & ls \\
\hline & & & 529.405 & 49.1 & 188938 & 3 & 1 & 20 & 0.027 & 0.14 & -1.09 & $\mathrm{D}-$ & ls \\
\hline & & & 529.627 & 49.1 & 188858 & 3 & 5 & 4.9 & 0.034 & 0.18 & -0.99 & D- & ls \\
\hline & & & 529.343 & 0.0 & 188910 & 1 & 3 & 6.5 & 0.082 & 0.14 & -1.09 & $\mathrm{D}-$ & $l s$ \\
\hline 11 & & $\begin{array}{c}{ }^{1} \mathrm{D}-{ }^{1} \mathrm{D}^{\circ} \\
(10 \mathrm{uv})\end{array}$ & 582.150 & 15316 & 187092 & 5 & 5 & 13 & 0.064 & 0.61 & -0.50 & $\mathrm{D}-$ & 2 \\
\hline 12 & & $\begin{array}{c}{ }^{1} \mathrm{D}-{ }^{1} \mathrm{~F}^{\circ} \\
(1 \mathrm{l} \mathbf{u v})\end{array}$ & 574.650 & 15316 & 189336 & 5 & 7 & 35 & 0.24 & 2.3 & 0.08 & $\mathrm{D}-$ & 2 \\
\hline 13 & & ${ }^{1} \mathrm{D}-{ }^{1} \mathrm{P}^{0}$ & [572.07] & 15316 & 190121 & 5 & 3 & 0.97 & 0.0029 & 0.0270 & -1.84 & $\mathrm{D}-$ & 2 \\
\hline 14 & & $\begin{array}{c}{ }^{1} S-{ }^{1} P^{\circ} \\
(13 u v)\end{array}$ & 635.180 & 32687 & 190121 & 1 & 3 & 18 & 0.32 & 0.68 & -0.49 & $\mathrm{D}-$ & 2 \\
\hline \multirow[t]{7}{*}{15} & $\begin{array}{l}2 p 3 s- \\
2 p\left(^{2} \mathrm{P}^{\circ} 3 p\right.\end{array}$ & ${ }^{3} \mathrm{P}^{\circ}-{ }^{3} \mathrm{D}$ & 5679.4 & 149013 & 166616 & 9 & 15 & 0.56 & 0.452 & 76 & 0.61 & C & $3,4, c a$ \\
\hline & & & 5679.56 & 149077 & 166679 & 5 & 7 & 0.56 & 0.380 & 35.5 & 0.278 & C & ls \\
\hline & & & 5666.64 & 148941 & 166583 & 3 & 5 & 0.423 & 0.339 & 19.0 & 0.008 & C & $l s$ \\
\hline & & & 5676.02 & 148909 & 166522 & 1 & 3 & 0.310 & 0.450 & 8.4 & -0.347 & $\mathrm{C}$ & ls \\
\hline & & & 5710.76 & 149077 & 166583 & 5 & 5 & 0.137 & 0.067 & 6.3 & -0.475 & C & ls \\
\hline & & & 5686.21 & 148941 & 166522 & 3 & 3 & 0.231 & 0.112 & 6.3 & -0.473 & C & ls \\
\hline & & & & & & & & & & & & & \\
\hline \multirow{4}{*}{16} & & ${ }^{3} \mathrm{P}^{\circ}-{ }^{3} \mathrm{~S}$ & 5028.8 & 149013 & 168893 & 9 & 3 & 0.76 & 0.097 & 14.4 & -0.061 & C & $3,4, c a$ \\
\hline & & & 5045.10 & 149077 & 168893 & 5 & 3 & 0.410 & 0.094 & 7.8 & -0.328 & C & $3 n$ \\
\hline & & & 5010.62 & 148941 & 168893 & 3 & 3 & 0.268 & 0.101 & 5.0 & -0.52 & C & $3 n$ \\
\hline & & & 5002.69 & 148909 & 168893 & 1 & 3 & 0.085 & 0.095 & 1.57 & -1.021 & $\mathrm{C}$ & $3 n$ \\
\hline \multirow[t]{7}{*}{17} & & ${ }^{3} \mathrm{P}^{\circ}-{ }^{3} \mathrm{P}$ & 4623.2 & 149013 & 170637 & 9 & 9 & 1.05 & 0.337 & 46.1 & 0.481 & C & $3,4, c a$ \\
\hline & & & 4630.54 & 149077 & 170667 & 5 & 5 & 0.84 & 0.269 & 20.5 & 0.129 & C & $3 n$ \\
\hline & & & 4613.87 & 148941 & 170609 & 3 & 3 & 0.196 & 0.063 & 2.85 & -0.73 & C & $3 n$ \\
\hline & & & 4643.09 & 149077 & 170609 & 5 & 3 & 0.466 & 0.090 & 6.9 & -0.345 & C. & $3 n$ \\
\hline & & & 4621.39 & 148941 & 170573 & 3 & 1 & 0.90 & 0.096 & 4.37 & -0.54 & C & $3 n$ \\
\hline & & & 4601.48 & 148941 & 170667 & 3 & 5 & 0.270 & 0.143 & 6.5 & -0.368 & C & $3 n$ \\
\hline & & & 4607.16 & 148909 & 170609 & 1 & 3 & 0.340 & 10.325 & 4.93 & -0.488 & C & $3 n$ \\
\hline
\end{tabular}


N II. Allowed Transitions-Continued

\begin{tabular}{|c|c|c|c|c|c|c|c|c|c|c|c|c|c|}
\hline No. & $\begin{array}{c}\text { Transition } \\
\text { Array }\end{array}$ & Multiplet & $\lambda(\AA)$ & $E_{i}\left(\mathrm{~cm}^{-1}\right)$ & $E_{k}\left(\mathrm{~cm}^{-1}\right)$ & $g_{i}$ & $g_{k}$ & $A_{k i}\left(10^{8} \sec ^{-1}\right)$ & $f_{i k}$ & $S$ (at.u.) & $\log g f$ & $\begin{array}{c}\text { Accu- } \\
\text { racy }\end{array}$ & Source \\
\hline 18 & & $\begin{array}{c}{ }^{1} \mathrm{P}^{\circ}-{ }^{1} \mathrm{P} \\
(8)\end{array}$ & 6482.07 & 149189 & 164612 & 3 & 3 & 0.365 & 0.230 & 14.7 & -0.162 & C & $4, c a$ \\
\hline 19 & & $\begin{array}{c}{ }^{1} \mathrm{P}^{\circ}-{ }^{1} \mathrm{D} \\
(12)\end{array}$ & 3995.00 & 149189 & 174213 & 3 & 5 & 1.58 & 0.63 & 24.8 & 0.276 & C & $4, c a$ \\
\hline 20 & & $\begin{array}{c}{ }^{1} \mathrm{P}^{\circ}-{ }^{1} \mathrm{~S} \\
(13)\end{array}$ & 3437.16 & 149189 & 178274 & 3 & 1 & 2.40 & 0.142 & 4.82 & -0.371 & $\mathrm{C}$ & $4, c a$ \\
\hline \multirow[t]{8}{*}{21} & \multirow{8}{*}{$\left|\begin{array}{l}2 s 2 p^{2} 3 s- \\
2 s 2 p^{2}\left({ }^{4} \mathrm{P}\right) 3 p\end{array}\right|$} & \multirow{8}{*}{$\begin{array}{c}{ }^{5} \mathrm{P}-{ }^{-5} \mathrm{D}^{\circ} \\
(63)\end{array}$} & 5537.4 & 205677 & 223731 & 15 & 25 & 0.56 & 0.432 & 118 & 0.81 & C & $c a$ \\
\hline & & & 5535.39 & 205726 & 223787 & 7 & 9 & 0.56 & 0.334 & 42.6 & 0.368 & C & $l s$ \\
\hline & & & 5530.27 & 205656 & 223733 & 5 & $\begin{array}{l}7 \\
5\end{array}$ & 0.377 & 0.242 & 22.0 & 0.083 & C & ls \\
\hline & & & $\begin{array}{l}5526.26 \\
555195\end{array}$ & 205600 & 223690 & 3 & 5 & $\begin{array}{l}0.198 \\
0.187\end{array}$ & 0.151 & 8.3 & $\begin{array}{l}-0.343 \\
-0.910\end{array}$ & $\mathrm{C}$ & ls \\
\hline & & & $\begin{array}{l}5501.93 \\
5543.49\end{array}$ & $\begin{array}{l}205 / 20 \\
205656\end{array}$ & $\begin{array}{l}223733 \\
223690\end{array}$ & 5 & 5 & $\begin{array}{l}0.187 \\
0.327\end{array}$ & $\mid \begin{array}{l}0.086 \\
0.151\end{array}$ & $\begin{array}{l}11.0 \\
13.8\end{array}$ & $\begin{array}{l}-0.219 \\
-0.123\end{array}$ & $\stackrel{\mathrm{C}}{\mathrm{C}}$ & $\begin{array}{l}l s \\
l s\end{array}$ \\
\hline & & & 5535.39 & 205600 & 223660 & 3 & 3 & 0.422 & 0.194 & 10.6 & -0.235 & C & ls \\
\hline & & & $\begin{array}{l}5565.30 \\
555254\end{array}$ & $\begin{array}{l}205726 \\
205656\end{array}$ & $\begin{array}{l}223690 \\
223660\end{array}$ & $\begin{array}{l}7 \\
5\end{array}$ & $\begin{array}{l}5 \\
3\end{array}$ & $\begin{array}{l}0.0370 \\
0140\end{array}$ & $\begin{array}{l}0.0123 \\
0.0387\end{array}$ & $\begin{array}{l}1.57 \\
3.54\end{array}$ & $\begin{array}{l}-1.066 \\
-0.71\end{array}$ & C & $l s$ \\
\hline & & & 5540.16 & 205600 & 223645 & 3 & 1 & 0.56 & 0.086 & 4.71 & -0.59 & $\mathrm{C}$ & $l s$ \\
\hline \multirow[t]{7}{*}{22} & & \multirow[t]{7}{*}{$\begin{array}{c}{ }^{5} \mathrm{P}-5 \mathrm{P}^{\circ} \\
(64)\end{array}$} & 5006.9 & 205677 & 225644 & 15 & 15 & 0.77 & 0.291 & 72 & 0.64 & C & $c a$ \\
\hline & & & 5012.03 & 205726 & $\begin{array}{l}225673 \\
25620\end{array}$ & $\begin{array}{l}7 \\
5\end{array}$ & 7 & 0.51 & 0.194 & 22.4 & $\begin{array}{r}0.132 \\
-0.0 ?\end{array}$ & C & ls \\
\hline & & & $\begin{array}{l}5005.14 \\
4997.23\end{array}$ & $\begin{array}{l}205656 \\
205600\end{array}$ & $\begin{array}{l}225629 \\
225605\end{array}$ & $\begin{array}{l}5 \\
3\end{array}$ & $\begin{array}{l}5 \\
3\end{array}$ & $\begin{array}{l}0.064 \\
0.194\end{array}$ & $\begin{array}{l}0.0242 \\
0.073\end{array}$ & $\begin{array}{l}2.00 \\
3.59\end{array}$ & $\begin{array}{l}-0.92 \\
-0.66\end{array}$ & $\begin{array}{l}\mathrm{C} \\
\mathrm{C}\end{array}$ & $\begin{array}{l}l s \\
l s\end{array}$ \\
\hline & & & 5023.11 & 205726 & 225629 & 7 & 5 & 0.358 & 0.097 & 11.2 & -0.170 & $\mathrm{C}$ & $l s$ \\
\hline & & & 5011.24 & 205656 & 225605 & 5 & 3 & 0.58 & 0.131 & 10.8 & -0.185 & C & $l s$ \\
\hline & & & 4994.36 & 205656 & 225673 & 5 & 7 & 0.260 & 0.136 & 11.2 & -0.170 & C & $l s$ \\
\hline & & & 4991.22 & 205600 & 225629 & 3 & 5 & 0.351 & 0.218 & 10.8 & -0.184 & C & $l s$ \\
\hline \multirow[t]{3}{*}{23} & & \multirow{3}{*}{$\begin{array}{c}{ }^{5} \mathrm{P}-{ }^{5} \mathrm{~S}^{\circ} \\
(65)\end{array}$} & 4137.4 & 205677 & 229840 & 15 & 5 & 1.37 & 0.117 & 24.0 & 0.244 & C & $c a$ \\
\hline & & & $\begin{array}{l}4145.76 \\
4133.67\end{array}$ & $\begin{array}{l}205726 \\
205656\end{array}$ & $\begin{array}{l}229840 \\
229840\end{array}$ & $\begin{array}{l}7 \\
5\end{array}$ & $\begin{array}{l}5 \\
5\end{array}$ & \begin{tabular}{|l|}
0.64 \\
0.458
\end{tabular} & 0.117 & $\begin{array}{r}11.2 \\
80\end{array}$ & $\begin{array}{l}-0.087 \\
-0.0232\end{array}$ & $\mathrm{C}$ & $l s$ \\
\hline & & & 4124.08 & 205600 & 229840 & 3 & 5 & 0.276 & 0.117 & $\begin{array}{l}0.0 \\
4.78\end{array}$ & -0.453 & C & $l s$ \\
\hline 24 & $\begin{array}{l}2 p 3 s- \\
2 p\left({ }^{2} \mathrm{P}^{\circ}\right) 4 p\end{array}$ & $\begin{array}{l}{ }^{1} \mathrm{P}^{\circ}-{ }^{-1} \mathrm{P} \\
(14 \mathrm{uv})\end{array}$ & 1886.82 & 149189 & 202170 & 3 & 3 & 0.52 & 0.028 & 0.52 & -1.08 & D & 4 \\
\hline 25 & $\begin{array}{l}2 p 3 p- \\
2 p\left({ }^{2} \mathrm{P}^{\circ}\right) 3 d\end{array}$ & $\begin{array}{c}{ }^{1} \mathrm{P}-{ }^{1} \mathrm{D}^{\circ} \\
(15)\end{array}$ & 4447.03 & 164612 & 187092 & 3 & 5 & 1.30 & 0.642 & 28.2 & 0.285 & $\mathrm{C}+$ & $3,4, c a$ \\
\hline 26 & & $\begin{array}{c}{ }^{1} \mathrm{P}-{ }^{-1} \mathrm{P}^{\circ} \\
(17)\end{array}$ & 3919.01 & 164612 & 190121 & 3 & 3 & 1.00 & 0.231 & 8.93 & -0.160 & $\mathrm{C}+$ & $3,4, c a$ \\
\hline \multirow[t]{6}{*}{27} & & \multirow{6}{*}{$\begin{array}{c}{ }^{3} \mathrm{D}-{ }^{3} \mathrm{~F}^{\circ} \\
(19)\end{array}$} & 5004.5 & 166616 & 186593 & 15 & 21 & 1.22 & 0.639 & 158 & 0.982 & $\mathrm{C}+$ & $3,4, c a$ \\
\hline & & & 5005.14 & 166679 & 186653 & 7 & 9 & 1.22 & 0.588 & 67.8 & 0.614 & $\mathrm{C}+$ & $l s$ \\
\hline & & & 5001.47 & 166583 & & 5 & 7 & 1.0 & & 46.8 & 0.454 & $\mathrm{C}+$ & ls \\
\hline & & & 5001.13 & 166522 & 186512 & 3 & 5 & 1.02 & 0.640 & 31.6 & 0.283 & $C+1$ & ls \\
\hline & & & $\begin{array}{l}5025.67 \\
5016\end{array}$ & $\begin{array}{l}166679 \\
1\end{array}$ & 186572 & $\begin{array}{l}7 \\
5\end{array}$ & 7 & 0.134 & 0.0506 & 5.86 & -0.451 & $\mathrm{C}+$ & ls \\
\hline & & & $\begin{array}{l}5016.39 \\
5040.76\end{array}$ & $\begin{array}{l}166583 \\
166679\end{array}$ & $\begin{array}{l}186512 \\
186512\end{array}$ & $\begin{array}{l}5 \\
7\end{array}$ & $\begin{array}{l}5 \\
5\end{array}$ & $\mid \begin{array}{l}0.188 \\
0.00525\end{array}$ & $\mid \begin{array}{l}0.0710 \\
0.00143\end{array}$ & $\begin{array}{l}5.86 \\
0.166\end{array}$ & $\begin{array}{l}-0.450 \\
-1.999\end{array}$ & $\begin{array}{l}\mathrm{C}+ \\
\mathrm{C}+\end{array}$ & $\begin{array}{l}l s \\
l s\end{array}$ \\
\hline \multirow[t]{7}{*}{28} & & \multirow{7}{*}{$\begin{array}{c}{ }^{3} \mathrm{D}-{ }^{-3} \mathrm{D}^{\circ} \\
(20)\end{array}$} & 4793.5 & 166616 & 187472 & 15 & 15 & 0.356 & 0.123 & 29.0 & 0.264 & $\mathrm{C}+$ & $3,4, c a$ \\
\hline & & & 4803.27 & 166679 & 187493 & 7 & 7 & 0.313 & 0.108 & 12.0 & -0.120 & $\mathrm{C}+$ & ls \\
\hline & & & 4788.13 & 166583 & 187462 & 5 & 5 & 0.2 & 0.0854 & 6.73 & -0.370 & $\mathrm{C}+$ & ls \\
\hline & & & 4779.71 & 166522 & 187438 & 3 & 3 & 0.269 & 0.0921 & 4.35 & -0.558 & $\mathrm{C}+$ & ls \\
\hline & & & $\begin{array}{l}4810.29 \\
4702\end{array}$ & 166679 & 187462 & $\begin{array}{l}7 \\
5\end{array}$ & \begin{tabular}{l|l|l}
5 & \\
2 &
\end{tabular} & 0.0550 & 0.0136 & 1.51 & -1.021 & $\begin{array}{l}\mathrm{C}+ \\
\mathrm{C}+\end{array}$ & ls \\
\hline & & & $\begin{array}{l}4793.66 \\
4781.17\end{array}$ & $\begin{array}{l}166583 \\
166583\end{array}$ & $\begin{array}{l}187438 \\
187493\end{array}$ & $\begin{array}{l}5 \\
5\end{array}$ & $\begin{array}{l}3 \\
7\end{array}$ & $\begin{array}{l}0.0889 \\
0.0400\end{array}$ & $\begin{array}{l}0.0184 \\
0.0192\end{array}$ & $\begin{array}{l}1.45 \\
1.51\end{array}$ & $\begin{array}{l}-1.037 \\
-1.018\end{array}$ & $\begin{array}{l}\mathrm{C} \\
\mathrm{C}+\end{array}$ & ls \\
\hline & & & 4774.22 & 166522 & 187462 & 3 & 51 & 0.0540 & 0.0308 & 1.45 & -1.035 & $\mathrm{C}+$ & $l s$ \\
\hline
\end{tabular}


N II. Allowed Transitions-Continued

\begin{tabular}{|c|c|c|c|c|c|c|c|c|c|c|c|c|c|}
\hline No. & $\begin{array}{l}\text { Transition } \\
\text { Array }\end{array}$ & Multiplet & $\lambda(\AA)$ & $E_{i}\left(\mathrm{~cm}^{-1}\right)$ & $E_{k}\left(\mathrm{~cm}^{-1}\right)$ & $g_{i}$ & $g_{k}$ & $A_{k i}\left(10^{\kappa} \sec ^{-1}\right)$ & $f_{i k}$ & $S$ (at.u.) & $\log g f$ & $\begin{array}{c}\text { Accu- } \\
\text { racy }\end{array}$ & Source \\
\hline \multirow[t]{6}{*}{29} & & $\begin{array}{c}{ }^{3} \mathrm{D}-{ }^{3} \mathrm{P}^{\circ} \\
(2 \mathrm{l})\end{array}$ & 4489.4 & 166616 & 188884 & 15 & 9 & 0.0460 & 0.0083 & 1.85 & -0.90 & C & $4, c a$ \\
\hline & & & 4507.56 & 166679 & 188858 & 7 & 5 & 0.0381 & 0.0083 & 0.86 & -1.237 & C & ls \\
\hline & & & 4477.74 & 166583 & 188910 & 5 & 3 & 0.0348 & 0.0063 & 0.463 & -1.50 & C & ls \\
\hline & & & 4459.96 & 166522 & 188938 & 3 & 1 & 0.0468 & 0.00465 & 0.205 & -1.86 & $\mathrm{C}$ & ls \\
\hline & & & 4488.15 & 166583 & 188858 & 5 & 5 & 0.0069 & 0.00208 & 0.154 & -1.98 & C & ls \\
\hline & & & $\begin{array}{r}4465.54 \\
{[4475.8]}\end{array}$ & $\begin{array}{l}166522 \\
166522\end{array}$ & $\begin{array}{l}188910 \\
188858\end{array}$ & 3 & $\begin{array}{l}3 \\
5\end{array}$ & $\begin{array}{l}0.0117 \\
4.65 \times 10^{-4}\end{array}$ & $\begin{array}{l}0.00349 \\
2.33 \times 10^{-4}\end{array}$ & 0.154 & -1.98 & C & ls \\
\hline & & & & & & & & & & & 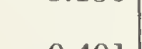 & & ¿s \\
\hline \multirow{4}{*}{30} & & $\begin{array}{c}{ }^{3} \mathrm{~S}-{ }^{3} \mathrm{P}^{0} \\
(24)\end{array}$ & 5000.8 & 168893 & 188884 & 3 & 9 & 0.75 & 0.84 & 41.4 & 0.401 & C & $3,4, c a$ \\
\hline & & & 5007.32 & 168893 & 188858 & 3 & 5 & 0.77 & 0.483 & 23.9 & 0.161 & C & $3 n$ \\
\hline & & & 4994.36 & 168893 & 188910 & 3 & 3 & 0.74 & 0.276 & 13.6 & -0.082 & C & $3 n$ \\
\hline & & & 4.987 .38 & 168893 & 188938 & 3 & 1 & 0.63 & 0.078 & 3.83 & -0.63 & C & $3 n$ \\
\hline \multirow[t]{6}{*}{31} & & $\begin{array}{c}{ }^{3} \mathrm{P}-{ }^{3} \mathrm{D}^{\circ} \\
(28)\end{array}$ & 5938.5 & 170637 & 187472 & 9 & 15 & 0.565 & 0.498 & 87.6 & 0.651 & $\mathrm{C}+$ & $3,4, c a$ \\
\hline & & & 5941.67 & 170667 & 187493 & 5 & 7 & 0.564 & 0.418 & 40.9 & 0.320 & $\mathrm{C}+$ & ls \\
\hline & & & 5931.79 & 170609 & 187462 & 3 & 5 & 0.425 & 0.374 & 21.9 & 0.050 & $\mathrm{C}+$ & ls \\
\hline & & & 5927.82 & 170573 & 187438 & 1 & 3 & 0.315 & 0.498 & 9.72 & -0.303 & C+ & ls \\
\hline & & & 5952.39 & 170667 & 187462 & 5 & 5 & 0.140 & 0.0745 & 7.30 & -0.429 & $\mathrm{C}+$ & ls \\
\hline & & & $\begin{array}{l}5940.25 \\
5960.93\end{array}$ & $\begin{array}{l}170609 \\
170667\end{array}$ & $\begin{array}{l}187438 \\
187438\end{array}$ & $\begin{array}{l}3 \\
5\end{array}$ & 3 & $\begin{array}{l}0.235 \\
0.0155\end{array}$ & 0.124 & $\begin{array}{l}7.30 \\
0.87\end{array}$ & -0.428 & $\begin{array}{l}\mathrm{C}+ \\
\mathrm{C}+\end{array}$ & ls \\
\hline \multirow{7}{*}{32} & & & & & & & & & & & & & \\
\hline & & $\begin{array}{c}3-3 p \\
(29)\end{array}$ & 5478.8 & 170637 & 188884 & 9 & 9 & 0.400 & 0.180 & 29.2 & 0.209 & C & $4, c a$ \\
\hline & & & 5495.70 & 170667 & 188858 & 5 & 5 & 0.298 & 0.135 & 12.2 & -0.171 & C & ls \\
\hline & & & 5462.62 & 170609 & 188910 & 3 & 3 & 0.101 & 0.0450 & 2.43 & -0.87 & C & ls \\
\hline & & & $\begin{array}{l}5480.10 \\
5454.26\end{array}$ & $\begin{array}{l}170667 \\
170609\end{array}$ & $\begin{array}{l}188910 \\
188938\end{array}$ & $\begin{array}{l}5 \\
3\end{array}$ & $\begin{array}{l}3 \\
1\end{array}$ & $\begin{array}{l}0.167 \\
0.405\end{array}$ & $\begin{array}{l}0.0450 \\
0.060\end{array}$ & $\begin{array}{l}4.06 \\
3.24\end{array}$ & $\begin{array}{l}-0.65 \\
-0.74\end{array}$ & $\mathrm{C}$ & $\begin{array}{l}l s \\
l s\end{array}$ \\
\hline & & & 5478.13 & 170609 & 188858 & 3 & 5 & 0.100 & 0.075 & 4.06 & -0.65 & $\mathrm{C}$ & ls \\
\hline & & & 5452.12 & 170573 & 188910 & 1 & 3 & 0.135 & 0.181 & 3.24 & -0.74 & $\mathrm{C}$ & ls \\
\hline 33 & & $\begin{array}{c}{ }^{1} \mathrm{D}-{ }^{1} \mathbf{F}^{\circ} \\
(31)\end{array}$ & 6610.58 & 174213 & 189336 & 5 & 7 & 0.59 & 0.54 & 59 & 0.433 & C & $4, c a$ \\
\hline 34 & & $\begin{array}{c}{ }^{1} \mathrm{D}-{ }^{1} \mathrm{P}^{0} \\
(32)\end{array}$ & 6284.30 & 174213 & 190121 & 5 & 3 & 0.0188 & 0.0067 & 0.69 & -1.477 & C & $4, c a$ \\
\hline \multirow[t]{12}{*}{35} & $\begin{array}{c}2 s 2 p^{2} 3 p- \\
2 s 2 p^{2}\left({ }^{4} \mathrm{P}\right) 3 d\end{array}$ & ${ }^{5} \mathrm{D}^{\circ}-{ }^{5} \mathrm{~F}$ & 5177.8 & 223731 & 243039 & 25 & 35 & 1.02 & 0.57 & 244 & 1.154 & C & $c a$ \\
\hline & & & 5179.50 & 223787 & 243088 & 9 & 11 & 1.02 & 0.50 & 70 & 0.65 & C & $l s$ \\
\hline & & & 5175.89 & 223733 & 243048 & 7 & 9 & 0.85 & 0.440 & 52 & 0.488 & C & ls \\
\hline & & & 5173.37 & 223690 & 243014 & 5 & 7 & 0.70 & 0.394 & 33.5 & 0.294 & C & ls \\
\hline & & & 5172.32 & 223660 & 242989 & 3 & 5 & 0.57 & 0.382 & 19.5 & 0.060 & C & ls \\
\hline & & & 5172.32 & 223645 & 242973 & 1 & 3 & 0.476 & 0.57 & 9.8 & -0.242 & C & ls \\
\hline & & & 5190.42 & 223787 & 243048 & 9 & 9 & 0.169 & 0.068 & 10.5 & -0.211 & C & ls \\
\hline & & & $\begin{array}{l}5184.96 \\
5180.34\end{array}$ & $\begin{array}{l}223733 \\
223690\end{array}$ & $\begin{array}{l}243014 \\
242989\end{array}$ & $\begin{array}{l}7 \\
5\end{array}$ & $\begin{array}{l}7 \\
5\end{array}$ & $\begin{array}{l}0.305 \\
0.407\end{array}$ & $\begin{array}{l}0.123 \\
0.164\end{array}$ & $\begin{array}{l}14.7 \\
14.0\end{array}$ & $\begin{array}{l}-0.066 \\
-0.087\end{array}$ & $\stackrel{C}{C}$ & ls \\
\hline & & & 5177.06 & 223660 & 242973 & 3 & 3 & 0.475 & 0.191 & 9.8 & -0.242 & $\mathrm{C}$ & ls \\
\hline & & & 5199.50 & 223787 & 243014 & 9 & 7 & 0.0144 & 0.00454 & 7.0 & -1.388 & C & $l s$ \\
\hline & & & 5191.97 & 223733 & 242989 & 7 & 5 & 0.0405 & 0.0117 & 1.40 & -1.088 & C & ls \\
\hline & & & 5184.96 & 223690 & 242973 & 5 & 3 & 0.068 & 0.0163 & 1.40 & -1.088 & C & ls \\
\hline \multirow[t]{7}{*}{36} & & $\begin{array}{c}{ }^{5} \mathrm{P}^{\circ}-{ }^{5} \mathrm{P} \\
(69)\end{array}$ & 5335.8 & & 244380 & 15 & 15 & 0.417 & 0.178 & 46.8 & 0.427 & C & $c a$ \\
\hline & & & 5351.21 & 225673 & 244355 & 7 & 7 & 0.275 & 0.118 & 14.6 & -0.082 & C & ls \\
\hline & & & 5327.45 & 225629 & 244393 & 5 & 5 & 0.0349 & 0.0148 & 1.30 & -1.129 & C & ls \\
\hline & & & 5313.43 & 225605 & 244419 & 3 & 3 & 0.106 & 0.0447 & 2.34 & -0.87 & C & $l s$ \\
\hline & & & 5340.20 & 225673 & 244393 & 7 & 5 & 0.194 & 0.059 & 7.3 & -0.382 & C & ls \\
\hline & & & 5320.96 & 225629 & 244419 & 5 & 3 & 0.315 & 0.080 & 7.0 & -0.396 & $\mathrm{C}$ & ls \\
\hline & & & $\begin{array}{l}5338.66 \\
5320.96\end{array}$ & $\begin{array}{l}225629 \\
225605\end{array}$ & $\begin{array}{l}244355 \\
244393\end{array}$ & $\begin{array}{l}5 \\
3\end{array}$ & $\begin{array}{l}7 \\
5\end{array}$ & $\begin{array}{l}0.139 \\
0.189\end{array}$ & $\begin{array}{l}0.083 \\
0.134\end{array}$ & $\begin{array}{l}7.3 \\
7.0\end{array}$ & $\begin{array}{l}-0.383 \\
-0.397\end{array}$ & $\begin{array}{l}\mathrm{C} \\
\mathrm{C}\end{array}$ & $\begin{array}{l}l s \\
l s\end{array}$ \\
\hline
\end{tabular}


N II. Allowed Transitions-Continued

\begin{tabular}{|c|c|c|c|c|c|c|c|c|c|c|c|c|c|}
\hline No. & $\begin{array}{c}\text { Transition } \\
\text { Array }\end{array}$ & Multiplet & $\lambda(\AA ̊)$ & $E_{i}\left(\mathrm{~cm}^{-1}\right)$ & $E_{k}\left(\mathrm{~cm}^{-1}\right)$ & $g_{i}$ & $g_{k}$ & $A_{k i}\left(10^{8} \mathrm{sec}^{-1}\right)$ & $f_{i k}$ & $S$ (at.u.) & $\log g f$ & $\begin{array}{l}\text { Accu- } \\
\text { racy }\end{array}$ & Source \\
\hline \multirow[t]{8}{*}{37} & & ${ }^{5} P^{\circ}-{ }^{5} \mathrm{D}$ & 5175.9 & 225644 & 244959 & 15 & 25 & 0.83 & 0.56 & 142 & 0.92 & C & $c a$ \\
\hline & & & 5179.50 & 225673 & 244974 & 7 & 9 & 0.83 & 0.429 & 51 & 0.478 & C & $l s$ \\
\hline & & & 5171.46 & $\begin{array}{l}225629 \\
205605\end{array}$ & 244960 & 5 & 7 & 0.56 & 0.312 & 26.5 & 0.193 & $\mathrm{C}$ & ls \\
\hline & & & $\begin{array}{l}5168.24 \\
5183.21\end{array}$ & $\begin{array}{l}225000 \\
225673\end{array}$ & 244960 & $\begin{array}{l}3 \\
7\end{array}$ & $\begin{array}{l}3 \\
7\end{array}$ & $\begin{array}{l}0.292 \\
0.276\end{array}$ & $\mid \begin{array}{l}0.195 \\
0.111\end{array}$ & $\begin{array}{r}9.9 \\
13.3\end{array}$ & $\begin{array}{l}-0.233 \\
-0.109\end{array}$ & $\begin{array}{l}\mathrm{C} \\
\mathrm{C}\end{array}$ & ls \\
\hline & & & 5174.46 & 225629 & 244949 & 5 & 5 & 0.485 & 0.195 & 16.6 & -0.012 & C & ls \\
\hline & & & $\begin{array}{l}5170.08 \\
518617\end{array}$ & $\begin{array}{l}225605 \\
295673\end{array}$ & 244941 & $\begin{array}{l}3 \\
7\end{array}$ & $\begin{array}{l}3 \\
5 \\
\end{array}$ & 0.62 & $\begin{array}{l}0.250 \\
0.0150\end{array}$ & 12.8 & -0.124 & C & ls \\
\hline & & & 5176.56 & 225629 & 244941 & 5 & $\begin{array}{l}3 \\
3\end{array}$ & $\begin{array}{l}0.055 \\
0.208\end{array}$ & \begin{tabular}{|l}
0.0159 \\
0.050
\end{tabular} & $\begin{array}{l}1.90 \\
4.26\end{array}$ & $\begin{array}{l}-0.95 \\
-0.60\end{array}$ & $\begin{array}{l}\mathrm{C} \\
\mathrm{C}\end{array}$ & is \\
\hline & & & 5171.30 & 225605 & 244937 & 3 & 1 & 0.83 & 0.111 & 5.7 & -0.477 & $\mathrm{C}$ & ls \\
\hline 38 & \multirow[t]{7}{*}{$\begin{array}{l}2 p 3 p- \\
2 p\left({ }^{2} \mathrm{P}^{\circ}\right) 4 s\end{array}$} & $\begin{array}{c}{ }^{1} \mathrm{P}-{ }^{1} \mathrm{P}^{\circ} \\
(18)\end{array}$ & 3006.86 & 164612 & 197859 & 3 & 3 & 0.54 & 0.073 & 2.17 & -0.66 & C & $c a$ \\
\hline 39 & & ${ }^{3} \mathrm{D}-{ }^{3} \mathrm{P}^{\circ}$ & 3328.3 & 166616 & 196653 & 15 & 9 & 1.11 & 0.111 & 18.2 & 0.220 & C & $c a$ \\
\hline & & & 3328.79 & 166679 & 196712 & 7 & 5 & 0.93 & 0.111 & 8.5 & -0.110 & $\mathrm{C}$ & $l s$ \\
\hline & & & 3331.32 & 166583 & 196593 & 5 & 3 & 0.83 & 0.083 & 4.55 & -0.382 & C & ls \\
\hline & & & 3330.30 & 166522 & 196541 & 3 & 1 & 1.11 & 0.061 & 2.02 & -0.73 & C & ls \\
\hline & & & $\begin{array}{l}3318.14 \\
3324.58\end{array}$ & 166583 & $\begin{array}{l}196712 \\
196593\end{array}$ & $\begin{array}{l}5 \\
3\end{array}$ & $\begin{array}{l}5 \\
3\end{array}$ & $\begin{array}{l}0.169 \\
0.279\end{array}$ & $\begin{array}{l}0.0278 \\
0.0463\end{array}$ & $\begin{array}{l}1.52 \\
1.52\end{array}$ & $\begin{array}{l}-0.86 \\
-0.86\end{array}$ & $\mathrm{C}$ & ls \\
\hline & & & [3311.4] & 166522 & 196712 & 3 & 5 & 0.0113 & 0.00309 & 0.101 & -2.033 & C & $l s$ \\
\hline \multirow[t]{4}{*}{40} & & ${ }^{3} \mathrm{~S}-{ }^{-3} \mathrm{P}^{\circ}$ & 3601.3 & 168893 & 196653 & 3 & 9 & 0.230 & 0.134 & 4.77 & -0.395 & C & $c a$ \\
\hline & & & 3593.60 & 168893 & 196712 & 3 & 5 & 0.231 & 0.075 & 2.65 & -0.65 & C & $l s$ \\
\hline & & & 3609.09 & 168893 & 196593 & 3 & 3 & 0.228 & 0.0446 & 1.59 & -0.87 & C & ls \\
\hline & & & 3615.88 & 168893 & 196541 & 3 & 1 & 0.227 & 0.0148 & 0.53 & -1.351 & C & $l s$ \\
\hline \multirow[t]{6}{*}{41} & & $\begin{array}{c}{ }^{3} \mathbf{P}-{ }^{3} \mathbf{P}^{\circ} \\
(30)\end{array}$ & 3842.7 & 170637 & 196653 & 9 & 9 & 0.69 & 0.153 & 17.4 & 0.138 & C & $c a$ \\
\hline & & & $\begin{array}{l}3838.39 \\
3847.38\end{array}$ & $\begin{array}{l}170667 \\
170609\end{array}$ & 196712 & $\begin{array}{l}5 \\
3\end{array}$ & $\begin{array}{l}5 \\
3\end{array}$ & $\begin{array}{l}0.52 \\
0.172\end{array}$ & $\begin{array}{l}0.116 \\
0.0382\end{array}$ & 7.3 & -0.238 & $C$ & ls \\
\hline & & & 3856.07 & 170667 & 196593 & $\begin{array}{l}0 \\
5\end{array}$ & $\begin{array}{l}0 \\
3\end{array}$ & $\begin{array}{l}0.182 \\
0.285\end{array}$ & $\begin{array}{l}0.0382 \\
0.0381\end{array}$ & $\begin{array}{l}1.45 \\
2.42\end{array}$ & $\begin{array}{l}-0.94 \\
-0.72\end{array}$ & C & ls \\
\hline & & & 3855.08 & 170609 & 196541 & 3 & 1 & 0.68 & 0.051 & 1.93 & -0.82 & $\mathrm{C}$ & $l s$ \\
\hline & & & $\begin{array}{l}3829.80 \\
2840\end{array}$ & 170609 & 196712 & 3 & 5 & 0.1 & 0.064 & 2.42 & -0.72 & C & ls \\
\hline & & & 3842.20 & 170573 & 196593 & 1 & 3 & 0.230 & 0.153 & 1.93 & -0.82 & C & ls \\
\hline 42 & & $\begin{array}{c}{ }^{1} \mathrm{D}-{ }^{-1} \mathrm{P}^{\circ} \\
(33)\end{array}$ & 4227.75 & 174213 & 197859 & 5 & 3 & 1.06 & 0.171 & 11.9 & -0.068 & C & $c a$ \\
\hline 43 & & $\begin{array}{c}{ }^{1} \mathrm{~S}-{ }^{1} \mathrm{P}^{\circ} \\
(34)\end{array}$ & 5104.45 & 178274 & 197859 & 1 & 3 & 0.189 & 0.222 & 3.73 & -0.65 & C & $c a$ \\
\hline 44 & $\begin{array}{l}2 p 3 p- \\
2 p\left({ }^{\left(\mathbf{P}^{\circ}\right.}\right) 4 d\end{array}$ & $\begin{array}{l}{ }^{1} \mathrm{P}-{ }^{1} \mathrm{D}^{\circ} \\
(15 \mathrm{uv})\end{array}$ & 2206.10 & 164612 & 209927 & 3 & 5 & 0.49 & 0.060 & 1.3 & -0.75 & $\mathrm{D}-$ & 4 \\
\hline \multirow[t]{6}{*}{45} & & $\begin{array}{l}{ }^{3} \mathrm{D}-{ }^{3} \mathrm{~F}^{\circ} \\
\text { (16uv) }\end{array}$ & 2317.1 & 166616 & 209761 & 15 & 21 & 0.56 & 0.063 & 7.2 & -0.03 & $\mathrm{D}-$ & 4 \\
\hline & & & $\begin{array}{l}2317.01 \\
2316.46\end{array}$ & $\begin{array}{l}166679 \\
166583\end{array}$ & $\begin{array}{l}209825 \\
209740\end{array}$ & $\begin{array}{l}7 \\
5\end{array}$ & $\begin{array}{l}9 \\
7\end{array}$ & $\begin{array}{l}0.56 \\
0.9\end{array}$ & 0.058 & $3.1^{\circ}$ & -0.39 & $\mathrm{D}-$ & $l s$ \\
\hline & & & $\begin{array}{l}2316.46 \\
2316.65\end{array}$ & $\begin{array}{l}106583 \\
166522\end{array}$ & $\begin{array}{l}209440 \\
209675\end{array}$ & $\begin{array}{l}5 \\
3\end{array}$ & 5 & $\begin{array}{l}0.49 \\
0.46\end{array}$ & & $\begin{array}{l}2.1 \\
1.4\end{array}$ & $\begin{array}{l}-0.56 \\
-0.74\end{array}$ & $\begin{array}{l}\mathrm{D}- \\
\mathrm{D}-\end{array}$ & ls \\
\hline & & & 2321.65 & 166679 & 209740 & 7 & 7 & 0.062 & 0.0 & 0. & -1 & & ls \\
\hline & & & $\begin{array}{l}2319.94 \\
\end{array}$ & 166583 & 209675 & 5 & 5 & 0.088 & 0.0071 & 0.27 & -1.45 & D- & ls \\
\hline & & & 2325.16 & 166679 & 209675 & 7 & 5 & 0.0024 & $1.4 \times 10^{-4}$ & 0.0076 & -3.00 & D- & ls \\
\hline \multirow[t]{5}{*}{46} & & $\begin{array}{c}{ }^{3} \mathrm{P}-{ }^{3} \mathrm{D}^{\circ} \\
(19 \mathrm{uv})\end{array}$ & 2521.9 & 170637 & 210278 & 9 & 15 & 0.33 & 0.052 & 3.9 & -0.33 & $\mathrm{D}-$ & 4 \\
\hline & & & $\begin{array}{l}2522.27 \\
2520.85\end{array}$ & $\begin{array}{l}170667 \\
170609\end{array}$ & $\begin{array}{l}210302 \\
210266\end{array}$ & $\begin{array}{l}5 \\
3\end{array}$ & $\begin{array}{l}7 \\
5\end{array}$ & $\begin{array}{l}0.32 \\
0.25\end{array}$ & $\begin{array}{l}0.043 \\
0.039\end{array}$ & $\begin{array}{l}1.8 \\
0.97\end{array}$ & $\begin{array}{l}-0.66 \\
-0.93\end{array}$ & $\begin{array}{l}\mathrm{D}- \\
\mathrm{D}-\end{array}$ & ls \\
\hline & & & 2520.27 & 170573 & 210240 & 1 & 3 & 0.18 & 0.052 & 0.43 & -1.29 & $\mathrm{D}-$ & ls \\
\hline & & & $\begin{array}{l}2524.49 \\
2522\end{array}$ & 170667 & 210266 & 5 & 5 & 0.081 & 0.0077 & 0.32 & -1.41 & D- & $l s$ \\
\hline & & & $\begin{array}{l}2522.46 \\
2526.17\end{array}$ & $\begin{array}{l}170609 \\
170667\end{array}$ & $\begin{array}{l}210240 \\
210240\end{array}$ & $\begin{array}{l}3 \\
5\end{array}$ & $\begin{array}{l}3 \\
3\end{array}$ & $\begin{array}{l}0.13 \\
0.0092\end{array}$ & $\begin{array}{l}0.013 \\
5.3 \times 10^{-4}\end{array}$ & $\begin{array}{l}0.32 \\
0.022\end{array}$ & $\begin{array}{l}-1.41 \\
-2.58\end{array}$ & $\begin{array}{l}D- \\
D-\end{array}$ & ls \\
\hline
\end{tabular}


N II. Allowed Transitions-Continued

\begin{tabular}{|c|c|c|c|c|c|c|c|c|c|c|c|c|c|}
\hline No. & $\begin{array}{c}\text { Transition } \\
\text { Array }\end{array}$ & Multiplet & $\lambda(\AA)$ & $E_{i}\left(\mathrm{~cm}^{-1}\right)$ & $E_{k}\left(\operatorname{cin}^{-1}\right)$ & $g_{i}$ & $g_{k}$ & $A_{k i}\left(10^{4} \sec ^{-1}\right)$ & $f_{i k}$ & $S(a t . u)$. & $\log g f$ & $\begin{array}{l}\text { Accu- } \\
\text { racy }\end{array}$ & Source \\
\hline \multirow[t]{6}{*}{47} & & $\begin{array}{c}{ }^{3} \mathrm{P}-3 \mathrm{P}^{\circ} \\
(20 \mathrm{uv})\end{array}$ & 249.3 .5 & 1706.37 & 210729 & 9 & 9 & 0.19 & 0.018 & 1.3 & -0.80 & $\mathrm{D}-$ & 4 \\
\hline & & & 2496.88 & 170667 & 210705 & 5 & 5 & 0.14 & 0.013 & 0.54 & -1.18 & D - & ls \\
\hline & & & 2490.37 & 170609 & 210752 & 3 & 3 & 0.048 & 0.0045 & 0.11 & -1.87 & D - & ls \\
\hline & & & 2493.94 & 170667 & 210752 & 5 & 3 & 0.078 & 0.0044 & 0.18 & -1.66 & $D-$ & \\
\hline & & & $\begin{array}{l}2488.75 \\
2493.16\end{array}$ & $\begin{array}{l}170609 \\
170609\end{array}$ & $\begin{array}{l}210777 \\
210705\end{array}$ & $\begin{array}{l}3 \\
3\end{array}$ & $\begin{array}{l}1 \\
5\end{array}$ & $\begin{array}{l}0.18 \\
0.047\end{array}$ & $\begin{array}{l}0.0057 \\
0.0073\end{array}$ & $\begin{array}{l}0.14 \\
0.18\end{array}$ & $\begin{array}{l}-1.77 \\
-1.66\end{array}$ & $\begin{array}{l}D- \\
D-\end{array}$ & Is \\
\hline & & & 2488.12 & 170573 & 210752 & 1 & 3 & 0.061 & 0.017 & 0.14 & -1.77 & $\mathrm{D}-$ & ls \\
\hline 48 & & $\begin{array}{l}{ }^{1} \mathrm{D}-{ }^{1} \mathrm{D}^{\circ} \\
(2 \mathrm{luv})\end{array}$ & 2799.20 & 174213 & 209927 & 5 & 5 & 0.079 & 0.0093 & 0.43 & -1.33 & $\mathrm{D}-$ & 4 \\
\hline 49 & & $\begin{array}{c}{ }^{1} \mathrm{D}-{ }^{1} \mathrm{~F}^{\circ} \\
(22 \mathrm{uv})\end{array}$ & 2709.82 & 174213 & 211031 & 5 & 7 & 0.35 & 0.054 & 2.4 & -0.57 & $\mathrm{D}-$ & 4 \\
\hline 50 & & $\begin{array}{c}{ }^{1} \mathrm{~S}-{ }^{1} \mathrm{P}^{\circ} \\
(35)\end{array}$ & 3023.80 & 178274 & 211336 & 1 & 3 & 0.14 & 0.057 & 0.57 & -1.24 & $\mathrm{D}-$ & 4 \\
\hline 51 & $\begin{array}{l}2 p 3 p- \\
\quad 2 p\left({ }^{2} \mathrm{P}^{\circ}\right) 5 s\end{array}$ & $\begin{array}{c}{ }^{1} \mathrm{D}-{ }^{1} \mathrm{P}^{\circ} \\
(23 \mathrm{uv})\end{array}$ & 2461.30 & 174213 & 214828 & 5 & 3 & 0.353 & 0.0193 & 0.78 & -1.017 & C & $4, c a$ \\
\hline \multirow[t]{6}{*}{52} & $\begin{array}{l}2 p 3 d- \\
\quad 2 p\left({ }^{2} \mathrm{P}^{\circ}\right) 4 p\end{array}$ & $\begin{array}{c}{ }^{3} \mathrm{~F}^{\circ}-{ }^{3} \mathrm{D} \\
\quad(36)\end{array}$ & 6168.1 & 186593 & 202801 & 21 & 15 & 0.363 & 0.148 & 63 & 0.492 & $\mathrm{C}$ & $4, c a$ \\
\hline & & & 6167.82 & 186653 & 202862 & 9 & 7 & 0.333 & 0.148 & 27.0 & 0.124 & C & $l s$ \\
\hline & & & 6173.40 & 186572 & 202766 & 7 & 5 & 0.320 & 0.131 & 18.6 & -0.039 & C & ls \\
\hline & & & 6170.16 & $\begin{array}{l}186512 \\
186570\end{array}$ & 202715 & 5 & 3 & 0.362 & 0.124 & 12.6 & -0.207 & $\mathrm{C}$ & Is \\
\hline & & & $\begin{array}{l}0136.89 \\
6150.76\end{array}$ & $\begin{array}{l}186512 \\
186512\end{array}$ & $\begin{array}{l}202862 \\
202766\end{array}$ & $\begin{array}{l}7 \\
5\end{array}$ & $\begin{array}{l}7 \\
5\end{array}$ & $\begin{array}{l}0.0293 \\
0.0407\end{array}$ & $\begin{array}{l}0.0165 \\
0.0231\end{array}$ & $\begin{array}{l}2.34 \\
2.34\end{array}$ & $\begin{array}{l}-0.94 \\
-0.94\end{array}$ & $\stackrel{\mathrm{C}}{\mathrm{C}}$ & $\begin{array}{l}l s \\
l s\end{array}$ \\
\hline & & & 6114.6 & 186512 & 202862 & 5 & 7 & $8.4 \times 10^{-4}$ & $6.6 \times 10^{-4}$ & 0.066 & -2.48 & $\mathrm{C}$ & ls \\
\hline 53 & & $\begin{array}{c}{ }^{1} \mathrm{D}^{\circ}-{ }^{1} \mathrm{P} \\
(41)\end{array}$ & 6629.80 & 187092 & 202170 & 5 & 3 & 0.283 & 0.112 & 12.2 & -0.253 & C & $4, c a$ \\
\hline \multirow[t]{7}{*}{54} & & $\begin{array}{c}{ }^{3} \mathrm{D}^{\circ}-{ }^{3} \mathrm{D} \\
(45)\end{array}$ & 6521.8 & 187472 & 202801 & 15 & 15 & 0.058 & 0.0373 & 12.0 & -0.253 & C & $4, c a$ \\
\hline & & & 6504.61 & 187493 & 202862 & 7 & 7 & 0.052 & 0.0332 & 4.98 & -0.63 & C & ls \\
\hline & & & $\begin{array}{l}6532.55 \\
6544.16\end{array}$ & $\begin{array}{l}187462 \\
187438\end{array}$ & $\begin{array}{l}202766 \\
202715\end{array}$ & $\begin{array}{l}5 \\
3\end{array}$ & $\begin{array}{l}5 \\
3\end{array}$ & $\begin{array}{l}0.0404 \\
0.0434\end{array}$ & $\begin{array}{l}0.0259 \\
0.0278\end{array}$ & $\begin{array}{l}2.78 \\
1.80\end{array}$ & $\begin{array}{l}-0.89 \\
-1.078\end{array}$ & $\stackrel{\mathrm{C}}{\mathrm{C}}$ & Is \\
\hline & & & 6545.53 & 187493 & 202766 & 7 & 5 & 0.0090 & 0.00411 & 0.62 & -1.54 & C & ls \\
\hline & & & 6554.47 & 187462 & 202715 & 5 & 3 & 0.0144 & 0.0056 & 0.60 & -1.56 & C & Is \\
\hline & & & 6491.79 & 187462 & 202862 & 5 & 7 & 0.0066 & 0.0058 & 0.62 & -1.54 & C & Is \\
\hline & & & 6522.39 & 187438 & 202766 & 3 & 5 & 0.0088 & 0.0093 & 0.60 & -1.55 & C & ls \\
\hline \multirow[t]{6}{*}{55} & & $\begin{array}{c}{ }^{3} \mathrm{D}^{\circ}-{ }^{3} \mathrm{P} \\
(46)\end{array}$ & 6345.8 & 187472 & 203226 & 15 & 9 & 0.306 & 0.111 & 34.7 & 0.220 & C & $4, c a$ \\
\hline & & & 6340.57 & 187493 & 203260 & 7 & 5 & 0.258 & 0.111 & 16.2 & -0.110 & $C$ & Is \\
\hline & & & 6356.55 & 187462 & 203189 & 5 & 3 & 0.229 & 0.083 & 8.7 & -0.381 & C & Is \\
\hline & & & $\begin{array}{l}6357.57 \\
6328.39\end{array}$ & $\begin{array}{l}187438 \\
187462\end{array}$ & $\begin{array}{l}203165 \\
203260\end{array}$ & $\begin{array}{l}3 \\
5\end{array}$ & $\begin{array}{l}1 \\
5\end{array}$ & $\begin{array}{l}0.304 \\
0.0462\end{array}$ & $\begin{array}{l}0.061 \\
0.0277\end{array}$ & $\begin{array}{l}3.85 \\
2.89\end{array}$ & $\begin{array}{l}-0.74 \\
-0.86\end{array}$ & C & ls \\
\hline & & & 6346.86 & 187438 & 203189 & 3 & 3 & 0.076 & 0.0461 & 2.89 & -0.86 & $\mathrm{C}$ & ls \\
\hline & & & 6318.80 & 187438 & 203260 & 3 & 5 & 0.00310 & 0.00310 & 0.193 & -2.033 & C & 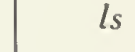 \\
\hline \multirow[t]{6}{*}{56} & & $\begin{array}{c}{ }^{3} \mathrm{P}^{\circ}-{ }^{3} \mathrm{D} \\
(52)\end{array}$ & 7183.5 & 188884 & 202801 & 9 & 15 & 0.00324 & 0.00418 & 0.89 & -1.424 & C & 4, ca \\
\hline & & & 7138.87 & 188858 & 202862 & 5 & 7 & 0.00332 & 0.00355 & 0.417 & -1.75 & C & Is \\
\hline & & & 7215.06 & 188910 & 202766 & 3 & 5 & 0.00242 & 0.00314 & 0.224 & -2.026 & $\mathrm{C}$ & Is \\
\hline & & & 7256.53 & 188938 & 202715 & 1 & 3 & 0.00175 & 0.00414 & 0.099 & -2.383 & C & ls \\
\hline & & & $\begin{array}{r}7188.20 \\
{[72418]}\end{array}$ & $\begin{array}{l}188858 \\
188910\end{array}$ & $\begin{array}{l}202766 \\
202715\end{array}$ & $\begin{array}{l}5 \\
3\end{array}$ & $\begin{array}{l}5 \\
3\end{array}$ & $\begin{array}{l}8.1 \times 10^{-4} \\
0.00132\end{array}$ & $\begin{array}{l}6.3 \times 10^{-4} \\
0.00103\end{array}$ & 0.074 & $\begin{array}{l}-2.50 \\
-25\end{array}$ & $\mathrm{C}$ & Is \\
\hline & & & {$[7214.6]$} & 188858 & 202715 & 5 & 3 & $8.9 \times 10^{-5}$ & $4.18 \times 10^{-5}$ & 0.00497 & -3.68 & $\mathrm{C}$ & is \\
\hline \multirow[t]{4}{*}{57} & & $\begin{array}{c}{ }^{3} \mathrm{P}^{\circ}-3 \mathrm{P} \\
(53)\end{array}$ & 6970.6 & 188884 & 203226 & 9 & 9 & 0.086 & 0.062 & 12.9 & -0.250 & C & 4. $c a$ \\
\hline & & & 6941.75 & 188858 & 203260 & 5 & 5 & 0.065 & 0.0473 & 5.4 & -0.63 & C & ls \\
\hline & & & 7003.0 & 188910 & 203189 & 3 & 3 & 0.0224 & 0.0165 & 1.14 & -1.306 & C & is \\
\hline & & & 6975.64 & 188858 & 203189 & 5 & 3 & 0.0356 & 0.0156 & 1.79 & -1.108 & C & ls \\
\hline
\end{tabular}


N II. Allowed Transitions - Continued

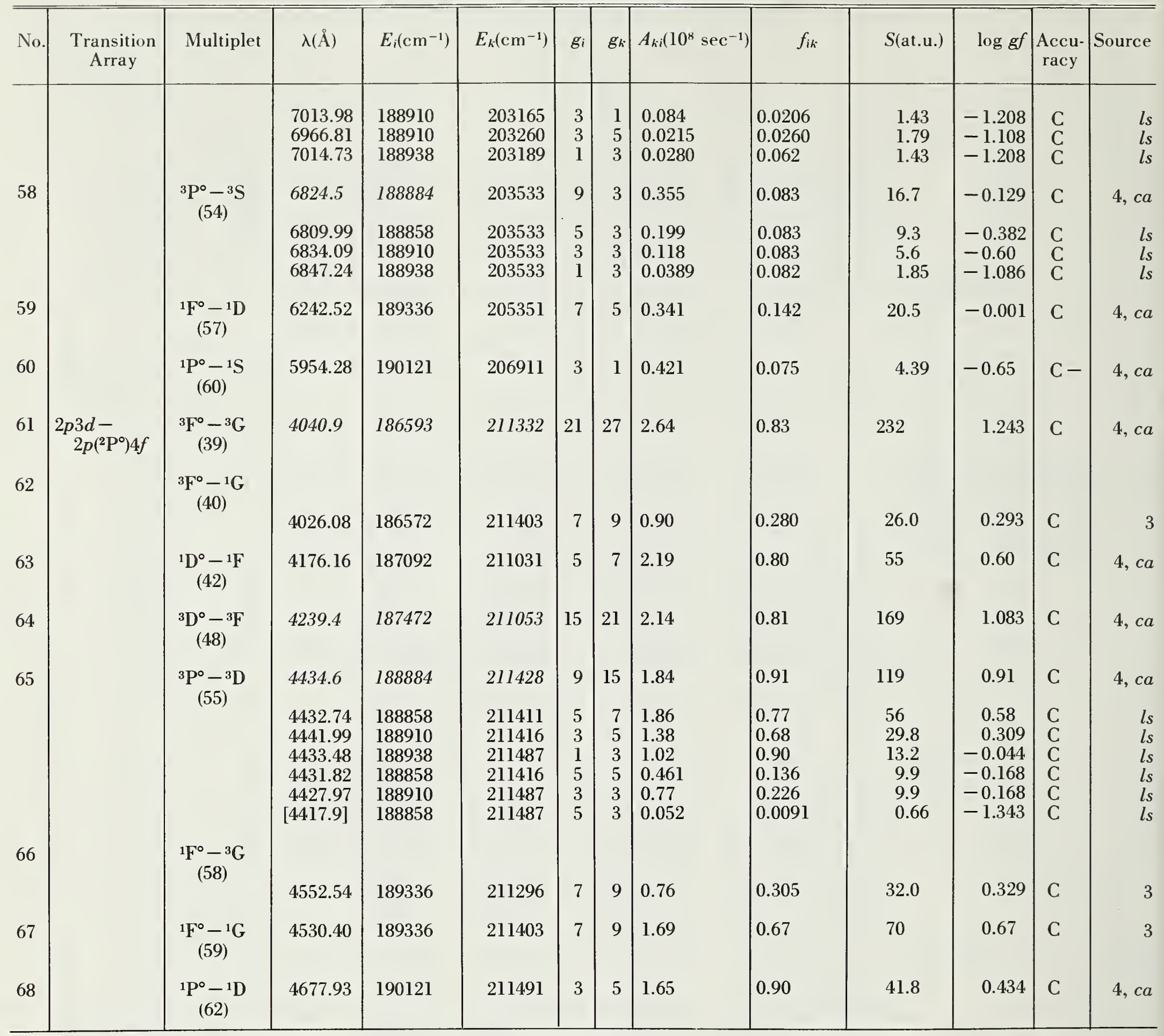

\section{Forbidden Transitions}

The adopted values represent, as in the case of CI, the work of Garstang [1], Naqvi [2], and Yamanouchi and Horie [3], who have independently done essentially the same calculations and arrived at very similar results. For the selection of values, the same considerations as for CI are applied.

\section{References}

[1] Garstang, R. H., Monthly Notices Roy. Astron. Soc. 111 , 115-124 (1951).

[2] Naqvi, A. M., Thesis Harvard (1951).

[3] Yamanouchi, T., and Horie, H., J. Phys. Soc. Japan 7, 52-56 (1952). 
N II. Forbidden Transitions

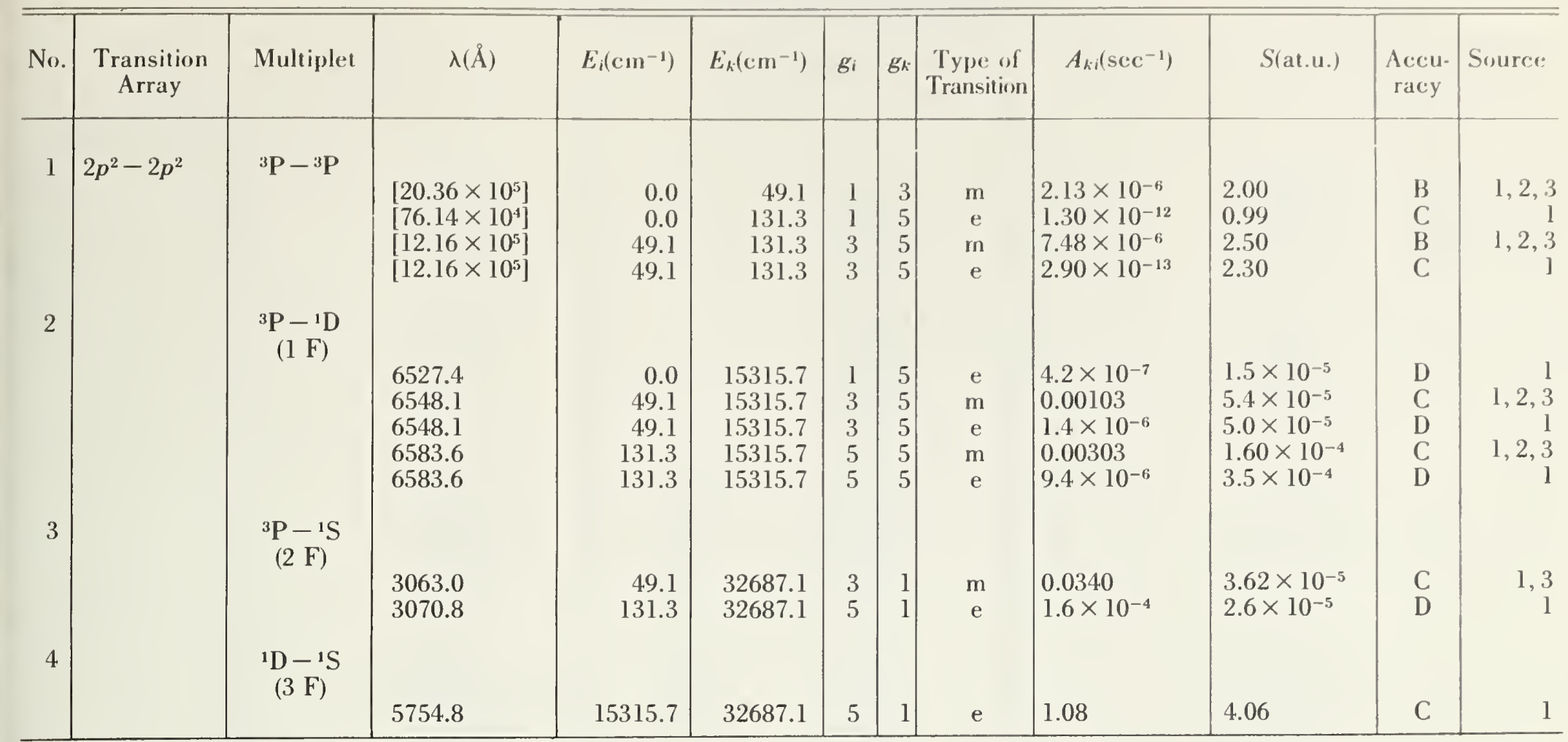

N III

Ground State

Ionization Potential
$1 s^{2} 2 s^{2} 2 p^{2} \mathrm{P}_{1 / 2}^{\circ}$

$47.426 \mathrm{eV}=382625.5 \mathrm{~cm}^{-1}$

\section{Allowed Transitions}

List of tabulated lines:

\begin{tabular}{l|c||c|c||c|c}
\hline \hline Wavelength $[\AA]$ & No. & Wavelength $[\AA]$ & No. & Wavelength $[\AA]$ & No. \\
\hline 374.204 & 11 & 772.93 & 6 & 1804.3 & 24 \\
374.44 & 11 & 772.975 & 6 & 1805.5 & 24 \\
374.441 & 11 & 979.77 & 5 & 1885.25 & 27 \\
451.869 & 10 & 979.842 & 5 & 1908.11 & 28 \\
452.226 & 10 & 979.919 & 5 & 1917.7 & 29 \\
684.996 & 3 & 980.01 & 5 & 1918.7 & 29 \\
685.513 & 3 & 989.790 & 1 & 1919.5 & 29 \\
685.816 & 3 & 991.514 & 1 & 1919.7 & 29 \\
686.335 & 3 & 1006.0 & 1 & 1920.0 & 29 \\
763.340 & 2 & 1183.03 & 9 & 1921.49 & 29 \\
764.357 & 2 & 1184.54 & 9 & 2063.50 & 29 \\
771.544 & 4 & 1747.86 & 8 & 2063.99 & 30 \\
771.901 & 4 & 1751.24 & 8 & 2068.25 & 30 \\
772.385 & 4 & 1751.75 & & 22.7 .7 & 25
\end{tabular}


List of tabulated lines-Continued

\begin{tabular}{l|l||c|c||c|c}
\hline \hline & & & & \\
Wavelength $[\AA]$ & No. & Wavelength $[\AA]$ & No. & Wavelength $[\AA]$ & No. \\
\hline & & & & & \\
2247.92 & 25 & 3771.08 & 14 & 4527.86 & 22 \\
2248.88 & 25 & 3934.41 & 18 & 4530.84 & 13 \\
2453.85 & 26 & 3938.52 & 18 & 4534.57 & 13 \\
2459.2 & 26 & 394.78 & 18 & 4535.11 & 22 \\
2462.56 & 26 & 3998.69 & 31 & 4546.36 & 22 \\
2462.9 & 26 & 4003.6 & 31 & 4547.34 & 13 \\
2466.3 & 26 & 4003.64 & 31 & 4858.74 & 20 \\
2468.36 & 26 & 4097.31 & 12 & 4858.88 & 20 \\
2469.1 & 26 & 4103.37 & 12 & 4861.33 & 20 \\
2471.2 & 26 & 4195.70 & 16 & 4867.18 & 20 \\
2972.60 & 19 & 4200.02 & 16 & 4873.58 & 20 \\
2977.3 & 19 & 4215.69 & 16 & 4881.81 & 20 \\
2978.8 & 19 & 4321.37 & 21 & 4884.14 & 20 \\
2983.58 & 19 & 4323.93 & 21 & 4896.71 & 20 \\
3342.77 & 17 & 4328.15 & 21 & 6445.05 & 23 \\
3353.78 & 15 & 4330.14 & & & \\
3354.29 & 15 & 4330.44 & 21 & 6450.78 & 23 \\
3355.47 & 17 & 4335.53 & 21 & 6453.95 & 23 \\
3358.72 & 15 & 4339.52 & 21 & 6463.03 & 23 \\
3361.90 & 15 & 4348.36 & 21 & 6468.86 & 23 \\
3365.79 & 15 & 4353.66 & 21 & & 23 \\
3367.36 & 15 & 4510.92 & 13 & 6478.69 & 23 \\
3374.06 & 15 & 4514.89 & 13 & & 23 \\
3745.83 & 14 & 4518.18 & 13 & & \\
3754.62 & 14 & 4523.60 & 13 & & \\
\hline
\end{tabular}

As in the case of analogous configurations, the data for the lower excited transitions, which are obtained from the calculations by Bolotin and Yutsis [1] and Kelly [2], are quite uncertain because of the strong effects of configuration interaction. The latter are either crudely taken into account (Bolotin and Yutsis) or entirely neglected (Kelly). For some higher excited transitions self-consistent field calculations by Kelly [3] including exchange affects are available and are averaged with the results of the Coulomb approximation. For other prominent transitions the Coulomb approximation is applied whenever it is expected to give reliable results.

\section{References}

[1] Bolotin, A. B., and Yutsis, A. P., Zhur. Eksptl. i Teoret. Fiz. 24, 537-543 (1953) (Translated in "Optical Transition Probabilities", Office of Technical Services, U.S. Department of Commerce, Washington, D.C.).

[2] Kelly, P. S., Astrophys. J. 140, 1247-1268 (1964).

[3] Kelly, P. S., J. Quant. Spectrosc. Radiat. Transfer 4, 117-148 (1964). 
N III. Allowed Transitions

\begin{tabular}{|c|c|c|c|c|c|c|c|c|c|c|c|c|c|}
\hline No. & $\begin{array}{l}\text { Transition } \\
\text { Array }\end{array}$ & Multiplet & $\lambda(\AA)$ & $E_{i}\left(\mathrm{~cm}^{-1}\right)$ & $E_{k}\left(\mathrm{~cm}^{-1}\right)$ & $\mathrm{Bi}_{i}$ & $g_{k}$ & $A_{k i}\left(10^{\kappa} \sec ^{-1}\right)$ & $f_{i k}$ & $S($ al.u. $)$ & $\log g f$ & $\begin{array}{c}\text { Accu- } \\
\text { racy }\end{array}$ & Sourcer \\
\hline \multirow[t]{4}{*}{1} & \multirow[t]{12}{*}{$2 s^{2} 2 p-2 s 2 p^{2}$} & \multirow{4}{*}{$\begin{array}{c}{ }^{2} \mathrm{P}^{\circ}-{ }^{2} \mathrm{D} \\
(\mathrm{luv})\end{array}$} & 990.98 & 116.3 & 101027 & 6 & 10 & 7.3 & 0.18 & 3.5 & 0.03 & E. & 1 \\
\hline & & & 991.579 & 174.5 & 101024 & 4 & 6 & 7.3 & 0.16 & 2.1 & -0.19 & $\mathrm{E}$ & ls \\
\hline & & & 989.790 & 0.0 & 101032 & 2 & 4 & 6.3 & 0.18 & 1.2 & -0.43 & E & Is \\
\hline & & & 991.514 & 174.5 & 101032 & 4 & 4 & 1.2 & 0.018 & 0.2 .3 & -1.15 & E & ls \\
\hline \multirow[t]{3}{*}{2} & & \multirow{3}{*}{$\begin{array}{c}{ }^{2} \mathrm{P}^{\circ}-{ }^{2} \mathrm{~S} \\
(2 \mathrm{uv})\end{array}$} & 764.01 & 116.3 & 131004 & 6 & 2 & 39 & 0.11 & 1.7 & -0.17 & $\mathrm{E}$ & 1 \\
\hline & & & 764.357 & 174.5 & 131004 & 4 & 2 & 25 & 0.11 & 1.1 & -0.36 & E & \\
\hline & & & 763.340 & 0.0 & 131004 & 2 & 2 & 13 & 0.11 & 0.57 & -0.64 & $\mathrm{E}$ & ls \\
\hline \multirow[t]{5}{*}{3} & & \multirow{5}{*}{$\begin{array}{c}{ }^{2} \mathrm{P}^{\circ}-{ }^{2} \mathrm{P} \\
(3 \mathrm{uv})\end{array}$} & 685.71 & 116.3 & 145950 & 6 & 6 & 64 & 0.45 & 6.1 & 0.43 & E & 1 \\
\hline & & & 685.816 & 174.5 & 145987 & 4 & 4 & 53 & 0.38 & 3.4 & 0.18 & $\mathrm{E}$ & \\
\hline & & & 685.513 & 0.0 & 145876 & 2 & 2 & 44 & 0.31 & 1.4 & -0.21 & $\mathrm{E}$ & ls \\
\hline & & & $\begin{array}{l}686.335 \\
684.996\end{array}$ & $\begin{array}{r}174.5 \\
0.0\end{array}$ & 145876 & 4 & 2 & 21 & 0.075 & 0.68 & $\begin{array}{l}-0.52 \\
-0.52\end{array}$ & E & ls \\
\hline & & & 004.990 & & 145987 & 2 & 4 & 11 & 0.15 & 0.68 & -0.52 & E & ls \\
\hline \multirow[t]{3}{*}{4} & \multirow[t]{8}{*}{$2 s 2 p^{2}-2 p^{3}$} & \multirow{3}{*}{$\begin{array}{c}{ }^{4} \mathrm{P}-{ }^{4} \mathrm{~S}^{\circ} \\
(8 \mathrm{uv})\end{array}$} & 772.09 & 57283 & 186802 & 12 & 4 & 56 & 0.17 & 5.1 & 0.30 & $\mathrm{E}$ & 2 \\
\hline & & & 772.385 & 57333 & 186802 & 6 & 4 & 29 & 0.17 & 2.6 & 0.01 & $\mathrm{E}$ & is \\
\hline & & & $\begin{array}{l}771.901 \\
771.544\end{array}$ & $\begin{array}{l}57252 \\
57192\end{array}$ & $\begin{array}{l}186802 \\
186802\end{array}$ & $\begin{array}{l}4 \\
2\end{array}$ & $\begin{array}{l}4 \\
4\end{array}$ & $\begin{array}{l}19 \\
9.4\end{array}$ & $\mid \begin{array}{l}0.17 \\
0.17\end{array}$ & $\begin{array}{l}1.7 \\
0.85\end{array}$ & $\begin{array}{l}-0.17 \\
-0.48\end{array}$ & $\begin{array}{l}\text { E } \\
E\end{array}$ & ls \\
\hline \multirow{5}{*}{5} & & \multirow{5}{*}{$\begin{array}{l}{ }^{2} \mathrm{D}-{ }^{2} \mathrm{D}^{\circ} \\
(12 \mathrm{uv})\end{array}$} & 97989 & 101027 & 203079 & 10 & 10 & 14 & | & 66 & 0.31 & 5 & \\
\hline & & & 80.0 & 101028 & 20.067 & 10 & 10 & 14 & 0.20 & 0.0 & 0.31 & $\mathrm{E}$ & \\
\hline & & & 979.919 & 101024 & 203072 & 6 & 6 & 13 & 0.19 & 3.7 & 0.06 & E & \\
\hline & & & $\begin{array}{r}979.842 \\
{[979771}\end{array}$ & $\begin{array}{l}101032 \\
101024\end{array}$ & $\begin{array}{l}203089 \\
203089\end{array}$ & $\begin{array}{l}4 \\
6\end{array}$ & $\begin{array}{l}4 \\
4\end{array}$ & 13 & $\begin{array}{l}0.19 \\
0.013\end{array}$ & 2.4 & $\begin{array}{l}-0.13 \\
-109\end{array}$ & $\begin{array}{l}\mathrm{E} \\
\mathrm{E}\end{array}$ & ls \\
\hline & & & [980.01] & 101032 & 203072 & 4 & 6 & 0.93 & 0.020 & 0.26 & -1.09 & E & $i s$ \\
\hline \multirow[t]{4}{*}{6} & & \multirow{4}{*}{$\begin{array}{l}{ }^{2} \mathrm{D}-{ }^{-2} \mathrm{P}^{\circ} \\
(13 \mathrm{uv})\end{array}$} & 772.91 & 101027 & 230408 & 10 & 6 & 16 & 0.086 & 2.2 & -0.06 & E & \\
\hline & & & 772.891 & 101024 & 230409 & 6 & 4 & 14 & 0.085 & $1 .:$ & -0.29 & E & \\
\hline & & & 772.975 & 101032 & 230405 & 4 & 2 & 16 & 0.072 & 0.73 & -0.54 & E & \\
\hline & & & [772.93] & 101032 & 230409 & 4 & 4 & 1.6 & 0.015 & 0.15 & -1.23 & E & \\
\hline \multirow[t]{5}{*}{7} & & $\begin{array}{l}{ }^{2} \mathrm{~S}-{ }^{2} \mathrm{P}^{\circ} \\
(17 \mathrm{uv})\end{array}$ & 1006.0 & 131004 & 230408 & 2 & 6 & 6.0 & 0.27 & 1.8 & -0.26 & E & \\
\hline & & ${ }^{2} \mathrm{P}-{ }^{2} \mathrm{D}^{\circ}$ & 1750.4 & 145950 & 203079 & 6 & 10 & 2.6 & 0.20 & 6.9 & 0.08 & $\mathrm{E}$ & \\
\hline & & & 1751.75 & 145987 & 203072 & 4 & 6 & 2.6 & 0.18 & 4.1 & -0.15 & $\mathrm{E}$ & \\
\hline & & & 1747.86 & 145876 & 203089 & 2 & 4 & 2.2 & 0.2 & 2. & -0.40 & E & \\
\hline & & & 1751.24 & 145987 & 203089 & 4 & 4 & 0.43 & 0.020 & 0.46 & -1.10 & $\mathrm{E}$ & \\
\hline \multirow[t]{5}{*}{9} & & ${ }^{2} \mathrm{P}-{ }^{2} \mathrm{P}^{\circ}$ & 1184.0 & 145950 & 230408 & 6 & 6 & 8.5 & 0.18 & 4.2 & 0.03 & E & 2 \\
\hline & & & 1184.54 & 145987 & 230409 & 4 & 4 & 7.0 & 0.15 & 2.3 & -0.23 & $\mathrm{E}$ & ls \\
\hline & & & 1183.03 & 145876 & 230405 & 2 & 2 & 5.7 & & 0.93 & -0.62 & $\mathrm{E}$ & $l$ \\
\hline & & & 1184.54 & 145987 & 230405 & 4 & 2 & 2.9 & 0.030 & 0.47 & -0.92 & $\mathrm{E}$ & \\
\hline & & & 1183.03 & 145876 & 230409 & 2 & 4 & 1.4 & 0.060 & 0.47 & -0.92 & $\mathrm{E}$ & \\
\hline \multirow[t]{3}{*}{10} & \multirow[t]{3}{*}{$2 p-\left({ }^{1} \mathrm{~S}\right) 3 s$} & ${ }^{2} \mathrm{P}^{\circ}-{ }^{2} \mathrm{~S}$ & 452.11 & 116.3 & 221302 & 6 & 2 & 45 & 0.046 & 0.41 & -0.56 & $\mathrm{E}$ & \\
\hline & & & 452.226 & 174.5 & 221302 & 4 & 2 & 30 & 0.045 & 0.27 & -0.74 & $\mathrm{E}$ & ls \\
\hline & & & 451.869 & 0.0 & 221302 & 2 & 2 & 15 & 0.047 & 0.14 & -1 & $\mathrm{E}$ & \\
\hline 11 & $2 p-\left({ }^{1} \mathrm{~S}\right) 3 d$ & ${ }^{2} \mathrm{P}^{\circ}-{ }^{2} \mathrm{D}$ & 374.36 & 116.3 & 267242 & 6 & 10 & 110 & 0.39 & 2.9 & 0.37 & $\mathrm{D}-$ & \\
\hline & & & 374.441 & 174.5 & 267244 & 4 & 6 & 110 & 0.3 & 1.7 & 0.14 & $\mathrm{D}-$ & \\
\hline & & & 374.204 & 0.0 & 267239 & 2 & 4 & 94 & 0.39 & 0.97 & -0.10 & $\mathrm{D}-$ & ls \\
\hline & & & [374.44] & 174.5 & 267239 & 4 & 4 & 18 & 0.039 & 0.19 & -0.81 & $0-$ & \\
\hline 12 & $3 s-\left({ }^{1} \mathrm{~S}\right) 3 p$ & ${ }^{2} \mathrm{~S}-{ }^{2} \mathrm{P}^{\circ}$ & 4099.2 & 221302 & 245690 & 2 & 6 & 0.97 & 0.73 & 19.7 & 0.164 & C & 3. ca \\
\hline & & & 4097.31 & 221302 & 245702 & 2 & 4 & 0.9 & 0.486 & 13.1 & -0.013 & C & ls $s$ \\
\hline & & & 4103.37 & 221302 & 245666 & 2 & 2 & 0.97 & $0.2+4$ & 6.6 & -0.311 & C & \\
\hline
\end{tabular}


N III. Allowed Transitions - Continued

\begin{tabular}{|c|c|c|c|c|c|c|c|c|c|c|c|c|c|}
\hline No. & $\begin{array}{c}\text { Transition } \\
\text { Array }\end{array}$ & Multiplet & $\lambda(\AA)$ & $E_{i}\left(\mathrm{~cm}^{-1}\right)$ & $E_{k}\left(\mathrm{~cm}^{-1}\right)$ & $g_{i}$ & $g_{k}$ & $A_{k i}\left(10^{*} \sec ^{-1}\right)$ & $f_{i k}$ & $S$ (at.u.) & $\log g f$ & $\begin{array}{l}\text { Accu- } \\
\text { racy }\end{array}$ & Source \\
\hline \multirow[t]{8}{*}{13} & \multirow{8}{*}{$\begin{array}{l}2 s 2 p 3 s- \\
2 s 2 p\left({ }^{(3} \mathrm{P}^{\circ}\right) 3 p\end{array}$} & \multirow{8}{*}{$\begin{array}{c}{ }^{4} \mathrm{P}^{\circ}-{ }^{4} \mathrm{D} \\
\quad(3)\end{array}$} & 4517.3 & 287646 & 309777 & 12 & 20 & 0.70 & 0.357 & 64 & 0.63 & $\mathrm{C}$ & \\
\hline & & & 4514.89 & 287714 & 309857 & 6 & 8 & 0.70 & 0.286 & 25.5 & 0.235 & $\mathrm{C}$ & \\
\hline & & & 4510.92 & 287598 & 309761 & 4 & 6 & 0.492 & 0.225 & 13.4 & -0.046 & $\mathrm{C}$ & s \\
\hline & & & 2 & 287536 & 309698 & 2 & 4 & 0.293 & 0.179 & 5.3 & -0.446 & $\mathrm{C}$ & \\
\hline & & & $\begin{array}{l}4534.57 \\
4523.60\end{array}$ & $\begin{array}{l}287714 \\
287598\end{array}$ & $\begin{array}{l}309761 \\
309698\end{array}$ & $\begin{array}{l}6 \\
4\end{array}$ & $\begin{array}{l}6 \\
4\end{array}$ & $\begin{array}{l}0.208 \\
0.372\end{array}$ & $\mid \begin{array}{l}0.064 \\
0.114\end{array}$ & $\begin{array}{l}5.7 \\
6.8\end{array}$ & $\begin{array}{l}-0.416 \\
-0.341\end{array}$ & $\stackrel{\mathrm{C}}{\mathrm{C}}$ & \\
\hline & & & 4518.18 & 287536 & 309663 & 2 & 2 & 0.58 & 0.178 & 5.3 & -0.449 & $\mathrm{C}$ & \\
\hline & & & 4547.34 & 287714 & 309698 & 6 & 4 & 0.0344 & 0.0071 & 0.64 & -1.371 & $\mathrm{C}$ & \\
\hline & & & & 287598 & 309663 & 4 & 2 & 0.116 & 0.0178 & 1.06 & -1.148 & $\mathrm{C}$ & \\
\hline \multirow[t]{4}{*}{14} & & \multirow{4}{*}{$\begin{array}{c}{ }^{4} \mathrm{P}^{\circ}-{ }^{4} \mathrm{~S} \\
\quad(4)\end{array}$} & 3761.4 & 287646 & 314224 & 12 & 4 & 1.24 & 0.088 & 13.1 & 0.024 & $\mathrm{C}$ & \\
\hline & & & 3771.08 & 287714 & 314224 & 6 & 4 & 0.61 & 0.087 & 6.5 & -0.282 & $\mathrm{C}$ & \\
\hline & & & 3754.62 & 287598 & 314224 & 4 & 4 & 0.416 & 0.088 & 4.35 & -0.454 & $\mathrm{C}$ & \\
\hline & & & 3745.83 & 287536 & 314224 & 2 & 4 & 0.209 & 0.088 & 2.17 & -0.75 & $\mathrm{C}$ & \\
\hline \multirow[t]{7}{*}{15} & & \multirow{7}{*}{${ }^{4} \mathrm{P}^{\circ}-{ }^{4} \mathrm{P}$} & 3363.9 & 287646 & 317365 & 12 & 12 & 1.74 & 0.296 & 39.3 & 0.55 & $\mathrm{C}$ & \\
\hline & & & 3367.36 & 287714 & 317402 & 6 & 6 & 1.22 & 0.207 & 13.8 & 0.094 & $\mathrm{C}$ & \\
\hline & & & 3361.90 & 287598 & 317343 & 4 & 4 & 0.233 & 0.0395 & 1. & -0.80 & $\mathrm{C}$ & \\
\hline & & & 8.72 & 287536 & 317300 & 2 & 2 & 0.292 & 0.0494 & 1.09 & -1.005 & $\mathrm{C}$ & \\
\hline & & & $\begin{array}{l}3374.06 \\
236570\end{array}$ & 287714 & 317343 & 6 & 4 & 0.78 & 0.089 & 5.9 & -0.273 & $\mathrm{C}$ & \\
\hline & & & $\begin{array}{l}3365.79 \\
3354.29\end{array}$ & $\begin{array}{l}287598 \\
287598\end{array}$ & $\begin{array}{l}317300 \\
317402\end{array}$ & $\begin{array}{l}4 \\
4\end{array}$ & $\begin{array}{l}2 \\
6\end{array}$ & $\begin{array}{l}1.45 \\
0.53\end{array}$ & $\mid \begin{array}{l}0.123 \\
0.134\end{array}$ & $\begin{array}{l}5.5 \\
5.9\end{array}$ & $\begin{array}{l}-0.308 \\
-0.271\end{array}$ & $\stackrel{\mathrm{C}}{\mathrm{C}}$ & \\
\hline & & & 3353.78 & 287536 & 317343 & 2 & 4 & 0.73 & 0.247 & 5.5 & -0.306 & $\mathrm{C}$ & \\
\hline \multirow[t]{4}{*}{16} & & \multirow{4}{*}{$\begin{array}{c}{ }^{2} \mathrm{P}^{\circ}-{ }^{2} \mathrm{D} \\
(6)\end{array}$} & 4199.6 & 297225 & 321030 & 6 & 10 & 1.00 & 0.442 & 36.7 & 0.424 & $\mathrm{C}$ & \\
\hline & & & 4200.02 & 297263 & 321066 & 4 & 6 & 1.00 & 0.398 & 22.0 & 0.202 & $\mathrm{C}$ & \\
\hline & & & 4195.70 & 297150 & 320977 & 2 & 4 & $\mid 0.8$ & 0.442 & 12.2 & -0.054 & C & \\
\hline & & & 4215.69 & 297263 & 320977 & 4 & 4 & $\mid 0.165$ & 0.0440 & 2.44 & -0.75 & C & \\
\hline \multirow[t]{3}{*}{17} & & \multirow{3}{*}{${ }^{2 \mathrm{P}^{\circ}-{ }^{2} \mathrm{~S}}(7)$} & 3351.1 & 297225 & 327057 & 6 & 2 & 2.00 & 0.112 & 7.4 & -0.173 & $\mathrm{C}$ & \\
\hline & & & 3355.47 & 297263 & 327057 & 4 & 2 & 1.33 & 0.112 & 4.95 & -0.349 & C & \\
\hline & & & 3342.77 & 297150 & 327057 & 2 & 2 & 0.67 & 0.113 & 2.49 & -0.65 & C & \\
\hline \multirow[t]{4}{*}{18} & \multirow{19}{*}{$\begin{array}{l}2 s 2 p 3 p- \\
2 s 2 p\left({ }^{3} \mathrm{P}^{\circ}\right) 3 d\end{array}$} & \multirow{4}{*}{$\begin{array}{c}{ }^{2} \mathrm{P}-{ }^{2} \mathrm{D}^{\circ} \\
(8)\end{array}$} & 3937.4 & 309168 & 334558 & 6 & 10 & 0.96 & 0.372 & 28.9 & 0.349 & $\mathrm{C}$ & \\
\hline & & & 3938.52 & 309186 & 334569 & 4 & 6 & 0.9 & I & 17.4 & 0.127 & $\mathrm{C}$ & \\
\hline & & & 3934.41 & 309133 & 334542 & 2 & 4 & $\mid 0.8$ & 0.372 & 9. & -0.128 & $\mathrm{C}$ & \\
\hline & & & 3942.78 & 309186 & 334542 & 4 & 4 & $\mid 0.160$ & 0.0372 & 1.93 & -0.83 & C & \\
\hline \multirow[t]{5}{*}{19} & & \multirow{5}{*}{$\mid \begin{array}{c}{ }^{2} \mathrm{P}-{ }^{2} \mathrm{P}^{\circ} \\
(25 \mathrm{uv})\end{array}$} & 2979.9 & 309168 & 342717 & 6 & 6 & 1.38 & 0.184 & 10.8 & 0.043 & C & \\
\hline & & & 2983.58 & 309186 & 342693 & 4 & 4 & 1.14 & 0.153 & 6.0 & -0.214 & C & \\
\hline & & & 2972.60 & 309133 & 342764 & 2 & 2 & 0.9 & & 2.40 & -0.61 & C & \\
\hline & & & [2977.3] & 309186 & 342764 & 4 & 2 & $\mid 0.461$ & 0.0307 & 1.20 & -0.91 & C & \\
\hline & & & [2978.8] & 309133 & 342693 & 2 & 4 & 0.230 & 0.061 & 1.20 & -0.91 & C & \\
\hline \multirow[t]{10}{*}{20} & & \multirow{10}{*}{${ }_{(9)}^{4} \mathrm{D}-{ }^{4} \mathrm{~F}^{\circ}$} & 4864.8 & 309777 & 330327 & 20 & 28 & 0.63 & 0.312 & 100 & 0.80 & $\mathrm{C}$ & \\
\hline & & & 4867.18 & 309857 & 330397 & 8 & 10 & 0.63 & 0.2 & 35.8 & 0.349 & C & \\
\hline & & & 4861.33 & 309761 & 330325 & 6 & 8 & 0.5 & 0.2 & 24.5 & 0.1 & C & \\
\hline & & & 4858.88 & 309698 & 330274 & 4 & 6 & 0.471 & 0.250 & 16.0 & 0.000 & C & \\
\hline & & & 4858.74 & 309663 & 330238 & 2 & 4 & 0.441 & 0.312 & 10.0 & -0.205 & $\mathrm{C}$ & \\
\hline & & & 4884.14 & 309857 & 330325 & 8 & 8 & 0.08 & 0.0318 & 4.09 & -0. & C & \\
\hline & & & 4873.58 & 309761 & 330274 & 6 & 6 & 0.152 & 0.054 & 5.2 & -0.490 & C & ? \\
\hline & & & 4867.18 & 309698 & 330238 & 4 & 4 & 0.175 & 0.062 & 3.97 & -0.61 & C & $y$ \\
\hline & & & 4896.71 & 309857 & 330274 & 8 & 6 & 0.0059 & 0.00158 & 0.204 & -1.90 & C & \\
\hline & & & 4881.81 & 309761 & 330238 & 6 & 4 & 0.0124 & 0.0029 & 0.285 & -1.75 & C & \\
\hline
\end{tabular}


N III. Allowed Transitions-Continued

\begin{tabular}{|c|c|c|c|c|c|c|c|c|c|c|c|c|c|}
\hline No. & $\begin{array}{c}\text { Transition } \\
\text { Array }\end{array}$ & Multiplet & $\lambda(\AA)$ & $E_{i}\left(\mathrm{~cm}^{-1}\right)$ & $E_{k}\left(\mathrm{~cm}^{-1}\right)$ & $g_{i}$ & $g_{k}$ & $A_{k i}\left(10^{K} \sec ^{-1}\right)$ & $f_{i k}$ & $S($ at.u.) & $\log g f$ & $\begin{array}{c}\text { Accu- } \\
\text { racy }\end{array}$ & Source \\
\hline \multirow[t]{10}{*}{21} & & ${ }^{4} \mathrm{D}-{ }^{4} \mathrm{D}^{\circ}$ & 43335.7 & 309777 & 3.328 .35 & 20 & 20 & 0.234 & 0.066 & 18.8 & 0.121 & $\mathrm{C}$ & $c a$ \\
\hline & & & 4348.36 & 309857 & 332860 & 8 & 8 & 0.198 & 0.056 & 6.4 & -0.349 & C & ls \\
\hline & & & 4335.53 & 309761 & 332832 & 6 & 6 & 0.134 & 0.0378 & 3.24 & -0.64 & $\mathrm{C}$ & ls \\
\hline & & & 4328.15 & 309698 & 332810 & 4 & 4 & 0.094 & 0.0263 & 1.50 & -0.98 & $\mathrm{C}$ & ls \\
\hline & & & 4323.93 & 309663 & 332797 & 2 & 2 & 0.117 & 0.0329 & 0.94 & -1.182 & $\mathrm{C}$ & ls \\
\hline & & & 4353.66 & 309857 & 332832 & 8 & 6 & 0.0436 & 0.0093 & 1.07 & -1.128 & $\mathrm{C}$ & ls \\
\hline & & & 4339.52 & $\begin{array}{l}309761 \\
300609\end{array}$ & $\begin{array}{l}332810 \\
322707\end{array}$ & 6 & 4 & 0.081 & 0.0153 & 1.31 & -1.037 & C & ls \\
\hline & & & 4330.14 & 309761 & 332860 & $\begin{array}{l}4 \\
6\end{array}$ & $\begin{array}{l}2 \\
8\end{array}$ & $\begin{array}{l}0.117 \\
0.0334\end{array}$ & $\begin{array}{l}0.0164 \\
0.0125\end{array}$ & $\begin{array}{l}0.94 \\
1.07\end{array}$ & $\begin{array}{l}-1.183 \\
-1.125\end{array}$ & $\begin{array}{l}\mathrm{C} \\
\mathrm{C}\end{array}$ & $\begin{array}{l}l s \\
l s\end{array}$ \\
\hline & & & 4323.93 & 309698 & 332832 & 4 & 6 & 0.056 & 0.0236 & 1.34 & -1.025 & $\mathrm{C}$ & ls \\
\hline & & & 4321.37 & 309663 & 332810 & 2 & 4 & 0.059 & 0.0329 & 0.94 & -1.182 & $\mathrm{C}$ & ls \\
\hline \multirow[t]{4}{*}{22} & & ${ }^{4} \mathrm{~S}-{ }^{4} \mathrm{P}^{\circ}$ & 4539.6 & 314224 & 336246 & 4 & 12 & 0.99 & 0.91 & 54 & 0.56 & C & $c a$ \\
\hline & & & 4546.36 & 314224 & 336213 & 4 & 6 & 0.99 & 0.456 & 27.2 & 0.261 & C & ls \\
\hline & & & 4535.11 & 314224 & 336268 & 4 & 4 & 0.99 & 0.304 & 18.2 & 0.085 & $\mathrm{C}$ & ls \\
\hline & & & 4527.86 & 314224 & 336303 & 4 & 2 & 0.99 & 0.152 & 9.1 & -0.216 & $\mathrm{C}$ & ls \\
\hline \multirow[t]{8}{*}{23} & & $\begin{array}{c}{ }^{4} \mathrm{P}-{ }^{4} \mathrm{D}^{\circ} \\
(14)\end{array}$ & 6462.3 & 317365 & 332835 & 12 & 20 & 0.432 & 0.451 & 115 & 0.73 & C & $c a$ \\
\hline & & & 6466.86 & 317402 & 332860 & 6 & 8 & 0.432 & 0.361 & 46.1 & 0.336 & $\mathrm{C}$ & Is \\
\hline & & & 6453.95 & 317343 & 332832 & 4 & 6 & 0.304 & 0.285 & 24.2 & 0.057 & $\mathrm{C}$ & Is \\
\hline & & & 6445.05 & 317300 & 332810 & 2 & 4 & 0.181 & 0.226 & 9.6 & -0.345 & $\mathrm{C}$ & Is \\
\hline & & & 6478.69 & 317402 & 332832 & 6 & 6 & 0.129 & 0.081 & 10.4 & -0.313 & C & ls \\
\hline & & & 6463.03 & 317343 & 332810 & 4 & 4 & 0.232 & 0.145 & 12.3 & -0.237 & C & ls \\
\hline & & & 6450.78 & 317300 & 332797 & 2 & 2 & 0.362 & 0.226 & 9.6 & -0.345 & C & ls \\
\hline & & & $\begin{array}{l}6487.55 \\
6468.77\end{array}$ & $\begin{array}{l}317402 \\
317343\end{array}$ & $\begin{array}{l}332810 \\
332797\end{array}$ & $\begin{array}{l}6 \\
4\end{array}$ & $\begin{array}{l}4 \\
2\end{array}$ & $\begin{array}{l}0.0214 \\
0.072\end{array}$ & $\begin{array}{l}0.0090 \\
0.0226\end{array}$ & $\begin{array}{l}1.15 \\
1.93\end{array}$ & $\begin{array}{l}-1.268 \\
-1.044\end{array}$ & $\begin{array}{l}\mathrm{C} \\
\mathrm{C}\end{array}$ & $\begin{array}{l}\text { ls } \\
\text { ls }\end{array}$ \\
\hline \multirow[t]{3}{*}{24} & $3 p-\left({ }^{1} \mathrm{~S}\right) 4 s$ & ${ }^{2} \mathrm{P}^{\circ}-{ }^{2} \mathrm{~S}$ & 1805.1 & 245690 & 301088 & 6 & 2 & 6.8 & 0.110 & 3.93 & -0.180 & C & $3, c a$ \\
\hline & & & 1805.5 & 245702 & 301088 & 4 & 2 & 4.51 & 0.110 & 2.62 & -0.356 & $\mathrm{C}$ & ls \\
\hline & & & 1804.3 & 245666 & 301088 & 2 & 2 & 2.26 & 0.110 & 1.31 & -0.66 & $\mathrm{C}$ & ls \\
\hline \multirow[t]{4}{*}{25} & $3 d-\left({ }^{1} \mathrm{~S}\right) 4 p$ & ${ }^{2} \mathrm{D}-{ }^{2} \mathrm{P}^{\circ}$ & 2248.2 & 267242 & 311708 & 10 & 6 & 1.6 & 0.074 & 5.5 & -0.13 & D & $3, c a$ \\
\hline & & & 2247.92 & 267244 & 311716 & 6 & 4 & 1.5 & 0.074 & 3.3 & -0.35 & D & Is \\
\hline & & & 2248.88 & 267239 & 311691 & 4 & 2 & 1.6 & 0.062 & 1.8 & -0.61 & D & ls \\
\hline & & & [224.7.7] & 267239 & 311716 & 4 & 4 & 0.16 & 0.012 & 0.37 & -1.30 & D & ls \\
\hline \multirow[t]{8}{*}{26} & $\begin{array}{l}2 s 2 p 3 d- \\
\quad 2 s 2 p\left({ }^{3} \mathrm{P}^{\circ}\right) 4 p\end{array}$ & $\begin{array}{l}{ }^{4} \mathrm{P}^{\circ}-{ }^{4} \mathrm{D} \\
(28 \mathrm{uv})\end{array}$ & 2460.4 & 336246 & 376877 & 12 & 20 & 0.014 & 0.0022 & 0.21 & -1.59 & D & $c a$ \\
\hline & & & 2453.85 & 336213 & 376953 & 6 & 8 & 0.015 & 0.0018 & 0.085 & -1.98 & D & Is \\
\hline & & & 2462.56 & 336268 & 376864 & 4 & 6 & 0.010 & 0.0014 & 0.046 & -2.25 & D & ls \\
\hline & & & 2468.36 & 336303 & 376803 & 2 & 4 & 0.0062 & 0.0011 & 0.018 & -2.65 & D & Is \\
\hline & & & [2459.2] & 336213 & 376864 & 6 & 6 & 0.0044 & $4.0 \times 10^{-4}$ & 0.019 & -2.62 & D & Is \\
\hline & & & $\begin{array}{l}{[2466.3]} \\
{[2471.2]}\end{array}$ & $\begin{array}{l}336268 \\
336303\end{array}$ & $\begin{array}{l}376803 \\
376757\end{array}$ & $\begin{array}{l}4 \\
2\end{array}$ & $\begin{array}{l}4 \\
2\end{array}$ & $\begin{array}{l}0.0079 \\
0.012\end{array}$ & $\begin{array}{l}7.2 \times 10^{-4} \\
0.0011\end{array}$ & $\begin{array}{l}0.023 \\
0.018\end{array}$ & $\begin{array}{l}-2.54 \\
-2.64\end{array}$ & $\begin{array}{l}\mathrm{D} \\
\mathrm{D}\end{array}$ & Is \\
\hline & & & [24.62.9] & 336213 & 376803 & 6 & 4 & $7.4 \times 10^{-4}$ & $4.5 \times 10^{-5}$ & 0.0022 & -3.57 & D & ls \\
\hline & & & [2469.1] & 336268 & 376757 & 4 & 2 & 0.0025 & $1.1 \times 10^{-4}$ & 0.0037 & -3.34 & D & ls \\
\hline 27 & $3 d-\left({ }^{1} \mathrm{~S}\right) 4 f$ & $\begin{array}{c}{ }^{2} \mathrm{D}-{ }^{2} \mathrm{~F}^{\circ} \\
(24 \mathrm{uv})\end{array}$ & 1885.25 & 267242 & 320285 & 10 & 14 & 11.9 & 0.89 & 55 & 0.95 & $\mathrm{C}$ & 3. $c a$ \\
\hline 28 & $\begin{array}{l}2 s 2 p 3 d- \\
\quad 2 s 2 p\left({ }^{3} \mathrm{P}^{\circ}\right) 4 f\end{array}$ & $\begin{array}{c}{ }^{2} \mathrm{D}^{\circ}-{ }^{2} \mathrm{~F} \\
(27 \mathrm{uv})\end{array}$ & 1908.11 & 334.558 & 386965 & 10 & 14 & 11.0 & 0.84 & 53 & 0.92 & C & $c a$ \\
\hline
\end{tabular}


N III. Allowed Transitions - Continued

\begin{tabular}{|c|c|c|c|c|c|c|c|c|c|c|c|c|c|}
\hline No. & $\begin{array}{c}\text { Transition } \\
\text { Array }\end{array}$ & Multiplet & $\lambda(\AA)$ & $E_{i}\left(\mathrm{~cm}^{-1}\right)$ & $E_{k}\left(\mathrm{~cm}^{-1}\right)$ & $g_{i}$ & $g_{k}$ & $A_{k i}\left(10^{8} \sec ^{-1}\right)$ & $f_{i k}$ & $S$ (at.u.) & $\log g f$ & $\begin{array}{c}\text { Accu- } \\
\text { racy }\end{array}$ & Source \\
\hline \multirow[t]{9}{*}{29} & & ${ }^{4} \mathrm{P}^{\circ}-{ }^{4} \mathrm{D}$ & 1920.6 & 336246 & 388313 & 12 & 20 & 10.3 & 0.95 & 72 & 1.057 & C & $c a$ \\
\hline & & & 1920.86 & 336213 & 388273 & 6 & 8 & 10.3 & 0.76 & 28.7 & 0.66 & C & $l s$ \\
\hline & & & 1921.49 & 336268 & 388311 & 4 & 6 & 7.2 & 0.60 & 15.1 & 0.38 & C & $l s$ \\
\hline & & & 1920.86 & 336303 & 388359 & 2 & 4 & 4.27 & 0.472 & $6.0^{\circ}$ & -0.025 & C & $l s$ \\
\hline & & & [1919.5] & 336213 & 388311 & 6 & 6 & 3.08 & 0.170 & 6.4 & 0.009 & C & $l s$ \\
\hline & & & [1919.7] & 336268 & 388359 & 4 & 4 & 5.5 & 0.302 & 7.6 & 0.082 & C & $l s$ \\
\hline & & & [1920.0] & 336303 & 388387 & 2 & 2 & 8.5 & 0.471 & 6.0 & -0.026 & C & $l s$ \\
\hline & & & [1917.7] & 336213 & 388359 & 6 & 4 & 0.51 & 0.0188 & 0.71 & -0.95 & C & $l s$ \\
\hline & & & [1918.7] & 336268 & 388387 & 4 & 2 & 1.71 & 0.0471 & 1.19 & -0.72 & C & $l s$ \\
\hline \multirow[t]{4}{*}{30} & & ${ }^{2} \mathrm{~F}^{\circ}-{ }^{2} \mathrm{G}$ & 2063.8 & 339808 & 388246 & 14 & 18 & 11.3 & 0.93 & 88 & 1.115 & C & $c a$ \\
\hline & & & 2063.99 & 339856 & 388290 & 8 & 10 & 11.3 & 0.90 & 48.9 & 0.86 & C & $l s$ \\
\hline & & & 2063.50 & 339744 & 388190 & 6 & 8 & 10.9 & 0.93 & 37.9 & 0.75 & C & $l s$ \\
\hline & & & 2068.25 & 339856 & 388190 & 8 & 8 & 0.402 & 0.0258 & 1.41 & -0.69 & C & $l s$ \\
\hline \multirow[t]{4}{*}{31} & $4 d-\left({ }^{1} \mathrm{~S}\right) 5 f$ & ${ }^{2} \mathrm{D}-{ }^{2} \mathrm{~F}^{\circ}$ & 4001.8 & 317770 & 342752 & 10 & 14 & 2.11 & 0.709 & 93.4 & 0.851 & $\mathrm{C}+$ & $c a$ \\
\hline & & & 4003.64 & 317782 & 342752 & 6 & 8 & 2.10 & 0.674 & 53.3 & 0.609 & $\mathrm{C}+$ & $l s$ \\
\hline & & & 3998.69 & 317751 & 342752 & 4 & 6 & 1.98 & 0.710 & 37.4 & 0.454 & $\mathrm{C}+$ & $l s$ \\
\hline & & & [4003.6] & 317782 & 342752 & 6 & 6 & 0.140 & 0.0338 & 2.67 & -0.693 & $\mathrm{C}+$ & $l s$ \\
\hline
\end{tabular}

\section{Forbidden Transitions}

Naqvi's calculation [1] of the one possible transition in the ground state configuration $2 p$ is the only available source. The line strength should be quite accurate, since it does not sensitively depend on the choice of the interaction parameters.

Reference

[1] Naqvi, A. M., Thesis Harvard (1951).

N III. Forbidden Transitions

\begin{tabular}{|c|c|c|c|c|c|c|c|c|c|c|c|c|}
\hline No. & $\begin{array}{c}\text { Transition } \\
\text { Array }\end{array}$ & Multiplet & $\lambda(\AA)$ & $E_{i}\left(\mathrm{~cm}^{-1}\right)$ & $E_{k}\left(\mathrm{~cm}^{-1}\right)$ & $g_{i}$ & $g_{k}$ & $\begin{array}{l}\text { Type of } \\
\text { Transi- } \\
\text { tion }\end{array}$ & $A_{k i}\left(\sec ^{-1}\right)$ & $S($ at.u. $)$ & $\begin{array}{l}\text { Accu- } \\
\text { racy }\end{array}$ & Source \\
\hline 1 & $2 p-2 p$ & ${ }^{2} \mathbf{P}^{\circ}-{ }^{2} \mathbf{P}^{\circ}$ & {$\left[57.29 \times 10^{4}\right]$} & 0 & 174.5 & 2 & 4 & m & $4.77 \times 10^{-5}$ & 1.33 & B & 1 \\
\hline
\end{tabular}




\section{Allowed Transitions}

List of tabulated lines:

\begin{tabular}{l|l||l|l||l|l}
\hline \hline Wavelength $[\AA]$ & No. & Wavelength $[\AA]$ & No. & Wavelength $[\AA]$ & No. \\
\hline & & & & & \\
225.025 & 10 & 923.211 & 3 & 4685.4 & 20 \\
225.098 & 10 & 923.669 & 3 & 4723 & 20 \\
225.13 & 10 & 924.274 & 3 & 4733 & 20 \\
225.136 & 10 & 955.335 & 5 & 4740 & 20 \\
225.20 & 10 & 1718.52 & 4 & 4752 & 20 \\
247.205 & 2 & 3443 & 15 & 4762 & 20 \\
283.420 & 8 & 3445 & 15 & 5073 & 13 \\
283.47 & 8 & 3454 & 15 & 5236 & 13 \\
283.470 & 8 & 3461.34 & 15 & 5245 & 13 \\
283.579 & 8 & 3463.36 & 15 & 5280.9 & 13 \\
283.59 & 8 & 3474.56 & 15 & 5281 & 13 \\
322.503 & 6 & 3478.69 & 11 & 5303.9 & 19 \\
322.570 & 6 & 3482.98 & 11 & 5349.8 & 12 \\
322.724 & 6 & 3484.90 & 11 & 5734 & \\
335.050 & 9 & 3747.66 & 16 & 6383 & 18 \\
387.353 & 7 & 4057.80 & 17 & 7103.28 & 18 \\
765.140 & 1 & 4479 & 14 & 7109.48 & 18 \\
921.982 & 3 & 4495 & 14 & 7111.28 & 18 \\
922.507 & 3 & 4528 & 20 & 7123.10 & 18.21 \\
923.045 & 3 & 4678.6 & & 7129 & \\
& & & & & \\
\hline
\end{tabular}

Values for the $2 s^{2}-2 s 2 p$ and $2 s 2 p-2 p^{2}$ transition arrays are taken from the self-consistent field calculations of Weiss [1]. These calculations do not include the important effects of configuration interaction; hence large uncertainties must be expected. The average of the dipole length and velocity approximations is adopted [1]. Accuracies within $50 \%$ are indicated by the following comparison: Weiss [1] has undertaken refined calculations, including configuration interaction, for the same transitions in $\mathrm{Be} I$ - the first member of this isoelectronic sequence - in addition to calculations of the type done for this ion. In all cases the agreement with the average of the dipole length and velocity approximations is close.

For the remaining low-lying transitions Kelly's approximate Hartree-Fock calculations [2] are adopted, while for the moderately excited transitions Kelly's values are averaged with the Coulomb approximation, with which they agree quite well.

\section{References}

[1] Weiss, A. W., private communication (1964).

[2] Kelly, P. S., J. Quant. Spectrosc. Radiat. Transfer 4, 117-148 (1964). 
N IV. Allowed Transitions

\begin{tabular}{|c|c|c|c|c|c|c|c|c|c|c|c|c|c|}
\hline No. & $\begin{array}{c}\text { Transition } \\
\text { Array }\end{array}$ & Multiplet & $\lambda(\AA)$ & $E_{i}\left(\mathrm{~cm}^{-1}\right)$ & $E_{k}\left(\mathrm{~cm}^{-1}\right)$ & $g_{i}$ & $g_{k}$ & $A_{k i}\left(10^{8} \mathrm{sec}^{-1}\right)$ & $f_{i k}$ & $S($ at.u.) & $\log g f$ & $\begin{array}{c}\text { Accu- } \\
\text { racy }\end{array}$ & Source \\
\hline 1 & $\begin{array}{l}2 s^{2}- \\
2 s\left({ }^{2} \mathrm{~S}\right) 2 p\end{array}$ & $\begin{array}{c}{ }^{1} \mathrm{~S}-{ }^{1} \mathrm{P}^{\circ} \\
(1 \mathrm{uv})\end{array}$ & 765.140 & 0 & 130695 & 1 & 3 & 24 & 0.64 & 1.6 & -0.19 & D & 1 \\
\hline 2 & $\begin{array}{l}2 s^{2}- \\
2 s\left({ }^{2} \mathrm{~S}\right) 3 p\end{array}$ & $\begin{array}{c}{ }^{1} \mathrm{~S}-{ }^{1} \mathrm{P}^{\circ} \\
(2 \mathrm{uv})\end{array}$ & 247.205 & 0 & 404521 & 1 & 3 & 200 & 0.55 & 0.45 & -0.26 & D & 2 \\
\hline \multirow[t]{6}{*}{3} & \multirow[t]{8}{*}{$\begin{array}{c}2 s 2 p- \\
2 p^{2}\end{array}$} & \multirow[t]{6}{*}{$\begin{array}{c}{ }^{3} \mathrm{P}^{\circ}-{ }^{3} \mathrm{P} \\
(3 \mathrm{uv})\end{array}$} & 923.15 & [67273] & [175598] & 9 & 9 & 17 & 0.21 & 5.8 & 0.28 & $\mathrm{D}$ & 1 \\
\hline & & & 923.211 & [67344] & [175662] & 5 & 5 & 13 & 0.16 & 2.4 & -0.10 & $\mathrm{D}$ & $l s$ \\
\hline & & & 923.045 & [67200] & [175537] & 3 & 3 & 4.2 & 0.053 & 0.49 & -0.80 & D & ls \\
\hline & & & $\begin{array}{l}924.274 \\
923.669\end{array}$ & $\begin{array}{l}{[67344]} \\
{[67200]}\end{array}$ & {$[175537]$} & $\begin{array}{l}5 \\
3\end{array}$ & $\begin{array}{l}3 \\
1\end{array}$ & $\begin{array}{c}6.9 \\
17\end{array}$ & $\begin{array}{l}0.053 \\
0.071\end{array}$ & $\begin{array}{l}0.81 \\
0.65\end{array}$ & $\begin{array}{l}-0.58 \\
-0.67\end{array}$ & D & ls \\
\hline & & & 921.982 & [67200] & [175662] & 3 & 5 & 4.2 & 0.089 & 0.81 & -0.57 & D & ls \\
\hline & & & 922.507 & [67136] & {$[175537]$} & 1 & 3 & 5.6 & 0.21 & 0.65 & -0.67 & $\mathrm{D}$ & ls \\
\hline 4 & & $\begin{array}{c}{ }^{1} \mathrm{P}^{\circ}-{ }^{1} \mathrm{D} \\
(7 \mathrm{uv})\end{array}$ & 1718.52 & 130695 & 188885 & 3 & 5 & 5.1 & 0.38 & 6.4 & 0.05 & D & 1 \\
\hline 5 & & $\begin{array}{c}{ }^{1} \mathrm{P}^{\circ}-{ }^{1} \mathrm{~S} \\
(8 \mathrm{uv})\end{array}$ & 955.335 & 130695 & 235370 & 3 & 1 & 16 & 0.074 & 0.70 & -0.66 & $\mathrm{D}$ & 1 \\
\hline \multirow[t]{3}{*}{6} & \multirow[t]{4}{*}{$\begin{array}{l}2 s 2 p- \\
\quad 2 s\left({ }^{2} \mathrm{~S}\right) 3 s\end{array}$} & \multirow[t]{3}{*}{$\begin{array}{c}{ }^{3} \mathrm{P}^{\circ}-{ }^{3} \mathrm{~S} \\
(4 \mathrm{uv})\end{array}$} & 322.65 & {$[67273]$} & [377206] & 9 & 3 & 113 & 0.059 & 0.56 & -0.278 & $\mathrm{C}$ & 2 \\
\hline & & & 322.724 & [67344] & [377206] & 5 & 3 & 62 & 0.059 & 0.311 & -0.53 & $\mathrm{C}$ & ls \\
\hline & & & $\begin{array}{l}322.570 \\
322.503\end{array}$ & $\begin{array}{l}{[67200]} \\
{[67136]}\end{array}$ & $\begin{array}{l}{[377206]} \\
{[377206]}\end{array}$ & $\begin{array}{l}3 \\
1\end{array}$ & $\begin{array}{l}3 \\
3\end{array}$ & $\begin{array}{l}37.6 \\
12.5\end{array}$ & $\begin{array}{l}0.059 \\
0.058\end{array}$ & $\begin{array}{l}0.187 \\
0.062\end{array}$ & $\begin{array}{l}-0.75 \\
-1.234\end{array}$ & $\begin{array}{l}\mathrm{C} \\
\mathrm{C}\end{array}$ & $\begin{array}{l}l s \\
l s\end{array}$ \\
\hline 7 & & $\begin{array}{c}{ }^{1} \mathrm{P}^{\circ}-{ }^{1} \mathrm{~S} \\
(9 \mathrm{uv})\end{array}$ & 387.353 & 130695 & 388858 & 3 & 1 & 65 & 0.0489 & 0.187 & -0.83 & $\mathrm{C}$ & 2 \\
\hline \multirow[t]{6}{*}{8} & \multirow{7}{*}{$\begin{array}{l}2 s 2 p- \\
2 s\left({ }^{2} \mathrm{~S}\right) 3 d\end{array}$} & \multirow{6}{*}{$\begin{array}{c}{ }^{3} \mathrm{P}^{\circ}-{ }^{3} \mathrm{D} \\
(5 \mathrm{uv})\end{array}$} & 283.53 & [67273] & [419974] & 9 & 15 & 314 & 0.63 & 5.3 & 0.75 & C & 2 \\
\hline & & & 283.579 & [67344] & [419979] & 5 & 7 & 31.1 & 0.52 & 2.45 & 0.419 & $\mathrm{C}$ & ls \\
\hline & & & 283.470 & {$[67200]$} & {$[419971]$} & 3 & 5 & 235 & 0.471 & 1.32 & 0.151 & $\mathrm{C}$ & $l s$ \\
\hline & & & 283.420 & [67136] & [419968] & 1 & 3 & 172 & 0.62 & 0.58 & -0.207 & C & $l s$ \\
\hline & & & $\begin{array}{l}{[283.59]} \\
{[283.47]}\end{array}$ & $\begin{array}{l}{[67344]} \\
{[67200]}\end{array}$ & $\begin{array}{l}{[419971]} \\
{[419968]}\end{array}$ & $\begin{array}{l}5 \\
3\end{array}$ & $\begin{array}{l}5 \\
3\end{array}$ & $\begin{array}{r}78 \\
130\end{array}$ & $\begin{array}{l}0.094 \\
0.156\end{array}$ & $\begin{array}{l}0.438 \\
0.438\end{array}$ & $\begin{array}{l}-0.329 \\
-0.329\end{array}$ & $\stackrel{\mathrm{C}}{\mathrm{C}}$ & ls \\
\hline & & & [283.59] & [67344] & [419968] & 5 & 3 & 8.6 & 0.0063 & 0.0292 & -1.50 & $\mathrm{C}$ & ls \\
\hline 9 & & $\begin{array}{l}{ }^{1} \mathrm{P}^{\circ}-{ }^{1} \mathrm{D} \\
(10 \mathrm{uv})\end{array}$ & 335.050 & 130695 & 429158 & 3 & 5 & 189 & 0.53 & 1.75 & 0.200 & $\mathrm{C}$ & 2 \\
\hline \multirow[t]{6}{*}{10} & \multirow[t]{6}{*}{$\begin{array}{l}2 s 2 p- \\
2 s\left({ }^{2} \mathrm{~S}\right) 4 d\end{array}$} & \multirow[t]{6}{*}{$\begin{array}{c}{ }^{3} \mathrm{P}^{\circ}-{ }^{3} \mathrm{D} \\
(6 \mathrm{uv})\end{array}$} & 225.17 & [67273] & [511382] & 9 & 15 & 92 & 0.117 & 0.78 & 0.022 & C & 2 \\
\hline & & & 225.025 & [67344] & [511384] & 5 & 7 & 92 & 0.098 & 0.364 & -0.309 & $\mathrm{C}$ & ls \\
\hline & & & 225.136 & [67200] & [511376] & 3 & 5 & 69 & 0.088 & 0.195 & -0.58 & C & ls \\
\hline & & & 225.098 & [67136] & [511387] & 1 & 3 & 52 & 0.117 & 0.087 & -0.93 & C & ls \\
\hline & & & $\begin{array}{l}{[225.20]} \\
{[225.13]}\end{array}$ & $\begin{array}{l}{[67344]} \\
{[67200]}\end{array}$ & $\begin{array}{l}{[511376]} \\
{[511387]}\end{array}$ & $\begin{array}{l}5 \\
3\end{array}$ & $\begin{array}{l}5 \\
3\end{array}$ & $\begin{array}{l}23.1 \\
38.5\end{array}$ & $\begin{array}{l}0.0175 \\
0.0292\end{array}$ & 0.065 & $\begin{array}{l}-1.057 \\
-1.057\end{array}$ & $\begin{array}{l}\mathrm{C} \\
\mathrm{C}\end{array}$ & $\begin{array}{l}l s \\
l s\end{array}$ \\
\hline & & & [225.20] & [67344] & [511387] & 5 & 3 & 2.56 & 0.00117 & 0.00433 & -2.234 & $\mathrm{C}$ & ls \\
\hline \multirow[t]{3}{*}{11} & \multirow[t]{4}{*}{$\begin{array}{l}2 s 3 s- \\
2 s\left({ }^{2} \mathrm{~S}\right) 3 p\end{array}$} & \multirow[t]{3}{*}{$\begin{array}{c}{ }^{3} \mathrm{~S}-{ }^{3} \mathrm{P}^{\circ} \\
(1)\end{array}$} & 3480.8 & [377206] & [405927] & 3 & 9 & 1.16 & 0.634 & 21.8 & 0.279 & $\mathrm{C}+$ & $2, c a$ \\
\hline & & & 3478.69 & [377206] & [405944] & 3 & 5 & 1.16 & 0.352 & 12.1 & 0.024 & $\mathrm{C}+$ & $l s$ \\
\hline & & & $\begin{array}{l}3482.98 \\
3484.90\end{array}$ & $\begin{array}{l}{[377206]} \\
{[377206]}\end{array}$ & $\begin{array}{l}{[405909]} \\
{[405893]}\end{array}$ & $\begin{array}{l}3 \\
3\end{array}$ & $\begin{array}{l}3 \\
1\end{array}$ & $\begin{array}{l}1.16 \\
1.16\end{array}$ & $\begin{array}{l}0.211 \\
0.0703\end{array}$ & $\begin{array}{l}7.27 \\
2.42\end{array}$ & $\begin{array}{l}-0.198 \\
-0.676\end{array}$ & $\begin{array}{l}\mathrm{C}+ \\
\mathrm{C}+\end{array}$ & $\begin{array}{l}l s \\
l s\end{array}$ \\
\hline 12 & & $\begin{array}{c}{ }^{1} \mathrm{~S}-{ }^{1} \mathrm{P}^{\circ} \\
(2)\end{array}$ & 6383 & 388858 & 404521 & 1 & 3 & 0.193 & 0.353 & 7.42 & -0.452 & $\mathrm{C}+$ & $2, c a$ \\
\hline \multirow[t]{6}{*}{13} & \multirow[t]{6}{*}{$\begin{array}{l}2 p 3 s- \\
2 p\left({ }^{2} \mathrm{P}^{\circ}\right) 3 p\end{array}$} & \multirow{6}{*}{${ }^{3} \mathrm{P}^{\circ}-{ }^{3} \mathrm{D}$} & 5255.1 & [465382] & [484406] & 9 & 15 & 0.343 & 0.237 & 36.9 & 0.329 & C & $c a$ \\
\hline & & & 5245 & [465463] & [484525] & 5 & 7 & 0.345 & 0.199 & 17.2 & -0.002 & $\mathrm{C}$ & $l s$ \\
\hline & & & 5236 & [465301] & [484394] & 3 & 5 & 0.261 & 0.178 & 9.2 & -0.271 & $\mathrm{C}$ & ls \\
\hline & & & $\begin{array}{c}5281 \\
{[5280.9]}\end{array}$ & {$\left[\begin{array}{l}{[465223]} \\
{[465463]}\end{array}\right.$} & $\begin{array}{l}{[484150]} \\
{[4843941]}\end{array}$ & $\begin{array}{l}1 \\
5\end{array}$ & $\begin{array}{l}3 \\
5\end{array}$ & $\begin{array}{l}0.188 \\
0.085\end{array}$ & $\begin{array}{l}0.236 \\
0.0354\end{array}$ & $\begin{array}{l}4.10 \\
3.08\end{array}$ & $\begin{array}{l}-0.63 \\
-0.75\end{array}$ & $\begin{array}{l}\mathrm{C} \\
\mathrm{C}\end{array}$ & $\begin{array}{l}l s \\
l s\end{array}$ \\
\hline & & & [5303.9] & [465301] & [484150] & 3 & 3 & 0.139 & 0.059 & 3.08 & -0.75 & C & ls \\
\hline & & & [5349.8] & {$[465463$} & [484150] & 5 & 3 & 0.0090 & 0.00233 & 0.205 & -1.93 & $\mathrm{C}$ & ls \\
\hline
\end{tabular}


N IV. Allowed Transitions-Continued

\begin{tabular}{|c|c|c|c|c|c|c|c|c|c|c|c|c|c|}
\hline No & $\begin{array}{c}\text { Transition } \\
\text { Array }\end{array}$ & Multiplet & $\lambda(\AA)$ & $E_{i}\left(\mathrm{~cm}^{-1}\right)$ & $E_{k}\left(\mathrm{em}^{-1}\right)$ & $g_{i}$ & $g_{k}$ & $A_{k i}\left(10^{8} \sec ^{-1}\right)$ & $f_{i k}$ & $S($ at.u. $)$ & $\log g f$ & $\begin{array}{l}\text { Accu- } \\
\text { racy }\end{array}$ & Source \\
\hline \multirow[t]{3}{*}{14} & & ${ }^{3} \mathrm{P}^{0}-{ }^{3} \mathrm{~S}$ & 4511.4 & [465382] & [487542] & 9 & 3 & 0.56 & 0.056 & 7.5 & -0.29 .5 & C & $c a$ \\
\hline & & & 4528 & [465463] & [487542] & 5 & 3 & 0.305 & 0.056 & 4.19 & -0.55 & C & ls \\
\hline & & & $\begin{array}{l}4495 \\
4479\end{array}$ & $\begin{array}{l}{[465301]} \\
{[465223]}\end{array}$ & $\begin{array}{l}{[487542]} \\
{[487542]}\end{array}$ & $\begin{array}{l}3 \\
1\end{array}$ & $\begin{array}{l}3 \\
3\end{array}$ & $\begin{array}{l}0.189 \\
0.063\end{array}$ & $\begin{array}{l}0.057 \\
0.057\end{array}$ & $\begin{array}{l}2.54 \\
0.84\end{array}$ & $\begin{array}{l}-0.77 \\
-1.245\end{array}$ & $\begin{array}{l}\mathrm{C} \\
\mathrm{C}\end{array}$ & $\begin{array}{l}l_{s} \\
l_{s}\end{array}$ \\
\hline \multirow[t]{6}{*}{15} & & ${ }^{3} \mathrm{P}^{\circ}-{ }^{3} \mathrm{P}$ & 3456.7 & [465382] & [494303] & 9 & 9 & 1.27 & 0.227 & 23.2 & 0.310 & C & $c a$ \\
\hline & & & 3463.36 & [465463] & [494338] & 5 & 5 & 0.94 & 0.170 & 9.7 & -0.072 & C & $l s$ \\
\hline & & & 3454 & [465301] & [494240] & 3 & 3 & 0.316 & 0.057 & 1.93 & -0.77 & C & ls \\
\hline & & & 3474.56 & [465301] & [494338] & 3 & 5 & 0.311 & 0.094 & 3.22 & -0.55 & C & ls \\
\hline & & & 3461.34 & [465223] & [494240] & 1 & 3 & 0.420 & 0.226 & 2.58 & -0.65 & C & ls \\
\hline & & & $\begin{array}{l}3443 \\
3445\end{array}$ & $\begin{array}{l}{[465463]} \\
{[465301]}\end{array}$ & $\begin{array}{l}{[494240]} \\
{[494320]}\end{array}$ & $\begin{array}{l}5 \\
3\end{array}$ & $\begin{array}{l}3 \\
1\end{array}$ & $\begin{array}{l}0.53 \\
1.28\end{array}$ & $\begin{array}{l}0.057 \\
0.076\end{array}$ & $\begin{array}{l}3.22 \\
2.58\end{array}$ & $\begin{array}{l}-0.55 \\
-0.64\end{array}$ & $\begin{array}{l}\mathrm{C} \\
\mathrm{C}\end{array}$ & $\begin{array}{l}l s \\
l s\end{array}$ \\
\hline 16 & & ${ }^{1} \mathrm{P}^{\circ}-{ }^{1}{ }^{1} \mathrm{D}$ & 3747.66 & 473032 & 499708 & 3 & 5 & 1.06 & 0.371 & 13.7 & 0.047 & C & $c a$ \\
\hline 17 & $\begin{array}{l}2 s 3 p- \\
2 s\left({ }^{2} S\right) 3 d\end{array}$ & $\begin{array}{c}{ }^{1} \mathrm{P}^{\circ}-{ }^{-1} \mathrm{D} \\
(3)\end{array}$ & 4057.80 & 388858 & 429158 & 3 & 5 & 0.758 & 0.312 & 12.5 & -0.029 & $C+$ & $2, c a$ \\
\hline \multirow[t]{6}{*}{18} & & ${ }^{3} \mathrm{P}^{\circ}-{ }^{3} \mathrm{D}$ & 7117.0 & [405927] & [419974] & 9 & 15 & 0.132 & 0.167 & 35.3 & 0.178 & $\mathrm{C}+$ & $2, c a$ \\
\hline & & & 7123.10 & [405944] & [419979] & 5 & 7 & 0.132 & 0.141 & 16.5 & -0.153 & $C+$ & $l s$ \\
\hline & & & 7109.48 & [405909] & [419971] & 3 & 5 & 0.0995 & 0.126 & 8.82 & -0.424 & $\mathrm{C}+$ & $l s$ \\
\hline & & & $\begin{array}{l}7103.28 \\
7127.21\end{array}$ & $\begin{array}{l}{[405893]} \\
{[405944]}\end{array}$ & [419968] & $\begin{array}{l}1 \\
5\end{array}$ & $\begin{array}{l}3 \\
5\end{array}$ & $\begin{array}{l}0.0739 \\
0.0329\end{array}$ & $\begin{array}{l}0.168 \\
0.0251\end{array}$ & $\begin{array}{l}3.92 \\
2.94\end{array}$ & $\begin{array}{l}-0.776 \\
-0.902\end{array}$ & $\begin{array}{l}\mathrm{C}+ \\
\mathrm{C}+\end{array}$ & ls \\
\hline & & & 7111.28 & [405909] & [419968] & 3 & 3 & 0.0552 & 0.0419 & 2.94 & -0.901 & $\mathrm{C}+$ & ls \\
\hline & & & 7129 & {$[405944]$} & [4.19968] & 5 & 3 & 0.00365 & 0.00167 & 0.196 & -2.078 & $C+$ & $l s$ \\
\hline 19 & $\begin{array}{l}2 p 3 p- \\
2 p\left({ }^{2} \mathrm{P}^{\circ}\right) 3 d\end{array}$ & $\begin{array}{c}{ }^{1} \mathrm{P}-{ }^{1} \mathrm{D}^{\circ} \\
(9)\end{array}$ & 5734 & 480880 & 498315 & 3 & 5 & 0.178 & 0.146 & 8.3 & -0.359 & C & $c a$ \\
\hline \multirow[t]{6}{*}{20} & & ${ }^{3} \mathrm{D}-{ }^{3} \mathrm{D}^{\circ}$ & 4732.2 & [484406] & [505532] & 15 & 15 & 0.116 & 0.0389 & 9.1 & -0.234 & C & $c a$ \\
\hline & & & 4752 & [484525] & [505561] & 7 & 7 & 0.102 & 0.0344 & 3.77 & -0.62 & C & $l_{s}$ \\
\hline & & & $\begin{array}{c}4733 \\
{[4685.4]}\end{array}$ & $\begin{array}{l}{[484394]} \\
{[484150]}\end{array}$ & $\begin{array}{l}{[505518]} \\
{[505487]}\end{array}$ & $\begin{array}{l}5 \\
3\end{array}$ & $\begin{array}{l}5 \\
3\end{array}$ & $\begin{array}{l}0.081 \\
0.089\end{array}$ & $\begin{array}{l}0.0271 \\
0.0294\end{array}$ & 2.11 & $\begin{array}{l}-0.87 \\
-1.055\end{array}$ & C & Is \\
\hline & & & 4762 & [484525] & [505518] & 7 & 5 & 0.0177 & 0.00431 & 0.473 & -1.52 & $\mathrm{C}$ & $l s$ \\
\hline & & & 4740 & [484394] & [505487] & 5 & 3 & 0.0289 & 0.0058 & 0.455 & -1.54 & $\mathrm{C}$ & ls \\
\hline & & & $\begin{array}{c}4723 \\
{[4678.6]}\end{array}$ & $\begin{array}{l}{[484394]} \\
{[484150]}\end{array}$ & [505561] & $\begin{array}{l}5 \\
3\end{array}$ & $\begin{array}{l}7 \\
5\end{array}$ & $\begin{array}{l}0.0130 \\
0.0180\end{array}$ & $\begin{array}{l}0.0061 \\
0.0098\end{array}$ & $\begin{array}{l}0.473 \\
0.455\end{array}$ & $\begin{array}{l}-1.52 \\
-1.53\end{array}$ & $\begin{array}{l}\mathrm{C} \\
\mathrm{C}\end{array}$ & $\begin{array}{l}l s \\
l s\end{array}$ \\
\hline 21 & & ${ }^{1} \mathrm{D}-\mathrm{P}^{\circ}$ & 5073 & 499708 & 519414 & 5 & 3 & 0.0127 & 0.00295 & 0.246 & -1.83 & C & $c a$ \\
\hline
\end{tabular}

\section{Forbidden Transitions}

Naqvi's calculations [1] are the only available source. The results for the ${ }^{3} \mathrm{P}^{\circ}-{ }^{3} \mathrm{P}^{\circ}$ transitions are essentially independent of the choice of the interaction parameters. For the ${ }^{3} \mathrm{P}^{\circ}-{ }^{1} \mathrm{P}^{\circ}$ transitions, Naqvi has used empirical term intervals, i.e., the effects of configuration interaction should be partially included.

Reference

[1] Naqvi, A. M., Thesis Harvard (1951). 
N IV. Forbidden Transitions

\begin{tabular}{|c|c|c|c|c|c|c|c|c|c|c|c|c|}
\hline No. & $\begin{array}{c}\text { Transition } \\
\text { Array }\end{array}$ & Multiplet & $\lambda(\AA)$ & $E_{i}\left(\mathrm{~cm}^{-1}\right)$ & $E_{k}\left(\mathrm{~cm}^{-1}\right)$ & $g_{i}$ & $g_{k}$ & $\begin{array}{c}\text { Type of } \\
\text { Transi- } \\
\text { tion }\end{array}$ & $A_{k i}\left(\sec ^{-1}\right)$ & $S$ (at.u.) & $\begin{array}{l}\text { Accu: } \\
\text { racy }\end{array}$ & Source \\
\hline 1 & $\begin{array}{l}2 s 2 p- \\
\quad 2 s\left({ }^{2} \mathrm{~S}\right) 2 p\end{array}$ & ${ }^{3} \mathrm{P}^{\circ}-{ }^{3} \mathrm{P}^{\circ}$ & $\begin{array}{l}{\left[15.82 \times 10^{5}\right]} \\
{\left[69.33 \times 10^{4}\right]}\end{array}$ & $\begin{array}{l}{[67136.4]} \\
{[67199.6]}\end{array}$ & $\begin{array}{l}{[67199.6]} \\
{[67343.8]}\end{array}$ & $\begin{array}{l}1 \\
3\end{array}$ & $\begin{array}{l}3 \\
5\end{array}$ & $\begin{array}{l}\mathrm{m} \\
\mathrm{m}\end{array}$ & $\begin{array}{l}4.56 \times 10^{-6} \\
4.05 \times 10^{-5}\end{array}$ & $\begin{array}{l}2.00 \\
2.50\end{array}$ & $\begin{array}{l}\text { B } \\
\text { B }\end{array}$ & $\begin{array}{l}1 \\
1\end{array}$ \\
\hline 2 & & ${ }^{3} \mathrm{P}^{\circ}-{ }^{1} \mathrm{P}^{\circ}$ & $\begin{array}{l}{[1573.4]} \\
{[1574.9]} \\
{[1578.5]}\end{array}$ & $\begin{array}{l}{[67136.4]} \\
{[67199.6]} \\
{[67343.8]}\end{array}$ & $\begin{array}{l}130695 \\
130695 \\
130695\end{array}$ & $\begin{array}{l}1 \\
3 \\
5\end{array}$ & $\begin{array}{l}3 \\
3 \\
3\end{array}$ & $\begin{array}{l}\mathrm{m} \\
\mathrm{m} \\
\mathrm{m}\end{array}$ & $\begin{array}{l}0.0118 \\
5.5 \\
0.0146\end{array}$ & $\begin{array}{l}5.1 \times 10^{-6} \\
0.00240 \\
6.4 \times 10^{-6}\end{array}$ & $\begin{array}{l}\mathrm{C} \\
\mathrm{C} \\
\mathrm{C}\end{array}$ & $\begin{array}{l}1 \\
1 \\
1\end{array}$ \\
\hline
\end{tabular}

Ground State

Ionization Potential
$1 s^{2} 2 s^{2} \mathrm{~S}_{1 / 2}$

$97.863 \mathrm{eV}=789532.9 \mathrm{~cm}^{-1}$

Allowed Transitions

List of tabulated lines:

\begin{tabular}{c|c||c|c||c|c}
\hline \hline Wavelength $[\AA]$ & No. & Wavelength $[\AA]$ & No. & Wavelength $[\AA]$ & No. \\
\hline & & & & & \\
162.562 & 3 & 266.375 & 4 & 5067 & 13 \\
186.070 & 6 & 1238.81 & 1 & 5273 & 11 \\
186.153 & 6 & 1242.80 & 1 & 6719 & 16 \\
186.16 & 6 & 4335 & 9 & 7330 & 17 \\
209.270 & 2 & 46035 & 15203 & 8 \\
209.303 & 2 & 4603.83 & 7 & 15258 & 8 \\
247.563 & 5 & 475.9 & 7 & & \\
247.710 & 5 & 4933 & 12 & & \\
266.192 & 4 & 4952 & 15 & & \\
\hline
\end{tabular}

The values taken from Weiss' calculations [1] are estimated to be accurate to within 10 percent because of the very close agreement between his dipole length and dipole velocity approximations. The values calculated with the length approximation are adopted. The Coulomb approximation should be quite reliable for the highly excited transitions and is given preference over Kelly's approximate Hartree-Fock calculations [2], with which it sometimes disagrees.

\section{References}

[1] Weiss, A. W., Astrophys. J. 138, 1262-1276 (1963).

[2] Kelly, P. S., J. Quant. Spectrosc. Radiat. Transfer 4, 117 (1964). 
N V. Allowed Transitions

\begin{tabular}{|c|c|c|c|c|c|c|c|c|c|c|c|c|c|}
\hline No. & $\begin{array}{c}\text { Transition } \\
\text { Array }\end{array}$ & Multiplet & $\lambda(\AA)$ & $E_{i}\left(\mathrm{~cm}^{-1}\right)$ & $E_{k}\left(\mathrm{~cm}^{-1}\right)$ & $g_{i}$ & $g_{k}$ & $A_{k i}\left(10^{H} \sec ^{-1}\right)$ & $f_{i k}$ & $S($ at.u. $)$ & $\log g f$ & $\begin{array}{l}\text { Accu- } \\
\text { racy }\end{array}$ & Source \\
\hline \multirow[t]{3}{*}{1} & $2 s-2 p$ & ${ }^{2} \mathrm{~S}-{ }^{2} \mathrm{P}^{\circ}$ & 1240.1 & 0.0 & 806.37 & 2 & 6 & 3.38 & 0.234 & 1.91 & -0.330 & $A$ & 1 \\
\hline & & & 1238.81 & 0.0 & 80723 & 2 & 4 & 3.38 & 0.156 & 1.27 & -0.507 & $A$ & ls \\
\hline & & & 1242.80 & 0.0 & 80465 & 2 & 2 & 3.36 & 0.0778 & 0.637 & -0.808 & A & ls \\
\hline \multirow[t]{3}{*}{2} & $2 s-3 p$ & ${ }^{2} \mathrm{~S}-{ }^{2} \mathrm{P}^{\circ}$ & 209.28 & 0.0 & 477826 & 2 & 6 & 120 & 0.235 & 0.324 & -0.327 & $\mathrm{~B}+$ & 1 \\
\hline & & & 209.270 & 0.0 & 477851 & 2 & 4 & 119 & 0.157 & 0.216 & -0.504 & $B+$ & ls \\
\hline & & & 209.303 & 0.0 & 477777 & 2 & 2 & 119 & 0.0784 & 0.108 & -0.805 & $\mathrm{~B}+$ & ts \\
\hline 3 & $2 s-4 p$ & $\begin{array}{c}{ }^{2} \mathrm{~S}-{ }^{2} \mathrm{P}^{\circ} \\
(3 \mathrm{uv})\end{array}$ & 162.562 & 0.0 & 615150 & 2 & 6 & 56.3 & 0.0669 & 0.0716 & -0.874 & B & $c a$ \\
\hline \multirow[t]{3}{*}{4} & $2 p-3 s$ & ${ }^{2} \mathrm{P}^{\circ}-{ }^{2} \mathrm{~S}$ & 266.31 & 80637 & 456134 & 6 & 2 & 90.9 & 0.0322 & 0.169 & -0.714 & $B+$ & 1 \\
\hline & & & 266.375 & 80723 & 456134 & 4 & 2 & 60.6 & 0.0322 & 0.113 & -0.890 & $\mathrm{~B}+$ & ls \\
\hline & & & 266.192 & 80465 & 456134 & 2 & 2 & 30.2 & 0.0321 & 0.0563 & -1.192 & $\mathrm{~B}+$ & ls \\
\hline \multirow[t]{4}{*}{5} & $2 p-3 d$ & ${ }^{2} \mathrm{P}^{\circ}-{ }^{2} \mathrm{D}$ & 247.66 & 80637 & 484417 & 6 & 10 & 429 & 0.658 & 3.22 & 0.596 & $\mathrm{~B}+$ & 1 \\
\hline & & & 247.710 & 80723 & 484427 & 4 & 6 & 4.29 & 0.592 & 1.93 & 0.374 & $\mathrm{~B}+$ & ls \\
\hline & & & 247.563 & 80465 & 484403 & 2 & 4 & 357 & 0.656 & 1.07 & 0.118 & $\mathrm{~B}+$ & ls \\
\hline & & & {$[247.72]$} & 80723 & 484403 & 4 & 4 & 71.6 & 0.0659 & 0.215 & -0.579 & $B+$ & ls \\
\hline \multirow[t]{4}{*}{6} & $2 p-4 d$ & ${ }^{2} \mathrm{P}^{\circ}-{ }^{2} \mathrm{D}$ & 186.13 & 806.37 & 617905 & 6 & 10 & 140 & 0.121 & 0.445 & -0.139 & B & $c a$ \\
\hline & & & 186.153 & 80723 & 617905 & 4 & 6 & 140 & 0.109 & 0.267 & -0.361 & B & ls \\
\hline & & & 186.070 & 80465 & 617905 & 2 & 4 & 116 & 0.121 & 0.148 & -0.617 & B & ls \\
\hline & & & [186.16] & 80723 & 617905 & 4 & 4 & 23.3 & 0.0121 & 0.0297 & -1.315 & B & ls \\
\hline \multirow[t]{3}{*}{7} & $3 s-3 p$ & ${ }^{2} \mathrm{~S}-{ }^{2} \mathrm{P}^{\circ}$ & 4608.7 & 456134 & 477826 & 2 & 6 & 0.413 & 0.395 & 12.0 & -0.103 & B & 1 \\
\hline & & & 4603.83 & 456134 & 477851 & 2 & 4 & 0.415 & 0.264 & 8.00 & -0.278 & B & ls \\
\hline & & & 4619.9 & 456134 & 477777 & 2 & 2 & 0.411 & 0.131 & 4.00 & -0.580 & B & ls \\
\hline \multirow[t]{4}{*}{8} & $3 p-3 d$ & ${ }^{2} \mathrm{P}^{\circ}-{ }^{2} \mathrm{D}$ & 15168 & 477826 & 484417 & 6 & 10 & 0.00946 & 0.0544 & 16.3 & -0.486 & B & 1 \\
\hline & & & [15203] & 477851 & 484427 & 4 & 6 & 0.00940 & 0.0489 & 9.78 & -0.709 & B & ls \\
\hline & & & [15088] & 477777 & 484403 & 2 & 4 & 0.00801 & 0.0547 & 5.43 & -0.961 & B & ls \\
\hline & & & [15258] & 477851 & 484403 & 4 & 4 & 0.00155 & 0.00542 & 1.09 & -1.664 & B & ls \\
\hline 9 & $5 p-6 s$ & $\begin{array}{c}{ }^{2} \mathrm{P}^{\circ}-{ }^{2} \mathrm{~S} \\
(2)\end{array}$ & 3161 & 678297 & [709947] & 6 & 2 & 3.06 & 0.153 & 9.55 & -0.037 & B & $c a$ \\
\hline 10 & $6 s-7 p$ & $\begin{array}{c}{ }^{2} \mathrm{~S}-{ }^{2} \mathrm{P}^{\circ} \\
(3)\end{array}$ & 4335 & {$[709947]$} & 732993 & 2 & 6 & 0.376 & 0.318 & 9.08 & -0.197 & B & $c a$ \\
\hline 11 & $6 p-7 s$ & ${ }^{2 P^{\circ}-{ }^{2} S}$ & 5273 & 712464 & [731432] & 6 & 2 & 1.41 & 0.196 & 20.4 & 0.070 & B & $c a$ \\
\hline 12 & $6 p-7 d$ & ${ }_{(5)}^{2 P^{\circ}-{ }^{2} \mathrm{D}}$ & 4751 & 712464 & [733516] & 6 & 10 & 0.963 & 0.543 & 51.0 & 0.513 & B & $c a$ \\
\hline 13 & $6 d-7 p$ & ${ }_{(6)}^{2 D-{ }^{2} P^{\circ}}$ & 5067 & 713289 & 732993 & 10 & 6 & 0.423 & 0.0977 & 16.3 & -0.010 & B & $c a$ \\
\hline 14 & $6 d-7 f$ & $\begin{array}{c}{ }^{2} \mathrm{D}-{ }^{2} \mathrm{~F}^{\circ} \\
(7)\end{array}$ & 4933 & 713289 & [733547] & 10 & 14 & 1.62 & 0.828 & 134 & 0.918 & B & $c a$ \\
\hline 15 & $6 f-7 d$ & $\begin{array}{c}{ }^{2} \mathrm{~F}^{\circ}-{ }^{2} \mathrm{D} \\
(8)\end{array}$ & 4952 & [713327] & [733516] & 14 & 10 & 0.161 & 0.0423 & 9.66 & -0.227 & B & $c a$ \\
\hline 16 & $7 s-8 p$ & $\begin{array}{c}{ }^{2} \mathrm{~S}-{ }^{2} \mathrm{P}^{\circ} \\
(11)\end{array}$ & 6719 & [731432] & [746311] & 2 & 6 & 0.171 & 0.348 & 15.4 & -0.157 & B & $c a$ \\
\hline 17 & $7 p-8 d$ & ${ }_{(12)}^{2 P^{\circ}-{ }^{2} \mathrm{D}}$ & 7330 & 732993 & [746649] & 6 & 10 & 0.454 & 0.610 & 88.3 & 0.564 & B & $c a$ \\
\hline
\end{tabular}


Ground State

$1 s^{2}{ }^{1} \mathrm{~S}_{0}$

Ionization Potential

$551.925 \mathrm{eV}=4452800 \mathrm{~cm}^{-1}$

\section{Allowed Transitions}

The results of extensive non-relativistic variational calculations by Weiss [1] are used. Values have been calculated in both the dipole length and dipole velocity approximations and agree to within $1 \%$, except for the $3 p^{1} \mathrm{P}^{\circ}-3 d^{1} \mathrm{D}$ transition where agreement is not as good. The average of the two approximations is adopted [1].

\section{Reference}

[1] Weiss, A. W., private communication (1964).

N VI. Allowed Transitions

\begin{tabular}{|c|c|c|c|c|c|c|c|c|c|c|c|c|c|}
\hline No. & $\begin{array}{c}\text { Transition } \\
\text { Array }\end{array}$ & Multiplet & $\lambda(\AA)$ & $E_{i}\left(\mathrm{~cm}^{-1}\right)$ & $E_{k}\left(\mathrm{~cm}^{-1}\right)$ & $g_{i}$ & $g_{k}$ & $A_{k i}\left(10^{8} \mathrm{sec}^{-1}\right)$ & $f_{i k}$ & $S$ (at.u.) & $\log g f$ & Accuracy & Source \\
\hline 1 & $1 s^{2}-1 s 2 p$ & ${ }^{1} \mathrm{~S}-{ }^{1} \mathrm{P}^{\circ}$ & [28.787] & 0 & 3473790 & 1 & 3 & 18100 & 0.674 & 0.0639 & -0.171 & A & 1 \\
\hline 2 & $1 s^{2}-1 s 3 p$ & ${ }^{1} \mathrm{~S}-{ }^{1} \mathrm{P}^{0}$ & [24.898] & 0 & 4016390 & 1 & 3 & 5160 & 0.144 & 0.0118 & -0.842 & A & 1 \\
\hline 3 & $1 s 2 s-1 s 2 p$ & ${ }^{1} \mathrm{~S}-{ }^{1} \mathrm{P}^{\circ}$ & [2914.6] & [3439490] & 3473790 & 1 & 3 & 0.206 & 0.0786 & 0.754 & -1.105 & A & 1 \\
\hline 4 & $1 s 2 s-1 s 3 p$ & ${ }^{1} \mathrm{~S}-{ }^{1} \mathrm{P}^{0}$ & [173.34] & [3439490] & 4016390 & 1 & 3 & 269 & 0.364 & 0.208 & -0.439 & A & 1 \\
\hline 5 & $1 s 2 p-1 s 3 d$ & ${ }^{1} \mathrm{P}^{\circ}-{ }^{1} \mathrm{D}$ & [185.09] & 3473790 & [4014057] & 3 & 5 & 825 & 0.706 & 1.29 & 0.326 & A & 1 \\
\hline 6 & $1 s 3 d-1 s 3 p$ & ${ }^{1} \mathrm{D}-{ }^{1} \mathrm{P}^{\circ}$ & {$[42850] ?$} & [4014057] & 4016390 & 5 & 3 & $5.44 \times 10^{-4}$ & 0.00899 & 6.34 & -1.347 & $\mathrm{C}+$ & 1 \\
\hline 7 & $1 s 2 s--1 s 2 p$ & ${ }^{3} \mathrm{~S}-{ }^{3} \mathrm{P}^{\circ}$ & [1901.5] & [3385890] & [3438480] & 3 & 9 & 0.678 & 0.110 & 2.07 & -0.481 & A & 1 \\
\hline 8 & $1 s 2 s-1 s 3 p$ & ${ }^{3} \mathrm{~S}-{ }^{3} \mathrm{P}^{\circ}$ & [161.40] & [3385890] & [4005471] & 3 & 9 & 285 & 0.334 & 0.533 & 0.001 & A & 1 \\
\hline 9 & $1 s 2 p-1 s 3 d$ & ${ }^{3} \mathrm{P}^{\circ}-{ }^{3} \mathrm{D}$ & [173.98] & {$[3438480]$} & [4013259] & 9 & 15 & 876 & 0.662 & 3.41 & 0.775 & A & 1 \\
\hline 10 & $1 s 3 p-1 s 3 d$ & ${ }^{3} \mathrm{P}^{\circ}-{ }^{3} \mathrm{D}$ & [12837]? & [4005471] & [4013259] & 9 & 15 & 0.0102 & 0.0419 & 15.9 & -0.424 & A & 1 \\
\hline
\end{tabular}




\section{Allowed Transitions}

List of tabulated lines:

\begin{tabular}{l|r||c|c||c|c}
\hline \hline Wavelength $[\AA]$ & No. & Wavelength $[\AA]$ & No. & Wavelength $[\AA]$ & No. \\
\hline & & & & & \\
811.37 & 9 & 5435.16 & 40 & 7943.15 & 14 \\
877.804 & 5 & 5435.76 & 40 & 7947.20 & 14 \\
877.885 & 5 & 5436.83 & 40 & 7947.56 & 14 \\
878.979 & 5 & 6046.4 & 41 & 7950.83 & 14 \\
879.027 & 5 & 6106.5 & 34 & 7952.18 & 14 \\
879.108 & 5 & 6155.99 & 32 & 7981.97 & 27 \\
879.553 & 5 & 6156.78 & 32 & 7982.3 & 27 \\
936.011 & 10 & 6158.19 & 32 & 7982.41 & 27 \\
988.581 & 3 & 6242.5 & 15 & 7987.00 & 27 \\
988.658 & 3 & 6259.6 & 36 & 7987.34 & 27 \\
988.777 & 3 & 6269.4 & 35 & 7995.12 & 27 \\
990.132 & 3 & 6453.64 & 37 & 8073.7 & 19 \\
990.120 & 3 & 6454.48 & 37 & 8221.84 & 13 \\
990.799 & 3 & 6456.01 & 37 & 8227.64 & 13 \\
999.494 & 6 & 6653.78 & 24 & 8230.01 & 13 \\
& & & & \\
1025.77 & 8 & 7002.1 & 33 & 8232.99 & 13 \\
1027.42 & 8 & 7156.80 & 17 & 8235.31 & 13 \\
1028.16 & 8 & 7194.6 & 21 & 8446.5 & 12 \\
115.16 & 4 & 7254.4 & 38 & 8508.63 & 22 \\
1217.64 & 7 & 7471.36 & 20 & 8820.45 & 16 \\
1302.17 & 2 & 7473.23 & 20 & 9260.88 & 28 \\
1304.87 & 2 & 7476.45 & 20 & 9262.73 & 28 \\
1306.04 & 2 & 7477.21 & 20 & 9265.99 & 28 \\
1355.61 & 1 & 7479.06 & 20 & 9391.2 & 18 \\
1358.52 & 1 & 7480.66 & 20 & 11287 & 29 \\
3947.29 & 25 & 7771.96 & 11 & 11295.0 & 30 \\
4368.30 & 26 & 7774.18 & 11 & 11297.5 & 30 \\
5328.98 & 39 & 7775.40 & 11 & 11302.2 & 30 \\
5329.59 & 39 & 7886.31 & 23 & 13164 & 31 \\
5330.66 & 39 & 7939.49 & 14 & & \\
\hline & & & & & \\
\hline
\end{tabular}

The data for the ultraviolet lines are taken from the theoretical work by Garstang [1] and Kelly [2], with the exception of one multiplet where an experimental value by Prag and Clark [3] is available. Even though the agreement between theory and experiment is quite good in this case, namely within $25 \%$, this may be accidental, since the extensive comparisons between theory and experiment for similar transitions of $\mathrm{C} I$ and $\mathrm{N}$ I reveal many strong discrepancies. As in the case of the other two atoms, one must again expect that the theoretical values are drastically affected by configuration interaction which is entirely neglected in the calculations. Thus, only the.most prominent transitions are listed, with an accuracy rating of "E". 
Considerable material is available for the lines in the visible and near infrared region of the spectrum. The tabulated values are taken from Kelly's earlier self-consistent field calculations [4] (which include exchange effects in an approximate way), Vainshtein's semi-empirical calculations [5], the Coulomb approximation by Bates and Damgaard, and experimental work by Jürgens [6], Foster [7], Doherty [8], Buttrey and Gibson [9], Wiese and Shumaker [10], and Solarski and Wiese [11]. In all experiments the emission of thermal plasmas generated in stabilized arcs or shock tubes has been studied. The agreement between the various theoretical and experimental methods is often quite remarkable. This is particularly true for the multiplets at 6157,6455 , $6654,7157,7477,7773,7886,8227$, and $8446 \AA$ for which the spread between the highest and lowest result is only $25 \%$ or less. Based on this good agreement, an accuracy of $10 \%$ for the averaged values is indicated. In arriving at best values, theoretical and experimental methods have usually been equally weighted, but among the experimental methods, the recent more advanced work $[8,9,10,11]$ is regarded as superseding the earlier work $[6,7]$. For the multiplets of the moderately excited $3 s-3 p$ array, the advanced experimental methods are used exclusively, since the theoretical papers do not take into account the existence of weak intercombination lines from the upper levels. The theoretical methods are also not too reliable for two other multiplets at 3947 and 4368 $\AA$, since cancellation in the transition integral occurs. In these cases, the experimental results are chosen. In the case of the strong multiplet $3 p^{3} \mathrm{P}-3 s^{\prime}{ }^{3} \mathrm{D}^{\circ}$ at $7989 \AA$ recourse is taken to a calculation by Petrie [12] because the use of approximate, hydrogen-like wave functions in this paper has given reasonable results for $3 s-3 p$ transitions, but appears to fail otherwise.

\section{References}

[1] Garstang, R. H., Proc. Cambridge Phil. Soc. 57, 115-120 (1961).

[2] Kelly, P. S., Astrophys. J. 140, 1247-1268 (1964).

[3] Prag, A. B., and Clark, K. C., Phys: Rev. Letters 12, 34-35 (1964).

[4] Kelly, P. S., J. Quant. Spectrosc. Radiat. Transfer 4, 117-148 (1964).

[5] Vainshtein, L. A., Optika i Spektroskopiya 3, 313-321 (1957).

[6] Jürgens, G., Z. Physik 138, 613-622 (1954).

[7] Foster, E. W., Proc. Phys. Soc. London A 79, 94-104 (1962).

[8] Doherty, L. R., Thesis Michigan (1961).

[9] Buttrey, D. E., and Gibson, J. B., Technical Documentary Report No. RTD-TDR-63-3047 (1964).

[10] Wiese, W. L., and Shumaker, Jr., J. B., J. Opt. Soc. Am. 5 1, 937-942 (1961).

[11] Solarski, J., and Wiese, W. L., Phys. Rev. 135, A1236-A124l (1964).

[12] Petrie, W., J. Geophys. Research 55, 143-151 (1950).

\section{O I. Allowed Transitions}

\begin{tabular}{|c|c|c|c|c|c|c|c|c|c|c|c|c|c|}
\hline No. & $\begin{array}{c}\text { Transition } \\
\text { Array }\end{array}$ & Multiplet & $\lambda(\AA)$ & $E_{i}\left(\mathrm{~cm}^{-1}\right)$ & $E_{k}\left(\mathrm{~cm}^{-1}\right)$ & $g_{i}$ & $g_{k}$ & $A_{k i}\left(10^{8} \sec ^{-1}\right)$ & $f_{i k}$ & $S$ (at.u.) & $\log g f$ & $\begin{array}{l}\text { Accu } \\
\text { racy }\end{array}$ & Source \\
\hline \multirow[t]{3}{*}{1} & \multirow{6}{*}{$\begin{array}{l}2 p^{4}- \\
2 p^{3}\left({ }^{4} S^{\circ}\right) 3 s\end{array}$} & \multirow{3}{*}{$\begin{array}{c}{ }^{3} \mathrm{P}-{ }^{5} \mathrm{~S}^{\circ} \\
(1 \mathrm{uv})\end{array}$} & & & & & & & & & & & \\
\hline & & & 1355.61 & 0.0 & 73768 & 5 & 5 & $1.3 \times 10^{-5}$ & $3.6 \times 10^{-7}$ & $8.0 \times 10^{-6}$ & -5.74 & $\mathrm{E}$ & 1 \\
\hline & & & 1358.52 & 158.5 & 73768 & 3 & 5 & $3.8 \times 10^{-6}$ & $1.8 \times 10^{-7}$ & $2.4 \times 10^{-6}$ & -6.27 & $\mathrm{E}$ & $\begin{array}{llll} & \end{array}$ \\
\hline \multirow[t]{4}{*}{2} & & \multirow{3}{*}{$\begin{array}{c}{ }^{3} \mathrm{P}-{ }^{3} \mathrm{~S}^{\circ} \\
(2 \mathrm{uv})\end{array}$} & 1303.5 & 78.0 & 76795 & 9 & 3 & 3.8 & 0.031 & 1.2 & -0.54 & D & $1,2,3$ \\
\hline & & & 1302.17 & 0.0 & 76795 & 5 & 3 & 2.1 & 0.031 & 0.68 & -0.80 & D & $\ln$ \\
\hline & & & 1304.87 & 158.5 & 76795 & 3 & $\begin{array}{l}3 \\
2\end{array}$ & 1.3 & 0.032 & 0.41 & -1.02 & $\mathrm{D}$ & $\ln$ \\
\hline & \multirow{8}{*}{$\begin{array}{l}2 p^{4}- \\
2 p^{3}\left({ }^{2} \mathrm{D}^{\circ}\right) 3 s^{\prime}\end{array}$} & \multirow{7}{*}{$\begin{array}{c}{ }^{3} \mathrm{P}-{ }^{3} \mathrm{D}^{\circ} \\
(5 \mathrm{uv})\end{array}$} & & & & & & & & & & & \\
\hline \multirow[t]{6}{*}{3} & & & 989.46 & 78.0 & 101143 & 9 & 15 & 2.3 & 0.056 & 1.6 & -0.30 & E & 1,2 \\
\hline & & & 988.777 & $\begin{array}{r}0.0 \\
1585\end{array}$ & 101135 & 5 & 7 & 2.3 & 0.047 & 0.76 & -0.63 & $\underset{F}{E}$ & $\ln n$ \\
\hline & & & $\begin{array}{l}990.210 \\
990.799\end{array}$ & $\begin{array}{l}158.5 \\
226.5\end{array}$ & $\begin{array}{l}101147 \\
101155\end{array}$ & $\begin{array}{l}3 \\
1\end{array}$ & $\begin{array}{l}5 \\
3\end{array}$ & $\begin{array}{l}1.6 \\
1.2\end{array}$ & $\mid \begin{array}{l}0.042 \\
0.054\end{array}$ & $\begin{array}{l}0.42 \\
0.18\end{array}$ & $\begin{array}{l}-0.90 \\
-1.27\end{array}$ & $\mathrm{E}$ & ln $n$ \\
\hline & & & 988.658 & 0.0 & 101147 & 5 & 5 & 0.58 & 0.0085 & 0.14 & -1.37 & $\mathrm{E}$ & $\ln$ \\
\hline & & & 990.132 & 158.5 & 101155 & 3 & 3 & 0.95 & 0.014 & 0.14 & $\begin{array}{l}-1.38 \\
-2.54\end{array}$ & E & $1 n$ \\
\hline & & & 988.581 & 0.0 & 101155 & 5 & 3 & 0.066 & $5.8 \times 10^{-5}$ & 0.0094 & -2.34 & $\mathbf{L}$ & $1 n$ \\
\hline 4 & & $\begin{array}{c}{ }^{1} \mathrm{D}-{ }^{1} \mathrm{D}^{\circ} \\
(6 \mathrm{uv})\end{array}$ & 1152.16 & 15868 & 102662 & 5 & 5 & 4.5 & 0.090 & 1.7 & -0.35 & E & 1,2 \\
\hline
\end{tabular}


O I. Allowed Transitions-Continued

\begin{tabular}{|c|c|c|c|c|c|c|c|c|c|c|c|c|c|}
\hline Vo. & $\begin{array}{c}\text { Transition } \\
\text { Array }\end{array}$ & Multiplet & $\lambda(\AA)$ & $E_{i}\left(\mathrm{~cm}^{-1}\right)$ & $E_{k}\left(\mathrm{~cm}^{-1}\right)$ & $g_{i}$ & $g_{k}$ & $A_{k i}\left(10^{8} \sec ^{-1}\right)$ & $f_{i k}$ & $S$ (at.u.) & $\log g f$ & $\begin{array}{l}\text { Accu- } \\
\text { racy }\end{array}$ & Source \\
\hline \multirow[t]{7}{*}{5} & \multirow{9}{*}{$\begin{array}{l}2 p^{4}- \\
2 p^{3}\left({ }^{2} \mathrm{P}^{\circ}\right) 3 s^{\prime \prime}\end{array}$} & \multirow[t]{7}{*}{${ }^{3} \mathrm{P}-{ }^{3} \mathrm{P}^{\circ}$} & 878.45 & 78.0 & 11.3916 & 9 & 9 & 3.2 & 0.037 & 0.96 & -0.48 & $\mathrm{E}$ & 1,2 \\
\hline & & & 877.885 & 0.0 & 113910 & 5 & 5 & 2.4 & 0.027 & 0.39 & -0.87 & E & $1 n$ \\
\hline & & & 879.027 & 158.5 & 113921 & 3 & 3 & 0.79 & 0.0091 & 0.079 & -1.56 & E & $1 n$ \\
\hline & & & 877.804 & 0.0 & 113921 & 5 & 3 & 1.3 & 0.0092 & 0.13 & -1.34 & $\mathrm{E}$ & $1 n$ \\
\hline & & & 878.979 & 158.5 & 113927 & 3 & 1 & 3.2 & 0.012 & 0.11 & -1.43 & $\mathrm{E}$ & $\ln$ \\
\hline & & & 879.108 & 158.5 & 113910 & 3 & 5 & 0.83 & 0.016 & 0.14 & -1.32 & $\mathrm{E}$ & $\ln n$ \\
\hline & & & 879.553 & 226.5 & 113921 & 1 & 3 & 1.1 & 0.038 & 0.11 & -1.42 & $\mathrm{E}$ & $\ln$ \\
\hline 6 & & $\begin{array}{c}{ }^{1} \mathrm{D}-{ }^{1} \mathrm{P}^{\circ} \\
(7 \mathrm{uv})\end{array}$ & 999.494 & 15868 & 115918 & 5 & 3 & 3.9 & 0.035 & 0.57 & -0.76 & $\mathrm{E}$ & 1,2 \\
\hline 7 & & $\begin{array}{c}{ }^{1} S-{ }^{1} \mathrm{p}^{\circ} \\
(9 \mathrm{uv})\end{array}$ & 1217.64 & 33792 & 115918 & 1 & 3 & 2.0 & 0.13 & 0.53 & -0.88 & $\mathrm{E}$ & 1,2 \\
\hline \multirow[t]{7}{*}{8} & \multirow[t]{7}{*}{$\begin{array}{l}2 p^{4}- \\
2 p^{3}\left({ }^{4} \mathrm{~S}^{\circ}\right) 3 d\end{array}$} & \multirow[t]{7}{*}{$\begin{array}{c}{ }^{3} \mathrm{P}-{ }^{3} \mathrm{D}^{\circ} \\
(4 \mathrm{uv})\end{array}$} & 1026.6 & 78.0 & 97488 & 9 & 15 & 0.39 & 0.010 & 0.31 & -1.04 & $\mathrm{D}-$ & 2 \\
\hline & & & 1025.77 & 0.0 & 97488 & 5 & 7 & 0.39 & 0.0086 & 0.15 & -1.36 & $\mathrm{D}-$ & ls \\
\hline & & & 1027.42 & 158.5 & 97488 & 3 & 5 & 0.29 & 0.0077 & 0.078 & -1.65 & $\mathrm{D}-$ & ls \\
\hline & & & 1028.16 & 226.5 & 97488 & 1 & 3 & 0.20 & 0.010 & 0.035 & -1.98 & $\mathrm{D}-$ & ls \\
\hline & & & 1025.77 & 0.0 & 97488 & 5 & 5 & 0.097 & 0.0015 & 0.026 & -2.12 & $\mathrm{D}-$ & ls \\
\hline & & & 1027.42 & 158.5 & 97488 & 3 & 3 & 0.16 & 0.0026 & 0.026 & -2.12 & $\mathrm{D}-$ & ls \\
\hline & & & 1025.77 & 0.0 & 97488 & 5 & 3 & 0.011 & $1.0 \times 10^{-4}$ & 0.0017 & -3.29 & $\mathrm{D}-$ & ls \\
\hline 9 & \multirow[t]{2}{*}{$\begin{array}{l}2 p^{4}- \\
2 p^{3}\left({ }^{2} \mathrm{D}^{\circ}\right) 3 d^{\prime}\end{array}$} & ${ }^{3} \mathrm{P}-{ }^{3} \mathrm{P}^{\circ}$ & 811.37 & 78.0 & 123326 & 9 & 9 & 0.78 & 0.0077 & 0.18 & -1.16 & $\mathrm{D}-$ & 2 \\
\hline 10 & & $\begin{array}{c}{ }^{1} \mathrm{D}-{ }^{1} \mathrm{~F}^{\circ} \\
(8 \mathrm{uv})\end{array}$ & 936.011 & 15868 & 124326 & 5 & 7 & 0.83 & 0.015 & 0.23 & -1.12 & $\mathrm{D}-$ & 2 \\
\hline \multirow[t]{4}{*}{11} & \multirow[t]{5}{*}{$\begin{array}{l}2 p^{3} 3 s- \\
2 p^{3}\left({ }^{4} \mathrm{~S}^{\circ}\right) 3 p\end{array}$} & \multirow[t]{4}{*}{$\begin{array}{c}{ }^{5} \mathrm{~S}^{\circ}-{ }^{5} \mathrm{P} \\
(1)\end{array}$} & 7773.4 & 73768 & 86629 & 5 & 15 & 0.340 & 0.922 & 118 & 0.664 & B & \multirow{4}{*}{$\begin{array}{r}4,5,8 \\
11, c a \\
l s \\
l s \\
l s\end{array}$} \\
\hline & & & 7771.96 & 73768 & 86631 & 5 & 7 & 0.340 & 0.431 & 55.1 & 0.333 & B & \\
\hline & & & 7774.18 & 73768 & 86627 & 5 & 5 & 0.340 & 0.307 & 39.3 & 0.186 & B & \\
\hline & & & 7775.40 & 73768 & 86625 & 5 & 3 & 0.340 & 0.184 & 23.6 & -0.035 & B & \\
\hline 12 & & ${ }^{3} \mathrm{~S}^{\circ}-{ }^{3} \mathrm{P}$ & 8446.5 & 76795 & 88631 & 3 & 9 & 0.280 & 0.898 & 74.9 & 0.430 & B & $\begin{array}{r}4,8,11 \\
c a\end{array}$ \\
\hline \multirow[t]{7}{*}{13} & \multirow[t]{13}{*}{$\begin{array}{l}2 p^{3} 3 s^{\prime}- \\
\quad 2 p^{3}\left({ }^{2} \mathrm{D}^{\circ}\right) 3 p^{\prime}\end{array}$} & \multirow[t]{7}{*}{$\begin{array}{c}{ }^{3} \mathrm{D}^{\circ}-{ }^{3} \mathrm{D} \\
(34)\end{array}$} & 8226.8 & 101143 & 113295 & 15 & 15 & 0.323 & 0.327 & 133 & 0.691 & B & \multirow[t]{7}{*}{$4,6, c a$} \\
\hline & & & 8221.84 & 101135 & 113294 & 7 & 7 & 0.292 & 0.296 & 56.1 & 0.316 & B & \\
\hline & & & 8230.01 & 101147 & 113295 & 5 & 5 & 0.211 & 0.214 & 29.0 & 0.029 & B & \\
\hline & & & 8232.99 & 101155 & 113298 & 3 & 3 & 0.261 & 0.266 & 21.6 & -0.099 & B & \\
\hline & & & 8221.84 & 101135 & 113295 & 7 & 5 & 0.0663 & 0.0480 & 9.09 & -0.474 & B & \\
\hline & & & 8227.64 & 101147 & 113298 & 5 & 3 & 0.0834 & 0.0508 & 6.88 & -0.595 & B & \\
\hline & & & $\begin{array}{l}8230.01 \\
8235.31\end{array}$ & 101147 & 113294 & $\begin{array}{l}5 \\
3\end{array}$ & $\begin{array}{l}7 \\
5\end{array}$ & $\begin{array}{l}0.0261 \\
0.0432\end{array}$ & $\begin{array}{l}0.0371 \\
0.0732\end{array}$ & 5.03 & -0.732 & $\begin{array}{l}\mathrm{B} \\
\mathrm{B}\end{array}$ & \\
\hline \multirow[t]{6}{*}{14} & & \multirow[t]{6}{*}{$\begin{array}{c}{ }^{3} \mathrm{D}^{\circ}-{ }^{3} \mathrm{~F} \\
(35)\end{array}$} & 7949.3 & 101143 & 113719 & 15 & 21 & 0.373 & 0.495 & 194 & 0.87 & $\mathrm{C}$ & \multirow{6}{*}{$\begin{array}{l}l s \\
l s \\
l s \\
l s \\
l s \\
l s\end{array}$} \\
\hline & & & 7947.56 & 101135 & 113714 & 7 & 9 & 0.373 & 0.454 & 83 & 0.50 & C & \\
\hline & & & 7950.83 & 101147 & 113721 & 5 & 7 & 0.331 & 0.439 & 58 & 0.342 & C & \\
\hline & & & 7952.18 & 101155 & 113727 & 3 & 5 & 0.313 & 0.495 & 38.8 & 0.171 & C & \\
\hline & & & 7943.15 & 101135 & 113721 & 7 & 7 & 0.0417 & 0.0394 & 7.2 & -0.56 & C & \\
\hline & & & $\begin{array}{l}7947.20 \\
7939.49\end{array}$ & $\begin{array}{l}101147 \\
101135\end{array}$ & $\begin{array}{l}113727 \\
113727\end{array}$ & $\begin{array}{l}5 \\
7\end{array}$ & $\begin{array}{l}5 \\
5\end{array}$ & $\begin{array}{l}0.058 \\
0.00165\end{array}$ & $\begin{array}{l}0.055 \\
0.00111\end{array}$ & $\begin{array}{l}7.2 \\
0.203\end{array}$ & $\begin{array}{l}-0.56 \\
-2.109\end{array}$ & $\begin{array}{l}\mathrm{C} \\
\mathrm{C}\end{array}$ & \\
\hline 15 & & ${ }^{3} \mathrm{D}^{\circ}-{ }^{3} \mathrm{P}$ & {$[6242.5] ?$} & 101143 & [117158]? & 15 & 9 & 0.73 & 0.257 & 79 & 0.59 & $\mathrm{C}$ & 4 \\
\hline 16 & & $\begin{array}{c}{ }^{1} \mathrm{D}^{\circ}-{ }^{1} \mathrm{~F} \\
(37)\end{array}$ & 8820.45 & 102662 & 113996 & 5 & 7 & 0.261 & 0.426 & 62 & 0.328 & C & 4. $c a$ \\
\hline 17 & & $\begin{array}{c}{ }^{1} \mathrm{D}^{\circ}-{ }^{1} \mathrm{D} \\
(38)\end{array}$ & 7156.80 & 102662 & 116631 & 5 & 5 & 0.473 & 0.363 & 42.8 & 0.259 & B & $4,11, c a$ \\
\hline 18 & & ${ }^{1} \mathrm{D}^{\circ}-{ }^{1} \mathrm{P}$ & [9391.2]? & 102662 & [113307]? & 5 & 3 & 0.215 & 0.171 & 26.4 & -0.069 & C & 4 \\
\hline
\end{tabular}


O I. Allowed Transitions-Continued

\begin{tabular}{|c|c|c|c|c|c|c|c|c|c|c|c|c|c|}
\hline No. & $\begin{array}{c}\text { Transition } \\
\text { Array }\end{array}$ & Multiplet & $\lambda(\AA)$ & $E_{i}\left(\mathrm{~cm}^{-1}\right)$ & $E_{k}\left(\mathrm{~cm}^{-1}\right)$ & $g_{i}$ & $g_{k}$ & $A_{k i}\left(10^{8} \mathrm{sec}^{-1}\right)$ & $f_{i k}$ & $S$ (at.u.) & $\log g f$ & $\begin{array}{c}\text { Accu- } \\
\text { racy }\end{array}$ & Source \\
\hline 19 & \multirow{10}{*}{$\begin{array}{l}2 p^{3} 3 s^{\prime \prime}- \\
2 p^{3}\left({ }^{2} \mathrm{P}^{\mathrm{O}}\right) 3 p^{\prime \prime}\end{array}$} & ${ }^{3} \mathrm{P}^{0}-{ }^{3} \mathrm{~S}$ & {$[8073.7] ?$} & 113916 & [126298]? & 9 & 3 & 0.339 & 0.110 & 26.4 & -0.003 & $\mathrm{C}$ & 4 \\
\hline \multirow[t]{5}{*}{20} & & \multirow[t]{5}{*}{$\begin{array}{c}{ }^{3} \mathrm{P}^{\circ}-{ }^{3} \mathrm{D} \\
(55)\end{array}$} & 7477.3 & 113916 & 127286 & 9 & 15 & 0.408 & 0.570 & 126 & 0.710 & B & \multirow{5}{*}{$l s$} \\
\hline & & & $\begin{array}{l}7476.45 \\
7479.06\end{array}$ & $\begin{array}{l}113910 \\
113921\end{array}$ & $\begin{array}{l}127282 \\
127288\end{array}$ & $\begin{array}{l}5 \\
3\end{array}$ & $\begin{array}{l}7 \\
5\end{array}$ & $\begin{array}{l}0.408 \\
0.306\end{array}$ & $\begin{array}{l}0.479 \\
0.428\end{array}$ & $\begin{array}{l}58.9 \\
31.6\end{array}$ & $\begin{array}{l}0.379 \\
0.108\end{array}$ & $\begin{array}{l}\mathrm{B} \\
\mathrm{B}\end{array}$ & \\
\hline & & & 7480.66 & 113927 & 127291 & 1 & 3 & 0.226 & 0.570 & 14.0 & -0.244 & B & \\
\hline & & & 7473.23 & 113910 & 127288 & 5 & 5 & 0.102 & 0.0856 & 10.5 & -0.369 & B & \\
\hline & & & $\begin{array}{l}7477.21 \\
7471.36\end{array}$ & $\begin{array}{l}113921 \\
113910\end{array}$ & $\begin{array}{l}127291 \\
127291\end{array}$ & $\begin{array}{l}3 \\
5\end{array}$ & $\begin{array}{l}3 \\
3\end{array}$ & $\begin{array}{l}0.170 \\
0.0114\end{array}$ & $\begin{array}{l}0.143 \\
0.00571\end{array}$ & $\begin{array}{c}10.5 \\
0.702\end{array}$ & $\begin{array}{l}-0.369 \\
-1.545\end{array}$ & $\begin{array}{l}\mathrm{B} \\
\mathrm{B}\end{array}$ & \\
\hline 21 & & ${ }^{3} \mathrm{P}^{0}-{ }^{3} \mathrm{P}$ & [7194.6]? & 113916 & [127811]? & 9 & 9 & 0.478 & 0.371 & 79 & 0.52 & C & 4 \\
\hline 22 & & ${ }^{1} \mathrm{P}^{0}-{ }^{1} \mathrm{P}$ & 8508.63 & 115918 & 127668 & 3 & 3 & 0.289 & 0.314 & 26.4 & -0.026 & $\mathrm{C}$ & 4 \\
\hline 23 & & $\begin{array}{c}{ }^{1} \mathrm{P}^{\circ}-{ }^{1} \mathrm{D} \\
(64)\end{array}$ & 7886.31 & 115918 & 128595 & 3 & 5 & 0.370 & 0.575 & 44.8 & 0.236 & B & $4,6, c a$ \\
\hline 24 & & $\begin{array}{c}{ }^{1} \mathrm{P}^{\circ}-{ }^{1} \mathrm{~S} \\
(65)\end{array}$ & 6653.78 & 115918 & 130943 & 3 & 1 & 0.600 & 0.133 & 8.72 & -0.400 & B & $4,11, c a$ \\
\hline 25 & \multirow[t]{2}{*}{$\begin{array}{l}2 p^{3} 3 s- \\
2 p^{3}\left({ }^{4} \mathrm{~S}^{\circ}\right) 4 p\end{array}$} & $\begin{array}{c}{ }^{5} S^{\circ}-{ }^{5} P \\
(3)\end{array}$ & 3947.29 & 73768 & 99095 & 5 & 15 & 0.00326 & 0.00229 & 0.149 & -1.94 & C & 4,7 \\
\hline 26 & & ${ }^{3} \mathrm{~S}^{\circ}-^{-3} \mathrm{P}$ & 4368.30 & 76795 & 99680 & 3 & 9 & 0.0066 & 0.0056 & 0.242 & -1.77 & $\mathrm{C}$ & 9,11 \\
\hline \multirow[t]{6}{*}{27} & \multirow{6}{*}{$\begin{array}{r}2 p^{3}\left(^{4} \mathrm{~S}^{\circ}\right) 3 p- \\
\quad 2 p^{3}\left({ }^{2} \mathrm{D}^{\circ}\right) 3 s^{\prime}\end{array}$} & \multirow{6}{*}{$\begin{array}{c}{ }^{3} P-{ }^{3} D^{\circ} \\
(19)\end{array}$} & 7989.9 & 88631 & 101143 & 9 & 15 & 0.29 & 0.46 & 110 & 0.61 & D & 12 \\
\hline & & & 7995.12 & 88631 & 101135 & 5 & 7 & 0.29 & 0.38 & 50 & 0.28 & D & ls \\
\hline & & & $\begin{array}{l}7987.00 \\
7002\end{array}$ & 30 & 101147 & 3 & $\begin{array}{l}5 \\
2\end{array}$ & 0.21 & $0.5^{\circ}$ & 27 & $\begin{array}{r}0.01 \\
-0.01\end{array}$ & D & ls \\
\hline & & & $\begin{array}{l}7982.41 \\
7987.34\end{array}$ & $\begin{array}{l}88631 \\
88631\end{array}$ & $\begin{array}{l}101155 \\
101147\end{array}$ & $\begin{array}{l}1 \\
5\end{array}$ & $\begin{array}{l}3 \\
5\end{array}$ & \begin{tabular}{|l}
0.16 \\
0.072
\end{tabular} & $\begin{array}{l}0.46 \\
0.068\end{array}$ & $\begin{array}{l}12 \\
9.0\end{array}$ & $\begin{array}{l}-0.34 \\
-0.47\end{array}$ & $\begin{array}{l}\mathrm{D} \\
\mathrm{D}\end{array}$ & $\begin{array}{l}l s \\
l s\end{array}$ \\
\hline & & & 7981.97 & 88631 & 101155 & 3 & 3 & & & 9.0 & -0.47 & D & 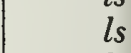 \\
\hline & & & [7982.3] & 88631 & 101155 & 5 & 3 & 0.0080 & 0.0046 & 0.60 & -1.64 & D & \\
\hline \multirow[t]{8}{*}{28} & \multirow{8}{*}{$\begin{array}{l}2 p^{3} 3 p- \\
\quad 2 p^{3}\left({ }^{4} S^{\circ}\right) 3 d\end{array}$} & \multirow{8}{*}{$\begin{array}{c}{ }^{5} \mathrm{P}-{ }^{5} \mathrm{D}^{\circ} \\
(8)\end{array}$} & 9263.9 & 86629 & 97420 & 15 & 25 & 0.419 & 0.90 & 412 & 1.130 & C & $4, c a$ \\
\hline & & & $\begin{array}{l}9265.99 \\
9262.73\end{array}$ & $\begin{array}{l}86631 \\
86627\end{array}$ & $\begin{array}{l}97420 \\
97420\end{array}$ & $\begin{array}{l}7 \\
5\end{array}$ & $\begin{array}{l}9 \\
7\end{array}$ & $\begin{array}{l}0.419 \\
0.280\end{array}$ & 0.69 & 148 & 0.69 & $\stackrel{\mathrm{C}}{\mathrm{C}}$ & $\begin{array}{ll}l s \\
l s\end{array}$ \\
\hline & & & $\begin{array}{l}9262.73 \\
9260.88\end{array}$ & $\begin{array}{l}86627 \\
86625\end{array}$ & $\begin{array}{l}97420 \\
97420\end{array}$ & $\begin{array}{l}5 \\
3\end{array}$ & $\begin{array}{l}7 \\
5\end{array}$ & $\begin{array}{l}0.2 \\
0.1\end{array}$ & $\begin{array}{l}0 . \\
0 .\end{array}$ & 28.8 & $\begin{array}{r}0.401 \\
-0.025\end{array}$ & $\mathrm{C}$ & ls \\
\hline & & & $\begin{array}{l}9265.99 \\
\end{array}$ & 86631 & 97420 & 7 & 7 & 0.140 & 0.180 & 38.4 & 0.100 & $\mathrm{C}$ & es \\
\hline & & & $\begin{array}{l}9262.73 \\
9260.88\end{array}$ & $\begin{array}{l}86627 \\
86625\end{array}$ & $\begin{array}{l}97420 \\
97421\end{array}$ & $\begin{array}{l}5 \\
3\end{array}$ & $\begin{array}{l}5 \\
3\end{array}$ & $\begin{array}{l}0.2 \\
0.3\end{array}$ & $\begin{array}{l}0.315 \\
0.405\end{array}$ & $\begin{array}{l}48.0 \\
37.0\end{array}$ & $\begin{array}{l}0.197 \\
0.085\end{array}$ & $\begin{array}{l}\mathrm{C} \\
\mathrm{C}\end{array}$ & ls \\
\hline & & & 9265.99 & 86631 & 97420 & 7 & 5 & 0.0279 & 0.0257 & 5.5 & -0.75 & C & 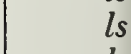 \\
\hline & & & 9262.73 & 86627 & 97421 & 5 & 3 & 0.11 & & 12. & -0.393 & $\mathrm{C}$ & Is \\
\hline & & & 9260.88 & 86625 & 97421 & 3 & 1 & 0.420 & 0.0180 & 16.5 & -0.268 & $\mathrm{C}$ & \\
\hline 29 & \multirow{4}{*}{$\begin{array}{l}2 p^{3} 3 p- \\
\quad 2 p^{3}\left({ }^{\circ} \mathrm{S}^{\circ}\right) 4 s\end{array}$} & ${ }^{3} \mathrm{P}-{ }^{3} \mathrm{D}^{\circ}$ & 11287 & 88631 & 97488 & 9 & 15 & 0.235 & 0.75 & 250 & 0.83 & C & 4 \\
\hline \multirow[t]{3}{*}{30} & & \multirow{3}{*}{$\begin{array}{l}{ }^{5} \mathrm{P}-{ }^{-5} \mathrm{~S}^{\circ} \\
(7)\end{array}$} & 11299 & 86629 & 95476 & 15 & 5 & 0.272 & 0.173 & 97 & 0.415 & C & $4, c a$ \\
\hline & & & 11302.2 & 86631 & 95476 & 7 & 5 & 0.127 & 0.173 & 45.1 & 0.084 & C & ls \\
\hline & & & \begin{tabular}{|l|}
11297.5 \\
11295.0
\end{tabular} & $\begin{array}{l}86627 \\
86625\end{array}$ & $\begin{array}{l}95476 \\
95476\end{array}$ & $\begin{array}{l}5 \\
3\end{array}$ & $\begin{array}{l}5 \\
5\end{array}$ & $\begin{array}{l}0.091 \\
0.054\end{array}$ & $\begin{array}{l}0.173 \\
0.173\end{array}$ & $\begin{array}{l}32.2 \\
19.3\end{array}$ & $\begin{array}{l}-0.062 \\
-0.284\end{array}$ & $\begin{array}{l}\mathrm{C} \\
\mathrm{C}\end{array}$ & $l s$ \\
\hline $31 \mid$ & & ${ }^{3} \mathrm{P}-{ }^{3} \mathrm{~S}^{\circ}$ & 13164 & 88631 & 96226 & 9 & 31 & $\mid 0.188$ & 0.163 & 63 & 0.165 & $\mathrm{C}$ & $1, c a$ \\
\hline
\end{tabular}


O I. Allowed Transitions-Continued

\begin{tabular}{|c|c|c|c|c|c|c|c|c|c|c|c|c|c|}
\hline No. & $\begin{array}{l}\text { Transition } \\
\text { Array }\end{array}$ & Multiplet & $\lambda(\AA)$ & $E_{i}\left(\mathrm{~cm}^{-1}\right)$ & $E_{k}\left(\mathrm{~cm}^{-1}\right)$ & $g_{i}$ & $g_{k}$ & $A_{k i}\left(10^{8} \sec ^{-1}\right)$ & $f_{i k}$ & $S($ at.u. $)$ & $\log g f$ & $\begin{array}{c}\text { Accu- } \\
\text { racy }\end{array}$ & Source \\
\hline \multirow[t]{8}{*}{32} & \multirow{10}{*}{\begin{tabular}{|c|}
$2 p^{3} 3 p-$ \\
$2 p^{3}\left({ }^{4} S^{\circ}\right) 4 d$
\end{tabular}} & \multirow{8}{*}{$\begin{array}{c}{ }^{5} \mathrm{P}-{ }^{-5} \mathrm{D}^{\circ} \\
(10)\end{array}$} & 6157.3 & 86629 & 102865 & 15 & 25 & 0.0701 & 0.0664 & 20.2 & -0.001 & B & $4,11, c a$ \\
\hline & & & 6158.19 & 86631 & 102865 & 7 & 9 & 0.0701 & 0.0512 & 7.27 & -0.445 & B & \\
\hline & & & 6156.78 & 86627 & 102865 & 5 & 7 & 0.0468 & 0.0372 & 3.77 & -0.731 & B & ls \\
\hline & & & $\begin{array}{l}6155.99 \\
6158.19\end{array}$ & $\begin{array}{l}86625 \\
86631\end{array}$ & $\begin{array}{l}102865 \\
10285\end{array}$ & $\begin{array}{l}3 \\
7 \\
\end{array}$ & 5 & 0.0245 & 0.0232 & 1.41 & -1.158 & B & is \\
\hline & & & 6156.78 & 86627 & 102865 & 5 & 5 & $\begin{array}{l}0.0234 \\
0.0410\end{array}$ & $\begin{array}{l}0.0133 \\
0.0233\end{array}$ & $\begin{array}{l}1.89 \\
2.36\end{array}$ & $\begin{array}{l}-1.031 \\
-0.934\end{array}$ & $\begin{array}{l}\mathrm{B} \\
\mathrm{B}\end{array}$ & $\begin{array}{l}\text { ls } \\
\text { ls }\end{array}$ \\
\hline & & & 6155.99 & 86625 & 102865 & 3 & 3 & 0.0527 & 0.0299 & 1.82 & -1.047 & B & ls \\
\hline & & & 6158.19 & 86631 & 102865 & 7 & 5 & 0.00467 & 0.00190 & 0.269 & -1.877 & B & ls \\
\hline & & & $\begin{array}{l}6156.78 \\
6155.99\end{array}$ & $\begin{array}{l}86627 \\
86625\end{array}$ & $\begin{array}{l}102865 \\
102865\end{array}$ & $\begin{array}{l}5 \\
3\end{array}$ & $\begin{array}{l}3 \\
1\end{array}$ & $\begin{array}{l}0.0175 \\
0.0702\end{array}$ & $\begin{array}{l}0.00598 \\
0.0133\end{array}$ & $\begin{array}{l}0.606 \\
0.808\end{array}$ & $\begin{array}{l}-1.524 \\
-1.399\end{array}$ & $\begin{array}{l}\mathrm{B} \\
\mathrm{B}\end{array}$ & $\begin{array}{l}l s \\
l s\end{array}$ \\
\hline 33 & & ${ }^{3} \mathrm{P}-{ }^{3} \mathrm{D}^{\circ}$ & 7002.1 & 88631 & 102908 & 9 & 15 & 0.0325 & 0.0398 & 8.3 & -0.446 & $\mathrm{C}$ & $4,6, c a$ \\
\hline & & & & & & & & & & & & & \\
\hline 34 & \multirow[t]{3}{*}{$\begin{array}{l}2 p^{3} 3 p^{\prime}- \\
\quad 2 p^{3}\left({ }^{2} \mathrm{D}^{\circ}\right) 4 d^{\prime}\end{array}$} & $\begin{array}{c}{ }^{3} \mathrm{D}-{ }^{3} \mathrm{~F}^{\circ} \\
(43)\end{array}$ & [6106.5] & 113295 & [129667] & 15 & 21 & 0.0460 & 0.0360 & 10.9 & -0.268 & C & $4,6, c a$ \\
\hline 35 & & $\begin{array}{c}{ }^{3} \mathrm{~F}-{ }^{3} \mathrm{~F}^{\circ} \\
(48)\end{array}$ & {$[6269.4]$} & 113719 & [129667] & 21 & 21 & 0.0198 & 0.0117 & 5.1 & -0.61 & C & $4,6, c a$ \\
\hline 36 & & $\begin{array}{c}{ }^{3} \mathrm{~F}-{ }^{-3} \mathrm{G}^{\circ} \\
(50)\end{array}$ & 6259.6 & 113719 & 129690 & 21 & 27 & 0.063 & 0.0475 & 20.6 & -0.001 & C & $4,6, c a$ \\
\hline \multirow[t]{3}{*}{37} & \multirow{4}{*}{$\begin{array}{l}2 p^{3} 3 p- \\
\quad 2 p^{3}\left(\mathrm{~S}^{\circ}\right) 5 s\end{array}$} & $\begin{array}{c}{ }^{5} \mathrm{P}-{ }^{5} \mathrm{~S}^{\circ} \\
(9)\end{array}$ & 6455.0 & 86629 & 102116 & 15 & 5 & 0.0710 & 0.0148 & 4.71 & -0.654 & B & $4,11, c a$ \\
\hline & & & $\begin{array}{l}6456.01 \\
6454.48\end{array}$ & $\begin{array}{l}86631 \\
86627\end{array}$ & $\begin{array}{l}102116 \\
102116\end{array}$ & $\begin{array}{l}7 \\
5\end{array}$ & $\begin{array}{l}5 \\
5\end{array}$ & $\begin{array}{l}0.0331 \\
0.0237\end{array}$ & $\begin{array}{l}0.0148 \\
0.0148\end{array}$ & $\begin{array}{l}2.20 \\
1.57\end{array}$ & -0.985 & $\stackrel{\mathrm{B}}{\mathrm{B}}$ & ls \\
\hline & & & 6453.64 & 86625 & 102116 & 3 & 5 & 0.0142 & 0.0148 & 0.942 & -1.353 & B & ls \\
\hline 38 & & $\begin{array}{c}{ }^{3} \mathrm{P}-{ }^{3} \mathrm{~S}^{\circ} \\
(20)\end{array}$ & 7254.4 & 88631 & 102412 & 9 & 3 & 0.062 & 0.0162 & 3.48 & -0.84 & $\mathrm{C}$ & $4,6, c a$ \\
\hline \multirow[t]{8}{*}{39} & \multirow[t]{8}{*}{$\begin{array}{l}2 p^{33} 3 p- \\
\quad 2 p^{3}\left({ }^{4} \mathrm{~S}^{\circ}\right) 5 d\end{array}$} & \multirow[t]{8}{*}{$\begin{array}{c}{ }^{5} \mathrm{P}-{ }^{-5} \mathrm{D}^{\circ} \\
(12)\end{array}$} & 5330.0 & 86629 & 105385 & 15 & 25 & 0.0197 & 0.0140 & 3.68 & -0.68 & $\mathrm{C}$ & $\begin{array}{r}4,6,7, \\
c a\end{array}$ \\
\hline & & & $\begin{array}{l}5330.66 \\
532050\end{array}$ & $\begin{array}{l}86631 \\
86627\end{array}$ & 105385 & 7 & 9 & 0.0196 & 0.0107 & 1.32 & -1.124 & $\mathrm{C}$ & ls \\
\hline & & & $\begin{array}{l}5329.59 \\
5328.98\end{array}$ & $\begin{array}{l}86627 \\
86652\end{array}$ & & 5 & 7 & & & & -1.407 & $\mathrm{C}$ & is \\
\hline & & & 5330.66 & $\begin{array}{l}80025 \\
86631\end{array}$ & $\begin{array}{l}105385 \\
105385\end{array}$ & $\begin{array}{l}3 \\
7\end{array}$ & $\begin{array}{l}5 \\
7\end{array}$ & $\begin{array}{l}0.0069 \\
0.0066\end{array}$ & $\begin{array}{l}0.00490 \\
0.00279\end{array}$ & $\begin{array}{l}0.258 \\
0.343\end{array}$ & $\begin{array}{l}-1.83 \\
-1.71\end{array}$ & C & ls \\
\hline & & & 5329.59 & 86627 & 105385 & 5 & 5 & 0.0115 & 0.00489 & & -1.61 & C & Is \\
\hline & & & 5328.98 & 86625 & 105385 & 3 & 3 & 0.0148 & 0.0063 & 0.3 & -1.72 & C & ls \\
\hline & & & $\begin{array}{l}5330.66 \\
5329.59\end{array}$ & $\begin{array}{l}86631 \\
86627\end{array}$ & $\begin{array}{l}105385 \\
105385\end{array}$ & $\begin{array}{l}7 \\
5\end{array}$ & $\begin{array}{l}5 \\
3\end{array}$ & $\begin{array}{l}0.00131 \\
0.00491\end{array}$ & $\begin{array}{l}4.00 \times 10^{-4} \\
0.00125\end{array}$ & $\begin{array}{l}0.0491 \\
0.110\end{array}$ & $\begin{array}{l}-2.55 \\
-2.203\end{array}$ & C & ls \\
\hline & & & 5328.98 & 86625 & 105385 & 3 & 1 & 0.0197 & 0.00279 & 0.147 & -2.077 & C & 1 \\
\hline \multirow[t]{3}{*}{40} & \multirow[t]{4}{*}{$\begin{array}{l}2 p^{3} 3 p- \\
\quad 2 p^{3}\left({ }^{4} \mathrm{~S}^{\circ}\right) 6 s\end{array}$} & \multirow[t]{3}{*}{$\begin{array}{l}{ }^{5} \mathrm{P}-{ }^{5} \mathrm{~S}^{\circ} \\
(11)\end{array}$} & 5436.1 & 86629 & 105019 & 15 & 5 & 0.0305 & 0.00450 & 1.21 & -1.170 & C & $\begin{array}{r}4,5,6, \\
7, c a\end{array}$ \\
\hline & & & \begin{tabular}{|l|}
5436.83 \\
5435.76
\end{tabular} & $\begin{array}{l}86631 \\
86627\end{array}$ & $\begin{array}{l}105019 \\
105019\end{array}$ & $\begin{array}{l}7 \\
5\end{array}$ & $\begin{array}{l}5 \\
5\end{array}$ & $\begin{array}{l}0.0142 \\
0.0102\end{array}$ & $\begin{array}{l}0.00451 \\
0.00450\end{array}$ & $\begin{array}{l}0.57 \\
0.403\end{array}$ & $\begin{array}{l}-1.50 \\
-165\end{array}$ & C & \\
\hline & & & 5435.16 & 86625 & 105019 & 3 & 5 & 0.0061 & 0.00451 & 0.242 & -1.87 & $\mathrm{C}$ & ls \\
\hline 41 & & $\begin{array}{c}{ }^{3} \mathrm{P}-{ }^{3} \mathrm{~S}^{\circ} \\
(22)\end{array}$ & 6046.4 & 88631 & 105165 & 9 & 3 & 0.0234 & 0.00427 & 0.77 & -1.415 & C & $4,6, c a$ \\
\hline
\end{tabular}




\section{Forbidden Transitions}

The list of forbidden lines for $\mathrm{O} 1$ is a very interesting one since it contains some of the very few cases of experimental determinations of forbidden line strengths. This fortunate circumstance is due to the appearance of strong forbidden lines in the aurora, so that the latter could be used as a "light source." Extensive measurements by Omholt [1] have given, for the $5577 \AA$ line, a transition probability of $1.43 \mathrm{sec}^{-1} \pm 14 \%$, whereas Garstang [2] with a refined calculation has obtained $1.25 \mathrm{sec}^{-1}$. For another case, namely the lifetime for the ${ }^{1} \mathrm{D}$ state, the averaged experimental result $[3,4]$ is approximately $160 \mathrm{sec}$, whereas the theory [5, 7] gives $135 \mathrm{sec}$. In both instances the mean value is adopted. Attempts have also been made in the laboratory to obtain experimental results, but they are all subject to some doubt and are therefore omitted in this compilation.

Aside from applications in atmospheric physics, the importance of the above lines lies in the circumstance that the experimental determinations give a reliable indication of the uncertainties in the theory of forbidden line strengths which in itself does not allow error estimates.

A number of other transitions in the $p^{4}$ configuration have been investigated by several authors. All electric quadrupole line strengths are taken from Garstang [6], since his estimate of the quadrupole integral $s_{q}$ is the most advanced one available. Naqvi [5], and Yamanouchi and Horie [7], in their calculations of magnetic dipole line strengths, retain the spin-spin and spin-other-orbit parameter in the transformation coefficients, while Garstang neglects it. Thus, their values are used for the ${ }^{3} \mathrm{P}-{ }^{1} \mathrm{D}$ and ${ }^{3} \mathrm{P}-{ }^{1} \mathrm{~S}$ transitions, where this gives an improvement of about $15 \%$. For the latter line, however, only Yamanouchi and Horie's [7] data are employed, since Naqvi's treatment of configuration interaction effects, which are import ant for this line, appears to be inadequate (see also general introduction).

\section{References}

[1] Omholt, A., Geofys. Publikasjoner Norske Videnskaps. Akad. Oslo 21, 1-38 (1959).

[2] Garstang, R. H., The Airglow and the Aurorae, 324-327 (ed. Armstrong and Dalgarno, Pergamon Press, New York, 1956).

[3] Stoffregen, W., and Derblom, H., Nature 185, 28-29 (1960).

[4] Omholt, A., Planetary and Space Science 2, 246-248 (1960).

[5] Naqvi, A. M., Thesis Harvard (1951).

[6] Garstang, R. H., Monthly Notices Roy. Astron. Soc. 111, 115-124 (1951).

[7] Yamanouchi, T., and Horie, H., J. Phys. Soc. Japan 7, 52-56 (1952).

O I. Forbidden Transitions

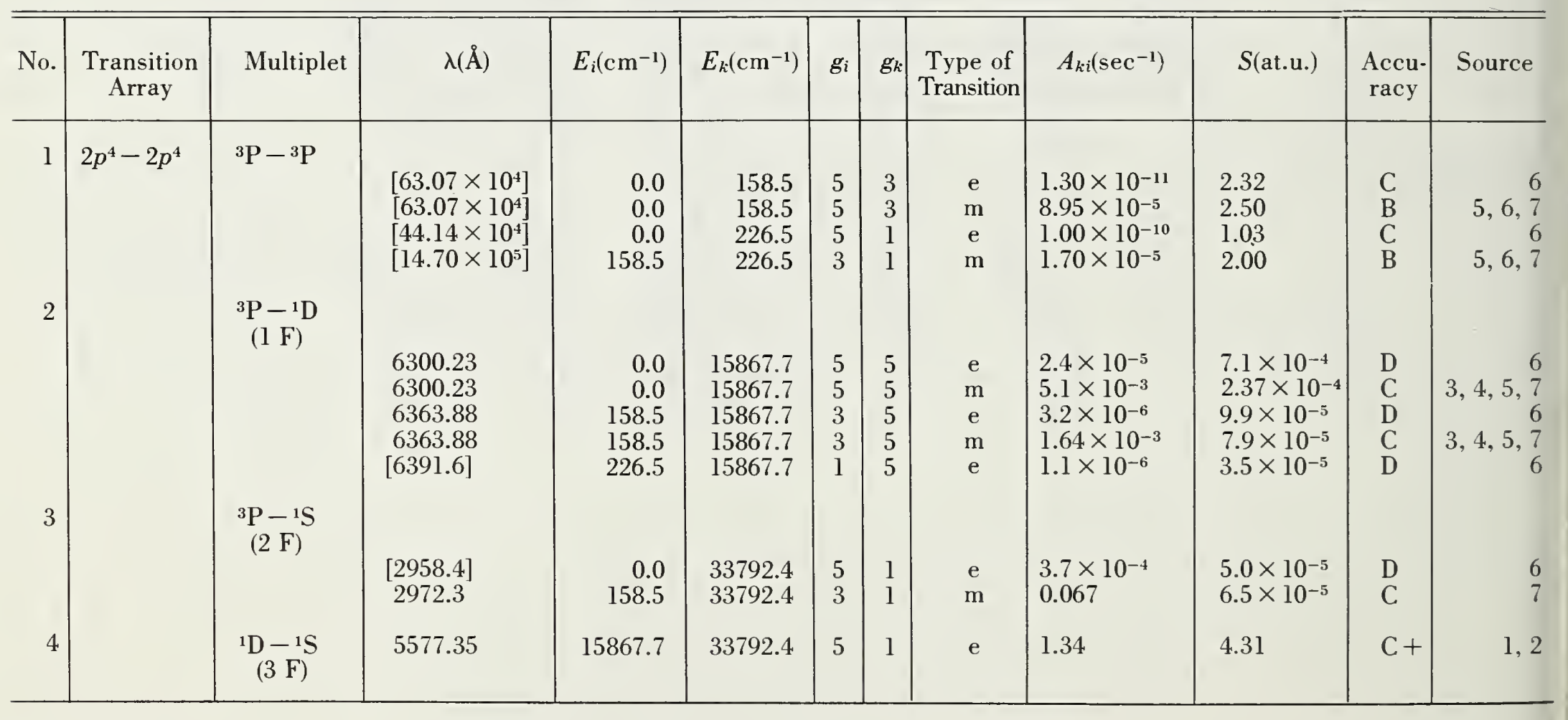




\section{O II}

Ground State

Ionization Potential
$1 s^{2} 2 s^{2} 2 p^{3}{ }^{4} S_{3 / 2}^{\circ}$

$35.108 \mathrm{eV}=283244 \mathrm{~cm}^{-1}$

\section{Allowed Transitions}

List of tabulated lines:

\begin{tabular}{|c|c|c|c|c|c|}
\hline Wavelength $[\AA]$ & No. & Wavelength $[\AA]$ & No. & Wavelength $[\AA]$ & No. \\
\hline 424.66 & 22 & 3007.4 & 84 & 3488.18 & 36 \\
\hline 429.918 & 10 & 3007.74 & 85 & 3496.27 & 36 \\
\hline 430.041 & 10 & 3008.28 & 85 & 3712.75 & 25 \\
\hline 430.177 & 10 & 3008.8 & 84 & 3727.33 & 25 \\
\hline 440.58 & 18 & 3009.7 & 85 & 3729.34 & 73 \\
\hline 442.03 & 17 & 3009.81 & 85 & 3735.9 & 73 \\
\hline 445.62 & 16 & 3010.0 & 85 & 3735.94 & 73 \\
\hline 464.78 & 21 & 3010.5 & 85 & 3739.92 & 69 \\
\hline 468.77 & 20 & 3012.8 & 85 & 3749.49 & 25 \\
\hline 470.41 & 19 & 3013.0 & 85 & 3762.63 & 69 \\
\hline 481.66 & 13 & 3013.37 & 84 & 3777.60 & 69 \\
\hline 483.752 & 12 & 3014.0 & 84 & 3794.48 & 70 \\
\hline 483.796 & 12 & 3019.8 & 84 & 3803.14 & 70 \\
\hline 484.03 & 12 & 3032.08 & 86 & 3821.68 & 70 \\
\hline 485.086 & 11 & 3032.50 & 86 & 3830.45 & 70 \\
\hline 485.47 & 11 & 3047.5 & 86 & 3833.10 & 43 \\
\hline 485.515 & 11 & 3113.71 & 66 & 3842.82 & 42 \\
\hline 515.498 & 15 & 3122.62 & 66 & 3843.58 & 43 \\
\hline 515.640 & 15 & 3124.02 & 66 & 3847.89 & 42 \\
\hline 517.937 & 14 & 3129.44 & 66 & 3850.81 & 42 \\
\hline 518.242 & 14 & 3134.32 & 66 & 3851.04 & 42 \\
\hline 539.086 & 5 & 3134.82 & 66 & 3851.47 & 43 \\
\hline 539.547 & 5 & 3138.44 & 66 & 3856.16 & 42 \\
\hline 539.853 & 5 & 3139.77 & 66 & 3857.18 & 43 \\
\hline 555.056 & 8 & 3270.9 & 71 & 3863.50 & 42 \\
\hline 555.121 & 8 & 3270.98 & 71 & 3864.13 & 41 \\
\hline 600.585 & 9 & 3273.52 & 71 & 3864.45 & 42 \\
\hline 616.291 & 6 & 3277.69 & 67 & 3864.68 & 42 \\
\hline 616.363 & 6 & 3282.0 & 39 & 3872.45 & 41 \\
\hline 617.051 & 6 & 3287.59 & 67 & 3874.10 & 41 \\
\hline 644.148 & 4 & 3290.13 & 67 & 3875.82 & 43 \\
\hline 672.948 & 7 & 3295.13 & 67 & 3882.20 & 42 \\
\hline 673.768 & 7 & 3301.56 & 67 & 3882.45 & 41 \\
\hline 718.484 & 2 & 3305.15 & 67 & 3883.15 & 42 \\
\hline 718.562 & 2 & 3306.60 & 67 & 3893.53 & 41 \\
\hline 796.661 & 3 & 3377.20 & 38 & 3896.30 & 41 \\
\hline 832.754 & 1 & 3390.25 & 38 & 3907.45 & 41 \\
\hline 833.326 & 1 & 3407.38 & 72 & 3911.96 & 33 \\
\hline 834.462 & 1 & 3409.84 & 72 & 3912.09 & 33 \\
\hline 2733.34 & 65 & 3447.98 & 68 & 3919.29 & 33 \\
\hline 2747.46 & 65 & 3460.6 & 26 & 3926.58 & 41 \\
\hline 3000.1 & 84 & 3470.42 & 68 & 3945.05 & 30 \\
\hline 3002.2 & 84 & 3470.81 & 68 & 3954.37 & 30 \\
\hline 3006.0 & 84 & 3474.94 & 37 & 3967.44 & 47 \\
\hline 3007.08 & 85 & 3479.7 & 26 & 3973.26 & 30 \\
\hline
\end{tabular}


List of tabulated lines-Continued

\begin{tabular}{|c|c|c|c|c|c|}
\hline Wavelength $[\AA ̊]$ & No. & Wavelength $[\AA]]$ & No. & Wavelength $[\AA]$ & No. \\
\hline 3982.72 & 30 & 4272.3 & 34 & 4596.17 & 31 \\
\hline 3985.46 & 47 & 4275.52 & 78 & 4598.2 & 50 \\
\hline 4060.8 & 82 & 4276.71 & 77 & 4602.11 & 81 \\
\hline 4069.64 & 40 & 4276.71 & 78 & & \\
\hline 4069.90 & 40 & & & 4609.42 & 81 \\
\hline & & 4277.40 & 78 & 4613.11 & 81 \\
\hline 4072.16 & 40 & 4277.90 & 78 & 4638.85 & 23 \\
\hline 4075.87 & 40 & 4281.40 & 77 & 4641.81 & 23 \\
\hline 4078.86 & 40 & 4282.82 & 77 & 4649.14 & 23 \\
\hline 4084.66 & 46 & 4282.96 & 78 & & \\
\hline 4085.12 & 40 & 428313 & & 4650.84 & 23 \\
\hline 4087.16 & 76 & $\begin{array}{l}4283.13 \\
4283.75\end{array}$ & $\begin{array}{l}78 \\
78\end{array}$ & $\begin{array}{l}4661.64 \\
4673.75\end{array}$ & $\begin{array}{l}23 \\
23\end{array}$ \\
\hline 4089.30 & 76 & 4284.0 & 78 & 4676.23 & 23 \\
\hline 4092.94 & 40 & 4284.4 & 78 & 4690.97 & 62 \\
\hline 4094.18 & 40 & 4288.8 & 77 & & \\
\hline 4095.63 & 76 & & & 4691.47 & 62 \\
\hline & & 4288.83 & 77 & 4696.36 & 23 \\
\hline 4096.18 & 76 & 4294.82 & 77 & 4698.48 & 60 \\
\hline 4096.54 & 46 & 4295.5 & 29 & 4699.21 & 49 \\
\hline 4097.26 & 45 & 4303.82 & 77 & 4699.21 & 60 \\
\hline 4097.26 & 76 & 4317.14 & 24 & & \\
\hline 4103.02 & 45 & & & 4701.23 & 62 \\
\hline 4104.74 & 4.5 & $\begin{array}{l}4319.63 \\
4319.93\end{array}$ & $\begin{array}{l}24 \\
63\end{array}$ & $\begin{array}{l}4701.76 \\
4703.18\end{array}$ & $\begin{array}{l}62 \\
60\end{array}$ \\
\hline $\begin{array}{l}4104.74 \\
4105.00\end{array}$ & 45 & 4325.77 & $\begin{array}{l}03 \\
24\end{array}$ & & 49 \\
\hline $\begin{array}{l}4105.00 \\
4106.03\end{array}$ & 40 & 4328.62 & 63 & 4710.04 & 48 \\
\hline $\begin{array}{l}4106.03 \\
4108.75\end{array}$ & 76 & 4329.0 & 29 & & \\
\hline 4109.3 & 76 & & & 4741.71 & 49 \\
\hline & & 4336.87 & 24 & 4751.34 & 48 \\
\hline 4109.8 & 59 & 4340.36 & 79 & 4752.70 & 48 \\
\hline 4110.20 & 59 & 4342.00 & 79 & 4845.0 & 54 \\
\hline 4110.80 & 45 & 4345.56 & 24 & 4856.49 & 53 \\
\hline 4112.03 & 46 & 4347.43 & 32 & & \\
\hline 4113.82 & 59 & & & 4856.76 & 53 \\
\hline & & 4349.1 & 32 & $\begin{array}{l}4861.03 \\
4864.95\end{array}$ & $\begin{array}{l}61 \\
52\end{array}$ \\
\hline 4114.4 & 76 & $\begin{array}{l}4349.43 \\
\end{array}$ & 24 & $\begin{array}{l}4864.95 \\
4871.58\end{array}$ & 53 \\
\hline 4119.22 & 45 & $\begin{array}{l}4351.27 \\
4351.5\end{array}$ & $\begin{array}{l}32 \\
32\end{array}$ & $\begin{array}{l}4871.58 \\
4872.2\end{array}$ & 61 \\
\hline $\begin{array}{l}4120.28 \\
4120.55\end{array}$ & $\begin{array}{l}45 \\
45\end{array}$ & $\begin{array}{l}4351.5 \\
4359.38\end{array}$ & $\begin{array}{l}32 \\
51\end{array}$ & 4872.2 & \\
\hline $\begin{array}{l}4120.55 \\
4121.48\end{array}$ & 44 & 4.59 .00 & & 4890.93 & 52 \\
\hline & & 4366.90 & 24 & 4906.88 & 52 \\
\hline 4126.1 & 76 & 4369.28 & 51 & 4924.60 & 52 \\
\hline 4129.34 & 44 & 4371.3 & 79 & 4941.12 & 56 \\
\hline 4132.81 & 44 & 4395.95 & 51 & 4943.06 & 56 \\
\hline 4140.74 & 44 & 4406.02 & 51 & & \\
\hline 4141.96 & 64 & & & $\begin{array}{l}4955.78 \\
5160.02\end{array}$ & 56 \\
\hline & & 4414.91 & $\begin{array}{l}28 \\
28\end{array}$ & $\begin{array}{l}5160.02 \\
5176.00\end{array}$ & $\begin{array}{l}55 \\
55\end{array}$ \\
\hline 4142.08 & 64 & $\begin{array}{l}4416.98 \\
4443.05\end{array}$ & $\begin{array}{l}28 \\
57\end{array}$ & 5190.56 & $\begin{array}{l}53 \\
55\end{array}$ \\
\hline $\begin{array}{l}4142.24 \\
4143.4\end{array}$ & $\begin{array}{l}64 \\
64\end{array}$ & $\begin{array}{l}4443.05 \\
4443.7\end{array}$ & $\begin{array}{l}37 \\
57\end{array}$ & 5206.73 & 55 \\
\hline 4143.52 & 64 & 4447.7 & 57 & & \\
\hline 4143.77 & 64 & & & 6627.62 & 75 \\
\hline & & 4448.21 & 57 & 6640.90 & 27 \\
\hline 4145.6 & 64 & 4452.38 & 28 & 6666.94 & 75 \\
\hline 4145.90 & 64 & 4465.40 & 35 & 6678.19 & 75 \\
\hline 4146.09 & 64 & 4466.6 & 80 & 6718.1 & 75 \\
\hline 4153.30 & 44 & 4467.88 & 35 & & \\
\hline 4156.54 & 44 & 4469.32 & 35 & $\begin{array}{l}6721.35 \\
6810.6\end{array}$ & $\begin{array}{l}27 \\
74\end{array}$ \\
\hline 416923 & 44 & 4489.48 & 80 & 6844.1 & 74 \\
\hline 4185.46 & 58 & 4491.25 & 80 & 6846.97 & 74 \\
\hline 4189.6 & 58 & 4539.6 & 50 & 6869.74 & 74 \\
\hline 4189.79 & 58 & 4563.2 & 50 & 689507 & \\
\hline 4253.9 & 83 & 4590.97 & 31 & $\begin{array}{l}6885.07 \\
6895.29\end{array}$ & 74 \\
\hline & & 4596.0 & 31 & 6906.54 & 74 \\
\hline 4263.2 & 78 & & & 6908.11 & $\begin{array}{l}74 \\
74\end{array}$ \\
\hline
\end{tabular}


Most data on the vaeuum ultraviolet lines are taken from the self-consistent field calculations by Kelly [1] in which exehange effeets have been considered. The data for the strong $2 s^{2} 2 p^{3}$ $-2 s 2 p^{4}$ and $2 p^{3}-2 p^{2} 3 s$ transitions are probably quite uncertain sinee these are sensitively affected by eonfiguration interaction which has not been taken into aceount. Two transitions of the $2 s^{2} 2 p^{3}$ $-2 s 2 p^{4}$ array eould be taken, however, from the calculations of Levinson et al. [2] in which the effects of eonfiguration interaction have been approximately included.

For the multiplets in the visible four data sourees have been selected: simplified self-consistent field caleulations by Kelly [3] (with exchange effeets approximately taken into aceount), the Coulomb approximation by Bates and Damgaard, emission measurements by Mastrup and Wiese [4], and intermediate eoupling caleulations by Garstang [5]. The absolute multiplet values have been obtained by averaging the results of the first three methods mentioned. The agreement is quite remarkable, often within $30 \%$. For the breakdown of the multiplets into lines the intermediate eoupling ealeulations by Garstang [5] and, whenever available, the experimental results by Mastrup and Wiese [4] are employed, both normalized to the absolute total multiplet values. The two sets of data agree very well, while LS-coupling values show large deviations for some multiplets of the $3 p-3 d$ array.

The absolute values of the intercombination lines are obtained by normalizing Garstang's coupling ealculations [5] to the Coulomb approximation, as he has proposed in his paper.

\section{Referenees}

[1] Kelly, P. S., Astrophys. J. 140, 1247-1268 (1964).

[2] Levinson, I. B., Bolotin, A. B., and Levin, L. I., Trudy Vil'nyusskogo Univ. 5, 49-55 (1956).

[3] Kelly, P. S., J. Quant. Spectrosc. Radiat. Transfer 4, 117-148 (1964).

[4] Mastrup, F., and Wiese, W. L., Z. Astrophys. 44, 259-279 (1958).

[5] Garstang, R. H., Monthly Notices Roy. Astron. Soc. 114, 118-133 (1954).

O II. Allowed Transitions

\begin{tabular}{|c|c|c|c|c|c|c|c|c|c|c|c|c|c|}
\hline No. & $\begin{array}{c}\text { Transition } \\
\text { Array }\end{array}$ & Multiplet & $\lambda(\AA)$ & $E_{i}\left(\mathrm{~cm}^{-1}\right)$ & $E_{k}\left(\mathrm{~cm}^{-1}\right)$ & $g_{i}$ & $g_{k}$ & $A_{k i}\left(10^{8} \sec ^{-1}\right)$ & $f_{i k}$ & $S$ (at.u.) & $\log g f$ & $\begin{array}{c}\text { Accu- } \\
\text { racy }\end{array}$ & Source \\
\hline \multirow[t]{4}{*}{1} & $\begin{array}{r}2 s^{2} 2 p^{3}- \\
2 s 2 p^{4}\end{array}$ & $\begin{array}{c}{ }^{4} \mathrm{~S}^{\circ}-{ }^{4} \mathrm{P} \\
(1 \mathrm{uv})\end{array}$ & 833.80 & 0.0 & 119933 & 4 & 12 & 14 & 0.43 & 4.7 & 0.23 & E & 1 \\
\hline & & & 834.462 & 0.0 & 119838 & 4 & 6 & 13 & 0.21 & 2.3 & -0.08 & $\mathrm{E}$ & ls \\
\hline & & & 833.326 & 0.0 & 120001 & 4 & 4 & 14 & 0.15 & 1.6 & -0.23 & $\mathrm{E}$ & ls \\
\hline & & & 832.754 & 0.0 & 120084 & 4 & 2 & 14 & 0.07 & 0.77 & -0.55 & $\mathrm{E}$ & ls \\
\hline \multirow[t]{5}{*}{2} & & ${ }^{2} \mathrm{D}^{\circ}-{ }^{2} \mathrm{D}$ & 718.54 & 26817 & 165991 & 10 & 10 & 32 & 0.25 & 5.9 & 0.40 & E & 1 \\
\hline & & & 718.484 & 26808 & 165988 & 6 & 6 & 30 & 0.23 & 3.3 & 0.14 & E & ls \\
\hline & & & 718.562 & 26829 & 165996 & 4 & 4 & 29 & 0.22 & 2.1 & -0.05 & $\mathrm{E}$ & ls \\
\hline & & & 718.484 & 26808 & 165996 & 6 & 4 & 3.1 & 0.016 & 0.23 & -1.01 & $\mathrm{E}$ & ls \\
\hline & & & 718.562 & 26829 & 165988 & 4 & 6 & 2.1 & 0.024 & 0.23 & -1.01 & $\mathrm{E}$ & ls \\
\hline 3 & & $\begin{array}{c}{ }^{2} \mathrm{P}^{\circ}-{ }^{2} \mathrm{D} \\
(11 \mathrm{uv})\end{array}$ & 796.661 & 40467 & 165991 & 6 & 10 & 4.4 & 0.070 & 1.1 & -0.38 & E & 2 \\
\hline 4 & & $\begin{array}{c}{ }^{2} \mathrm{P}^{\circ}-{ }^{2} \mathrm{~S} \\
(13 \mathrm{uv})\end{array}$ & 644.148 & 40467 & 195710 & 6 & 2 & 72 & 0.15 & 1.9 & -0.05 & E & 2 \\
\hline \multirow[t]{4}{*}{5} & $2 p^{3}-$ & ${ }^{4} \mathrm{~S}^{\circ}-{ }^{4} \mathrm{P}$ & 5.39 .37 & 0.0 & 185402 & 4 & 12 & 8.6 & 0.11 & 0.80 & -0.35 & E & 1 \\
\hline & & & 539.086 & 0.0 & 185499 & 4 & 6 & 8.6 & 0.056 & 0.40 & -0.65 & E & ls \\
\hline & & & 539.547 & 0.0 & 185341 & 4 & 4 & 8.6 & 0.037 & 0.27 & -0.82 & $\mathrm{E}$ & ls \\
\hline & & & 539.853 & 0.0 & 185235 & 4 & 2 & 8.6 & 0.019 & 0.13 & -1.13 & E & ls \\
\hline \multirow[t]{4}{*}{6} & & ${ }^{2} \mathrm{D}^{\circ}-{ }^{2} \mathrm{P}$ & 616.56 & 26817 & 189008 & 10 & 6 & 18 & 0.061 & 1.2 & -0.21 & $\mathrm{E}$ & l \\
\hline & & & 616.291 & 26808 & 189068 & 6 & 4 & 16 & 0.061 & 0.74 & -0.44 & E & ls \\
\hline & & & 617.051 & 26829 & 188888 & 4 & 2 & 18 & 0.051 & 0.41 & -0.69 & $\mathrm{E}$ & ls \\
\hline & & & 616.363 & 26829 & 189068 & 4 & 4 & 1.8 & 0.010 & 0.083 & -1.39 & $\mathrm{E}$ & ls \\
\hline
\end{tabular}


O II. Allowed Transitions-Continued

\begin{tabular}{|c|c|c|c|c|c|c|c|c|c|c|c|c|c|}
\hline No. & $\begin{array}{c}\text { Transition } \\
\text { Array }\end{array}$ & Multiplet & $\lambda(\AA)$ & $E_{i}\left(\mathrm{~cm}^{-1}\right)$ & $E_{k}\left(\mathrm{~cm}^{-1}\right)$ & $g_{i}$ & $g_{k}$ & $A_{k i}\left(10^{8} \sec ^{-1}\right)$ & $f_{i k}$ & $S$ (at.u.) & $\log g f$ & $\begin{array}{l}\text { Accu- } \\
\text { racy }\end{array}$ & Source \\
\hline \multirow[t]{4}{*}{7} & & ${ }^{2} \mathrm{P}^{\circ}-{ }^{2} \mathrm{P}$ & 673.22 & 40467 & 189008 & 6 & 6 & 9.2 & 0.063 & 0.84 & -0.42 & $\mathrm{E}$ & 1 \\
\hline & & & 672.948 & 40467 & 189068 & 4 & 4 & 7.7 & 0.052 & 0.46 & -0.68 & $\mathrm{E}$ & $l s$ \\
\hline & & & 673.768 & 40468 & 188888 & 2 & 2 & 6.2 & 0.042 & 0.19 & -1.08 & $\mathrm{E}$ & ls \\
\hline & & & $\begin{array}{l}672.948 \\
672.108\end{array}$ & 40468 & $\begin{array}{l}188888 \\
189068\end{array}$ & $\begin{array}{l}4 \\
2\end{array}$ & $\begin{array}{l}2 \\
4\end{array}$ & $\begin{array}{l}3.1 \\
1.5\end{array}$ & $\begin{array}{l}0.010 \\
0.021\end{array}$ & $\begin{array}{l}0.093 \\
0.093\end{array}$ & $\begin{array}{l}-1.38 \\
-1.38\end{array}$ & $\stackrel{\mathrm{E}}{\mathrm{E}}$ & $\begin{array}{l}l s \\
l s\end{array}$ \\
\hline \multirow[t]{4}{*}{8} & $2 p^{3}-$ & ${ }^{2} \mathrm{D}^{\circ}-{ }^{2} \mathrm{D}$ & 555.08 & 26817 & 206972 & 10 & 10 & 15 & 0.067 & 1.2 & -0.17 & $\mathrm{E}$ & 1 \\
\hline & & & 555.056 & 26808 & 206971 & 6 & 6 & 14 & 0.063 & 0.69 & -0.42 & $\mathrm{E}$ & $l s$ \\
\hline & & & 555.121 & 26829 & 206972 & 4 & 4 & 13 & 0.061 & 0.44 & -0.62 & $\mathrm{E}$ & $l s$ \\
\hline & & & $\begin{array}{l}555.121 \\
5550\end{array}$ & $\begin{array}{l}20000 \\
26829\end{array}$ & 206971 & 4 & $\begin{array}{l}4 \\
6\end{array}$ & $\begin{array}{l}1.5 \\
0.97\end{array}$ & 0.0067 & $\begin{array}{l}0.049 \\
0.049\end{array}$ & -1.57 & $\mathrm{~L}$ & $l s$ \\
\hline 9 & & $\begin{array}{c}{ }^{2} \mathrm{P}^{\circ}-{ }^{2} \mathrm{D} \\
(14 \mathrm{uv})\end{array}$ & 600.585 & 40467 & 206972 & 6 & 10 & 4.3 & 0.039 & 0.46 & -0.63 & $\mathrm{E}$ & 1 \\
\hline \multirow[t]{4}{*}{10} & $2 p^{3}-$ & ${ }^{4} \mathrm{~S}^{\circ}-{ }^{4} \mathrm{P}$ & 430.09 & 0.0 & 232511 & 4 & 12 & 39 & 0.32 & 1.8 & 0.11 & D- & 1 \\
\hline & & & 430.177 & 0.0 & 232463 & 4 & 6 & 39 & 0.16 & 0.91 & -0.19 & $\mathrm{D}-$ & $l s$ \\
\hline & & & 430.041 & 0.0 & 232536 & 4 & 4 & 39 & 0.1 & 0.61 & -0.37 & D- & $l s$ \\
\hline & & & 429.918 & 0.0 & 232603 & 4 & 2 & 39 & 0.054 & 0.31 & -0.67 & $\mathrm{D}-$ & ls \\
\hline \multirow[t]{4}{*}{11} & & ${ }^{2} \mathrm{D}^{\circ}-{ }^{2} \mathrm{~F}$ & 485.27 & 26817 & 232889 & 10 & 14 & 25 & 0.12 & 1.9 & 0.08 & D- & $\begin{array}{lll}1 & & l \\
\end{array}$ \\
\hline & & & 485.086 & 26808 & 232959 & 6 & 8 & 25 & 0.12 & 1.1 & -0.16 & $\mathrm{D}-$ & ls \\
\hline & & & 485.515 & 26829 & 232796 & 4 & 6 & 23 & 0.12 & 0.78 & -0.31 & D - & $l s$ \\
\hline & & & [485.47] & 26808 & 232796 & 6 & 6 & 1.6 & 0.0058 & 0.056 & -1.46 & D- & $l s$ \\
\hline \multirow[t]{3}{*}{12} & & ${ }^{2} \mathrm{D}^{\circ}-{ }^{-2} \mathrm{P}$ & 483.91 & 26817 & 233468 & 10 & 6 & 0.84 & 0.0018 & 0.028 & -1.75 & D- & 1 \\
\hline & & & 483.976 & 26808 & 233430 & 6 & 4 & 0.76 & 0.0018 & 0.017 & -1.97 & D- & $l s$ \\
\hline & & & $\begin{array}{r}483.752 \\
{[484.03]}\end{array}$ & $\begin{array}{l}26829 \\
26829\end{array}$ & 233544 & 4 & 2 & $\begin{array}{l}0.84 \\
0.884\end{array}$ & $\begin{array}{l}0.0015 \\
3.0 \times 10^{-4}\end{array}$ & $\begin{array}{l}0.0094 \\
0.0019\end{array}$ & $\begin{array}{l}-2.23 \\
-2.93\end{array}$ & D- & ls \\
\hline 13 & & & & & & & & & & & & & \\
\hline 13 & & $\begin{array}{l}2^{2} D^{\circ}-{ }^{-2} D \\
(10 \text { uv })\end{array}$ & 481.66 & 26817 & 234434 & 10 & 10 & 5.4 & 0.019 & 0.30 & -0.73 & $\mathbf{D}-$ & 1 \\
\hline \multirow[t]{5}{*}{14} & & ${ }^{2} \mathrm{P}^{\circ}-{ }^{2} \mathrm{P}$ & 518.13 & 40467 & 233468 & 6 & 6 & 11 & 0.045 & 0.46 & -0.57 & $\mathrm{D}-$ & 1 \\
\hline & & & 518.242 & 40467 & 233430 & 4 & 4 & 9.4 & 0.038 & 0.26 & -0.82 & D- & $l s$ \\
\hline & & & 517.937 & 40468 & 233544 & 2 & 2 & 7.5 & 0.030 & & -1.22 & D- & ls \\
\hline & & & 517.937 & 40467 & 233544 & 4 & 2 & 3.7 & 0.0075 & 0.051 & -1.52 & D- & $l s$ \\
\hline & & & 518.242 & 40468 & 233430 & 2 & 4 & 1.9 & 0.015 & 0.051 & -1.52 & $\mathrm{D}-$ & $l s$ \\
\hline \multirow[t]{3}{*}{15} & & ${ }^{2} \mathrm{P}^{\circ}-{ }^{-2} \mathrm{D}$ & 515.55 & 40467 & 234434 & 6 & 10 & 15 & 0.097 & 0.98 & -0.24 & D - & 1 \\
\hline & & & 515.498 & 40467 & 234454 & 4 & 6 & 15 & 0.087 & 0.59 & -0.46 & D- & $l s$ \\
\hline & & & $\begin{array}{l}515.640 \\
515.640\end{array}$ & $\begin{array}{l}40468 \\
40467\end{array}$ & $\begin{array}{l}234402 \\
234402\end{array}$ & $\begin{array}{l}2 \\
4\end{array}$ & $\begin{array}{l}4 \\
4\end{array}$ & $\begin{array}{l}12 \\
2.4\end{array}$ & \begin{tabular}{|l|}
0.097 \\
0.0097
\end{tabular} & $\begin{array}{l}0.33 \\
0.066\end{array}$ & $\begin{array}{l}-0.71 \\
-1.41\end{array}$ & $\begin{array}{l}\mathrm{D}- \\
\mathrm{D}-\end{array}$ & $\begin{array}{l}l s \\
l s\end{array}$ \\
\hline 16 & $\begin{array}{l}2 p^{3}- \\
2 p^{2}\left({ }^{1} \mathrm{D}\right) 3 d^{\prime}\end{array}$ & ${ }^{2} \mathrm{D}^{\circ}-{ }^{2} \mathrm{~F}$ & 445.62 & 26817 & 251222 & 10 & 14 & 26 & 0.11 & 1.6 & 0.04 & D- & 1 \\
\hline 17 & & ${ }^{2} \mathrm{D}^{\circ}-{ }^{2} \mathrm{D}$ & 442.03 & 26817 & 253048 & 10 & 10 & 21 & 0.063 & 0.91 & -0.20 & $\mathrm{D}-$ & 1 \\
\hline 18 & & ${ }^{2} \mathrm{D}^{\circ}-{ }^{2} \mathrm{P}$ & 440.58 & 26817 & 253791 & 10 & 6 & 7.7 & 0.013 & 0.20 & -0.87 & $\mathrm{D}-$ & 1 \\
\hline 19 & & ${ }^{2} \mathrm{P}^{\circ}-{ }^{\circ} \mathrm{D}$ & 470.41 & 40467 & 253048 & 6 & 10 & 6.5 & 0.036 & 0.33 & -0.67 & $\mathrm{D}-$ & 1 \\
\hline 20 & & ${ }^{2} \mathrm{P}^{\circ}-{ }^{2} \mathrm{P}$ & 468.77 & 40467 & 253791 & 6 & 6 & 12 & 0.039 & 0.36 & -0.64 & $\mathrm{D}-$ & 1 \\
\hline 21 & & ${ }^{2} \mathrm{P}^{\circ}-{ }^{2} \mathrm{~S}$ & 464.78 & 40467 & 255622 & 6 & 2 & 16 & 0.017 & 0.16 & -0.99 & $\mathrm{D}-$ & 1 \\
\hline 22 & $2 p^{3}-$ & ${ }^{2} \mathrm{P}^{\circ}-{ }^{2} \mathrm{D}$ & [424.66] & 40467 & [275951] & 6 & $|10|$ & 19 & 0.086 & 0.72 & -0.29 & $\mathrm{D}-$ & 1 \\
\hline
\end{tabular}


O 11. Allowed Transitions-Continued

\begin{tabular}{|c|c|c|c|c|c|c|c|c|c|c|c|c|c|}
\hline No. & $\begin{array}{l}\text { Transition } \\
\text { Array }\end{array}$ & Multiplet & $\lambda(\AA \AA)$ & $E_{i}\left(\mathrm{~cm}^{-1}\right)$ & $E_{k}\left(\mathrm{~cm}^{-1}\right)$ & $g_{i}$ & $g_{k}$ & $A_{k i}\left(10^{k} \sec ^{-1}\right)$ & $f_{i k}$ & $S$ (at.u.) & $\log g f$ & $\begin{array}{l}\text { Accu- } \\
\text { racy }\end{array}$ & Source \\
\hline \multirow[t]{9}{*}{23} & \multirow{15}{*}{$\begin{array}{l}2 p^{2} 3 s- \\
2 p^{2}\left({ }^{3} \mathrm{P}\right) 3 p\end{array}$} & \multirow{8}{*}{${ }^{4} \mathrm{P}-{ }^{4} \mathrm{D}^{\circ}$} & 4651.5 & 185402 & 206895 & 12 & 20 & 1.02 & 0.55 & 101 & 0.82 & C & $3,4, c a$ \\
\hline & & & 4649.14 & 185499 & 207003 & 6 & 8 & 1.04 & 0.448 & 41.1 & 0.429 & C & $4 n, 5 n$ \\
\hline & & & 4641.81 & 185341 & 206878 & 4 & 6 & 0.79 & 0.381 & 23.3 & 0.183 & $\mathrm{C}$ & $4 n, 5 n$ \\
\hline & & & 4638.85 & 185235 & 206786 & 2 & 4 & 0.422 & 0.272 & 8.3 & -0.264 & C & $4 n, 5 n$ \\
\hline & & & 4676.23 & 185499 & 206878 & 6 & 6 & 0.257 & 0.084 & 7.8 & -0.296 & C & $4 n, 5 n$ \\
\hline & & & 4661.64 & 185341 & 206786 & 4 & 4 & 0.52 & 0.169 & 10.4 & -0.169 & C & $4 n, 5 n$ \\
\hline & & & 4650.84 & 185235 & 206731 & 2 & 2 & 0.82 & 0.265 & 8.1 & -0.277 & C & $4 n, 5 n$ \\
\hline & & & $\begin{array}{l}4696.36 \\
4673.75\end{array}$ & $\begin{array}{l}185499 \\
185341\end{array}$ & $\begin{array}{l}206786 \\
206731\end{array}$ & $\begin{array}{l}6 \\
4\end{array}$ & $\begin{array}{l}4 \\
2\end{array}$ & $\begin{array}{l}0.0372 \\
0.131\end{array}$ & $\begin{array}{l}0.0082 \\
0.0214\end{array}$ & $\begin{array}{l}0.76 \\
1.32\end{array}$ & $\begin{array}{l}-1.308 \\
-1.067\end{array}$ & $\begin{array}{l}\mathrm{C} \\
\mathrm{C}\end{array}$ & $\begin{array}{l}4 n, 5 n \\
4 n, 5 n\end{array}$ \\
\hline & & \multirow{7}{*}{${ }_{(2)}^{4} \mathrm{P}-{ }^{4} \mathrm{P}^{\circ}$} & 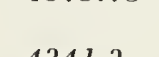 & & & & & & D. & & & & \\
\hline \multirow{7}{*}{24} & & & 4341.3 & 185402 & 208431 & 12 & 12 & 1.05 & 0.297 & 51 & 0.55 & C & $3,4, c a$ \\
\hline & & & 4349.43 & $\begin{array}{l}185499 \\
185341\end{array}$ & 208484 & 6 & 6 & 0.74 & $\begin{array}{l}0.211 \\
0.0462\end{array}$ & $\begin{array}{c}18.1 \\
264\end{array}$ & $\begin{array}{r}0.102 \\
-0.73\end{array}$ & C & $4 n, 5 n$ \\
\hline & & & $\begin{array}{l}4336.81 \\
4325.77\end{array}$ & $\begin{array}{l}185341 \\
185235\end{array}$ & 208346 & $\begin{array}{l}4 \\
2\end{array}$ & $\begin{array}{r}4 \\
2\end{array} \mid$ & $\begin{array}{l}0.164 \\
0.155\end{array}$ & $\begin{array}{l}0.0462 \\
0.0435\end{array}$ & $\begin{array}{l}2.04 \\
1.24\end{array}$ & & C & $\begin{array}{l}4 n, 5 n \\
4 n, 5 n\end{array}$ \\
\hline & & & 4366.90 & 185499 & 208392 & 6 & 4 & 0.50 & 0.096 & 8.3 & -0.239 & $\mathrm{C}$ & $\begin{array}{l}4 n, 3 n \\
4 n, 5 n\end{array}$ \\
\hline & & & 4345.56 & 185341 & 208346 & 4 & 2 & 0.89 & 0.125 & 7.2 & -0.300 & $\mathrm{C}$ & $4 n, 5 n$ \\
\hline & & & 4319.63 & $\begin{array}{l}185341 \\
185235\end{array}$ & 208484 & 4. & $\begin{array}{l}6 \\
4\end{array}$ & 0.284 & 0.119 & 6.8 & $\begin{array}{l}-0.321 \\
-0.325\end{array}$ & C & $4 n, 5 n$ \\
\hline & & \multirow{4}{*}{ 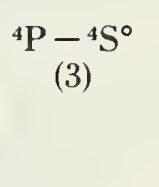 } & 4 & 100200 & 200392 & & 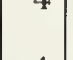 & 0.424 & 0.206 & & 25 & & \\
\hline \multirow{3}{*}{25} & & & 3735.9 & 185402 & 212162 & 12 & 4 & 1.77 & 0.123 & 18.2 & 0.170 & C & $3,4, c a$ \\
\hline & & & $\begin{array}{l}3749.49 \\
3797.33\end{array}$ & 185499 & 212162 & 6 & 4 & $\begin{array}{l}0.90 \\
0.59\end{array}$ & 0.127 & 9.4 & -0.119 & C & $4 n, 5 n$ \\
\hline & & & 3712.75 & 185235 & 212162 & $\begin{array}{l}4 \\
2\end{array}$ & 4 & $\begin{array}{l}0.09 \\
0.280\end{array}$ & 0.116 & $\begin{array}{l}.0 \\
2.83\end{array}$ & $\begin{array}{l}-0.312 \\
-0.64\end{array}$ & C & $\begin{array}{l}4 n, 5 n \\
4 n, 5 n\end{array}$ \\
\hline \multirow{2}{*}{26} & & \multirow{2}{*}{${ }^{4} \mathrm{P}-{ }^{2} \mathrm{P}^{\circ}$} & [3479.7] & 185499 & 214229 & 6 & 4 & 0.00101 & $1.22 \times 10^{-4}$ & 0.0084 & -3.135 & C & 5 \\
\hline & & & {$[3460.6]$} & 185341 & 214229 & 4 & 4 & 0.00154 & $2.76 \times 10^{-4}$ & 0.0126 & -2.96 & C & 5 \\
\hline \multirow[t]{3}{*}{27} & & \multirow{3}{*}{$\mid \begin{array}{c}2 \mathrm{P}-{ }^{2} \mathrm{~S}^{\circ} \\
(4)\end{array}$} & 6694.4 & 189008 & 203942 & 6 & 2 & 0.287 & 0.064 & 8.5 & -0.414 & C & $3, c a$ \\
\hline & & & 6721.35 & 189068 & 203942 & 4 & 2 & 0.189 & 0.064 & 5. & -0.59 & C & $l s$ \\
\hline & & & 6640.90 & 188888 & 203942 & 2 & 2 & 0.098 & 0.065 & 2.8 & -0.89 & $\mathrm{C}$ & 1 \\
\hline \multirow[t]{3}{*}{28} & & \multirow[t]{3}{*}{${ }^{2} \mathrm{P}-{ }^{2} \mathrm{D}^{\circ}$} & 4418.1 & 189008 & 211636 & 6 & 10 & 1.13 & 0.55 & 48.1 & 0.52 & C & $3,4, c a$ \\
\hline & & & $\begin{array}{l}4414.91 \\
4416698\end{array}$ & $\begin{array}{l}189068 \\
180980\end{array}$ & 211713 & 4 & 6 & 1.15 & 0.50 & 29.3 & 0.305 & C & $4 n, 5 n$ \\
\hline & & & $\begin{array}{l}4416.98 \\
4452.38\end{array}$ & $\begin{array}{l}188888 \\
189068\end{array}$ & $\begin{array}{l}211522 \\
211522\end{array}$ & $\begin{array}{l}2 \\
4\end{array}$ & $\begin{array}{l}4 \\
4\end{array}$ & $\begin{array}{l}0.95 \\
0.154\end{array}$ & $\mid \begin{array}{l}0.55 \\
0.0457\end{array}$ & $\begin{array}{c}16.1 \\
2.68\end{array}$ & $\begin{array}{c}0.044 \\
-0.74\end{array}$ & $\begin{array}{l}\mathrm{C} \\
\mathrm{C}\end{array}$ & $\begin{array}{l}4 n, 5 n \\
4 n, 5 n\end{array}$ \\
\hline \multirow[t]{2}{*}{29} & & \multirow[t]{2}{*}{${ }^{2} \mathrm{P}-{ }^{4} \mathrm{~S}^{\circ}$} & & & & & & & & & & & \\
\hline & & & $\begin{array}{l}{[4329.0]} \\
{[4295.5]}\end{array}$ & $\begin{array}{l}189068 \\
188888\end{array}$ & $\begin{array}{l}212162 \\
212162\end{array}$ & $\begin{array}{l}4 \\
2\end{array}$ & $\begin{array}{l}4 \\
4\end{array}$ & $\begin{array}{l}0.00157 \\
0.268 \times 10^{-3}\end{array}$ & $\begin{array}{l}4.42 \times 10^{-4} \\
1.48 \times 10^{-4}\end{array}$ & $\begin{array}{l}0.0252 \\
0.00420\end{array}$ & $\begin{array}{l}-2.75 \\
-3.53\end{array}$ & C & 5 \\
\hline \multirow[t]{4}{*}{30} & & \multirow{4}{*}{${ }^{2} \mathrm{P}-{ }^{2} \mathrm{P}^{\circ}$} & 3966.9 & 189008 & 214210 & 6 & 6 & 1.46 & 0.343 & 26.9 & 0.314 & $\mathrm{C}$ & $3,4, c a$ \\
\hline & & & 3973.26 & 189068 & 214229 & 4 & 4 & 1.27 & 0.300 & 15.7 & 0.080 & C & $4 n, 5 n$ \\
\hline & & & $\begin{array}{l}3954.37 \\
\text { 309? }\end{array}$ & 188888 & 214170 & 2 & 2 & 0.95 & 0.222 & $\begin{array}{l}5.8 \\
? 70\end{array}$ & -0.353 & C & $4 n, 5 n$ \\
\hline & & & $\begin{array}{l}3982.72 \\
3945.05\end{array}$ & $\begin{array}{l}189068 \\
188888\end{array}$ & $\begin{array}{l}214170 \\
214229\end{array}$ & $\begin{array}{l}4 \\
2\end{array}$ & $\begin{array}{l}2 \\
4\end{array}$ & $\begin{array}{l}0.447 \\
0.217\end{array}$ & $\mid \begin{array}{l}0.053 \\
0.101\end{array}$ & $\begin{array}{l}2.79 \\
2.63\end{array}$ & $\begin{array}{l}-0.67 \\
-0.69\end{array}$ & $\begin{array}{l}\mathrm{C} \\
\mathrm{C}\end{array}$ & $\begin{array}{l}4 n, 5 n \\
4 n, 5 n\end{array}$ \\
\hline \multirow[t]{3}{*}{31} & \multirow{7}{*}{$\begin{array}{l}2 p^{2} 3 s^{\prime}- \\
2 p^{2}\left({ }^{1} \mathrm{D}\right) 3 p^{\prime}\end{array}$} & \multirow{3}{*}{$\begin{array}{c}{ }^{2} \mathrm{D}-{ }^{2} \mathrm{~F}^{\circ} \\
(15)\end{array}$} & 4593.2 & 206972 & 228737 & 10 & 14 & 1.11 & 0.490 & 74 & 0.69 & C & $3,4, c a$ \\
\hline & & & 4590.97 & 206971 & 228747 & 6 & 8 & 1.11 & 0.46 & 42.3 & 0.447 & C & $5 n$ \\
\hline & & & $\begin{array}{r}4596.17 \\
{[4596.0]}\end{array}$ & $\begin{array}{l}206972 \\
206971\end{array}$ & $\begin{array}{l}228723 \\
228723\end{array}$ & 4 & 6 & \begin{tabular}{|l|}
1.03 \\
0.079
\end{tabular} & $\begin{array}{l}0.487 \\
0.0251\end{array}$ & $\begin{array}{c}29.5 \\
2.28\end{array}$ & $\begin{array}{r}0.290 \\
-0.82\end{array}$ & $\begin{array}{l}\mathrm{C} \\
\mathrm{C}\end{array}$ & $5 n$ \\
\hline \multirow[t]{4}{*}{32} & & \multirow{4}{*}{$\begin{array}{c}{ }^{2} \mathrm{D}-{ }^{2} \mathrm{D}^{\circ} \\
(16)\end{array}$} & 4349.7 & 206972 & 229955 & 10 & 10 & 1.04 & 0.296 & 42.4 & 0.471 & C & $3,4, c a$ \\
\hline & & & 4351.27 & 206971 & 229947 & 6 & 6 & & & 236 & 0.217 & $C$ & \\
\hline & & & 4347.43 & 206972 & 229968 & 4 & $\begin{array}{l}0 \\
4\end{array}$ & $\begin{array}{l}0.97 \\
0.94\end{array}$ & 0.267 & 15.3 & $\begin{array}{l}0.218 \\
0.029\end{array}$ & C & $\begin{array}{l}5 n \\
5 n\end{array}$ \\
\hline & & & $\begin{array}{l}{[4349.1]} \\
{[4351.5]}\end{array}$ & $\begin{array}{l}206971 \\
206972\end{array}$ & $\begin{array}{l}229968 \\
229947\end{array}$ & $\begin{array}{l}6 \\
4\end{array}$ & $\begin{array}{l}4 \\
6\end{array}$ & $\begin{array}{l}0.102 \\
0.075\end{array}$ & $\begin{array}{l}0.0192 \\
0.0318\end{array}$ & $\begin{array}{l}1.65 \\
1.82\end{array}$ & $\begin{array}{l}-0.94 \\
-0.90\end{array}$ & $\mathrm{C}$ & $5 n$ \\
\hline
\end{tabular}


O II. Allowed Transitions-Continued

\begin{tabular}{|c|c|c|c|c|c|c|c|c|c|c|c|c|c|}
\hline No. & $\begin{array}{c}\text { Transition } \\
\text { Array }\end{array}$ & Multiplet & $\lambda(\AA)$ & $E_{i}\left(\mathrm{~cm}^{-1}\right)$ & $E_{h}\left(\mathrm{~cm}^{-1}\right)$ & $g_{i}$ & $g_{k}$ & $A_{k i}\left(10^{8} \sec ^{-1}\right)$ & $f_{i k}$ & $S$ (at.u.) & $\log g f$ & $\begin{array}{l}\text { Accu- } \\
\text { racy }\end{array}$ & Source \\
\hline \multirow[t]{3}{*}{33} & & ${ }^{2} \mathrm{D}-{ }^{2} \mathrm{P}^{\circ}$ & 3914.4 & 206972 & 232511 & 10 & 6 & 1.40 & 0.193 & 24.9 & 0.286 & C & $3,4, c a$ \\
\hline & & & 3911.96 & 206971 & 232527 & 6 & 4 & 1.27 & 0.194 & 15.0 & 0.066 & C & $5 n$ \\
\hline & & & $\begin{array}{l}3919.29 \\
3912.09\end{array}$ & $\begin{array}{l}206972 \\
206972\end{array}$ & $\begin{array}{l}232480 \\
232527\end{array}$ & $\begin{array}{l}4 \\
4\end{array}$ & $\begin{array}{l}2 \\
4\end{array}$ & \begin{tabular}{|l|}
1.40 \\
0.137
\end{tabular} & $\begin{array}{l}0.161 \\
0.0314\end{array}$ & $\begin{array}{l}8.3 \\
1.62\end{array}$ & $\begin{array}{l}-0.192 \\
-0.90\end{array}$ & $\begin{array}{l}\mathrm{C} \\
\mathrm{C}\end{array}$ & $\begin{array}{l}5 n \\
5 n\end{array}$ \\
\hline 34 & $\begin{array}{c}2 p^{2} 3 s^{\prime \prime}- \\
2 p^{2}(1 \mathrm{~S}) 3 p^{\prime \prime}\end{array}$ & ${ }^{2} \mathrm{~S}-{ }^{2} \mathrm{P}^{\circ}$ & [4272.3] & [226851] & [250251] & 2 & 6 & 1.08 & 0.89 & 25.0 & 0.250 & C & $3, c a$ \\
\hline \multirow[t]{4}{*}{35} & $\begin{array}{c}2 s 2 p^{3} 3 s^{\prime \prime \prime \prime}- \\
2 s 2 p^{3}\left({ }^{5} \mathrm{~S}^{\circ}\right)\end{array}$ & $\begin{array}{c}{ }^{6} \mathrm{~S}^{\circ}-{ }^{6} \mathrm{P} \\
(94)\end{array}$ & 4467.2 & {$[245396]$} & [267775] & 6 & 18 & 0.92 & 0.83 & 73 & 0.70 & C & $c a$ \\
\hline & & & 4465.40 & [245396] & [267783] & 6 & 8 & 0.92 & 0.367 & 32.4 & 0.343 & C & $l s$ \\
\hline & & & $\begin{array}{l}4467.88 \\
446932\end{array}$ & {$[245396]$} & [267771] & 6 & 6 & 0.92 & 0.275 & 24.3 & 0.218 & C & ls \\
\hline & & & 4409.02 & [245390] & [26/763] & 6 & 4 & 0.92 & 0.184 & 16.2 & 0.042 & c & $l s$ \\
\hline \multirow[t]{2}{*}{36} & $\begin{array}{c}2 p^{2} 3 p- \\
2 p^{2}\left({ }^{3} \mathrm{P}\right) 3 d\end{array}$ & $\begin{array}{c}{ }^{2} \mathrm{~S}^{\circ}-{ }^{4} \mathrm{P} \\
(7)\end{array}$ & & & & & & & & & & & \\
\hline & & & $\begin{array}{l}3496.27 \\
3488.18\end{array}$ & $\begin{array}{l}203942 \\
203942\end{array}$ & $\begin{array}{l}232536 \\
232603\end{array}$ & $\begin{array}{l}2 \\
2\end{array}$ & $\begin{array}{l}4 \\
2\end{array}$ & $\begin{array}{l}0.0111 \\
0.0084\end{array}$ & $\mid \begin{array}{l}0.00408 \\
0.00152\end{array}$ & $\begin{array}{l}0.094 \\
0.0350\end{array}$ & $\begin{array}{l}-2.088 \\
-2.52\end{array}$ & $\begin{array}{l}\mathrm{C} \\
\mathrm{C}\end{array}$ & $\begin{array}{l}5 \\
5\end{array}$ \\
\hline \multirow[t]{2}{*}{37} & & $\begin{array}{l}{ }^{2} \mathrm{~S}^{\circ}-{ }^{4} \mathrm{D} \\
(8)\end{array}$ & & & & & & & & & & & \\
\hline & & & 3474.94 & 203942 & 232712 & 2 & 2 & 0.0085 & 0.00153 & 0.0351 & -2.51 & C & 5 \\
\hline \multirow[t]{2}{*}{38} & & $\begin{array}{c}{ }^{2} \mathrm{~S}^{\circ}-{ }^{2} \mathrm{P} \\
(9)\end{array}$ & 3385.9 & 203942 & 233468 & 2 & 6 & 1.86 & 0.96 & 21.4 & 0.283 & C & $3, c a$ \\
\hline & & & $\begin{array}{l}3390.25 \\
3377.20\end{array}$ & 203942 & 233430 & 2 & 4 & 1.86 & 0.64 & 14.3 & 0.108 & C & 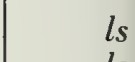 \\
\hline \multirow{2}{*}{39} & & $2 \mathrm{~S}^{\circ}-{ }^{2} \mathrm{D}$ & & & & & & & & & -0.20 & 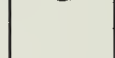 & \\
\hline & & -5 & [3282.0] & 203942 & 234402 & 2 & 4 & 0.0168 & 0.0054 & 0.117 & -1.97 & C & 5 \\
\hline \multirow[t]{8}{*}{40} & & $\begin{array}{c}{ }^{4} \mathrm{D}^{\circ}-{ }^{4} \mathrm{~F} \\
(10)\end{array}$ & 4074.8 & 206895 & 231429 & 20 & 28 & 1.98 & 0.69 & 185 & 1.140 & C & $3, c a$ \\
\hline & & & 4075.87 & $\begin{array}{l}207003 \\
206878\end{array}$ & 231530 & 8 & $\begin{array}{r}10 \\
8\end{array}$ & 1.98 & 0.62 & 66 & 0.69 & C & $l s$ \\
\hline & & & $\begin{array}{l}4072.16 \\
4069.90\end{array}$ & $\begin{array}{l}206878 \\
206786\end{array}$ & $\begin{array}{l}231428 \\
231350\end{array}$ & $\begin{array}{l}6 \\
4\end{array}$ & \begin{tabular}{|l|}
8 \\
6 \\
\end{tabular} & $\begin{array}{l}1.70 \\
1.49\end{array}$ & $\begin{array}{l}0 . \\
0 .\end{array}$ & $\begin{array}{l}45 . \\
29 .\end{array}$ & $\begin{array}{l}0.53 \\
0.346\end{array}$ & $\begin{array}{l}\mathrm{C} \\
\mathrm{C}\end{array}$ & $\begin{array}{l}l s \\
l s\end{array}$ \\
\hline & & & 4069.64 & 206731 & 231296 & 2 & 4 & 1.39 & 0. & 18.5 & 0.140 & C & ls \\
\hline & & & $\begin{array}{l}4092.94 \\
4085.12\end{array}$ & $\begin{array}{l}207003 \\
206878\end{array}$ & $\begin{array}{l}231428 \\
231350\end{array}$ & $\begin{array}{l}8 \\
6\end{array}$ & $\begin{array}{l}8 \\
6\end{array}$ & $\begin{array}{l}0.278 \\
0.478\end{array}$ & $\mid \begin{array}{l}0.070 \\
0.120\end{array}$ & $\begin{array}{l}7.5 \\
9.7\end{array}$ & $\begin{array}{l}-0.253 \\
-0.144\end{array}$ & C & ls \\
\hline & & & 4078.86 & 206786 & $\begin{array}{l}231350 \\
231296\end{array}$ & 4 & 4 & 0.55 & 0.138 & 7.4 & -0.259 & $\mathrm{C}$ & 6 \\
\hline & & & $\begin{array}{l}4106.03 \\
4094.18\end{array}$ & $\begin{array}{l}207003 \\
206878\end{array}$ & $\begin{array}{l}231350 \\
231296\end{array}$ & 8 & $\begin{array}{l}6 \\
4\end{array}$ & $\begin{array}{l}0.0187 \\
0.0390\end{array}$ & $\begin{array}{l}0.00354 \\
0.0065\end{array}$ & 0.383 & $\begin{array}{l}-1.55 \\
-1.406\end{array}$ & $\mathrm{C}$ & ls \\
\hline & & & 4094.18 & $2068 / 8$ & 231296 & 0 & 4 & 0.0390 & 0.0065 & 0.53 & -1.406 & C & is \\
\hline \multirow[t]{7}{*}{41} & & $\begin{array}{c}{ }^{4} \mathrm{D}^{\circ}-{ }^{4} \mathrm{P} \\
(11)\end{array}$ & 3902.7 & 206895 & 232511 & 20 & 12 & 0.063 & 0.0086 & 2.21 & -0.76 & C & $3, c a$ \\
\hline & & & \begin{tabular}{|l|}
3926.58 \\
3896.30
\end{tabular} & $\begin{array}{l}207003 \\
206878\end{array}$ & $\begin{array}{l}232463 \\
232536\end{array}$ & $\begin{array}{l}8 \\
6\end{array}$ & $\begin{array}{l}6 \\
4\end{array}$ & $\begin{array}{l}0.0493 \\
0.0397\end{array}$ & $\begin{array}{l}0.0085 \\
0.0060\end{array}$ & $\begin{array}{l}0.88 \\
0.464\end{array}$ & $\begin{array}{l}-1.165 \\
-1.442\end{array}$ & $\begin{array}{l}\mathrm{C} \\
\mathrm{C}\end{array}$ & $l s$ \\
\hline & & & 3872.45 & 206786 & 232603 & 4 & $\begin{array}{l}4 \\
2\end{array}$ & 0.0321 & 0.00 & $\begin{array}{l}0.404 \\
0.184\end{array}$ & $\begin{array}{l}-1.842 \\
-1.84\end{array}$ & ${ }_{\mathrm{C}}^{\mathrm{C}}$ & $\begin{array}{l}l s \\
l s\end{array}$ \\
\hline & & & 3907.45 & 206878 & 232463 & 6 & 6 & 0.0113 & 0.00258 & 0.199 & -1.81 & $\mathrm{C}$ & $l s$ \\
\hline & & & 3882.45 & 206786 & 232536 & 4 & 4 & 0.0204 & & 0.2 & -1.73 & C & ls \\
\hline & & & 3864.13 & 206731 & 232603 & 2 & 2 & 0.0323 & 0.0072 & 0.184 & -1.84 & C & ls \\
\hline & & & \begin{tabular}{|l|}
3893.53 \\
3874.10
\end{tabular} & $\begin{array}{l}206786 \\
206731\end{array}$ & $\begin{array}{l}232463 \\
232536\end{array}$ & $\begin{array}{l}4 \\
2\end{array}$ & $\begin{array}{l}6 \\
4\end{array}$ & $\begin{array}{l}0.00126 \\
0.00321\end{array}$ & $\begin{array}{l}4.31 \times 10^{-4} \\
0.00144\end{array}$ & $\begin{array}{l}0.0221 \\
0.0368\end{array}$ & $\begin{array}{l}-2.76 \\
-2.54\end{array}$ & $\begin{array}{l}\mathrm{C} \\
\mathrm{C}\end{array}$ & $l s$ \\
\hline \multirow[t]{9}{*}{42} & & $\begin{array}{c}{ }^{4} \mathrm{D}^{\circ}-{ }^{4} \mathrm{D} \\
(12)\end{array}$ & 3867.2 & 206895 & 232746 & 20 & 20 & 0.58 & 0.130 & 33.2 & 0.416 & C & $3, c a$ \\
\hline & & & 3882.20 & 207003 & 232754 & 8 & 8 & 0.493 & 0.111 & 11.4 & -0.050 & C & $l s$ \\
\hline & & & \begin{tabular}{|l}
3864.45 \\
3851.04
\end{tabular} & $\begin{array}{l}206878 \\
206786\end{array}$ & $\begin{array}{l}232748 \\
232746\end{array}$ & $\begin{array}{l}6 \\
4\end{array}$ & $\begin{array}{l}6 \\
4\end{array}$ & $\begin{array}{l}0.334 \\
0.236\end{array}$ & $\mid \begin{array}{l}0.075 \\
0.052\end{array}$ & $\begin{array}{l}5.7 \\
2.66\end{array}$ & $\begin{array}{l}-0.348 \\
-0.68\end{array}$ & $\begin{array}{l}\mathrm{C} \\
\mathrm{C}\end{array}$ & $l s$ \\
\hline & & & 3847.89 & 206731 & 232712 & 2 & 2 & 0.295 & 0.066 & 1.66 & -0.88 & $\mathrm{C}$ & ls \\
\hline & & & 3883.15 & 207003 & 232748 & 8 & 6 & 0.109 & 0.0185 & 1.89 & -0.83 & C & ls \\
\hline & & & 3864.68 & 206878 & 232746 & 6 & 4 & 0.204 & 0.0304 & 2.32 & -0.74 & C & ls \\
\hline & & & $\begin{array}{l}3856.16 \\
3863.50\end{array}$ & $\begin{array}{l}206786 \\
206878\end{array}$ & $\begin{array}{l}232712 \\
232754\end{array}$ & 4 & 2 & $\begin{array}{l}0.293 \\
0.083\end{array}$ & $\begin{array}{l}0.0327 \\
0.0248\end{array}$ & $\begin{array}{l}1.66 \\
1.89\end{array}$ & $\begin{array}{l}-0.88 \\
-0.83\end{array}$ & $\stackrel{\mathrm{C}}{\mathrm{C}}$ & ls \\
\hline & & & $\begin{array}{l}3863.50 \\
3850.81\end{array}$ & $\begin{array}{l}206878 \\
206786\end{array}$ & $\begin{array}{l}232754 \\
232748\end{array}$ & $\begin{array}{l}6 \\
4\end{array}$ & $\begin{array}{l}8 \\
6\end{array}$ & $\begin{array}{l}0.083 \\
0.137\end{array}$ & $\begin{array}{l}0.0248 \\
0.0458\end{array}$ & $\begin{array}{l}1.89 \\
2.32\end{array}$ & -0.83 & $\mathrm{C}$ & $l s$ \\
\hline & & & 3842.82 & 206731 & 232746 & 2 & & 0.148 & 066 & 1.66 & 0.88 & C & $l s$ \\
\hline
\end{tabular}


O II. Allowed Transitions - Continued

\begin{tabular}{|c|c|c|c|c|c|c|c|c|c|c|c|c|c|}
\hline No. & $\begin{array}{c}\text { Transition } \\
\text { Array }\end{array}$ & Multiplet & $\lambda(\AA)$ & $E_{i}\left(\mathrm{~cm}^{-1}\right)$ & $E_{k}\left(\mathrm{~cm}^{-1}\right)$ & $g_{i}$ & $g_{k}$ & $A_{k i}\left(10^{8} \sec ^{-1}\right)$ & $f_{i k}$ & $S$ (at.u.) & $\log g f$ & $\begin{array}{l}\text { Accu- } \\
\text { racy }\end{array}$ & Source \\
\hline \multirow[t]{5}{*}{43} & & $\begin{array}{c}{ }^{4} \mathrm{D}^{\circ}-{ }^{2} \mathrm{~F} \\
(13)\end{array}$ & & & & & & & & & & & \\
\hline & & & 3851.47 & 207003 & 232959 & 8 & 8 & 0.0223 & 0.0050 & 0.50 & -1.402 & C & \\
\hline & & & $\begin{array}{l}3857.18 \\
387589\end{array}$ & 206878 & 232796 & $\begin{array}{l}6 \\
8\end{array}$ & $\begin{array}{l}6 \\
6\end{array}$ & 0.0448 & $\begin{array}{l}0.0100 \\
0.0016\end{array}$ & $\begin{array}{l}0.76 \\
0.164\end{array}$ & $\begin{array}{l}-1.222 \\
-1.89\end{array}$ & C & \\
\hline & & & 3833.10 & $\begin{array}{l}201003 \\
206878\end{array}$ & $\begin{array}{l}232190 \\
232959\end{array}$ & $\begin{array}{l}8 \\
6\end{array}$ & $\begin{array}{l}6 \\
8\end{array}$ & $\begin{array}{l}0.0095 \\
0.0095\end{array}$ & $\begin{array}{l}0.00161 \\
0.00279\end{array}$ & $\begin{array}{l}0.164 \\
0.211\end{array}$ & $\begin{array}{l}-1.89 \\
-1.78\end{array}$ & $\stackrel{\mathrm{C}}{\mathrm{C}}$ & \\
\hline & & & 3843.58 & 206786 & 232796 & 4 & 6 & 0.0222 & 0.0074 & 0.374 & -1.53 & $\mathrm{C}$ & \\
\hline \multirow[t]{7}{*}{44} & & ${ }^{4} \mathrm{P}^{\circ}-{ }^{4} \mathrm{P}$ & 4151.7 & 208431 & 232511 & 12 & 12 & 1.01 & 0.261 & 42.8 & 0.496 & C & $3,4 \mathrm{ca}$ \\
\hline & & & 4169.28 & 208484 & 232463 & 6 & 6 & 0.220 & 0.057 & 4.73 & -0.463 & C & \\
\hline & & & 4140.74 & 208392 & 232536 & 4 & 4 & 0.0236 & 0.0061 & 0.331 & -1.61 & $\mathrm{C}$ & \\
\hline & & & 4121.48 & 208346 & 232603 & 2 & 2 & $\begin{array}{l}0.93 \\
0.157\end{array}$ & 0.237 & 6.4 & $\begin{array}{l}-0.324 \\
-079\end{array}$ & C & $5 r$ \\
\hline & & & $\begin{array}{l}4150.54 \\
4129.34\end{array}$ & $\begin{array}{l}208484 \\
208392\end{array}$ & $\begin{array}{l}232530 \\
232603\end{array}$ & $\begin{array}{l}0 \\
4\end{array}$ & 2 & $\begin{array}{l}0.158 \\
0.150\end{array}$ & $\left\{\begin{array}{l}.02190 \\
0.019\end{array}\right.$ & $\begin{array}{l}2.22 \\
1.04\end{array}$ & -1.116 & C & \\
\hline & & & 4153.30 & 208392 & 232463 & 4 & 6 & 0.77 & 0.298 & 16.3 & 0.076 & C & \\
\hline & & & 4132.81 & 208346 & 232536 & 2 & 4 & $\mid 0.84$ & 0.430 & 11.7 & -0.066 & C & \\
\hline \multirow[t]{8}{*}{45} & & ${ }^{4} \mathrm{P}^{\circ}-{ }^{4} \mathrm{D}$ & 4111.4 & 208431 & 232746 & 12 & 20 & $\mid 1.49$ & 0.63 & 102 & 0.88 & C & $3, c a$ \\
\hline & & & 4119.22 & 208484 & 232754 & 6 & 8 & \begin{tabular}{|l|l|}
1.48 \\
\end{tabular} & 0.50 & 40.8 & 0.478 & C & \\
\hline & & & 4104.74 & 208392 & 232748 & 4 & 6 & 1.04 & 0.3 & 21.4 & & $\mathrm{C}$ & l \\
\hline & & & 4097.26 & 208346 & 232746 & 2 & 4 & 0.63 & 0.315 & 8.5 & -0.201 & C & \\
\hline & & & 4120.28 & 208484 & 232748 & 6 & 6 & 0.443 & 0.113 & 9.2 & -0.170 & C & \\
\hline & & & 4105.00 & 408392 & 232746 & 4 & 4 & 0.80 & 0.202 & 10.9 & -0.093 & C & \\
\hline & & & $\begin{array}{l}4103.02 \\
410055\end{array}$ & 208346 & 232712 & 2 & 2 & 1.25 & 0.315 & 8.5 & -0.201 & C & 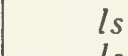 \\
\hline & & & $\begin{array}{l}4120.55 \\
4110.80\end{array}$ & $\begin{array}{l}208484 \\
208392\end{array}$ & $\begin{array}{l}232746 \\
232712\end{array}$ & $\begin{array}{l}6 \\
4\end{array}$ & $\begin{array}{l}4 \\
2\end{array}$ & $\begin{array}{l}0.074 \\
0.248\end{array}$ & $\begin{array}{l}0.0125 \\
0.0314\end{array}$ & $\begin{array}{l}1.02 \\
1.70\end{array}$ & $\begin{array}{l}-1.124 \\
-0.90\end{array}$ & $\begin{array}{l}\mathrm{C} \\
\mathrm{C}\end{array}$ & ls \\
\hline \multirow[t]{4}{*}{46} & & ${ }^{4} \mathrm{P}^{\circ}-{ }^{2} \mathrm{~F}$ & & & & & & & & & & & \\
\hline & & $(2$ & 4084.66 & 208484 & 232959 & 6 & 8 & 0.065 & 0.0216 & 1.74 & -0.89 & C & \\
\hline & & & 4096.54 & 208392 & 232796 & 4 & 6 & 0.092 & & 1. & -0.86 & $\mathrm{C}$ & \\
\hline & & & 4112.03 & 208484 & 232796 & 6 & 6 & $\mid 0.109$ & 0.0275 & 2.23 & -0.78 & C & \\
\hline \multirow[t]{3}{*}{47} & & ${ }^{4} \mathrm{P}^{0}-{ }^{2} \mathrm{P}$ & & & & & & & & & & & \\
\hline & & & 3967.44 & 208346 & 233544 & 2 & 2 & 0.0133 & 0.00314 & 0.082 & -2.203 & C & \\
\hline & & & 3985.46 & 208346 & 233430 & 2 & 4 & 0.0084 & 0.00400 & 0.105 & -2.097 & $\mathrm{C}$ & \\
\hline \multirow[t]{4}{*}{48} & & ${ }^{2} \mathrm{D}^{\circ}-{ }^{4} \mathrm{D}$ & & & & & & & & & & & \\
\hline & & & 4751.34 & 211713 & 232754 & 6 & 8 & 0.059 & 0.0264 & 2.48 & -0.80 & C & \\
\hline & & & 4710.04 & 211522 & 232748 & 4 & 6 & 0.17 & 0.08 & 5.3 & -0.469 & $\mathrm{C}$ & \\
\hline & & & 4752.70 & 211713 & 232748 & 6 & 6 & 0.0088 & 0.00300 & 0.281 & -1.75 & $\mathrm{C}$ & \\
\hline \multirow[t]{4}{*}{49} & & ${ }^{2} \mathrm{D}^{\circ}-{ }^{2} \mathbf{F}$ & 4703.9 & 211636 & 232889 & 10 & 14 & 1.38 & 0.64 & 99 & 0.81 & C & $3, c a$ \\
\hline & & & 4705.36 & 211713 & 232959 & 6 & 8 & 1.38 & 0.61 & 57 & 0.56 & C & \\
\hline & & & 46 & 211522 & 232796 & 4 & 6 & 1.2 & & 39. & 0.408 & C & ls \\
\hline & & & 4741.71 & 211713 & 232796 & 6 & 6 & $\mid 0.090$ & 0.0302 & 2.83 & -0.74 & C & \\
\hline \multirow[t]{3}{*}{50} & & ${ }^{2} \mathrm{D}^{\circ}-{ }^{2} \mathrm{P}$ & 4579.2 & 211636 & 233468 & 10 & 6 & 0.0418 & 0.0079 & 1.19 & -1.103 & C & $3, c a$ \\
\hline & & & [4598.2] & 211713 & 233430 & 6 & 4 & 0.0372 & 0.0079 & 0.7 & -1.326 & C & \\
\hline & & & $\begin{array}{l}{[4539.6]} \\
{[4563.2]}\end{array}$ & $\begin{array}{l}211522 \\
211522\end{array}$ & $\begin{array}{l}233544 \\
233430\end{array}$ & 4 & $\begin{array}{l}2 \\
4\end{array}$ & $\begin{array}{l}0.0430 \\
0.0423\end{array}$ & $\begin{array}{l}0.0066 \\
0.00132\end{array}$ & $\begin{array}{l}0.397 \\
0.070\end{array}$ & $\begin{array}{l}-1.58 \\
-2.278\end{array}$ & C & $l s$ \\
\hline \multirow{5}{*}{51} & & ${ }^{2} \mathrm{D}^{\circ}-{ }^{2} \mathrm{D}$ & & & & & & & 0.0010 & & 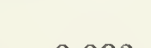 & & \\
\hline & & (26) & T500.0 & 211000 & 234434 & 10 & & 0.430 & 0.124 & 17.9 & 0.093 & L & $3, c a$ \\
\hline & & & 4395.95 & 211713 & 234454 & 6 & 6 & \begin{tabular}{|l|l|} 
& 0.398
\end{tabular} & 0.1 & 10.0 & -0.161 & C & l. \\
\hline & & & $\begin{array}{l}4369.28 \\
4406.02\end{array}$ & $\begin{array}{l}211522 \\
\end{array}$ & 234402 & 4 & 4 & 0.391 & 0.1 & 6.4 & -0.349 & C & ls \\
\hline & & & 4359.38 & $\begin{array}{l}211113 \\
211522\end{array}$ & $\begin{array}{l}234402 \\
234454\end{array}$ & $\begin{array}{l}0 \\
4\end{array}$ & $\begin{array}{l}4 \\
6\end{array}$ & 0.0292 & $\mid \begin{array}{l}0.0082 \\
0.0125\end{array}$ & $\begin{array}{l}0.72 \\
0.72\end{array}$ & $\begin{array}{l}-1.307 \\
-1.302\end{array}$ & ${ }_{\mathrm{C}}^{\mathrm{C}}$ & \\
\hline
\end{tabular}


O II. Allowed Transitions-Continued

\begin{tabular}{|c|c|c|c|c|c|c|c|c|c|c|c|c|c|}
\hline No. & $\begin{array}{l}\text { Transition } \\
\text { Array }\end{array}$ & Multiplet & $\lambda(\AA)$ & $E_{i}\left(\mathrm{~cm}^{-1}\right)$ & $E_{k}\left(\mathrm{~cm}^{-1}\right)$ & $g_{i}$ & $g_{k}$ & $A_{k i}\left(10^{8} \sec ^{-1}\right)$ & $f_{i k}$ & $S($ at.u.) & $\log g f$ & $\begin{array}{l}\text { Accu- } \\
\text { racy }\end{array}$ & Source \\
\hline \multirow[t]{4}{*}{52} & & ${ }^{4} \mathrm{~S}^{\circ}-{ }^{4} \mathrm{P}$ & 4913.0 & 212162 & 232511 & 4 & 12 & 0.67 & 0.73 & 47.3 & 0.466 & $\mathrm{C}$ & $3, c a$ \\
\hline & & & 4924.60 & 212162 & 232463 & 4 & 6 & 0.67 & 0.365 & 23.7 & 0.165 & $\mathrm{C}$ & \\
\hline & & & 4906.88 & 212162 & 232536 & 4 & 4 & 0.68 & 0.245 & 15.8 & -0.011 & $\mathrm{C}$ & $l s$ \\
\hline & & & 4890.93 & 212162 & 232603 & 4 & 2 & 0.68 & 0.122 & 7.9 & -0.310 & C & ls \\
\hline \multirow[t]{3}{*}{53} & & 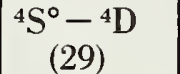 & & & & & & & & & & & \\
\hline & & & 4856.49 & 212162 & 232748 & 4 & 6 & 0.094 & 0.050 & 3.19 & -0.70 & C & \\
\hline & & & \begin{tabular}{|l|}
4856.76 \\
4864.95
\end{tabular} & $\begin{array}{l}212162 \\
212162\end{array}$ & $\begin{array}{l}232746 \\
232712\end{array}$ & $\begin{array}{l}4 \\
4\end{array}$ & $\begin{array}{l}4 \\
2\end{array}$ & $\begin{array}{l}0.176 \\
0.235\end{array}$ & $\mid \begin{array}{l}0.062 \\
0.0417\end{array}$ & $\begin{array}{l}3.97 \\
2.67\end{array}$ & $\begin{array}{l}-0.61 \\
-0.78\end{array}$ & $\stackrel{\mathrm{C}}{\mathrm{C}}$ & $\begin{array}{l}5 \\
5\end{array}$ \\
\hline \multirow[t]{2}{*}{54} & & ${ }^{4} S^{\circ}-$ & & & & & & & & & & & \\
\hline & & & 4845.0 & 212162 & 232796 & 4 & 6 & 0.0094 & 0.0050 & 0.316 & -1.70 & $\mathrm{C}$ & 5 \\
\hline \multirow[t]{5}{*}{55} & & ${ }^{2} \mathrm{P}^{\circ}-{ }^{2} \mathrm{P}$ & 5191.1 & 214210 & 233468 & 6 & 6 & 0.53 & 0.213 & 21.8 & 0.106 & C & $3,4, c a$ \\
\hline & & & 5206.73 & 214229 & 233430 & 4 & 4 & 0.391 & 0.160 & 10.9 & -0.197 & $\mathrm{C}$ & $4 n, 5 n$ \\
\hline & & & 5160.02 & 214170 & 233544 & 2 & 2 & 0.350 & 0.140 & 4.75 & -0.55 & $\mathrm{C}$ & $4 n, 5 n$ \\
\hline & & & 5176.00 & 214229 & 233544 & 4 & 2 & 0.171 & 0.0343 & 2.34 & -0.86 & $\mathrm{C}$ & $4 n, 5 n$ \\
\hline & & & 5190.56 & 214170 & 233430 & 2 & 4 & 0.137 & 0.111 & 3.78 & -0.66 & $\mathrm{C}$ & $4 n, 5 n$ \\
\hline \multirow[t]{4}{*}{56} & & ${ }^{2} \mathrm{P}^{\circ}-{ }^{2} \mathrm{D}$ & 4943.2 & 214210 & 234434 & 6 & 10 & 1.07 & 0.65 & 64 & 0.59 & $\mathrm{C}$ & $3,4, c$ \\
\hline & & & 4943.06 & 214229 & 234454 & 4 & 6 & 1.06 & 0.58 & 37.9 & 0.367 & $\mathrm{C}$ & $5 n$ \\
\hline & & & 4941.12 & 214170 & & 2 & 4 & 0.8 & & 19.7 & 0.083 & C & $5 r$ \\
\hline & & & 4955.18 & 214229 & 234402 & & 4 & 0.2 & 0.094 & b. & -0.424 & $\mathrm{C}$ & \\
\hline \multirow[t]{4}{*}{57} & $2 p^{2} 3 p^{\prime}-$ & ${ }^{2} \mathrm{~F}^{\circ}-{ }^{2} \mathrm{~F}$ & 4446.1 & 228737 & 251222 & 14 & 14 & 0.59 & 0.176 & 36.0 & 0.391 & $\mathrm{C}$ & $3,4, c a$ \\
\hline & & & 4448.21 & 228747 & 251221 & 8 & 8 & 0.57 & 0.169 & 19.8 & 0.131 & $\mathrm{C}$ & ls \\
\hline & & & $\begin{array}{r}4443.05 \\
{[44477]}\end{array}$ & $\begin{array}{l}228723 \\
228747\end{array}$ & $\begin{array}{l}251224 \\
251224\end{array}$ & $\begin{array}{l}6 \\
8\end{array}$ & $\begin{array}{l}6 \\
6\end{array}$ & $\begin{array}{l}0.57 \\
0.0282\end{array}$ & $\begin{array}{l}0.167 \\
0.0063\end{array}$ & 14.7 & $\begin{array}{r}0.002 \\
-1000\end{array}$ & $\mathrm{C}$ & ls \\
\hline & & & [4443.7] & 228723 & $\begin{array}{l}201224 \\
251221\end{array}$ & $\begin{array}{l}8 \\
6\end{array}$ & 8 & 0.0212 & $\begin{array}{l}0.0003 \\
0.0084\end{array}$ & 0.74 & $\begin{array}{l}-1.299 \\
-1.299\end{array}$ & C & ls \\
\hline \multirow[t]{3}{*}{58} & & ${ }^{2} \mathrm{~F}^{\circ}-{ }^{3} \mathrm{G}$ & 4187.9 & 228737 & 252608 & 14 & 18 & 2.51 & 0.85 & 164 & 1.075 & $\mathrm{C}$ & $3,4, c a$ \\
\hline & & & 4189.79 & 228747 & 252608 & 8 & 10 & 2.51 & 0.83 & 91 & 0.82 & $\mathrm{C}$ & \\
\hline & & & $\begin{array}{r}4185.46 \\
{[4189.6]}\end{array}$ & $\begin{array}{l}228723 \\
228747\end{array}$ & $\begin{array}{l}252609 \\
252609\end{array}$ & $\begin{array}{l}6 \\
0\end{array}$ & $\begin{array}{l}8 \\
0 \\
0\end{array}$ & $\begin{array}{l}2.43 \\
0.09\end{array}$ & & $\stackrel{70}{260}$ & $\begin{array}{r}0.71 \\
\end{array}$ & $\mathrm{C}$ & ls \\
\hline \multirow{4}{*}{59} & & $25^{\circ}-2 \mathrm{D}$ & & & & & & & & & & & \\
\hline & & $\begin{array}{c}{ }^{2} F^{0}-{ }^{2} \mathrm{D} \\
(37)\end{array}$ & 4112.3 & 228737 & 253048 & 14 & 10 & 0.132 & 0.0239 & 4.53 & -0.476 & $\mathrm{C}$ & 3, \\
\hline & & & 4113.82 & 228747 & 253048 & 8 & 6 & 0.126 & 0.0239 & 2.59 & -0.72 & C & ls \\
\hline & & & $\begin{array}{r}4110.20 \\
{[4109.8]}\end{array}$ & $\begin{array}{l}228723 \\
228723\end{array}$ & $\begin{array}{l}253046 \\
253048\end{array}$ & $\begin{array}{l}6 \\
6\end{array}$ & $\begin{array}{l}4 \\
6\end{array}$ & $\begin{array}{l}0.132 \\
0.0063\end{array}$ & $\begin{array}{l}0.0223 \\
0.00159\end{array}$ & $\begin{array}{l}1.81 \\
0.129\end{array}$ & $\begin{array}{l}-0.87 \\
-2.021\end{array}$ & $\begin{array}{l}\mathrm{C} \\
\mathrm{C}\end{array}$ & $\begin{array}{l}l s \\
l s\end{array}$ \\
\hline \multirow[t]{4}{*}{60} & & ${ }^{2} \mathrm{D}^{\circ}-{ }^{2} \mathrm{~F}$ & 4700.8 & 229955 & 251222 & 10 & 14 & 0.88 & 0.410 & 63 & 0.61 & $\mathrm{C}$ & $3, c a$ \\
\hline & & & 4699.21 & 229947 & 251221 & 6 & 8 & 0.8 & 0.35 & 36 & 0.369 & $\mathrm{C}$ & ls \\
\hline & & & & 229968 & 251224 & 4 & 6 & 0.8 & & 25. & 0.215 & $\mathrm{C}$ & 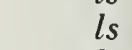 \\
\hline & & & 4698 & 229947 & 251224 & 6 & 6 & 0.059 & 0.0 & 18 & -0.93 & C & $l s$ \\
\hline \multirow[t]{3}{*}{61} & & ${ }^{2} \mathrm{P}^{\circ}-{ }^{2} \mathrm{D}$ & 4868.1 & 232511 & 253048 & 6 & 10 & 0.437 & 0.259 & 24.9 & 0.191 & $\mathrm{C}$ & $3, c a$ \\
\hline & & & 4871.58 & 232527 & 253048 & 4 & 6 & 0.435 & 0.232 & 14.9 & -0.032 & $\mathrm{C}$ & ls \\
\hline & & & $\begin{array}{l}4861.03 \\
{[4872.2]}\end{array}$ & $\begin{array}{l}232480 \\
232527\end{array}$ & $\begin{array}{l}253046 \\
253046\end{array}$ & $\begin{array}{l}2 \\
4\end{array}$ & $\begin{array}{l}4 \\
4\end{array}$ & $\mid \begin{array}{l}0.366 \\
0.073\end{array}$ & $\begin{array}{l}0.259 \\
0.0259\end{array}$ & $\begin{array}{l}8.3 \\
1.66\end{array}$ & $\begin{array}{l}-0.285 \\
-0.99\end{array}$ & $\begin{array}{l}\mathrm{C} \\
\mathrm{C}\end{array}$ & $J_{s}$ \\
\hline \multirow[t]{4}{*}{62} & & ${ }^{2} \mathrm{P}^{\circ}-{ }^{2} \mathrm{P}$ & 4698.0 & 232511 & 253791 & 6 & 6 & 1.05 & 0.347 & 33.2 & 0.319 & C & $3, c a$ \\
\hline & & & 4701.23 & 232527 & 253792 & 4 & 4 & 0.87 & 0.289 & 17.9 & 0.063 & C & $l s$ \\
\hline & & & & 232480 & & 2 & 2 & & & 7. & & C & $l s$ \\
\hline & & & 4690.97 & 232480 & $\begin{array}{l}253790 \\
253792\end{array}$ & $\begin{array}{l}4 \\
2\end{array}$ & 4 & 0 & $\begin{array}{l}0.058 \\
0.116\end{array}$ & $\begin{array}{l}3.00 \\
3.58\end{array}$ & $\begin{array}{l}-0.64 \\
-0.64\end{array}$ & $\begin{array}{l}\mathrm{C} \\
\mathrm{C}\end{array}$ & $f_{s}$ \\
\hline
\end{tabular}


O II. Allowed Transitions-Continued

\begin{tabular}{|c|c|c|c|c|c|c|c|c|c|c|c|c|c|}
\hline No. & $\begin{array}{c}\text { Transition } \\
\text { Array }\end{array}$ & Multiplet & $\lambda(\AA)$ & $E_{i}\left(\mathrm{~cm}^{-1}\right)$ & $E_{k}\left(\mathrm{~cm}^{-1}\right)$ & $g_{i}$ & $g_{k}$ & $A_{k i}\left(10^{k} \sec ^{-1}\right)$ & $f_{i k}$ & S(at.u.) & $\log g f$ & $\begin{array}{c}\text { Accu- } \\
\text { racy }\end{array}$ & Source \\
\hline \multirow[t]{2}{*}{63} & & $\begin{array}{c}{ }^{2} \mathrm{P}^{\circ}-{ }^{2} \mathrm{~S} \\
(61)\end{array}$ & 4325.7 & 232511 & 255622 & 6 & 2 & 1.81 & 0.170 & 14.5 & 0.008 & $\mathrm{C}$ & $3, c a$ \\
\hline & & & $\begin{array}{l}4328.62 \\
4319.93\end{array}$ & $\begin{array}{l}232527 \\
232480\end{array}$ & $\begin{array}{l}255622 \\
255622\end{array}$ & $\begin{array}{l}4 \\
2\end{array}$ & $\begin{array}{l}2 \\
2\end{array}$ & $\begin{array}{l}1.21 \\
0.61\end{array}$ & $\begin{array}{l}0.170 \\
0.170\end{array}$ & $\begin{array}{l}9.7 \\
4.83\end{array}$ & $\begin{array}{l}-0.168 \\
-0.469\end{array}$ & $\stackrel{\mathrm{C}}{\mathrm{C}}$ & ls \\
\hline \multirow[t]{9}{*}{64} & $\begin{array}{c}2 s 2 p^{3} 3 p^{\prime \prime \prime}- \\
2 s 2 p^{3}\left(5 S^{\circ}\right)\end{array}$ & $\begin{array}{c}{ }^{6} \mathrm{P}-{ }^{6} \mathrm{D}^{\circ} \\
(106)\end{array}$ & 4144.4 & [267775] & [291897] & 18 & 30 & 0.211 & 0.090 & 22.2 & 0.211 & C & $c a$ \\
\hline & & & 4146.09 & [267783] & [291896] & 8 & 10 & 0.210 & 0.068 & 7.4 & -0.266 & C & $l s$ \\
\hline & & & 4143.77 & [267771] & [291897] & 6 & 8 & 0.135 & 0.0464 & 3.80 & -0.55 & C & ls \\
\hline & & & 4142.24 & [267763] & [291898] & 4 & 6 & 0.066 & 0.0253 & 1.38 & -0.99 & C & $l s$ \\
\hline & & & 4145.90 & [267783] & [291897] & 8 & 8 & 0.075 & 0.0194 & 2.12 & -0.81 & C & $l s$ \\
\hline & & & & {$[267771]$} & [291898] & 6 & 6 & 0.129 & 0.0331 & 2.71 & -0.70 & C & ls \\
\hline & & & [4145.6] & {$[267783]$} & {$[291899]$} & $\begin{array}{l}4 \\
8\end{array}$ & $\begin{array}{l}4 \\
6\end{array}$ & $\begin{array}{l}0.148 \\
0.0167\end{array}$ & $\begin{array}{l}0.0380 \\
0.00323\end{array}$ & $\begin{array}{l}2.07 \\
0.353\end{array}$ & $\begin{array}{l}-0.82 \\
-1.59\end{array}$ & $\stackrel{\mathrm{C}}{\mathrm{C}}$ & $\begin{array}{l}\text { ls } \\
l s\end{array}$ \\
\hline & & & {$[4143.4]$} & [267771] & [291899] & 6 & 4 & 0.063 & 0.0108 & 0.89 & -1.186 & $\mathrm{C}$ & $l s$ \\
\hline & & & 4142.08 & [267763] & [291900] & 4 & 2 & 0.211 & 0.0271 & 1.48 & -0.96 & $\mathrm{C}$ & $l s$ \\
\hline \multirow[t]{3}{*}{65} & $2 p^{2} 3 p-$ & ${ }^{2} \mathrm{~S}^{\circ}-{ }^{2} \mathrm{P}$ & 2738.0 & 203942 & 240454 & 2 & 6 & 0.36 & 0.12 & 2.2 & -0.61 & D & $3, c a$ \\
\hline & & & 2733.34 & 203942 & 240516 & 2 & 4 & 0.37 & 0.082 & 1.5 & -0.79 & D & $l s$ \\
\hline & & & 2747.46 & 203942 & 240329 & 2 & 2 & 0.36 & 0.041 & 0.74 & -1.09 & D & ls \\
\hline \multirow[t]{8}{*}{66} & & ${ }^{4} \mathrm{D}^{\circ}-{ }^{4} \mathrm{P}$ & 3133.9 & 206895 & 238795 & 20 & 12 & 1.53 & 0.135 & 27.9 & 0.432 & C & $3, c a$ \\
\hline & & & 3134.82 & 207003 & 238893 & 8 & 6 & 1.23 & 0.136 & 11.2 & 0.036 & C & $l s$ \\
\hline & & & 3138.44 & 206878 & 238732 & 6 & 4 & 0.96 & 0.095 & 5.9 & -0.246 & $\mathrm{C}$ & ls \\
\hline & & & 3139.77 & 206786 & 238626 & 4 & 2 & 0.76 & 0.056 & 2.33 & -0.65 & $\mathrm{C}$ & $l s$ \\
\hline & & & 3122.62 & 206878 & 238893 & 6 & 6 & 0.278 & 0.0407 & 2.51 & -0.61 & C & $l s$ \\
\hline & & & 3129.44 & 206786 & 238732 & 4 & 4 & 0.493 & 0.072 & 2.98 & -0.54 & C & ls \\
\hline & & & 3134.32 & 206731 & 238626 & 2 & 2 & 0.77 & 0.113 & 2.33 & -0.65 & C & ls \\
\hline & & & $\begin{array}{l}3113.71 \\
3124.02\end{array}$ & $\begin{array}{l}206 / 86 \\
206731\end{array}$ & $\begin{array}{l}238893 \\
238732\end{array}$ & $\begin{array}{l}4 \\
2\end{array}$ & $\begin{array}{l}6 \\
4\end{array}$ & $\begin{array}{l}0.0312 \\
0.077\end{array}$ & 0.0068 & $\begin{array}{l}0.279 \\
0.465\end{array}$ & $\begin{array}{l}-1.5 \\
-1.345\end{array}$ & $\stackrel{\mathrm{C}}{\mathrm{C}}$ & $\begin{array}{l}l s \\
l s\end{array}$ \\
\hline \multirow[t]{7}{*}{67} & & ${ }^{4} \mathrm{P}^{\circ}-{ }^{4} \mathrm{P}$ & 3292.4 & 208431 & 238795 & 12 & 12 & 0.85 & 0.138 & 18.0 & 0.220 & C & $3, c a$ \\
\hline & & & 3287.59 & 208484 & 238893 & 6 & 6 & 0.60 & 0.097 & 6.3 & -0.235 & C & $l s$ \\
\hline & & & 3295.13 & 208392 & 238732 & 4 & 4 & 0.113 & 0.0184 & 0.80 & -1.132 & $\mathrm{C}$ & ls \\
\hline & & & 3301.56 & 208346 & 238626 & 2 & 2 & 0.141 & 0.0230 & 0.50 & -1.337 & C & ls \\
\hline & & & 3305.15 & 208484 & 238732 & 6 & 4 & 0.379 & 0.0414 & 2.70 & -0.61 & C & ls \\
\hline & & & 3306.60 & 208392 & 238626 & 4 & 2 & 0.70 & 0.057 & 2.50 & -0.64 & C & $l s$ \\
\hline & & & $\begin{array}{l}3277.69 \\
3290.13\end{array}$ & $\begin{array}{l}208392 \\
208346\end{array}$ & $\begin{array}{l}238893 \\
238732\end{array}$ & $\begin{array}{l}4 \\
2\end{array}$ & $\begin{array}{l}6 \\
4\end{array}$ & $\begin{array}{l}0.259 \\
0.356\end{array}$ & $\begin{array}{l}0.063 \\
0.115\end{array}$ & $\begin{array}{l}2.70 \\
2.50\end{array}$ & $\begin{array}{l}-0.60 \\
-0.64\end{array}$ & $\begin{array}{l}\mathrm{C} \\
\mathrm{C}\end{array}$ & $\begin{array}{l}\text { ls } \\
\text { ls }\end{array}$ \\
\hline \multirow[t]{4}{*}{68} & & $2 D^{\circ}-2 P$ & 34691 & 211636 & 240454 & 10 & 6 & 125 & 135 & 154 & 0130 & $C$ & $3 \mathrm{co}$ \\
\hline & & $(27)$ & 3409.1 & & 240454 & 10 & 0 & 1.25 & 0.135 & 15.4 & 0.130 & C & $3, c a$ \\
\hline & & & 3470.81 & 211713 & 240516 & 6 & 4 & 1.12 & 0.135 & 9.2 & -0.092 & C & $l s$ \\
\hline & & & $\begin{array}{l}3470.42 \\
3447.98\end{array}$ & $\begin{array}{l}211522 \\
211522\end{array}$ & $\begin{array}{l}240329 \\
240516\end{array}$ & $\begin{array}{l}4 \\
4\end{array}$ & $\begin{array}{l}2 \\
4\end{array}$ & $\begin{array}{l}1.24 \\
0.127\end{array}$ & $\begin{array}{l}0.112 \\
0.0227\end{array}$ & $\begin{array}{l}5.1 \\
1.03\end{array}$ & $\begin{array}{l}-0.348 \\
-1.04 .2\end{array}$ & $\stackrel{\mathrm{C}}{\mathrm{C}}$ & $\begin{array}{l}l s \\
l s\end{array}$ \\
\hline \multirow{5}{*}{69} & & ${ }^{4} \mathrm{~S}^{\circ}-{ }^{4} \mathrm{P}$ & 3753.7 & 212162 & 238795 & 4. & 12 & 0265 & 0168 & 83 & -0173 & $C$ & $34 \mathrm{ca}$ \\
\hline & & $(31)$ & 3800.6 & & & $\Psi$ & 12 & 0.200 & 0.100 & 0.5 & 0. & U & $3,4, c a$ \\
\hline & & & 3739.92 & 212162 & 238893 & 4 & 6 & 0.267 & 0.084 & 4.14 & -0.473 & C & $4 n, l s$ \\
\hline & & & 3762.63 & 212162 & 238732 & 4 & 4 & 0.269 & 0.057 & 2.83 & -0.64 & $\mathrm{C}$ & $4 n, l s$ \\
\hline & & & 3777.60 & 212162 & 238626 & 4 & 2 & 0.252 & 0.0269 & 1.34 & -0.97 & C & $4 n, l s$ \\
\hline \multirow[t]{5}{*}{70} & & $\begin{array}{c}{ }^{2} \mathrm{P}^{\circ}-{ }^{2} \mathrm{P} \\
(34)\end{array}$ & 3809.3 & 214210 & 240454 & 6 & 6 & 0.65 & 0.142 & 10.7 & -0.069 & C & $3, c a$ \\
\hline & & & 3803.14 & 214230 & 240516 & 4 & 4 & 0.55 & 0.119 & 5.9 & -0.324 & C & ls \\
\hline & & & 3821.68 & 214170 & 240329 & 2 & 2 & 0.432 & 0.095 & 2.38 & -0.72 & C & ls \\
\hline & & & 3830.45 & 214230 & 240329 & 4 & 2 & 0.215 & 0.0236 & 1.19 & -1.025 & C & ls \\
\hline & & & 3794.48 & 214170 & 240516 & 2 & 4 & 0.110 & 0.0476 & 1.19 & -1.021 & $\mathrm{C}$ & ls \\
\hline
\end{tabular}


O II. Allowed Transitions-Continued

\begin{tabular}{|c|c|c|c|c|c|c|c|c|c|c|c|c|c|}
\hline No. & $\begin{array}{c}\text { Transition } \\
\text { Array }\end{array}$ & Multiplet & $\lambda(\AA)$ & $E_{i}\left(\mathrm{~cm}^{-1}\right)$ & $E_{k}\left(\mathrm{~cm}^{-1}\right)$ & $g_{i}$ & $g_{k}$ & $A_{k i}\left(10^{8} \sec ^{-1}\right)$ & $f_{i k}$ & $S$ (at.u.) & $\log g f$ & $\begin{array}{c}\text { Accu- } \\
\text { racy }\end{array}$ & Source \\
\hline \multirow[t]{3}{*}{71} & \multirow{10}{*}{$\begin{array}{l}2 p^{2} 3 p^{\prime}- \\
\quad 2 p^{2}\left({ }^{1} \mathrm{D}\right) 4 s^{\prime}\end{array}$} & \multirow{3}{*}{$\begin{array}{c}{ }^{2} \mathrm{~F}^{\circ}-{ }^{2} \mathrm{D} \\
(39)\end{array}$} & 3272.4 & 228737 & 259287 & 14 & 10 & 1.20 & 0.137 & 20.7 & 0.284 & $\mathrm{C}$ & $3, c a$ \\
\hline & & & 3273.52 & 228747 & 259286 & 8 & 6 & 1.14 & 0.137 & 11.8 & 0.039 & C & 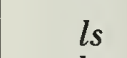 \\
\hline & & & \begin{tabular}{|c}
3270.98 \\
{$[3270.9]$}
\end{tabular} & $\begin{array}{l}228723 \\
228723\end{array}$ & $\begin{array}{l}259287 \\
259287\end{array}$ & $\begin{array}{l}6 \\
6\end{array}$ & $\begin{array}{l}4 \\
6\end{array}$ & $\begin{array}{l}1.20 \\
0.057\end{array}$ & $\begin{array}{l}0.128 \\
0.0091\end{array}$ & $\begin{array}{l}8.3 \\
0.59\end{array}$ & $\begin{array}{l}-0.114 \\
-1.261\end{array}$ & $\begin{array}{l}\mathrm{C} \\
\mathrm{C}\end{array}$ & $\begin{array}{l}l s \\
l s\end{array}$ \\
\hline \multirow[t]{4}{*}{72} & & \multirow{4}{*}{$\begin{array}{c}{ }^{2} \mathrm{D}^{\circ}-{ }^{2} \mathrm{D} \\
(44)\end{array}$} & 3408.4 & 229956 & 259287 & 10 & 10 & 0.80 & 0.140 & 15.7 & 0.146 & C & $3, c a$ \\
\hline & & & 3407.38 & 229947 & 259286 & 6 & 6 & 0.75 & 0.131 & 8.8 & -0.106 & $\mathrm{C}$ & ls \\
\hline & & & 3409.84 & 229968 & 259287 & 4 & 4 & 0.72 & 0.126 & 5.7 & -0.298 & $\mathrm{C}$ & ls \\
\hline & & & \begin{tabular}{|l|}
34.07 .38 \\
3409.84
\end{tabular} & $\begin{array}{l}229947 \\
229968\end{array}$ & $\begin{array}{l}259287 \\
259286\end{array}$ & $\begin{array}{l}6 \\
4\end{array}$ & $\begin{array}{l}4 \\
6\end{array}$ & $\begin{array}{l}0.080 \\
0.054\end{array}$ & $\begin{array}{l}0.0093 \\
0.0140\end{array}$ & 0.63 & $\begin{array}{l}-1.252 \\
-1.252\end{array}$ & $\mathrm{C}$ & ls \\
\hline \multirow[t]{3}{*}{73} & & \multirow{3}{*}{$\mid \begin{array}{c}{ }^{2} \mathrm{P}^{\circ}-{ }^{2} \mathrm{D} \\
(62)\end{array}$} & 3733.9 & 232513 & 259287 & 6 & 10 & 0.416 & 0.145 & 10.7 & -0.060 & C & $3, c a$ \\
\hline & & & 3735.94 & 232527 & 259286 & 4 & 6 & 0.416 & 0.130 & 6.4 & -0.282 & $\mathrm{C}$ & ls \\
\hline & & & $\begin{array}{c}3729.34 \\
{[3735.9]}\end{array}$ & $\begin{array}{l}232480 \\
232527\end{array}$ & $\begin{array}{l}259287 \\
259287\end{array}$ & $\begin{array}{l}2 \\
4\end{array}$ & $\begin{array}{l}4 \\
4\end{array}$ & $\begin{array}{l}0.349 \\
0.069\end{array}$ & $\begin{array}{l}0.145 \\
0.0145\end{array}$ & $\begin{array}{l}3.57 \\
0.71\end{array}$ & $\begin{array}{l}-0.54 \\
-1.237\end{array}$ & $\begin{array}{l}\mathrm{C} \\
\mathrm{C}\end{array}$ & $\begin{array}{l}l s \\
l s\end{array}$ \\
\hline \multirow[t]{9}{*}{74} & \multirow{13}{*}{$\begin{array}{l}2 p^{2} 3 d- \\
\quad 2 p^{2}\left({ }^{3} \mathrm{P}\right) 4 p\end{array}$} & \multirow{9}{*}{$\begin{array}{c}{ }^{4} \mathrm{~F}-{ }^{4} \mathrm{D}^{\circ} \\
(45)\end{array}$} & 6897.5 & 231429 & 245923 & 28 & 20 & 0.333 & 0.170 & 108 & 0.68 & C & $3, c a$ \\
\hline & & & 6895.29 & 231530 & 246029 & 10 & 8 & 0.298 & 0.170 & 38.6 & 0.231 & C & $l s$ \\
\hline & & & 6906.54 & 231428 & 245903 & 8 & 6 & 0.272 & 0.146 & 26.5 & 0.067 & $\mathrm{C}$ & $l s$ \\
\hline & & & 6910.75 & 231350 & 245816 & 6 & 4 & 0.267 & 0.127 & 17.4 & -0.117 & $\mathrm{C}$ & $l s$ \\
\hline & & & 6908.11 & 231296 & 245768 & 4 & 2 & 0.332 & 0.119 & 10.8 & -0.323 & $\mathrm{C}$ & ls \\
\hline & & & $\begin{array}{l}6846.97 \\
686974\end{array}$ & $\begin{array}{l}231428 \\
231350\end{array}$ & $\begin{array}{l}246029 \\
245903\end{array}$ & $\begin{array}{l}8 \\
6\end{array}$ & 8 & $\begin{array}{l}0.0347 \\
0.059\end{array}$ & 0.0244 & 4.40 & $\begin{array}{l}-0.71 \\
-066\end{array}$ & C & ls \\
\hline & & & 6885.07 & 231296 & 245816 & 4 & 4 & 0.067 & 0.0476 & $\begin{array}{l}4.0 \\
4.32\end{array}$ & -0.72 & C & ls \\
\hline & & & [6810.6] & 231350 & 246029 & 6 & 8 & 0.00180 & 0.00167 & 0.224 & -2.000 & C & ls \\
\hline & & & [6844.1] & 231296 & 245903 & 4 & 6 & 0.00325 & 0.00343 & 0.309 & -1.86 & C & Is \\
\hline \multirow[t]{4}{*}{75} & & \multirow{4}{*}{$\begin{array}{c}{ }^{2} \mathrm{P}-{ }^{2} \mathrm{P}^{\circ} \\
(85)\end{array}$} & 6657.3 & 233468 & 248485 & 6 & 6 & 0.105 & 0.070 & 9.2 & -0.377 & $\mathrm{C}$ & $3, c a$ \\
\hline & & & $\begin{array}{r}6627.62 \\
677811\end{array}$ & 233430 & 248514 & 4 & 4 & 0.089 & 0.059 & 5.1 & -0.63 & $\mathrm{C}$ & ls \\
\hline & & & $\frac{[0618.1]}{6678.19}$ & $\begin{array}{l}233544 \\
233544\end{array}$ & $\begin{array}{l}248425 \\
248514\end{array}$ & $\begin{array}{l}2 \\
2\end{array}$ & $\begin{array}{l}2 \\
4\end{array}$ & \begin{tabular}{|l|}
0.068 \\
0.0173
\end{tabular} & $\begin{array}{l}0.0461 \\
0.0232\end{array}$ & $\begin{array}{l}2.04 \\
1.02\end{array}$ & $\begin{array}{l}-1.035 \\
-1.334\end{array}$ & $\begin{array}{l}\mathrm{C} \\
\mathrm{C}\end{array}$ & $\begin{array}{ll}l s \\
l s\end{array}$ \\
\hline & & & 6666.94 & 233430 & 248425 & 4 & 2 & 0.0349 & 0.0116 & 1.02 & -1.333 & $\mathrm{C}$ & ls \\
\hline \multirow[t]{8}{*}{76} & \multirow[t]{17}{*}{$\begin{array}{l}2 p^{2} 3 d- \\
2 p^{2}\left({ }^{3} \mathrm{P}\right) 4 f\end{array}$} & \multirow[t]{8}{*}{$\begin{array}{c}{ }^{4} \mathrm{~F}-{ }^{4} \mathrm{G}^{\circ} \\
(48)\end{array}$} & 4093.7 & 231429 & 255850 & 28 & 36 & 2.60 & 0.84 & 317 & 1.372 & C & $3, c a$ \\
\hline & & & 4089.30 & 231530 & 255978 & 10 & 12 & 2.62 & 0.79 & 106 & 0.90 & $\mathrm{C}$ & $l s$ \\
\hline & & & $\begin{array}{l}4097.26 \\
4095.63\end{array}$ & $\begin{array}{l}231428 \\
231350\end{array}$ & $\begin{array}{l}255828 \\
255759\end{array}$ & $\begin{array}{l}8 \\
6\end{array}$ & $\begin{array}{r}10 \\
8\end{array}$ & $\begin{array}{l}2.37 \\
2.23\end{array}$ & $\mid \begin{array}{l}0.75 \\
0.75\end{array}$ & $\begin{array}{l}81 \\
61\end{array}$ & $\begin{array}{l}0.78 \\
0.65\end{array}$ & $\begin{array}{l}\text { C } \\
\text { C }\end{array}$ & ls \\
\hline & & & 4087.16 & 231296 & 255756 & 4 & 6 & 2.24 & 0.84 & 45.3 & 0.53 & $\mathrm{C}$ & $l s$ \\
\hline & & & [4114.4] & 231530 & 255828 & 10 & 10 & 0.212 & 0.054 & 7.3 & -0.269 & C & ls \\
\hline & & & 4108.75 & 231428 & 255759 & 8 & 8 & 0.349 & 0.088 & 9.6 & -0.151 & C & $l s$ \\
\hline & & & $\begin{array}{r}4096.18 \\
{[4126.1]}\end{array}$ & $\begin{array}{l}231350 \\
231530\end{array}$ & $\begin{array}{l}255756 \\
255759\end{array}$ & $\begin{array}{r}6 \\
10\end{array}$ & $\begin{array}{l}6 \\
8\end{array}$ & $\begin{array}{l}0.359 \\
0.0077\end{array}$ & 0.090 & 7.3 & -0.267 & $\mathrm{C}$ & is \\
\hline & & & $\begin{array}{l}{[4.26 .1]} \\
{[4109.3]}\end{array}$ & $\begin{array}{l}231530 \\
231428\end{array}$ & $\begin{array}{l}255579 \\
255756\end{array}$ & $\begin{array}{r}10 \\
8\end{array}$ & $\begin{array}{l}8 \\
6\end{array}$ & $\begin{array}{l}0.0017 \\
0.0128\end{array}$ & $\mid \begin{array}{l}0.00158 \\
0.00244\end{array}$ & $\begin{array}{l}0.214 \\
0.264\end{array}$ & $\begin{array}{l}-1.80 \\
-1.71\end{array}$ & $\begin{array}{l}\mathrm{C} \\
\mathrm{C}\end{array}$ & Is \\
\hline \multirow[t]{9}{*}{77} & & \multirow{9}{*}{$\frac{{ }^{4} \mathrm{P}-{ }^{4} \mathrm{D}^{\circ}}{(54)}$} & 4293.8 & 232511 & 255794 & 12 & 20 & 1.98 & 0.91 & 155 & 1.040 & C & $3, c a$ \\
\hline & & & 4303.82 & 232463 & 255691 & 6 & 8 & 1.97 & 0.7 & 62 & 0.6 & $\mathrm{C}$ & $l s$ \\
\hline & & & 4294.82 & 232536 & 255813 & 4 & 6 & 1.39 & 0.57 & 32.5 & 0.362 & C & ls \\
\hline & & & [4288.8] & 232603 & 255913 & 2 & 4 & 0.83 & 0.457 & 12.9 & -0.039 & $\mathrm{C}$ & 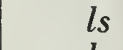 \\
\hline & & & 4281.40 & 232463 & 255813 & 6 & 6 & 0.60 & 0.164 & 13.9 & -0.006 & C & ls \\
\hline & & & 4282.82 & 232536 & 255913 & 4 & 4 & 1.06 & 0.293 & 16.5 & -0.068 & $\mathrm{C}$ & ls \\
\hline & & & 4288.83 & 232603 & 255912 & 2 & 2 & 1.66 & 0.457 & 12.9 & -0.039 & C & ls \\
\hline & & & $\begin{array}{r}{[4263.2]} \\
49767]\end{array}$ & $\begin{array}{l}232463 \\
222536\end{array}$ & $\begin{array}{c}255913 \\
25501\end{array}$ & 6 & 4 & 0.101 & 0.0184 & 1.55 & -0 & $\mathrm{C}$ & ls \\
\hline & & & 4276.71 & 232536 & 255912 & 4 & 2 & 0.334 & | 0.0458 & 2.58 & -0.74 & C & ls \\
\hline
\end{tabular}


O II. Allowed Transitions - Continued

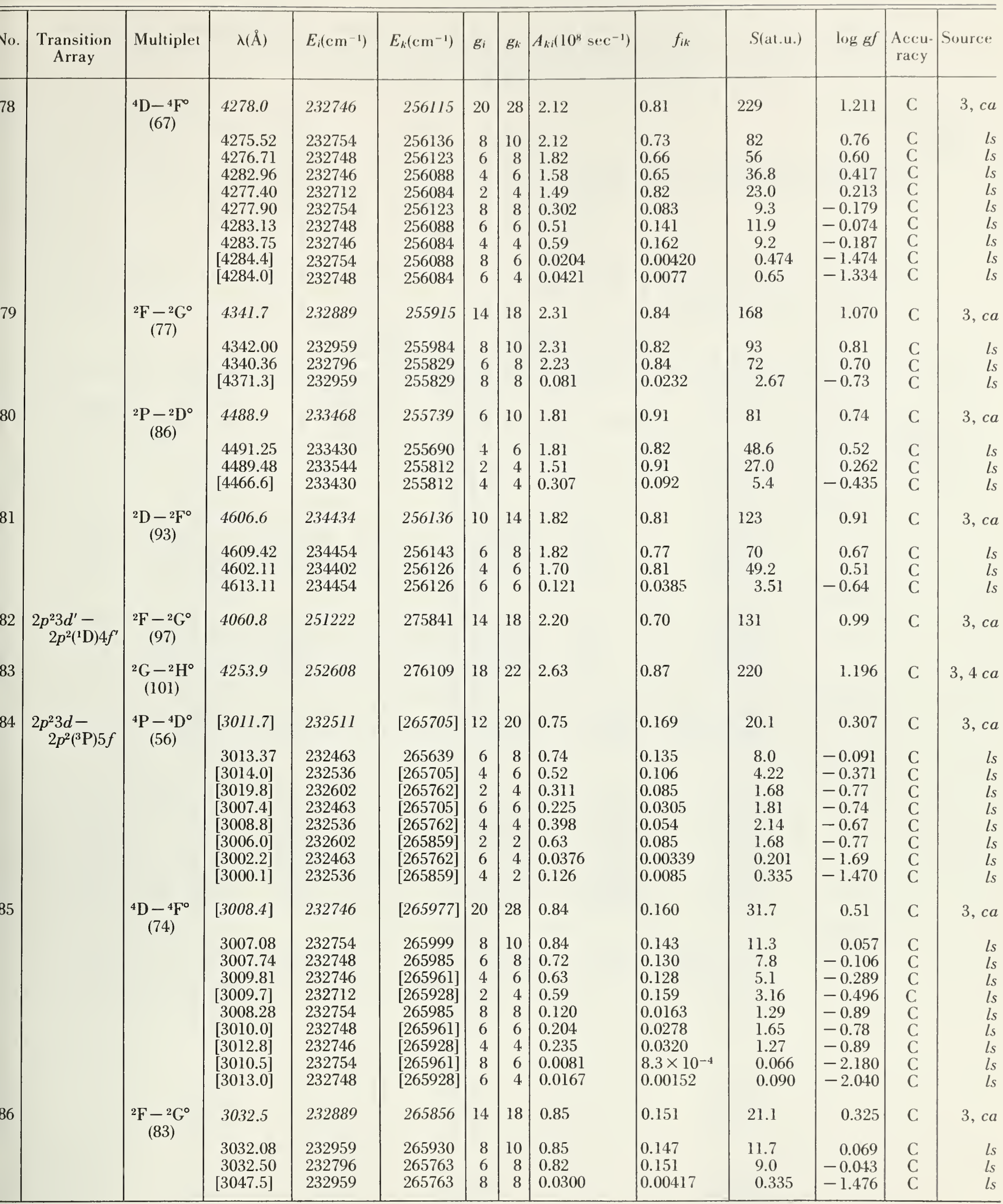




\section{Forbidden Transitions}

The adopted values are exclusively from Seaton and Osterbrock's calculations [1]. The important effects of configuration interaction are partially taken into account and a reliable estimate of the quadrupole integral is given (see also general introduction).

\section{Reference}

[1] Seaton, M. J., and Osterbrock, D. E., Astrophys. J. 125, 66-83 (1957).

O III. Forbidden Transitions

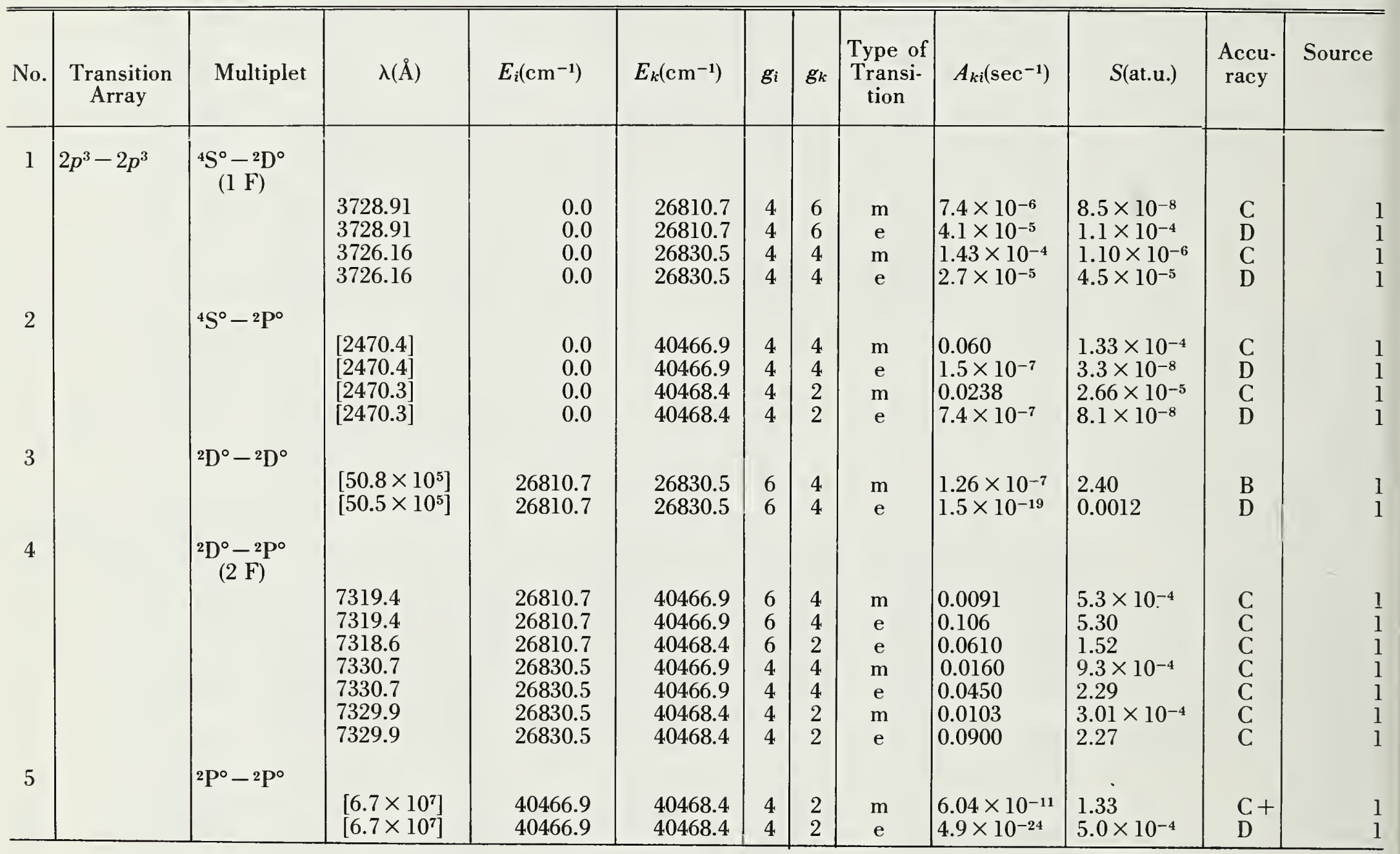




\section{O III}

Ground State

Ionization Potential
$1 s^{2} 2 s^{2} 2 p^{23} \mathrm{P}_{0}$

$54.886 \mathrm{eV}=442807 \mathrm{~cm}^{-1}$

\section{Allowed Transitions}

List of tabulated lines:

\begin{tabular}{|c|c|c|c|c|c|}
\hline Wavelength $[\AA]$ & No. & Wavelength $[\AA]$ & No. & Wavelength $[\AA ̊]$ & No. \\
\hline $\begin{array}{l}302.34 \\
303.411 \\
303.460 \\
303.515 \\
303.621\end{array}$ & $\begin{array}{l}14 \\
11 \\
11 \\
11 \\
11\end{array}$ & $\begin{array}{l}2683.65 \\
2686.14 \\
2687.53 \\
2695.49 \\
2983.78\end{array}$ & $\begin{array}{l}36 \\
24 \\
36 \\
36 \\
20\end{array}$ & $\begin{array}{l}3284.57 \\
3299.36 \\
3305.77 \\
3312.30 \\
3326.16\end{array}$ & $\begin{array}{l}27 \\
17 \\
27 \\
17 \\
40\end{array}$ \\
\hline $\begin{array}{l}303.693 \\
303.799 \\
305.596 \\
305.656 \\
305.703\end{array}$ & $\begin{array}{l}11 \\
11 \\
10 \\
10 \\
10\end{array}$ & $\begin{array}{l}2992.11 \\
2996.51 \\
2997.71 \\
3004.35 \\
3008.79\end{array}$ & $\begin{array}{l}28 \\
28 \\
28 \\
28 \\
28\end{array}$ & $\begin{array}{l}3330.40 \\
3332.49 \\
3333.00 \\
3333.40 \\
3336.78\end{array}$ & $\begin{array}{l}40 \\
40 \\
23 \\
23 \\
23\end{array}$ \\
\hline $\begin{array}{l}305.769 \\
305.836 \\
305.879 \\
320.979 \\
328.448\end{array}$ & $\begin{array}{l}10 \\
10 \\
10 \\
13 \\
12\end{array}$ & $\begin{array}{l}3017.63 \\
3023.45 \\
3024.36 \\
3024.57 \\
3035.43\end{array}$ & $\begin{array}{l}28 \\
18 \\
28 \\
18 \\
18\end{array}$ & $\begin{array}{l}3336.78 \\
3340.74 \\
3344.26 \\
3344.26 \\
3348.05\end{array}$ & $\begin{array}{l}40 \\
17 \\
23 \\
40 \\
40\end{array}$ \\
\hline $\begin{array}{l}345.309 \\
373.805 \\
374.005 \\
374.075 \\
374.165\end{array}$ & $\begin{array}{r}15 \\
7 \\
7 \\
7 \\
7\end{array}$ & $\begin{array}{l}3043.02 \\
3047.13 \\
3059.30 \\
3065.01 \\
3068.06\end{array}$ & $\begin{array}{l}18 \\
18 \\
18 \\
38 \\
38\end{array}$ & $\begin{array}{l}3350.68 \\
3350.99 \\
3355.92 \\
3362.38 \\
3376.4\end{array}$ & $\begin{array}{l}23 \\
23 \\
40 \\
23 \\
39\end{array}$ \\
\hline $\begin{array}{l}374.331 \\
374.436 \\
395.558 \\
434.975 \\
507.391\end{array}$ & $\begin{array}{l}7 \\
7 \\
8 \\
9 \\
3\end{array}$ & $\begin{array}{l}3068.48 \\
3068.68 \\
3074.15 \\
3074.68 \\
3075.19\end{array}$ & $\begin{array}{l}38 \\
38 \\
38 \\
38 \\
38\end{array}$ & $\begin{array}{l}3376.82 \\
3377.3 \\
3382.69 \\
3383.5 \\
3383.85\end{array}$ & $\begin{array}{l}39 \\
39 \\
39 \\
39 \\
39\end{array}$ \\
\hline $\begin{array}{l}507.683 \\
508.182 \\
525.795 \\
597.818 \\
599.598\end{array}$ & $\begin{array}{l}3 \\
3 \\
5 \\
6 \\
4\end{array}$ & $\begin{array}{l}3075.95 \\
3083.65 \\
3084.63 \\
3088.04 \\
3095.81\end{array}$ & $\begin{array}{l}38 \\
38 \\
38 \\
38 \\
38\end{array}$ & $\begin{array}{l}3384.95 \\
3394.26 \\
3395.5 \\
3405.74 \\
3408.13\end{array}$ & $\begin{array}{l}39 \\
39 \\
39 \\
31 \\
31\end{array}$ \\
\hline $\begin{array}{l}702.332 \\
702.822 \\
702.899 \\
703.850 \\
832.927\end{array}$ & $\begin{array}{l}2 \\
2 \\
2 \\
2 \\
1\end{array}$ & $\begin{array}{l}3115.73 \\
3121.71 \\
3132.86 \\
3198.2 \\
3200.95\end{array}$ & $\begin{array}{l}29 \\
29 \\
29 \\
42 \\
42\end{array}$ & $\begin{array}{l}3415.29 \\
3428.67 \\
3430.60 \\
3444.10 \\
3446.73\end{array}$ & $\begin{array}{l}31 \\
31 \\
31 \\
31 \\
37\end{array}$ \\
\hline $\begin{array}{c}833.742 \\
835.096 \\
835.292 \\
2454.99 \\
2558.06\end{array}$ & $\begin{array}{r}1 \\
1 \\
1 \\
21 \\
46\end{array}$ & $\begin{array}{l}3202.2 \\
3207.12 \\
3210.2 \\
3215.97 \\
3221.2\end{array}$ & $\begin{array}{l}42 \\
42 \\
42 \\
42 \\
42\end{array}$ & $\begin{array}{l}3447.22 \\
3448.05 \\
3450.94 \\
3451.33 \\
3454.90\end{array}$ & $\begin{array}{l}37 \\
37 \\
37 \\
37 \\
37\end{array}$ \\
\hline $\begin{array}{l}2597.69 \\
2605.41 \\
2609.6 \\
2665.69 \\
2674.57\end{array}$ & $\begin{array}{l}45 \\
45 \\
45 \\
24 \\
24\end{array}$ & $\begin{array}{l}3260.98 \\
3265.46 \\
3267.31 \\
3279.97 \\
3281.94\end{array}$ & $\begin{array}{l}27 \\
27 \\
27 \\
47 \\
27\end{array}$ & $\begin{array}{l}3455.12 \\
3459.52 \\
3459.98 \\
3466.15 \\
3466.90\end{array}$ & $\begin{array}{l}37 \\
37 \\
37 \\
37 \\
37\end{array}$ \\
\hline
\end{tabular}


List of tabulated lines-Continued

\begin{tabular}{|c|c|c|c|c|c|}
\hline Wavelength $[\AA]]$ & No. & Wavelength $[\AA]$ & No. & Wavelength $[\AA]$ & No. \\
\hline $\begin{array}{l}3475.2 \\
3520.7 \\
3530.7 \\
3532.8 \\
3534.3\end{array}$ & $\begin{array}{l}37 \\
26 \\
26 \\
26 \\
26\end{array}$ & $\begin{array}{l}3712.48 \\
3714.03 \\
3715.08 \\
3720.86 \\
3721.95\end{array}$ & $\begin{array}{l}22 \\
30 \\
30 \\
22 \\
22\end{array}$ & $\begin{array}{l}3816.75 \\
3961.59 \\
4072.3 \\
4073.90 \\
4081.10\end{array}$ & $\begin{array}{l}34 \\
33 \\
25 \\
25 \\
25\end{array}$ \\
\hline $\begin{array}{l}3555.3 \\
3556.92 \\
3638.70 \\
3645.20 \\
3646.84\end{array}$ & $\begin{array}{l}26 \\
26 \\
44 \\
44 \\
44\end{array}$ & $\begin{array}{l}3725.30 \\
3728.49 \\
3728.82 \\
3729.70 \\
3732.13\end{array}$ & $\begin{array}{l}30 \\
41 \\
41 \\
41 \\
30\end{array}$ & $\begin{array}{l}4088.5 \\
4103.8 \\
4118.6 \\
4440.1 \\
4447.82\end{array}$ & $\begin{array}{l}25 \\
25 \\
25 \\
43 \\
43\end{array}$ \\
\hline $\begin{array}{l}3649.20 \\
3650.70 \\
3653.00 \\
3695.37 \\
3698.70\end{array}$ & $\begin{array}{l}44 \\
44 \\
44 \\
22 \\
22\end{array}$ & $\begin{array}{l}3734.80 \\
3742.0 \\
3747.6 \\
3754.67 \\
3757.21\end{array}$ & $\begin{array}{l}22 \\
41 \\
41 \\
16 \\
16\end{array}$ & $\begin{array}{l}4461.56 \\
5268.06 \\
5500.11 \\
5592.37\end{array}$ & $\begin{array}{l}43 \\
35 \\
32 \\
19\end{array}$ \\
\hline $\begin{array}{l}3702.75 \\
3703.37 \\
3704.73 \\
3707.24 \\
3709.52 \\
\end{array}$ & $\begin{array}{l}30 \\
22 \\
22 \\
30 \\
22 \\
\end{array}$ & $\begin{array}{l}3759.87 \\
3761.2 \\
3774.00 \\
3791.26 \\
3810.96\end{array}$ & $\begin{array}{l}16 \\
41 \\
16 \\
16 \\
16\end{array}$ & & \\
\hline
\end{tabular}

Values for the strong $2 s^{2} 2 p^{2}-2 s 2 p^{3}$ transitions, which are very sensitive to configuration interaction, are taken from the calculations of Bolotin et al. [1]. These authors have used analytical one-electron wave functions and include configuration interaction in a crude manner. Thus large uncertainties must be expected. This applies also for the $2 p^{2}-2 p 3 s$ and to a lesser extent to the $2 p^{2}-2 p 3 d$ transitions, for which only Kelly's self-consistent field calculations [2] are available. In these, configuration interaction has been entirely neglected. For many other transitions the simplified self-consistent field calculations by Kelly [3], in which exchange effects are approximately taken into account, are applied. The results agree within a few percent with the values of the Coulomb approximation and the averaged values are adopted. The accuracy rating of "C" is supported by the good agreement with relative $f$-value measurements of Berg et al. [4] done with a magnetically driven shock tube.

\section{References}

[1] Bolotin, A. B., Levinson, I. B., and Levin, L. I., Soviet Phys. -JETP 2, 391-395 (1956).

[2] Kelly, P. S., Astrophys. J. 140, 1247-1268 (1964).

[3] Kelly, P. S., J. Quant. Spectrosc. Radiat. Transfer 4, 117-148 (1964).

[4] Berg, H. F., Eckerle, K. L., Burris, R. W., and Wiese, W. L., Astrophys. J. 139, 751-757 (1964).

OIII. Allowed Transitions

\begin{tabular}{|c|c|c|c|c|c|c|c|c|c|c|c|c|c|}
\hline No. & $\begin{array}{c}\text { Transition } \\
\text { Array }\end{array}$ & Multiplet & $\lambda(\AA)$ & $E_{i}\left(\mathrm{~cm}^{-1}\right)$ & $E_{k}\left(\mathrm{~cm}^{-1}\right)$ & $g_{i}$ & $g_{k}$ & $\begin{array}{c}A_{k i}\left(10^{8}\right. \\
\left.\sec ^{-1}\right)\end{array}$ & $f_{i k}$ & $S$ (at.u.) & $\log g f$ & $\begin{array}{c}\text { Accu- } \\
\text { racy }\end{array}$ & Source \\
\hline 1 & $\begin{array}{r}2 s^{2} 2 p^{2}- \\
2 s 2 p^{3}\end{array}$ & $\begin{array}{c}{ }^{3} \mathrm{P}-{ }^{3} \mathrm{D}^{\circ} \\
(1 \mathrm{uv})\end{array}$ & $\begin{array}{l}834.50 \\
835.292 \\
833.742 \\
832.927 \\
835.096 \\
833.742 \\
835.096\end{array}$ & $\begin{array}{r}208.2 \\
306.8 \\
113.4 \\
0.0 \\
306.8 \\
113.4 \\
306.8\end{array}$ & $\begin{array}{l}120041 \\
120025 \\
120053 \\
120059 \\
120053 \\
120059 \\
120059\end{array}$ & $\begin{array}{l}9 \\
\\
5 \\
3 \\
1 \\
5 \\
3 \\
5\end{array}$ & $\begin{array}{l}15 \\
\\
7 \\
5 \\
3 \\
5 \\
3 \\
3\end{array}$ & $\begin{array}{l}8.4 \\
\\
8.4 \\
6.3 \\
4.7 \\
2.1 \\
3.5 \\
0.23\end{array}$ & $\begin{array}{l}0.15 \\
\\
0.12 \\
0.11 \\
0.15 \\
0.022 \\
0.036 \\
0.0015\end{array}$ & $\begin{array}{l}3.6 \\
1.7 \\
0.90 \\
0.40 \\
0.30 \\
0.30 \\
0.020\end{array}$ & $\begin{array}{r}0.12 \\
-0.21 \\
-0.48 \\
-0.84 \\
-0.96 \\
-0.96 \\
-2.14\end{array}$ & $\begin{array}{l}\mathrm{E} \\
\mathrm{E} \\
\mathrm{E} \\
\mathrm{E} \\
\mathrm{E} \\
\mathrm{E} \\
\mathrm{E}\end{array}$ & $\begin{array}{l}1 \\
l s \\
l s \\
l s \\
l s \\
l s \\
l s\end{array}$ \\
\hline
\end{tabular}


O III. Allowed Transitions-Continued

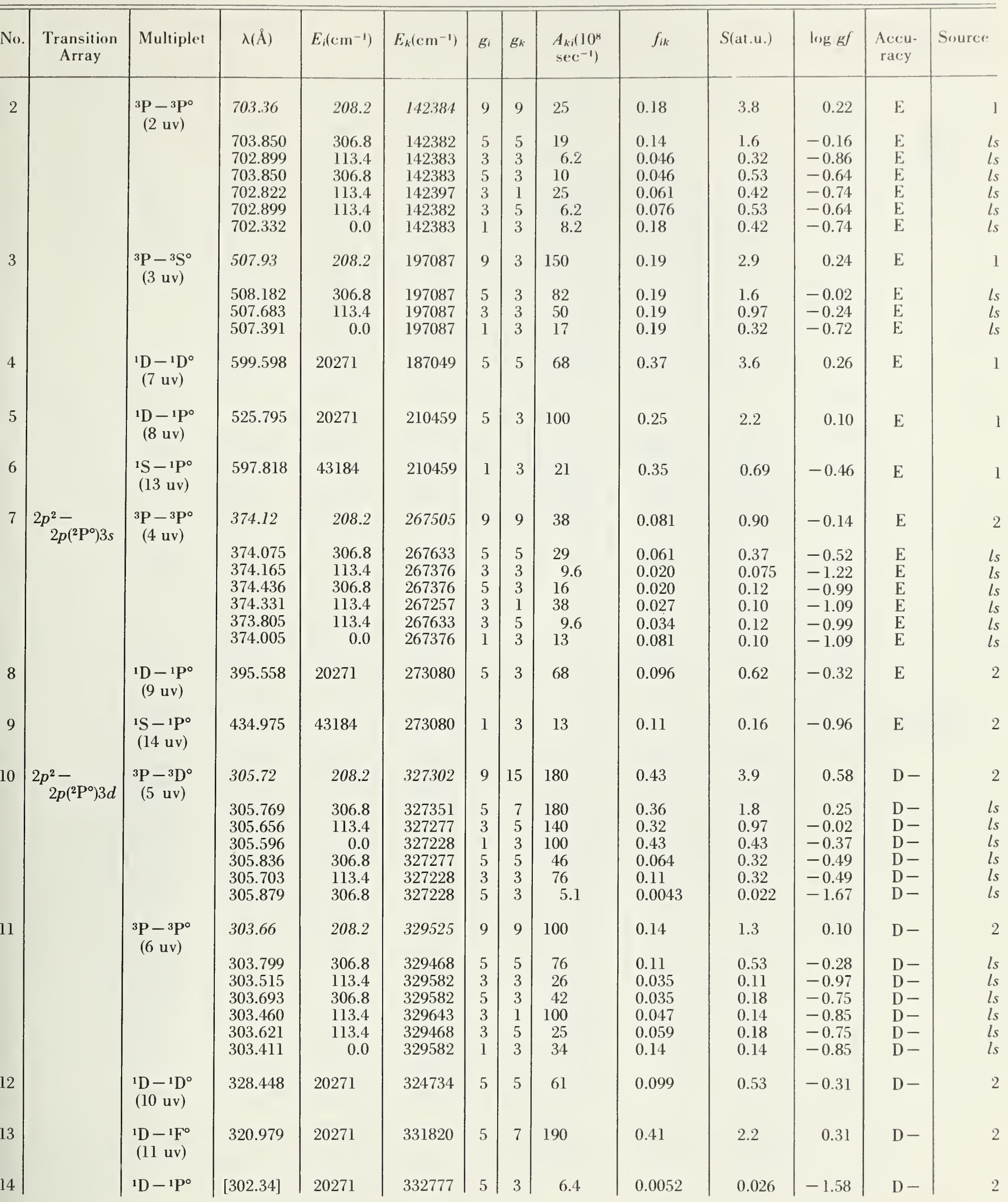


O III. Allowed Transitions-Continued

\begin{tabular}{|c|c|c|c|c|c|c|c|c|c|c|c|c|c|}
\hline No. & $\begin{array}{l}\text { Transition } \\
\text { Array }\end{array}$ & Multiplet & $\lambda(\AA)$ & $E_{i}\left(\mathrm{~cm}^{-1}\right)$ & $E_{k}\left(\mathrm{~cm}^{-1}\right)$ & $g_{i}$ & $g_{k}$ & $\begin{array}{c}A_{k i}\left(10^{8}\right. \\
\left.\sec ^{-1}\right)\end{array}$ & $f_{i k}$ & $S$ (at.u.) & $\log g f$ & $\begin{array}{l}\text { Accu- } \\
\text { racy }\end{array}$ & Source \\
\hline 15 & & $\begin{array}{l}{ }^{1} \mathrm{~S}-{ }^{1} \mathrm{P}^{0} \\
(15 \mathrm{uv})\end{array}$ & 345.309 & 43184 & 332777 & 1 & 3 & 98 & 0.53 & 0.60 & -0.28 & $\mathrm{D}-$ & 2 \\
\hline \multirow[t]{7}{*}{16} & $2 p 3 s-$ & ${ }^{3} \mathrm{P}^{\circ}-{ }^{3} \mathrm{D}$ & 3762.3 & 267505 & 294077 & 9 & 15 & 1.07 & 0.377 & 42.0 & 0.53 & C & $3, c a$ \\
\hline & & & 3759.87 & 267633 & 294222 & 5 & 7 & 1.07 & 0.317 & 19.6 & 0.200 & C & $l s$ \\
\hline & & & 3754.67 & 267376 & 294002 & 3 & 5 & 0.80 & 0.283 & 10.5 & -0.071 & C & $l s$ \\
\hline & & & 3757.21 & 267257 & 293865 & 1 & 3 & 0.59 & 0.378 & 4.67 & -0.423 & C & $l s$ \\
\hline & & & 3791.26 & 267633 & 294002 & 5 & 5 & 0.260 & 0.056 & 3.50 & -0.55 & C & ls \\
\hline & & & 3774.00 & 267376 & 293865 & 3 & 3 & 0.440 & 0.094 & 3.50 & -0.55 & C & $l s$ \\
\hline & & & 3810.96 & 267633 & 293865 & 5 & 3 & 0.0284 & 0.00371 & 0.233 & -1.73 & C & $l s$ \\
\hline \multirow[t]{4}{*}{17} & & ${ }^{3} \mathrm{P}^{0}-{ }^{3} \mathrm{~S}$ & 3326.6 & 267505 & 297558 & 9 & 3 & 1.56 & 0.086 & 8.5 & -0.110 & C & $3, c a$ \\
\hline & & & 3340.74 & 267633 & 297558 & 5 & 3 & 0.85 & 0.085 & 4.70 & -0.369 & C & $l s$ \\
\hline & & & 3312.30 & 267376 & 297558 & 3 & 3 & 0.52 & 0.086 & 2.82 & -0.59 & C & ls \\
\hline & & & 3299.36 & 267257 & 297558 & 1 & 3 & 0.177 & 0.087 & 0.94 & -1.063 & C & $l s$ \\
\hline \multirow[t]{7}{*}{18} & & ${ }^{3} \mathrm{P}^{\circ}-{ }^{3} \mathrm{P}$ & 3041.5 & 267505 & 300374 & 9 & 9 & 2.04 & 0.283 & 25.5 & 0.406 & C & $3, c a$ \\
\hline & & & 3047.13 & 267633 & 300441 & 5 & 5 & 1.52 & 0.211 & 10.6 & 0.024 & C & $l s$ \\
\hline & & & 3035.43 & 267376 & 300310 & 3 & 3 & 0.51 & 0.071 & 2.12 & -0.67 & C & ls \\
\hline & & & 3059.30 & 267633 & 300310 & 5 & 3 & 0.84 & 0.070 & 3.54 & -0.454 & C & $l s$ \\
\hline & & & 3043.02 & 267376 & 300228 & 3 & 1 & 2.03 & 0.094 & 2.83 & -0.55 & C & $l s$ \\
\hline & & & 3023.45 & 267376 & 300441 & 3 & 5 & 0.52 & 0.119 & 3.54 & -0.449 & C & $l s$ \\
\hline & & & 3024.57 & 267257 & 300310 & 1 & 3 & 0.69 & 0.284 & 2.83 & -0.55 & C & $l s$ \\
\hline 19 & & ${ }^{1 P^{\circ}-{ }^{1} \mathrm{P}}$ & 5592.37 & 273080 & 290957 & 3 & 3 & 0.328 & 0.154 & 8.5 & -0.336 & C & $3, c a$ \\
\hline 20 & & ${ }^{1 \mathrm{P}^{\circ}-{ }^{1} \mathrm{D}}$ & 2983.78 & 273080 & 306585 & 3 & 5 & 2.24 & 0.499 & 14.7 & 0.175 & C & $3, c a$ \\
\hline 21 & & $\begin{array}{r}{ }^{1} \mathrm{P}^{\circ}-{ }^{1} \mathrm{~S} \\
(19 \mathrm{uv})\end{array}$ & 2454.99 & 273080 & 313801 & 3 & 1 & 4.00 & 0.120 & 2.92 & -0.442 & C & $3, c a$ \\
\hline \multirow[t]{9}{*}{22} & 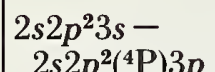 & ${ }^{5} \mathrm{P}-{ }^{5} \mathrm{D}^{\circ}$ & 3706.1 & 338741 & 365716 & 15 & 25 & 1.09 & 0.375 & 69 & 0.75 & C & $c a$ \\
\hline & & & 3703.37 & 338852 & 365846 & 7 & 9 & 1.10 & 0.290 & 24.7 & 0.308 & C & $l s$ \\
\hline & & & 3698.70 & 338690 & 365719 & 5 & 7 & 0.73 & 0.210 & 12.8 & 0.021 & C & $l s$ \\
\hline & & & 3695.37 & 338566 & 365619 & 3 & 5 & 0.384 & 0.131 & 4.78 & -0.406 & C & $l s$ \\
\hline & & & 3720.86 & 338852 & 365719 & 7 & 7 & 0.361 & 0.075 & 6.4 & -0.280 & C & $l s$ \\
\hline & & & 3712.48 & 338690 & 365619 & 5 & 5 & 0.63 & 0.131 & 8.0 & -0.184 & C & ls \\
\hline & & & $\begin{array}{l}3704.73 \\
3734.80\end{array}$ & 338566 & 365551 & 3 & 3 & 0.81 & 0.168 & 6.1 & -0.298 & C & $l s$ \\
\hline & & & $\begin{array}{l}3734.80 \\
3721.95\end{array}$ & $\begin{array}{l}338852 \\
338690\end{array}$ & $\begin{array}{l}365619 \\
365551\end{array}$ & 5 & $\begin{array}{l}5 \\
3\end{array}$ & $\begin{array}{l}0.061 \\
0.270\end{array}$ & $\begin{array}{l}0.0106 \\
0.0336\end{array}$ & $\begin{array}{l}0.91 \\
2.06\end{array}$ & $\begin{array}{l}-1.130 \\
-0.77\end{array}$ & $\mathrm{C}$ & $l s$ \\
\hline & & & 3709.52 & 338566 & 365516 & 3 & 1 & 1.09 & 0.075 & 2.75 & -0.65 & $\mathrm{C}$ & $l s$ \\
\hline \multirow[t]{8}{*}{23} & & $\begin{array}{c}{ }^{5} \mathbf{P}-{ }^{5} \mathrm{P}^{0} \\
(22)\end{array}$ & 3345.9 & 338741 & 368620 & 15 & 15 & 1.50 & 0.252 & 41.6 & 0.58 & C & $c a$ \\
\hline & & & 3350.99 & 338852 & 368685 & 7 & 7 & 1.00 & 0.168 & 13.0 & 0.070 & C. & $l s$ \\
\hline & & & 3344.26 & 338690 & 368584 & 5 & 5 & 0.125 & 0.0210 & 1.16 & -0.98 & $\mathrm{C}$ & $l s$ \\
\hline & & & 3336.78 & 338566 & 368526 & 3 & 3 & 0.377 & 0.063 & 2.08 & -0.72 & C & $l s$ \\
\hline & & & 3362.38 & 338852 & 368584 & 7 & 5 & 0.69 & 0.084 & 6.5 & -0.233 & C & $l s$ \\
\hline & & & 3350.68 & 338690 & 368526 & 5 & 3 & 0.112 & 0.113 & 6.2 & -0.248 & C & $l s$ \\
\hline & & & 3333.00 & 338690 & 368685 & 5 & 7 & 0.51 & 0.118 & 6.5 & -0.229 & C & $l s$ \\
\hline & & & 3333.40 & 338566 & 368584 & 3 & 5 & 0.68 & 0.189 & 6.2 & -0.246 & C & $l s$ \\
\hline \multirow[t]{4}{*}{24} & & ${ }^{5} \mathrm{P}-{ }^{5} \mathrm{~S}^{\circ}$ & 2678.2 & 338741 & 376068 & 15 & 5 & 2.99 & 0.107 & 14.2 & 0.206 & C & $c a$ \\
\hline & & & 2686.14 & 338852 & 376068 & 7 & 5 & 1.38 & 0.107 & 6.6 & -0.126 & C & $l s$ \\
\hline & & & 2674.57 & 338690 & 376068 & 5 & 5 & 1.00 & 0.107 & 4.71 & -0.272 & C & $l s$ \\
\hline & & & 2665.69 & 338566 & 376068 & 3 & 5 & 0.60 & 0.107 & 2.82 & -0.494 & C & $l s$ \\
\hline
\end{tabular}


O III. Allowed Transitions-Continued

\begin{tabular}{|c|c|c|c|c|c|c|c|c|c|c|c|c|c|}
\hline No. & $\begin{array}{c}\text { Transition } \\
\text { Array }\end{array}$ & Multiplet & $\lambda(\AA)$ & $E_{i}\left(\mathrm{~cm}^{-1}\right)$ & $E_{k}\left(\mathrm{~cm}^{-1}\right)$ & $g_{i}$ & $g_{k}$ & $\begin{array}{l}A_{k i}\left(10^{\mathrm{k}}\right. \\
\left.\mathrm{sec}^{-1}\right)\end{array}$ & $f_{i k}$ & $S($ at.u. $)$ & $\log g f$ & $\begin{array}{l}\text { Accu- } \\
\text { racy }\end{array}$ & Source \\
\hline \multirow[t]{6}{*}{25} & & ${ }^{3} \mathrm{P}-{ }^{3} \mathrm{D}^{\circ}$ & 4081.0 & 350212 & 374709 & 9 & 15 & 0.94 & 0.391 & 47.3 & 0.5 .5 & $\mathrm{C}$ & $c a$ \\
\hline & & & 4081.10 & 350302 & 374799 & 5 & 7 & 0.94 & 0.329 & 22.1 & 0.217 & $\mathrm{C}$ & ls \\
\hline & & & 4073.90 & 350123 & 374663 & 3 & 5 & 0.71 & 0.294 & 11.8 & -0.05 .5 & $\mathrm{C}$ & ls \\
\hline & & & [4072.3] & 350026 & 374575 & 1 & 3 & 0.52 & 0.392 & 5.2 & -0.407 & $\mathrm{C}$ & ls \\
\hline & & & [4.103.8] & 350302 & 374663 & 5 & 5 & 0.232 & 0.058 & 3.95 & -0.53 & $\mathrm{C}$ & ls \\
\hline & & & $\begin{array}{l}{[4088.5]} \\
{[4118.6]}\end{array}$ & $\begin{array}{l}350123 \\
350302\end{array}$ & $\begin{array}{l}374575 \\
374575\end{array}$ & $\begin{array}{l}3 \\
5\end{array}$ & $\begin{array}{l}3 \\
3\end{array}$ & $\begin{array}{l}0.389 \\
0.0254\end{array}$ & $\begin{array}{l}0.098 \\
0.00388\end{array}$ & $\begin{array}{l}3.94 \\
0.263\end{array}$ & $\begin{array}{l}-0.53 \\
-1.71\end{array}$ & $\begin{array}{l}\mathrm{C} \\
\mathrm{C}\end{array}$ & ls \\
\hline \multirow[t]{7}{*}{26} & & ${ }^{3} \mathrm{P}-{ }^{3} \mathrm{P}^{0}$ & 3544.6 & 350212 & 378416 & 9 & 9 & 1.46 & 0.274 & 28.8 & 0.392 & C & $c a$ \\
\hline & & & 3556.92 & 350302 & 378409 & 5 & 5 & 1.08 & 0.205 & 12.0 & 0.010 & C & ls \\
\hline & & & [3532.8] & 350123 & 378421 & 3 & 3 & 0.367 & 0.069 & 2.39 & -0.69 & $\mathrm{C}$ & ls \\
\hline & & & [3555.3] & 350302 & 378421 & 5 & 3 & 0.60 & 0.068 & 4.00 & -0.467 & $\mathrm{C}$ & $l s$ \\
\hline & & & {$[3530.7]$} & 350123 & 378438 & 3 & 1 & 1.47 & 0.092 & 3.19 & -0.56 & $\mathrm{C}$ & ls \\
\hline & & & [3534.3] & 350123 & 378409 & 3 & 5 & 0.366 & 0.114 & 3.99 & -0.465 & C & ls \\
\hline & & & {$[3520.7]$} & 350026 & 378421 & 1 & 3 & 0.493 & 0.275 & 3.19 & -0.56 & $\mathrm{C}$ & ls \\
\hline \multirow[t]{7}{*}{27} & $2 p 3 p-$ & ${ }^{3} \mathrm{D}-{ }^{3} \mathrm{~F}^{\circ}$ & 3265.9 & 294007 & 324688 & 15 & 21 & 2.08 & 0.465 & 75 & 0.84 & $\mathrm{C}$ & $3, c a$ \\
\hline & & & 3265.46 & 294222 & 324836 & 7 & 9 & 2.07 & 0.425 & 32.0 & 0.474 & C & ls \\
\hline & & & 3260.98 & 294002 & 324658 & 5 & 7 & 1.84 & 0.412 & 22.1 & 0.314 & $\mathrm{C}$ & ls \\
\hline & & & .3267 .31 & 293865 & 324462 & 3 & 5 & 1.73 & 0.462 & 14.9 & 0.142 & $\mathrm{C}$ & ls \\
\hline & & & 3284.57 & 294222 & 324658 & 7 & 7 & 0.226 & 0.0366 & 2.77 & -0.59 & $\mathrm{C}$ & ls \\
\hline & & & 3281.94 & 294002 & 324462 & 5 & 5 & 0.317 & 0.051 & 2.77 & -0.59 & $\mathrm{C}$ & $l s$ \\
\hline & & & 3305.77 & 294222 & 324462 & 7 & 5 & 0.0093 & 0.00109 & 0.083 & -2.118 & C & $l s$ \\
\hline \multirow[t]{7}{*}{28} & & $\begin{array}{c}{ }^{3} \mathrm{D}-{ }^{3} \mathrm{D}^{\circ} \\
(10)\end{array}$ & 3002.6 & 294007 & 327302 & 15 & 15 & 0.68 & 0.092 & 13.6 & 0.139 & C & $3, c a$ \\
\hline & & & 3017.63 & 294222 & 327351 & 7 & 7 & 0.59 & 0.081 & 5.6 & -0.249 & $\mathrm{C}$ & $l s$ \\
\hline & & & 3004.35 & 294002 & 327277 & 5 & 5 & 0.472 & 0.064 & 3.16 & -0.496 & $\mathrm{C}$ & $l s$ \\
\hline & & & 2996.51 & 293865 & 327228 & 3 & 3 & 0.51 & 0.069 & 2.04 & -0.68 & C & $l s$ \\
\hline & & & $\begin{array}{l}3024.36 \\
3008.79\end{array}$ & $\begin{array}{l}294222 \\
294002\end{array}$ & $\begin{array}{l}327277 \\
327228\end{array}$ & $\begin{array}{l}7 \\
5\end{array}$ & $\begin{array}{l}5 \\
3\end{array}$ & $\begin{array}{l}0.104 \\
0.134\end{array}$ & $\begin{array}{l}0.0102 \\
0.0109\end{array}$ & $\begin{array}{l}0.71 \\
0.54\end{array}$ & $\begin{array}{l}-1.147 \\
-1.264\end{array}$ & $\stackrel{\mathrm{C}}{\mathrm{C}}$ & ls \\
\hline & & & 2997.71 & 294002 & 327351 & 5 & 7 & 0.076 & 0.0144 & 0.71 & -1.143 & $\mathrm{C}$ & ls \\
\hline & & & 2992.11 & 293865 & 327277 & 3 & 5 & 0.082 & 0.0183 & 0.54 & -1.261 & $\mathrm{C}$ & ls \\
\hline \multirow[t]{4}{*}{29} & & ${ }^{3} \mathrm{~S}-{ }^{3} \mathrm{P}^{\circ}$ & 3127.3 & 297558 & 329525 & 3 & 9 & 1.37 & 0.60 & 18.6 & 0.257 & $\mathrm{C}$ & $3, c a$ \\
\hline & & & 3132.86 & 297558 & 329468 & 3 & 5 & 1.36 & 0.333 & 10.3 & -0.001 & C & $l s$ \\
\hline & & & 3121.71 & 297558 & 329582 & 3 & 3 & 1.38 & 0.201 & 6.2 & -0.220 & C & ls \\
\hline & & & 3115.73 & 297558 & 329643 & 3 & 1 & 1.39 & 0.067 & 2.07 & -0.70 & $\mathrm{C}$ & $l s$ \\
\hline \multirow[t]{7}{*}{30} & & ${ }^{3} \mathrm{P}-{ }^{3} \mathrm{D}^{0}$ & 3712.5 & 300374 & 327302 & 9 & 15 & 1.10 & 0.379 & 41.7 & 0.53 & C & $3, c a$ \\
\hline & & & 3715.08 & 300441 & 327351 & 5 & 7 & 1.10 & 0.319 & 19.5 & 0.203 & $\mathrm{C}$ & $l s$ \\
\hline & & & 3707.24 & 300310 & 327277 & 3 & 5 & 0.83 & 0.284 & 10.4 & -0.070 & $\mathrm{C}$ & ls \\
\hline & & & 3702.75 & 300228 & 327228 & 1 & 3 & 0.62 & 0.380 & 4.63 & -0.420 & $\mathrm{C}$ & $l s$ \\
\hline & & & 3725.30 & 300441 & 327277 & 5 & 5 & 0.273 & 0.057 & 3.48 & -0.55 & $\mathrm{C}$ & $l s$ \\
\hline & & & 3714.03 & 300310 & 327228 & 3 & 3 & 0.459 & 0.095 & 3.48 & -0.55 & C & $l s$ \\
\hline & & & 3732.13 & 300441 & 327228 & 5 & 3 & 0.0301 & 0.00378 & 0.232 & -1.72 & C & $l s$ \\
\hline \multirow[t]{7}{*}{31} & & ${ }^{3} \mathrm{P}-3 \mathrm{P}^{\circ}$ & 3429.4 & 300374 & 329525 & 9 & 9 & 0.79 & 0.140 & 14.2 & 0.100 & $\mathrm{C}$ & $3, c a$ \\
\hline & & & 3444.10 & 300441 & 329468 & 5 & 5 & 0.59 & 0.104 & 5.9 & -0.284 & C & ls \\
\hline & & & 3415.29 & 300310 & 329582 & 3 & 3 & 0.200 & 0.0350 & 1.18 & -0.98 & $\mathrm{C}$ & $l s$ \\
\hline & & & 3430.60 & 300441 & 329582 & 5 & 3 & 0.330 & 0.0349 & 1.97 & -0.76 & $\mathrm{C}$ & ls \\
\hline & & & 3408.13 & 300310 & 329643 & 3 & 1 & 0.81 & 0.0469 & 1.58 & -0.85 & C & ls \\
\hline & & & 3428.67 & 300310 & 329468 & 3 & 5 & 0.198 & 0.058 & 1.97 & -0.76 & C & ls \\
\hline & & & 3405.74 & 300228 & 329582 & 1 & 3 & 0.270 & 0.141 & 1.58 & -0.85 & $\mathrm{C}$ & $l s$ \\
\hline 32 & & $\begin{array}{c}{ }^{1} \mathrm{D}-{ }^{1} \mathrm{D}^{\circ} \\
(16)\end{array}$ & 5500.11 & 306585 & 324734 & 5 & 5 & 0.112 & 0.051 & 4.58 & -0.60 & $\mathrm{C}$ & $3, c a$ \\
\hline 33 & & ${ }^{1} \mathrm{D}-{ }^{1} \mathrm{~F}^{\circ}$ & 3961.59 & 306585 & 331820 & 5 & 7 & 1.28 & 0.422 & 27.5 & 0.324 & $\mathrm{C}$ & $3, c a$ \\
\hline
\end{tabular}


O III. Allowed Transitions-Continued

\begin{tabular}{|c|c|c|c|c|c|c|c|c|c|c|c|c|c|}
\hline No. & $\begin{array}{l}\text { Transition } \\
\text { Array }\end{array}$ & Multiplet & $\lambda(\AA)$ & $E_{i}\left(\mathrm{~cm}^{-1}\right)$ & $E_{k}\left(\mathrm{~cm}^{-1}\right)$ & $g_{i}$ & $g_{k}$ & $\begin{array}{l}A_{k i}\left(10^{8}\right. \\
\left.\sec ^{-1}\right)\end{array}$ & $f_{i k}$ & $S($ at.u.) & $\log g f$ & $\begin{array}{l}\text { Accu- } \\
\text { racy }\end{array}$ & Source \\
\hline 34 & & $\begin{array}{c}{ }^{1} \mathrm{D}-{ }^{1} \mathrm{P}^{\circ} \\
(18)\end{array}$ & 3816.75 & 306585 & 332777 & 5 & 3 & 0.0402 & 0.0053 & 0.331 & -1.58 & C & $3, c a$ \\
\hline 35 & & $\begin{array}{c}{ }^{1} \mathrm{~S}-{ }^{1} \mathrm{P}^{0} \\
(19)\end{array}$ & 5268.06 & 313801 & 332777 & 1 & 3 & 0.311 & 0.389 & 6.7 & -0.411 & C & $3, c a$ \\
\hline \multirow[t]{4}{*}{36} & $\begin{array}{l}2 s 2 p^{2} 3 p- \\
2 s 2 p^{2}\left({ }^{4} \mathrm{P}\right) 3 d\end{array}$ & $\begin{array}{l}{ }^{3} \mathrm{~S}^{\circ}-{ }^{3} \mathrm{P} \\
(23 \mathrm{uv})\end{array}$ & 2691.5 & 363267 & 400410 & 3 & 9 & 2.10 & 0.68 & 18.2 & 0.312 & C & $c a$ \\
\hline & & & 2695.49 & 363267 & 400355 & 3 & 5 & 2.09 & 0.379 & 10.1 & 0.056 & C & $l s$ \\
\hline & & & 2687.53 & 363267 & 400465 & 3 & 3 & 2.11 & 0.229 & 6.1 & -0.163 & $\mathrm{C}$ & ls \\
\hline & & & 2683.65 & 363267 & 400518 & 3 & 1 & 2.12 & 0.076 & 2.02 & -0.64 & C & ls \\
\hline \multirow[t]{12}{*}{37} & & ${ }^{5} \mathrm{D}^{\circ}-{ }^{5} \mathrm{~F}$ & 3453.0 & 365716 & 394668 & 25 & 35 & 1.68 & 0.420 & 119 & 1.021 & C & $c a$ \\
\hline & & & 3455.12 & 365846 & 394780 & 9 & 11 & 1.67 & 0.365 & 37.4 & 0.52 & C & $l s$ \\
\hline & & & 3450.94 & 365719 & 394688 & 7 & 9 & 1.40 & 0.321 & 25.5 & 0.351 & C & ls \\
\hline & & & 3448.05 & 365619 & 394613 & 5 & 7 & 1.15 & 0.287 & 16.3 & 0.157 & C & ls \\
\hline & & & 3446.73 & 365551 & 394555 & 3 & 5 & 0.94 & 0.279 & 9.5 & -0.077 & C & ls \\
\hline & & & 3447.22 & 365516 & 394516 & 1 & 3 & 0.78 & 0.419 & 4.76 & -0.377 & $\mathrm{C}$ & $l s$ \\
\hline & & & 3466.15 & 365846 & 394688 & 9 & 9 & 0.276 & 0.0497 & 5.1 & -0.350 & C & ls \\
\hline & & & 3459.98 & 365719 & 394613 & 7 & 7 & 0.496 & 0.089 & 7.1 & -0.205 & C & ls \\
\hline & & & $\begin{array}{l}3454.90 \\
3451.33\end{array}$ & $\begin{array}{l}365619 \\
365551\end{array}$ & $\begin{array}{l}394555 \\
394516\end{array}$ & $\begin{array}{l}5 \\
3\end{array}$ & $\begin{array}{l}5 \\
3\end{array}$ & $\begin{array}{l}0.67 \\
0.78\end{array}$ & $\begin{array}{l}0.120 \\
0.140\end{array}$ & $\begin{array}{l}6.8 \\
4.76\end{array}$ & $\begin{array}{l}-0.223 \\
-0.378\end{array}$ & $\stackrel{\mathrm{C}}{\mathrm{C}}$ & $\begin{array}{l}l s \\
l s\end{array}$ \\
\hline & & & {$[3475.2]$} & 365846 & 394613 & 9 & 7 & 0.0234 & 0.00330 & 0.340 & -1.53 & $\mathrm{C}$ & ls \\
\hline & & & 3466.90 & 365719 & 394555 & 7 & 5 & 0.066 & 0.0085 & 0.68 & -1.225 & C & ls \\
\hline & & & 3459.52 & 365619 & 394516 & 5 & 3 & 0.111 & 0.0119 & 0.68 & -1.224 & C & ls \\
\hline \multirow[t]{12}{*}{38} & & ${ }^{5} \mathrm{D}^{\circ}-{ }^{5} \mathrm{D}$ & 3081.0 & 365716 & 398164 & 25 & 25 & 0.62 & 0.089 & 22.5 & 0.345 & C & $c a$ \\
\hline & & & 3088.04 & 365846 & 398219 & 9 & 9 & 0.52 & 0.074 & 6.8 & -0.178 & C & $l s$ \\
\hline & & & 3083.65 & 365719 & 398137 & 7 & 7 & 0.311 & 0.0443 & 3.15 & -0.51 & $\mathrm{C}$ & $l s$ \\
\hline & & & 3075.19 & 365619 & 398127 & 5 & 5 & 0.157 & 0.0222 & 1.12 & -0.95 & $\mathrm{C}$ & ls \\
\hline & & & 3068.48 & 365551 & 398131 & 3 & 3 & 0,234 & 0.0330 & 0.225 & -1.004 & $\mathrm{C}$ & ls \\
\hline & & & 3095.81 & 365846 & 398137 & 9 & 7 & 0.132 & 0.0147 & 1.35 & -0.88 & C & ls \\
\hline & & & 3084.63 & 365719 & 398127 & 7 & 5 & 0.248 & 0.0253 & 1.80 & -0.75 & C & ls \\
\hline & & & 3074.68 & 365619 & 398131 & 5 & 3 & 0.365 & 0.0310 & 1.57 & -0.81 & C & ls \\
\hline & & & $\begin{array}{l}3068.06 \\
3075.95\end{array}$ & $\begin{array}{l}365551 \\
365719\end{array}$ & $\begin{array}{l}398135 \\
398219\end{array}$ & 3 & 1 & 0.63 & 0.0296 & 0.90 & -1.052 & C & ls \\
\hline & & & 3074.15 & $\begin{array}{l}365719 \\
365619\end{array}$ & $\begin{array}{l}398219 \\
398137\end{array}$ & 5 & $\begin{array}{l}9 \\
7\end{array}$ & $\begin{array}{l}0.104 \\
0.180\end{array}$ & $\begin{array}{l}0.0190 \\
0.0355\end{array}$ & $\begin{array}{l}1.35 \\
1.80\end{array}$ & $\begin{array}{l}-0.88 \\
-0.75\end{array}$ & C & $\begin{array}{l}l s \\
l s\end{array}$ \\
\hline & & & 3068.68 & 365551 & 398127 & 3 & 5 & 0.220 & 0.052 & 1.57 & -0.81 & $\mathrm{C}$ & ls \\
\hline & & & 3065.01 & 365516 & 398131 & 1 & 3 & 0.210 & 0.089 & 0.90 & -1.051 & $\mathrm{C}$ & ls \\
\hline \multirow[t]{9}{*}{39} & & $\begin{array}{c}{ }^{5} \mathrm{P}^{\circ}-{ }^{-5} \mathrm{D} \\
(27)\end{array}$ & 3383.8 & 368620 & 398164 & 15 & 25 & 1.45 & 0.416 & 69 & 0.80 & C & $c a$ \\
\hline & & & 3384.95 & 368685 & 398219 & 7 & 9 & 1.45 & 0.321 & 25.0 & 0.351 & $\mathrm{C}$ & ls \\
\hline & & & 3382.69 & 368584 & 398137 & 5 & 7 & 0.97 & 0.233 & 13.0 & 0.066 & C & ls \\
\hline & & & [3377.3] & 368526 & 398127 & 3 & 5 & 0.51 & 0.146 & 4.85 & -0.360 & C & $l s$ \\
\hline & & & 3394.26 & 368685 & 398137 & 7 & 7 & 0.480 & 0.083 & 6.5 & -0.237 & C & ls \\
\hline & & & $\begin{array}{l}3383.85 \\
3376.82\end{array}$ & $\begin{array}{l}368584 \\
368526\end{array}$ & $\begin{array}{l}398127 \\
398131\end{array}$ & $\begin{array}{l}5 \\
3\end{array}$ & $\begin{array}{l}5 \\
3\end{array}$ & $\begin{array}{l}0.85 \\
1.09\end{array}$ & $\begin{array}{l}0.145 \\
0.187\end{array}$ & $\begin{array}{l}8.1 \\
6.2\end{array}$ & $\begin{array}{l}-0.139 \\
-0.251\end{array}$ & C & ls \\
\hline & & & [3395.5] & 368685 & 398127 & 7 & 5 & 0.096 & 0.0118 & 0.93 & -1.082 & $\mathrm{C}$ & ls \\
\hline & & & [3383.5] & 368584 & 398131 & 5 & 3 & 0.363 & 0.0374 & 2.08 & -0.73 & $\mathrm{C}$ & ls \\
\hline & & & {$[3376.4]$} & 368526 & 398135 & 3 & 1 & 1.46 & 0.083 & 2.77 & -0.60 & C & $l s$ \\
\hline \multirow[t]{8}{*}{40} & & ${ }^{5} \mathrm{P}^{\circ}-{ }^{5} \mathrm{P}$ & 3343.6 & 368620 & 398519 & 15 & 15 & 0.84 & 0.140 & 23.1 & 0.322 & $\mathrm{C}$ & $c a$ \\
\hline & & & 3355.92 & 368685 & 398474 & 7 & 7 & 0.55 & 0.093 & 7.2 & -0.187 & C & ls \\
\hline & & & 3336.78 & 368584 & 398544 & 5 & 5 & 0.070 & 0.0117 & 0.64 & -1.233 & C & ls \\
\hline & & & 3326.16 & 368526 & 398583 & 3 & 3 & 0.212 & 0.0352 & 1.16 & -0.98 & C & ls \\
\hline & & & 3348.05 & 368685 & 398544 & 7 & 5 & 0.388 & 0.0466 & 3.60 & -0.487 & C & ls \\
\hline & & & 3332.49 & 368584 & 398583 & 5 & 3 & 0.63 & 0.063 & 3.47 & -0.50 & C & ls \\
\hline & & & 3344.26 & 368584 & 398474 & 5 & 7 & 0.278 & 0.065 & 3.59 & -0.487 & C & $l s$ \\
\hline & & & 3330.40 & 368526 & 398544 & 3 & 5 & 0.379 & 0.105 & 3.45 & -0.50 & $\mathrm{C}$ & $l s$ \\
\hline
\end{tabular}


O III. Allowed Transitions-Continued

\begin{tabular}{|c|c|c|c|c|c|c|c|c|c|c|c|c|c|}
\hline No. & $\begin{array}{c}\text { Transition } \\
\text { Array }\end{array}$ & Multiplet & $\lambda(\AA)$ & $E_{i}\left(\mathrm{~cm}^{-1}\right)$ & $E_{k}\left(\mathrm{~cm}^{-1}\right)$ & $g_{i}$ & $g_{k}$ & $\begin{array}{l}A_{k i}\left(10^{4}\right. \\
\left.\sec ^{-1}\right)\end{array}$ & $f_{i k}$ & S(at.u.) & $\log g f$ & $\begin{array}{l}\text { Accu- } \\
\text { racy }\end{array}$ & Source \\
\hline \multirow[t]{6}{*}{41} & & ${ }^{3} \mathrm{D}^{\circ}-{ }^{-3} \mathrm{~F}$ & .37 .30 .1 & .374709 & 401510 & 15 & 21 & 1.58 & 0.460 & 85 & 0.84 & C & $c a$ \\
\hline & & & 3728.82 & 374799 & 401609 & 7 & 9 & 1.58 & 0.424 & 36.4 & 0.472 & C & ls \\
\hline & & & $\begin{array}{l}3728.49 \\
379090\end{array}$ & $\begin{array}{l}374663 \\
374575\end{array}$ & 401475 & 5 & 7 & 1.41 & 0.411 & 25.2 & 0.312 & C & ls \\
\hline & & & [3747.6] & 374799 & 401475 & 7 & 7 & 0.174 & 0.0366 & 3.16 & -0.59 & $\mathrm{C}$ & ls \\
\hline & & & [3742.0] & 374663 & 401379 & 5 & 5 & 0.244 & 0.051 & 3.16 & -0.59 & C & ls \\
\hline & & & [3761.2] & 374799 & 401379 & 7 & 5 & 0.0072 & 0.00108 & 0.094 & -2.120 & C & is \\
\hline \multirow[t]{6}{*}{42} & & $\begin{array}{c}{ }^{3} \mathrm{D}^{\circ}-{ }^{3} \mathrm{D} \\
(31)\end{array}$ & 3210.2 & 374709 & 405851 & 15 & 15 & 0.66 & 0.102 & 16.2 & 0.186 & C & $c a$ \\
\hline & & & 3215.97 & 374799 & 405883 & 7 & 7 & 0.58 & 0.091 & 6.7 & -0.198 & C & ls \\
\hline & & & $\begin{array}{l}3207.12 \\
3000.05\end{array}$ & 374663 & 405834 & 5 & 5 & 0.460 & 0.071 & 3.74 & -0.451 & C & ls \\
\hline & & & $\begin{array}{r}3200.95 \\
{[3221.2]}\end{array}$ & $\begin{array}{l}374575 \\
374799\end{array}$ & $\begin{array}{l}405805 \\
405834\end{array}$ & 3 & $\begin{array}{l}3 \\
5\end{array}$ & 0.499 & $\begin{array}{l}0.077 \\
0.0113\end{array}$ & 2.42 & $\begin{array}{l}-0.64 \\
-102\end{array}$ & C & ls \\
\hline & & & {$[3210.2]$} & 374.663 & 405805 & 5 & 3 & 0.165 & 0.0153 & 0.81 & -1.117 & $\mathrm{C}$ & ls \\
\hline & & & {$[3202.2]$} & $\begin{array}{l}374663 \\
374575\end{array}$ & $\begin{array}{l}405883 \\
405834\end{array}$ & 5 & 7 & 0.074 & 0.0159 & 0.84 & -1.099 & $\underset{c}{C}$ & ls \\
\hline \multirow{4}{*}{43} & & ${ }^{5} \mathrm{~S}^{\circ}-5 \mathrm{P}$ & & & & & & & & & & & \\
\hline & & (33) & 4432.9 & 580000 & 390319 & 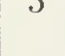 & 12 & 0.490 & 0.438 & 32.0 & 0.340 & $\mathrm{C}^{2}$ & $c a$ \\
\hline & & & $\begin{array}{l}4461.56 \\
44799\end{array}$ & $\begin{array}{l}376068 \\
276060\end{array}$ & 398474 & 5 & 7 & 0.486 & 0.203 & 14.9 & 0.007 & C & ls \\
\hline & & & 4440.1 & $\begin{array}{l}370008 \\
376068\end{array}$ & $\begin{array}{l}396544 \\
398583\end{array}$ & $\begin{array}{l}5 \\
5\end{array}$ & $\begin{array}{l}5 \\
3\end{array}$ & $\begin{array}{l}0.492 \\
0.495\end{array}$ & $\begin{array}{l}0.140 \\
0.088\end{array}$ & $\begin{array}{r}0.1 \\
6.4\end{array}$ & -0.1358 & C & $\begin{array}{ll}l s \\
l s\end{array}$ \\
\hline \multirow[t]{6}{*}{44} & & ${ }^{3} \mathrm{P}^{\circ}-{ }^{3} \mathrm{D}$ & 3643.9 & 378416 & 405851 & 9 & 15 & 1.39 & 0.461 & 49.8 & 0.62 & C & $c a$ \\
\hline & & & 3638.70 & 378409 & 405883 & 5 & 7 & 1.40 & 0.388 & 23.2 & 0.288 & C & ls \\
\hline & & & 3646.84 & 378421 & 405834 & 3 & 5 & 1.04 & 0.345 & 12.4 & 0.015 & C & ls \\
\hline & & & 3653.00 & 378438 & 405805 & 1 & 3 & 0.77 & 0.460 & 5. & -0.337 & C & ls \\
\hline & & & $\begin{array}{l}3645.20 \\
3650.70\end{array}$ & $\begin{array}{l}378409 \\
378421\end{array}$ & $\begin{array}{l}405834 \\
405805\end{array}$ & $\begin{array}{l}5 \\
3\end{array}$ & 5 & 0.347 & 0.069 & 4.15 & $\begin{array}{l}-0.462 \\
-0.462\end{array}$ & C & ls \\
\hline & & & 3649.20 & 378409 & 405805 & 5 & 3 & 0.0384 & 0.00460 & 0.276 & -1.64 & $\mathrm{C}$ & ls \\
\hline \multirow[t]{3}{*}{45} & $\begin{array}{l}2 p 3 d- \\
\left.2 p p^{(2} \mathrm{P}^{\circ}\right) 4 p\end{array}$ & ${ }^{3} \mathrm{P}^{\circ}-{ }^{3} \mathrm{~S}$ & 2601.6 & 329525 & 367952 & 9 & 3 & 1.73 & 0.059 & 4.52 & -0.278 & C & $3, c a$ \\
\hline & & & 2597.69 & 329468 & 367952 & 5 & 3 & 0.97 & 0.059 & 2.51 & -0.53 & C & ls \\
\hline & & & $\begin{array}{r}2605.41 \\
{[2609.6]}\end{array}$ & $\begin{array}{l}329582 \\
329643\end{array}$ & $\begin{array}{l}367952 \\
367952\end{array}$ & $\begin{array}{l}3 \\
1\end{array}$ & $\begin{array}{l}3 \\
3\end{array}$ & $\begin{array}{l}0.58 \\
0.190\end{array}$ & $\begin{array}{l}0.059 \\
0.058\end{array}$ & $\begin{array}{l}1.51 \\
0.50\end{array}$ & $\begin{array}{l}-0.75 \\
-1.235\end{array}$ & $\begin{array}{l}\mathrm{C} \\
\mathrm{C}\end{array}$ & $\begin{array}{l}l s \\
l s\end{array}$ \\
\hline 46 & & $\begin{array}{l}{ }^{1} \mathrm{~F}^{\circ}-{ }^{1} \mathrm{D} \\
(21 \mathrm{uv})\end{array}$ & 2558.06 & 331820 & 370901 & 7 & 5 & 1.16 & 0.081 & 4.79 & -0.245 & C & $3, c a$ \\
\hline 47 & $\begin{array}{l}2 p 4 p- \\
2 p\left({ }^{2} \mathrm{P}^{\circ}\right) 5 d\end{array}$ & $\begin{array}{c}{ }^{1} \mathrm{~S}-{ }^{-1} \mathrm{P}^{\circ} \\
(29)\end{array}$ & 3279.97 & 373046 & 403526 & 1 & 3 & 0.241 & 0.117 & 1.26 & -0.93 & C & $3, c a$ \\
\hline
\end{tabular}




\section{Forbidden Transitions}

The adopted values represent, as in the case of C I, the work of Garstang [1], Naqvi [2], and Yamanouchi and Horie [3], who have independently done essentially the same calculations and arrived at very similar results. For the selection of values, the same considerations as for $\mathrm{C}$ I have been applied. (Yamanouchi and Horie's result for the ${ }^{3} \mathrm{P}_{1}-{ }^{1} \mathrm{D}_{2}$ transition apparently contains a numerical error and is not used).

\section{References}

[1] Garstang, R. H., Monthly Notices Roy. Astron. Soc. 111, 115-124 (1951).

[2] Naqvi, A. M., Thesis Harvard (1951).

[3] Yamanouchi, T., and Horie, H., J. Phys. Soc. Japan 7, 52-56 (1952).

O III. Forbidden Transitions

\begin{tabular}{|c|c|c|c|c|c|c|c|c|c|c|c|c|}
\hline No. & $\begin{array}{c}\text { Transition } \\
\text { Array }\end{array}$ & Multiplet & $\lambda(\AA)$ & $E_{i}\left(\mathrm{~cm}^{-1}\right)$ & $E_{k}\left(\mathrm{~cm}^{-1}\right)$ & $g_{i}$ & $g_{k}$ & $\begin{array}{l}\text { Type of } \\
\text { Transi- } \\
\text { tion }\end{array}$ & $A_{k i}\left(\sec ^{-1}\right)$ & $S$ (at.u.) & $\begin{array}{l}\text { Accu- } \\
\text { racy }\end{array}$ & Source \\
\hline 1 & $2 p^{2}-2 p^{2}$ & ${ }^{3} \mathrm{P}-{ }^{3} \mathrm{P}$ & $\begin{array}{l}{\left[51.69 \times 10^{4}\right]} \\
{\left[51.69 \times 10^{4}\right]} \\
{\left[32.59 \times 10^{4}\right]} \\
{\left[88.16 \times 10^{4}\right]}\end{array}$ & $\begin{array}{c}113.4 \\
113.4 \\
0 \\
0\end{array}$ & $\begin{array}{l}306.8 \\
306.8 \\
306.8 \\
113.4\end{array}$ & $\begin{array}{l}3 \\
3 \\
1 \\
1\end{array}$ & $\begin{array}{l}5 \\
5 \\
5 \\
3\end{array}$ & $\begin{array}{l}\mathrm{m} \\
\mathrm{e} \\
\mathrm{e} \\
\mathrm{m}\end{array}$ & $\begin{array}{l}9.75 \times 10^{-5} \\
7.8 \times 10^{-12} \\
3.50 \times 10^{-11} \\
2.62 \times 10^{-5}\end{array}$ & $\begin{array}{l}2.50 \\
0.086 \\
0.383 \\
2.00\end{array}$ & $\begin{array}{l}\mathrm{B} \\
\mathrm{C} \\
\mathrm{C} \\
\mathrm{B}\end{array}$ & $\begin{array}{r}1,2,3 \\
1 \\
1 \\
1,2,3\end{array}$ \\
\hline 2 & & $\begin{array}{c}{ }^{3} \mathrm{P}-{ }^{1} \mathrm{D} \\
(1 \mathrm{~F})\end{array}$ & $\begin{array}{l}5006.84 \\
5006.84 \\
4958.91 \\
4958.91 \\
4931.8\end{array}$ & $\begin{array}{c}306.8 \\
306.8 \\
113.4 \\
113.4 \\
0\end{array}$ & $\begin{array}{l}20271 \\
20271 \\
20271 \\
20271 \\
20271\end{array}$ & $\begin{array}{l}5 \\
5 \\
3 \\
3 \\
1\end{array}$ & $\begin{array}{l}5 \\
5 \\
5 \\
5 \\
5\end{array}$ & $\begin{array}{l}\mathrm{m} \\
\mathrm{e} \\
\mathrm{m} \\
\mathrm{e} \\
\mathrm{e}\end{array}$ & $\begin{array}{l}0.0210 \\
4.1 \times 10^{-5} \\
0.0071 \\
6.2 \times 10^{-6} \\
1.9 \times 10^{-6}\end{array}$ & $\begin{array}{l}4.88 \times 10^{-4} \\
3.8 \times 10^{-4} \\
1.61 \times 10^{-4} \\
5.5 \times 10^{-5} \\
1.7 \times 10^{-5}\end{array}$ & $\begin{array}{l}\text { C } \\
\text { D } \\
\text { C } \\
\text { D } \\
\text { D }\end{array}$ & $\begin{array}{r}1,2,3 \\
1 \\
1,2 \\
1 \\
1\end{array}$ \\
\hline 3 & & ${ }^{3} \mathrm{P}-{ }^{1} \mathrm{~S}$ & $\begin{array}{l}{[2331.6]} \\
{[2321.1]}\end{array}$ & $\begin{array}{l}306.8 \\
113.4\end{array}$ & $\begin{array}{l}43183.5 \\
43183.5\end{array}$ & $\begin{array}{l}5 \\
3\end{array}$ & $\begin{array}{l}1 \\
1\end{array}$ & $\begin{array}{l}\mathrm{e} \\
\mathrm{m}\end{array}$ & $\begin{array}{l}7.1 \times 10^{-4} \\
0.230\end{array}$ & $\begin{array}{l}2.9 \times 10^{-5} \\
1.07 \times 10^{-4}\end{array}$ & $\begin{array}{l}\text { D } \\
\text { C }\end{array}$ & 1,3 \\
\hline 4 & & $\begin{array}{c}{ }^{1} \mathrm{D}-{ }^{1} \mathrm{~S} \\
(2 \mathrm{~F})\end{array}$ & 4363.21 & 20271 & 43183.5 & 5 & 1 & e & 1.60 & 1.51 & C & 1 \\
\hline
\end{tabular}


Ground State

Ionization Potential
$1 s^{2} 2 s^{2} 2 p^{2} \mathrm{P}_{\mathrm{i} / 2}^{\circ}$

$77.394 \mathrm{eV}=624396.5 \mathrm{~cm}^{-1}$

\section{Allowed Transitions}

List of tabulated lines:

\begin{tabular}{|c|c|c|c|c|c|}
\hline Wavelength $[\AA]]$ & No. & Wavelength $[\AA]$ & No. & Wavelength $[\AA ̊]$ & No. \\
\hline $\begin{array}{l}238.361 \\
238.573 \\
238.58 \\
279.633 \\
279.937\end{array}$ & $\begin{array}{l}6 \\
6 \\
6 \\
5 \\
5\end{array}$ & $\begin{array}{l}3216.31 \\
3348.08 \\
3349.11 \\
3354.31 \\
3362.63\end{array}$ & $\begin{array}{r}14 \\
9 \\
9 \\
15 \\
15\end{array}$ & $\begin{array}{l}3774.38 \\
3930.63 \\
3942.14 \\
3945.29 \\
3956.82\end{array}$ & $\begin{array}{l}13 \\
17 \\
17 \\
17 \\
17\end{array}$ \\
\hline $\begin{array}{l}553.328 \\
554.074 \\
554.514 \\
555.262 \\
608.395\end{array}$ & $\begin{array}{l}3 \\
3 \\
3 \\
3 \\
2\end{array}$ & $\begin{array}{l}3375.50 \\
3378.09 \\
3381.28 \\
3381.33 \\
3385.55\end{array}$ & $\begin{array}{r}15 \\
9 \\
8 \\
8 \\
8\end{array}$ & $\begin{array}{l}3974.66 \\
3977.10 \\
3995.17 \\
4568 \\
4652.5\end{array}$ & $\begin{array}{l}17 \\
17 \\
17 \\
21 \\
20\end{array}$ \\
\hline $\begin{array}{l}609.829 \\
624.617 \\
625.130 \\
625.852 \\
787.710\end{array}$ & $\begin{array}{l}2 \\
4 \\
4 \\
4 \\
1\end{array}$ & $\begin{array}{l}3390.37 \\
3396.83 \\
3403.58 \\
3405.97 \\
3409.75\end{array}$ & $\begin{array}{r}8 \\
8 \\
12 \\
8 \\
8\end{array}$ & $\begin{array}{l}4685.4 \\
4772.57 \\
4779.09 \\
4783.43 \\
4794.22\end{array}$ & $\begin{array}{l}20 \\
16 \\
16 \\
16 \\
16\end{array}$ \\
\hline $\begin{array}{l}790.103 \\
790.203 \\
2494.8 \\
2511.4 \\
3063.46\end{array}$ & $\begin{array}{r}1 \\
1 \\
10 \\
10 \\
7\end{array}$ & $\begin{array}{l}3411.76 \\
3413.71 \\
3425.57 \\
3489.84 \\
3492.2\end{array}$ & $\begin{array}{r}12 \\
12 \\
8 \\
11 \\
11\end{array}$ & $\begin{array}{l}4798.25 \\
4800.77 \\
4813.07 \\
4823.93 \\
5290.1\end{array}$ & $\begin{array}{l}16 \\
16 \\
16 \\
16 \\
18\end{array}$ \\
\hline $\begin{array}{l}3071.66 \\
3177.80 \\
3180.72 \\
3180.98 \\
3185.72\end{array}$ & $\begin{array}{r}7 \\
14 \\
14 \\
14 \\
14\end{array}$ & $\begin{array}{l}3492.24 \\
3560.42 \\
3563.36 \\
3593.1 \\
3725.81\end{array}$ & $\begin{array}{l}11 \\
19 \\
19 \\
19 \\
13\end{array}$ & $\begin{array}{l}5305.3 \\
5362.4 \\
5378.3\end{array}$ & $\begin{array}{l}18 \\
18 \\
18\end{array}$ \\
\hline $\begin{array}{l}3188.17 \\
3188.65 \\
3194.75 \\
3199.53 \\
3209.64\end{array}$ & $\begin{array}{l}14 \\
14 \\
14 \\
14 \\
14\end{array}$ & $\begin{array}{l}3729.03 \\
3736.78 \\
3744.73 \\
3755.82 \\
3758.45 \\
\end{array}$ & $\begin{array}{l}13 \\
13 \\
13 \\
13 \\
13 \\
\end{array}$ & & \\
\hline
\end{tabular}

Values for the $2 s^{2} 2 p-2 s 2 p^{2}$ transitions are taken from the calculations of Bolotin and Yutsis [1] who employ analytical one-electron wave functions and include configuration interaction with a relatively crude approximation. For these as well as the $2 s 2 p^{2}-2 p^{3}$ and $2 p-3 s$ transitions large uncertainties must be expected because they are very sensitive to the effects of configuration interaction.

For several other transitions Kelly's self-consistent field calculations [2, 3] (which include exchange effects) are available. In the case of the highly excited lines, they agree within a few percent with the results of the Coulomb approximation and the averaged values are adopted.

\section{References}

[1] Bolotin, A. B., and Yutsis, A. P., Zhur. Eksptl. i Teoret. Fiz. 24, 537-543 (1953). (Translated in "Optical Transition

Probabilities," Office of Technical Services, U.S. Department of Commerce, Washington. D.C.)

[2] Kelly, P. S., Astrophys. J. 140, 1247-1268 (1964).

[3] Kelly, P. S., J. Quant. Spectrosc. Radiat. Transfer 4, 117-148 (1964). 
O IV. Allowed Transitions

\begin{tabular}{|c|c|c|c|c|c|c|c|c|c|c|c|c|c|}
\hline No & $\begin{array}{c}\text { Transition } \\
\text { Array }\end{array}$ & Multiplet & $\lambda(\AA)$ & $E_{i}\left(\mathrm{~cm}^{-1}\right)$ & $E_{k}\left(\mathrm{~cm}^{-1}\right)$ & $g_{i}$ & $g_{k}$ & $\begin{array}{l}A_{k i}\left(10^{8}\right. \\
\left.\sec ^{-1}\right)\end{array}$ & $f_{i k}$ & $S$ (at.u.) & $\log g f$ & $\begin{array}{l}\text { Accu- } \\
\text { racy }\end{array}$ & Source \\
\hline \multirow[t]{3}{*}{1} & \multirow{10}{*}{$\begin{array}{l}2 s^{2} 2 p- \\
2 s\left({ }^{1} \mathrm{~S}\right) 2 p^{2}\end{array}$} & \multirow{3}{*}{$\begin{array}{c}{ }^{2} \mathrm{P}^{\circ}-{ }^{2} \mathrm{D} \\
(1 \mathrm{uv})\end{array}$} & 789.36 & 257.7 & 126942 & 6 & 10 & 9.5 & 0.15 & 2.3 & -0.05 & $\mathrm{E}$ & \\
\hline & & & 790.203 & 386.5 & 126936 & 4 & 6 & 9.6 & 0.13 & 1.4 & -0.27 & $\mathrm{E}$ & \\
\hline & & & $\begin{array}{l}787.710 \\
790.103\end{array}$ & $\begin{array}{r}0.0 \\
386.5\end{array}$ & $\begin{array}{l}126950 \\
126950\end{array}$ & $\begin{array}{l}2 \\
4\end{array}$ & $\begin{array}{l}4 \\
4\end{array}$ & $\begin{array}{l}8.0 \\
1.5\end{array}$ & & 0.77 & $\begin{array}{l}-0.53 \\
-1.24\end{array}$ & E & \\
\hline \multirow{3}{*}{2} & & \multirow{3}{*}{$\begin{array}{c}{ }^{2} \mathrm{P}^{\circ}-{ }^{2} \mathrm{~S} \\
(2 \mathrm{uv})\end{array}$} & & & & & & & & & & & \\
\hline & & & 609.35 & 257.7 & 164367 & 6 & 2 & 54 & 0.10 & 1.2 & -0.22 & $\mathrm{E}$ & \\
\hline & & & $\begin{array}{l}609.829 \\
608.395\end{array}$ & $\begin{array}{r}386.5 \\
0.0\end{array}$ & $\begin{array}{l}164367 \\
164367\end{array}$ & $\begin{array}{l}4 \\
2\end{array}$ & $\begin{array}{l}2 \\
2\end{array}$ & $\begin{array}{l}36 \\
18\end{array}$ & $\begin{array}{l}0.10 \\
0.10\end{array}$ & 0.80 & $\begin{array}{l}-0.40 \\
-0.70\end{array}$ & $\mathrm{E}$ & \\
\hline \multirow[t]{4}{*}{3} & & \multirow{4}{*}{$\begin{array}{c}{ }^{2} \mathrm{P}^{\circ}-{ }^{2} \mathrm{P} \\
(3 \mathrm{uv})\end{array}$} & 554.37 & 257.7 & 180644 & 6 & 6 & 83 & 0.38 & 4.2 & 0.36 & $\mathrm{E}$ & \\
\hline & & & 554.514 & 386.5 & 180725 & 4 & 4 & 68 & 0.31 & 2.3 & 0.10 & $\mathrm{E}$ & \\
\hline & & & 554.074 & 0.0 & 180481 & 2 & 2 & 55 & 0.2 & 0.93 & -0.29 & $\mathrm{E}$ & \\
\hline & & & $\begin{array}{l}555.262 \\
553.328\end{array}$ & $\begin{array}{r}386.5 \\
0.0\end{array}$ & 180481 & 4 & 2 & 28 & 0.6 & 0.47 & -0.59 & $\mathrm{E}$ & \\
\hline \multirow{4}{*}{4} & \multirow{4}{*}{$2 s 2 p^{2}-2 p^{3}$} & \multirow{4}{*}{$\begin{array}{c}{ }^{4} \mathrm{P}-{ }^{4} \mathrm{~S}^{\circ} \\
(6 \mathrm{uv})\end{array}$} & & & & & & & & & & & \\
\hline & & & 023.41 & {$[11369]$} & {$[23126]$} & 12 & 4 & 12 & 0.14 & 0.5 & 0.23 & $\mathrm{E}$ & \\
\hline & & & 625.852 & [71493] & [231275] & 6 & 4 & 37 & 0.15 & 1.8 & -0.06 & $\mathrm{E}$ & \\
\hline & & & $\begin{array}{l}624.130 \\
624.617\end{array}$ & [71177] & [231275] & 2 & 4 & $\begin{array}{l}25 \\
12\end{array}$ & $\begin{array}{l}0.15 \\
0.14\end{array}$ & $\begin{array}{l}1.2 \\
0.58\end{array}$ & $\begin{array}{l}-0.23 \\
-0.55\end{array}$ & $\stackrel{\mathrm{E}}{\mathrm{E}}$ & \\
\hline \multirow[t]{3}{*}{5} & \multirow[t]{3}{*}{$2 p-\left({ }^{1} \mathrm{~S}\right) 3 s$} & \multirow{3}{*}{$\begin{array}{c}{ }^{2} \mathrm{P}^{\circ}-{ }^{2} \mathrm{~S} \\
(4 \mathrm{uv})\end{array}$} & 279.83 & 257.7 & 357615 & 6 & 2 & 130 & 0.050 & 0.28 & -0.52 & $\mathrm{E}$ & \\
\hline & & & 279.937 & 386.5 & 357615 & 4 & 2 & 85 & 0.050 & 0.1 & -0.70 & $\mathrm{E}$ & \\
\hline & & & 279.633 & 0.0 & 357615 & 2 & 2 & 43 & 0.050 & 0.092 & -1.00 & $\mathrm{E}$ & \\
\hline \multirow[t]{4}{*}{6} & \multirow[t]{4}{*}{$2 p-\left({ }^{1} \mathrm{~S}\right) 3 d$} & \multirow{4}{*}{$\begin{array}{c}{ }^{2} \mathrm{P}^{\circ}-{ }^{2} \mathrm{D} \\
(5 \mathrm{uv})\end{array}$} & 238.50 & 257.7 & 419544 & 6 & 10 & 350 & 0.50 & 2.4 & 0.48 & $\mathrm{D}-$ & \\
\hline & & & 238.573 & 386.5 & 419550 & 4 & 6 & 350 & 0.45 & 1.4 & 0.26 & D- & \\
\hline & & & 238.361 & 0.0 & 419534 & 2 & 4 & 300 & 0.5 & 0. & 0.0 & D- & \\
\hline & & & [238.58] & 386.5 & 419534 & 4 & 4 & 59 & 0.050 & 0.16 & -0.70 & $\mathrm{D}-$ & \\
\hline \multirow[t]{3}{*}{7} & \multirow[t]{3}{*}{$3 s-\left({ }^{1} \mathrm{~S}\right) 3 p$} & \multirow{3}{*}{$\begin{array}{c}{ }^{2} \mathrm{~S}-{ }^{2} \mathrm{P}^{\circ} \\
\text { (1) }\end{array}$} & 3066.2 & 357615 & 390219 & 2 & 6 & 1.48 & 0.62 & 12.6 & 0.096 & C & 3, \\
\hline & & & 3063.46 & 357615 & 390248 & 2 & 4 & 1.48 & 0.416 & 8.4 & -0.079 & C & \\
\hline & & & 3071.66 & 357615 & 390161 & 2 & 2 & 1.47 & 0.208 & 4.20 & -0 & $\mathrm{C}$ & \\
\hline \multirow[t]{8}{*}{8} & \multirow{14}{*}{$\begin{array}{l}2 s 2 p 3 s- \\
\left.2 s^{(}{ }^{\circ} \mathrm{P}^{\circ}\right) 2 p 3 p\end{array}$} & ${ }^{4} \mathrm{P}^{\circ}-{ }^{4} \mathrm{D}$ & 3374.3 & [438698] & [468325] & 12 & 20 & 1.07 & 0.303 & 40.4 & 0.56 & C & \\
\hline & & & 3385.55 & [438589] & [468499] & 6 & 8 & 1.06 & 0.242 & 16.2 & 0.162 & C & \\
\hline & & & 3381.28 & [438724] & {$[468290]$} & 4 & 6 & 0.7 & 0.1 & 8. & -0.1 & $\mathrm{C}$ & \\
\hline & & & 3381.33 & [438971] & [468154] & 2 & 4 & 0.4 & 0.1 & 3. & -0 & C & \\
\hline & & & & [438589] & & 6 & 6 & & & & & C & \\
\hline & & & & {$[438971]$} & [468075] & 2 & 2 & & & 3. & & C & \\
\hline & & & & [438589] & [468154] & 6 & 4 & 0.0 & 0.0 & 0.404 & -1.4 & $\mathrm{C}$ & \\
\hline & & & 3405.97 & [438724] & [468075] & 4 & 2 & 0.172 & 0.0149 & 0.67 & -1.224 & $\mathrm{C}$ & \\
\hline 9 & & ${ }^{2} \mathrm{P}^{\circ}-{ }^{2} \mathrm{D}$ & 3350.7 & 452985 & 482821 & 6 & 10 & 1.23 & 0.346 & 22.9 & 0.317 & C & \\
\hline & & & 3349.11 & 453073 & 482923 & 4 & 6 & 1.2 & 0.3 & 13.7 & 0.094 & $\mathrm{C}$ & \\
\hline & & & $\begin{array}{l}3348.08 \\
3378.09\end{array}$ & $\begin{array}{l}452808 \\
453073\end{array}$ & 482668 & 2 & 4 & 1.8 & & 7.6 & -0.1 & C. & \\
\hline & & & 356.09 & 453013 & 482000 & & 4 & 0.201 & 0.0344 & & -1 & $\mathrm{C}$ & \\
\hline 10 & & ${ }^{2} \mathrm{P}^{\circ}-{ }^{2} \mathrm{~S}$ & 2505.8 & 452985 & 492880 & 6 & 2 & 3.04 & 0.095 & 4.72 & -0.243 & C & \\
\hline & & & $\begin{array}{l}{[2511.4]} \\
{[2494.8]}\end{array}$ & $\begin{array}{l}453073 \\
452808\end{array}$ & $\begin{array}{l}492880 \\
492880\end{array}$ & $\begin{array}{l}4 \\
2\end{array}$ & 2 & 2.01 & 0.095 & 3.15 & $\begin{array}{l}-0.420 \\
-072\end{array}$ & C & \\
\hline 11 & & & 3490.9 & 518688 & 547326 & 6 & 10 & 000 & 0301 & 20 & 257 & 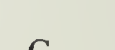 & \\
\hline & $2 s 2 p\left({ }^{1} \mathrm{P}^{\circ}\right) 3 p^{\prime}$ & (14) & & J10000 & 347020 & 0 & & 0.99 & 0.501 & 20.8 & 0.257 & $\mathrm{C}$ & \\
\hline & & & 3489.84 & 518690 & 547336 & 4 & 6 & 0.99 & 0.272 & 12.5 & 0.037 & C & \\
\hline & & & [3492.2] & 518684 & $\begin{array}{l}547311 \\
547311\end{array}$ & \begin{tabular}{|l}
2 \\
4
\end{tabular} & $\begin{array}{l}4 \\
4\end{array}$ & $\begin{array}{l}0.82 \\
0.165\end{array}$ & 0.0302 & $\begin{array}{l}6.9 \\
1.39\end{array}$ & $\begin{array}{l}-0.222 \\
-0.92\end{array}$ & $\begin{array}{l}\mathrm{C} \\
\mathrm{C}\end{array}$ & \\
\hline
\end{tabular}


O IV. Allowed Transitions-Continued

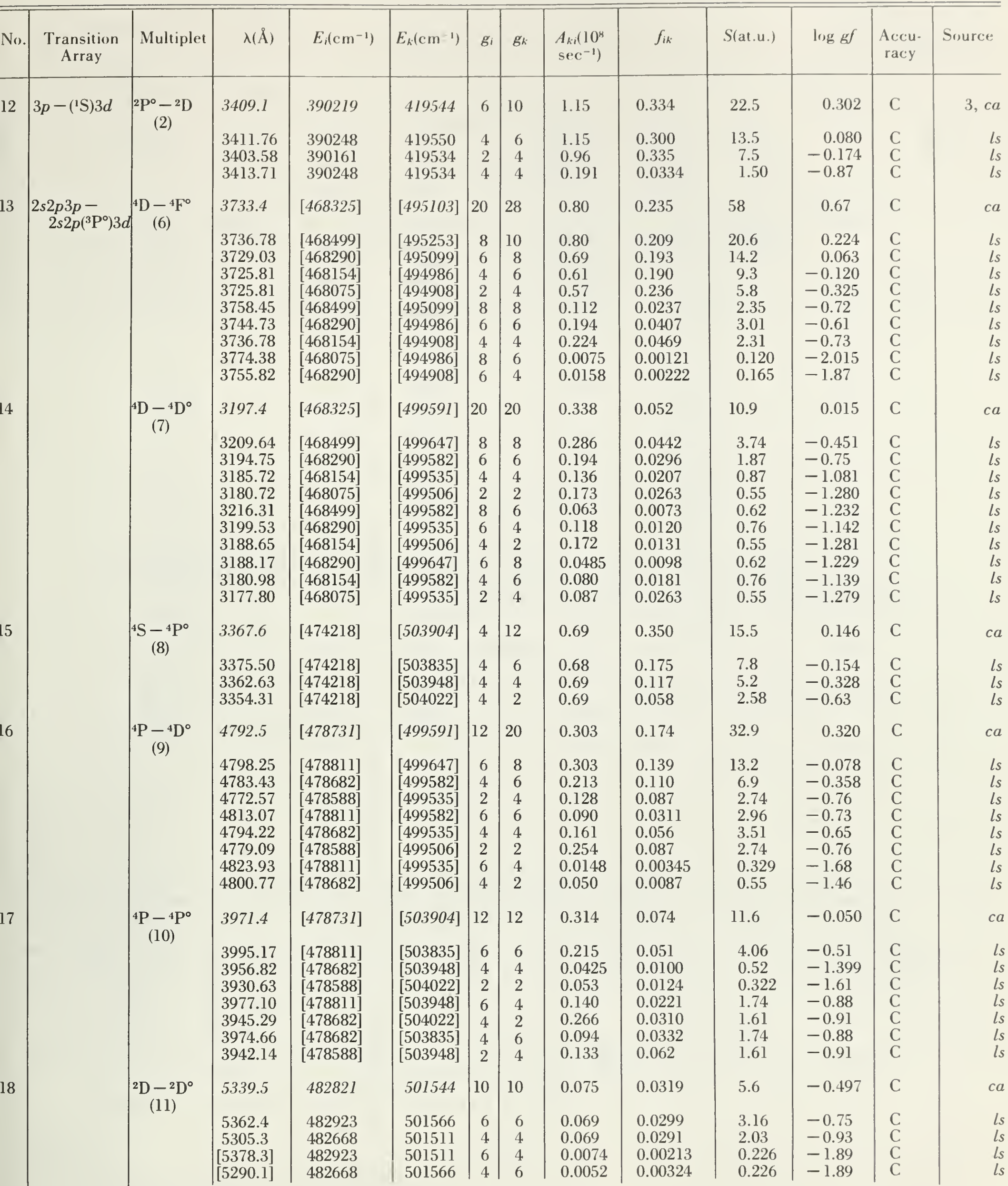


O IV. Allowed Transitions-Continued

\begin{tabular}{|c|c|c|c|c|c|c|c|c|c|c|c|c|c|}
\hline No. & $\begin{array}{c}\text { Transition } \\
\text { Array }\end{array}$ & Multiplet & $\lambda(\AA)$ & $E_{i}\left(\mathrm{~cm}^{-1}\right)$ & $E_{k}\left(\mathrm{~cm}^{-1}\right)$ & $g_{i}$ & $g_{k}$ & $\begin{array}{l}A_{k i}\left(10^{8}\right. \\
\left.\sec ^{-1}\right)\end{array}$ & $f_{i k}$ & $S$ (at.u.) & $\log g f$ & $\begin{array}{l}\text { Accu- } \\
\text { racy }\end{array}$ & Source \\
\hline \multirow[t]{3}{*}{19} & & ${ }^{2} \mathrm{D}-{ }^{2} \mathrm{~F}^{\circ}$ & 3563.0 & 482821 & 510879 & 10 & 14 & 1.15 & 0.306 & 35.9 & 0.486 & C & $c a$ \\
\hline & & & 3563.36 & 482923 & 510979 & 6 & 8 & 1.15 & 0.291 & 20.5 & 0.242 & $\mathrm{C}$ & \\
\hline & & & $\begin{array}{c}3560.42 \\
{[3593.1]}\end{array}$ & $\begin{array}{l}482668 \\
482923\end{array}$ & $\begin{array}{l}510746 \\
510746\end{array}$ & $\begin{array}{l}4 \\
6\end{array}$ & $\begin{array}{l}6 \\
6\end{array}$ & $\begin{array}{l}1.08 \\
0.075\end{array}$ & $\begin{array}{l}0.307 \\
0.0145\end{array}$ & $\begin{array}{l}14.4 \\
1.03\end{array}$ & $\begin{array}{r}0.089 \\
-1.060\end{array}$ & $\begin{array}{l}\mathrm{C} \\
\mathrm{C}\end{array}$ & $l_{s}$ \\
\hline \multirow[t]{2}{*}{20} & & ${ }^{2} \mathrm{~S}-{ }^{2} \mathrm{P}^{\circ}$ & 4674.4 & 492880 & 514267 & 2 & 6 & 0.297 & 0.292 & 9.0 & -0.234 & C & $c a$ \\
\hline & & & [4685.4] & 492880 & 514217 & 2 & 4 & 0.295 & 0.194 & 6.0 & -0.411 & C & $l$ \\
\hline 21 & $5 f-\left({ }^{1} \mathrm{~S}\right) 6 d$ & $\begin{array}{c}{ }^{2} \mathrm{~F}^{\circ}-{ }^{2} \mathrm{D} \\
(15)\end{array}$ & 4568 & 552490 & [574375] & 14 & 10 & 0.124 & 0.0278 & 5.9 & -0.410 & $\mathrm{C}$ & $c c$ \\
\hline
\end{tabular}

\section{Forbidden Transitions}

Naqvi's calculation [1] of the one possible transition in the ground state configuration $2 p$ is the only available source. The line strength should be quite accurate, since it does not sensitively depend on the choice of the interaction parameters.

\section{Reference}

[1] Naqvi, A. M., Thesis Harvard (1951).

O IV. Forbidden Transitions

\begin{tabular}{|c|c|c|c|c|c|c|c|c|c|c|c|c|}
\hline No. & $\begin{array}{c}\text { Transition } \\
\text { Array }\end{array}$ & Multiplet & $\lambda(\AA)$ & $E_{i}\left(\mathrm{~cm}^{-1}\right)$ & $E_{k}\left(\mathrm{~cm}^{-1}\right)$ & $g_{i}$ & $g_{k}$ & $\begin{array}{c}\text { Type of } \\
\text { Transi- } \\
\text { tion }\end{array}$ & $A_{k i}\left(\sec ^{-1}\right)$ & S(at.u.) & $\begin{array}{l}\text { Accu- } \\
\text { racy }\end{array}$ & Source \\
\hline 1 & $2 p-2 p$ & ${ }^{2} \mathrm{P}^{\circ}-{ }^{2} \mathrm{P}^{\circ}$ & {$\left[25.87 \times 10^{4}\right]$} & 0 & 386.5 & 2 & 4 & $\mathrm{~m}$ & $5.18 \times 10^{-4}$ & 1.33 & B & 1 \\
\hline
\end{tabular}




\section{Allowed Transitions}

List of tabulated lines:

\begin{tabular}{l|l||l|l||l|l}
\hline \hline Wavelength $[\AA]$ & No. & Wavelength $[\AA]$ & No. & Wavelength $[\AA]$ & No. \\
\hline 172.168 & 2 & 3230 & 18 & 5114 & 10 \\
192.751 & 8 & 3239 & 12 & 5343 & 21 \\
192.80 & 8 & 3245 & 18 & 5352 & 21 \\
192.800 & 8 & 3249 & 18 & 5376 & 21 \\
192.906 & 8 & 3264 & 18 & 5417 & 21 \\
192.91 & 8 & 3275.67 & 12 & 5432 & 21 \\
192.92 & 8 & 3298 & 18 & 5473 & 21 \\
215.034 & 6 & 3692 & 17 & 5573 & 15 \\
215.104 & 6 & 3701 & 17 & 5582 & 15 \\
215.245 & 6 & 3703 & 17 & 5584 & \\
220.352 & 9 & 3717 & 17 & 5600 & 15 \\
248.459 & 7 & 3726 & 17 & 5606 & 15 \\
629.732 & 1 & 3747 & 17 & 5608 & 22 \\
758.677 & 3 & 3762 & 17 & 6329 & 20 \\
759.440 & 3 & 4120 & 11 & 6767 & 20 \\
760.229 & 3 & 4121.7 & 19 & 6790 & 20 \\
760.445 & 3 & 4123 & 11 & 6819 & 20 \\
761.131 & 3 & 4123.90 & 11 & 6830 & 20 \\
762.001 & 3 & 4135.9 & 19 & 6878 & \\
774.522 & 5 & 4151 & 11 & 6909 & \\
1371.29 & 4 & 4158.76 & 19 & 7438 & \\
3058.68 & 13 & 4179 & 11 & & \\
3144.68 & 14 & 4211 & 11 & & \\
3222 & 12 & 4522 & 23 & & \\
3222 & 18 & 4554.28 & 16 & & \\
\hline
\end{tabular}

Values for the $2 s^{2}-2 s 2 p$ and $2 s 2 p-2 p^{2}$ transition arrays are taken from the self-consistent field calculations of Weiss [1]. These calculations do not include the important effects of configuration interaction; hence large uncertainties must be expected. The average of the dipole length and velocity approximations is adopted [1]. Accuracies within 50 percent are indicated by the following comparison: Weiss [1] has undertaken refined calculations, including configuration interaction, for the same transitions in Be I-the first member of this isoelectronic sequence-in addition to calculations of the type done for this ion. In all cases the agreement with the average of the dipole length and velocity approximations is close.

For the remaining low-lying transitions Kelly's approximate Hartree-Fock calculations [2] are adopted, while for the moderately excited transitions Kelly's values are averaged with the Coulomb approximation, with which they agree quite well.

\section{References}

[1] Weiss, A. W., private communication (1964).

[2] Kelly, P. S., J. Quant. Spectrosc. Radiat. Transfer 4, 117-148 (1964). 
Ov. Allowed Transitions

\begin{tabular}{|c|c|c|c|c|c|c|c|c|c|c|c|c|c|}
\hline No. & $\begin{array}{c}\text { Transition } \\
\text { Array }\end{array}$ & Multiplet & $\lambda(\AA)$ & $E_{i}\left(\mathrm{~cm}^{-1}\right)$ & $E_{k}\left(\mathrm{~cm}^{-1}\right)$ & $g_{i}$ & $g_{k}$ & $A_{k i}\left(10^{8} \sec ^{-1}\right)$ & $f_{i k}$ & $S$ (at.u.) & $\log g f$ & $\begin{array}{c}\text { Accu- } \\
\text { racy }\end{array}$ & Source \\
\hline 1 & 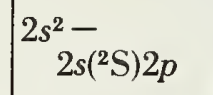 & $\begin{array}{l}{ }^{1} S-{ }^{-1} \mathbf{P}^{\circ} \\
(1 \text { uv })\end{array}$ & 629.732 & 0 & 158798 & 1 & 3 & 30 & 0.53 & 1.1 & -0.28 & D & 1 \\
\hline 2 & 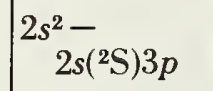 & $\begin{array}{l}{ }^{1} \mathrm{~S}-{ }^{-1} \mathrm{P}^{\circ} \\
(2 \mathrm{uv})\end{array}$ & 172.168 & 0 & 580826 & 1 & 3 & 450 & 0.59 & 0.34 & -0.23 & D & 2 \\
\hline \multirow[t]{5}{*}{3} & \multirow[t]{5}{*}{$2 s 2 p-2 p^{2}$} & \multirow{5}{*}{$\begin{array}{c}{ }^{3} \mathbf{P}^{\circ}-3 \mathbf{P} \\
(3 \mathrm{uv})\end{array}$} & 760.36 & [82413] & [213929] & 9 & 9 & 21 & 0.18 & 4.1 & 0.21 & D & 1 \\
\hline & & & $\begin{array}{l}760.445 \\
760229\end{array}$ & {$[82564]$} & {$[214066]$} & $\begin{array}{l}5 \\
3 \\
\end{array}$ & 5 & 16 & 0.14 & 1.7 & -0.17 & $\mathrm{D}$ & ls \\
\hline & & & 762.001 & [83564] & [213797] & $\begin{array}{l}3 \\
5\end{array}$ & $\begin{array}{l}3 \\
3\end{array}$ & $\begin{array}{l}5.2 \\
8.6\end{array}$ & $\begin{array}{l}0.045 \\
0.045\end{array}$ & $\begin{array}{l}. .34 \\
0.56\end{array}$ & $\begin{array}{l}-0.87 \\
-0.65\end{array}$ & $\begin{array}{l}\mathrm{D} \\
\mathrm{D}\end{array}$ & $\begin{array}{l}l s \\
l s\end{array}$ \\
\hline & & & 761.131 & [82258] & [213642] & 3 & 1 & 21 & 0.060 & 0.45 & -0.75 & D & ls \\
\hline & & & $\begin{array}{l}758.677 \\
759.440\end{array}$ & $\begin{array}{l}{[82258]} \\
{[82121]}\end{array}$ & $\begin{array}{l}{[214066]} \\
{[213797]}\end{array}$ & $\begin{array}{l}3 \\
1\end{array}$ & $\begin{array}{l}5 \\
3\end{array}$ & $\begin{array}{l}5.2 \\
6.9\end{array}$ & $\begin{array}{l}0.075 \\
0.18\end{array}$ & $\begin{array}{l}0.56 \\
0.45\end{array}$ & $\begin{array}{l}-0.65 \\
-0.74\end{array}$ & $\begin{array}{l}\mathrm{D} \\
\mathrm{D}\end{array}$ & $l s$ \\
\hline 4 & & $\begin{array}{c}{ }^{1 P^{\circ}-{ }^{1} D} \\
(7 \text { uv })\end{array}$ & $\mid 1371.29$ & 158798 & 231722 & 3 & 5 & 6.7 & 0.32 & 4.3 & -0.03 & D & 1 \\
\hline 5 & & $\begin{array}{c}{ }^{1} \mathrm{P}^{\circ}-1 \mathrm{~S} \\
(8 \mathrm{uv})\end{array}$ & 774.522 & 158798 & 287909 & 3 & 1 & 21 & 0.062 & 0.48 & -0.73 & D & 1 \\
\hline \multirow[t]{2}{*}{6} & \multirow[t]{3}{*}{$\begin{array}{l}2 s 2 p- \\
2 s\left({ }^{2} \mathrm{~S}\right) 3 s\end{array}$} & \multirow[t]{2}{*}{$\begin{array}{c}{ }^{3} \mathrm{P}^{\circ}-{ }^{3} \mathrm{~S} \\
(4 \mathrm{uv})\end{array}$} & 215.18 & [82413] & [547150] & 9 & 3 & 211 & 0.0488 & 0.311 & -0.358 & C & 2 \\
\hline & & & $\begin{array}{l}215.245 \\
215.104 \\
215.034\end{array}$ & $\begin{array}{l}{[82564]} \\
{[82258]} \\
{[82121]}\end{array}$ & $\begin{array}{l}{[547150]} \\
{[547150]} \\
{[547150]}\end{array}$ & $\begin{array}{l}5 \\
3 \\
1\end{array}$ & $\begin{array}{l}3 \\
3 \\
3\end{array}$ & $\begin{array}{l}117 \\
71 \\
23.5\end{array}$ & $\begin{array}{l}0.0488 \\
0.0490 \\
0.0489\end{array}$ & $\begin{array}{l}0.173 \\
0.104 \\
0.0346\end{array}$ & $\begin{array}{l}-0.61 \\
-0.83 \\
-1.311\end{array}$ & $\begin{array}{l}\mathrm{C} \\
\mathrm{C} \\
\mathrm{C}\end{array}$ & $\begin{array}{l}l s \\
l s \\
l s\end{array}$ \\
\hline 7 & & $\begin{array}{c}{ }^{1} \mathrm{P}^{\circ}-{ }^{-1} \mathrm{~S} \\
(9 \mathrm{uv})\end{array}$ & 248.459 & 158798 & 561278 & 3 & 1 & 137 & 0.0424 & 0.104 & -0.90 & C & 2 \\
\hline \multirow[t]{5}{*}{8} & \multirow{5}{*}{$\begin{array}{l}2 s 2 p- \\
2 s\left({ }^{2} \mathrm{~S}\right) 3 d\end{array}$} & \multirow[t]{5}{*}{$\begin{array}{c}{ }^{3} \mathrm{P}^{\circ}-{ }^{3} \mathrm{D} \\
(5 \mathrm{uv})\end{array}$} & 192.85 & [82413] & [600943] & 9 & 15 & 680 & 0.64 & 3.63 & 0.76 & C & 2 \\
\hline & & & $\begin{array}{l}192.906 \\
192.800\end{array}$ & $\begin{array}{l}{[82564]} \\
{[82258]}\end{array}$ & $\begin{array}{l}{[600956]} \\
{[600936]}\end{array}$ & $\begin{array}{l}5 \\
3\end{array}$ & $\begin{array}{l}7 \\
5\end{array}$ & $\begin{array}{l}680 \\
510\end{array}$ & $\begin{array}{l}0.53 \\
0.478\end{array}$ & $\begin{array}{l}1.69 \\
0.91\end{array}$ & $\begin{array}{l}0.425 \\
0.157\end{array}$ & $\begin{array}{l}\mathrm{C} \\
\mathrm{C}\end{array}$ & $l s$ \\
\hline & & & 192.751 & [82121] & [600926] & 1 & 3 & 380 & 0.64 & 0.403 & -0.197 & $\mathrm{C}$ & $l s$ \\
\hline & & & [192.91] & {$[82564]$} & [600936] & 5 & 5 & 171 & 0.095 & 0.303 & -0.321 & $\mathrm{C}$ & $l s$ \\
\hline & & & $\begin{array}{l}{[192.80]} \\
{[192.92]}\end{array}$ & $\begin{array}{l}{[82258]} \\
{[82564]}\end{array}$ & [600926] & $\begin{array}{l}3 \\
5\end{array}$ & $\begin{array}{l}3 \\
3\end{array}$ & $\begin{array}{l}286 \\
19.0\end{array}$ & $\begin{array}{l}0.159 \\
0.0064\end{array}$ & $\begin{array}{l}0.303 \\
0.0202\end{array}$ & $\begin{array}{l}-0.321 \\
-1.498\end{array}$ & $\begin{array}{l}\mathrm{C} \\
\mathrm{C}\end{array}$ & $\begin{array}{l}l s \\
l s\end{array}$ \\
\hline 9 & & $\begin{array}{l}{ }^{1 P^{\circ}-1}-{ }^{1} D \\
(10 \text { uv })\end{array}$ & 220.352 & 158798 & 612617 & 3 & 5 & 458 & 0.56 & 1.21 & 0.222 & C & 2 \\
\hline 10 & $\begin{array}{l}2 s 3 s- \\
2 s\left({ }^{2} S\right) 3 p\end{array}$ & $\begin{array}{c}{ }^{1} \mathrm{~S}-{ }^{-1} \mathrm{P}^{\circ} \\
(\mathrm{l})\end{array}$ & 5114 & 547150 & 580826 & 1 & 3 & 0.253 & 0.298 & 5.01 & -0.526 & $\mathrm{C}+$ & 2, $c a$ \\
\hline \multirow[t]{5}{*}{11} & \multirow[t]{5}{*}{$\begin{array}{l}2 p 3 s- \\
2 p\left({ }^{2} \mathrm{P}^{\circ}\right) 3 p\end{array}$} & \multirow[t]{5}{*}{$\begin{array}{l}{ }^{3} \mathrm{P}^{\circ}-{ }^{3} \mathrm{D} \\
(4)\end{array}$} & 4130.4 & [653435] & [677639] & 9 & 15 & 0.483 & 0.206 & 25.2 & 0.268 & C & $c a$ \\
\hline & & & $\begin{array}{l}4123.90 \\
4120\end{array}$ & $\begin{array}{l}{[653605]} \\
{[653262]}\end{array}$ & $\begin{array}{l}{[677847]} \\
{[677532]}\end{array}$ & $\begin{array}{l}5 \\
3\end{array}$ & $\begin{array}{l}7 \\
5\end{array}$ & $\begin{array}{l}0.487 \\
0.365\end{array}$ & $\begin{array}{l}0.174 \\
0.155\end{array}$ & $\begin{array}{r}11.8 \\
6.3\end{array}$ & $\begin{array}{l}-0.061 \\
-0.333\end{array}$ & $\begin{array}{l}\mathrm{C} \\
\mathrm{C}\end{array}$ & $\begin{array}{l}l s \\
l s\end{array}$ \\
\hline & & & 4123 & [653100] & {$[677333]$} & 1 & 3 & 0.270 & 0.206 & 2.80 & -0.69 & C & $l s$ \\
\hline & & & $\begin{array}{l}4179 \\
4151\end{array}$ & [653605] & $\begin{array}{l}{[677532]} \\
{[677333]}\end{array}$ & $\begin{array}{l}5 \\
3\end{array}$ & $\begin{array}{l}5 \\
3\end{array}$ & $\begin{array}{l}0.117 \\
0.198\end{array}$ & $\begin{array}{l}0.0305 \\
0.051\end{array}$ & $\begin{array}{l}2.10 \\
2.10\end{array}$ & $\begin{array}{l}-0.82 \\
-0.81\end{array}$ & $\begin{array}{l}\mathrm{C} \\
\mathrm{C}\end{array}$ & $\begin{array}{l}l s \\
l s\end{array}$ \\
\hline & & & 4211 & [653605] & [677333] & $\begin{array}{l}0 \\
5\end{array}$ & $\begin{array}{l}3 \\
3\end{array}$ & 0.0127 & 0.00202 & 0.140 & -1.99 & C & $l s$ \\
\hline \multirow[t]{3}{*}{12} & & \multirow{3}{*}{${ }^{3} \mathrm{P}^{\circ}-{ }^{3} \mathrm{~S}$} & 3257.5 & [653435] & [684124] & 9 & 3 & 1.01 & 0.053 & 5.2 & -0.318 & C & $c a$ \\
\hline & & & $\begin{array}{l}3275.67 \\
3239\end{array}$ & [653605] & [684124] & 5 & $\begin{array}{l}3 \\
3\end{array}$ & 0.5 & 0.053 & 2.87 & $\begin{array}{l}-0.57 \\
-070\end{array}$ & $\mathrm{C}$ & $l s$ \\
\hline & & & $\mid \begin{array}{l}3259 \\
3222\end{array}$ & [653100] & [684124] & $\begin{array}{l}3 \\
1\end{array}$ & $\begin{array}{l}3 \\
3\end{array}$ & $\begin{array}{l}0.342 \\
0.116\end{array}$ & $\begin{array}{l}0.054 \\
0.054\end{array}$ & 0.57 & -1.268 & $\mathrm{C}$ & $l s$ \\
\hline 13 & & ${ }^{1} \mathrm{P}^{\circ}-{ }^{-1} \mathrm{D}$ & \begin{tabular}{|l|}
3058.68 \\
\end{tabular} & 664486 & 697170 & 3 & 5 & 1.30 & 0.305 & 9.2 & -0.039 & C & $c a$ \\
\hline 14 & $\mid \begin{array}{l}2 s 3 p- \\
2 s\left({ }^{2} \mathrm{~S}\right) 3 d\end{array}$ & $\begin{array}{c}{ }^{1} \mathrm{P}^{\circ}-{ }^{1} \mathrm{D} \\
\text { (2) }\end{array}$ & | 3144.68 & 580826 & 612617 & $3 \mid$ & 5 & 1.05 & 0.258 & 8.02 & -0.111 & $\mathrm{C}+$ & $2, c a$ \\
\hline
\end{tabular}


Ov. Allowed Transitions-Continued

\begin{tabular}{|c|c|c|c|c|c|c|c|c|c|c|c|c|c|}
\hline No. & $\begin{array}{c}\text { Transition } \\
\text { Array }\end{array}$ & Multiplet & $\lambda(\AA \AA)$ & $E_{i}\left(\mathrm{~cm}^{-1}\right)$ & $E_{k}\left(\mathrm{~cm}^{-1}\right)$ & $g_{i}$ & $g_{k}$ & $A_{k i}\left(10^{*} \sec ^{-1}\right)$ & $f_{i k}$ & $S$ (at.u.) & $\log g f$ & $\begin{array}{l}\text { Accu. } \\
\text { racy }\end{array}$ & Source \\
\hline \multirow[t]{5}{*}{15} & & ${ }^{3} \mathrm{P}^{\circ}-{ }^{3} \mathrm{D}$ & 5590.0 & [583059] & [600943] & 9 & 15 & 0.176 & 0.138 & 22.8 & 0.093 & $\mathrm{C}+$ & $2, c a$ \\
\hline & & & 5600 & [583097] & [600956] & 5 & 7 & 0.175 & 0.115 & 10.6 & -0.240 & $\mathrm{C}+$ & 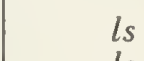 \\
\hline & & & $\begin{array}{l}5582 \\
5573\end{array}$ & $\begin{array}{l}{[583020]} \\
{[582984]}\end{array}$ & $\begin{array}{l}{[600936]} \\
{[600926]}\end{array}$ & $\begin{array}{l}3 \\
1\end{array}$ & $\begin{array}{l}5 \\
3\end{array}$ & $\mid \begin{array}{l}0.133 \\
0.0987\end{array}$ & & $\begin{array}{l}5.70 \\
2.53\end{array}$ & $\begin{array}{l}-0.508 \\
-0.861\end{array}$ & $\begin{array}{l}\mathrm{C}+ \\
\mathrm{C}+\end{array}$ & $\begin{array}{l}l s \\
l s\end{array}$ \\
\hline & & & 5606 & [583097] & [600936] & 5 & 5 & 0.0437 & 0.0206 & 1.90 & -0.987 & $\mathrm{C}+$ & \\
\hline & & & $\begin{array}{l}5584 \\
5608\end{array}$ & {$[583020]$} & [600926] & 3 & $\begin{array}{l}3 \\
2\end{array}$ & 0.0737 & & 1. & -0.986 & $\begin{array}{l}\mathrm{C}+ \\
\mathrm{C}+\end{array}$ & $l_{s}$ \\
\hline 16 & & & 45 & & & & & & & & & & \\
\hline & $\begin{array}{l}2 p 3 p- \\
2 p\left({ }^{2} \mathrm{P}^{\circ}\right) 3 d\end{array}$ & (7) & 4554.28 & 672965 & 694646 & 3 & 5 & 0.233 & 0.121 & 5.4 & -0.440 & $\mathrm{C}$ & $\mathrm{ca}$ \\
\hline & & ${ }^{3} \mathrm{D}-{ }^{3} \mathrm{D}^{\circ}$ & 3727.5 & [667639] & [704459] & 15 & 15 & 0.155 & 0.0323 & 6.0 & -0.314 & C & c \\
\hline & & & 3747 & [677847] & [704527] & 7 & 7 & 0.136 & 0.0286 & 2.47 & -0.70 & C & \\
\hline & & & 3717 & [677532] & [704424] & 5 & 5 & 0.109 & 0.0226 & 1.38 & -0.95 & C & 1 \\
\hline & & & $\begin{array}{l}3701 \\
3762\end{array}$ & $\begin{array}{l}{[677333]} \\
{[677847]}\end{array}$ & {$[704360]$} & \begin{tabular}{|l}
3 \\
7
\end{tabular} & $\begin{array}{l}3 \\
5\end{array}$ & $\begin{array}{l}0.119 \\
0.0235\end{array}$ & $\begin{array}{l}44 \\
356\end{array}$ & $\begin{array}{l}0.89 \\
0.309\end{array}$ & $\begin{array}{l}-1.135 \\
-1.60\end{array}$ & $\begin{array}{l}\mathrm{C} \\
\mathrm{C}\end{array}$ & l. \\
\hline & & & 3726 & [677532] & [704360] & 5 & 3 & 0.0388 & 0.00484 & 0.297 & -1.62 & $\mathrm{C}$ & \\
\hline & & & $\begin{array}{l}3703 \\
3692\end{array}$ & {$[677532]$} & [704527] & $\begin{array}{l}5 \\
3\end{array}$ & 7 & 0.0 & 0.0051 & 0.309 & -1.60 & C & \\
\hline \multirow{6}{*}{18} & & & & & & & & & & & - 10 & & \\
\hline & & $\begin{array}{c}{ }^{3} \mathrm{D}-{ }^{-3} \mathrm{P}^{\circ} \\
(9)\end{array}$ & 3268.4 & [677639] & [708226] & 15 & 9 & 0.0264 & 0.00254 & 0.410 & -1.419 & C & $c a$ \\
\hline & & & $\begin{array}{l}3298 \\
3249\end{array}$ & $\begin{array}{l}{[677847]} \\
{[677532]}\end{array}$ & $\begin{array}{l}\text { [708154] } \\
{[708296]}\end{array}$ & $\begin{array}{l}7 \\
5\end{array}$ & $\begin{array}{l}5 \\
3\end{array}$ & $\begin{array}{l}0.0216 \\
0.0201\end{array}$ & $\mid \begin{array}{l}0.00251 \\
0.00191\end{array}$ & $\begin{array}{l}0.191 \\
0.102\end{array}$ & $\begin{array}{l}-1.75 \\
-2.021\end{array}$ & C & ls \\
\hline & & & $\begin{array}{l}5249 \\
3222\end{array}$ & [677333] & {$[708379]$} & \begin{tabular}{|l}
0 \\
3
\end{tabular} & $\begin{array}{l}3 \\
1\end{array}$ & $\begin{array}{l}0.0201 \\
0.0276\end{array}$ & 0.00143 & $\begin{array}{l}0.102 \\
0.0455\end{array}$ & \begin{tabular}{|l}
-2.021 \\
-2.368
\end{tabular} & $\stackrel{\mathrm{C}}{\mathrm{C}}$ & ls \\
\hline & & & 3264 & [677532] & [708154] & 5 & 5 & 0.00397 & $6.3 \times 10^{-4}$ & 0.0341 & -2.499 & C & ls \\
\hline & & & $\begin{array}{l}3230 \\
3245\end{array}$ & $\begin{array}{l}{[677333]} \\
{[677333]}\end{array}$ & $\begin{array}{l}{[708296]} \\
{[708379]}\end{array}$ & $\begin{array}{l}3 \\
3\end{array}$ & $\begin{array}{l}3 \\
5\end{array}$ & $\begin{array}{l}0.0068 \\
2.70 \times 10^{-4}\end{array}$ & $\mid \begin{array}{l}0.00107 \\
7.1 \times 10^{-5}\end{array}$ & $\begin{array}{l}0.0341 \\
0.00228\end{array}$ & $\begin{array}{l}-2.494 \\
-3.67\end{array}$ & $\begin{array}{l}\mathrm{C} \\
\mathrm{C}\end{array}$ & $\begin{array}{l}l s \\
l s\end{array}$ \\
\hline \multirow[t]{4}{*}{19} & & ${ }^{3} \mathrm{~S}-{ }^{3} \mathrm{P}^{\circ}$ & 4147.9 & [684124] & [708226] & 3 & 9 & 0.259 & 0.200 & 8.2 & -0.222 & C & $c a$ \\
\hline & & & 4158.76 & [684124] & [708154] & 3 & 5 & 0.257 & 0.1 & 4.56 & -0.478 & $\mathrm{C}$ & \\
\hline & & & $\begin{array}{l}4135.9 \\
41917\end{array}$ & [684124] & [708296] & 3 & 3 & 0.261 & 0.8 & 2.73 & -0.70 & C & ls \\
\hline & & & 4.121 .7 & [684.124] & [708379] & 3 & 1 & 0.264 & 0.0224 & 0.91 & -1.173 & C & ls \\
\hline \multirow[t]{6}{*}{20} & & $\begin{array}{l}{ }^{3} \mathrm{P}-{ }^{-3} \mathrm{D}^{\circ} \\
(12)\end{array}$ & 6816.6 & [689793] & [704459] & 9 & 15 & 0.075 & 0.088 & 17.7 & -0.103 & C & $c a$ \\
\hline & & & 6830 & [689890] & [704527] & 5 & 7 & 0.075 & 0.073 & 8.3 & -0.435 & C & \\
\hline & & & $\begin{array}{l}6790 \\
6767\end{array}$ & $\begin{array}{l}{[689700]} \\
{[689586]}\end{array}$ & $\begin{array}{l}{[704.424]} \\
{[704360]}\end{array}$ & $\begin{array}{l}3 \\
1\end{array}$ & $\begin{array}{l}5 \\
3\end{array}$ & $\begin{array}{l}0.057 \\
0.0430\end{array}$ & $\mid \begin{array}{l}0.066 \\
0.088\end{array}$ & $\begin{array}{l}4.42 \\
1.97\end{array}$ & $\begin{array}{l}-0.70 \\
-1.053\end{array}$ & $\begin{array}{l}\mathrm{C} \\
\mathrm{C}\end{array}$ & ls \\
\hline & & & 6878 & [689890] & [704424] & 5 & 5 & 0.0183 & & & -1.188 & $\mathrm{C}$ & t \\
\hline & & & 6819 & [689700] & [704360] & 3 & 3 & 0.03 & 0.0218 & 1.4 & -1.184 & C & so \\
\hline & & & 6909 & [689890] & [704360] & 5 & 3 & 0.00201 & $8.6 \times 10^{-4}$ & 0.098 & -2.364 & C & is \\
\hline \multirow[t]{6}{*}{21} & & $\begin{array}{l}{ }^{3} \mathrm{P}-{ }^{3} \mathrm{P}^{\circ} \\
(13)\end{array}$ & 5423.6 & [689793] & [708226] & 9 & 9 & 0.87 & 0.384 & 62 & 0.54 & C & $c a$ \\
\hline & & & 5473 & [689890] & [708154] & 5 & 5 & 0.6 & 0.285 & 25.7 & 0.154 & C & ls \\
\hline & & & 5376 & [689700] & [708296] & 3 & 3 & 0.2 & & 5.1 & & C & ls \\
\hline & & & $\begin{array}{l}5432 \\
5352\end{array}$ & $\begin{array}{l}{[689890]} \\
{[689700]}\end{array}$ & $\begin{array}{l}{[708296]} \\
{[708379]}\end{array}$ & $\begin{array}{l}5 \\
3\end{array}$ & 3 & 0.361 & 0.096 & $\begin{array}{l}8.6 \\
6.9\end{array}$ & $\begin{array}{l}-0.320 \\
-0.410\end{array}$ & C & $l s$ \\
\hline & & & 5417 & {$[689700]$} & [708154] & 3 & $\frac{1}{5}$ & $\begin{array}{l}0.91 \\
0.218\end{array}$ & $\begin{array}{l}0.130 \\
0.160\end{array}$ & 8.6 & -0.318 & $\mathrm{C}$ & 15 \\
\hline & & & 534 & [689586] & [708296] & 1 & 3 & 0.304 & 0.390 & 6.9 & -0.409 & C & $l s$ \\
\hline 22 & & $\begin{array}{c}{ }^{1} \mathrm{D}-{ }^{1} \mathrm{~F}^{\circ} \\
(14)\end{array}$ & 6329 & 697170 & 712967 & 5 & 7 & 0.136 & 0.114 & 11.9 & -0.243 & C & $c a$ \\
\hline 23 & & $\begin{array}{c}{ }^{1} \mathrm{D}-{ }^{1} \mathrm{P}^{\circ} \\
(15)\end{array}$ & 4522 & 697170 & 719277 & 5 & 3 & 0.0110 & 0.00203 & 0.151 & -1.99 & C & $c a$ \\
\hline 24 & $\begin{array}{l}2 s 4 s- \\
\quad 2 s\left({ }^{2} \mathrm{~S}\right) 4 p\end{array}$ & $\begin{array}{c}{ }^{3} \mathrm{~S}-{ }^{-3} \mathrm{P}^{\circ} \\
(17)\end{array}$ & 7438 & [722666] & [736107] & 3 & 9 & 0.287 & 0.715 & 52.5 & 0.331 & $\mathrm{C}+$ & $2, c a$ \\
\hline
\end{tabular}




\section{Forbidden Transitions}

Naqvi's calculations [1] are the only available source. The results for the ${ }^{3} \mathrm{P}^{\circ}-{ }^{3} \mathrm{P}^{\circ}$ transitions are essentially independent of the choice of the interaction parameters. For the ${ }^{3} \mathrm{P}^{\circ}-{ }^{1} \mathrm{P}^{\circ}$ transitions, Naqvi has used empirical term intervals, i.e., the effects of configuration interaction should be partially included.

\section{Reference}

[1] Naqvi, A. M., Thesis Harvard (1951).

Ov. Forbidden Transitions

\begin{tabular}{|c|c|c|c|c|c|c|c|c|c|c|c|c|}
\hline No. & $\begin{array}{c}\text { Transition } \\
\text { Array }\end{array}$ & Multiplet & $\lambda(\AA)$ & $E_{i}\left(\mathrm{~cm}^{-1}\right)$ & $E_{k}\left(\mathrm{~cm}^{-1}\right)$ & $g_{i}$ & $g_{k}$ & $\begin{array}{l}\text { Type of } \\
\text { Transition }\end{array}$ & $A_{k i}\left(\sec ^{-1}\right)$ & $S$ (at.u.) & Accuracy & Source \\
\hline 1 & $\begin{array}{l}2 s 2 p- \\
\quad 2 s\left({ }^{2} \mathrm{~S}\right) 2 p\end{array}$ & ${ }^{3} \mathrm{P}^{\circ}-{ }^{3} \mathrm{P}^{\circ}$ & $\begin{array}{l}{\left[73.13 \times 10^{4}\right]} \\
{\left[32.65 \times 10^{4}\right]}\end{array}$ & $\begin{array}{l}{[82121.2]} \\
{[82257.9]}\end{array}$ & $\begin{array}{l}{[82257.9]} \\
{[82564.1]}\end{array}$ & $\begin{array}{l}1 \\
3\end{array}$ & $\begin{array}{l}3 \\
5\end{array}$ & $\begin{array}{l}\mathrm{m} \\
\mathrm{m}\end{array}$ & $\begin{array}{l}4.60 \times 10^{-5} \\
3.87 \times 10^{-4}\end{array}$ & $\begin{array}{l}2.00 \\
2.50\end{array}$ & $\begin{array}{l}\mathrm{B} \\
\mathrm{B}\end{array}$ & 1 \\
\hline 2 & & ${ }^{3} \mathrm{P}^{\circ}-{ }^{1} \mathrm{P}^{\circ}$ & $\begin{array}{l}{[1304.2]} \\
{[1306.5]} \\
{[1311.8]}\end{array}$ & $\begin{array}{l}{[82121.2]} \\
{[82257.9]} \\
{[82564.1]}\end{array}$ & $\begin{array}{l}158798 \\
158798 \\
158798\end{array}$ & $\begin{array}{l}1 \\
3 \\
5\end{array}$ & $\begin{array}{l}3 \\
3 \\
3\end{array}$ & $\begin{array}{l}\mathrm{m} \\
\mathrm{m} \\
\mathrm{m}\end{array}$ & $\begin{array}{c}0.064 \\
16.9 \\
0.078\end{array}$ & $\begin{array}{l}1.57 \times 10^{-5} \\
0.00420 \\
1.96 \times 10^{-5}\end{array}$ & $\begin{array}{l}\mathrm{C} \\
\mathrm{C} \\
\mathrm{C}\end{array}$ & 1 \\
\hline
\end{tabular}

\section{O VI}

Ground State

Ionization Potential
$1 s^{2} 2 s^{2} \mathrm{~S}_{1 / 2}$

$138.080 \mathrm{eV}=1113999.5 \mathrm{~cm}^{-1}$

\section{Allowed Transitions}

List of tabulated lines:

\begin{tabular}{l|c||c|c||c|c}
\hline \hline Wavelength $[\AA]$ & No. & Wavelength $[\AA]$ & No. & Wavelength $[\AA]$ & No. \\
\hline 129.786 & 5 & 1031.95 & 1 & 4751 & \\
129.87 & 5 & 1037.63 & 1 & 5112 & 14 \\
129.872 & 5 & 3068 & 8 & 5279 & 16 \\
150.088 & 2 & 3314 & 10 & 5298 & 19 \\
150.124 & 2 & 3426 & 12 & 5410 & 17 \\
172.935 & 4 & 3438 & 13 & 5602 & 15 \\
173.082 & 4 & 3509 & 11 & 11744 & 7 \\
173.09 & 4 & 3622 & 9 & 11892 & 7 \\
183.937 & 3 & 3811.35 & 6 & 11964 & 7 \\
\hline 184.117 & 3 & 3834.24 & 6 & & \\
\hline
\end{tabular}

The values taken from Weiss' calculations [1] are estimated to be accurate to within $10 \%$ because of the very close agreement between his dipole length and dipole velocity approximations. The values calculated with the length approximation are adopted. The Coulomb approximation should be quite reliable for the highly excited transitions and is given perference over Kelly's approximate Hartree-Fock calculations [2], with which it sometimes disagrees.

\section{References}

[1] Weiss, A. W., Astrophys. J. 138, 1262-1276 (1963).

[2] Kelly, P. S., J. Quant. Spectrosc. Radiat. Transfer 4, 117-148 (1964). 
O VI. Allowed Transitions

\begin{tabular}{|c|c|c|c|c|c|c|c|c|c|c|c|c|}
\hline $\begin{array}{c}\text { Transition } \\
\text { Array }\end{array}$ & Multiplet & $\lambda(\AA)$ & $E_{i}\left(\mathrm{~cm}^{-1}\right)$ & $E_{k}\left(\mathrm{~cm}^{-1}\right)$ & $g_{i}$ & $g_{k}$ & $\begin{array}{l}A_{k i}\left(10^{4}\right. \\
\left.\sec ^{-1}\right)\end{array}$ & $f_{i k}$ & $S($ at.u.) & $\log g f$ & $\begin{array}{l}\text { Accu- } \\
\text { racy }\end{array}$ & Source \\
\hline \multirow[t]{3}{*}{$2 s-2 p$} & ${ }^{2} \mathrm{~S}-{ }^{2} \mathrm{P}^{\circ}$ & 1033.8 & 0.0 & 96730 & 2 & 6 & 4.08 & 0.196 & 1.33 & -0.407 & A & 1 \\
\hline & & 1031.95 & 0.0 & 96908 & 2 & 4 & 4.09 & 0.131 & 0.887 & -0.583 & A & ls \\
\hline & & 1037.63 & 0.0 & 96375 & 2 & 2 & 4.02 & 0.0648 & 0.443 & -0.887 & A & ls \\
\hline \multirow[t]{2}{*}{$2 s-3 p$} & ${ }^{2} \mathrm{~S}-{ }^{2} \mathrm{P}^{0}$ & 150.10 & 0.0 & 666218 & 2 & 6 & 259 & 0.262 & 0.259 & -0.281 & $\mathrm{~B}+$ & 1 \\
\hline & & $\begin{array}{l}150.088 \\
150.124\end{array}$ & $\begin{array}{l}0.0 \\
0.0\end{array}$ & $\begin{array}{l}666270 \\
666113\end{array}$ & $\begin{array}{l}2 \\
2\end{array}$ & $\begin{array}{l}4 \\
2\end{array}$ & $\begin{array}{l}259 \\
259\end{array}$ & $\begin{array}{l}0.175 \\
0.0874\end{array}$ & $\begin{array}{l}0.173 \\
0.0864\end{array}$ & $\begin{array}{l}-0.456 \\
-0.757\end{array}$ & $\mathrm{~B}+$ & $l s$ \\
\hline \multirow[t]{3}{*}{$2 p-3 s$} & ${ }^{2} \mathrm{P}^{\circ}-{ }^{2} \mathrm{~S}$ & 184.06 & 96730 & 640040 & 6 & 2 & 170 & 0.0287 & 0.104 & -0.764 & $\mathrm{~B}+$ & 1 \\
\hline & & 184.117 & 96908 & 640040 & 4 & 2 & 113 & 0.0287 & 0.093 & -0.939 & $B+$ & ls \\
\hline & & | 100.206 & & 040040 & 2 & 2 & & 0.0208 & 0.0046 & -1.241 & & is \\
\hline \multirow[t]{4}{*}{$2 p-3 d$} & ${ }^{2} \mathrm{P}^{\circ}-{ }^{2} \mathrm{D}$ & 173.03 & 96730 & 674657 & 6 & 10 & 884 & 0.662 & 2.26 & 0.599 & $B+$ & 1 \\
\hline & & 173.082 & 96908 & 674677 & 4 & 6 & 886 & 0.597 & 1.36 & 0.378 & $B+$ & ls \\
\hline & & 172.935 & 96375 & 674626 & 2 & 4 & 737 & 0.661 & 0.753 & 0.121 & $\mathrm{~B}+$ & ls \\
\hline & & [173.09] & 96908 & 674626 & 4 & 4 & 147 & 0.0662 & 0.151 & -0.577 & $\mathrm{~B}+$ & ls \\
\hline \multirow[t]{3}{*}{$2 p-4 d$} & ${ }^{2} \mathrm{P}^{\circ}-{ }^{2} \mathrm{D}$ & 129.84 & 96730 & 866893 & 6 & 10 & 287 & 0.121 & 0.310 & -0.139 & B & $c a$ \\
\hline & & 129.872 & 96908 & 866902 & 4 & 6 & 285 & 0.108 & 0.185 & -0.364 & B & $l s$ \\
\hline & & $\begin{array}{r}129.786 \\
{[129.87]}\end{array}$ & $\begin{array}{l}96375 \\
96908\end{array}$ & $\begin{array}{l}866880 \\
866880\end{array}$ & \begin{tabular}{|l|}
2 \\
4
\end{tabular} & $\begin{array}{l}4 \\
4\end{array}$ & $\begin{array}{c}239 \\
47.6\end{array}$ & $\begin{array}{l}0.121 \\
0.0120\end{array}$ & $\begin{array}{l}0.103 \\
0.0206\end{array}$ & $\begin{array}{l}-0.618 \\
-1.317\end{array}$ & $\stackrel{B}{B}$ & ls \\
\hline \multirow[t]{3}{*}{$3 s-3 p$} & ${ }^{2} \mathrm{~S}-{ }^{2} \mathrm{P}^{\circ}$ & 3818.9 & 640040 & 666218 & 2 & 6 & 0.510 & 0.335 & 8.41 & -0.175 & B & l \\
\hline & & 3811.35 & 640040 & 666270 & 2 & 4 & 0.513 & 0.224 & 5.61 & -0.350 & B & $l s$ \\
\hline & & 3834.24 & 640040 & 666113 & 2 & 2 & 0.503 & 0.111 & 2.80 & -0.654 & B & ls \\
\hline \multirow[t]{4}{*}{$3 p-3 d$} & ${ }^{2} \mathrm{P}^{0}-{ }^{2} \mathrm{D}$ & 11847 & 666218 & 674657 & 6 & 10 & 0.0137 & 0.0481 & 11.3 & -0.540 & B & l \\
\hline & & [11892] & 666270 & 674677 & 4 & 6 & 0.0136 & 0.0433 & 6.78 & -0.762 & B & ls \\
\hline & & [11744] & 666113 & 674626 & 2 & 4 & 0.0118 & 0.0488 & 3.7 & -1.0 & B & $l s$ \\
\hline & & [111964] & 666270 & 674626 & 4 & 4 & 0.00223 & 0.00478 & 0.753 & -1.719 & B & ls \\
\hline $6 s-7 p$ & $\begin{array}{c}{ }^{2} S-{ }^{2} \mathrm{P}^{\circ} \\
(2)\end{array}$ & 3068 & 1000080 & 1032630 & 2 & 6 & 0.874 & 0.370 & 7.47 & -0.131 & B & $c a$ \\
\hline $6 p-7 s$ & ${ }^{2} \mathrm{P}^{\circ}-{ }^{2} \mathrm{~S}$ & 3622 & 1003130 & 1030780 & 6 & 2 & 2.72 & 0.178 & 12.7 & 0.029 & B & $c a$ \\
\hline $6 p-7 d$ & ${ }^{2} \mathrm{P}^{\circ}-{ }^{2} \mathrm{D}$ & 3314 & 1003130 & 1033324 & 6 & 10 & 2.02 & 0.554 & 36.3 & 0.522 & B & $c a$ \\
\hline $6 d-7 p$ & ${ }^{2} \mathrm{D}-{ }^{2} \mathrm{P}^{\circ}$ & 3509 & 1004178 & 1032630 & 10 & 6 & 0.860 & 0.0952 & 11.0 & -0.021 & B & $c a$ \\
\hline $6 d-7 f$ & ${ }^{2} \mathrm{D}-{ }^{2} \mathrm{~F}^{\circ}$ & 3426 & 1004178 & [1033382] & 10 & 14 & 3.34 & 0.824 & \begin{tabular}{|l}
92.9 \\
\end{tabular} & 0.916 & B & $c a$ \\
\hline $6 f-7 d$ & ${ }^{2} \mathrm{~F}^{\circ}-{ }^{2} \mathrm{D}$ & 3438 & [1004265] & 1033324 & 14 & 10 & 0.337 & 0.0426 & 6.75 & -0.225 & B & $\mathrm{ca}$ \\
\hline $7 s-8 p$ & ${ }^{2} \mathrm{~S}-{ }^{2} \mathrm{P}^{\circ}$ & 4751 & 1030780 & 1051724 & 2 & 6 & 0.423 & 0.429 & 13.4 & -0.067 & B & $\mathrm{ca}$ \\
\hline & $(1)$ & & & & & & & & & & & \\
\hline $7 p-8 s$ & $2 \mathrm{P}^{\circ}-{ }^{2} \mathrm{~S}$ & 5602 & 1032630 & [1050543] & 6 & 2 & 1.38 & 0.216 & 23.9 & 0.113 & B & $c a$ \\
\hline
\end{tabular}


O VI. Allowed Transitions - Continued

\begin{tabular}{|c|c|c|c|c|c|c|c|c|c|c|c|c|c|}
\hline No. & $\begin{array}{c}\text { Transition } \\
\text { Array }\end{array}$ & Multiplet & $\lambda(\AA)$ & $E_{i}\left(\mathrm{~cm}^{-1}\right)$ & $E_{k}\left(\mathrm{~cm}^{-1}\right)$ & $g_{i}$ & $g_{k}$ & $\begin{array}{l}A_{k i}\left(10^{8}\right. \\
\left.\sec ^{-1}\right)\end{array}$ & $f_{i k}$ & $S$ (at.u.) & $\log g f$ & $\begin{array}{l}\text { Accu- } \\
\text { racy }\end{array}$ & Source \\
\hline 16 & $7 p-8 d$ & $\begin{array}{c}{ }^{2} \mathrm{P}^{\circ}-{ }^{2} \mathrm{D} \\
(12)\end{array}$ & 5112 & 1032630 & 1052296 & 6 & 10 & 0.923 & 0.603 & 60.9 & 0.559 & B & $c a$ \\
\hline 17 & $7 d-8 p$ & $\begin{array}{c}{ }^{2} \mathrm{D}-{ }^{-2} \mathrm{P}^{\circ} \\
(13)\end{array}$ & 5410 & 1033324 & 1051724 & 10 & 6 & 0.491 & 0.129 & 23.0 & 0.111 & B & $c a$ \\
\hline 18 & $7 d-8 f$ & $\begin{array}{c}{ }^{2} \mathrm{D}-{ }^{-2} \mathrm{~F}^{\circ} \\
(14)\end{array}$ & 5279 & 1033324 & {$[1052280]$} & 10 & 14 & 1.64 & 0.960 & 167 & 0.982 & B & $c a$ \\
\hline 19 & $7 f-8 d$ & $\begin{array}{c}{ }^{2} \mathrm{~F}^{\circ}-{ }^{2} \mathrm{D} \\
(15)\end{array}$ & 5298 & [1033382] & 1052296 & 14 & 10 & 0.255 & 0.0766 & 18.7 & 0.030 & B & 2 \\
\hline
\end{tabular}

\section{O VIII}

Ground State

Ionization Potential
$1 s^{2} \mathrm{~S}_{0}$

$739.114 \mathrm{eV}=5963000 \mathrm{~cm}^{-1}$

\section{Allowed Transitions}

The results of extensive non-relativistic variational calculations by Weiss [1] are used. Values have been calculated in both the dipole length and dipole velocity approximations and agree to within 1 percent, except for the $3 p^{1} \mathrm{P}^{\circ}-3 d^{1} \mathrm{D}$ transition where agreement is not as good. The average of the two approximations is adopted [1].

Reference

[1] Weiss, A. W., private communication (1964).

O VII. Allowed Transitions

\begin{tabular}{r|c|c|c|c|c|c|c|c|c|c|c|c|c}
\hline \hline No. & $\begin{array}{c}\text { Transition } \\
\text { Array }\end{array}$ & Multiplet & $\lambda(\AA)$ & $E_{i}\left(\mathrm{~cm}^{-1}\right)$ & $E_{k}\left(\mathrm{~cm}^{-1}\right)$ & $g_{i}$ & $g_{k}$ & $A_{k i}\left(10^{8} \mathrm{sec}^{-1}\right)$ & $f_{i k}$ & $S($ at. u. $)$ & $\log g f$ & $\begin{array}{c}\text { Accu- } \\
\text { racy }\end{array}$ & $\begin{array}{l}\text { Source } \\
1\end{array}$ \\
\hline & $1 s^{2}-1 s 2 p$ & ${ }^{1} \mathrm{~S}-{ }^{1} \mathrm{P}^{\circ}$ & {$[21.602]$} & 0 & 4629200 & 1 & 3 & 33000 & 0.694 & 0.0494 & -0.158 & $\mathrm{~A}$ & 1 \\
2 & $1 s^{2}-1 s 3 p$ & ${ }^{1} \mathrm{~S}-{ }^{1} \mathrm{P}^{\circ}$ & {$[18.627]$} & 0 & 5368550 & 1 & 3 & 9370 & 0.146 & 0.00897. & -0.835 & $\mathrm{~A}$ & 1 \\
3 & $1 s 2 s-1 s 2 p$ & ${ }^{1} \mathrm{~S}-{ }^{1} \mathrm{P}^{\circ}$ & {$[2475.4]$} & {$[4588814]$} & 4629200 & 1 & 3 & 0.246 & 0.0679 & 0.553 & -1.168 & $\mathrm{~A}$ & 1 \\
4 & $1 s 2 s-1 s 3 p$ & ${ }^{1} \mathrm{~S}-{ }^{1} \mathrm{P}^{\circ}$ & {$[128.25]$} & {$[4588814]$} & 5368550 & 1 & 3 & 504 & 0.373 & 0.158 & -0.428 & $\mathrm{~A}$ & 1 \\
5 & $1 s 2 p-1 s 3 d$ & ${ }^{1} \mathrm{P}^{\circ}-{ }^{1} \mathrm{D}$ & {$[135.77]$} & 4629200 & {$[5365734]$} & 3 & 5 & 1530 & 0.705 & 0.945 & 0.325 & $\mathrm{~A}$ & 1 \\
6 & $1 s 3 d-1 s 3 p$ & ${ }^{1} \mathrm{D}-{ }^{1} \mathrm{P}^{\circ}$ & {$[35500] ?$} & {$[5365734]$} & 5368550 & 5 & 3 & $6.99 \times 10^{-4}$ & 0.00792 & 4.62 & -1.402 & $\mathrm{C}+$ & 1 \\
7 & $1 s 2 s-1 s 2 p$ & ${ }^{3} \mathrm{~S}-{ }^{3} \mathrm{P}^{\circ}$ & {$[1630.3]$} & 4525270 & {$[4586610]$} & 3 & 9 & 0.794 & 0.0949 & 1.53 & -0.546 & $\mathrm{~A}$ & 1 \\
8 & $1 s 2 s-1 s 3 p$ & ${ }^{3} \mathrm{~S}-{ }^{3} \mathrm{P}^{\circ}$ & {$[120.33]$} & 4525270 & 5356300 & 3 & 9 & 533 & 0.347 & 0.413 & 0.018 & $\mathrm{~A}$ & 1 \\
9 & $1 s 2 p-1 s 3 d$ & ${ }^{3} \mathrm{P}^{\circ}-{ }^{3} \mathrm{D}$ & {$[128.46]$} & {$[4586610]$} & 5365070 & 9 & 15 & 1620 & 0.666 & 2.54 & 0.778 & $\mathrm{~A}$ & 1 \\
10 & $1 s 3 p-1 s 3 d$ & ${ }^{3} \mathrm{P}^{\circ}-{ }^{3} \mathrm{D}$ & {$[11399] ?$} & 5356300 & 5365070 & 9 & 15 & 0.0113 & 0.0367 & 12.4 & -0.481 & $\mathrm{~A}$ & 1 \\
\hline
\end{tabular}




\section{FLUORINE}

Ground State

Ionization Potential
F I.

$$
1 s^{2} 2 s^{2} 2 p^{5}{ }^{2} \mathrm{P}_{3 / 2}^{\circ}
$$

$17.418 \mathrm{eV}=140524.5 \mathrm{~cm}^{-1}$

Allowed Transitions

List of tabulated lines:

\begin{tabular}{l|l||c|c||c|c}
\hline \hline Wavelength $[\AA]$ & No. & Wavelength $[\AA]$ & No. & Wavelength $[\AA]$ & No. \\
\hline 6239.64 & 3 & 6909.82 & 2 & 7489.14 & \\
6348.50 & 3 & 6966.35 & 6 & 7514.93 & 1 \\
6413.66 & 3 & 7037.45 & 6 & 7552.24 & 1 \\
6708.27 & 2 & 7127.88 & 6 & 7573.41 & 1 \\
6773.97 & 2 & 7202.37 & 6 & 7607.17 & 4 \\
6795.52 & 2 & 7311.02 & 5 & 7754.70 & 4 \\
6834.26 & 2 & 7331.95 & 1 & 7800.22 & 4 \\
6856.02 & 2 & 7398.68 & 1 & & \\
680.22 & 2 & 7425.64 & 1 & & \\
6902.46 & 2 & 7482.72 & 1 & & \\
\hline
\end{tabular}

Since there are no numerical data available for this spectrum, values for the prominent lines have been calculated from the Coulomb approximation by Bates and Damgaard. This method is expected to give fairly reliable results as judged from other atomic systems of similar complexity, where comparison data have been available.

F I. Allowed Transitions

\begin{tabular}{|c|c|c|c|c|c|c|c|c|c|c|c|c|c|}
\hline No. & $\begin{array}{c}\text { Transition } \\
\text { Array }\end{array}$ & $\begin{array}{l}\text { Multi- } \\
\text { plet }\end{array}$ & $\lambda(\AA)$ & $E_{i}\left(\mathrm{~cm}^{-1}\right)$ & $E_{k}\left(\mathrm{~cm}^{-1}\right)$ & $g_{i}$ & $g_{k}$ & $\begin{array}{c}A_{k i}\left(10^{8}\right. \\
\left.\mathrm{sec}^{-1}\right)\end{array}$ & $f_{i k}$ & $S($ at.u.) & $\log g x$ & $\begin{array}{l}\text { Accu- } \\
\text { racy }\end{array}$ & Source \\
\hline \multirow[t]{8}{*}{1} & \multirow[t]{17}{*}{$\begin{array}{l}2 p^{4} 3 s- \\
2 p^{4}\left({ }^{3} \mathrm{P}\right) 3 p\end{array}$} & \multirow[t]{8}{*}{${ }_{(1)}^{4} \mathrm{P}-{ }^{4} \mathrm{P}^{\circ}$} & 7490.3 & 102571 & 115918 & 12 & 12 & 0.35 & 0.29 & 86 & 0.54 & D & $c a$ \\
\hline & & & 7398.68 & 102407 & 115919 & 6 & 6 & 0.25 & 0.21 & 30 & 0.09 & D & Is \\
\hline & & & 7482.72 & 102681 & 116042 & 4 & 4 & 0.047 & 0.039 & 3.9 & -0.81 & D & ls \\
\hline & & & 7514.93 & 102841 & 116144 & 2 & 2 & 0.058 & 0.049 & 2.4 & -1.01 & D & Is \\
\hline & & & 7331.95 & 102407 & 116042 & 6 & 4 & 0.17 & 0.089 & 13 & -0.27 & D & ls \\
\hline & & & 7425.64 & 102681 & 116144 & 4 & 2 & 0.30 & 0.12 & 12 & -0.31 & D & ls \\
\hline & & & 7552.24 & 102681 & 115919 & 4 & 6 & 0.10 & 0.13 & 13 & -0.28 & D & ls \\
\hline & & & 7573.41 & 102841 & 116042 & 2 & 4 & 0.14 & 0.24 & 12 & -0.31 & $\mathrm{D}$ & Is \\
\hline \multirow[t]{9}{*}{2} & & \multirow{9}{*}{${ }_{(2)}^{4} \mathrm{P}-{ }^{4} \mathrm{D}^{\circ}$} & 6859.2 & 102571 & 117146 & 12 & 20 & 0.45 & 0.53 & 140 & 0.80 & $\mathrm{D}$ & $c a$ \\
\hline & & & 6856.02 & 102407 & 116988 & 6 & 8 & 0.45 & 0.42 & 57 & 0.40 & D & ls \\
\hline & & & 6902.46 & 102681 & 117165 & 4 & 6 & 0.31 & 0.33 & 30 & 0.12 & D & is \\
\hline & & & 6909.82 & 102841 & 117309 & 2 & 4 & 0.18 & 0.26 & 12 & -0.28 & D & ls \\
\hline & & & 6773.97 & 102407 & 117165 & 6 & 6 & 0.14 & 0.095 & 13 & -0.24 & D & ls \\
\hline & & & 6834.26 & 102681 & 117309 & 4 & 4 & 0.24 & 0.17 & 15 & -0.17 & D & is \\
\hline & & & 6870.22 & 102841 & 117393 & 2 & 2 & 0.38 & 0.27 & 12 & -0.27 & $\mathrm{D}$ & is \\
\hline & & & 6708.27 & 102407 & 117309 & 6 & 4 & 0.024 & 0.011 & 1.4 & -1.19 & $\mathrm{D}$ & ls \\
\hline & & & 6795.52 & 102681 & 117393 & 4 & 2 & 0.077 & 0.027 & 2.4 & -0.97 & $\mathrm{D}$ & ls \\
\hline
\end{tabular}


Allowed Transitions - Continued

\begin{tabular}{|c|c|c|c|c|c|c|c|c|c|c|c|c|c|}
\hline No. & $\begin{array}{c}\text { Transition } \\
\text { Array }\end{array}$ & $\begin{array}{c}\text { Multi- } \\
\text { plet }\end{array}$ & $\lambda(\AA)$ & $E_{i}\left(\mathrm{~cm}^{-1}\right)$ & $E_{k}\left(\mathrm{~cm}^{-1}\right)$ & $g$ & $g_{k}$ & $\begin{array}{r}A_{k i}\left(10^{8}\right. \\
\left.\sec ^{-1}\right)\end{array}$ & $f_{i k}$ & $S$ (at.u.) & $\log g f$ & $\begin{array}{c}\text { Accu- } \\
\text { racy }\end{array}$ & Source \\
\hline \multirow[t]{4}{*}{3} & & ${ }^{4} \mathrm{P}-{ }^{4} \mathrm{~S}^{\circ}$ & 6304.2 & 102571 & 1184.29 & 12 & 4 & 0.56 & 0.11 & 28 & 0.12 & D & $c a$ \\
\hline & & & 6239.64 & 102407 & 118429 & 6 & 4 & 0.29 & 0.11 & 14 & -0.18 & D & $l s$ \\
\hline & & & 6348.50 & 102681 & 118429 & 4 & 4 & 0.18 & 0.11 & 9.3 & -0.35 & D & ls \\
\hline & & & 6413.66 & 102841 & 118429 & 2 & 4 & 0.090 & 0.11 & 4.7 & -0.65 & D & ls \\
\hline \multirow[t]{4}{*}{4} & & ${ }^{2} \mathrm{P}-{ }^{2} \mathrm{D}^{\circ}$ & 7759.4 & 104840 & 117724 & 6 & 10 & 0.35 & 0.53 & 80 & 0.50 & D & $c a$ \\
\hline & & & 7754.70 & 104732 & 117624 & 4 & 6 & 0.35 & 0.47 & 48 & 0.27 & D & ls \\
\hline & & & 7800.22 & 105057 & 117874 & 2 & 4 & 0.29 & 0.53 & 27 & 0.02 & D & ls \\
\hline & & & 7607.17 & 104732 & 117874 & 4 & 4 & 0.061 & 0.053 & 5.3 & -0.67 & D & $l s$ \\
\hline \multirow[t]{3}{*}{5} & & ${ }^{2} \mathrm{P}-{ }^{2} \mathrm{~S}^{\circ}$ & 7369.3 & 104840 & 118406 & 6 & 2 & 0.40 & 0.11 & 16 & -0.18 & D & $c a$ \\
\hline & & & 7311.02 & 104732 & 118406 & 4 & 2 & 0.27 & 0.11 & 11 & -0.36 & D & $l s$ \\
\hline & & & 7489.14 & 105057 & 118406 & 2 & 2 & 0.13 & 0.11 & 5.4 & -0.66 & D & $l s$ \\
\hline \multirow[t]{5}{*}{6} & & ${ }^{2} \mathrm{P}-{ }^{2} \mathrm{P}^{\circ}$ & 7067.02 & 104840 & 118986 & 6 & 6 & 0.46 & 0.34 & 48 & 0.31 & D & $c a$ \\
\hline & & & 7037.45 & 104732 & 118938 & 4 & 4 & 0.38 & 0.28 & 26 & 0.05 & D & $l s$ \\
\hline & & & 7127.88 & 105057 & 119083 & 2 & 2 & 0.30 & 0.23 & 11 & -0.35 & D & ls \\
\hline & & & 6966.35 & 104732 & 119083 & 4 & 2 & 0.16 & 0.056 & 5.2 & -0.65 & D & $l s$ \\
\hline & & & 7202.37 & 105057 & 118938 & 2 & 4 & 0.072 & 0.11 & 5.3 & -0.65 & D & ls \\
\hline
\end{tabular}

\section{Forbidden Transitions}

Naqvi's calculation [1] of the one possible transition in the ground state configuration $2 p^{5}$ is the only available source. The line strength should be quite accurate, since it does not sensitively depend on the choice of the interaction parameters.

Reference

[1] Naqvi, A. M., Thesis Harvard (1951).

F I. Forbidden Transitions

\begin{tabular}{|c|c|c|c|c|c|c|c|c|c|c|c|c|}
\hline No. & $\begin{array}{c}\text { Transition } \\
\text { Array }\end{array}$ & Multiplet & $\lambda(\AA)$ & $E_{i}\left(\mathrm{~cm}^{-1}\right)$ & $E_{k}\left(\mathrm{~cm}^{-1}\right)$ & $g_{i}$ & $g_{k}$ & $\begin{array}{l}\text { Type of } \\
\text { Transition }\end{array}$ & $A_{k i}\left(\mathrm{sec}^{-1}\right)$ & $S($ at. u. $)$ & $\begin{array}{l}\text { Accu- } \\
\text { racy }\end{array}$ & Source \\
\hline
\end{tabular}




\section{Allowed Transitions}

List of tabulated lines:

\begin{tabular}{|c|c|c|c|c|c|}
\hline Wavelength $[\AA]]$ & No. & Wavelength $[\AA]$ & No. & Wavelength $[\AA]$ & No. \\
\hline $\begin{array}{l}605.67 \\
606.27 \\
606.81 \\
606.95 \\
607.48 \\
\\
608.06 \\
3202.74 \\
3501.42 \\
3501.5 \\
3501.6 \\
3502.9 \\
3502.95 \\
3503.10 \\
3505.4 \\
3505.6 \\
3505.61\end{array}$ & $\begin{array}{l}1 \\
1 \\
1 \\
1 \\
1 \\
1 \\
7 \\
8 \\
8 \\
8 \\
8 \\
8 \\
8 \\
8 \\
8 \\
8\end{array}$ & $\begin{array}{l}3535.2 \\
3536.84 \\
3538.6 \\
3541.77 \\
3544.5 \\
3546.1 \\
3640.89 \\
3640.9 \\
3641.99 \\
3642.80 \\
3847.09 \\
3889.99 \\
3851.67 \\
4024.73 \\
4025.01 \\
4025.50\end{array}$ & $\begin{array}{r}5 \\
5 \\
5 \\
5 \\
\\
5 \\
5 \\
10 \\
10 \\
10 \\
10 \\
2 \\
2 \\
2 \\
3 \\
\\
3 \\
3\end{array}$ & $\begin{array}{l}4103.09 \\
4103.3 \\
4103.53 \\
4103.72 \\
4103.87 \\
4104.1 \\
4109.17 \\
4112.7 \\
4113.1 \\
4116.55 \\
4117.1 \\
4118.8 \\
4119.22 \\
4246.16 \\
4299.18 \\
4446.9\end{array}$ & $\begin{array}{r}9 \\
9 \\
9 \\
9 \\
9 \\
9 \\
4 \\
4 \\
4 \\
4 \\
4 \\
4 \\
4 \\
11 \\
6 \\
12\end{array}$ \\
\hline
\end{tabular}

For one of the strongest ultraviolet transitions a value calculated by Varsavsky [1] from a screening approximation is available and listed. Because the strong effects of configuration interaction have been neglected, this number should be quite uncertain. (In general, Varsavsky's values have a tendency to be too large.) Values obtained with the Coulomb approximation are presented for all prominent transitions in the visible and near ultraviolet. The indicated accuracies are estimated from extrapolation with equivalent transitions of $\mathrm{O} \mathrm{I}$, for which experimental and theoretical comparison data are available.

\section{Reference}

[1] Varsavsky, C. M., Astrophys. J. Suppl. Ser. 6, 75-108 (1961).

F II. Allowed Transitions

\begin{tabular}{|c|c|c|c|c|c|c|c|c|c|c|c|c|c|}
\hline No. & $\begin{array}{c}\text { Transition } \\
\text { Array }\end{array}$ & $\begin{array}{l}\text { Multi- } \\
\text { plet }\end{array}$ & $\lambda(\AA)$ & $E_{i}\left(\mathrm{~cm}^{-1}\right)$ & $E_{k}\left(\mathrm{~cm}^{-1}\right)$ & $g_{i}$ & $g_{k}$ & $\begin{array}{c}A_{k i}\left(10^{8}\right. \\
\left.\sec ^{-1}\right)\end{array}$ & $f_{i k}$ & $S($ at.u. $)$ & $\log g f$ & $\begin{array}{l}\text { Accu- } \\
\text { racy }\end{array}$ & Source \\
\hline \multirow[t]{7}{*}{1} & $2 s^{2} 2 p^{4}-$ & ${ }^{3} \mathrm{P}-{ }^{3} \mathrm{P}^{\circ}$ & 606.85 & 169 & 164955 & 9 & 9 & 100 & 0.56 & 10 & 0.70 & $\mathrm{E}$ & 1 \\
\hline & & & 606.81 & 0 & 164798 & 5 & 5 & 78 & 0.43 & 4.3 & 0.33 & $\mathrm{E}$ & Is \\
\hline & & & 606.95 & 342 & 165107 & 3 & 3 & 26 & 0.14 & 0.86 & -0.37 & $\mathrm{E}$ & Is \\
\hline & & & 605.67 & 0 & 165107 & 5 & 3 & 43 & 0.14 & 1.4 & -0.15 & $\mathrm{E}$ & Is \\
\hline & & & 606.27 & 342 & 165281 & 3 & 1 & 100 & 0.18 & $\overline{1} . \overline{1}$ & -0.26 & $\mathrm{E}$ & Is \\
\hline & & & 608.06 & 342 & 164798 & 3 & 5 & 25 & 0.23 & 1.4 & -0.16 & $\mathrm{E}$ & Is \\
\hline & & & 607.48 & 491 & 165107 & 1 & 3 & 33 & 0.55 & 1.1 & -0.26 & $\mathrm{E}$ & ls \\
\hline \multirow[t]{4}{*}{2} & $2 p^{3} 3 s-$ & ${ }^{5} \mathrm{~S}^{\circ}-{ }^{5} \mathrm{P}$ & 3848.9 & [176654] & {$[20 \overline{2628]}$} & 5 & 15 & 1.3 & 0.84 & 54 & 0.62 & $\mathrm{D}$ & $c a$ \\
\hline & & & 3847.09 & [176654] & {$[202641]$} & 5 & 7 & 1.3 & 0.39 & 25 & 0.29 & D & Is \\
\hline & & & 3849.99 & [176654] & [202621] & 5 & 5 & 1.3 & 0.28 & 18 & 0.15 & D & ls \\
\hline & & & 3851.67 & [176654] & {$[202610]$} & 5 & 3 & 1.3 & 0.17 & 11 & -0.08 & D & ls \\
\hline
\end{tabular}


F II. Allowed Transitions-Continued

\begin{tabular}{|c|c|c|c|c|c|c|c|c|c|c|c|c|c|}
\hline No. & $\begin{array}{c}\text { Transition } \\
\text { Array }\end{array}$ & $\begin{array}{l}\text { Multi- } \\
\text { plet }\end{array}$ & $\lambda(\AA)$ & $E_{i}\left(\mathrm{~cm}^{-1}\right)$ & $E_{k}\left(\mathrm{~cm}^{-1}\right)$ & $g_{i}$ & $g_{k}$ & $\begin{array}{r}A_{k i}\left(10^{8}\right. \\
\left.\sec ^{-1}\right)\end{array}$ & $f_{i k}$ & $S$ (at.u.) & $\log g f$ & $\begin{array}{c}\text { Accu- } \\
\text { racy }\end{array}$ & Source \\
\hline \multirow[t]{4}{*}{3} & & \multirow{4}{*}{$\begin{array}{c}{ }^{3} \mathrm{~S}^{\circ}{ }^{3} \mathrm{P} \\
(2)\end{array}$} & 4025.0 & 182865 & 207703 & 3 & 9 & 1.2 & 0.90 & 36 & 0.43 & D & $c a$ \\
\hline & & & 4024.73 & 182865 & 207705 & 3 & 5 & 1.2 & 0.50 & 20 & 0.18 & $\mathrm{D}$ & ls \\
\hline & & & 4025.50 & 182865 & 207700 & 3 & 3 & 1.2 & 0.30 & 12 & -0.04 & D & ls \\
\hline & & & 4025.01 & 182865 & 207703 & 3 & 1 & 1.2 & 0.10 & 4.0 & -0.52 & D & $l s$ \\
\hline \multirow[t]{7}{*}{4} & \multirow{7}{*}{$\begin{array}{l}2 p^{3} 3 s^{\prime}- \\
2 p^{3}\left({ }^{2} \mathrm{D}^{\circ}\right) 3 p^{\prime}\end{array}$} & \multirow{7}{*}{$\begin{array}{c}{ }^{3} \mathrm{D}^{\circ}-{ }^{3} \mathrm{D} \\
\quad(5)\end{array}$} & 4113.7 & 211881 & 236183 & 15 & 15 & 1.8 & 0.45 & 92 & 0.83 & D & $c a$ \\
\hline & & & 4109.17 & 211867 & 236196 & 7 & 7 & 1.6 & 0.40 & 38 & 0.45 & D & ls \\
\hline & & & 4116.55 & 211888 & 236173 & 5 & 5 & 1.2 & 0.32 & 21 & 0.20 & D & $l s$ \\
\hline & & & 4119.22 & 211901 & 236170 & 3 & 3 & 1.3 & 0.34 & 14 & 0.01 & D & $l s$ \\
\hline & & & [41113.1] & 211867 & 236173 & 7 & 5 & 0.28 & 0.050 & 4.8 & -0.45 & D & ls \\
\hline & & & [4117.1] & 211888 & 236170 & 5 & 3 & 0.45 & 0.068 & 4.6 & -0.47 & D & $l s$ \\
\hline & & & {$\left[\begin{array}{l}{[4112.7]} \\
{[4118.8]}\end{array}\right.$} & $\begin{array}{l}211888 \\
211901\end{array}$ & $\begin{array}{l}236196 \\
236173\end{array}$ & $\begin{array}{l}5 \\
3\end{array}$ & $\begin{array}{l}7 \\
5\end{array}$ & $\begin{array}{l}0.20 \\
0.27\end{array}$ & $\begin{array}{l}0.071 \\
0.11\end{array}$ & $\begin{array}{l}4.8 \\
4.6\end{array}$ & $\begin{array}{l}-0.45 \\
-0.47\end{array}$ & $\begin{array}{l}\mathrm{D} \\
\mathrm{D}\end{array}$ & $\begin{array}{l}l s \\
l s\end{array}$ \\
\hline \multirow[t]{7}{*}{5} & & \multirow{7}{*}{$\begin{array}{c}{ }^{3} \mathrm{D}^{\circ}-{ }^{3} \mathrm{P} \\
(6)\end{array}$} & 3539.8 & 211881 & 240123 & 15 & 9 & 2.1 & 0.23 & 40 & 0.54 & D & $c a$ \\
\hline & & & 3541.77 & 211867 & 240093 & 7 & 5 & 1.7 & 0.23 & 19 & 0.21 & D & ls \\
\hline & & & 3536.84 & 211888 & 240153 & 5 & 3 & 1.5 & 0.17 & 10 & -0.06 & D & ls \\
\hline & & & [3535.2] & 211901 & 240180 & 3 & 1 & 2.1 & 0.13 & 4.5 & -0.42 & D & ls \\
\hline & & & [3544.5] & 211888 & 240093 & 5 & 5 & 0.31 & 0.058 & 3.4 & -0.54 & D & ls \\
\hline & & & [3538.6] & $\begin{array}{l}211901 \\
211901\end{array}$ & 240153 & 3 & $\begin{array}{l}3 \\
5\end{array}$ & 0.51 & 0.096 & 3.4 & -0.54 & D & $l s$ \\
\hline & & & [3546.1] & 211901 & 240093 & 3 & 5 & 0.021 & 0.0064 & 0.23 & -1.71 & D & $l s$ \\
\hline 6 & & $\begin{array}{c}{ }^{1} \mathrm{D}^{\circ}-1 \mathrm{~F} \\
(7)\end{array}$ & 4299.18 & 215070 & 238324 & 5 & 7 & 1.7 & 0.64 & 45 & 0.51 & D & $c a$ \\
\hline 7 & & $\begin{array}{c}1 D^{\circ}-{ }^{-1} \mathrm{D} \\
(8)\end{array}$ & 3202.74 & 215070 & 246284 & 5 & 5 & 1.4 & 0.21 & 11 & 0.02 & D & $c a$ \\
\hline \multirow[t]{9}{*}{8} & \multirow{16}{*}{$\begin{array}{l}2 p^{3} 3 p- \\
\quad 2 p^{3}\left({ }^{4} \mathrm{~S}^{\circ}\right) 3 d\end{array}$} & \multirow{9}{*}{$\begin{array}{c}{ }^{5} \mathrm{P}-{ }^{-5} \mathrm{D}^{\circ} \\
(3)\end{array}$} & 3504.0 & [202628] & [231159] & 15 & 25 & 2.86 & 0.88 & 152 & 1.120 & C & $c a$ \\
\hline & & & 3505.61 & [202641] & [231158] & 7 & 9 & 2.86 & 0.68 & 55 & 0.68 & $\mathrm{C}$ & ls \\
\hline & & & 3503.10 & [202621] & [231159] & 5 & 7 & 1.91 & 0.491 & 28.3 & 0.390 & C & ls \\
\hline & & & [3501.6] & [202610] & [231160] & 3 & 5 & 1.00 & 0.307 & 10.6 & -0.036 & C & $l s$ \\
\hline & & & [3505.6] & [202641] & [231159] & 7 & 7 & 0.95 & 0.175 & 14.2 & 0.089 & C & ls \\
\hline & & & 3502.95 & [202621] & {$[231160]$} & 5 & 5 & 1.67 & 0.307 & 17.7 & 0.186 & C & ls \\
\hline & & & [3501.5] & $\begin{array}{l}{[202610]} \\
{[202641]}\end{array}$ & $\begin{array}{l}{[231161]} \\
{[231160]}\end{array}$ & $\begin{array}{l}3 \\
7\end{array}$ & $\begin{array}{l}3 \\
5\end{array}$ & $\begin{array}{l}2.15 \\
0.190\end{array}$ & $\begin{array}{l}0.395 \\
0.0251\end{array}$ & $\begin{array}{l}13.7 \\
2.02\end{array}$ & $\begin{aligned} & 0.074 \\
- & 0.76\end{aligned}$ & $\begin{array}{l}\mathrm{C} \\
\mathrm{C}\end{array}$ & $\begin{array}{l}l s \\
l s\end{array}$ \\
\hline & & & [3502.9] & [202621] & [231161] & 5 & 3 & 0.72 & 0.079 & 4.55 & -0.404 & $\mathrm{C}$ & ls \\
\hline & & & 3501.42 & [202610] & {$[231161]$} & 3 & 1 & 2.86 & 0.175 & 6.1 & -0.279 & C & ls \\
\hline \multirow[t]{7}{*}{9} & & \multirow{7}{*}{ 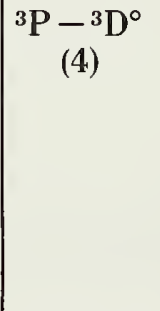 } & 4103.4 & 207703 & 232066 & 9 & 15 & 2.05 & 0.86 & 105 & 0.89 & C & $c a$ \\
\hline & & & 4103.53 & 207705 & 232067 & 5 & 7 & 2.05 & 0.72 & 48.8 & 0.56 & $\mathrm{C}$ & ls \\
\hline & & & 4103.09 & 207700 & 232065 & 3 & 5 & 1.54 & 0.65 & 26.2 & 0.287 & C & ls \\
\hline & & & 4103.72 & 207703 & 232064 & 1 & 3 & 1.14 & 0.86 & 11.6 & -0.065 & $\mathrm{C}$ & ls \\
\hline & & & 4103.87 & 207705 & 232065 & 5 & 5 & 0.51 & 0.129 & 8.7 & -0.190 & C & ls \\
\hline & & & [4103.3] & 207700 & 232064 & 3 & 3 & 0.85 & 0.215 & 8.7 & -0.190 & C & ls \\
\hline & & & [4104.1] & 207705 & 232064 & 5 & 3 & 0.057 & 0.0086 & 0.58 & -1.366 & C & ls \\
\hline \multirow[t]{7}{*}{10} & \multirow{7}{*}{$\begin{array}{l}2 p^{3} 3 p^{\prime}- \\
2 p^{3}\left({ }^{2} \mathrm{D}^{\circ}\right) 3 d^{\prime}\end{array}$} & \multirow{7}{*}{$\begin{array}{c}{ }^{3} \mathrm{~F}-{ }^{-3} \mathrm{~F}^{\circ} \\
(\overline{1} 1)\end{array}$} & 3641.7 & 237509 & 264961 & 21 & 21 & 0.147 & 0.0292 & 7.3 & -0.213 & $\mathrm{C}-$ & $c a$ \\
\hline & & & 3640.89 & 237508 & 264966 & 9 & 9 & 0.137 & 0.0273 & 2.95 & -0.61 & C- & $l s$ \\
\hline & & & $\begin{array}{l}3641.99 \\
364290\end{array}$ & $\begin{array}{l}237509 \\
237500\end{array}$ & 264959 & 7 & 7 & 0.123 & 0.0245 & 2.06 & -0.77 & $\mathrm{C}-$ & ls \\
\hline & & & 3642.80 & $\begin{array}{l}237509 \\
237508\end{array}$ & $\begin{array}{l}264953 \\
264959\end{array}$ & 5 & 5 & 0.130 & $\begin{array}{l}0.0259 \\
0.00182\end{array}$ & $\begin{array}{l}1.55 \\
0.197\end{array}$ & $\begin{array}{l}-0.89 \\
-1.79\end{array}$ & C- & $\begin{array}{l}l s \\
l s\end{array}$ \\
\hline & & & 3642.80 & $\begin{array}{l}237509 \\
237509\end{array}$ & $\begin{array}{l}264953 \\
26493\end{array}$ & 7 & 5 & $\begin{array}{l}0.01163 \\
0.0163\end{array}$ & 0.00231 & 0.194 & -1.79 & $\mathrm{C}-$ & $l s$ \\
\hline & & & [3640.9] & 237509 & 264966 & 7 & 9 & 0.0092 & 0.00234 & 0.196 & -1.79 & $\mathrm{C}-$ & $l s$ \\
\hline & & & 3641.99 & 237509 & 264959 & 5 & 7 & 0.0116 & 0.00324 & 0.194 & -1.79 & $\mathrm{C}-$ & $l s$ \\
\hline 11 & $\begin{array}{l}2 p^{3} 3 d- \\
\quad 2 p^{3}\left({ }^{4} S^{\circ}\right) 4 f\end{array}$ & $\begin{array}{c}{ }^{5} \mathrm{D}^{\circ}-{ }^{5} \mathrm{~F} \\
(9)\end{array}$ & 4246.16 & [231159] & {$[254703]$} & 25 & 25 & 2.47 & 0.93 & 326 & 1.368 & C & $c a$ \\
\hline 12 & & $\begin{array}{c}{ }^{3} \mathrm{D}^{\circ}-{ }^{3} \mathrm{~F} \\
(10)\end{array}$ & 4446.9 & 232066 & 254547 & 15 & 21 & 2.35 & 0.97 & 214 & 1.165 & C & $c a$ \\
\hline
\end{tabular}




\section{Forbidden Transitions}

The adopted values represent the results of calculations by Garstang [1] and Naqvi [2], which are very similar in character. Garstang's evaluation of the quadrupole integral $s_{q}$ is considered the more refined one; therefore, the quadrupole line strengths are taken from his paper. Naqvi, in his calculations of magnetic dipole lines, retains the spin-spin and spin-other-orbit integral in the transformation coefficients, while Garstang neglects it. Thus, Naqvi's values are chosen, whenever this becomes significant. When this refinement is not sensitive, the two authors agree. For the ${ }^{3} \mathrm{P}-{ }^{1} \mathrm{~S}$ transition the important effects of configuration interaction are most appropriately taken into account by Garstang's method, so that his values are used. (Further explanations are found in the general introduction.)

\section{References}

[1] Garstang, R. H., Monthly Notices Roy. Astron. Soc. 11 1, 115-124 (1951).

[2] Naqvi, A. M., Thesis Harvard (1951).

\section{F II. Forbidden Transitions}

\begin{tabular}{|c|c|c|c|c|c|c|c|c|c|c|c|c|}
\hline No. & $\begin{array}{c}\text { Transition } \\
\text { Array }\end{array}$ & $\begin{array}{l}\text { Multi- } \\
\text { plet }\end{array}$ & $\lambda(\AA)$ & $E_{i}\left(\mathrm{~cm}^{-1}\right)$ & $E_{k}\left(\mathrm{~cm}^{-1}\right)$ & $g_{i}$ & $g_{k}$ & $\begin{array}{c}\text { Type of } \\
\text { Transition }\end{array}$ & $A_{k i}\left(\mathrm{sec}^{-1}\right)$ & $S$ (at.u.) & $\begin{array}{l}\text { Accu- } \\
\text { racy }\end{array}$ & Source \\
\hline \multirow[t]{5}{*}{1} & $2 p^{4}-2 p^{4}$ & ${ }^{3} \mathrm{P}-{ }^{3} \mathrm{P}$ & & & & & & & & & & \\
\hline & & & {$\left[29.25 \times 10^{4}\right]$} & 0.0 & 341.8 & 5 & 3 & $\mathrm{~m}$ & $8.97 \times 10^{-4}$ & 2.50 & B & 1,2 \\
\hline & & & {$\left[29.25 \times 10^{4}\right]$} & 0.0 & 341.8 & 5 & 3 & $\mathrm{e}$ & $2.30 \times 10^{-10}$ & 0.88 & C & 1 \\
\hline & & & {$\left[20.38 \times 10^{4}\right]$} & 0.0 & 490.6 & 5 & 1 & $\mathrm{e}$ & $1.80 \times 10^{-9}$ & 0.376 & C & 1 \\
\hline & & & {$\left[67.19 \times 10^{4}\right]$} & 341.8 & 490.6 & 3 & 1 & $\mathrm{~m}$ & $1.78 \times 10^{-4}$ & 2.00 & B & 1,2 \\
\hline \multirow[t]{6}{*}{2} & & $\begin{array}{c}{ }^{3} \mathrm{P}-{ }^{1} \mathrm{D} \\
(1 \mathrm{~F})\end{array}$ & & & & & & & & & & \\
\hline & & & 4789.5 & 0.0 & 20873 & 5 & 5 & $\mathrm{~m}$ & 3.81 & $7.8 \times 10^{-4}$ & $\mathrm{C}$ & 2 \\
\hline & & & 4789.5 & 0.0 & 20873 & 5 & 5 & $\mathrm{e}$ & $9.6 \times 10^{-5}$ & $7.2 \times 10^{-4}$ & D & 1 \\
\hline & & & 4869.3 & 341.8 & 20873 & 3 & 5 & $\mathrm{~m}$ & 0.0121 & $2.59 \times 10^{-4}$ & C. & 2 \\
\hline & & & 4869.3 & 341.8 & 20873 & 3 & 5 & $\mathrm{e}$ & $1.3 \times 10^{-5}$ & $1.1 \times 10^{-4}$ & D & 1 \\
\hline & & & [4904.8] & 490.6 & 20873 & 1 & 5 & e & $4.1 \times 10^{-6}$ & $3.5 \times 10^{-5}$ & D & 1 \\
\hline \multirow[t]{3}{*}{3} & & ${ }^{3} \mathrm{P}-{ }^{1} \mathrm{~S}$ & & & & & & & & & & \\
\hline & & & [2225.5] & $\begin{array}{r}0.0 \\
0\end{array}$ & 44919 & 5 & 1 & e & 0.0016 & $5.2 \times 10^{-5}$ & D & 1 \\
\hline & & & & & & & & & & & & \\
\hline \multirow[t]{2}{*}{4} & & ${ }^{1} \underset{(2 \mathrm{~F})}{\mathrm{D}-{ }^{1} \mathrm{~S}}$ & & & & & & & & & & \\
\hline & & & 4157.5 & 20873 & 44919 & 5 & 1 & $\mathrm{e}$ & 2.10 & 1.55 & C & 1 \\
\hline
\end{tabular}

\section{F III.}

Ground State

Ionization Potential

\author{
$1 s^{2} 2 s^{2} 2 p^{3}{ }^{4} \mathrm{~S}_{3 / 2}^{\circ}$ \\ $62.646 \mathrm{eV}=505410 \mathrm{~cm}^{-1}$
}

\section{Allowed Transitions}

After having written introductory remarks for 9.2 elements, we must confess that this becomes a rather cumbersome exercise in style. Since we expect that this introduction will share the fate of most introductions (namely be ignored) and since there is nothing new to say on this ion (for scientific content see F I) we might as well give the few readers of this introduction some good advice:

If there is no other data source,

Use the Coulomb approximation, of course.

The results should be certainly fine

For any moderately or highly excited line. 
F III. Allowed Transitions

\begin{tabular}{|c|c|c|c|c|c|c|c|c|c|c|c|c|c|}
\hline No. & $\begin{array}{c}\text { Transition } \\
\text { Array }\end{array}$ & $\begin{array}{l}\text { Multi- } \\
\text { plet }\end{array}$ & $\lambda(\AA)$ & $E_{i}\left(\mathrm{~cm}^{-1}\right)$ & $E_{k}\left(\mathrm{~cm}^{-1}\right)$ & $g_{i}$ & $g_{k}$ & $\begin{array}{c}A_{k i}\left(10^{8}\right. \\
\left.\sec ^{-1}\right)\end{array}$ & $f_{i k}$ & $S$ (at.u.) & $\log g f$ & $\begin{array}{l}\text { Accu- } \\
\text { racy }\end{array}$ & Source \\
\hline \multirow[t]{9}{*}{1} & \multirow{13}{*}{$\mid \begin{array}{l}2 p^{23 s}- \\
2 p^{2}\left({ }^{3} \mathrm{P}\right) 3 p\end{array}$} & \multirow{9}{*}{${ }^{4} \mathrm{P}-{ }^{4} \mathrm{D}^{\circ}$} & 3124.4 & 317043 & 349040 & 12 & 20 & 1.6 & 0.40 & 48 & 0.67 & D & $c a$ \\
\hline & & & 3121.52 & 317238 & 349264 & 6 & 8 & 1.6 & 0.31 & 19 & 0.27 & D & ls \\
\hline & & & 3115.67 & 316919 & 349005 & 4 & 6 & 1.1 & 0.25 & 10 & -0.01 & D & $l s$ \\
\hline & & & 3113.58 & 316707 & 348815 & 2 & 4 & 0.67 & 0.19 & 4.0 & -0.41 & D & $l s$ \\
\hline & & & 3146.96 & 317238 & 349005 & 6 & 6 & 0.47 & 0.070 & 4.3 & -0.38 & D & $l s$ \\
\hline & & & 3134.21 & 316919 & 348815 & 4 & 4 & 0.84 & 0.12 & 5.1 & -0.30 & D & ls \\
\hline & & & 3124.76 & 316707 & 348701 & 2 & 2 & 1.3 & 0.19 & 4.0 & -0.41 & D & $l s$ \\
\hline & & & 3165.86 & 317238 & 348815 & 6 & 4 & 0.077 & 0.0077 & 0.48 & -1.34 & D & $l s$ \\
\hline & & & 3145.54 & 316919 & 348701 & 4 & 2 & 0.26 & 0.019 & 0.80 & -1.11 & D & $l s$ \\
\hline \multirow[t]{4}{*}{2} & & \multirow{4}{*}{${ }^{2} \mathrm{P}-{ }^{-2} \mathrm{D}^{\circ}$} & 3176.9 & 324746 & 356214 & 6 & 10 & 1.7 & 0.42 & 26 & 0.40 & D & $c a$ \\
\hline & & & 3174.13 & 324874 & 356370 & 4 & 6 & 1.7 & 0.38 & 16 & 0.18 & D & ls \\
\hline & & & 3174.73 & 324490 & 355980 & 2 & 4 & 1.4 & 0.42 & 8.7 & -0.08 & D & ls \\
\hline & & & 3213.97 & 324874 & 355980 & 4 & 4 & 0.27 & 0.041 & 1.8 & -0.78 & D & ls \\
\hline \multirow[t]{4}{*}{3} & \multirow{8}{*}{$\begin{array}{l}2 p^{2} 3 p^{\prime}- \\
2 p^{2}\left({ }^{1} \mathrm{D}\right) 3 d^{\prime}\end{array}$} & \multirow{4}{*}{${ }^{2} \mathrm{D}^{\circ}-{ }^{2} \mathrm{~F}$} & 3039.3 & 380265 & 413158 & 10 & 14 & 2.75 & 0.53 & 53 & 0.73 & C & $c a$ \\
\hline & & & 3039.25 & 380243 & 413136 & 6 & 8 & 2.75 & 0.51 & 30.4 & 0.483 & $\mathrm{C}$ & ls \\
\hline & & & 3039.75 & 380299 & 4.13187 & 4 & 6 & 2.56 & 0.53 & 21.3 & 0.329 & C & $l s$ \\
\hline & & & 3034.54 & 380243 & 413187 & 6 & 6 & 0.184 & 0.0254 & 1.52 & -0.82 & $\mathrm{C}$ & $l s$ \\
\hline \multirow[t]{4}{*}{4} & & \multirow{4}{*}{$\begin{array}{c}2 \mathrm{P}^{\circ}-{ }^{2} \mathrm{D} \\
(4)\end{array}$} & 3150.6 & 384440 & 416171 & 6 & 10 & 1.39 & 0.344 & 21.4 & 0.315 & $\mathrm{C}$ & $c a$ \\
\hline & & & 3154.39 & 384485 & 416178 & 4 & 6 & 1.38 & 0.309 & 12.9 & 0.093 & C & ls \\
\hline & & & 3142.78 & 384351 & 416161 & 2 & 4 & 1.16 & 0.344 & 7.1 & -0.162 & $\mathrm{C}$ & ls \\
\hline & & & 3156.11 & 384485 & 416161 & 4 & 4 & 0.230 & 0.0344 & 1.43 & -0.86 & C & ls \\
\hline
\end{tabular}

\section{Forbidden Transitions}

Naqvi's [1] calculations are the only available source. The values should not be quite as reliable as for other ions of the $p^{3}$ configuration ( $\mathrm{O}$ II, Ne IV) since the important configuration interaction effects (see general introduction) are neglected.

Reference

[1] Naqvi, A. M., Thesis Harvard (1951).

F III. Forbidden Transitions

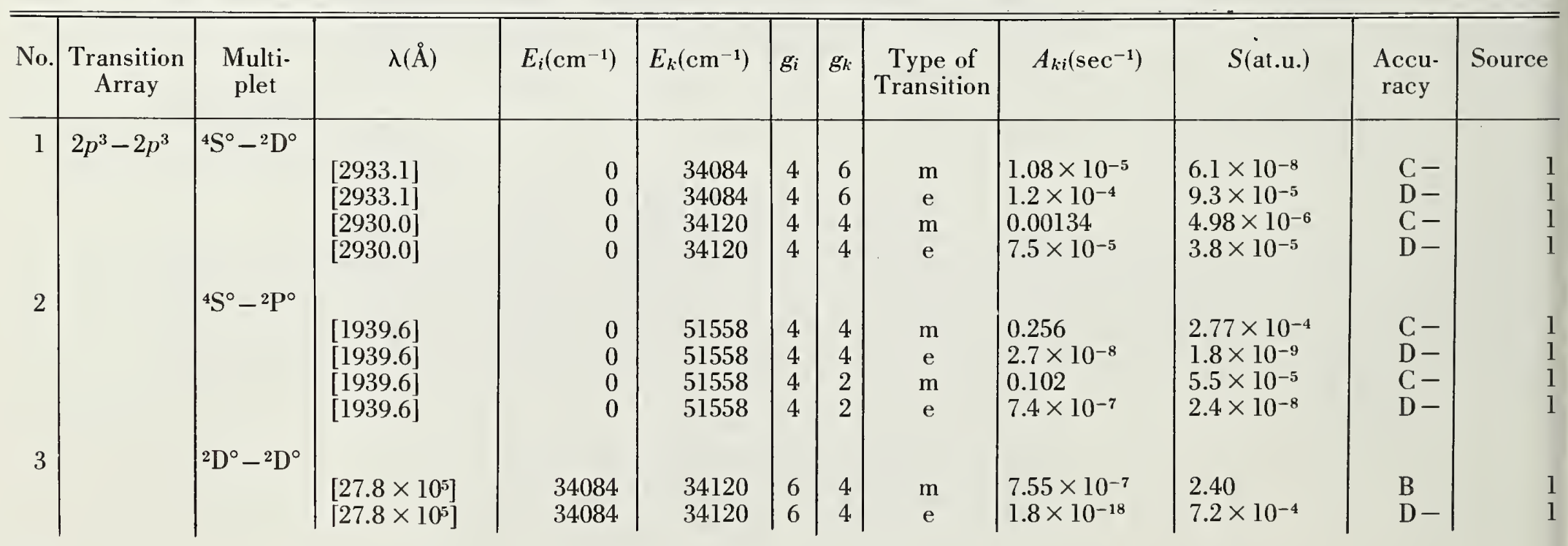


F III. Forbidden Transitions-Continued

\begin{tabular}{|c|c|c|c|c|c|c|c|c|c|c|c|c|}
\hline No. & $\begin{array}{c}\text { Transition } \\
\text { Array }\end{array}$ & Multiplet & $\lambda(\AA)$ & $E_{i}\left(\mathrm{~cm}^{-1}\right)$ & $E_{k}\left(\mathrm{~cm}^{-1}\right)$ & $g_{i}$ & $g_{k}$ & $\begin{array}{l}\text { Type of } \\
\text { Transition }\end{array}$ & $A_{k i}\left(\sec ^{-1}\right)$ & $S($ at.u.) & $\begin{array}{c}\text { Accu- } \\
\text { racy }\end{array}$ & Source \\
\hline 4 & & $\begin{array}{c}{ }^{2} \mathrm{D}^{\circ}-{ }^{2} \mathrm{P}^{\circ} \\
(\mathrm{IF})\end{array}$ & $\begin{array}{c} \\
5721.2 \\
5721.2 \\
{[5721.2]} \\
5733.0 \\
5733.0 \\
{[5733.0]} \\
{[5733.0]}\end{array}$ & $\begin{array}{l}34084 \\
34084 \\
34084 \\
34120 \\
34120 \\
34120 \\
34120\end{array}$ & $\begin{array}{l}51558 \\
51558 \\
51558 \\
51558 \\
51558 \\
51558 \\
51558\end{array}$ & $\begin{array}{l}6 \\
6 \\
6 \\
4 \\
4 \\
4 \\
4\end{array}$ & $\begin{array}{l}4 \\
4 \\
2 \\
4 \\
4 \\
2 \\
2\end{array}$ & $\begin{array}{c}\mathrm{m} \\
\mathrm{e} \\
\mathrm{e} \\
\mathrm{m} \\
\mathrm{e} \\
\mathrm{m} \\
\mathrm{e}\end{array}$ & $\begin{array}{l}0.0280 \\
0.15 \\
0.088 \\
0.0494 \\
0.065 \\
0.0309 \\
0.13\end{array}$ & $\begin{array}{l}7.8 \times 10^{-4} \\
2.2 \\
0.64 \\
0.00138 \\
0.96 \\
4.32 \times 10^{-4} \\
0.96\end{array}$ & $\begin{array}{l}\mathrm{C}- \\
D \\
D \\
\mathrm{C}- \\
\mathrm{D} \\
\mathrm{C}- \\
\mathrm{D}-\end{array}$ & $\begin{array}{l}1 \\
1 \\
1 \\
1 \\
1 \\
1 \\
1 \\
1\end{array}$ \\
\hline
\end{tabular}

F IV.

Ground State

Ionization Potential
$1 s^{2} 2 s^{2} 2 p^{2}{ }^{3} \mathrm{P}_{0}$

$87.14 \mathrm{eV}=703020 \mathrm{~cm}^{-1}$

\section{Allowed Transitions}

The results of calculations by Bolotin et al. [1] are listed. These authors have employed relatively simple wave functions and include the important effects of configuration interaction in a crude manner. Thus, their results should be quite uncertain, but they are nevertheless included since these transitions are expected to be among the strongest for this ion.

\section{Reference}

[1] Bolotin, A. B., Levinson, I. B., and Levin, L. I., Soviet Phys.-JETP 2, 391-395 (1956).

F IV. Allowed Transitions

\begin{tabular}{|c|c|c|c|c|c|c|c|c|c|c|c|c|c|}
\hline No. & $\begin{array}{c}\text { Transition } \\
\text { Array }\end{array}$ & $\begin{array}{l}\text { Multi- } \\
\text { plet }\end{array}$ & $\lambda(\AA)$ & $E_{i}\left(\mathrm{~cm}^{-1}\right)$ & $E_{k}\left(\mathrm{~cm}^{-1}\right)$ & $g_{i}$ & $g_{k}$ & $\begin{array}{c}A_{k i}\left(10^{8}\right. \\
\left.\sec ^{-1}\right)\end{array}$ & $f_{i k}$ & S(at.u.) & $\log g f$ & $\begin{array}{l}\text { Accu- } \\
\text { racy }\end{array}$ & Source \\
\hline \multirow[t]{7}{*}{1} & \multirow{21}{*}{$\begin{array}{r}2 s^{2} 2 p^{2}- \\
2 s 2 p^{3}\end{array}$} & \multirow[t]{7}{*}{${ }^{3} \mathrm{P}-{ }^{3} \mathrm{D}^{\circ}$} & 678.18 & 416 & 147870 & 9 & 15 & 11 & 0.13 & 2.6 & 0.07 & $\mathrm{E}$ & 1 \\
\hline & & & [679.21] & 613 & 147842 & 5 & 7 & 11 & 0.11 & 1.2 & -0.27 & $\mathrm{E}$ & Is \\
\hline & & & {$[677.21]$} & 225 & 147889 & 3 & 5 & 8.5 & 0.097 & 0.65 & -0.54 & $\mathrm{E}$ & ls \\
\hline & & & {$[676.12]$} & 0 & 147902 & 1 & 3 & 6.3 & 0.13 & 0.29 & -0.89 & $\mathrm{E}$ & ls \\
\hline & & & {$[679.00]$} & 613 & 147889 & 5 & 5 & 2.8 & 0.020 & 0.22 & -1.01 & $\mathrm{E}$ & ls \\
\hline & & & {$[677.15]$} & 225 & 147902 & 3 & 3 & 4.8 & 0.033 & 0.22 & -1.01 & $\mathrm{E}$ & ls \\
\hline & & & {$[678.94]$} & 613 & 147902 & 5 & 3 & 0.30 & 0.0013 & 0.014 & -2.20 & $\mathrm{E}$ & ls \\
\hline 2 & & \multirow[t]{7}{*}{${ }^{3} \mathrm{P}-{ }^{3} \mathrm{P}^{\circ}$} & 572.00 & 416 & 175242 & 9 & 9 & 32 & 0.16 & 2.7 & 0.16 & $\mathrm{E}$ & 1 \\
\hline & & & [572.66] & 613 & 175237 & 5 & 5 & 24 & 0.12 & 1.1 & -0.23 & $\mathrm{E}$ & ls \\
\hline & & & [571.37] & 225 & 175242 & 3 & 3 & 8.3 & 0.041 & 0.23 & -0.91 & $\mathrm{E}$ & ls \\
\hline & & & {$[572.64]$} & 613 & 175242 & 5 & 3 & 14 & 0.040 & 0.38 & -0.70 & $\mathrm{E}$ & ls \\
\hline & & & {$[571.30]$} & 225 & 175264 & 3 & 1 & 33 & 0.053 & 0.30 & -0.80 & $\mathrm{E}$ & Is \\
\hline & & & [571.39] & 225 & 175237 & 3 & 5 & 8.3 & 0.067 & 0.38 & -0.69 & $\mathrm{E}$ & ls \\
\hline & & & {$[570.64]$} & 0 & 175242 & 1 & 3 & 11 & 0.16 & 0.30 & -0.80 & $\mathrm{E}$ & ls \\
\hline \multirow[t]{4}{*}{3} & & \multirow[t]{4}{*}{${ }^{3} \mathrm{P}-{ }^{3} \mathrm{~S}^{\circ}$} & 420.38 & 416 & 238297 & 9 & 3 & 180 & 0.16 & 2.0 & 0.16 & $\mathrm{E}$ & 1 \\
\hline & & & [420.73] & 613 & 238297 & 5 & 3 & 100 & 0.16 & 1.1 & -0.10 & $\mathrm{E}$ & ls \\
\hline & & & {$[420.04]$} & 225 & 238297 & 3 & 3 & 61 & 0.16 & 0.67 & -0.31 & $\mathrm{E}$ & ls \\
\hline & & & {$[419.64]$} & 0 & 238297 & 1 & 3 & 20 & 0.16 & 0.22 & -0.80 & $\mathrm{E}$ & ls \\
\hline 4 & & ${ }^{1} \mathrm{D}-{ }^{1} \mathrm{D}^{\circ}$ & {$[491.00]$} & 25241 & 228908 & 5 & 5 & 86 & 0.31 & 2.5 & 0.19 & $\mathrm{E}$ & 1 \\
\hline 5 & & ${ }^{1} \mathrm{D}-{ }^{1} \mathrm{P}^{\circ}$ & {$[430.76]$} & 25241 & 257390 & 5 & 3 & 130 & 0.21 & 1.5 & 0.02 & $\mathrm{E}$ & l \\
\hline 6 & & ${ }^{1} \mathrm{~S}-{ }^{1} \mathrm{P}^{\circ}$ & {$[490.57]$} & 53544 & 257390 & 1 & 3 & 27 & 0.30 & 0.48 & -0.53 & $\mathrm{E}$ & 1 \\
\hline
\end{tabular}




\section{Forbidden Transitions}

The adopted values represent the results of calculations by Garstang [1] and Naqvi [2], which are very similar in character. Garstang's evaluation of the quadrupole integral $s_{q}$ is considered the more refined one; therefore, the quadrupole line strengths are taken from his paper. The results for the magnetic dipole lines agree, except for the ${ }^{3} \mathrm{P}-{ }^{1} \mathrm{~S}$ transition. For this line, the important effects of configuration interaction are more appropriately taken into account by Garstang's approach of using the experimental term intervals, so that his values are used. (Further explanations are found in the general introduction.)

\section{References}

[1] Garstang, R. H., Monthly Notices Roy. Astron. Soc. 111, 115-124 (1951).

[2] Naqvi, A. M., Thesis Harvard (1951).

F IV. Forbidden Transitions

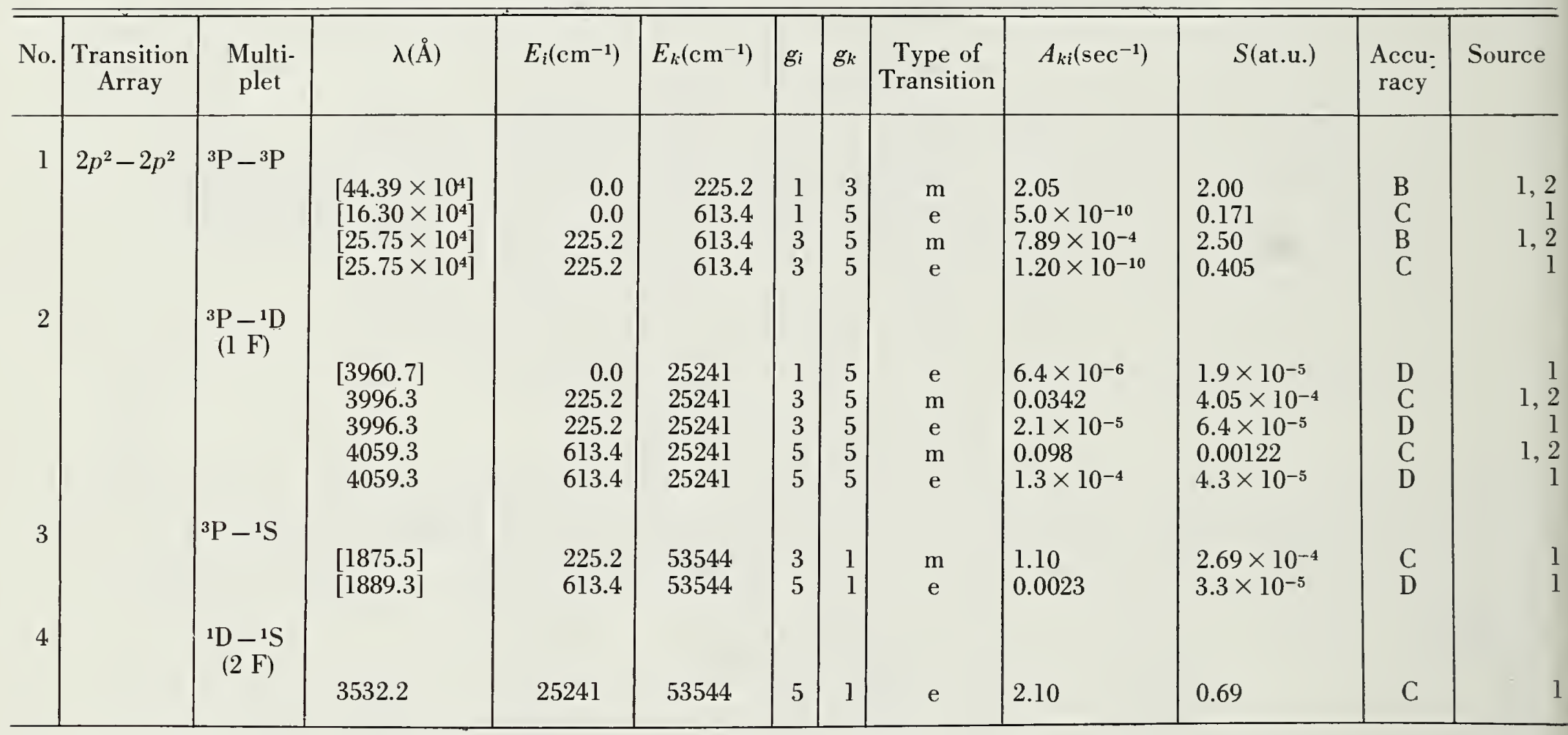

Ground State

Ionization Potential
FV.

$$
\cdot s^{2} 2 s^{2} 2 p^{2} \mathrm{P}_{1 / 2}^{\circ}
$$

$$
114.214 \mathrm{eV}=921450 \mathrm{~cm}^{-1}
$$

\section{Allowed Transitions}

The two available sources are quantum mechanical calculations by Bolotin and Yutsis [1] and Naqvi and Victor [2]. The important transitions covered by Bolotin and Yutsis are very sensitive to the effects of configuration interaction, which are only approximately included in their calculations. For the result of Naqvi and Victor, obtained from the charge expansion method, an accuracy within 25 apercent is indicated from comparisons of this work with other material for equivalent transitions within this isoelectronic sequence. 
[1] Bolotin, A. B., and Yutsis, A. P., Zhur. Eksptl. i Teoret. Fiz. 24, 537-543 (1953) (Translated in "Optical Transition

Probabilities," Office of Technical Services, U.S. Department of Commerce, Washington, D.C.).

[2] Naqvi, A. M., and Victor, G. A., Technical Documentary Report No. RTD TDR-63-3118 (1964).

F v. Allowed Transitions

\begin{tabular}{|c|c|c|c|c|c|c|c|c|c|c|c|c|c|}
\hline No. & $\begin{array}{c}\text { Transition } \\
\text { Array }\end{array}$ & $\begin{array}{c}\text { Multi- } \\
\text { plet }\end{array}$ & $\lambda(\AA)$ & $E_{i}\left(\mathrm{~cm}^{-1}\right)$ & $E_{k}\left(\mathrm{~cm}^{-1}\right)$ & $g_{i}$ & $g_{k}$ & $\begin{array}{l}A_{k i}\left(10^{8}\right. \\
\left.\sec ^{-1}\right)\end{array}$ & $f_{i k}$ & $S$ (at.u.) & $\log g f$ & $\begin{array}{l}\text { Accu- } \\
\text { racy }\end{array}$ & Source \\
\hline \multirow[t]{4}{*}{1} & \multirow[t]{11}{*}{$\begin{array}{r}2 s^{2} 2 p- \\
2 s 2 p^{2}\end{array}$} & \multirow[t]{4}{*}{${ }^{2} \mathrm{P}^{\circ}-{ }^{2} \mathrm{D}$} & 656.22 & 497 & 152885 & 6 & 10 & 12 & 0.13 & 1.7 & -0.10 & $\mathrm{E}$ & 1 \\
\hline & & & [657.33] & 746 & 152876 & 4 & 6 & 12 & 0.12 & 1.0 & -0.34 & $\mathrm{E}$ & \\
\hline & & & {$[654.03]$} & 0 & 152898 & 2 & 4 & 10 & 0.13 & 0.57 & -0.58 & $\mathrm{E}$ & Is \\
\hline & & & {$[656.24]$} & 746 & 152898 & 4 & 4 & 2.0 & 0.013 & 0.11 & -1.29 & E & Is \\
\hline 2 & & \multirow[t]{3}{*}{${ }^{2} \mathrm{P}^{0}-{ }^{2} \mathrm{~S}$} & 507.44 & 497 & 197565 & 6 & 2 & 67 & 0.086 & 0.86 & -0.29 & E & l \\
\hline & & & [508.08] & 746 & 197565 & 4 & 2 & 44 & 0.085 & 0.57 & -0.47 & E & 15 \\
\hline & & & [506.16] & 0 & 197565 & 2 & 2 & 23 & 0.087 & 0.29 & -0.76 & E & \\
\hline \multirow[t]{4}{*}{3} & & \multirow[t]{4}{*}{${ }^{2} \mathrm{P}^{\circ}-{ }^{2} \mathrm{P}$} & 465.78 & 497 & 215192 & 6 & 6 & 100 & 0.33 & 3.0 & 0.29 & E & l \\
\hline & & & [465.98] & 746 & 215348 & 4 & 4 & 85 & 0.28 & 1.7 & 0.04 & $\mathrm{E}$ & \\
\hline & & & {$[465.37]$} & 0 & 214881 & 2 & 2 & 67 & 0.22 & 0.67 & -0.36 & $\mathrm{E}$ & ds \\
\hline & & & $\begin{array}{l}{[467.00]} \\
{[464.36]}\end{array}$ & $\begin{array}{r}746 \\
0\end{array}$ & $\begin{array}{l}214881 \\
215348\end{array}$ & $\begin{array}{l}4 \\
2\end{array}$ & $\begin{array}{l}2 \\
4\end{array}$ & $\begin{array}{l}33 \\
17\end{array}$ & $\begin{array}{l}0.054 \\
0.11\end{array}$ & $\begin{array}{l}0.33 \\
0.33\end{array}$ & $\begin{array}{l}-0.67 \\
-0.67\end{array}$ & $\begin{array}{l}\mathrm{E} \\
\mathrm{E}\end{array}$ & ls \\
\hline \multirow[t]{4}{*}{4} & \multirow{4}{*}{$3 s-\left({ }^{1} \mathrm{~S}\right) 3 p$} & \multirow{4}{*}{${ }^{2} \mathrm{~S}-{ }^{2} \mathrm{P}^{\circ}$} & 2454.2 & $52475]$ & 565485 & 2 & 6 & 14 & 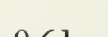 & & & & \\
\hline & & & & & (20) & 2 & 0 & 2.24 & 0.01 & 9.8 & 0.084 & C & \\
\hline & & & {$[2450.7]$} & 524751 & 565544 & 2 & 4 & 2.24 & 0.403 & 6. & -0.094 & C & ls \\
\hline & & & {$[24$} & 524751 & 565367 & 2 & 2 & 2.22 & 0.202 & 3.27 & -0.394 & C & \\
\hline
\end{tabular}

\section{Forbidden Transitions}

Naqvi's calculation [1] of the one possible transition in the ground state configuration $2 p$ is the only available source. The line strength should be quite accurate, since it does not sensitively depend on the choice of the interaction parameters.

\section{Reference}

[1] Naqvi, A. M., Thesis Harvard (1951).

F V. Forbidden Transitions

\begin{tabular}{|c|c|c|c|c|c|c|c|c|c|c|c|c|}
\hline No. & $\begin{array}{c}\text { Transition } \\
\text { Array }\end{array}$ & Multiplet & $\lambda(\AA)$ & $E_{i}\left(\mathrm{~cm}^{-1}\right)$ & $E_{k}\left(\mathrm{~cm}^{-1}\right)$ & $g_{i}$ & $g_{k}$ & $\begin{array}{c}\text { Type of } \\
\text { Transition }\end{array}$ & $A_{k i}\left(\sec ^{-1}\right)$ & $S($ at. u. $)$ & $\begin{array}{l}\text { Accu- } \\
\text { racy }\end{array}$ & Source \\
\hline 1 & $2 p-2 p$ & ${ }^{2} \mathrm{P}^{\circ}-{ }^{2} \mathrm{P}^{\circ}$ & $13.40 \times 10^{4}$ & 0 & 746 & 2 & 4 & $\mathrm{~m}$ & 0.00373 & 1.33 & B & l \\
\hline
\end{tabular}




\section{Allowed Transitions}

The results of the charge expansion calculations by Naqvi and Victor [1] are utilized whenever comparison of this work with other data in the isoelectronic sequence indicates a fair reliability of this material. Values for the $2 s 2 p-2 p^{2}$ transition array are available from the self-consistent field calculations by Weiss [2]. These calculations do not include the important effects of configuration interaction; hence, fairly large uncertainties must be expected. The average of the dipole length and velocity approximations [2] is adopted and accuracies within 50 percent are indicated from comparisons possible for the first member of this isoelectronic sequence.

\section{References}

[1] Naqvi, A. M., and Victor, G. A., Technical Documentary Report No. RTD TDR-63-3118 (1964).

[2] Weiss, A. W., private communication (1964).

Fvi. Allowed Transitions

\begin{tabular}{|c|c|c|c|c|c|c|c|c|c|c|c|c|c|}
\hline No. & $\begin{array}{l}\text { Transition } \\
\text { Array }\end{array}$ & Multiplet & $\lambda(\AA)$ & $E_{i}\left(\mathrm{~cm}^{-1}\right)$ & $E_{k}\left(\mathrm{~cm}^{-1}\right)$ & $g_{i}$ & $g_{k}$ & $\begin{array}{l}A_{k i}\left(10^{8}\right. \\
\left.\operatorname{sce}^{-1}\right)\end{array}$ & $f_{i k}$ & $S$ (at.u.) & $\log g f$ & $\begin{array}{l}\text { Accu- } \\
\text { racy }\end{array}$ & Source \\
\hline 1 & $2 s^{2}-2 s\left({ }^{2} \mathbf{S}\right) 2 p$ & ${ }^{1} \mathrm{~S}-{ }^{1} \mathrm{P}^{\circ}$ & [535.21] & 0 & 186841 & 1 & 3 & 48 & 0.62 & 1.1 & -0.20 & $\mathrm{E}$ & 1 \\
\hline 2 & $2 s^{2}-2 s\left({ }^{2} \mathrm{~S}\right) 3 p$ & ${ }^{1} \mathrm{~S}-{ }^{1} \mathrm{P}^{\circ}$ & [126.93] & 0 & 787833 & 1 & 3 & 660 & 0.48 & 0.20 & -0.32 & $\mathrm{E}$ & 1 \\
\hline \multirow[t]{6}{*}{3} & $2 s 2 p-2 p^{2}$ & ${ }^{3} \mathrm{P}^{\circ}-{ }^{3} \mathrm{P}$ & 646.27 & 97152 & 251886 & 9 & 9 & 25 & 0.16 & 3.0 & 0.15 & D & 2 \\
\hline & & & [646.38] & 97437 & 252145 & 5 & 5 & 18 & 0.11 & 1.2 & -0.25 & D & ls \\
\hline & & & {$[646.10]$} & 96861 & 251635 & 3 & 3 & 6.3 & 0.039 & 0.25 & -0.93 & D & ls \\
\hline & & & {$\left[\begin{array}{l}648.52] \\
{[647}\end{array}\right.$} & $\begin{array}{l}97437 \\
96861\end{array}$ & $\begin{array}{l}251635 \\
251341\end{array}$ & $\begin{array}{l}5 \\
3\end{array}$ & 3 & $\begin{array}{l}10 \\
25\end{array}$ & $\begin{array}{l}0.039 \\
0.052\end{array}$ & $\begin{array}{l}0.42 \\
0.33\end{array}$ & $\begin{array}{l}-0.71 \\
-0.81\end{array}$ & D & ls \\
\hline & & & [643.98] & $\begin{array}{l}90001 \\
96861\end{array}$ & 252145 & $\begin{array}{l}5 \\
3\end{array}$ & $\begin{array}{l}1 \\
5\end{array}$ & $\begin{array}{c}25 \\
6.4\end{array}$ & $\begin{array}{l}0.052 \\
0.066\end{array}$ & $\begin{array}{l}0.33 \\
0.42\end{array}$ & $\begin{array}{l}-0.81 \\
-0.70\end{array}$ & $\begin{array}{l}\text { D } \\
\text { D }\end{array}$ & is \\
\hline & & & [645.02] & 96601 & 251635 & 1 & 3 & 8.3 & 0.16 & 0.33 & -0.81 & D & ls \\
\hline 4 & & ${ }^{1} \mathrm{P}^{\circ}-{ }^{1} \mathrm{D}$ & [1139.5] & 186841 & 274597 & 3 & 5 & 8.2 & 0.27 & 3.0 & -0.10 & D & 2 \\
\hline 5 & & ${ }^{1} \mathrm{P}^{\circ}-{ }^{1} \mathrm{~S}$ & [651.11] & 186841 & 340424 & 3 & 1 & 26 & 0.054 & 0.35 & -0.79 & D & 2 \\
\hline 6 & $2 s 3 s-2 s\left({ }^{2} \mathrm{~S}\right) 3 p$ & ${ }^{1} \mathrm{~S}-{ }^{1} \mathrm{P}^{\circ}$ & [4264.8] & 764392 & 787833 & 1 & 3 & 0.326 & 0.267 & 3.75 & -0.57 & C & 1 \\
\hline
\end{tabular}

\section{Forbidden Transitions}

Naqvi's calculations [1] are the only available source. The results for the ${ }^{3} \mathrm{P}^{\circ}-{ }^{3} \mathrm{P}^{\circ}$ transitions are essentially independent of the choice of the interaction parameters. For the ${ }^{3} \mathrm{P}^{\circ}-{ }^{1} \mathrm{P}^{\circ}$ transitions, Naqvi uses empirical term intervals, i.e., the effects of configuration interaction should be partially included.

[1] Naqvi, A. M., Thesis Harvard (1951).

Reference 


\section{F vi . Forbidden Transitions}

\begin{tabular}{|c|c|c|c|c|c|c|c|c|c|c|c|c|}
\hline No. & $\begin{array}{c}\text { Transition } \\
\text { Array }\end{array}$ & Multiplet & $\lambda(\AA)$ & $E_{i}\left(\mathrm{~cm}^{-1}\right)$ & $E_{k}\left(\mathrm{~cm}^{-1}\right)$ & $g_{i}$ & $g_{k}$ & $\begin{array}{c}\text { Type of } \\
\text { Transition }\end{array}$ & $A_{k i}\left(\sec ^{-1}\right)$ & S(at. u.) & $\begin{array}{l}\text { Accu- } \\
\text { racy }\end{array}$ & Srource \\
\hline 1 & $\begin{array}{l}2 s 2 p- \\
2 s\left({ }^{2} \mathrm{~S}\right) 2 p\end{array}$ & ${ }^{3} \mathrm{P}^{\circ}-{ }^{3} \mathrm{P}^{\circ}$ & $\begin{array}{l}{\left[38.5 \times 10^{4}\right]} \\
{\left[17.36 \times 10^{4}\right]}\end{array}$ & $\begin{array}{l}96601 \\
96861\end{array}$ & $\begin{array}{l}96861 \\
97437\end{array}$ & $\begin{array}{l}1 \\
3\end{array}$ & $\begin{array}{l}3 \\
5\end{array}$ & $\begin{array}{l}m \\
m\end{array}$ & $\begin{array}{l}3.16 \times 10^{-4} \\
0.00258\end{array}$ & $\begin{array}{l}2.00 \\
2.50\end{array}$ & $\begin{array}{l}B \\
B\end{array}$ & 1 \\
\hline 2 & & 1 & 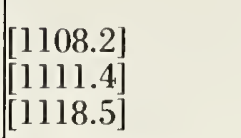 & $\begin{array}{l}96601 \\
96861 \\
97437\end{array}$ & $\begin{array}{l}186841 \\
186841 \\
186841\end{array}$ & $\begin{array}{l}1 \\
3 \\
5\end{array}$ & $\begin{array}{l}3 \\
3 \\
3\end{array}$ & $\begin{array}{l}\mathrm{m} \\
\mathrm{m} \\
\mathrm{m}\end{array}$ & $\begin{array}{c}0.268 \\
44.2 \\
0.324\end{array}$ & $\begin{array}{l}4.05 \times 10^{-5} \\
0.0068 \\
5.1 \times 10^{-5}\end{array}$ & $\begin{array}{l}\mathrm{C} \\
\mathrm{C} \\
\mathrm{C}\end{array}$ & ] \\
\hline
\end{tabular}

F VII.

Ground State

Ionization Potential
$1 s^{2} 2 s^{2} \mathrm{~S}_{1 / 2}$

$185.139 \mathrm{eV}=1493656 \mathrm{~cm}^{-1}$

\section{Allowed Transitions}

List of tabulated lines:

\begin{tabular}{c|c||c|c||c|r}
\hline \hline Wavelength $[\AA]$ & No. & Wavelength $[\AA]$ & No. & Wavelength $[\AA]$ & No. \\
\hline & 3 & 127.81 & 5 & 890.762 & 1 \\
86.732 & 6 & 134.71 & 4 & 3246.6 & 7 \\
97.262 & 6 & 134.88 & 4 & 3276.6 & 7 \\
97.354 & 2 & 335.17 & 8 & 7958.9 & 11 \\
112.94 & 2 & 391.73 & 10 & 9524.8 & 9 \\
127.98 & 5 & 392.16 & 10 & 9702.3 & 9 \\
127.80 & 5 & 883.097 & 1 & 9787.8 & 9 \\
\hline
\end{tabular}

For F VII, an ion of the Lithium sequence, theoretical data from the self-consistent field calculations by Weiss [1] and the variational calculations by Flannery and Stewart [2], both done in the dipole length and velocity approximations, as well as an experimental result from the lifetime measurement of Berkner et al. [3], are available. The agreement for the $2 s-2 p$ transition, which is covered by all three methods, is within an impressive 10 percent. Errors smaller than 25 percent are estimated from the close agreement between the dipole length and velocity approximations. The dipole length values are chosen.

\section{References}

[1] Weiss, A. W., Astrophys. J. 138, 1262-1276 (1963).

[2] Flannery, M. R., and Stewart, A. L., Monthly Notices Roy. Astron. Soc. 126, 387-392 (1963).

[3] Berkner, K. H., Cooper, W. L., Kaplan, S. N., and Pyle, R. V., Phys. Letters 16, 35 (1965). 
F vir. Allowed Transitions

\begin{tabular}{|c|c|c|c|c|c|c|c|c|c|c|c|c|c|}
\hline No. & $\begin{array}{l}\text { Transition } \\
\text { Array }\end{array}$ & $\begin{array}{l}\text { Multi- } \\
\text { plet }\end{array}$ & $\lambda(\AA)$ & $E_{i}\left(\mathrm{~cm}^{-1}\right)$ & $E_{k}\left(\mathrm{~cm}^{-1}\right)$ & $g_{i}$ & $g_{k}$ & $\begin{array}{l}A_{k i}\left(10^{8}\right. \\
\left.\sec ^{-1}\right)\end{array}$ & $f_{i k}$ & $S$ (at.u.) & $\log g f$ & $\begin{array}{l}\text { Accu- } \\
\text { racy }\end{array}$ & Source \\
\hline \multirow[t]{2}{*}{1} & $2 s-2 p$ & ${ }^{2} \mathrm{~S}-{ }^{2} \mathrm{P}^{0}$ & 885.64 & 0 & 112913 & 2 & 6 & 4.77 & 0.168 & 0.981 & -0.473 & B & $1,2,3$ \\
\hline & & & $\begin{array}{l}883.097 \\
890.762\end{array}$ & $\begin{array}{l}0 \\
0\end{array}$ & $\begin{array}{l}113238 \\
112263\end{array}$ & $\begin{array}{l}2 \\
2\end{array}$ & $\begin{array}{l}4 \\
2\end{array}$ & $\begin{array}{l}4.81 \\
4.69\end{array}$ & $\begin{array}{l}0.112 \\
0.0558\end{array}$ & $\begin{array}{l}0.654 \\
0.327\end{array}$ & $\begin{array}{l}-0.648 \\
-0.953\end{array}$ & $\begin{array}{l}\mathrm{B} \\
\mathrm{B}\end{array}$ & $\begin{array}{l}l s \\
l s\end{array}$ \\
\hline \multirow[t]{2}{*}{2} & $2 s-3 p$ & ${ }^{2} \mathrm{~S}-{ }^{2} \mathrm{P}^{\circ}$ & 112.95 & 0 & 885324 & 2 & 6 & 499 & 0.286 & 0.213 & -0.242 & B + & 1,2 \\
\hline & & & {$\left[\begin{array}{l}{[112.94]} \\
{[112.98]}\end{array}\right.$} & $\begin{array}{l}0 \\
0\end{array}$ & $\begin{array}{l}885418 \\
885136\end{array}$ & $\begin{array}{l}2 \\
2\end{array}$ & $\begin{array}{l}4 \\
2\end{array}$ & $\begin{array}{l}499 \\
499\end{array}$ & $\begin{array}{l}0.191 \\
0.0954\end{array}$ & $\begin{array}{l}0.142 \\
0.0710\end{array}$ & $\begin{array}{l}-0.418 \\
-0.719\end{array}$ & $\begin{array}{l}\mathrm{B}+ \\
\mathrm{B}+\end{array}$ & $\begin{array}{l}l s \\
l s\end{array}$ \\
\hline 3 & $2 s-4 p$ & ${ }^{2} \mathrm{~S}-{ }^{2} \mathrm{P}^{\circ}$ & {$[86.732]$} & 0 & 1152977 & 2 & 6 & 214 & 0.0725 & 0.0414 & -0.839 & $\mathrm{C}+$ & 2 \\
\hline \multirow[t]{2}{*}{4} & $2 p-3 s$ & ${ }^{2} \mathrm{P}^{\circ}-{ }^{2} \mathrm{~S}$ & 134.82 & 112913 & 854625 & 6 & 2 & 264 & 0.0240 & 0.0638 & -0.842 & $\mathrm{C}+$ & 1,2 \\
\hline & & & {$[134.88]$} & $\begin{array}{l}113238 \\
112263\end{array}$ & $\begin{array}{l}854625 \\
854625\end{array}$ & $\begin{array}{l}4 \\
2\end{array}$ & $\begin{array}{l}2 \\
2\end{array}$ & $\begin{array}{c}175 \\
88.3\end{array}$ & $\begin{array}{l}0.0239 \\
0.0240\end{array}$ & $\begin{array}{l}0.0425 \\
0.0213\end{array}$ & $\begin{array}{l}-1.019 \\
-1.319\end{array}$ & $\begin{array}{l}\mathrm{C}+ \\
\mathrm{C}+\end{array}$ & $\begin{array}{l}l s \\
l s\end{array}$ \\
\hline \multirow[t]{4}{*}{5} & $2 p-3 d$ & ${ }^{2} \mathrm{P}^{\circ}-{ }^{2} \mathrm{D}$ & 127.75 & 112913 & 895686 & 6 & 10 & 1630 & 0.666 & 1.68 & 0.602 & B & 1 \\
\hline & & & [127.80] & 113238 & 895722 & 4 & 6 & 1630 & 0.600 & 1.01 & 0.380 & B & $l s$ \\
\hline & & & [127.65] & 112263 & 895632 & 2 & 4 & 1360 & 0.666 & 0.560 & 0.125 & B & ls \\
\hline & & & [127.81] & 113238 & 895632 & 4 & 4 & 272 & 0.0665 & 0.112 & -0.575 & B & $l s$ \\
\hline \multirow[t]{2}{*}{6} & $2 p-4 s$ & ${ }^{2} \mathrm{P}^{\circ}-{ }^{2} \mathrm{~S}$ & 97.323 & 112913 & 1140416 & 6 & 2 & 99 & 0.00468 & 0.0090 & -1.55 & C & 2 \\
\hline & & & $\begin{array}{l}{[97.354]} \\
{[97.262]}\end{array}$ & $\begin{array}{l}113238 \\
112263\end{array}$ & $\begin{array}{l}1140416 \\
1140416\end{array}$ & $\begin{array}{l}4 \\
2\end{array}$ & $\begin{array}{l}2 \\
2\end{array}$ & $\begin{array}{l}66 \\
33.0\end{array}$ & $\begin{array}{l}0.00468 \\
0.00468\end{array}$ & $\begin{array}{l}0.0060 \\
0.00300\end{array}$ & $\begin{array}{l}-1.73 \\
-2.028\end{array}$ & $\begin{array}{l}\mathrm{C} \\
\mathrm{C}\end{array}$ & $\begin{array}{l}l s \\
l s\end{array}$ \\
\hline \multirow[t]{2}{*}{7} & $3 s-3 p$ & ${ }^{2} \mathrm{~S}-{ }^{2} \mathrm{P}^{\mathrm{o}}$ & 3256.5 & 854625 & 885324 & 2 & 6 & 0.604 & 0.288 & 6.18 & -0.239 & $\mathrm{~B}+$ & 1,2 \\
\hline & & & $\begin{array}{l}{[3246.6]} \\
{[3276.6]}\end{array}$ & $\begin{array}{l}854625 \\
854625\end{array}$ & $\begin{array}{l}885418 \\
885136\end{array}$ & $\begin{array}{l}2 \\
2\end{array}$ & $\begin{array}{l}4 \\
2\end{array}$ & $\begin{array}{l}0.610 \\
0.593\end{array}$ & $\begin{array}{l}0.193 \\
0.0955\end{array}$ & $\begin{array}{l}4.12 \\
2.06\end{array}$ & $\begin{array}{l}-0.414 \\
-0.719\end{array}$ & $\begin{array}{l}\mathrm{B}+ \\
\mathrm{B}+\end{array}$ & $\begin{array}{l}l s \\
l s\end{array}$ \\
\hline 8 & $3 s-4 p$ & ${ }^{2} \mathrm{~S}-{ }^{2} \mathrm{P}^{\mathrm{o}}$ & [335.17] & 854625 & 1152977 & 2 & 6 & 63.7 & 0.322 & 0.710 & -0.192 & B & 2 \\
\hline \multirow[t]{4}{*}{9} & $3 p-3 d$ & ${ }^{2} \mathrm{P}^{\circ}-{ }^{2} \mathrm{D}$ & 9648.0 & 885324 & 895686 & 6 & 10 & 0.0186 & 0.0432 & 8.23 & -0.587 & B & 1 \\
\hline & & & [9702.3] & 885418 & 895722 & 4 & 6 & 0.0183 & 0.0387 & 4.94 & -0.811 & B & $l s$ \\
\hline & & & & & 895632 & 2 & 4 & 0.0161 & 0.0437 & 2.7 & -1.059 & B & ls \\
\hline & & & [9787.8] & 885418 & 895632 & 4 & 4 & 0.00297 & 0.00426 & 0.549 & -1.769 & B & ls \\
\hline \multirow[t]{2}{*}{10} & $3 p-4 s$ & ${ }^{2} \mathrm{P}^{\circ}-{ }^{2} \mathrm{~S}$ & 392.02 & 885324 & 1140416 & 6 & 2 & 58.3 & 0.0448 & 0.347 & -0.571 & B & 2 \\
\hline & & & $\begin{array}{l}{[392.16]} \\
{[391.73]}\end{array}$ & $\begin{array}{l}885418 \\
885136\end{array}$ & $\begin{array}{l}1140416 \\
1140416\end{array}$ & $\begin{array}{l}4 \\
2\end{array}$ & $\begin{array}{l}2 \\
2\end{array}$ & $\begin{array}{l}38.8 \\
19.5\end{array}$ & $\begin{array}{l}0.0447 \\
0.0450\end{array}$ & $\begin{array}{l}0.231 \\
0.116\end{array}$ & $\begin{array}{l}-0.747 \\
-1.046\end{array}$ & $\begin{array}{l}\mathrm{B} \\
\mathrm{B}\end{array}$ & $\begin{array}{l}l s \\
l s\end{array}$ \\
\hline 11 & $4 s-4 p$ & ${ }^{2} \mathrm{~S}-{ }^{2} \mathrm{P}^{\circ}$ & [7958.9] & 1140416 & 1152977 & 2 & 6 & 0.140 & 0.399 & 20.9 & -0.098 & B & 2 \\
\hline
\end{tabular}


Ground State

F VIII.

Ionization Potential

$$
1 s^{2}{ }^{1} \mathrm{~S}_{0}
$$

$953.60 \mathrm{eV}=7693400 \mathrm{~cm}^{-1}$

\section{Allowed Transitions}

The results of extensive non-relativistic variational calculations by Weiss [1] are used. Values have been calculated in both the dipole length and dipole velocity approximations and agree to within 1 percent, except for the $3 p^{1} \mathrm{P}^{\circ}-3 d^{1} \mathrm{D}$ transition where agreement is not as good. The average of the two approximations is adopted [1].

\section{Reference}

[1] Weiss, A. W., private communication (1964).

F VIII. Allowed Transitions

\begin{tabular}{|c|c|c|c|c|c|c|c|c|c|c|c|c|c|}
\hline No. & $\begin{array}{c}\text { Transition } \\
\text { Array }\end{array}$ & Multiplet & $\lambda(\AA)$ & $E_{i}\left(\mathrm{~cm}^{-1}\right)$ & $E_{k}\left(\mathrm{~cm}^{-1}\right)$ & $g:$ & $g_{k}$ & $A_{k i}\left(10^{8} \sec ^{-1}\right)$ & $f_{i k}$ & $S($ at.u.) & $\log g f$ & $\begin{array}{l}\text { Accu- } \\
\text { racy }\end{array}$ & Source \\
\hline 1 & $\begin{array}{l}1 s^{2}- \\
1 s 2 p\end{array}$ & ${ }^{1} \mathrm{~S}-{ }^{1} \mathrm{P}^{\circ}$ & [16.807] & 0 & 5949900 & 1 & 3 & $5.59 \times 10^{4}$ & 0.710 & 0.0393 & -0.149 & A & 1 \\
\hline 2 & $\begin{array}{c}1 s^{2}- \\
1 s 3 p\end{array}$ & ${ }^{1} \mathrm{~S}-{ }^{1} \mathrm{P}^{\circ}$ & [14.458] & 0 & 6916590 & 1 & 3 & $1.57 \times 10^{4}$ & 0.148 & 0.00704 & -0.830 & A & 1 \\
\hline 3 & $\begin{array}{c}1 s 2 s- \\
1 s 2 p\end{array}$ & ${ }^{1} \mathrm{~S}-{ }^{1} \mathrm{P}^{\circ}$ & [2149.9] & {$[5903400]$} & 5949900 & 1 & 3 & 0.288 & 0.0599 & 0.424 & -1.223 & A & 1 \\
\hline 4 & $\begin{array}{c}1 s 2 s- \\
1 s 3 p\end{array}$ & ${ }^{1} \mathrm{~S}-{ }^{1} \mathrm{P}^{0}$ & [98.698] & {$[5903400]$} & 6916590 & 1 & 3 & 867 & 0.380 & 0.123 & -0.420 & A & 1 \\
\hline 5 & $\begin{array}{c}1 s 2 p- \\
1 s 3 d\end{array}$ & ${ }^{1} \mathrm{P}^{\circ}-{ }^{1} \mathrm{D}$ & [103.80] & 5949900 & {$[6913300]$} & 3 & 5 & 2610 & 0.703 & 0.721 & 0.324 & A & 1 \\
\hline 6 & $\begin{array}{c}1 s 3 d- \\
1 s 3 p\end{array}$ & ${ }^{1} \mathrm{D}-{ }^{1} \mathrm{P}^{\circ}$ & {$[30390] ?$} & [6913300] & 6916590 & 5 & 3 & $8.52 \times 10^{-4}$ & 0.00708 & 3.54 & -1.451 & $C+$ & 1 \\
\hline 7 & $\begin{array}{c}1 s 2 s- \\
1 s 2 p\end{array}$ & ${ }^{3} \mathrm{~S}-{ }^{3} \mathrm{P}^{\circ}$ & [1422.9] & [5829640] & [5899920] & 3 & 9 & 0.915 & 0.0833 & 1.17 & -0.602 & A & 1 \\
\hline 8 & $\begin{array}{c}1 s 2 s- \\
1 s 3 p\end{array}$ & ${ }^{3} \mathrm{~S}-{ }^{3} \mathrm{P}^{\circ}$ & [93.213] & [5829640] & [6902450] & 3 & 9 & 914 & 0.357 & 0.329 & 0.030 & A & 1 \\
\hline 9 & $\begin{array}{l}1 s 2 p- \\
1 s 3 d\end{array}$ & ${ }^{3} \mathrm{P}^{\circ}-{ }^{3} \mathrm{D}$ & [98.756] & [5899920] & 6912520 & 9 & 15 & 2750 & 0.669 & 1.96 & 0.780 & A & 1 \\
\hline 10 & $\begin{array}{c}1 s 3 p- \\
1 s 3 d\end{array}$ & ${ }^{3} \mathrm{P}^{\circ}-{ }^{3} \mathrm{D}$ & [9927.8] & {$[6902450]$} & 6912520 & 9 & 15 & 0.0133 & 0.0327 & 9.62 & -0.531 & A & 1 \\
\hline
\end{tabular}


Ne I.

Ground State $1 s^{2} 2 s^{2} 2 p^{6}{ }^{1} \mathrm{~S}_{0}$

Ionization Potential

$21.559 \mathrm{eV}=173932 \mathrm{~cm}^{-1}$

\section{Allowed Transitions}

List of tabulated lines:

\begin{tabular}{c|c||c|c||c|c}
\hline \hline Wavelength $[\AA]$ & No. & Wavelength $[\AA]$ & No. & Wavelength $[\AA]$ & No. \\
\hline 735.89 & 2 & & & & \\
743.70 & 1 & 6717.04 & 6 & 23709 & 14 \\
3454.19 & 7 & 7032.47 & 6 & 23956 & 16 \\
3472.57 & 7 & 7173.94 & 6 & 24249 & 16 \\
3520.47 & 8 & 7245.17 & 4 & 24339 & 13 \\
5852.49 & 6 & 7438.90 & 5 & 25524 & 14 \\
5881.90 & 3 & 7488.87 & 9 & 25855 & 13 \\
5944.83 & 3 & 8377.61 & 10 & 26861 & 14 \\
5975.53 & 3 & 8495.36 & 11 & 27521 & 15 \\
6030.00 & 4 & 8654.38 & 12 & 28533 & 16 \\
6074.34 & 4 & 18210 & 14 & 28744 & 16 \\
6096.16 & 4 & 19577 & 13 & 29714 & 16 \\
6128.45 & 4 & 19772 & 13 & 31860 & 15 \\
6143.06 & 3 & 20350 & 14 & & \\
6163.59 & 5 & 20354 & 14 & & \\
6217.28 & 3 & 20565 & 14 & & \\
6266.50 & 5 & 21041 & 16 & & \\
6304.79 & 4 & 21708 & 14 & & \\
6334.43 & 3 & 22530 & 13 & & \\
6382.99 & 4 & 22662 & 13 & & \\
6402.25 & 3 & 23100 & 15 & & \\
6506.53 & 4 & 23260 & 13 & & \\
6532.88 & 5 & 23373 & 15 & & \\
6598.95 & 6 & 23565 & 14 & & \\
6678.28 & 6 & 23636 & 13 & & \\
& & & & & \\
\hline
\end{tabular}

In the vacuum uv region data are available for the two transitions of the $2 p-3 s$ array. They have been obtained experimentally from lifetime measurements by Schütz [1], Phelps [2], Korolev et al. [3], and theoretically from SCF calculations including exchange effects by Gold and Knox [4]. In the experiments only one of the two lines is obtained directly whereas the other one is derived using Shortley's [5] calculated intensity ratio. Since the total spread between the results is within a factor of two, uncertainties within 25 percent (class $C$ ) are estimated for the averaged values.

Extensive experimental work $[6,7]$ has been done on the lines of the prominent $3 s-3 p$ transition array. Ladenburg and Levy, and Pery-Thorne and Chamberlain have used the method of anomalous dispersion; Doherty and Friedrichs measured absolute emission intensities, the former with a conventional shock tube, the latter with a stabilized arc; with a discharge tube Garbuny determined relative intensities in emission and Krebs in absorption. Relative $f$-values have been obtained from the following procedure: the results of the three emission experiments (by Garbuny, Doherty, and Friedrichs) have been employed to obtain the line strengths within the groups of lines with the same upper but different lower levels, since for these cases errors are obviously minimized. 
Analogously, the absorption measurements of Krebs and the anomalous dispersion measurements of Ladenburg and Levy, and Pery-Thorne and Chamberlain have supplied the most accurate relative $f$-values for lines starting with the same lower level but ending in different upper levels. The averaged emission and absorption data have then been combined in a least squares fit pro. cedure to obtain a best set of relative data. It turns out that this set fits most of the original data and the $J$-file sum rule within 10 percent, but a few deviations of 30 percent are encountered.

Absolute values have been obtained by employing the results of recent extensive lifetime measurements of the $3 p$ levels undertaken by Klose [7] with a delayed coincidence technique. An averaged conversion factor has been obtained from fitting the levels $p_{1}, p_{2}, p_{5}, p_{6}, p_{7}, p_{8}$, and $p_{9}$ which were measured by Klose with a high degree of precision. The averaged conversion factor has a standard deviation of only 6 percent. Accuracies within 10 percent for most absolute $f$-values are indicated (a) from the high experimental precision obtained by Klose which is usually within 4 percent, (b) from the good consistency of the data with the $J$-file sum rule, (c) from the excellent agreement of the total line strength for this transition array, which is 224 , with a value of 230 obtained from the Coulomb approximation, and (d) from the very good agreement of Klose's lifetime results with some preliminary lifetime data by Bennett et al. [8].

For a few lines of the $3 s-4 p$ array, Klose's lifetime data for $4 p$ levels could be utilized to derive transition probabilities. However, these cannot be considered as accurate as before because the contribution of the respective $4 s-4 p$ transition to the lifetimes could be only approximately taken into account using the data discussed immediately below.

The Coulomb approximation has been applied for obtaining an absolute scale for the $4 s-4 p$ array. For the breakdown into individual lines the intermediate coupling calculations of Ufford [10] are available. The latter are estimated to be not too reliable, since similar calculations by Shortley [5] for the $3 s-3 p$ array, as judged from the many experimental comparisons, have had only a fair degree of success.

From Doherty's [9] shock tube experiment some further material is available for the $3 p-3 d$ array. His absolute values have been renormalized by using the same conversion factor as that for his values of the $3 s-3 p$ array.

\section{References}

[1] Schütz, W., Ann. Physik 18, 705-720 (1933).

[2] Phelps, A. V., Phys. Rev. 100, 1230 (1955).

[3] Korolev, F. A., Odintsov, V. I., and Fursova, E. V., Optics and Spectroscopy (U.S.S.R.) 1 6, 304-305 (1964).

[4] Gold, A. and Knox, R. S., Phys. Rev. 113, 834-839 (1959).

[5] Shortley, G. H., Phys. Rev. 47, 295-300 (1935).

[6] Krebs, K., Z. Physik 101, 604-642 (1936).

Ladenburg, R. and Levy, S., Z. Physik 88, 461-468 (1934).

Pery-Thorne, A. and Chamberlain, J. E., Proc. Phys. Soc. London A 82, 133-141 (1963).

Doherty, L. R., Thesis Michigan (1961).

Garbuny, M., Z. Physik 107, 362-368 (1937).

Friedrichs, H., Z. Astrophys. 60, 176-183 (1964).

[7] Klose, J. Z., Phys. Rev. 141, 181-186 (1966).

[8] Bennett, Jr., W. R., Kindlmann, P. J., and Mercer, G. N., Applied Optics Supplement 2 of Chemical Lasers, 34-57 (1965).

[9] Doherty, L. R., Thesis Michigan (1961).

[10] Ufford, C. W., Astrophys, J. 85, 249-250 (1937). 
Ne I. Allowed Transitions

\begin{tabular}{|c|c|c|c|c|c|c|c|c|c|c|c|c|c|}
\hline No. & $\begin{array}{c}\text { Transition } \\
\text { Array }\end{array}$ & Transition & $\lambda(\AA)$ & $E_{i}\left(\mathrm{~cm}^{-1}\right)$ & $E_{k}\left(\mathrm{~cm}^{-1}\right)$ & $g_{i}$ & $g_{k}$ & $\begin{array}{r}A_{k i}\left(10^{8}\right. \\
\left.\sec ^{-1}\right)\end{array}$ & $f_{i k}$ & $S$ (at.u.) & $\log g f$ & $\begin{array}{c}\text { Accu- } \\
\text { racy }\end{array}$ & Source \\
\hline 1 & $\begin{array}{l}2 p^{6}- \\
2 p^{5}\left({ }^{2} \mathrm{P}_{1^{1 / 2}}^{\circ}\right) 3 s\end{array}$ & ${ }^{1} \mathrm{~S}-\left[1^{1 / 2}\right]^{\circ}$ & 743.70 & 0 & 134461 & 1 & 3 & 0.476 & 0.0118 & 0.0290 & -1.93 & C & $1,2,3,4,5$ \\
\hline 2 & $\begin{array}{l}2 p^{6}- \\
2 p^{5}\left({ }^{2} \mathrm{P}_{1 / 2}^{\circ}\right) 3 s^{\prime}\end{array}$ & $\begin{array}{c}{ }^{1} \mathrm{~S}-\left[{ }^{1 / 2}\right]^{\circ} \\
(2 \mathrm{uv})\end{array}$ & 735.89 & 0 & 135891 & 1 & 3 & 6.64 & 0.162 & 0.392 & -0.79 & $\mathrm{C}$ & $1,2,3,4,5$ \\
\hline \multirow[t]{8}{*}{3} & \multirow{5}{*}{$\begin{array}{l}2 p^{5} 3 s- \\
2 p^{5}\left({ }^{2} \mathrm{P}_{1 / 2}^{\circ}\right) 3 p \\
(1)\end{array}$} & {$\left[1 \frac{1}{2}\right]^{\circ}-[1 / 2]$} & 7032.41 & 134044 & 148260 & 5 & 3 & 0.192 & 0.0854 & 9.89 & -0.370 & $\mathrm{~B}-$ & $6 n, 7$ \\
\hline & & {$\left[1^{1 / 2}\right]^{\circ}-\left[2^{1 / 2}\right]$} & 6402.25 & 134044 & 149659 & 5 & 7 & 0.433 & 0.373 & 39.3 & 0.270 & B & $6 n, 7$ \\
\hline & & {$[11 / 2]^{\circ}-[11 / 2]$} & 6334.43 & 134044 & 149826 & 5 & 5 & 0.136 & 0.0818 & 8.53 & -0.388 & $\mathrm{~B}-$ & $6 n, 7$ \\
\hline & & {$\left[1^{11 / 2}\right]^{\circ}-[11 / 2]$} & 6217.28 & 134044 & 150124 & 5 & 3 & 0.0777 & 0.0270 & 2.76 & -0.869 & B - & $6 n, 7$ \\
\hline & & {$[11 / 2]^{0}-[1 / 2]$} & 6143.06 & 134044 & 150318 & 5 & 5 & 0.216 & 0.122 & 12.4 & -0.214 & $\mathrm{~B}-$ & $6 n, 7$ \\
\hline & $2 p^{5}\left({ }^{2} \mathrm{P}_{1^{1 / 2} / 2}^{\circ}\right) 3 s-$ & {$[11 / 2]^{\circ}-[11 / 2]$} & 5975.53 & 134044 & 150774 & 5 & 3 & 0.0433 & 0.0139 & 1.37 & -1.158 & $\mathrm{~B}-$ & $6 n .7$ \\
\hline & $2 p^{5}\left({ }^{2} \mathrm{P}_{1 / 2}^{\mathrm{o}}\right) 3 p^{\prime}$ & {$[11 / 2]^{\circ}-[11 / 2]$} & 5944.83 & 134044 & 150860 & 5 & 5 & 0.105 & 0.0556 & 5.44 & -0.556 & $\mathrm{C}+$ & $6 n, 7$ \\
\hline & (1) & {$[1 / 2]^{\circ}-[1 / 2]$} & 5881.90 & 134044 & 151040 & 5 & 3 & 0.128 & 0.0398 & 3.86 & -0.701 & $B-$ & $6 n, 7$ \\
\hline \multirow[t]{8}{*}{4} & \multirow{5}{*}{$\begin{array}{l}2 p^{5} 3 s- \\
2 p^{5}\left({ }^{2} \mathrm{P}_{1 / 2}^{\circ}\right) 3 p \\
(3)\end{array}$} & {$\left[1 \frac{1 / 2}{0}\right]^{\circ}-[1 / 2]$} & 7245.17 & 134461 & 148260 & 3 & 3 & 0.0977 & 0.0769 & 5.50 & -0.637 & $\mathrm{~B}-$ & $6 n, 7$ \\
\hline & & {$[11 / 2]^{\circ}-[21 / 2]$} & 6506.53 & 134461 & 149826 & 3 & 5 & 0.232 & 0.245 & 15.8 & -0.133 & $\dot{\mathrm{B}}-$ & $6 n, 7$ \\
\hline & & {$[11 / 2]^{\circ}-[11 / 2]$} & 6382.99 & 134461 & 150124 & 3 & 3 & 0.279 & 0.170 & 10.7 & -0.291 & B - & $6 n, 7$ \\
\hline & & {$[11 / 2]^{\circ}-[11 / 2]$} & 6304.79 & 134461 & 150318 & 3 & 5 & 0.0507 & 0.0504 & 3.14 & -0.821 & $\mathrm{~B}-$ & $6 n .7$ \\
\hline & & {$[11 / 2]^{\circ}-[1 / 2]$} & 6074.34 & 134461 & 150919 & 3 & 1 & 0.617 & 0.114 & 6.83 & -0.467 & $\mathrm{C}+$ & $6 n .7$ \\
\hline & $2 p^{5}\left({ }^{2} \mathrm{P}_{1 / 2}^{\circ}\right) 3 s-$ & {$\left[11^{1 / 2}\right]^{\circ}-[1 / 2]$} & 6128.45 & 134461 & 150774 & 3 & 3 & 0.0327 & 0.0184 & 1.11 & -1.258 & $B-$ & $6 n .7$ \\
\hline & $2 p^{5}\left({ }^{2} \mathrm{P}_{1 / 2}^{\circ}\right) 3 p^{\prime}$ & {$[11 / 2]^{\circ}-\left[1 \frac{1}{1}\right]$} & 6096.16 & 134461 & 150860 & 3 & 5 & 0.169 & 0.157 & 9.45 & -0.327 & $\mathrm{C}+$ & $6 n, 7$ \\
\hline & (3) & {$\left[11^{1 / 2}\right]^{\circ}-[1 / 2]$} & 6030.00 & 134461 & 151040 & 3 & 3 & 0.0627 & 0.0342 & 2.04 & -0.989 & B - & $6 n, 7$ \\
\hline \multirow[t]{4}{*}{5} & $2 p^{5}\left({ }^{2} \mathrm{P}_{1 / 2}^{\circ}\right) 3 s^{\prime}-$ & {$\left[\begin{array}{l}1 / 2 \\
{[}\end{array}\right]^{\circ}-\left[\begin{array}{ll}1 / 2\end{array}\right]$} & 7438.90 & 134821 & 148260 & 1 & 3 & 0.0292 & 0.0727 & 1.78 & -1.139 & $\mathrm{~B}-$ & $6 n, 7$ \\
\hline & $\begin{array}{c}2 p^{5}\left({ }^{2} \mathrm{P}_{1 / 2}^{\circ}\right) 3 p \\
(5)\end{array}$ & {$[1 / 2]^{\circ}-[1 / 2]$} & 6532.88 & 134821 & 150124 & 1 & 3 & 0.128 & 0.246 & 5.28 & -0.610 & $\mathrm{~B}-$ & $6 n, 7$ \\
\hline & $2 p^{5} 3 s^{\prime}-$ & {$[1 / 2]^{\circ}-[11 / 2]$} & 6266.50 & 134821 & 150774 & 1 & 3 & 0.223 & 0.394 & 8.13 & -0.405 & $\mathrm{~B}-$ & $6 n, 7$ \\
\hline & $\begin{array}{c}2 p^{5}\left({ }^{2} \mathrm{P}_{1 / 2}^{\circ}\right) 3 p^{\prime} \\
(5)\end{array}$ & {$\left[\begin{array}{ll}1 / 2 & \circ\end{array}\right]^{\circ}-[1 / 2]$} & 6163.59 & 134821 & 151040 & 1 & 3 & 0.160 & 0.273 & 5.55 & -0.563 & $\mathrm{~B}-$ & $6 n, 7$ \\
\hline \multirow[t]{6}{*}{6} & $2 p^{5}\left({ }^{2} \mathrm{P}_{1 / 2_{2}}^{\circ}\right) 3 s^{\prime}-$ & {$[1 / 2]^{\circ}-[21 / 2]$} & 7173.94 & 135891 & 149826 & 3 & 5 & 0.0365 & 0.0469 & 3.33 & -0.851 & $\mathrm{~B}-$ & $6 n, 7$ \\
\hline & $\begin{array}{c}2 p^{5}\left({ }^{2} \mathrm{P}_{1 / 2}^{\circ}\right) 3 p \\
(6)\end{array}$ & $1 /]^{\circ}-[1 / \Omega$ & 6929.47 & 135891 & 150318 & 3 & 5 & 0.190 & 0.228 & 15.6 & -0.165 & $\mathrm{~B}-$ & $6 n, 7$ \\
\hline & $2 p^{5} 3 s^{\prime}-$ & $1 / 2]^{\circ}-\left[1^{1 / 2}\right]$ & 6717.04 & 135891 & 150774 & 3 & 3 & 0.234 & 0.158 & 10.5 & -0.323 & $B-$ & $6 n, 7$ \\
\hline & $2 p^{5}\left({ }^{2} \mathrm{P}_{1 / 2}^{\circ}\right) 3 p^{\prime}$ & {$[1 / 2]^{\circ}-[11 / 2]$} & 6678.28 & 135891 & 150860 & 3 & 5 & 0.238 & 0.265 & 17.5 & -0.099 & $\mathrm{C}+$ & $6 n, 7$ \\
\hline & (6) & $1 / 2]^{0}-\left[\begin{array}{l}1 / 2 \\
1 / 2\end{array}\right]$ & $\begin{array}{l}6598.95 \\
5859.40\end{array}$ & 135891 & 151040 & 3 & 3 & 0.251 & 0.164 & 10.7 & -0.308 & $\begin{array}{l}\mathrm{B}- \\
\mathrm{B}\end{array}$ & $6 n, 7$ \\
\hline & & $82]-\left[\begin{array}{ll}1 & 2\end{array}\right]$ & 5852.49 & 155891 & 152913 & 3 & 1 & 0.719 & 0.123 & 1.11 & -0.453 & D & on, r \\
\hline \multirow[t]{2}{*}{7} & $2 p^{5} 3 s-$ & {$\left[1^{1 / 2}\right]^{\circ}-\left[2^{1 / 2}\right]$} & 3472.57 & 134044 & 162833 & 5 & 7 & 0.099 & 0.0251 & 1.4 & -0.90 & C & 7 \\
\hline & $\begin{array}{c}2 p^{5}\left({ }^{2} \mathrm{P}_{1 / 2}^{\circ}\right) 4 p \\
(2)\end{array}$ & {$[1 / 1 /]^{\circ}$} & 3454.19 & 134461 & 163403 & 3 & 1 & 0.085 & 0.0051 & 0.173 & -1.82 & C & 7 \\
\hline 8 & $\begin{array}{l}2 p^{5} 3 s^{\prime}- \\
2 p^{5}\left({ }^{2} \mathrm{P}_{1 / 2}^{\mathrm{n}}\right) 4 p^{\prime}\end{array}$ & {$[1 / 2]^{\circ}-[1 / 2]$} & 3520.47 & 135891 & 164288 & 3 & 1 & 0.073 & 0.00449 & 0.156 & -1.87 & C & 7 \\
\hline
\end{tabular}


NeI. Allowed Transitions-Continued

\begin{tabular}{|c|c|c|c|c|c|c|c|c|c|c|c|c|c|}
\hline No. & $\begin{array}{c}\text { Transition } \\
\text { Array }\end{array}$ & Transition & $\lambda(\AA)$ & $E_{i}\left(\mathrm{~cm}^{-1}\right)$ & $E_{k}\left(\mathrm{~cm}^{-1}\right)$ & $g_{i}$ & $g_{k}$ & $\begin{array}{l}A_{k i}\left(10^{4}\right. \\
\left.\sec ^{-1}\right)\end{array}$ & $f_{i k}$ & S(at.u.) & $\log g f$ & $\begin{array}{l}\text { Accu- } \\
\text { racy }\end{array}$ & Source \\
\hline 9 & $\left|\begin{array}{l}2 p^{5} 3 p- \\
2 p^{5}\left({ }^{2} \mathrm{P}_{1 / 2}^{\circ}\right) 3 d\end{array}\right|$ & $\mid 1 / 2]-\left[1 \frac{1}{1}\right]^{\circ}$ & 7488.87 & 148260 & 161609 & 3 & 5 & 0.349 & 0.489 & 36.2 & 0.166 & C & ) \\
\hline 10 & & $\begin{array}{c}{\left[2^{1 / 2}\right]-\left[3^{1 / 2}\right]^{\circ}} \\
(12)\end{array}$ & 8377.61 & 149659 & 161592 & 7 & 9 & 0.51 & 0.69 & 134 & 0.69 & $\mathrm{C}$ & $9 n$ \\
\hline 11 & & $\begin{array}{c}{\left[2 \frac{1}{2}\right]-\left[3 \frac{1 / 2}{}\right]} \\
(18)\end{array}$ & 8495.36 & 149826 & 161594 & 5 & 7 & 0.357 & 0.54 & 76 & 0.432 & $\mathrm{C}$ & $9 n$ \\
\hline 12 & $\begin{array}{l}2 p^{5} 3 p^{\prime}- \\
2 p^{5}\left({ }^{2} \mathrm{P}_{1 / 2}^{\circ}\right) 3 d^{\prime}\end{array}$ & $\begin{array}{c}{\left[1^{1 / 2}\right]-[21 / 2]^{\circ}} \\
(33)\end{array}$ & 8654.38 & 150860 & 162412 & 5 & 7 & 0.44 .5 & 0.70 & 100 & 0.54 & C & $9 n$ \\
\hline 13 & 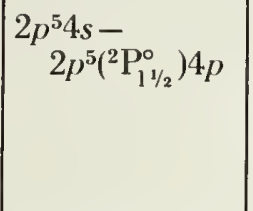 & $\begin{array}{l}{\left[1^{1 / 2}\right]^{\circ}-[1 / 2]} \\
{\left[1^{1 / 2}\right]^{\circ}-[21 / 2]} \\
{[11 / 2]^{\circ}-[21 / 2]} \\
{[11 / 2]^{\circ}-[11 / 2]} \\
{[11 / 2]^{\circ}-[11 / 2]}\end{array}$ & $\begin{array}{l}{[25524]} \\
{[23636]} \\
{[23260]} \\
{[22662]} \\
{[22530]}\end{array}$ & $\begin{array}{l}158603 \\
158603 \\
158603 \\
158603 \\
158603\end{array}$ & $\begin{array}{l}162520 \\
162833 \\
162901 \\
163015 \\
163040\end{array}$ & $\begin{array}{l}5 \\
5 \\
5 \\
5 \\
5\end{array}$ & $\begin{array}{l}3 \\
7 \\
5 \\
3 \\
5\end{array}$ & $\begin{array}{l}0.012 \\
0.057 \\
0.0025 \\
0.0058 \\
0.037\end{array}$ & $\begin{array}{l}0.073 \\
0.67 \\
0.020 \\
0.027 \\
0.28\end{array}$ & $\begin{array}{c}31 \\
260 \\
7.8 \\
10 \\
100\end{array}$ & $\begin{array}{r}-0.44 \\
0.52 \\
-0.99 \\
-0.87 \\
0.15\end{array}$ & $\begin{array}{l}\mathrm{D} \\
\mathrm{D}+ \\
\mathrm{D} \\
\mathrm{D} \\
\mathrm{D}\end{array}$ & $\begin{array}{l}10 n, c a \\
10 n . c a \\
10 n, c a \\
10 n, c a \\
10 n, c a\end{array}$ \\
\hline & $\mid \begin{array}{r}2 p^{5}\left({ }^{2} \mathrm{P}_{11 / 2}^{\circ}\right) 4 s- \\
2 p^{5}\left({ }^{2} \mathrm{P}_{1 / 2}^{\mathrm{o}}\right) 4 p^{\prime}\end{array}$ & {$\left[\begin{array}{l}{[11 / 2]^{\circ}-\left[1 \frac{1 / 2}{1}\right]} \\
{\left[1^{1 / 2}\right]^{\circ}-[11 / 2]} \\
{\left[1^{1 / 2}\right]^{\circ}-[1 / 2]}\end{array}\right.$} & $\begin{array}{l}{[19772]} \\
{[24339]} \\
{[19577]}\end{array}$ & $\begin{array}{l}158603 \\
158603 \\
158603\end{array}$ & $\begin{array}{l}163659 \\
163711 \\
163710\end{array}$ & $\begin{array}{l}5 \\
5 \\
5\end{array}$ & $\begin{array}{l}3 \\
5 \\
3\end{array}$ & $\begin{array}{l}0.0052 \\
0.021 \\
0.058\end{array}$ & $\begin{array}{l}0.018 \\
0.18 \\
0.20\end{array}$ & $\begin{array}{l}5.9 \\
73 \\
64\end{array}$ & $\begin{array}{l}-1.04 \\
-0.04 \\
-0.00\end{array}$ & $\begin{array}{l}\mathrm{D} \\
\mathrm{D} \\
\mathrm{D}\end{array}$ & $\begin{array}{l}10 n . c a \\
10 n . c a \\
10 n, c a\end{array}$ \\
\hline 14 & $\begin{array}{l}2 p^{5} 4 s- \\
\quad 2 p^{5}\left({ }^{2} \mathrm{P}_{1 / 2}^{\circ}\right) 4 p\end{array}$ & $\begin{array}{l}{\left[1^{1 / 2}\right]^{\circ}-[1 / 2]} \\
{\left[11^{1 / 2}\right]^{\circ}-\left[2^{1 / 2}\right]} \\
{[11 / 2]^{\circ}-[11 / 2]} \\
{[11 / 2]^{\circ}-[11 / 2]} \\
{[11 / 2]^{\circ}-[1 / 2]}\end{array}$ & $\begin{array}{l}{[26861]} \\
{[24365]} \\
{[23709]} \\
{[23565]} \\
{[21708]}\end{array}$ & $\begin{array}{l}158798 \\
158798 \\
158798 \\
158798 \\
158798\end{array}$ & $\begin{array}{l}162520 \\
162901 \\
163015 \\
163040 \\
163403\end{array}$ & $\begin{array}{l}3 \\
3 \\
3 \\
3 \\
3\end{array}$ & $\begin{array}{l}3 \\
5 \\
3 \\
5 \\
1\end{array}$ & $\begin{array}{l}0.015 \\
0.0015 \\
0.0019 \\
0.021 \\
0.068\end{array}$ & $\begin{array}{l}0.16 \\
0.022 \\
0.016 \\
0.29 \\
0.16\end{array}$ & $\begin{array}{l}43 \\
5.2 \\
3.1 \\
68 \\
34\end{array}$ & $\begin{array}{l}-0.31 \\
-1.19 \\
-1.32 \\
-0.06 \\
-0.32\end{array}$ & $\begin{array}{l}\mathrm{D} \\
\mathrm{D} \\
\mathrm{D} \\
\mathrm{D} \\
\mathrm{D}+\end{array}$ & $\begin{array}{l}10 n . c a \\
10 n . c a \\
10 n . c a \\
10 n . c a \\
10 n . c a\end{array}$ \\
\hline & $\mid \begin{array}{r}2 p^{5}\left({ }^{2} \mathrm{P}_{1}^{1 / 1}\right) 4 s- \\
2 p^{5}\left({ }^{2} \mathrm{P}_{1 / 2}^{\circ}\right) 4 p^{\prime}\end{array}$ & 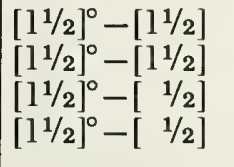 & $\begin{array}{l}{[20565]} \\
{[20350]} \\
{[20354]} \\
{[18210]}\end{array}$ & $\begin{array}{l}158798 \\
158798 \\
158798 \\
158798\end{array}$ & $\begin{array}{l}163659 \\
163711 \\
163710 \\
164289\end{array}$ & $\begin{array}{l}3 \\
3 \\
3 \\
3\end{array}$ & $\begin{array}{l}3 \\
5 \\
3 \\
1\end{array}$ & $\begin{array}{l}0.026 \\
0.054 \\
0.025 \\
0.0087\end{array}$ & $\begin{array}{l}0.16 \\
0.56 \\
0.15 \\
0.014\end{array}$ & $\begin{array}{r}33 \\
110 \\
31 \\
2.6\end{array}$ & $\begin{array}{r}-0.31 \\
0.22 \\
-0.34 \\
-1.36\end{array}$ & $\begin{array}{l}\mathrm{D} \\
\mathrm{D} \\
\mathrm{D} \\
\mathrm{D}\end{array}$ & $\begin{array}{l}10 n, c a \\
10 n . c a \\
10 n . c a \\
10 n . c a\end{array}$ \\
\hline 15 & $\begin{array}{r}2 p^{5}\left({ }^{2} \mathrm{P}_{1 / 2}^{\circ}\right) 4 s^{\prime}- \\
2 p^{5}\left({ }^{5} \mathrm{P}_{1 / 2}^{\circ}\right) 4 p\end{array}$ & $\begin{array}{l}{\left[\begin{array}{r}1 / 2 \\
1\end{array}\right]^{\circ}-\left[\begin{array}{r}1 / 2 \\
{[1 / 2}\end{array}\right]^{\circ}-\left[1 \frac{1}{1} 2\right]}\end{array}$ & $\begin{array}{l}{[31860]} \\
{[27521]}\end{array}$ & $\begin{array}{l}159382 \\
159382\end{array}$ & $\begin{array}{l}162520 \\
163015\end{array}$ & $\begin{array}{l}1 \\
1\end{array}$ & $\begin{array}{l}3 \\
3\end{array}$ & $\begin{array}{l}0.0054 \\
0.0075\end{array}$ & $\begin{array}{l}0.25 \\
0.26\end{array}$ & $\begin{array}{l}26 \\
23\end{array}$ & $\begin{array}{l}-0.61 \\
-0.59\end{array}$ & $\begin{array}{l}\mathrm{D} \\
\mathrm{D}\end{array}$ & $\begin{array}{l}10 n, c a \\
10 n, c a\end{array}$ \\
\hline & $\begin{array}{l}2 p^{5} 4 s^{\prime}- \\
\quad 2 p^{5}\left({ }^{2} \mathrm{P}_{1 / 2}^{\circ}\right) 4 p^{\prime}\end{array}$ & $\begin{array}{l}{\left[\begin{array}{l}1 / 2 \\
{[}\end{array}\right]^{\circ}-\left[\begin{array}{l}1 / 2 \\
1 / 2\end{array}\right]^{\circ}-[1 / 2]}\end{array}$ & $\begin{array}{l}{[23373]} \\
{[23100]}\end{array}$ & $\begin{array}{l}159382 \\
159382\end{array}$ & $\begin{array}{l}163659 \\
163710\end{array}$ & 1 & $\begin{array}{l}3 \\
3\end{array}$ & $\begin{array}{l}0.027 \\
0.0055\end{array}$ & $\begin{array}{l}0.67 \\
0.13\end{array}$ & $\begin{array}{l}52 \\
10\end{array}$ & $\begin{array}{l}-0.17 \\
-0.88\end{array}$ & $\begin{array}{l}\mathrm{D} \\
\mathrm{D}\end{array}$ & $\begin{array}{l}10 n . c a \\
10 n . c a\end{array}$ \\
\hline 16 & $\begin{array}{r}2 p^{5}\left({ }^{2} \mathrm{P}_{1 / 2}^{\circ}\right) 4 s^{\prime}- \\
2 p^{5}\left({ }^{2} \mathrm{P}_{1 / 2}^{\circ}\right) 4 p\end{array}$ & 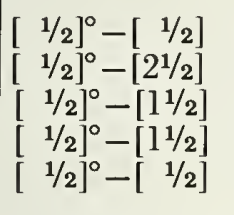 & $\begin{array}{l}{[33511]} \\
{[29714]} \\
{[28744]} \\
{[28533]} \\
{[25855]}\end{array}$ & $\begin{array}{l}159537 \\
159537 \\
159537 \\
159537 \\
159537\end{array}$ & $\begin{array}{l}162520 \\
162901 \\
163015 \\
163040 \\
163403\end{array}$ & $\begin{array}{l}3 \\
3 \\
3 \\
3 \\
3\end{array}$ & $\begin{array}{l}3 \\
5 \\
3 \\
5 \\
1\end{array}$ & $\begin{array}{l}0.0020 \\
0.027 \\
0.021 \\
0.0023 \\
0.0030\end{array}$ & $\begin{array}{l}0.034 \\
0.59 \\
0.26 \\
0.046 \\
0.010\end{array}$ & $\begin{array}{c}11 \\
170 \\
74 \\
13 \\
2.6\end{array}$ & $\begin{array}{r}-0.99 \\
0.25 \\
-0.11 \\
-0.86 \\
-1.52\end{array}$ & $\begin{array}{l}\mathrm{D} \\
\mathrm{D} \\
\mathrm{D} \\
\mathrm{D} \\
\mathrm{D}\end{array}$ & $\begin{array}{l}10 n, c a \\
10 n . c a \\
10 n, c a \\
10 n . c a \\
10 n . c a\end{array}$ \\
\hline & $\begin{array}{l}2 p^{5} 4 s^{\prime}- \\
2 p^{5}\left({ }^{2} \mathrm{P}_{1 / 2}^{\circ}\right) 4 p^{\prime}\end{array}$ & $\begin{array}{l}{\left[\begin{array}{l}1 / 2 \\
1\end{array}\right]^{\circ}-[11 / 2]} \\
1 / 2]^{\circ}-\left[\begin{array}{c}1 / 2 \\
{[1 / 2}\end{array}\right]^{\circ}-\left[\begin{array}{c}1 / 2\end{array}\right]\end{array}$ & $\begin{array}{l}{[24249]} \\
{[23956]} \\
{[21041]}\end{array}$ & $\begin{array}{l}159537 \\
159537 \\
159537\end{array}$ & $\begin{array}{l}163659 \\
163710 \\
164288\end{array}$ & $\begin{array}{l}3 \\
3 \\
3\end{array}$ & $\begin{array}{l}3 \\
3 \\
1\end{array}$ & $\begin{array}{l}0.0095 \\
0.0029 \\
0.075\end{array}$ & $\begin{array}{l}0.084 \\
0.025 \\
0.17\end{array}$ & $\begin{array}{l}20 \\
5.9 \\
34\end{array}$ & $\begin{array}{l}-0.60 \\
-1.12 \\
-0.30\end{array}$ & $\begin{array}{l}\mathrm{D} \\
\mathrm{D} \\
\mathrm{D}+\end{array}$ & $\begin{array}{l}10 n . c a \\
10 n . c a \\
10 n . c a\end{array}$ \\
\hline
\end{tabular}


Ne II.

Ground State

$1 s^{2} 2 s^{2} 2 p^{5}{ }^{2} \mathrm{P}_{3 / 2}^{\circ}$

Ionization Potential

$41.07 \mathrm{eV}=331350 \mathrm{~cm}^{-1}$

\section{Allowed Transitions}

List of tabulated lines:

\begin{tabular}{|c|c|c|c|c|c|}
\hline Wavelength $[\AA]$ & No. & Wavelength $[\AA ̊]$ & No. & Wavelength $[\AA]$ & No. \\
\hline $\begin{array}{l}460.725 \\
462.338 \\
2846.4 \\
2853.5 \\
2858.0\end{array}$ & $\begin{array}{r}1 \\
1 \\
23 \\
7 \\
22\end{array}$ & $\begin{array}{l}3166.2 \\
3169.30 \\
3173.58 \\
3176.16 \\
3187.60\end{array}$ & $\begin{array}{r}4 \\
28 \\
25 \\
28 \\
4\end{array}$ & $\begin{array}{l}3357.90 \\
3360.63 \\
3362.89 \\
3367.05 \\
3367.20\end{array}$ & $\begin{array}{r}24 \\
3 \\
24 \\
24 \\
31\end{array}$ \\
\hline $\begin{array}{l}2870.0 \\
2873.0 \\
2876.4 \\
2876.5\end{array}$ & $\begin{array}{l}19 \\
22 \\
22 \\
19\end{array}$ & $\begin{array}{l}3188.74 \\
3190.86 \\
3194.61 \\
3198.62 \\
3198.88\end{array}$ & $\begin{array}{l}26 \\
25 \\
28 \\
25 \\
25\end{array}$ & $\begin{array}{l}3371.87 \\
3374.10 \\
3378.28 \\
3379.39 \\
3386.24\end{array}$ & $\begin{array}{l}34 \\
24 \\
13 \\
24 \\
24\end{array}$ \\
\hline $\begin{array}{l}2878.1 \\
2888.4 \\
2889.0 \\
2891.5 \\
2895.0\end{array}$ & $\begin{array}{l}23 \\
19 \\
20 \\
22 \\
19\end{array}$ & $\begin{array}{l}3208.3 \\
3208.99 \\
3209.38 \\
3213.70 \\
3214.38\end{array}$ & $\begin{array}{l}27 \\
26 \\
28 \\
25 \\
26\end{array}$ & $\begin{array}{l}3388.46 \\
3390.56 \\
3392.7 \\
3392.78 \\
3393.2\end{array}$ & $\begin{array}{l}31 \\
24 \\
34 \\
13 \\
33\end{array}$ \\
\hline $\begin{array}{l}2896.3 \\
2897.1 \\
2906.8 \\
2907.7 \\
2910.4\end{array}$ & $\begin{array}{r}7 \\
19 \\
22 \\
20 \\
19\end{array}$ & $\begin{array}{l}3217.4 \\
3218.21 \\
3220.0 \\
3230.16 \\
3230.5\end{array}$ & $\begin{array}{l}28 \\
25 \\
27 \\
16 \\
16\end{array}$ & $\begin{array}{l}3397.5 \\
3414.82 \\
3416.87 \\
3417.71 \\
3453.10\end{array}$ & $\begin{array}{l}31 \\
32 \\
33 \\
32 \\
33\end{array}$ \\
\hline $\begin{array}{l}2916.2 \\
2925.7 \\
2933.7 \\
2934.3 \\
2935.3\end{array}$ & $\begin{array}{l}21 \\
22 \\
21 \\
20 \\
21\end{array}$ & $\begin{array}{l}3231.97 \\
3232.3 \\
3232.38 \\
3243.34 \\
3244.15\end{array}$ & $\begin{array}{l}16 \\
28 \\
16 \\
27 \\
26\end{array}$ & $\begin{array}{l}3456.68 \\
3463.1 \\
3475.25 \\
3477.69 \\
3479.5\end{array}$ & $\begin{array}{l}40 \\
12 \\
46 \\
33 \\
17\end{array}$ \\
\hline $\begin{array}{l}2951.1 \\
2953.0 \\
2955.7 \\
3001.65 \\
3015.7\end{array}$ & $\begin{array}{r}21 \\
21 \\
6 \\
6 \\
5\end{array}$ & $\begin{array}{l}3248.15 \\
3255.39 \\
3263.43 \\
3269.86 \\
3270.79\end{array}$ & $\begin{array}{r}27 \\
35 \\
27 \\
27 \\
3\end{array}$ & $\begin{array}{l}3480.8 \\
3481.96 \\
3503.61 \\
3522.72 \\
3538.3\end{array}$ & $\begin{array}{l}17 \\
11 \\
40 \\
46 \\
12\end{array}$ \\
\hline $\begin{array}{l}3017.34 \\
3027.04 \\
3028.7 \\
3028.84 \\
3034.48\end{array}$ & $\begin{array}{r}18 \\
18 \\
18 \\
6 \\
18\end{array}$ & $\begin{array}{l}3297.74 \\
3309.78 \\
3310.55 \\
3311.30 \\
3314.60\end{array}$ & $\begin{array}{r}3 \\
13 \\
35 \\
3 \\
34\end{array}$ & $\begin{array}{l}3542.90 \\
3544.2 \\
3546.22 \\
3551.2 \\
3554.39\end{array}$ & $\begin{array}{l}45 \\
30 \\
39 \\
37 \\
30\end{array}$ \\
\hline $\begin{array}{l}3037.73 \\
3045.58 \\
3047.57 \\
3054.69 \\
3097.5\end{array}$ & $\begin{array}{l}18 \\
18 \\
18 \\
18 \\
29\end{array}$ & $\begin{array}{l}3319.75 \\
3320.29 \\
3323.75 \\
3327.16 \\
3329.20\end{array}$ & $\begin{array}{r}15 \\
24 \\
13 \\
3 \\
24\end{array}$ & $\begin{array}{l}3557.84 \\
3561.23 \\
3565.84 \\
3568.53 \\
3571.26\end{array}$ & $\begin{array}{l}11 \\
42 \\
45 \\
14 \\
42\end{array}$ \\
\hline $\begin{array}{l}3118.02 \\
3132.22 \\
3135.8 \\
3135.82 \\
3136.5\end{array}$ & $\begin{array}{r}28 \\
25 \\
29 \\
4 \\
4\end{array}$ & $\begin{array}{l}3330.78 \\
3334.8 \\
3334.87 \\
3344.43 \\
3345.49\end{array}$ & $\begin{array}{r}31 \\
34 \\
3 \\
3 \\
15\end{array}$ & $\begin{array}{l}3574.23 \\
3574.3 \\
3574.64 \\
3590.47 \\
3594.18\end{array}$ & $\begin{array}{l}14 \\
39 \\
14 \\
43 \\
45\end{array}$ \\
\hline $\begin{array}{l}3151.16 \\
3154.82 \\
3160.0 \\
3164.46 \\
3165.70\end{array}$ & $\begin{array}{l}28 \\
26 \\
29 \\
25 \\
25\end{array}$ & $\begin{array}{l}3345.88 \\
3345.88 \\
3353.36 \\
3355.05 \\
3356.35\end{array}$ & $\begin{array}{r}24 \\
15 \\
35 \\
3 \\
32\end{array}$ & $\begin{array}{l}3612.35 \\
3628.06 \\
3632.75 \\
3643.89 \\
3644.86\end{array}$ & $\begin{array}{l}38 \\
51 \\
44 \\
10 \\
51\end{array}$ \\
\hline
\end{tabular}


Allowed Transitions - Continued

\begin{tabular}{|c|c|c|c|c|c|}
\hline Wavelength $[\AA]$ & No. & Wavelength $[\AA]$ & No. & Wavelength $[\AA]$ & No. \\
\hline $\begin{array}{l}3659.93 \\
3664.09 \\
3679.80 \\
3694.22 \\
3697.09\end{array}$ & $\begin{array}{r}44 \\
2 \\
51 \\
2 \\
51\end{array}$ & $\begin{array}{l}3790.96 \\
3800.02 \\
3806.30 \\
3818.44 \\
3829.77\end{array}$ & $\begin{array}{l}41 \\
49 \\
41 \\
49 \\
49\end{array}$ & $\begin{array}{l}4290.40 \\
4323.3 \\
4346.9 \\
4365.72 \\
4369.77\end{array}$ & $\begin{array}{l}54 \\
53 \\
54 \\
54 \\
53\end{array}$ \\
\hline $\begin{array}{l}3701.81 \\
3709.64 \\
3713.09 \\
3721.86 \\
3726.9\end{array}$ & $\begin{array}{r}50 \\
2 \\
10 \\
47 \\
50\end{array}$ & $\begin{array}{l}3903.9 \\
3942.3 \\
3999.5 \\
4217.15 \\
4219.76\end{array}$ & $\begin{array}{r}9 \\
9 \\
9 \\
52 \\
52\end{array}$ & $\begin{array}{l}4379.50 \\
4385.00 \\
4391.94 \\
4397.94 \\
4409.30\end{array}$ & $\begin{array}{l}53 \\
53 \\
54 \\
53 \\
54\end{array}$ \\
\hline $\begin{array}{l}3727.08 \\
3732.7 \\
3734.94 \\
3744.66 \\
3751.26\end{array}$ & $\begin{array}{r}10 \\
47 \\
2 \\
50 \\
2\end{array}$ & $\begin{array}{l}4220.92 \\
4224.75 \\
4231.60 \\
4234.3 \\
4239.95\end{array}$ & $\begin{array}{l}52 \\
52 \\
52 \\
52 \\
52\end{array}$ & $\begin{array}{l}4413.20 \\
4428.54 \\
4430.90 \\
4441.1 \\
4442.67\end{array}$ & $\begin{array}{r}54 \\
54 \\
53 \\
8 \\
53\end{array}$ \\
\hline $\begin{array}{l}3753.83 \\
3754.9 \\
3766.29 \\
3776.9 \\
3777.16\end{array}$ & $\begin{array}{r}48 \\
36 \\
2 \\
41 \\
2\end{array}$ & $\begin{array}{l}4242.20 \\
4249.2 \\
4250.68 \\
4257.82 \\
4280.3\end{array}$ & $\begin{array}{l}52 \\
54 \\
52 \\
52 \\
53\end{array}$ & $\begin{array}{l}4446.46 \\
4492.4 \\
4502.52\end{array}$ & $\begin{array}{l}53 \\
54 \\
53\end{array}$ \\
\hline
\end{tabular}

Aside from the multiplet $2 s^{2} 2 p^{5}{ }^{2} \mathrm{P}^{\circ}-2 s 2 p^{6}{ }^{2} \mathrm{~S}$ in the vacuum uv for which a quantum mechanical calculation (screening approximation) has been carried out by Varsavsky [1], the main source of theoretical information on the spectrum are the extensive calculations by Garstang [2] for lines of the $3 s-3 p$ and $3 p-3 d$ arrays. He has calculated the relative line strengths under the assumption of intermediate coupling and has used the Coulomb approximation to obtain the transition integrals for the absolute values. However, for the $3 s-3 p$ array Koopman's [3] relative line strengths obtained from intensity measurements with an electrically driven shock tube agree with LS-coupling better than with the intermediate coupling values. Therefore, Koopman's relative values are averaged with LS-coupling results and put on an absolute scale by using the Coulomb approximation. On the other hand, comparison with the very incomplete experimental intensity data [3] for the $3 p-3 d$ array indicates that intermediate coupling fits much better here than LScoupling and gives in may cases drastic improvements. Thus Garstang's results are exclusively used for this array as well as for all intercombination lines. Some lines marked D- should be considered inferior in quality to the rest, since Garstang finds them very sensitive to the choice of parameters.

In addition, the $f$-values for the three strongest multiplets of the $3 d-4 f$ array have been calculated with the Coulomb approximation using LS-coupling for the multiplet components.

\section{References}

[1] Varsavsky, C. M., Astrophys. J. Suppl. Ser. 6, No. 53, 75-108 (1961).

[2] Garstang, R. H., Monthly Notices Roy. Astron. Soc. 114, 118-133 (1954).

[3] Koopman, D. W., J. Opt. Soc. Am. 54, 1354-1358 (1964). 
Ne II. Allowed Transitions

\begin{tabular}{|c|c|c|c|c|c|c|c|c|c|c|c|c|c|}
\hline No. & $\begin{array}{c}\text { Transition } \\
\text { Array }\end{array}$ & $\begin{array}{l}\text { Multi- } \\
\text { plet }\end{array}$ & $\lambda(\AA)$ & $E_{i}\left(\mathrm{~cm}^{-1}\right)$ & $E_{k}\left(\mathrm{~cm}^{-1}\right)$ & $g_{i}$ & $g_{k}$ & $\begin{array}{c}A_{k i}\left(10^{8}\right. \\
\left.\sec ^{-1}\right)\end{array}$ & $f_{i k}$ & $S$ (at.u.) & $\log g f$ & $\begin{array}{c}\text { Accu- } \\
\text { racy }\end{array}$ & Source \\
\hline \multirow[t]{2}{*}{1} & \multirow{2}{*}{$\begin{array}{c}2 s^{2} 2 p^{5}- \\
2 s^{2} p^{6}\end{array}$} & \multirow[t]{2}{*}{$\begin{array}{c}{ }^{2} \mathrm{P}^{\circ}-{ }^{2} \mathrm{~S} \\
(1 \mathrm{uv})\end{array}$} & 461.28 & 260.07 & 217050 & 6 & 2 & 310 & 0.33 & 3.0 & 0.29 & E & 1 \\
\hline & & & $\begin{array}{l}460.725 \\
462.388\end{array}$ & $782^{0.00}$ & $\begin{array}{l}217050 \\
217050\end{array}$ & $\begin{array}{l}4 \\
2\end{array}$ & $\begin{array}{l}2 \\
2\end{array}$ & $\begin{array}{l}210 \\
100\end{array}$ & $\begin{array}{l}0.33 \\
0.33\end{array}$ & $\begin{array}{l}2.0 \\
1.0\end{array}$ & $\begin{array}{r}0.12 \\
-0.18\end{array}$ & $\begin{array}{l}\mathrm{E} \\
\mathrm{E}\end{array}$ & $\begin{array}{l}l s \\
l s\end{array}$ \\
\hline \multirow[t]{7}{*}{2} & \multirow{7}{*}{$\begin{array}{l}2 p^{4} 3 s- \\
\quad 2 p^{4}\left({ }^{3} \mathrm{P}\right) 3 p\end{array}$} & \multirow[t]{7}{*}{$\begin{array}{c}{ }^{4} \mathrm{P}-{ }^{4} \mathrm{P}^{\circ} \\
\text { (1) }\end{array}$} & $3717.2^{\prime}$ & 219442 & 246337 & 12 & 12 & 1.3 & 0.27 & 39 & 0.50 & D & $\mathrm{ca}$ \\
\hline & & & 3694.22 & 219133 & 246195 & 6 & 6 & 0.96 & 0.20 & 14 & 0.07 & D & $3, l s$ \\
\hline & & & $\begin{array}{l}3734.94 \\
3751.26\end{array}$ & $\begin{array}{l}219651 \\
219950\end{array}$ & $\begin{array}{l}246417 \\
246600\end{array}$ & $\begin{array}{l}4 \\
2\end{array}$ & $\begin{array}{l}4 \\
2\end{array}$ & $\begin{array}{l}0.20 \\
0.19\end{array}$ & $\begin{array}{l}0.041 \\
0.039\end{array}$ & $\begin{array}{l}2.0 \\
0.98\end{array}$ & $\begin{array}{l}-0.78 \\
-1.10\end{array}$ & $\begin{array}{l}\mathrm{D} \\
\mathrm{D}\end{array}$ & $\begin{array}{l}3, l s \\
3, l s\end{array}$ \\
\hline & & & 3664.09 & 219133 & 246417 & 6 & 4 & 0.67 & 0.090 & 6.6 & -0.27 & $\mathrm{D}$ & $3, l_{s}$ \\
\hline & & & 3709.64 & 219651 & 246600 & 4 & 2 & 1.1 & 0.11 & 5.6 & -0.34 & D & $3, l s$ \\
\hline & & & 3766.29 & 219651 & 246195 & 4 & 6 & 0.32 & 0.10 & 5.0 & -0.39 & D & $3, l s$ \\
\hline & & & 3777.16 & 219950 & 246417 & 2 & 4 & 0.43 & 0.18 & 4.6 & -0.43 & D & $3, l s$ \\
\hline \multirow[t]{8}{*}{3} & & \multirow{8}{*}{${ }^{4} \mathrm{P}-{ }^{4} \mathrm{D}^{\circ}$} & 3336.8 & 219442 & 249402 & 12 & 20 & 1.8 & 0.51 & 67 & 0.79 & D & $c a$ \\
\hline & & & 3334.87 & 219133 & 249111 & 6 & 8 & 1.8 & 0.41 & 27 & 0.39 & D & $3, l s$ \\
\hline & & & 3355.05 & 219651 & 249448 & 4 & 6 & 1.3 & 0.33 & 15 & 0.13 & D & $3, l s$ \\
\hline & & & 3360.63 & 219950 & 24.9698 & 2 & 4 & 0.73 & 0.25 & 5.5 & -0.30 & D & $3, l s$ \\
\hline & & & 3297.74 & 219133 & 249448 & 6 & 6 & 0.53 & 0.087 & 5.7 & -0.28 & D & 3, ls \\
\hline & & & 3327.16 & 219651 & 249618 & 4 & 4 & 0.98 & 0.17 & 7.0 & -0.17 & D & $3, l s$ \\
\hline & & & 3344.43 & 219950 & 249842 & 2 & 2 & 1.5 & 0.25 & 5.6 & -0.30 & D & $3, l s$ \\
\hline & & & $\begin{array}{l}3270.79 \\
3311.30\end{array}$ & $\begin{array}{l}219133 \\
219651\end{array}$ & $\begin{array}{l}249698 \\
249842\end{array}$ & $\begin{array}{l}6 \\
4\end{array}$ & $\begin{array}{l}4 \\
2\end{array}$ & $\begin{array}{l}0.12 \\
0.30\end{array}$ & $\begin{array}{l}0.013 \\
0.023\end{array}$ & $\begin{array}{l}0.85 \\
1.0\end{array}$ & $\begin{array}{l}-1.11 \\
-1.04\end{array}$ & $\begin{array}{l}\mathrm{D} \\
\mathrm{D}\end{array}$ & $\begin{array}{l}3, l s \\
3, l s\end{array}$ \\
\hline \multirow[t]{5}{*}{4} & & \multirow{5}{*}{${ }^{4} \mathrm{P}-{ }^{2} \mathrm{D}^{\circ}$} & & & & & & & & & & & \\
\hline & & & 3135.82 & 219133 & 251013 & 6 & 6 & 0.0065 & $9.6 \times 10^{-4}$ & 0.059 & -2.24 & D & 2 \\
\hline & & & [3136.5] & 219651 & 251525 & 4 & 4 & 0.0049 & $7.2 \times 10^{-4}$ & 0.030 & -2.54 & D & 2 \\
\hline & & & 3187.60 & 219651 & 251013 & 4 & 6 & 0.016 & 0.0036 & 0.15 & -1.84 & D & 2 \\
\hline & & & [3166.2] & 219950 & 251525 & 2 & 4 & 0.0042 & 0.0013 & 0.026 & -2.60 & D & 2 \\
\hline \multirow[t]{2}{*}{5} & & \multirow{2}{*}{${ }^{4} \mathrm{P}-{ }^{2} \mathrm{~S}^{\circ}$} & (20075 & 010651 & (25001 & 1 & 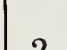 & 0012 & $01 \vee 10-4$ & 0026 & 241 & $\mathrm{D}$ & \\
\hline & & & [3015.7] & 219651 & 252801 & 4 & 2 & 0.013 & $9.1 \times 10^{-4}$ & 0.036 & -2.44 & D & 2 \\
\hline \multirow[t]{4}{*}{6} & & \multirow{4}{*}{${ }_{(4)}^{4} \mathrm{P}-{ }^{4} \mathrm{~S}^{\circ}$} & 2982.94 & 219442 & 252956 & 12 & 4 & 2.5 & 0.11 & 13 & 0.12 & D & $c a$ \\
\hline & & & [2955.7] & 219133 & 252956 & 6 & 4 & 1.2 & 0.10 & 5.9 & -0.22 & D & $3, l s$ \\
\hline & & & 3001.65 & 219651 & 252956 & 4 & 4 & 0.78 & 0.11 & 4.2 & -0.37 & D & $3, l s$ \\
\hline & & & 3028.84 & 219950 & 252956 & 2 & 4 & 0.57 & 0.16 & 3.1 & -0.50 & D & 3, ls \\
\hline \multirow[t]{2}{*}{7} & & ${ }^{4} \mathrm{P}-{ }^{2} \mathrm{P}^{\circ}$ & & & & & & & & & & & \\
\hline & & & $\begin{array}{l}{[2853.5]} \\
{[2896.3]}\end{array}$ & $\begin{array}{l}219133 \\
219651\end{array}$ & $\begin{array}{l}254167 \\
254167\end{array}$ & $\begin{array}{l}6 \\
4\end{array}$ & $\begin{array}{l}4 \\
4\end{array}$ & $\begin{array}{l}0.025 \\
0.020\end{array}$ & $\begin{array}{l}0.0020 \\
0.0025\end{array}$ & $\begin{array}{l}0.12 \\
0.096\end{array}$ & $\begin{array}{l}-1.91 \\
-2.00\end{array}$ & $\begin{array}{l}\mathrm{D} \\
\mathrm{D}\end{array}$ & $\begin{array}{l}2 \\
2\end{array}$ \\
\hline \multirow[t]{2}{*}{8} & & ${ }^{2} \mathrm{P}-{ }^{4} \mathrm{P}^{\circ}$ & & & & & & & & & & & \\
\hline & & & [4441.1] & 224089 & 246600 & 4 & 2 & 0.0034 & $5.1 \times 10^{-4}$ & 0.030 & -2.69 & D & 2 \\
\hline \multirow[t]{3}{*}{9} & & ${ }^{2} \mathrm{P}-{ }^{4} \mathrm{D}^{\circ}$ & {$[3942.3]$} & 224089 & 249448 & 4 & 6 & & 0.0041 & 0.21 & -1.79 & D & 2 \\
\hline & & & {$[3903.9]$} & $\begin{array}{l}224009 \\
224089\end{array}$ & $\begin{array}{l}249440 \\
249698\end{array}$ & 4 & 4 & $8.4 \times 10^{-4}$ & $1.9 \times 10^{-4}$ & 0.0099 & -3.11 & D & 2 \\
\hline & & & [3999.5] & 224702 & 249698 & 2 & 4 & 0.0037 & 0.0018 & 0.046 & -2.45 & D & 2 \\
\hline \multirow[t]{4}{*}{10} & & ${ }^{2} \mathrm{P}-{ }^{-2} \mathrm{D}^{\circ}$ & 3713.0 & 224293 & 251218 & 6 & 10 & 1.3 & 0.45 & 33 & 0.43 & D & $c a$ \\
\hline & & & 3713.09 & 224089 & 251013 & 4 & 6 & 1.3 & 0.40 & 20 & 0.21 & D & $3, l s$ \\
\hline & & & 3727.08 & 224702 & 251525 & 2 & 4 & 1.0 & 0.43 & 11 & -0.07 & D & $3, l s$ \\
\hline & & & 3643.89 & 224089 & 251525 & 4 & 4 & 0.23 & 0.046 & 2.2 & -0.74 & D & $3, l s$ \\
\hline \multirow[t]{3}{*}{11} & & ${ }^{2} \mathrm{P}-{ }^{2} \mathrm{~S}^{\circ}$ & 3506.9 & 224293 & 252801 & 6 & 2 & 1.5 & 0.095 & 6.6 & -0.25 & D & ca \\
\hline & & & $\begin{array}{l}3481.96 \\
3557.84\end{array}$ & $\begin{array}{l}224089 \\
224702\end{array}$ & $\begin{array}{l}252801 \\
252801\end{array}$ & 4 & 2 & $\begin{array}{l}1.2 \\
0.37\end{array}$ & 0.11 & 5.0 & $\begin{array}{l}-0.36 \\
-0.85\end{array}$ & D & $3, l s$ \\
\hline & & & & $J z$ & & 2 & & 0.37 & 0.070 & 1.6 & -0.85 & D & $3, l s$ \\
\hline
\end{tabular}


Ne II. Allowed Transitions-Continued

\begin{tabular}{|c|c|c|c|c|c|c|c|c|c|c|c|c|c|}
\hline No. & $\begin{array}{c}\text { Transition } \\
\text { Array }\end{array}$ & $\begin{array}{l}\text { Multi- } \\
\text { plet }\end{array}$ & $\lambda(\AA)$ & $E_{i}\left(\mathrm{~cm}^{-1}\right)$ & $E_{k}\left(\mathrm{~cm}^{-1}\right)$ & $g_{i}$ & $g_{k}$ & $\begin{array}{l}A_{k i}\left(10^{8}\right. \\
\left.\sec ^{-1}\right)\end{array}$ & $f_{i k}$ & $S(a t . u)$. & $\log g f$ & $\begin{array}{l}\text { Accu- } \\
\text { racy }\end{array}$ & Source \\
\hline 12 & & ${ }^{2} \mathrm{P}-{ }^{4} \mathrm{~S}^{\circ}$ & $\begin{array}{l}{[3463.1]} \\
{[3538.3]}\end{array}$ & $\begin{array}{l}224089 \\
224702\end{array}$ & $\begin{array}{l}2529.56 \\
252956\end{array}$ & $\begin{array}{l}4 \\
2\end{array}$ & $\begin{array}{l}4 \\
4\end{array}$ & $\begin{array}{l}0.028 \\
0.0090\end{array}$ & $\begin{array}{l}0.0051 \\
0.0034\end{array}$ & $\begin{array}{l}0.23 \\
0.079\end{array}$ & $\begin{array}{l}-1.69 \\
-2.17\end{array}$ & $\begin{array}{l}\mathrm{D} \\
\mathrm{D}\end{array}$ & $\begin{array}{l}2 \\
2\end{array}$ \\
\hline \multirow[t]{5}{*}{13} & & $\frac{{ }^{2} \mathrm{P}-{ }^{2} \mathrm{P}^{\circ}}{(7)}$ & 3341.8 & 224293 & 254209 & 6 & 6 & 1.8 & 0.30 & 19 & 0.25 & D) & $c a$ \\
\hline & & & 3323.75 & 224089 & 254167 & 4 & 4 & 1.4 & 0.24 & 11 & -0.02 & D & 3, ls \\
\hline & & & 3378.28 & 224702 & 254294 & 2 & 2 & 1.3 & 0.23 & 5.1 & 0.34 & D & 3, ls \\
\hline & & & 3309.78 & 224089 & 254294 & 4 & 2 & 0.46 & 0.037 & 1.6 & -0.83 & D & 3, ls \\
\hline & & & 3392.78 & 224702 & 254167 & 2 & 4 & 0.29 & 0.098 & & -0.71 & D & $3, l s$ \\
\hline \multirow[t]{4}{*}{14} & $\begin{array}{l}2 p^{4} 3 s^{\prime}- \\
2 p^{4}\left({ }^{1} \mathrm{D}\right) 3 p^{\prime}\end{array}$ & $\begin{array}{c}2 \mathrm{D}-{ }^{2} \mathrm{~F}^{\circ} \\
(9)\end{array}$ & 3571.1 & 246398 & 274392 & 10 & 14 & 1.3 & 0.36 & 42 & 0.56 & D & $c a$ \\
\hline & & & 3568.53 & 246397 & 274411 & 6 & 8 & 1.3 & 0.33 & 24 & 0.30 & D & $3, l s$ \\
\hline & & & 3574.64 & 246400 & 274367 & 4 & 6 & 1.3 & 0.37 & 18 & 0.17 & D & $3, / s$ \\
\hline & & & 3574.23 & 246397 & 274367 & 6 & 6 & 0.092 & 0.018 & 1.3 & -0.97 & D & 3, ls \\
\hline \multirow[t]{4}{*}{15} & & $\begin{array}{c}{ }^{2} \mathrm{D}-{ }^{2} \mathrm{P}^{\circ} \\
(10)\end{array}$ & 3336.9 & 246.398 & 276357 & 10 & 6 & 1.7 & 0.17 & 19 & 0.23 & D & $c a$ \\
\hline & & & 3345.49 & 246397 & 276279 & 6 & 4 & 1.5 & 0.17 & 11 & 0.01 & D & $3, l s$ \\
\hline & & & 3319.75 & 246400 & 276514 & 4 & 2 & 1.7 & 0.14 & 6.2 & -0.24 & D & $3, l s$ \\
\hline & & & 3345.88 & 246400 & 276279 & 4 & 4 & 0.17 & 0.028 & 1.3 & -0.95 & D & $3, l s$ \\
\hline \multirow[t]{5}{*}{16} & & $\begin{array}{c}{ }^{2} \mathrm{D}-{ }^{2} \mathrm{D}^{\circ} \\
(1 \mathrm{l})\end{array}$ & 3231.1 & 246398 & 277.3 .39 & 10 & 10 & 1.9 & 0.29 & 31 & 0.47 & D & $c a$ \\
\hline & & & 3230.16 & 246397 & 277346 & 6 & 6 & 1.8 & 0.27 & 18 & 0.22 & D & $3, l s$ \\
\hline & & & 3232.38 & 246400 & 277328 & 4 & 4 & 1.7 & 0.26 & 11 & 0.02 & D & $3, l s$ \\
\hline & & & 3231.97 & 246397 & 277328 & 6 & 4 & 0.19 & 0.020 & 1.3 & -0.93 & D & $3, l s$ \\
\hline & & & [3230.5] & 246400 & 277346 & 4 & 6 & 0.13 & 0.029 & 1.3 & -0.93 & D & $3, l s$ \\
\hline \multirow[t]{3}{*}{17} & $\begin{array}{l}2 p^{4} 3 s^{\prime \prime}- \\
2 p^{4}\left({ }^{1} S\right) 3 p^{\prime \prime}\end{array}$ & ${ }^{2} \mathrm{~S}-{ }^{2} \mathrm{P}^{\circ}$ & 3480.3 & 276678 & 30540.3 & 2 & 6 & 1.6 & 0.86 & 20 & 0.24 & D & $c a$ \\
\hline & & & [3480.8] & 276678 & 305399 & 2 & 4 & 1.6 & 0.58 & 13 & 0.06 & D & 2 \\
\hline & & & {$[3479.5]$} & 276678 & 305409 & 2 & 2 & 1.6 & 0.29 & 6.6 & -0.24 & D & 2 \\
\hline \multirow[t]{8}{*}{18} & $\begin{array}{l}2 p^{4} 3 p- \\
2 p^{4}\left({ }^{3} \mathrm{P}\right) 3 d\end{array}$ & ${ }^{4} \mathrm{P}^{\circ}-{ }^{4} \mathrm{D}$ & 3039.3 & 2463.37 & 279230 & 12 & 20 & 3.2 & 0.74 & 89 & 0.95 & D & $c a$ \\
\hline & & & 3034.48 & 246195 & 279139 & 6 & 8 & 3.1 & 0.57 & 34 & 0.53 & D & 2 \\
\hline & & & 3047.57 & 246417 & 279221 & 4 & 6 & 1.8 & 0.37 & 15 & 0.17 & D & 2 \\
\hline & & & 3054.69 & 246600 & 279327 & 2 & 4 & 0.93 & 0.26 & 5.2 & -0.28 & D & 2 \\
\hline & & & 3027.04 & 246195 & 279221 & 6 & 6 & 1.5 & 0.20 & 12 & 0.08 & D & 2 \\
\hline & & & 3037.73 & 246417 & 279327 & 4 & 4 & 2.0 & 0.28 & 11 & 0.05 & D & 2 \\
\hline & & & 3045.58 & 246600 & 279425 & 2 & 2 & 2.5 & 0.35 & 7.0 & -0.15 & D & 2 \\
\hline & & & $\begin{array}{c}3017.34 \\
{[3028.7]}\end{array}$ & $\begin{array}{l}246195 \\
246417\end{array}$ & $\begin{array}{l}279327 \\
279425\end{array}$ & $\begin{array}{l}6 \\
4\end{array}$ & $\begin{array}{l}4 \\
2\end{array}$ & $\begin{array}{l}0.35 \\
0.84\end{array}$ & $\begin{array}{l}0.032 \\
0.058\end{array}$ & $\begin{array}{l}1.9 \\
2.3\end{array}$ & $\begin{array}{l}-0.72 \\
-0.64\end{array}$ & $\begin{array}{l}\mathrm{D} \\
\mathrm{D}\end{array}$ & $\begin{array}{l}2 \\
2\end{array}$ \\
\hline \multirow[t]{6}{*}{19} & & ${ }^{4} \mathrm{P}^{\circ}-{ }^{4} \mathrm{~F}$ & & & & & & & & & & & \\
\hline & & & [2897.1] & 246195 & 280703 & 6 & 8 & 0.042 & 0.0071 & 0.41 & -1.37 & D & 2 \\
\hline & & & {$[2870.0]$} & 246195 & 281028 & 6 & 6 & 0.11 & 0.014 & 0.77 & -1.09 & D & 2 \\
\hline & & & [2876.5] & 246195 & 280950 & 6 & 4 & 0.18 & 0.015 & 0.86 & -1.04 & D & 2 \\
\hline & & & [2888.4] & 246417 & 281028 & 4 & 6 & 0.015 & 0.0029 & 0.11 & -1.94 & D & 2 \\
\hline & & & $\begin{array}{l}{[2895.0]} \\
{[2910.4]}\end{array}$ & $\begin{array}{l}246417 \\
246600\end{array}$ & $\begin{array}{l}280950 \\
280950\end{array}$ & $\begin{array}{l}4 \\
2\end{array}$ & $\begin{array}{l}4 \\
4\end{array}$ & $\begin{array}{l}0.016 \\
0.43\end{array}$ & 0.0021 & 0.079 & $\begin{array}{l}-2.08 \\
-0.66\end{array}$ & D & 2 \\
\hline \multirow[t]{4}{*}{20} & & ${ }^{4} \mathrm{P}^{\circ}-{ }^{2} \mathrm{~F}$ & & & & & & & & & $\infty$ & & - \\
\hline & & & [2934.3] & 246195 & 280264 & 6 & 8 & 0.0030 & $5.1 \times 10^{-3}$ & 0.030 & -2.51 & D & 2 \\
\hline & & & [2907.7] & 246417 & 280799 & 4 & 6 & 0.039 & 0.0075 & 0.29 & -1.52 & D & 2 \\
\hline & & & [2889.0] & 246195 & 280799 & 6 & 6 & 0.015 & 0.0019 & 0.11 & -1.94 & D & 2 \\
\hline \multirow[t]{5}{*}{21} & & ${ }^{4} P^{\circ}-{ }^{2} \mathrm{D}$ & {$[20327$} & & & & & & 00087 & & & & \\
\hline & & & [2916.2] & $\begin{array}{l}246195 \\
246195\end{array}$ & $\begin{array}{l}280271 \\
280476\end{array}$ & $\begin{array}{l}6 \\
6\end{array}$ & $\begin{array}{l}6 \\
4\end{array}$ & $\begin{array}{l}0.068 \\
0.047\end{array}$ & 0.0040 & $\begin{array}{l}0.51 \\
0.23\end{array}$ & $\begin{array}{l}-1.28 \\
-1.62\end{array}$ & $\begin{array}{l}D \\
D\end{array}$ & $\begin{array}{l}2 \\
2\end{array}$ \\
\hline & & & [2953.0] & 246417 & 280271 & 4 & 6 & 0.012 & 0.0023 & 0.089 & -2.04 & D & 2 \\
\hline & & & [2935.3] & 246417 & 280476 & 4 & 4 & 0.032 & 0.00 .1 & 0.16 & -1.79 & $\mathrm{D}$ & 2 \\
\hline & & & [2951.1] & 246600 & 280476 & 2 & 4 & 0.023 & 0.0061 & 0.12 & -1.92 & D & 2 \\
\hline
\end{tabular}


Ne II. Allowed Transitions-Continued

\begin{tabular}{|c|c|c|c|c|c|c|c|c|c|c|c|c|c|}
\hline No. & $\begin{array}{c}\text { Transition } \\
\text { Array }\end{array}$ & $\begin{array}{c}\text { Multi- } \\
\text { plet }\end{array}$ & $\lambda(\AA)$ & $E_{i}\left(\mathrm{~cm}^{-1}\right)$ & $E_{k}\left(\mathrm{~cm}^{-1}\right)$ & $g_{i}$ & $g_{k}$ & $\begin{array}{r}A_{k i}\left(10^{8}\right. \\
\left.\sec ^{-1}\right)\end{array}$ & $f_{i k}$ & $S$ (at.u.) & $\log g f$ & $\begin{array}{c}\text { Accu- } \\
\text { racy }\end{array}$ & Source \\
\hline \multirow[t]{8}{*}{22} & & ${ }^{4} \mathrm{P}^{\circ}-\mathrm{P}^{4}$ & 2880.2 & 246337 & 281046 & 12 & 12 & 1.7 & 0.21 & 24 & 0.40 & $\mathrm{D}$ & $c a$ \\
\hline & & & {$[2858.0]$} & 246195 & 281174 & 6 & 6 & 0.91 & 0.11 & 6.3 & -0.18 & D & 2 \\
\hline & & & [2891.5] & 246417 & 280992 & 4 & 4 & 0.097 & 0.012 & 0.47 & -1.31 & D & 2 \\
\hline & & & [2925.7] & 246600 & 280770 & 2 & 2 & 0.52 & 0.067 & 1.3 & -0.87 & D & 2 \\
\hline & & & {$[2873.0]$} & 246195 & 280992 & 6 & 4 & 0.46 & 0.038 & 2.2 & -0.64 & D & 2 \\
\hline & & & [2906.8] & 246417 & 280770 & 4 & 2 & 1.6 & 0.10 & 3.9 & -0.39 & D & 2 \\
\hline & & & {$[2876.4]$} & 246417 & 281174 & 4 & 6 & \begin{tabular}{|l|l|} 
& 0.84
\end{tabular} & 0.16 & 5.9 & -0.21 & D & 2 \\
\hline & & & {$[2906.8]$} & 246600 & 280992 & 2 & 4 & $\mid 0.75$ & 0.19 & 3.6 & -0.42 & D & 2 \\
\hline \multirow[t]{3}{*}{23} & & ${ }^{4} \mathrm{P}^{\circ}-{ }^{2} \mathrm{P}$ & & & & & & & & & & & \\
\hline & & & [2878.1] & 246600 & 281335 & 2 & 2 & 0.067 & 0.0083 & 0.16 & -1.78 & D & 0 \\
\hline & & & [2846.4] & 246600 & 281722 & 2 & 4 & 0.035 & 0.0084 & 0.16 & -1.77 & D & 2 \\
\hline \multirow[t]{11}{*}{24} & & ${ }^{4} \mathrm{D}^{\circ}-{ }^{4} \mathrm{D}$ & 3351.7 & 249402 & 279230 & 20 & 20 & 0.79 & 0.13 & 29 & 0.42 & D & $c a$ \\
\hline & & & 3329.20 & 249111 & 279139 & 8 & 8 & 0.87 & 0.15 & 13 & 0.07 & D & 2 \\
\hline & & & 3357.90 & 249448 & 279221 & 6 & 6 & 0.55 & 0.093 & 6.1 & -0.26 & D & 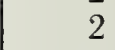 \\
\hline & & & 3374.10 & 249698 & 279327 & 4 & 4 & $\begin{array}{l}0.38 \\
\end{array}$ & 0.065 & 2.9 & -0.59 & D & 2 \\
\hline & & & 3379.39 & 249842 & 279425 & 2 & 2 & 0.35 & 0.060 & 1.3 & -0.92 & D & 2 \\
\hline & & & 3320.29 & 249111 & 279221 & 8 & 6 & 0.13 & 0.017 & 1.5 & -0.87 & D & 2 \\
\hline & & & 3345.88 & 249448 & 279327 & 6 & 4 & $\mid 0.22$ & 0.024 & 1.6 & -0.83 & D & 2 \\
\hline & & & 3362.89 & 249698 & 279425 & 4 & 2 & 0.30 & 0.025 & 1.1 & -0.99 & D & 2 \\
\hline & & & 3367.05 & 249448 & 279139 & 6 & 8 & 0.035 & 0.0079 & 0.53 & -1.32 & D & 0 \\
\hline & & & 3386.24 & 249698 & 279221 & 4 & 6 & 0.067 & 0.017 & 0.77 & -1.16 & $\mathrm{D}-$ & $?$ \\
\hline & & & 3390.56 & 249842 & 279327 & 2 & 4 & 0.078 & 0.027 & 0.60 & -1.27 & D & 2 \\
\hline \multirow[t]{10}{*}{25} & & ${ }^{4} \mathrm{D}^{\circ}-{ }^{4} \mathrm{~F}$ & 3202.5 & 249402 & 280619 & 20 & 28 & 2.5 & 0.55 & 120 & 1.04 & $\mathrm{D}$ & $c a$ \\
\hline & & & 3218.21 & 249111 & 280174 & 8 & 10 & 3.6 & 0.70 & 59 & 0.75 & D & \\
\hline & & & 3198.62 & 249448 & 280703 & 6 & 8 & 2.2 & 0.45 & 29 & 0.44 & D & 2 \\
\hline & & & 3190.86 & 249698 & 281028 & 4 & 6 & 0.73 & 0.17 & 7.0 & -0.18 & D & 2 \\
\hline & & & 3213.70 & 249842 & 280950 & 2 & 4 & 1.8 & 0.56 & 12 & 0.05 & D & 2 \\
\hline & & & 3164.46 & 249111 & 280703 & 8 & 8 & 0.22 & 0.033 & 2.7 & -0.58 & D & \\
\hline & & & 3165.70 & 249448 & 281028 & 6 & 6 & 0.19 & 0.028 & 1.7 & -0.78 & $\mathrm{D}-$ & 2 \\
\hline & & & 3198.88 & 249698 & 280950 & 4 & 4 & 0.59 & 0.0 & 3.8 & -0.44 & $\mathrm{D}-$ & 2 \\
\hline & & & 3132.22 & 249111 & 281028 & 8 & 6 & 0.022 & 0.0024 & 0.20 & -1.72 & $\mathrm{D}-$ & 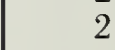 \\
\hline & & & 3173.58 & 249448 & 280950 & 6 & 4 & 0.0017 & $1.7 \times 10^{-4}$ & 0.011 & -2.98 & $\mathrm{D}-$ & \\
\hline \multirow[t]{5}{*}{26} & & ${ }^{4} \mathrm{D}^{\circ}-{ }^{2} \mathrm{~F}$ & & & & & & & & & & & \\
\hline & & & 3208.99 & 249111 & 280264 & 8 & 8 & 0.12 & 0.018 & 1.5 & -0.84 & D & \\
\hline & & & 3188.74 & 2494.48 & 280799 & 6 & 6 & 0.32 & 0.0 & 3. & -0.54 & D - & 2 \\
\hline & & & $\begin{array}{l}3154.82 \\
394415\end{array}$ & 249111 & 280799 & 8 & $\begin{array}{l}6 \\
0 \\
0\end{array}$ & 0.0022 & $2.5 \times 10^{-4}$ & 0.021 & -2.70 & D- & 2 \\
\hline & & & $\begin{array}{l}3244.15 \\
3214.38\end{array}$ & $\begin{array}{l}249448 \\
249698\end{array}$ & $\begin{array}{l}280264 \\
280799\end{array}$ & $\begin{array}{l}6 \\
4\end{array}$ & $\begin{array}{l}8 \\
6\end{array}$ & $\begin{array}{l}1.1 \\
1.6\end{array}$ & $\begin{array}{l}0.22 \\
0.38\end{array}$ & $\begin{array}{l}14 \\
16\end{array}$ & $\begin{array}{l}0.13 \\
0.18\end{array}$ & $\begin{array}{l}\mathrm{D} \\
\mathrm{D}-\end{array}$ & 2 \\
\hline \multirow[t]{7}{*}{27} & & ${ }^{4} \mathrm{D}^{\circ}-{ }^{2} \mathrm{D}$ & & & & & & & & & & & \\
\hline & & & 3243.34 & 249448 & 280271 & 6 & 6 & 0.18 & 0.029 & 1.8 & -0.76 & $\mathrm{D}-$ & \\
\hline & & & 3248.15 & 249698 & 280476 & 4 & 4 & 0.14 & 0.022 & 0. & -1.06 & D & 2 \\
\hline & & & 3269.86 & 249698 & 280271 & 4 & 6 & 0.48 & 0.1 & 5.8 & -0.33 & D- & 2 \\
\hline & & & 3263.43 & 249842 & 280476 & 2 & 4 & 0.36 & 0.12 & 2.5 & -0.64 & D & \\
\hline & & & {$\left[3222_{0} 0\right]$} & 249448 & 280476 & 6 & 4 & 0.020 & 0.0020 & 0.13 & -1.92 & D & \\
\hline & & & [3208.3] & 249111 & 280271 & 8 & 6 & 0.0091 & 0.0010 & 0.089 & -2.07 & D & \\
\hline \multirow[t]{9}{*}{28} & & ${ }^{4} \mathrm{D}^{\circ}-{ }^{4} \mathrm{P}$ & 3159.3 & 249402 & 281046 & 20 & 12 & 0.39 & 0.035 & 7.2 & -0.16 & D & $c a$ \\
\hline & & & 3118.02 & 249111 & 281174 & 8 & 6 & 0.11 & 0.013 & 1.0 & -0.99 & $\mathrm{D}$ & \\
\hline & & & 3169.30 & 249448 & 280992 & 6 & 4 & 0.17 & 0.0 & 1.7 & -0.99 & D - & 2 \\
\hline & & & [3217.4] & 249698 & 280770 & 4 & 2 & 0.1 & 0.0098 & 0.4 & -1.41 & D & 2 \\
\hline & & & 3151.16 & 249448 & 281174 & 6 & 6 & 0.066 & 0.0016 & 0.099 & -2 & D- & \\
\hline & & & 3194.61 & 249698 & 280992 & 4 & 4 & 0.1 & 0.02 & 0.088 & -1.08 & D - & \\
\hline & & & {$[3232.3]$} & 249842 & 280770 & 2 & 2 & 0.033 & 0.0051 & 0.11 & -1.99 & D & \\
\hline & & & 3176.16 & 2496 & 281174 & 4 & 6 & 0.034 & 0.0078 & 0.33 & -1.50 & D & \\
\hline & & & 3209.38 & 249842 & 280992 & $?$ & 4 & 0.51 & 0.16 & 3.3 & -0.50 & D. & \\
\hline
\end{tabular}


Ne II. Allowed Transitions-Continued

\begin{tabular}{|c|c|c|c|c|c|c|c|c|c|c|c|c|c|}
\hline No. & $\begin{array}{c}\text { Transition } \\
\text { Array }\end{array}$ & $\begin{array}{c}\text { Multi- } \\
\text { plet }\end{array}$ & $\lambda(\AA)$ & $E_{i}\left(\mathrm{~cm}^{-1}\right)$ & $E_{k}\left(\mathrm{~cm}^{-1}\right)$ & $g_{i}$ & $g_{k}$ & $\begin{array}{l}A_{k i}\left(10^{8}\right. \\
\left.\sec ^{-1}\right)\end{array}$ & $f_{i k}$ & $S$ (at.u.) & $\log g f$ & $\begin{array}{l}\text { Accu- } \\
\text { racy }\end{array}$ & Srource \\
\hline \multirow[t]{3}{*}{29} & & ${ }^{4} \mathrm{D}^{\circ}-{ }^{2} \mathrm{P}$ & & & & & & & & & & & \\
\hline & & & [3135.8] & 249842 & 281722 & 2 & 4 & 0.020 & 0.0058 & 0.12 & -1.94 & D & 2 \\
\hline & & & {$\left[\begin{array}{l}3160.0] \\
{[3097.5]}\end{array}\right.$} & $\begin{array}{l}249698 \\
249448\end{array}$ & 281335 & 4 & 2 & 0.019 & 0.0014 & 0.059 & -2.25 & D & 2 \\
\hline \multirow{3}{*}{30} & & $20^{\circ} 4$ & & & & & & & & & & & \\
\hline & & (18) & & & & & & & & & & & \\
\hline & & & $\begin{array}{l}3554.39 \\
{[3544.2]}\end{array}$ & $\begin{array}{l}251013 \\
251013\end{array}$ & $\begin{array}{l}279139 \\
279221\end{array}$ & $\begin{array}{l}6 \\
6\end{array}$ & $\begin{array}{l}8 \\
6\end{array}$ & $\begin{array}{l}0.013 \\
0.0037\end{array}$ & $\begin{array}{l}0.0034 \\
7.0 \times 10^{-4}\end{array}$ & $\begin{array}{l}0.24 \\
0.049\end{array}$ & $\begin{array}{l}-1.69 \\
-2.38\end{array}$ & $\begin{array}{l}\mathrm{D} \\
\mathrm{D}\end{array}$ & $\begin{array}{l}2 \\
2\end{array}$ \\
\hline \multirow[t]{5}{*}{31} & & ${ }^{2} \mathrm{D}^{\circ}-{ }^{4} \mathrm{~F}$ & & & & & & & & & & & \\
\hline & & & 3367.20 & 251013 & 280703 & 6 & 8 & 1.0 & 0.24 & 16 & 0.15 & D & 2 \\
\hline & & & 3388.46 & 251525 & 281028 & 4 & 6 & 2.0 & 0.51 & 23 & 0.31 & D & 2 \\
\hline & & & 3330.78 & 251013 & 281028 & 6 & 6 & 0.12 & 0.020 & 1.3 & -0.93 & D & 2 \\
\hline & & & [3397.5] & 251525 & 280950 & 4 & 4 & 0.049 & 0.0084 & 0.38 & -1.47 & $\mathrm{D}$ & 2 \\
\hline \multirow[t]{3}{*}{32} & & $\begin{array}{c}{ }^{2} \mathrm{D}^{\circ}-{ }^{2} \mathrm{~F} \\
(20)\end{array}$ & 3414.9 & 251218 & 280493 & 10 & 14 & 1.4 & 0.34 & 38 & 0.53 & D & $c a$ \\
\hline & & & 3417.71 & 251013 & 280264 & 6 & 8 & 2.0 & 0.47 & 32 & 0.45 & D & 2 \\
\hline & & & $\begin{array}{l}3414.82 \\
3356.35\end{array}$ & $\begin{array}{l}251525 \\
251013\end{array}$ & $\begin{array}{l}280799 \\
280799\end{array}$ & 4 & 6 & 0.41 & 0.11 & 4.8 & $\begin{array}{l}-0.37 \\
-0.93\end{array}$ & $\begin{array}{l}\mathrm{D}- \\
\mathrm{D}-\end{array}$ & 2 \\
\hline \multirow{5}{*}{33} & & & & 05120 & & & & & & & & D & \\
\hline & & $\begin{array}{c}{ }^{2} \mathrm{D}^{0}-{ }^{2} \mathrm{D} \\
(21)\end{array}$ & 3431.3 & 251218 & 280353 & 10 & 10 & 0.85 & 0.15 & 17 & 0.18 & D & $c a$ \\
\hline & & & 3416.87 & 251013 & 280271 & 6 & 6 & 0.67 & 0.12 & 7.9 & -0.15 & D & 2 \\
\hline & & & 3453.10 & $\begin{array}{l}251525 \\
251013\end{array}$ & 280476 & 4 & 4 & 0.59 & 0.11 & 4.8 & -0.38 & D & 2 \\
\hline & & & $\begin{array}{r}{[3393.2]} \\
3477.69\end{array}$ & $\begin{array}{l}251013 \\
251525\end{array}$ & $\begin{array}{l}280476 \\
280271\end{array}$ & $\begin{array}{l}6 \\
4\end{array}$ & $\begin{array}{l}4 \\
6\end{array}$ & $\begin{array}{l}0.022 \\
0.34\end{array}$ & $\begin{array}{l}0.0025 \\
0.091\end{array}$ & $\begin{array}{l}0.17 \\
4.2\end{array}$ & $\begin{array}{l}-1.82 \\
-0.44\end{array}$ & $\begin{array}{l}\mathrm{D} \\
\mathrm{D}-\end{array}$ & $\begin{array}{l}2 \\
2\end{array}$ \\
\hline \multirow[t]{4}{*}{34} & & ${ }^{2} \mathrm{D}^{\circ}-{ }^{4} \mathrm{P}$ & & & & & & & & & & & \\
\hline & & & 3314.60 & 251013 & 281174 & 6 & 6 & 0.026 & 0.0042 & 0.28 & -1.60 & D & 2 \\
\hline & & & 3371.87 & 251525 & 281174 & 4 & 6 & 0.12 & 0.032 & 1.4 & -0.89 & D & 2 \\
\hline & & & $\begin{array}{l}{[3392.7]} \\
{[3334.8]}\end{array}$ & $\begin{array}{l}251525 \\
251013\end{array}$ & $\begin{array}{l}280992 \\
280992\end{array}$ & $\begin{array}{l}4 \\
6\end{array}$ & $\begin{array}{l}4 \\
4\end{array}$ & $\begin{array}{l}0.14 \\
0.030\end{array}$ & $\begin{array}{l}0.025 \\
0.0033\end{array}$ & $\begin{array}{l}1.1 \\
0.22\end{array}$ & $\begin{array}{l}-1.00 \\
-1.70\end{array}$ & D & 2 \\
\hline \multirow[t]{4}{*}{35} & & ${ }^{2} \mathrm{D}^{\circ}-{ }^{2} \mathrm{P}$ & 3291.6 & 251218 & 281590 & 10 & 6 & 0.098 & 0.0095 & 1.0 & -1.02 & D & $c a$ \\
\hline & & $(2$ & 3255.39 & 251013 & 281722 & 6 & 4 & & 0.013 & 081 & -112 & $\mathrm{D}$ & \\
\hline & & & 3353.63 & 251525 & 281335 & 4 & 2 & $\begin{array}{l}0.12 \\
0.048\end{array}$ & 0.0040 & $\begin{array}{l}0.81 \\
0.18\end{array}$ & -1.79 & $\begin{array}{l}\text { D } \\
\text { D }\end{array}$ & $\begin{array}{l}2 \\
2\end{array}$ \\
\hline & & & 3310.55 & 251525 & 281722 & 4 & 4 & 0.0061 & 0.0010 & 0.044 & -2.40 & $\mathrm{D}$ & 2 \\
\hline 36 & & ${ }^{2} \mathrm{~S}^{\circ}-{ }^{4} \mathrm{D}$ & {$[3754.9]$} & 252801 & 270425 & 2 & 8 & 0005 & nonon & 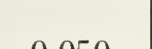 & 8 & & \\
\hline \multirow[t]{2}{*}{37} & & ${ }^{2} \mathrm{~S}^{\circ}-{ }^{4} \mathrm{~F}$ & & & & & & & & & 2.40 & & - \\
\hline & & & 3551.52 & 252801 & 280950 & 2 & 4 & 0.055 & 0.021 & 0.49 & -1.38 & D & 2 \\
\hline \multirow[t]{2}{*}{38} & & $\begin{array}{c}{ }^{2} \mathrm{~S}^{\circ}-{ }^{2} \mathrm{D} \\
(26)\end{array}$ & & & & & & & & & & & \\
\hline & & & 3612.35 & 252801 & 280476 & 2 & 4 & 0.22 & 0.087 & 2.1 & -0.76 & D & 2 \\
\hline \multirow[t]{2}{*}{39} & & $\begin{array}{c}{ }^{2} \mathrm{~S}^{\circ}-{ }^{4} \mathrm{P} \\
(27)\end{array}$ & & & & & & & & & & & \\
\hline & & & $\begin{array}{c}3546.22 \\
{[3574.3]}\end{array}$ & $\begin{array}{l}252801 \\
252801\end{array}$ & $\begin{array}{l}280992 \\
280770\end{array}$ & $\begin{array}{l}2 \\
2\end{array}$ & $\begin{array}{l}4 \\
2\end{array}$ & $\begin{array}{l}0.021 \\
0.046\end{array}$ & $\begin{array}{l}0.0081 \\
0.0088\end{array}$ & $\begin{array}{l}0.19 \\
0.21\end{array}$ & $\begin{array}{l}-1.79 \\
-1.75\end{array}$ & $\begin{array}{l}\mathrm{D} \\
\mathrm{D}\end{array}$ & $\frac{2}{2}$ \\
\hline \multirow[t]{2}{*}{40} & & ${ }^{2} \mathrm{~S}^{\circ}-{ }^{2} \mathrm{P}$ & 3472.5 & 252801 & 281590 & 2 & 6 & 1.3 & 0.70 & 16 & 0.15 & D & $c a$ \\
\hline & & & $\begin{array}{l}3456.68 \\
3503.61\end{array}$ & $\begin{array}{l}252801 \\
252801\end{array}$ & $\begin{array}{l}281722 \\
281335\end{array}$ & $\begin{array}{l}2 \\
2\end{array}$ & $\begin{array}{l}4 \\
2\end{array}$ & $\begin{array}{l}1.0 \\
1.9\end{array}$ & $\begin{array}{l}0.36 \\
0.34\end{array}$ & $\begin{array}{l}8.2 \\
7.9\end{array}$ & $\begin{array}{l}-0.14 \\
-0.16\end{array}$ & $\begin{array}{l}\mathrm{D} \\
\mathrm{D}\end{array}$ & $\frac{2}{2}$ \\
\hline
\end{tabular}


Ne II. Allowed Transitions-Continued

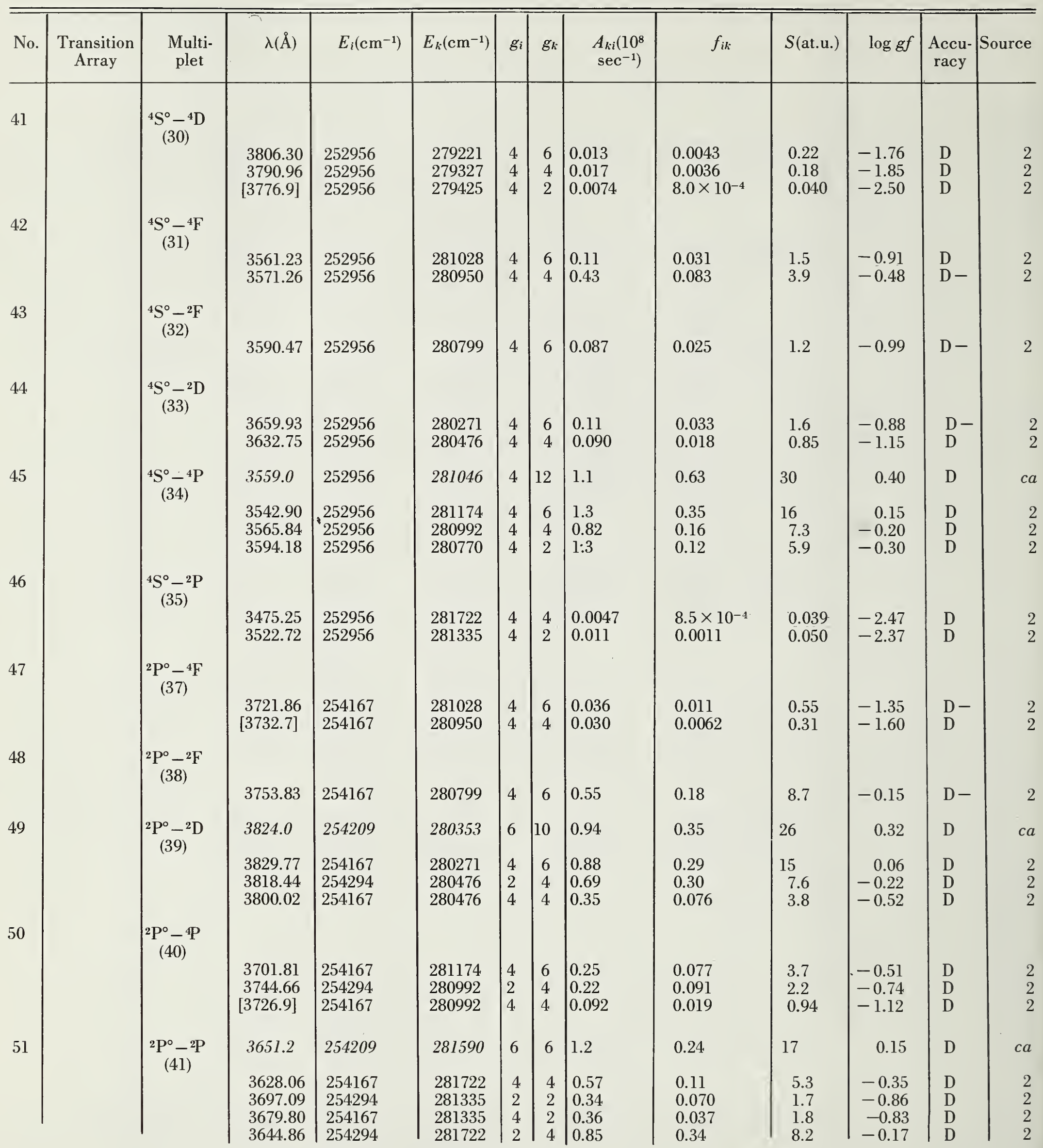


Ne II. Allowed Transitions-Continued

\begin{tabular}{|c|c|c|c|c|c|c|c|c|c|c|c|c|c|}
\hline No. & $\begin{array}{c}\text { Transition } \\
\text { Array }\end{array}$ & $\begin{array}{l}\text { Multi- } \\
\text { plet }\end{array}$ & $\lambda(\AA)$ & $E_{i}\left(\mathrm{~cm}^{-1}\right)$ & $E_{k}\left(\mathrm{~cm}^{-1}\right)$ & $g_{i}$ & $g_{k}$ & $\begin{array}{c}A_{k i}\left(10^{8}\right. \\
\left.\sec ^{-1}\right)\end{array}$ & $f_{i k}$ & $S$ (at.u.) & $\log g f$ & $\begin{array}{c}\text { Accu- } \\
\text { racy }\end{array}$ & Srource \\
\hline \multirow[t]{11}{*}{52} & \multirow[t]{31}{*}{$\begin{array}{l}2 p^{4} 3 d- \\
\quad 2 p^{4}\left({ }^{3} \mathrm{P}\right) 4 \mathrm{f}\end{array}$} & \multirow[t]{11}{*}{$\begin{array}{l}{ }^{4} \mathrm{D}-{ }^{4} \mathrm{D}^{\circ} \\
(52)\end{array}$} & 4229.6 & 2792.30 & .302866 & 20 & 20 & 0.39 & 0.10 & 29) & 0.32 & D & $c a$ \\
\hline & & & 4219.76 & 279139 & 302831 & 8 & 8 & 0.33 & 0.089 & 9.8 & -0.15 & D & Is \\
\hline & & & 4231.60 & 279221 & 302846 & 6 & 6 & 0.22 & 0.060 & 5.0 & -0.45 & D & Is \\
\hline & & & 4239.95 & 279327 & 302905 & 4. & 4 & 0.15 & 0.042 & 2.3 & -0.78 & D & Is \\
\hline & & & 4242.20 & 279425 & 302991 & 2 & 2 & 0.19 & 0.052 & 1.5 & -0.98 & D & Is \\
\hline & & & 4217.15 & 279139 & 302846 & 8 & 6 & 0.074 & 0.015 & 1.6 & -0.93 & D & Is \\
\hline & & & 4220.92 & 279221 & 302905 & 6 & 4 & 0.14 & 0.024 & 2.0 & -0.84 & D & ls \\
\hline & & & 4224.57 & 279327 & 302991 & 4 & 2 & 0.19 & 0.026 & 1.4 & -0.99 & D & Is \\
\hline & & & [4234.3] & 279221 & 302831 & 6 & 8 & 0.055 & 0.020 & 1.7 & -0.93 & D & ls \\
\hline & & & 4250.68 & 279327 & 302846 & 4. & 6 & 0.090 & 0.036 & 2.0 & -0.84 & D & Is \\
\hline & & & 4257.82 & 279425 & 302905 & 2 & 4 & 0.096 & 0.052 & 1.5 & -0.98 & D & ls \\
\hline \multirow[t]{10}{*}{53} & & \multirow[t]{10}{*}{$\begin{array}{c}{ }^{4} \mathrm{~F}-{ }^{4} \mathrm{~F}^{\circ} \\
(56)\end{array}$} & 4.394 .5 & 280619 & 3033368 & 28 & 28 & 0.26 & 0.076 & 31 & 0.33 & D & $c a$ \\
\hline & & & 4397.94 & 280174 & 302906 & 10 & 10 & 0.24 & 0.070 & 10 & -0.16 & D & ls \\
\hline & & & 4379.50 & 280703 & 303531 & 8 & 8 & 0.20 & 0.058 & 6.7 & -0.33 & D & ls \\
\hline & & & 4385.00 & 281028 & 303827 & 6 & 6 & 0.18 & 0.053 & 4.6 & -0.50 & D & Is \\
\hline & & & 4430.90 & 280950 & 303512 & 4 & 4 & 0.21 & 0.062 & 3.6 & -0.61 & D & Is \\
\hline & & & [4280.3] & 280174 & 303531 & 10 & 8 & 0.028 & 0.0062 & 0.88 & -1.21 & D & is \\
\hline & & & [4323.3] & 280703 & 303827 & 8 & 6 & 0.048 & 0.010 & 1.2 & -1.09 & D & is \\
\hline & & & $\begin{array}{l}4.40 .40 \\
4502.52\end{array}$ & 280703 & $\begin{array}{l}305512 \\
302906\end{array}$ & $\begin{array}{l}0 \\
8\end{array}$ & $\begin{array}{r}4 \\
10\end{array}$ & $\begin{array}{l}0.052 \\
0.021\end{array}$ & $\begin{array}{l}0.010 \\
0.0081\end{array}$ & $\begin{array}{l}0.91 \\
0.96\end{array}$ & $\begin{array}{l}-1.21 \\
-1.19\end{array}$ & D & is \\
\hline & & & 4442.67 & 281028 & 303531 & 6 & 8 & 0.035 & 0.014 & 1.2 & -1.08 & $\mathrm{D}$ & ls \\
\hline & & & 4369.77 & 280950 & 303827 & 4 & 6 & 0.035 & 0.015 & 0.88 & -1.21 & $\mathrm{D}$ & ls \\
\hline \multirow[t]{10}{*}{54} & & \multirow{10}{*}{$\begin{array}{c}{ }^{4} \mathrm{~F}-{ }^{4} \mathrm{G}^{0} \\
(57)\end{array}$} & 4360.8 & 280619 & 303544 & 28 & 36 & 2.4 & 0.89 & 360 & 1.39 & D & $c a$ \\
\hline & & & 4290.40 & 280174 & 303476 & 10 & 12 & 2.5 & 0.83 & 120 & 0.92 & D & Is \\
\hline & & & 4391.94 & 280703 & 303465 & 8 & 10 & 2.2 & 0.79 & 91 & 0.80 & $\mathrm{D}$ & ls \\
\hline & & & 4409.30 & 281028 & 303701 & 6 & 8 & 2.0 & 0.79 & 69 & 0.68 & D & ls \\
\hline & & & 4413.20 & 280950 & 303602 & 4 & 6 & 2.0 & 0.89 & 52 & 0.55 & D & ls \\
\hline & & & [4.292.4] & 280174 & 303465 & 10 & 10 & 0.20 & 0.056 & 7.9 & -0.25 & D & $l s$ \\
\hline & & & [4346.9] & 280703 & 303701 & 8 & 8 & 0.33 & 0.093 & 11 & -0.13 & D & ls \\
\hline & & & 4428.54 & 281028 & 303602 & 6 & 6 & 0.33 & 0.096 & 8.4 & -0.24 & $\mathrm{D}$ & $l s$ \\
\hline & & & [4249.2] & 280174 & 303701 & 10 & 8 & 0.0073 & 0.0016 & 0.22 & -1.80 & D & ls \\
\hline & & & 4365.72 & 280703 & 303602 & 8 & 6 & 0.012 & 0.0026 & 0.30 & -1.68 & D & $s$ \\
\hline
\end{tabular}

\section{Forbidden Transitions}

Naqvi's calculation [1] of the one possible transition in the ground state configuration $2 p^{5}$ is the only available source. The line strength should be quite accurate, since it does not sensitively depend on the choice of the interaction parameters.

\section{Reference}

[1] Naqvi, A. M., Thesis Harvard (1951).

Ne II. Forbidden Transitions

\begin{tabular}{|c|c|c|c|c|c|c|c|c|c|c|c|c|}
\hline No. & $\begin{array}{c}\text { Transition } \\
\text { Array }\end{array}$ & Multiplet & $\lambda(\AA)$ & $E_{i}\left(\mathrm{~cm}^{-1}\right)$ & $E_{k}\left(\mathrm{~cm}^{-1}\right)$ & $\dot{g}_{i}$ & $g_{k}$ & $\begin{array}{l}\text { Type of } \\
\text { Transition }\end{array}$ & $A_{k i}\left(\sec ^{-1}\right)$ & $S($ at.u.) & $\begin{array}{l}\text { Accu- } \\
\text { racy }\end{array}$ & Source \\
\hline 1 & $2 p^{5}-2 p^{5}$ & ${ }^{2} \mathrm{P}^{\circ}-{ }^{2} \mathrm{P}^{\circ}$ & {$\left[12.78 \times 10^{4}\right]$} & 0 & 782 & 4 & 2 & $\mathrm{~m}$ & 0.00859 & 1.33 & B & 1 \\
\hline
\end{tabular}




\section{Allowed Transitions}

List of tabulated lines:

\begin{tabular}{l|l|cc|c|c}
\hline \hline Wavelength $[\AA]$ & No. & Wavelength $[\AA]$ & No. & Wavelength $[\AA]$ & No. \\
\hline & & & & & \\
227.24 & 13 & 283.894 & 5 & 2163.5 & 17 \\
227.42 & 12 & 301.124 & 7 & 2163.7 & 17 \\
227.49 & 12 & 308.559 & 9 & 2163.77 & 17 \\
227.57 & 13 & 313.048 & 4 & 2412.73 & 18 \\
227.72 & 13 & 313.677 & 4 & 2412.94 & 18 \\
227.73 & 12 & 313.92 & 4 & 2413.18 & 18 \\
227.76 & 12 & 379.308 & 2 & 2413.54 & 18 \\
227.82 & 12 & 427.840 & 3 & 2413.78 & 18 \\
227.90 & 12 & 488.103 & 1 & 2590.04 & 14 \\
228.85 & 11 & 488.868 & 1 & 2593.60 & \\
228.88 & 11 & 489.501 & 1 & 2595.68 & 14 \\
228.91 & 11 & 489.641 & 1 & 2610.03 & 16 \\
229.19 & 11 & 490.310 & 1 & 2611.42 & 16 \\
229.22 & 11 & 491.050 & 1 & 2612.5 & 16 \\
229.34 & 11 & 2086.96 & 19 & 2613.41 & 16 \\
251.145 & 10 & 2087.44 & 19 & 2614.51 & 16 \\
251.558 & 10 & 2088.92 & 19 & 2615.87 & 16 \\
251.726 & 10 & 2089.43 & 19 & 2677.90 & 15 \\
267.059 & 6 & 2092.44 & 19 & 2678.64 & 15 \\
267.516 & 6 & 2095.54 & 19 & & \\
267.709 & 6 & 2159.44 & 17 & & \\
282.50 & 8 & 2159.60 & 17 & & \\
283.178 & 5 & 2160.88 & 17 & & \\
283.206 & 5 & 2161.04 & 17 & & \\
283.690 & 5 & 2161.22 & 17 & & \\
& & & & & \\
\hline
\end{tabular}

The values for the majority of the transitions are taken from the self-consistent field calculations (with exchange) by Weiss[1]. These calculations do not include the important effects of configuration interaction; hence, fairly large uncertainties must be expected in most cases. The average of the dipole length and velocity approximations is adopted [1].

For the $2 s^{2} 2 p^{4} \mathrm{~S}-2 s 2 p^{5} \mathrm{P}^{\circ}$ transition a value is available from the calculations of Bolotin et al. [2] which include configuration interaction in a limited way. Again, large uncertainties are to be expected.

\section{References}

[1] Weiss, A. W., private communication (1965).

[2] Bolotin, A. B., Shironas, I. I., and Braiman, M. Yu., Vilniaus Valstybinio v. Kapsuko vardo universiteto Mokslo Darbai, 33, matematika, fizika, 9, 107-112 (1960). 
Ne III. Allowed Transitions

\begin{tabular}{|c|c|c|c|c|c|c|c|c|c|c|c|c|c|}
\hline No. & $\begin{array}{c}\text { Transition } \\
\text { Array }\end{array}$ & $\begin{array}{l}\text { Multi- } \\
\text { plet }\end{array}$ & $\lambda(\AA)$ & $E_{i}\left(\operatorname{con}^{-1}\right)$ & $E_{k}\left(c \mathrm{~m}^{-1}\right)$ & $g_{i}$ & $g_{k}$ & $\begin{array}{l}A_{k i}\left(10^{8}\right. \\
\left.\sec ^{-1}\right)\end{array}$ & $f_{i k}$ & S(at.u.) & $\log g f$ & $\begin{array}{l}\text { Accu. } \\
\text { racy }\end{array}$ & Source \\
\hline \multirow[t]{6}{*}{1} & $\begin{array}{r}2 s^{2} 2 p^{4}- \\
2 s 2 p^{5}\end{array}$ & $\begin{array}{c}{ }^{3} \mathrm{P}-{ }^{3} \mathrm{P}^{\circ} \\
(1 \mathrm{uv})\end{array}$ & 489.54 & 317 & 204.589 & 9 & 9 & 71 & 0.26 & 3.7 & 0.36 & E & 1 \\
\hline & & & 489.501 & 0 & 204292 & 5 & 5 & 52 & 0.19 & 1.5 & -0.03 & $\mathrm{E}$ & Is \\
\hline & & & 489.641 & 643 & 204879 & 3 & 3 & 18 & 0.064 & 0.31 & -0.72 & $\mathrm{E}$ & Is \\
\hline & & . & 488.103 & 0 & 204879 & 5 & 3 & 30 & 0.063 & 0.51 & -0.50 & $\mathrm{E}$ & ls \\
\hline & & & 488.868 & 643 & 205204 & 3 & 1 & 71 & 0.085 & 0.41 & -0.59 & $\mathrm{E}$ & Is \\
\hline & & & $\begin{array}{l}491.050 \\
490.310\end{array}$ & $\begin{array}{l}643 \\
927\end{array}$ & $\begin{array}{l}204292 \\
204879\end{array}$ & $\begin{array}{l}3 \\
1\end{array}$ & $\begin{array}{l}5 \\
3\end{array}$ & $\begin{array}{l}17 \\
23\end{array}$ & $\begin{array}{l}0.11 \\
0.25\end{array}$ & $\begin{array}{l}0.51 \\
0.41\end{array}$ & $\begin{array}{l}-0.50 \\
-0.60\end{array}$ & $\begin{array}{l}\mathrm{E} \\
\mathrm{E}\end{array}$ & $\begin{array}{l}\text { Is } \\
\text { ls }\end{array}$ \\
\hline 2 & & $\begin{array}{c}{ }^{1} \mathrm{D}-{ }^{1} \mathrm{P}^{\circ} \\
(6 \mathrm{uv})\end{array}$ & 379.308 & 25841 & 289479 & 5 & 3 & 210 & 0.27 & 1.7 & 0.13 & $\mathrm{E}$ & 1 \\
\hline 3 & & $\begin{array}{c}{ }^{1} \mathrm{~S}-{ }^{1} \mathrm{P}^{\circ} \\
(9 \mathrm{uv})\end{array}$ & 427.840 & 55747 & 289479 & 1 & 3 & 16 & 0.13 & 0.19 & -0.87 & $\mathrm{E}$ & 2 \\
\hline \multirow[t]{4}{*}{4} & $\begin{array}{l}2 p^{4}- \\
2 p^{3}\left({ }^{4} \mathrm{~S}^{\circ}\right) 3 s\end{array}$ & $\begin{array}{c}{ }^{3} \mathrm{P}-{ }^{3} \mathrm{~S}^{\circ} \\
(2 u v)\end{array}$ & 313.35 & 317 & 319445 & 9 & 3 & 81 & 0.040 & 0.37 & -0.45 & $E$ & 1 \\
\hline & & & 313.048 & 0 & 319445 & 5 & 3 & 46 & 0.041 & 0.21 & -0.69 & $\mathrm{E}$ & ls \\
\hline & & & 313.677 & 643 & 319445 & 3 & 3 & 26 & 0.039 & 0.12 & -0.93 & $\mathrm{E}$ & ls \\
\hline & & & 313.92 & 927 & 319445 & 1 & 3 & 9.0 & 0.040 & 0.041 & -1.40 & $\mathrm{E}$ & ls \\
\hline \multirow[t]{6}{*}{5} & $\begin{array}{l}2 p^{4}- \\
2 p^{3}\left({ }^{2} \mathrm{D}^{\circ}\right) 3 s^{\prime}\end{array}$ & $\begin{array}{c}{ }^{3} \mathrm{P}-{ }^{3} \mathrm{D}^{\circ} \\
(3 \mathrm{uv})\end{array}$ & 283.41 & 317 & 353167 & 9 & 15 & 28 & 0.057 & 0.48 & -0.29 & $\mathrm{E}$ & 1 \\
\hline & & & 283.206 & 0 & 353148 & 5 & 7 & 28 & 0.047 & 0.22 & -0.63 & $\mathrm{E}$ & $l s$ \\
\hline & & & 283.690 & 643 & 353177 & 3 & 5 & 21 & 0.043 & 0.12 & -0.89 & $\mathrm{E}$ & ls \\
\hline & & & 283.894 & 927 & 353197 & 1 & 3 & 16 & 0.057 & 0.053 & -1.25 & $\mathrm{E}$ & is \\
\hline & & & $\begin{array}{l}283.178 \\
283.690\end{array}$ & $\begin{array}{r}0 \\
643\end{array}$ & $\begin{array}{l}353177 \\
353197\end{array}$ & $\begin{array}{l}5 \\
3\end{array}$ & $\begin{array}{l}5 \\
3\end{array}$ & $12^{7.1}$ & $\begin{array}{l}0.0086 \\
0.014\end{array}$ & $\begin{array}{l}0.040 \\
0.040\end{array}$ & $\begin{array}{l}-1.37 \\
-1.37\end{array}$ & $\begin{array}{l}\mathrm{E} \\
\mathrm{E}\end{array}$ & $\begin{array}{l}\text { Is } \\
\text { is }\end{array}$ \\
\hline & & & 283.178 & 0 & 353197 & $\begin{array}{l}5 \\
5\end{array}$ & $\begin{array}{l}0 \\
3\end{array}$ & 0.80 & $5.8 \times 10^{-4}$ & 0.0027 & -2.54 & $\mathrm{E}$ & ls \\
\hline \multirow[t]{6}{*}{6} & $2 p^{4}-$ & ${ }^{3} \mathrm{P}-{ }^{3} \mathrm{P}^{\circ}$ & 267.29 & 317 & 374448 & 9 & 9 & 32 & 0.034 & 0.27 & -0.51 & $\mathrm{E}$ & 1 \\
\hline & $2 p^{3}\left({ }^{2} \mathrm{P}^{\circ}\right) 3 s^{\prime \prime}$ & $(4-\mathrm{uv})$ & 267.059 & 0 & 374434 & 5 & 5 & 23 & 0.025 & 0.11 & -0.90 & $\mathrm{E}$ & ls \\
\hline & & & 267.516 & 643 & 374461 & 3 & 3 & 8.1 & 0.0087 & 0.023 & -1.58 & $\mathrm{E}$ & ls \\
\hline & & & 267.059 & 0 & 374461 & 5 & 3 & 13 & 0.0086 & 0.038 & -1.36 & $\mathrm{E}$ & ls \\
\hline & & & 267.516 & 643 & 374478 & 3 & 1 & 32 & 0.011 & 0.030 & -1.47 & E & ls \\
\hline & & & $\begin{array}{l}267.516 \\
267.709\end{array}$ & $\begin{array}{l}643 \\
927\end{array}$ & $\begin{array}{l}374434 \\
374461\end{array}$ & $\begin{array}{l}3 \\
1\end{array}$ & $\begin{array}{l}5 \\
3\end{array}$ & $\begin{array}{l}8.0 \\
11\end{array}$ & $\begin{array}{l}0.014 \\
0.034\end{array}$ & $\begin{array}{l}0.038 \\
0.030\end{array}$ & $\begin{array}{l}-1.37 \\
-1.47\end{array}$ & $\begin{array}{l}\mathrm{E} \\
\mathrm{E}\end{array}$ & ls \\
\hline 7 & $\begin{array}{l}2 p^{4}- \\
\quad 2 p^{3}\left({ }^{2} \mathrm{D}^{\circ}\right) 3 s^{\prime}\end{array}$ & $\begin{array}{c}{ }^{1} \mathrm{D}-{ }^{1} \mathrm{D}^{\circ} \\
(7 \mathrm{uv})\end{array}$ & 301.124 & 25841 & 357930 & 5 & 5 & 71 & 0.097 & 0.48 & -0.32 & $\mathrm{E}$ & 1 \\
\hline 8 & $\begin{array}{l}2 p^{4}- \\
\quad 2 p^{3}\left({ }^{2} \mathrm{P}^{\circ}\right) 3 s^{\prime \prime}\end{array}$ & $\begin{array}{c}{ }^{1} \mathrm{D}-{ }^{1} \mathrm{P}^{\circ} \\
(8 \mathrm{uv})\end{array}$ & 282.50 & 25841 & 379834 & 5 & 3 & 45 & 0.032 & 0.15 & -0.79 & $\mathrm{E}$ & 1 \\
\hline 9 & $\begin{array}{l}2 p^{4}- \\
\quad 2 p^{3}\left({ }^{2} \mathrm{P}^{\circ}\right) 3 s^{\prime \prime}\end{array}$ & $\begin{array}{l}{ }^{1} \mathrm{~S}-{ }^{1} \mathrm{P}^{\circ} \\
(10 \mathrm{uv})\end{array}$ & 308.559 & 55747 & 379834 & 1 & 3 & 32 & 0.14 & 0.14 & -0.86 & $\mathrm{E}$ & 1 \\
\hline \multirow[t]{6}{*}{10} & $2 p^{4}-$ & $\begin{array}{c}{ }^{3} \mathrm{P}-{ }^{3} \mathrm{D}^{\circ} \\
(5 \mathrm{uv})\end{array}$ & 251.33 & 317 & 398203 & 9 & 15 & 140 & 0.21 & 1.6 & 0.29 & $\mathrm{D}-$ & 1 \\
\hline & & & 251.145 & 0 & 398211 & 5 & 7 & 140 & 0.18 & 0.75 & -0.04 & $\mathrm{D}-$ & ls \\
\hline & & & 251.558 & 643 & 398197 & 3 & 5 & 100 & 0.16 & 0.40 & -0.32 & $\mathrm{D}-$ & ls \\
\hline & & & 251.726 & 927 & 398193 & 1 & 3 & 76 & 0.22 & 0.18 & -0.66 & $\mathrm{D}-$ & ls \\
\hline & & & $\begin{array}{l}251.145 \\
251.558\end{array}$ & $\begin{array}{r}0 \\
643\end{array}$ & $\begin{array}{l}398197 \\
398193\end{array}$ & $\begin{array}{l}5 \\
3\end{array}$ & $\begin{array}{l}5 \\
3\end{array}$ & $\begin{array}{l}33 \\
55\end{array}$ & $\begin{array}{l}0.031 \\
0.052\end{array}$ & $\begin{array}{l}0.13 \\
0.13\end{array}$ & $\begin{array}{l}-0.80 \\
-0.80\end{array}$ & $\begin{array}{l}D- \\
D-\end{array}$ & $\begin{array}{l}\text { ls } \\
\text { ls }\end{array}$ \\
\hline & & & 251.145 & 0 & 398193 & 5 & 3 & 3.8 & $\begin{array}{l}0.032 \\
0.0022\end{array}$ & 0.0089 & -1.97 & $\mathrm{D}-$ & ls \\
\hline \multirow[t]{6}{*}{11} & $\begin{array}{l}2 p^{4}- \\
2 p^{3}\left({ }^{2} \mathrm{D}^{\circ}\right) 3 d^{\prime}\end{array}$ & ${ }^{3} \mathrm{P}-{ }^{3} \mathrm{D}^{\circ}$ & 229.06 & .317 & 436891 & 9 & 15 & 73 & 0.096 & 0.65 & -0.06 & $\mathrm{D}-$ & 1 \\
\hline & & & [228.91] & 0 & 436845 & 5 & 7 & 72 & 0.080 & 0.30 & -0.40 & $\mathrm{D}-$ & Is \\
\hline & & & {$[229.22]$} & $\begin{array}{l}643 \\
927\end{array}$ & $\begin{array}{l}436914 \\
436959\end{array}$ & 3 & $\begin{array}{l}5 \\
3\end{array}$ & $\begin{array}{l}54 \\
40\end{array}$ & $\begin{array}{l}0.071 \\
0.095\end{array}$ & $\begin{array}{l}0.16 \\
0.072\end{array}$ & $\begin{array}{l}-0.67 \\
-102\end{array}$ & $\begin{array}{l}\mathrm{D}- \\
\mathrm{D}-\end{array}$ & is \\
\hline & & & {$[228.88]$} & 0 & 436914 & $\begin{array}{l}1 \\
5\end{array}$ & $\begin{array}{l}3 \\
5\end{array}$ & $\begin{array}{l}40 \\
18\end{array}$ & 0.014 & 0.054 & $\begin{array}{l}-1.02 \\
-1.14\end{array}$ & D- & ls \\
\hline & & & [229.19] & 643 & 436959 & 3 & 3 & 30 & 0.024 & 0.054 & -1.15 & $\mathrm{D}-$ & lis \\
\hline & & & [228.85] & 0 & 436959 & 5 & 3 & 2.0 & $9.6 \times 10^{-4}$ & 0.0036 & -2.32 & $\mathrm{D}-$ & ls \\
\hline
\end{tabular}


Ne III. Allowed Transitions - Continued

\begin{tabular}{|c|c|c|c|c|c|c|c|c|c|c|c|c|c|}
\hline No. & $\begin{array}{l}\text { Transition } \\
\text { Array }\end{array}$ & $\begin{array}{l}\text { Multi- } \\
\text { plet }\end{array}$ & $\lambda(\AA)$ & $E_{i}\left(\mathrm{~cm}^{-1}\right)$ & $E_{k}\left(\mathrm{~cm}^{-1}\right)$ & $g_{i}$ & $g_{k}$ & $\begin{array}{c}A_{k i}\left(10^{8}\right. \\
\left.\sec ^{-1}\right)\end{array}$ & $f_{i k}$ & $S$ (at.u.) & $\log g f$ & $\begin{array}{l}\text { Accu- } \\
\text { racy }\end{array}$ & Source \\
\hline \multirow[t]{7}{*}{12} & & ${ }^{3} \mathrm{P}-{ }^{3} \mathrm{P}^{\circ}$ & 227.62 & 317 & 439646 & 9 & 9 & 160 & 0.12 & 0.83 & 0.04 & $\mathrm{D}-$ & 1 \\
\hline & & & [227.49] & 0 & 439586 & 5 & 5 & 120 & 0.093 & 0.35 & -0.33 & $\mathrm{D}-$ & is \\
\hline & & & [227.76] & 643 & 439708 & 3 & 3 & 39 & 0.031 & 0.069 & -1.04 & $\mathrm{D}-$ & 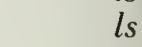 \\
\hline & & & {$[227.42]$} & 0 & 439708 & 5 & 3 & 69 & 0.032 & 0.12 & -0.80 & $\mathrm{D}-$ & ls \\
\hline & & & [227.73] & 643 & 439760 & 3 & 1 & 160 & 0.041 & 0.092 & -0.91 & $\mathrm{D}-1$ & 5 \\
\hline & & & [227.82] & 643 & 439586 & 3 & 5 & 41 & 0.053 & 0.12 & -0.80 & $\mathrm{D}-$ & 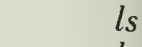 \\
\hline & & & [227.90] & 927 & 439708 & 1 & 3 & 52 & 0.12 & 0.092 & -0.91 & $\mathrm{D}-$ & $l s$ \\
\hline \multirow[t]{4}{*}{13} & & ${ }^{3} \mathrm{P}-{ }^{3} \mathrm{~S}^{\circ}$ & 227.40 & 317 & 440065 & 9 & 3 & 210 & 0.055 & 0.37 & -0.31 & $\mathrm{D}-$ & 1 \\
\hline & & & [227.24] & 0 & 440065 & 5 & 3 & 120 & 0.056 & 0.21 & -0.55 & $\mathrm{D}-$ & ls \\
\hline & & & [227.57] & 643 & 440065 & 3 & 3 & 69 & 0.053 & 0.12 & -0.80 & $\bar{D}-$ & ls \\
\hline & & & [227.72] & 927 & 440065 & 1 & 3 & 23 & 0.055 & 0.041 & -1.26 & $\mathrm{D}-$ & ls \\
\hline \multirow[t]{4}{*}{14} & $\begin{array}{l}2 p^{3} 3 s- \\
2 p^{3}\left({ }^{4} \mathrm{~S}^{\circ}\right) 3 p\end{array}$ & $\begin{array}{r}{ }^{5} \mathrm{~S}^{\circ}-{ }^{5} \mathrm{P} \\
(11 \mathrm{uv})\end{array}$ & 2592.3 & [314148] & [352712] & 5 & 15 & 2.5 & 0.76 & 32 & 0.58 & D & $c a$ \\
\hline & & & 2590.04 & [314148] & [352746] & 5 & 7 & 2.5 & 0.36 & 15 & 0.25 & D & Is \\
\hline & & & 2593.60 & [314148] & [352693] & $\begin{array}{l}5 \\
5\end{array}$ & 5 & 2.5 & 0.25 & 11 & 0.10 & D & ls \\
\hline & & & 2595.68 & {$[314148]$} & [352662] & 5 & 3 & 2.5 & 0.15 & 6.5 & -0.12 & D & $s$ \\
\hline \multirow[t]{4}{*}{15} & & $\begin{array}{l}{ }^{3} S^{\circ}-{ }^{3} P \\
(12 u v)\end{array}$ & 2678.2 & 319445 & 356773 & 3 & 9 & 2.4 & 0.78 & 20 & 0.37 & D & $c a$ \\
\hline & & & 2677.90 & 319445 & 356777 & 3 & 5 & 2.4 & 0.43 & 11 & 0.11 & D & $l s$ \\
\hline & & & 2678.64 & 319445 & 356766 & 3 & 3 & 2.4 & 0.26 & 6.8 & -0.11 & D & ls \\
\hline & & & 2677.90 & 319445 & 356777 & 3 & 1 & 2.4 & 0.086 & 2.3 & -0.59 & D & ls \\
\hline \multirow[t]{7}{*}{16} & $2 p^{3} 3 s^{\prime}-$ & ${ }^{3} \mathrm{D}^{\circ}-{ }^{3} \mathrm{~F}$ & 2612.4 & 353167 & 391435 & 15 & 21 & 2.4 & 0.34 & 44 & 0.71 & D & $c a$ \\
\hline & & & 2610.03 & 353148 & 391450 & 7 & 9 & 2.4 & 0.32 & 19 & 0.34 & D & $l s$ \\
\hline & & & 2613.41 & 353177 & 391430 & 5 & 7 & 2.1 & 0.31 & 13 & 0.18 & D & $l s$ \\
\hline & & & 2615.87 & 353197 & 391414 & 3 & 5 & 2.0 & 0.34 & 8.9 & 0.01 & D & $l s$ \\
\hline & & & 2611.42 & 353148 & 391430 & 7 & 7 & 0.27 & 0.027 & 1.6 & -0.72 & D & ls \\
\hline & & & 2614.51 & 353177 & 391414 & 5 & 5 & 0.37 & 0.038 & 1.6 & -0.72 & D & ls \\
\hline & & & [2612.5] & 353148 & 391414 & 7 & 5 & 0.011 & $7.8 \times 10^{-4}$ & 0.047 & -2.26 & D & $l s$ \\
\hline \multirow[t]{8}{*}{17} & $2 p^{3} 3 p-$ & ${ }^{5} \mathrm{P}-{ }^{5} \mathrm{D}^{\circ}$ & & & & & & & & & & & \\
\hline & $2 p^{3}\left({ }^{4} \mathrm{~S}^{\circ}\right) 3 d$ & & 2163.77 & [352746] & [398947] & 7 & 9 & 6.5 & 0.59 & 29.4 & 0.62 & $\mathrm{C}-$ & $c a, l s$ \\
\hline & & & 2161.22 & [352693] & [398949] & 5 & 7 & 4.36 & 0.428 & 15.2 & 0.330 & $\mathrm{C}-$ & $c a, l_{s}$ \\
\hline & & & $\begin{array}{r}2159.60 \\
{[2163.7]}\end{array}$ & $\begin{array}{l}{[352662]} \\
{[352746]}\end{array}$ & $\begin{array}{l}{[398952]} \\
{[398949]}\end{array}$ & $\begin{array}{l}3 \\
7\end{array}$ & $\begin{array}{l}5 \\
7\end{array}$ & $\begin{array}{l}2.30 \\
2.25\end{array}$ & $\begin{array}{l}0.268 \\
0.158\end{array}$ & $\begin{array}{l}5.7 \\
7.9\end{array}$ & $\begin{array}{r}-0.096 \\
0.044\end{array}$ & $\begin{array}{l}\mathrm{C}- \\
\mathrm{C}-\end{array}$ & $\begin{array}{l}c a, l s \\
c a, l s\end{array}$ \\
\hline & & & 2161.04 & [352693] & [398952] & 5 & 5 & 3.82 & 0.267 & 9.5 & 0.126 & $\mathrm{C}-$ & $\mathrm{ca}, \mathrm{ls}$ \\
\hline & & & 2159.44 & [352662] & [398956] & 3 & 3 & 4.92 & 0.344 & 7.3 & 0.014 & $\mathrm{C}-$ & $c a, l s$ \\
\hline & & & [2163.5] & {$[352746]$} & [398952] & 7 & 5 & 0.450 & 0.0227 & 1.12 & -0.80 & $\mathrm{C}-$ & $c a, l s$ \\
\hline & & & 2160.88 & [352693] & [398956] & 5 & 3 & 1.64 & 0.069 & 2.45 & -0.464 & $\mathrm{C}-$ & $c a, l s$ \\
\hline \multirow[t]{6}{*}{18} & & ${ }^{3} \mathrm{P}-{ }^{3} \mathrm{D}^{\circ}$ & 2413.0 & 356773 & 398203 & 9 & 15 & 4.87 & 0.71 & 51 & 0.80 & $\mathrm{C}-$ & $c a$ \\
\hline & & & 2412.73 & 356777 & 398212 & 5 & 7 & 4.87 & 0.59 & 23.6 & $\cdot 0.473$ & $\mathrm{C}-$ & $l s$ \\
\hline & & & 2412.94 & 356766 & 398197 & 3 & 5 & 3.65 & 0.53 & 12.7 & 0.202 & $\mathrm{C}-$ & ls \\
\hline & & & 2413.78 & 356777 & 398193 & 1 & 3 & 2.70 & 0.71 & 5.6 & -0.150 & C- & ls \\
\hline & & & 2413.54 & 356777 & 398197 & 5 & 5 & 1.22 & 0.106 & 4.22 & -0.275 & $\mathrm{C}-$ & ls \\
\hline & & & $\begin{array}{l}2413.18 \\
2413.78\end{array}$ & $\begin{array}{l}356766 \\
356777\end{array}$ & $\begin{array}{l}398193 \\
398193\end{array}$ & $\begin{array}{l}3 \\
5\end{array}$ & $\begin{array}{l}3 \\
3\end{array}$ & $\begin{array}{l}2.03 \\
0.135\end{array}$ & $\begin{array}{l}0.177 \\
0.0071\end{array}$ & $\begin{array}{l}4.22 \\
0.281\end{array}$ & $\begin{array}{l}-0.275 \\
-1.451\end{array}$ & $\begin{array}{l}\mathrm{C}- \\
\mathrm{C}-\end{array}$ & $\begin{array}{l}l s \\
l s\end{array}$ \\
\hline \multirow[t]{8}{*}{19} & $\begin{array}{l}2 p^{3} 3 p^{\prime}- \\
2 p^{3}\left({ }^{2} \mathrm{D}^{\circ}\right) 3 d^{\prime}\end{array}$ & ${ }^{3} \mathrm{D}-{ }^{3} \mathrm{D}^{\circ}$ & 2091.7 & 389099 & 436891 & 15 & 15 & 3.92 & 0.257 & 26.6 & 0.59 & $\mathrm{C}-$ & $c a$ \\
\hline & & & 2095.54 & 389139 & 436845 & 7 & 7 & 3.47 & 0.228 & 11.0 & 0.204 & $\mathrm{C}-$ & ls \\
\hline & & & 2089.43 & 389069 & 436914 & 5 & 5 & 2.73 & 0.179 & 6.2 & -0.048 & C- & ls \\
\hline & & & 2086.96 & 389058 & 436959 & 3 & 3 & 2.96 & 0.194 & 3.99 & -0.236 & C- & ls \\
\hline & & & 2092.44 & 389139 & 436914 & 7 & 5 & 0.61 & 0.0286 & 1.38 & -0.70 & $\mathrm{C}-$ & Is \\
\hline & & & 2087.44 & $\begin{array}{l}389069 \\
380060\end{array}$ & 436959 & 5 & 3 & 0.99 & 0.0387 & 1.33 & -0.71 & $\mathrm{C}-$ & Is \\
\hline & & & 2092.44 & 389069 & 436845 & 5 & 7 & 0.435 & 0.0400 & 1.38 & -0.70 & $\mathrm{C}-$ & ls \\
\hline & & & 2088.92 & 389058 & 436914 & 3 & 5 & 0.59 & 0.064 & 1.33 & -0.71 & $\mathrm{C}-$ & ls \\
\hline
\end{tabular}




\section{Forbidden Transitions}

The adopted valués represent, as in the case of F II, the work of Garstang [1] and Naqvi [2], who independently have done essentially the same calculations and have arrived at very similar results. For the selection of values, the same considerations as for F II have been applied.

\section{References}

[1] Garstang, R. H., Monthly Notices Roy. Astron. Soc. 11 1, 115-124 (1951).

[2] Naqvi, A. M., Thesis Harvard (1951).

Ne III. Forbidden Transitions

\begin{tabular}{|c|c|c|c|c|c|c|c|c|c|c|c|c|}
\hline No. & $\begin{array}{c}\text { Transition } \\
\text { Array }\end{array}$ & $\begin{array}{c}\text { Multi- } \\
\text { plet }\end{array}$ & $\lambda(\AA)$ & $E_{i}\left(\mathrm{~cm}^{-1}\right)$ & $E_{k}\left(\mathrm{~cm}^{-1}\right)$ & $g_{i}$ & $g_{k}$ & $\begin{array}{c}\text { Type of } \\
\text { Transition }\end{array}$ & $A_{k i}\left(\sec ^{-1}\right)$ & $S($ at.u. $)$ & $\begin{array}{l}\text { Accu- } \\
\text { racy }\end{array}$ & Source \\
\hline 1 & $2 p^{4}-2 p^{4}$ & $\begin{array}{l}{ }^{3 \mathrm{P}-{ }^{3} \mathrm{P}} \\
(1 \mathrm{~F}) \\
{ }^{3} \mathrm{P}-{ }^{1} \mathrm{D} \\
{ }^{1} \mathrm{D}-{ }^{1} \mathrm{~S} \\
(2 \mathrm{~F})\end{array}$ & $\begin{array}{l}{\left[15.55 \times 10^{4}\right]} \\
{\left[15.55 \times 10^{4}\right]} \\
{\left[10.79 \times 10^{4}\right]} \\
{\left[36.19 \times 10^{4}\right]} \\
\\
3868.74 \\
3868.74 \\
3967.51 \\
3967.51 \\
{[4012.7]} \\
{[1793.8]} \\
{[1814.8]} \\
\\
3342.9\end{array}$ & $\begin{array}{c}0 \\
0 \\
0 \\
642.9 \\
\\
0 \\
0 \\
642.9 \\
642.9 \\
927 \\
0 \\
642.9 \\
\\
\\
\\
\\
\\
\\
\end{array}$ & $\begin{array}{c}642.9 \\
642.9 \\
927 \\
927 \\
\\
\\
25841 \\
25841 \\
25841 \\
25841 \\
25841 \\
55747 \\
55747\end{array}$ & $\begin{array}{l}5 \\
5 \\
3 \\
3 \\
1 \\
5 \\
3\end{array}$ & $\begin{array}{l}5 \\
5 \\
5 \\
5 \\
5 \\
\\
1 \\
1\end{array}$ & $\begin{array}{l}\mathrm{m} \\
\mathrm{e} \\
\mathrm{m} \\
\mathrm{e} \\
\mathrm{e} \\
\mathrm{e} \\
\mathrm{m}\end{array}$ & $\begin{array}{l}0.00599 \\
2.60 \times 10^{-9} \\
2.03 \times 10^{-8} \\
0.00115 \\
\\
0.170 \\
3.0 \times 10^{-4} \\
0.052 \\
3.8 \times 10^{-5} \\
1.2 \times 10^{-5} \\
\\
0.0051 \\
2.20\end{array}$ & $\begin{array}{l}2.50 \\
0.422 \\
0.176 \\
2.00 \\
\\
0.00182 \\
7.7 \times 10^{-4} \\
6.1 \times 10^{-4} \\
1.1 \times 10^{-4} \\
3.7 \times 10^{-5} \\
5.6 \times 10^{-5} \\
4.87 \times 10^{-4} \\
\\
0.70\end{array}$ & $\begin{array}{l}\text { B } \\
\text { C } \\
\text { C } \\
\text { B } \\
\\
\text { C } \\
\text { D } \\
\text { C } \\
\text { D } \\
\text { D } \\
\text { D } \\
\text { C }\end{array}$ & $\begin{array}{r}1,2 \\
1 \\
1 \\
1,2 \\
\\
2 \\
1 \\
2 \\
1 \\
1 \\
- \\
1 \\
1\end{array}$ \\
\hline
\end{tabular}




\section{Allowed Transitions}

List of tabulated lines:

\begin{tabular}{|c|c|c|c|c|c|}
\hline Wavelength $[\AA]$ & No. & Wavelength $[\AA]$ & No. & Wavelength $[\AA]$ & No. \\
\hline $\begin{array}{l}208.485 \\
208.734 \\
208.899 \\
212.556 \\
357.831\end{array}$ & $\begin{array}{l}7 \\
7 \\
7 \\
8 \\
3\end{array}$ & $\begin{array}{c}541.124 \\
542.076 \\
543.884 \\
2018.44 \\
2022.19\end{array}$ & $\begin{array}{r}1 \\
1 \\
1 \\
13 \\
13\end{array}$ & $\begin{array}{l}2262.08 \\
2264.54 \\
2285.79 \\
2293.14 \\
2293.49\end{array}$ & $\begin{array}{l}14 \\
14 \\
12 \\
12 \\
12\end{array}$ \\
\hline $\begin{array}{l}358.70 \\
387.13 \\
388.23 \\
421.584 \\
469.77\end{array}$ & $\begin{array}{l}3 \\
6 \\
6 \\
5 \\
2\end{array}$ & $\begin{array}{l}2029.2 \\
2033.5 \\
2174.4 \\
2176.1 \\
2188.0\end{array}$ & $\begin{array}{l}13 \\
13 \\
10 \\
10 \\
10\end{array}$ & $\begin{array}{l}2350.84 \\
2352.52 \\
2357.96 \\
2362.68 \\
2363.28\end{array}$ & $\begin{array}{r}9 \\
9 \\
9 \\
9 \\
11\end{array}$ \\
\hline $\begin{array}{l}469.817 \\
469.865 \\
521.730 \\
521.74 \\
521.810\end{array}$ & $\begin{array}{l}2 \\
2 \\
4 \\
4 \\
4\end{array}$ & $\begin{array}{l}2192.6 \\
2203.88 \\
2206.4 \\
2220.81 \\
2258.02\end{array}$ & $\begin{array}{l}10 \\
10 \\
10 \\
10 \\
14\end{array}$ & $\begin{array}{l}2365.49 \\
2372.16 \\
2384.20 \\
2384.95 \\
2404.28 \\
2405.19\end{array}$ & $\begin{array}{r}11 \\
9 \\
9 \\
9 \\
11 \\
9\end{array}$ \\
\hline
\end{tabular}

The values for several of the transitions are taken from the self-consistent field calculations (with exchange) by Weiss [1]. These calculations do not include the important effects of configuration interaction; hence, fairly large uncertainties must be expected. The average of the dipole length and velocity approximations is adopted [1].

For the $2 s^{2} 2 p^{3}{ }^{2} \mathrm{P}^{\circ}-2 s 2 p^{4}{ }^{2} \mathrm{D},{ }^{2} \mathrm{~S}$ transitions values are available from the calculations of Levinson et al. [2] which include configuration interaction in a limited way. Again, large uncertainties are to be expected.

\section{References}

[1] Weiss, A. W., private communication (1965).

[2] Levinson, I. B., Bolotin, A. B., and Levin, L. I., Trudy Vil'nyusskogo un. 5, 49-55 (1956). 
Ne IV. Allowed Transitions

\begin{tabular}{|c|c|c|c|c|c|c|c|c|c|c|c|c|c|}
\hline No. & $\begin{array}{c}\text { Transition } \\
\text { Array }\end{array}$ & $\begin{array}{l}\text { Multi- } \\
\text { plet }\end{array}$ & $\lambda(\AA)$ & $E_{i}\left(\mathrm{~cm}^{-1}\right)$ & $E_{k}\left(\mathrm{~cm}^{-1}\right)$ & $g_{i}$ & $g_{k}$ & $\begin{array}{l}A_{k i}\left(10^{k}\right. \\
\left.\sec ^{-1}\right)\end{array}$ & $f_{i k}$ & $S($ at.u.) & $\log g f$ & $\begin{array}{l}\text { Accu- } \\
\text { racy }\end{array}$ & Source \\
\hline \multirow[t]{4}{*}{ l } & $\begin{array}{r}2 s^{2} 2 p^{3}- \\
2 s 2 p^{4}\end{array}$ & ${ }^{4} \mathrm{~S}^{\circ}-{ }^{4} \mathrm{P}$ & 542.82 & 0 & 184222 & 4 & 12 & 25 & 0.34 & 2.4 & 0.13 & $\mathrm{E}$ & \\
\hline & & & 543.884 & 0 & 183860 & 4 & 6 & 25 & 0.17 & 1.2 & -0.17 & $\mathrm{E}$ & $l$ \\
\hline & & & 542.076 & 0 & 184477 & 4 & 4 & 25 & 0.11 & 0.80 & -0.35 & $\mathrm{E}$ & l. \\
\hline & & & 541.124 & 0 & 184799 & 4 & 2 & 25 & 0.056 & 0.40 & -0.65 & $\mathrm{E}$ & $l$ \\
\hline \multirow[t]{5}{*}{2} & & ${ }^{2} \mathrm{D}^{\circ}-{ }^{2} \mathrm{D}$ & 469.84 & [40968] & {$[25.3808]$} & 10 & 10 & 51 & 0.17 & 2.6 & 0.23 & $\mathrm{E}$ & \\
\hline & & & 469.817 & [40950] & [253799] & 6 & 6 & 49 & 0.16 & 1.5 & -0.01 & $\mathrm{E}$ & 10 \\
\hline & & & 469.817 & [40995] & [253822] & 4 & 4 & 46 & 0.15 & 0.94 & -0.22 & $\mathrm{E}$ & i. \\
\hline & & & [469.77] & [4.0950] & [253822] & 6 & 4 & 4.9 & 0.011 & 0.10 & -1.19 & $\mathrm{E}$ & l: \\
\hline & & & 469.865 & [40995] & {$[253799]$} & 4 & 6 & 3.3 & 0.016 & 0.10 & -1.19 & $\mathrm{E}$ & ls \\
\hline \multirow[t]{4}{*}{3} & & ${ }^{2} \mathrm{D}^{\circ}-{ }^{2} \mathrm{P}$ & 358.40 & [40968] & [319985] & 10 & 6 & 150 & 0.18 & 2.1 & 0.25 & $\mathrm{E}$ & 1 \\
\hline & & & 358.70 & [40950] & {$[319751]$} & 6 & 4 & 140 & 0.18 & 1.3 & 0.04 & $\mathrm{E}$ & ls \\
\hline & & & 357.831 & [40995] & [320452] & 4 & 2 & 150 & 0.15 & 0.70 & -0.23 & $\mathrm{E}$ & ls \\
\hline & & & 358.70 & [40995] & [319751] & 4 & 4 & 15 & 0.030 & 0.14 & -0.93 & $\mathrm{E}$ & ls \\
\hline \multirow[t]{4}{*}{4} & & $\begin{array}{c}{ }^{2} \mathrm{P}^{\circ}-{ }^{2} \mathrm{D} \\
(8 \mathrm{uv})\end{array}$ & 521.78 & [62.155] & {$[253808]$} & 6 & 10 & 7.7 & 0.052 & 0.54 & -0.50 & $\mathrm{E}$ & 2 \\
\hline & & & 521.810 & [62157] & [253799] & 4 & 6 & 7.6 & 0.047 & 0.32 & -0.73 & $\mathrm{E}$ & ls \\
\hline & & & 521.730 & [62150] & {$[253822]$} & 2 & 4 & 6.4 & 0.052 & 0.18 & -0.98 & $\mathrm{E}$ & ls \\
\hline & & & [521.74] & [62157] & {$[253822]$} & 4 & 4 & 1.3 & 0.0052 & 0.036 & -1.68 & $\mathrm{E}$ & ls \\
\hline 5 & & $\begin{array}{c}{ }^{2} \mathrm{P}^{\circ}-{ }^{2} \mathrm{~S} \\
(9 \mathrm{uv})\end{array}$ & 421.584 & [62155] & [299351] & 6 & 2 & 120 & 0.11 & 0.92 & -0.18 & $\mathrm{E}$ & 2 \\
\hline \multirow[t]{5}{*}{6} & & $\begin{array}{l}{ }^{2} \mathrm{P}^{\circ}-{ }^{2} \mathrm{P} \\
(10 \mathrm{uv})\end{array}$ & 387.85 & [62155] & [319985] & 6 & 6 & 75 & 0.17 & 1.3 & 0.01 & $\mathrm{E}$ & 1 \\
\hline & & & 388.23 & [62157] & [319751] & 4 & 4 & 62 & 0.14 & 0.72 & -0.25 & $\mathrm{E}$ & ls \\
\hline & & & 387.13 & [62150] & {$[320452]$} & 2 & 2 & 51 & 0.11 & 0.29 & -0.64 & $\mathrm{E}$ & ls \\
\hline & & & 387.13 & [62157] & {$[320452]$} & 4 & 2 & 24 & 0.027 & 0.14 & -0.96 & $\mathrm{E}$ & ls \\
\hline & & & 388.23 & [62150] & [319751] & 2 & 4 & 12 & 0.055 & 0.14 & -0.96 & $\mathrm{E}$ & ls \\
\hline \multirow[t]{4}{*}{7} & $\begin{array}{l}2 p^{3}- \\
2 p^{2}\left({ }^{3} \mathrm{P}\right) 3 s\end{array}$ & $\begin{array}{c}{ }^{4} \mathrm{~S}^{\circ}-{ }^{4} \mathrm{P} \\
(2 \mathrm{uv})\end{array}$ & 208.63 & 0 & 479309 & 4 & 12 & 48 & 0.095 & 0.26 & -0.42 & $\mathrm{E}$ & l \\
\hline & & & 208.485 & 0 & 479662 & 4 & 6 & 48 & 0.047 & 0.13 & -0.72 & $\mathrm{E}$ & ls \\
\hline & & & 208.734 & 0 & 479083 & 4 & 4 & 48 & 0.032 & 0.087 & -0.90 & $\mathrm{E}$ & ls \\
\hline & & & 208.899 & 0 & 478701 & 4 & 2 & 48 & 0.016 & 0.043 & -1.20 & $\mathrm{E}$ & ls \\
\hline 8 & $\begin{array}{l}2 p^{3}- \\
2 p^{2}\left({ }^{1} \mathrm{D}\right) 3 s^{\prime}\end{array}$ & $\begin{array}{c}{ }^{2} \mathrm{D}^{\circ}-{ }^{2} \mathrm{D} \\
(6 \mathrm{uv})\end{array}$ & 212.556 & [40968] & {$[511681]$} & 10 & 10 & 74 & 0.050 & 0.35 & -0.30 & $\mathrm{E}$ & 1 \\
\hline \multirow[t]{9}{*}{9} & $2 p^{2} 3 s-$ & ${ }^{4} \mathrm{P}-{ }^{4} \mathrm{D}^{\circ}$ & 2361.5 & 479309 & 521643 & 12 & 20 & 2.5 & 0.34 & 32 & 0.62 & D & $c a$ \\
\hline & & & 2357.96 & 4.79662 & 522058 & 6 & 8 & 2.5 & 0.28 & 13 & 0.22 & D & ls \\
\hline & & & 2352.52 & 479083 & 521578 & 4 & 6 & 1.7 & 0.22 & 6.7 & -0.06 & D & ls \\
\hline & & & 2350.84 & 478701 & 521226 & 2 & 4 & 1.0 & 0.17 & 2.7 & -0.46 & D & ls \\
\hline & & & 2384.95 & 479662 & 521578 & 6 & 6 & 0.72 & 0.061 & 2.9 & -0.43 & $\mathrm{D}$ & ls \\
\hline & & & 2372.16 & 479083 & 521226 & 4 & 4 & 1.3 & 0.11 & 3.4 & -0.36 & D & ls \\
\hline & & & 2362.68 & 478701 & 521013 & 2 & 2 & 2.0 & 0.17 & 2.7 & -0.47 & D & ls \\
\hline & & & 2405.19 & 479662 & 521226 & 6 & 4 & 0.12 & 0.0067 & 0.32 & -1.39 & D & ls \\
\hline & & & 2384.20 & 479083 & 521013 & 4 & 2 & 0.40 & 0.017 & 0.53 & -1.17 & D & ls \\
\hline \multirow[t]{8}{*}{10} & & ${ }^{4} \mathrm{P}-{ }^{4} \mathrm{P}^{\circ}$ & 2197.5 & 479309 & 524802 & 12 & 12 & 3.1 & 0.23 & 20 & 0.43 & D & $c a$ \\
\hline & & & 2203.88 & 479662 & 525022 & 6 & 6 & 2.2 & 0.16 & 6.8 & -0.03 & D & ls \\
\hline & & & [2192.6] & 479083 & 524677 & 4 & 4 & 0.42 & 0.030 & 0.86 & -0.92 & D & ls \\
\hline & & & [2188.0] & 478701 & 524391 & 2 & 2 & 0.52 & 0.037 & 0.54 & -1.13 & D & ls \\
\hline & & & 2220.81 & 479662 & 524677 & 6 & 4 & 1.4 & 0.067 & 2.9 & -0.40 & D & Is \\
\hline & & & [2206.4] & 479083 & 524391 & 4 & 2 & 2.5 & 0.093 & 2.7 & -0.43 & D & ls \\
\hline & & & [2176.1] & 479083 & 525022 & 4 & 6 & 0.96 & 0.10 & 2.9 & -0.39 & D & ls \\
\hline & & & {$[2174.4]$} & 478701 & 524677 & 2 & 4 & 1.3 & 0.19 & 2.7 & -0.42 & D & Is \\
\hline
\end{tabular}


Ne IV. Allowed Transitions-Continued

\begin{tabular}{|c|c|c|c|c|c|c|c|c|c|c|c|c|c|}
\hline No. & $\begin{array}{l}\text { Transition } \\
\text { Array }\end{array}$ & $\begin{array}{l}\text { Multi- } \\
\text { plet }\end{array}$ & $\lambda(\AA)$ & $E_{i}\left(\mathrm{~cm}^{-1}\right)$ & $E_{k}\left(\mathrm{~cm}^{-1}\right)$ & $g_{i}$ & $g_{k}$ & $\begin{array}{c}A_{k i}\left(10^{8}\right. \\
\left.\sec ^{-1}\right)\end{array}$ & $f_{i k}$ & $S$ (at.u.) & $\log g f$ & $\begin{array}{l}\text { Accu- } \\
\text { racy }\end{array}$ & Source \\
\hline \multirow[t]{4}{*}{11} & & ${ }^{2} \mathrm{P}-{ }^{2} \mathrm{D}^{\circ}$ & 2366.7 & [4889.34] & [531174] & 6 & 10 & 2.6 & 0.37 & 17 & 0.35 & D & $c a$ \\
\hline & & & 2363.28 & [489161] & [531462] & 4 & 6 & 2.7 & 0.33 & 10 & 0.12 & D & ls \\
\hline & & & 2365.49 & [488479] & [530741] & 2 & 4 & 2.2 & 0.37 & 5.7 & -0.13 & D & ls \\
\hline & & & 2404.28 & [489161] & [530741] & 4 & 4 & 0.42 & 0.036 & 1.1 & -0.84 & $\mathrm{D}$ & ls \\
\hline \multirow[t]{3}{*}{12} & $2 p^{2} 3 s^{\prime}-$ & ${ }^{2} \mathrm{D}-{ }^{2} \mathrm{~F}^{\circ}$ & 2289.1 & {$[511681]$} & {$[555353]$} & 10 & 14 & 2.8 & 0.30 & 23 & 0.48 & D & $c a$ \\
\hline & & & 2285.79 & {$[511678]$} & [555413] & 6 & 8 & 2.8 & 0.29 & 13 & 0.24 & D & ls \\
\hline & & & $\begin{array}{l}2293.49 \\
2293.14\end{array}$ & $\begin{array}{l}{[511685]} \\
{[511678]}\end{array}$ & $\begin{array}{l}{[555273]} \\
{[555273]}\end{array}$ & $\begin{array}{l}4 \\
6\end{array}$ & $\begin{array}{l}6 \\
6\end{array}$ & $\begin{array}{l}2.6 \\
0.18\end{array}$ & $\begin{array}{l}0.30 \\
0.014\end{array}$ & $\begin{array}{l}9.2 \\
0.65\end{array}$ & $\begin{array}{r}0.08 \\
-1.06\end{array}$ & $\begin{array}{l}\mathrm{D} \\
\mathrm{D}\end{array}$ & ls \\
\hline \multirow[t]{4}{*}{13} & & ${ }^{2} \mathrm{D}-{ }^{2} \mathrm{D}^{\circ}$ & 2031.7 & [511681] & [560885] & 10 & 10 & 4.0 & 0.25 & 17 & 0.39 & D & $c a$ \\
\hline & & & 2022.19 & [511678] & [560846] & 6 & 6 & 3.8 & 0.23 & 9.3 & 0.14 & D & $l s$ \\
\hline & & & 2018.44 & [511685] & [560943] & 4 & 4 & 3.7 & 0.22 & 6.0 & -0.05 & D & $l s$ \\
\hline & & & $\begin{array}{l}{[2029.2]} \\
{[2033.5]}\end{array}$ & $\begin{array}{l}{[511678]} \\
{[511685]}\end{array}$ & $\begin{array}{l}{[560943]} \\
{[560846]}\end{array}$ & $\begin{array}{l}6 \\
4\end{array}$ & $\begin{array}{l}4 \\
6\end{array}$ & $\begin{array}{l}0.40 \\
0.27\end{array}$ & $\begin{array}{l}0.017 \\
0.025\end{array}$ & $\begin{array}{l}0.66 \\
0.66\end{array}$ & $\begin{array}{l}-1.00 \\
-1.00\end{array}$ & $\begin{array}{l}\mathrm{D} \\
\mathrm{D}\end{array}$ & $l s$ \\
\hline \multirow[t]{3}{*}{14} & $2 p^{2} 3 s^{\prime \prime}-$ & ${ }^{6} \mathrm{~S}-{ }^{6} \mathrm{P}^{\circ}$ & 2260.8 & [538500] & {$[582718]$} & 6 & 18 & 2.7 & 0.63 & 28 & 0.58 & D & $c a$ \\
\hline & & & 2258.02 & [538500] & [582773] & 6 & 8 & 2.7 & 0.28 & 12 & 0.23 & D & $l s$ \\
\hline & & & $\begin{array}{l}2262.08 \\
2264.54\end{array}$ & {$\left[\begin{array}{l}{[538500]} \\
{[538500]}\end{array}\right]$} & $\begin{array}{l}{[582693]} \\
{[582645]}\end{array}$ & $\begin{array}{l}6 \\
6\end{array}$ & $\begin{array}{l}6 \\
4\end{array}$ & $\begin{array}{l}2.7 \\
2.7\end{array}$ & $\begin{array}{l}0.21 \\
0.14\end{array}$ & $\begin{array}{l}9.4 \\
6.2\end{array}$ & $\begin{array}{r}0.10 \\
-0.08\end{array}$ & $\begin{array}{l}\mathrm{D} \\
\mathrm{D}\end{array}$ & ls \\
\hline & & & & & & & & & & & & & \\
\hline
\end{tabular}

\section{Forbidden Transitions}

Garstang's 1960 calculations [1] are exclusively used, since it is felt that the important effects of configuration interaction are partially taken into account in this work and a reliable estimate of the quadrupole integral is provided (see also general introduction).

Reference

[1] Garstang, R. H., Monthly Notices Roy. Astron. Soc. 120, 201 (1960).

Ne IV. Forbidden Transitions

\begin{tabular}{|c|c|c|c|c|c|c|c|c|c|c|c|c|}
\hline No. & $\begin{array}{c}\text { Transition } \\
\text { Array }\end{array}$ & $\begin{array}{c}\text { Multi- } \\
\text { plet }\end{array}$ & $\lambda(\AA ̊)$ & $E_{i}\left(\mathrm{~cm}^{-1}\right)$ & $E_{k}\left(\mathrm{~cm}^{-1}\right)$ & $g_{i}$ & $g_{k}$ & $\begin{array}{c}\text { Type of } \\
\text { Transition }\end{array}$ & $A_{k i}\left(\sec ^{-1}\right)$ & $S$ (at.u.) & $\begin{array}{l}\text { Accu- } \\
\text { racy }\end{array}$ & Source \\
\hline \multirow[t]{4}{*}{1} & $2 p^{3}-2 p^{3}$ & ${ }^{4} \mathrm{~S}^{\circ}-{ }^{2} \mathrm{D}^{\circ}$ & & & & & & & & & & \\
\hline & & & $\begin{array}{l}{[2441.3]} \\
{[2441.3]}\end{array}$ & $\begin{array}{l}0 \\
0\end{array}$ & $\begin{array}{l}40950 \\
40950\end{array}$ & $\begin{array}{l}4 \\
4\end{array}$ & $\begin{array}{l}6 \\
6\end{array}$ & $\begin{array}{c}\mathrm{m} \\
\mathrm{e}\end{array}$ & $\begin{array}{l}1.80 \times 10^{-4} \\
4.1 \times 10^{-4}\end{array}$ & $\begin{array}{l}5.8 \times 10^{-7} \\
1.3 \times 10^{-4}\end{array}$ & $\begin{array}{l}\text { C } \\
D\end{array}$ & $\begin{array}{l}1 \\
1\end{array}$ \\
\hline & & & [2438.6] & 0 & 40995 & 4 & 4 & $\mathrm{~m}$ & 0.0053 & $1.14 \times 10^{-5}$ & C & 1 \\
\hline & & & [2438.6] & 0 & 40995 & 4 & 4 & $\mathrm{e}$ & $2.7 \times 10^{-4}$ & $5.5 \times 10^{-5}$ & D & 1 \\
\hline \multirow[t]{4}{*}{2} & & ${ }^{4} \mathrm{~S}^{\circ}-{ }^{2} \mathrm{P}^{\circ}$ & & & & & & & & & & \\
\hline & & & {$[1609.0]$} & $\begin{array}{l}0 \\
0\end{array}$ & $\begin{array}{l}62150 \\
62150\end{array}$ & $\begin{array}{l}4 \\
4\end{array}$ & $\begin{array}{l}2 \\
2\end{array}$ & $\mathrm{~m}$ & $\begin{array}{l}0.53 \\
86 \times 10-6\end{array}$ & $1.64 \times 10^{-4}$ & $\begin{array}{l}C \\
D\end{array}$ & 1 \\
\hline & & & {$[1608.8]$} & 0 & 62157 & 4 & 4 & $\mathrm{~m}$ & 1.33 & $8.2 \times 10^{-4}$ & C & 1 \\
\hline & & & [1608.8] & 0 & 62157 & 4 & 4 & e & $1.5 \times 10^{-7}$ & $3.8 \times 10^{-9}$ & D & 1 \\
\hline \multirow[t]{2}{*}{3} & & ${ }^{2} \mathrm{D}^{\circ}-{ }^{2} \mathrm{D}^{\circ}$ & & & & & & & & & & \\
\hline & & & {$\left[22.2 \times 10^{5}\right]$} & 40950 & 40995 & 6 & 4 & $\mathrm{e}$ & $1.1 \times 10^{-17}$ & 0.0014 & D & 1 \\
\hline
\end{tabular}


Ne IV. Forbidden Transitions-Continued

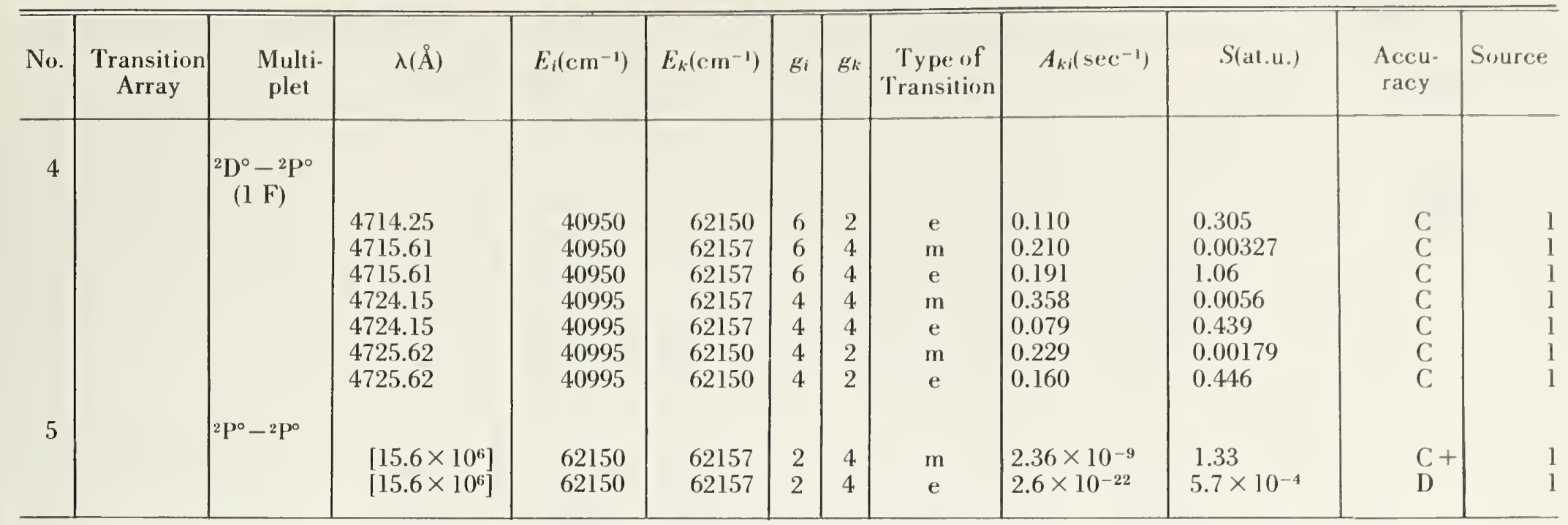

Ground State

Ionization Potential
Ne V.

$$
1 s^{2} 2 s^{2} 2 p^{2}{ }^{3} \mathrm{P}_{0}
$$

$126.3 \mathrm{eV}=1018634 \mathrm{~cm}^{-1}$

\section{Allowed Transitions}

List of tabulated lines:

\begin{tabular}{l|r||c|c||c|c}
\hline \hline Wavelength $[\AA]$ & No. & Wavelength $[\AA]$ & No. & Wavelength $[\AA]$ & No. \\
\hline & & & & & \\
142.44 & 8 & 173.932 & 6 & 572.336 & 1 \\
142.52 & 8 & 357.955 & 3 & 2224.12 & 14 \\
142.58 & 8 & 358.472 & 3 & 2227.42 & 14 \\
142.66 & 8 & 359.385 & 3 & 2232.41 & 14 \\
142.72 & 8 & 365.594 & 5 & 2236.29 & 14 \\
143.22 & 7 & 416.198 & 4 & 2245.48 & 14 \\
143.27 & 7 & 480.406 & 2 & 2256.05 & 14 \\
143.30 & 7 & 481.281 & 2 & 2257.9 & 14 \\
143.34 & 7 & 481.361 & 2 & 2259.57 & 13 \\
143.42 & 7 & 482.987 & 2 & 2263.39 & 13 \\
143.45 & 7 & 568.418 & 1 & & \\
147.13 & 11 & 569.759 & 1 & 2265.71 & 13 \\
148.78 & 10 & 569.830 & 1 & 228.54 & 14 \\
151.42 & 9 & 572.03 & 1 & 2306.61 & 13 \\
156.61 & 12 & 572.106 & 1 & 2330.3 & 13 \\
\hline
\end{tabular}

For the $2 s^{2} 2 p^{2}-2 s 2 p^{3}$ transition array, values are available from the calculations of Bolotin et al. [1] which include the important effects of configuration interaction only in a limited way. Hence, fairly large uncertainties must be expected.

The values for several other transitions are taken from the self-consistent field calculations (with exchange) by Weiss [2]. These calculations neglect the effects of configuration interaction entirely. The average of the dipole length and the velocity approximations is adopted [2].

\section{References}

[1] Bolotin, A. B., Levinson, I. B., and Levin, L. I., Soviet Phys.-JE'TP 2, 391-395 (1956).

[2] Weiss, A. W., private communication (1965). 
Ne V. Allowed Transitions

\begin{tabular}{|c|c|c|c|c|c|c|c|c|c|c|c|c|c|}
\hline No. & $\begin{array}{c}\text { Transition } \\
\text { Array }\end{array}$ & $\begin{array}{c}\text { Multi- } \\
\text { plet }\end{array}$ & $\lambda(\AA)$ & $E_{i}\left(\mathrm{~cm}^{-1}\right)$ & $E_{k}\left(\mathrm{~cm}^{-1}\right)$ & $g_{i}$ & $g_{k}$ & $\begin{array}{c}A_{k i}\left(10^{8}\right. \\
\left.\sec ^{-1}\right)\end{array}$ & $f_{i k}$ & $S$ (at.u.) & $\log g f$ & $\begin{array}{l}\text { Accu- } \\
\text { racy }\end{array}$ & Source \\
\hline \multirow[t]{6}{*}{1} & $2 s^{2} 2 p^{2}-$ & ${ }^{3} \mathrm{P}-{ }^{3} \mathrm{D}^{\circ}$ & 571.04 & 756 & 175876 & 9 & 15 & 14 & 0.11 & 1.9 & 0.00 & $\mathrm{E}$ & \\
\hline & & & 572.336 & 1112 & 175834 & 5 & 7 & 14 & 0.094 & 0.89 & -0.33 & $\mathrm{E}$ & ${ }_{t s}^{s}$ \\
\hline & & & 569.830 & 414 & 175905 & 3 & 5 & 10 & 0.084 & 0.47 & -0.60 & $\mathrm{E}$ & ls \\
\hline & & & 568.418 & $\begin{array}{l}0 \\
1112\end{array}$ & 175927 & 1 & $\begin{array}{l}3 \\
5\end{array}$ & $\begin{array}{l}7.7 \\
35\end{array}$ & & 0.21 & $\begin{array}{l}-0.95 \\
-107\end{array}$ & $\mathrm{E}$ & Is \\
\hline & & & $\begin{array}{l}572.106 \\
569.759\end{array}$ & $\begin{array}{r}1112 \\
414\end{array}$ & $\begin{array}{l}175905 \\
175927\end{array}$ & $\begin{array}{l}5 \\
3\end{array}$ & $\begin{array}{l}5 \\
3\end{array}$ & $\begin{array}{l}3.5 \\
5.8\end{array}$ & $\begin{array}{l}0.017 \\
0.028\end{array}$ & $\begin{array}{l}0.16 \\
0.16\end{array}$ & $\begin{array}{l}-1.07 \\
-1.07\end{array}$ & $\begin{array}{l}\mathrm{E} \\
\mathrm{E}\end{array}$ & $\begin{array}{l}l s \\
l s\end{array}$ \\
\hline & & & [572.03] & 1112 & 175927 & 5 & 3 & 0.40 & 0.0012 & 0.011 & -2.23 & $\overline{\mathrm{E}}$ & 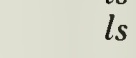 \\
\hline \multirow[t]{6}{*}{2} & & ${ }^{3} \mathrm{P}-{ }^{3} \mathrm{P}^{\circ}$ & 482.15 & 756 & 208161 & 9 & 9 & 40 & 0.14 & 2.0 & 0.10 & $\mathrm{E}$ & \\
\hline & & & 482.987 & 1112 & 208157 & 5 & 5 & 30 & 0.10 & 0.83 & -0.28 & $\mathrm{E}$ & \\
\hline & & & 481.361 & 414 & 208157 & 3 & 3 & 10 & 0.036 & 0.17 & -0.97 & $\mathrm{E}$ & 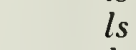 \\
\hline & & & 482.987 & 1112 & 208157 & 5 & 3 & 17 & 0.035 & 0.28 & -0.75 & $\mathrm{E}$ & $l_{s}$ \\
\hline & & & 481.281 & $\begin{array}{l}414 \\
414\end{array}$ & $\begin{array}{l}208193 \\
200857\end{array}$ & 3 & $\frac{1}{5}$ & $\begin{array}{l}40 \\
10\end{array}$ & $\begin{array}{l}0.046 \\
0.059\end{array}$ & 0.22 & $\begin{array}{l}-0.86 \\
-075\end{array}$ & $\mathrm{E}$ & c \\
\hline & & & 480.406 & $\begin{array}{r}414 \\
0\end{array}$ & 208157 & 1 & 3 & 13 & 0.14 & $\begin{array}{l}0.20 \\
0.22\end{array}$ & -0.86 & $\stackrel{\mathrm{L}}{\mathrm{E}}$ & \\
\hline \multirow[t]{4}{*}{3} & & ${ }^{3} \mathrm{P}-{ }^{3} \mathrm{~S}^{\circ}$ & 358.93 & 756 & 279365 & 9 & 3 & 220 & 0.14 & 1.5 & 0.10 & $\mathrm{E}$ & \\
\hline & & & 359.385 & 1112 & 279365 & 5 & 3 & 120 & 0.14 & 0.83 & -0.15 & $\mathrm{E}$ & \\
\hline & & & 358.472 & 414 & 279365 & 3 & 3 & 73 & 0.1 & 0. & -0.37 & $\mathrm{E}$ & \\
\hline & & & 357.955 & 0 & 279365 & 1 & 3 & 25 & 0.14 & 0.17 & -0.84 & $\bar{E}$ & \\
\hline 4 & & $\begin{array}{l}{ }^{1} \mathrm{D}-{ }^{-1} \mathrm{D}^{\circ} \\
\text { (4 uv) }\end{array}$ & 416.198 & 30294 & 270564 & 5 & 5 & 110 & 0.28 & 1.9 & 0.14 & $\mathrm{E}$ & \\
\hline 5 & & $\begin{array}{l}{ }^{1} \mathrm{D}-{ }^{-1} \mathrm{P}^{\circ} \\
(5 \mathrm{uv})\end{array}$ & 365.594 & 30294 & 303812 & 5 & 3 & 150 & 0.18 & 1.1 & -0.04 & $\mathrm{E}$ & \\
\hline 6 & $2 p^{2}-$ & ${ }^{1} \mathrm{D}-{ }^{1} \mathrm{P}^{\circ}$ & 173.932 & 30294 & 605231 & 5 & 3 & 230 & 0.063 & 0.18 & -0.50 & $\mathrm{E}$ & \\
\hline & $2 p\left({ }^{(2} \mathrm{P}^{\circ}\right) 3 s$ & $6 \mathrm{uv}$ & & & & & & & & & & & \\
\hline \multirow[t]{7}{*}{7} & $2 p^{2}-$ & ${ }^{3} \mathrm{P}-{ }^{-3} \mathrm{D}^{\circ}$ & 143.32 & 756 & 698517 & 9 & 15 & 1200 & 0.61 & 2.6 & 0.74 & $\mathrm{D}-$ & \\
\hline & & & {$[143.34]$} & 1112 & 698735 & 5 & 7 & 1200 & 0.51 & 1.2 & 0.41 & $\mathrm{D}-$ & \\
\hline & & & [143.27] & 414 & 698382 & 3 & 5 & 900 & 0.4 & 0.6 & 0.14 & $\mathrm{D}-$ & ls \\
\hline & & & [143.22] & 0 & 698231 & 1 & 3 & 670 & 0.6 & 0.2 & -0.21 & $\mathrm{D}-$ & ls \\
\hline & & & [143.42] & 1112 & 698382 & 5 & 5 & 300 & 0.1 & 0. & -0 & $\mathrm{D}$ & \\
\hline & & & [143.30] & 414 & 698231 & 3 & 3 & 500 & & 0. & -0 & $\mathrm{D}-$ & \\
\hline & & & [143.45] & 1112 & 698231 & 5 & 3 & 32 & 0.0059 & 0.014 & -1.53 & $\mathrm{D}-$ & \\
\hline \multirow[t]{7}{*}{8} & & ${ }^{3} \mathrm{P}-{ }^{3} \mathrm{P}^{\circ}$ & 142.61 & 756 & 701945 & 9 & 9 & 670 & 0.20 & 0.86 & 0.26 & $\mathrm{D}-$ & 2 \\
\hline & & & {$[142.72]$} & 1112 & 701765 & 5 & 5 & 500 & 0.1 & 0.3 & -0.12 & $\mathrm{D}-$ & \\
\hline & & & [142.52] & 414 & 702074 & 3 & 3 & 170 & 0.0 & 0.072 & -0.81 & D- & ls \\
\hline & & & [142.66] & 1112 & 702074 & 5 & 3 & 280 & 0.051 & 0.12 & -0.59 & D- & ls \\
\hline & & & {$[142.44]$} & 414 & 702459 & 3 & 1 & 670 & 0.068 & 0.0 & -0 & D & ls \\
\hline & & & [142.58] & 414 & 701765 & 3 & 5 & 170 & 0.085 & & -0.59 & $\mathrm{D}-$ & ls \\
\hline & & & [142.44] & 0 & 702074 & 1 & 3 & 220 & 0.20 & 0.096 & -0.69 & D- & \\
\hline 9 & & ${ }^{1} \mathrm{D}-{ }^{1} \mathrm{D}^{\circ}$ & [151.42] & 30294 & 690691 & 5 & 5 & 400 & 0.14 & 0.34 & -0.17 & $\mathrm{D}-$ & 2 \\
\hline 10 & & ${ }^{1} \mathrm{D}-{ }^{1} \mathrm{P}^{\circ}$ & [148.78] & 30294 & 702412 & 5 & 3 & 37 & 0.0073 & 0.018 & -1.43 & $\mathrm{D}-$ & 2 \\
\hline 11 & & ${ }^{1} \mathrm{D}-\mathrm{P}^{\circ}$ & [147.13] & 30294 & 709956 & 5 & 7 & 1300 & 0.58 & 1.4 & 0.46 & D- & 6 \\
\hline 12 & & ${ }^{1} \mathrm{~S}-{ }^{1} \mathrm{P}^{\circ}$ & [156.61] & 63900 & 702412 & 1 & 3 & 690 & 0.76 & 0.39 & -0.12 & $\mathrm{D}-$ & \\
\hline
\end{tabular}


Ne V. Allowed Transitions - Continued

\begin{tabular}{|c|c|c|c|c|c|c|c|c|c|c|c|c|c|}
\hline No. & $\begin{array}{c}\text { Transition } \\
\text { Array }\end{array}$ & $\begin{array}{c}\text { Multi- } \\
\text { plet }\end{array}$ & $\lambda(\AA)$ & $E_{i}\left(\mathrm{~cm}^{-1}\right)$ & $E_{k}\left(\mathrm{~cm}^{-1}\right)$ & $g_{i}$ & $g_{k}$ & $\begin{array}{l}A_{k i}\left(10^{8}\right. \\
\left.\sec ^{-1}\right)\end{array}$ & $f_{i k}$ & $S($ at.u.) & $\log g f$ & $\begin{array}{l}\text { Accu- } \\
\text { racy }\end{array}$ & Source \\
\hline \multirow[t]{7}{*}{13} & \multirow{7}{*}{$\begin{array}{l}2 p 3 s- \\
2 p\left({ }^{2} \mathrm{P}^{\circ}\right) 3 p\end{array}$} & \multirow[t]{7}{*}{${ }^{3} \mathrm{P}^{\circ}-{ }^{3} \mathrm{D}$} & & & 641142 & 9 & 15 & 2.2 & 0.28 & 19 & 0.40 & D & $c a$ \\
\hline & & & 2265.71 & 597523 & 641646 & 5 & 7 & 2.2 & 0.24 & 8.9 & 0.07 & D & ls \\
\hline & & & 2259.57 & 596626 & 640868 & 3 & 5 & 1.7 & 0.21 & 4.7 & -0.20 & D & Is \\
\hline & & & 2263.39 & 596254 & 640422 & 1 & 3 & 1.2 & 0.28 & 2.1 & -0.55 & D & Is \\
\hline & & & 2306.31 & 597523 & 640868 & 5 & 5 & 0.52 & 0.042 & 1.6 & -0.68 & D & Is \\
\hline & & & 2282.61 & 596626 & 640422 & 3 & 3 & 0.89 & 0.070 & 1.6 & -0.68 & D & 1 \\
\hline & & & [2330.3] & 597523 & 640422 & 5 & 3 & 0.056 & 0.0027 & 0.11 & -1.86 & D & $1 /$ \\
\hline \multirow[t]{9}{*}{14} & \multirow{9}{*}{$\begin{array}{l}2 s 2 p^{2} 3 s- \\
2 s 2 p^{2}\left({ }^{4} \mathrm{P}\right) 3 p\end{array}$} & \multirow{9}{*}{${ }^{5} \mathrm{P}-{ }^{5} \mathrm{D}^{\circ}$} & & & & & & & & & & & \\
\hline & & & 2232.41 & 698504 & 743285 & 7 & 9 & 0.20 & 0.019 & 0.96 & -0.88 & D & $c a, l s$ \\
\hline & & & 2227.42 & 697935 & 742816 & 5 & 7 & 0.13 & 0.01 & 0.5 & -1.17 & D & \\
\hline & & & 2224.12 & 697507 & 742455 & 3 & 5 & 0.069 & 0.0085 & 0.19 & -1.59 & D & $c a$, \\
\hline & & & 2256.05 & 698504 & 742816 & 7 & 7 & 0.063 & 0.0048 & 0.25 & -1.47 & D & $\mathrm{ca}$, ls \\
\hline & & & 2245.48 & 697935 & 742455 & 5 & 5 & 0.11 & 0.0084 & 0.31 & -1.37 & D & $\mathrm{ca}, \mathrm{ls}$ \\
\hline & & & 2236.29 & 697507 & 742210 & 3 & 3 & 0.15 & 0.011 & 0.24 & -1.49 & D & $\mathrm{ca}$, ls \\
\hline & & & 2274.54 & 698504 & 742455 & 7 & 5 & 0.012 & $6.8 \times 10^{-4}$ & 0.036 & -2.32 & D & $c a$, ls \\
\hline & & & [2257.9] & 697935 & $742210^{\circ}$ & 5 & 3 & 0.047 & 0.0022 & 0.080 & -1.97 & D & $\mathrm{ca}$, ls \\
\hline
\end{tabular}

\section{Forbidden Transitions}

The adopted values represent, as in the case of F IV, the work of Garstang [1] and Naqvi [2], who independently have done essentially the same calculations and have arrived at very similar results. For the selection of values, the same considerations as for F IV have been applied.

\section{References}

[1] Garstang, R. H., Monthly Notices Roy. Astron. Soc. 11 1, 115-124 (1951).

[2] Naqvi, A. M., Thesis Harvard (1951).

Ne v. Forbidden Transitions

\begin{tabular}{|c|c|c|c|c|c|c|c|c|c|c|c|c|}
\hline No. & $\begin{array}{c}\text { Transition } \\
\text { Array }\end{array}$ & $\begin{array}{c}\text { Multi- } \\
\text { plet }\end{array}$ & $\lambda(\AA)$ & $E_{i}\left(\mathrm{~cm}^{-1}\right)$ & $E_{k}\left(\mathrm{~cm}^{-1}\right)$ & $g_{i}$ & $g_{k}$ & $\begin{array}{l}\text { Type of } \\
\text { Transition }\end{array}$ & $A_{k i}\left(\sec ^{-1}\right)$ & $S$ (at.u.) & $\begin{array}{l}\text { Accu- } \\
\text { racy }\end{array}$ & Source \\
\hline 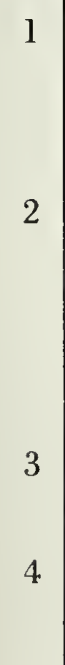 & $2 p^{2}-2 p^{2}$ & $\begin{array}{l}{ }^{3} \mathrm{P}-{ }^{3} \mathrm{P} \\
(1 \mathrm{~F}) \\
{ }^{3} \mathrm{P}-{ }^{1} \mathrm{~S} \\
{ }^{1} \mathrm{D}-{ }^{1 S} \mathrm{~S} \\
(2 \mathrm{~F})\end{array}$ & $\begin{array}{c}{\left[24.15 \times 10^{4}\right]} \\
{\left[8.990 \times 10^{4}\right]} \\
{\left[14.32 \times 10^{4}\right]} \\
{\left[14.32 \times 10^{4}\right]} \\
\\
{[3300.0]} \\
3345.9 \\
3345.9 \\
3425.8 \\
3425.8 \\
{[1575.2]} \\
{[1592.7]} \\
\\
2972\end{array}$ & $\begin{array}{r}0 \\
0 \\
414 \\
414 \\
\\
\\
0 \\
414 \\
414 \\
1112 \\
1112 \\
414 \\
1112 \\
\\
30294\end{array}$ & $\begin{array}{r}414 \\
1112 \\
1112 \\
1112 \\
\\
\\
30294 \\
30294 \\
30294 \\
30294 \\
30294 \\
63900 \\
63900 \\
\\
\\
63900\end{array}$ & $\begin{array}{l}1 \\
3 \\
3 \\
5 \\
5 \\
\\
3 \\
5\end{array}$ & $\begin{array}{l}5 \\
5 \\
5 \\
5 \\
5 \\
1 \\
1\end{array}$ & $\begin{array}{c}\mathrm{e} \\
\mathrm{m} \\
\mathrm{e} \\
\mathrm{m} \\
\mathrm{e} \\
\mathrm{m} \\
\mathrm{e}\end{array}$ & $\begin{array}{l}0.00129 \\
5.2 \times 10^{-9} \\
0.00459 \\
1.10 \times 10^{-9} \\
\\
1.9 \times 10^{-5} \\
0.138 \\
6.2 \times 10^{-5} \\
0.382 \\
3.9 \times 10^{-4} \\
4.20 \\
0.0068 \\
\\
2.60\end{array}$ & $\begin{array}{l}2.00 \\
0.091 \\
2.50 \\
0.197 \\
\\
2.2 \times 10^{-5} \\
9.6 \times 10^{-4} \\
7.7 \times 10^{-5} \\
0.00285 \\
5.5 \times 10^{-4} \\
6.1 \times 10^{-4} \\
4.1 \times 10^{-5} \\
\\
0.359\end{array}$ & $\begin{array}{l}\text { B } \\
\text { C } \\
B \\
C \\
\\
D \\
C \\
D \\
C \\
D \\
C \\
D\end{array}$ & $\begin{array}{r}1.2 \\
1 \\
1,2 \\
1 \\
\\
1 \\
1,2 \\
1 \\
1,2 \\
1 \\
1 \\
1\end{array}$ \\
\hline
\end{tabular}




\section{Ne VI.}

Ground State

Ionization Potential

$157.91 \mathrm{eV}=1274000 \mathrm{~cm}^{-1}$

\section{Allowed Transitions}

List of tabulated lines:

\begin{tabular}{l|l||l|l||l|l}
\hline \hline \multirow{2}{*}{ Wavelength $[\AA]$} & \multicolumn{1}{c}{ No. } & \multicolumn{1}{c}{ Wavelength $[\AA]$} & \multicolumn{1}{c}{ No. } & \multicolumn{1}{c}{ Wavelength $[\AA]$} & No. \\
\hline & & & & & \\
122.49 & 9 & 440.404 & 5 & 571.00 & 6 \\
122.69 & 9 & 440.46 & 5 & 637.90 & 7 \\
138.39 & 8 & 440.60 & 5 & 638.19 & 7 \\
138.64 & 8 & 451.843 & 4 & 641.26 & 7 \\
399.82 & 3 & 452.745 & 4 & 641.55 & 7 \\
401.14 & 3 & 454.072 & 4 & 2042.38 & 10 \\
401.93 & 3 & 558.59 & 1 & 2055.93 & 10 \\
403.26 & 3 & 562.71 & 1 & 2213.1 & 11 \\
433.176 & 2 & 562.80 & 1 & 2229.1 & 11 \\
435.649 & 2 & 570.77 & 6 & & \\
\hline
\end{tabular}

The transition probabilities are taken from the self-consistent field calculations (with exchange) by Weiss [1]. These calculations neglect the effects of configuration interaction entirely. The average of the dipole length and velocity approximations is adopted [1].

\section{Reference}

[1] Weiss, A. W., private communication (1965).

Ne VI. Allowed Transitions

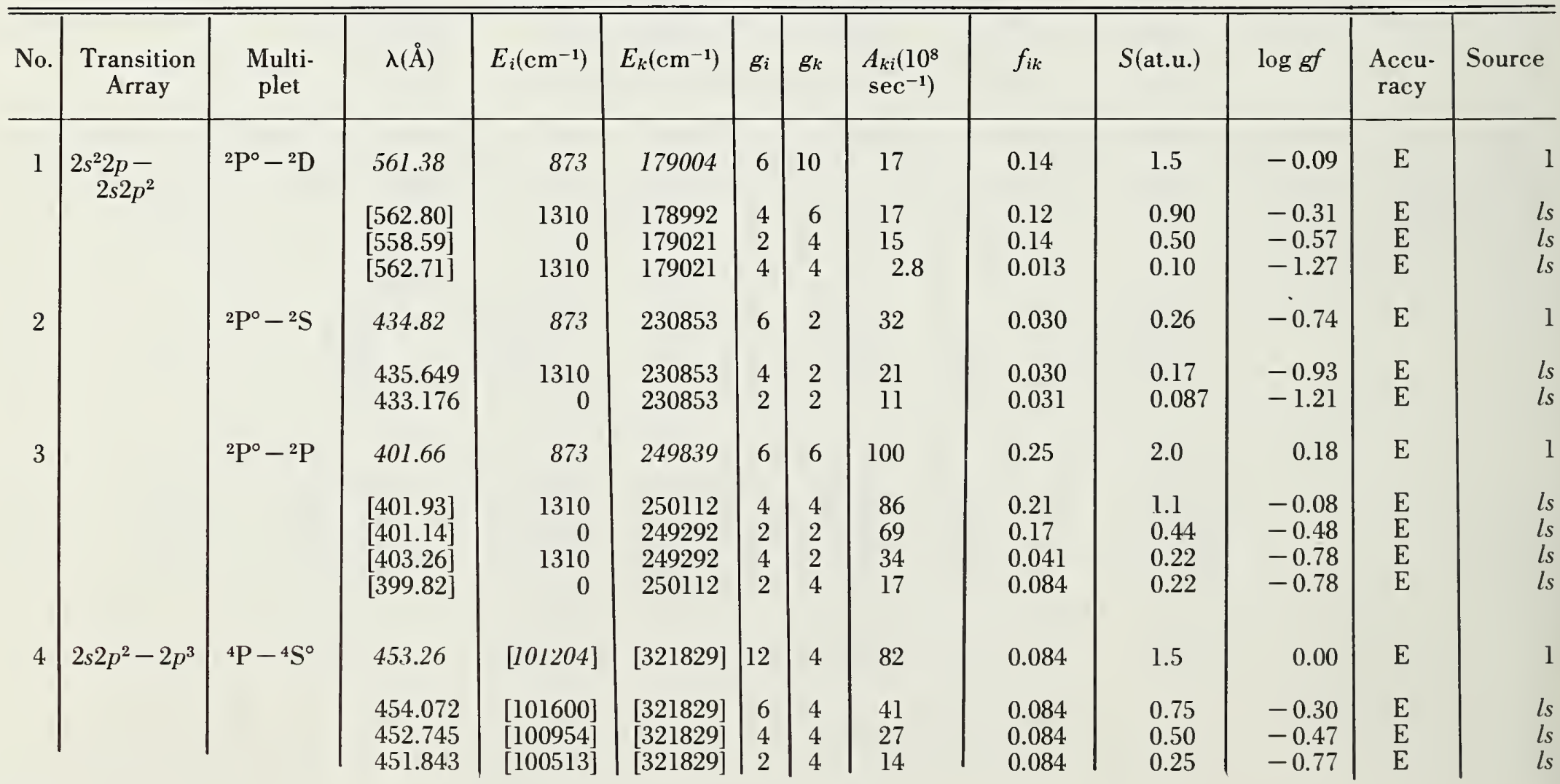


Ne VI. Allowed Transitions-Continued

\begin{tabular}{|c|c|c|c|c|c|c|c|c|c|c|c|c|c|}
\hline No & $\begin{array}{c}\text { Transition } \\
\text { Array }\end{array}$ & $\begin{array}{c}\text { Multi- } \\
\text { plet }\end{array}$ & $\lambda(\AA)$ & $E_{i}\left(\mathrm{~cm}^{-1}\right)$ & $E_{k}\left(\mathrm{~cm}^{-1}\right)$ & $g_{i}$ & $k_{k}$ & $\begin{array}{c}A_{\text {Liv }}\left(10^{4}\right. \\
\left.\sec ^{-1}\right)\end{array}$ & $f_{i k}$ & S(at.u.) & $\log g f$ & $\begin{array}{l}\text { Accu- } \\
\text { racy }\end{array}$ & Source \\
\hline \multirow[t]{4}{*}{5} & & ${ }^{2} \mathrm{D}-{ }^{2} \mathrm{P}^{\circ}$ & 440.47 & 179004 & 4060.32 & 10 & 6 & 25 & 0.044 & 0.64 & -0.36 & E & \\
\hline & & & 440.404 & 178992 & 406056 & 6 & 4 & 23 & 0.044 & 0.38 & -0.58 & $\mathrm{E}$ & \\
\hline & & & 440.60 & 179021 & 405984 & 4 & 2 & 25 & 0.036 & 0.21 & -0.84 & $\mathrm{E}$ & is \\
\hline & & & [440.46] & 179021 & 406056 & 4 & 4 & 25 & 0.0074 & 0.043 & -1.53 & E & l \\
\hline \multirow[t]{3}{*}{6} & & ${ }^{2} \mathrm{~S}-{ }^{2} \mathrm{P}^{\circ}$ & 570.84 & 230853 & 406032 & 2 & 6 & 12 & 0.17 & 0.64 & -0.47 & $\mathrm{E}$ & \\
\hline & & & {$[570.77]$} & 230853 & 406056 & 2 & 4 & 12 & 0.11 & 0.43 & -0.64 & E & Is \\
\hline & & & [571.00] & 230853 & 405984 & 2 & 2 & 12 & 0.056 & 0.21 & -0.95 & $E$ & 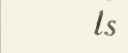 \\
\hline \multirow[t]{5}{*}{7} & & ${ }^{2} \mathrm{P}-{ }^{2} \mathrm{P}^{\circ}$ & 640.23 & 249839 & 406032 & 6 & 6 & 19 & 0.12 & 1.5 & -0.15 & $E$ & \\
\hline & & & [641.26] & 250112 & 406056 & 4 & 4 & 16 & 0.098 & 0.83 & -0.41 & $\mathrm{E}$ & \\
\hline & & & [638.19] & 249292 & 405984 & 2 & 2 & 13 & 0.079 & 0.33 & -0.80 & $\mathrm{E}$ & . \\
\hline & & & [641.55] & 250112 & 405984 & 4 & 2 & 6.5 & 0.020 & 0.17 & -1.09 & E & ls \\
\hline & & & [637.90] & 249292 & 406056 & 2 & 4 & 3.3 & 0.040 & 0.17 & -1.09 & $E$ & \\
\hline \multirow[t]{3}{*}{8} & $2 p-\left({ }^{1} \mathrm{~S}\right) 3 s$ & ${ }^{2} \mathrm{P}^{\circ}-{ }^{2} \mathrm{~S}$ & 138.55 & 873 & 722610 & 6 & 2 & 300 & 0.029 & 0.078 & -0.77 & $\mathrm{E}$ & \\
\hline & & & [138.64] & 1310 & 722610 & 4 & 2 & 200 & 0.028 & 0.052 & -0.94 & E & 5 \\
\hline & & & [138.39] & 0 & 722610 & 2 & 2 & 100 & 0.029 & 0.026 & -1.24 & $\mathrm{E}$ & \\
\hline \multirow[t]{3}{*}{9} & $2 p-\left({ }^{1} \mathrm{~S}\right) 3 d$ & ${ }^{2} \mathrm{P}^{\circ}-{ }^{2} \mathrm{D}$ & 122.62 & 873 & 816405 & 6 & 10 & 1400 & 0.54 & 1.3 & 0.51 & D & \\
\hline & & & [122.69] & 1310 & 816405 & 4 & 6 & 1400 & 0.48 & 0.78 & 0.29 & D & Is \\
\hline & & & $\begin{array}{l}{[122.49]} \\
{[122.69]}\end{array}$ & $\begin{array}{r}0 \\
1310\end{array}$ & $\begin{array}{l}816405^{\circ} \\
816405\end{array}$ & $\begin{array}{l}2 \\
4\end{array}$ & $\begin{array}{l}4 \\
4\end{array}$ & $\begin{array}{r}1200 \\
240\end{array}$ & $\begin{array}{l}0.53 \\
0.054\end{array}$ & $\begin{array}{l}0.43 \\
0.087\end{array}$ & $\begin{array}{r}0.03 \\
-0.67\end{array}$ & $\begin{array}{l}\mathrm{D} \\
\mathrm{D}\end{array}$ & ls \\
\hline \multirow[t]{3}{*}{10} & $3 s-\left({ }^{1} \mathrm{~S}\right) 3 p$ & ${ }^{2} \mathrm{~S}-{ }^{2} \mathrm{P}^{\circ}$ & 2046.9 & 722610 & 771449 & 2 & 6 & 2.72 & 0.51 & 6.9 & 0.010 & C & \\
\hline & & & 2042.38 & 722610 & 771557 & 2 & 4 & 2.73 & 0.342 & 4.60 & -0.165 & C & ls \\
\hline & & & 2055.93 & 722610 & 771234 & 2 & 2 & 2.68 & 0.170 & 2.30 & -0.469 & $\mathrm{C}$ & ls \\
\hline \multirow[t]{4}{*}{11} & $3 p-\left({ }^{1} \mathrm{~S}\right) 3 d$ & ${ }^{2} \mathrm{P}^{\circ}-{ }^{2} \mathrm{D}$ & 2223.7 & 771449 & 816405 & 6 & 10 & 1.82 & 0.225 & 9.9 & 0.131 & C & 1 \\
\hline & & & [2229.1] & 771557 & 816405 & 4 & 6 & 1.80 & 0.201 & 5.9 & -0.095 & C & \\
\hline & & & $\begin{array}{l}{[2213.1]} \\
{[2229.1]}\end{array}$ & $\begin{array}{l}771234 \\
771557\end{array}$ & $\begin{array}{l}816405 \\
816405\end{array}$ & $\begin{array}{l}2 \\
4\end{array}$ & $\begin{array}{l}4 \\
4\end{array}$ & $\begin{array}{l}1.54 \\
0.302\end{array}$ & $\begin{array}{l}0.226 \\
0.0225\end{array}$ & $\begin{array}{l}3.30 \\
0.66\end{array}$ & $\mid \begin{array}{l}-0.344 \\
-1.046\end{array}$ & $\begin{array}{l}\mathrm{C} \\
\mathrm{C}\end{array}$ & ls \\
\hline & & & & & & & & & & & & & \\
\hline
\end{tabular}

\section{Forbidden Transitions}

Naqvi's calculation [1] of the one possible transition in the ground state configuration $2 p$ is the only available source. The line strength should be quite accurate, since it does not sensitively -depend on the choice of the interaction parameters.

\section{Reference}

[1] Naqvi, A. M., Thesis Harvard (1951).

Ne VI. Forbidden Transitions

\begin{tabular}{c|c|c|c|r|r|r|r|r|r|r|r}
\hline \hline $\begin{array}{c}\text { No. } \\
\begin{array}{c}\text { Transition } \\
\text { Array }\end{array}\end{array}$ & Multiplet & $\lambda(\AA)$ & $E_{i}\left(\mathrm{~cm}^{-1}\right)$ & $E_{k}\left(\mathrm{~cm}^{-1}\right)$ & $g_{i}$ & $g_{k}$ & $\begin{array}{c}\text { Type of } \\
\text { Transition }\end{array}$ & $A_{k i}\left(\mathrm{sec}^{-1}\right)$ & $S(\mathrm{at} . \mathrm{u})$. & $\begin{array}{c}\text { Accu- } \\
\text { racy }\end{array}$ & $\begin{array}{c}\text { Source } \\
1\end{array}$ \\
\hline $2 p-2 p$ & ${ }^{2} \mathrm{P}^{\circ}-{ }^{2} \mathrm{P}^{\circ}$ & & & & & & & & & \\
\hline
\end{tabular}


Ground State

\section{Allowed Transitions}

The values are taken from the calculations of Veselov [1] who has used relatively simple wave functions and neglected the effects of configuration interaction entirely. Hence, large uncertainties are to be expected.

\section{Reference}

[1] Veselov, M. G., Zhur. Eksptl. i Teoret. Fiz. 19, 959-964 (1949).

Ne VII. Allowed Transitions

\begin{tabular}{|c|c|c|c|c|c|c|c|c|c|c|c|c|c|}
\hline No. & $\begin{array}{c}\text { Transition } \\
\text { Array }\end{array}$ & $\begin{array}{l}\text { Multi- } \\
\text { plet }\end{array}$ & $\lambda(\AA)$ & $E_{i}\left(\mathrm{~cm}^{-1}\right)$ & $E_{k}\left(\mathrm{~cm}^{-1}\right)$ & $g_{i}$ & $g_{k}$ & $\begin{array}{c}A_{k i}\left(10^{8}\right. \\
\left.\sec ^{-1}\right)\end{array}$ & $f_{i k}$ & $S$ (at.u.) & $\log g f$ & $\begin{array}{l}\text { Accu- } \\
\text { racy }\end{array}$ & Source \\
\hline 1 & $\begin{array}{l}2 s^{2}- \\
2 s\left({ }^{2} \mathrm{~S}\right) 2 p\end{array}$ & ${ }^{1} \mathrm{~S}-{ }^{1} \mathrm{P}^{\circ}$ & 465.221 & 0 & 214952 & 1 & 3 & 58 & 0.57 & 0.87 & -0.25 & $\mathrm{E}$ & 1 \\
\hline \multirow[t]{2}{*}{2} & \multirow[t]{2}{*}{$2 s 2 p-2 p^{2}$} & \multirow[t]{2}{*}{${ }^{3} \mathrm{P}^{\circ}-{ }^{3} \mathrm{P}$} & 561.59 & [112208] & [290273] & 9 & 9 & 33 & 0.16 & 2.6 & 0.14 & $\mathrm{E}$ & 1 \\
\hline & & & $\begin{array}{l}561.728 \\
561.378 \\
564.529 \\
562.992 \\
558.61 \\
559.947\end{array}$ & 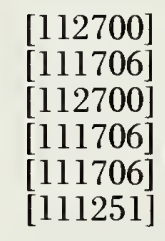 & $\begin{array}{l}{[290722]} \\
{[289839]} \\
{[289839]} \\
{[289328]} \\
{[290722]} \\
{[289839]}\end{array}$ & $\begin{array}{l}5 \\
3 \\
5 \\
3 \\
3 \\
1\end{array}$ & $\begin{array}{l}5 \\
3 \\
3 \\
1 \\
5 \\
3\end{array}$ & $\begin{array}{c}25 \\
8.2 \\
14 \\
32 \\
8.6 \\
11\end{array}$ & $\begin{array}{l}0.12 \\
0.039 \\
0.040 \\
0.051 \\
0.067 \\
0.15\end{array}$ & $\begin{array}{l}1.1 \\
0.21 \\
0.37 \\
0.28 \\
0.37 \\
0.28\end{array}$ & $\begin{array}{l}-0.23 \\
-0.94 \\
-0.70 \\
-0.81 \\
-0.70 \\
-0.81\end{array}$ & $\begin{array}{l}E \\
E \\
E \\
E \\
E \\
E\end{array}$ & $\begin{array}{l}l s \\
l s \\
l s \\
l s \\
l s \\
l s\end{array}$ \\
\hline \multirow[t]{4}{*}{3} & \multirow{4}{*}{$\begin{array}{l}2 s 3 s- \\
\quad 2 s\left({ }^{2} \mathrm{~S}\right) 3 p\end{array}$} & \multirow[t]{4}{*}{${ }^{3} \mathrm{~S}-{ }^{3} \mathrm{P}^{\circ}$} & 1987.0 & [978300] & [1028626] & 3 & 9 & 2.3 & 0.41 & 8.1 & 0.09 & $\mathrm{D}$ & $c a$ \\
\hline & & & 1981.97 & [978300] & [1028755] & 3 & 5 & 2.4 & 0.23 & 4.5 & -0.16 & D & ls \\
\hline & & & 1992.06 & {$[978300]$} & [1028499] & $\begin{array}{l}3 \\
3\end{array}$ & 3 & 2.3 & 0.14 & 2.7 & -0.38 & D & ls \\
\hline & & & 1991.05 & {$[9.0500]$} & {$[102836 /]$} & 3 & 1 & 2.3 & 0.040 & 0.90 & -0.80 & $D$ & \\
\hline
\end{tabular}

\section{Forbidden Transitions}

Naqvi's calculations [1] are the only available source. The results for the ${ }^{3} \mathrm{P}^{\circ}-{ }^{3} \mathrm{P}^{\circ}$ transitions are essentially independent of the choice of the interaction parameters. For the ${ }^{3} \mathrm{P}^{\circ}-{ }^{1} \mathrm{P}^{\circ}$ transitions, Naqvi uses empirical term intervals, i.e., the effects of configuration interaction should be partially included.

\section{Reference}

[1] Naqui, A. M., Thesis Harvard (1951). 
Ne VII. Forbidden Transitions

\begin{tabular}{|c|c|c|c|c|c|c|c|c|c|c|c|c|}
\hline No. & $\begin{array}{c}\text { Transition } \\
\text { Array }\end{array}$ & $\begin{array}{l}\text { Multi- } \\
\text { plet }\end{array}$ & $\lambda(\AA)$ & $E_{i}\left(\mathrm{~cm}^{-1}\right)$ & $E_{k}\left(\mathrm{~cm}^{-1}\right)$ & $g_{i}$ & $g_{k}$ & $\begin{array}{l}\text { Type of } \\
\text { Transition }\end{array}$ & $A_{k i}\left(\mathrm{sec}^{-1}\right)$ & S(at.u.) & $\begin{array}{l}\text { Accu- } \\
\text { racy }\end{array}$ & Source \\
\hline 1 & $\begin{array}{l}2 s 2 p- \\
2 s\left({ }^{2} \mathrm{~S}\right) 2 p\end{array}$ & ${ }^{3} P^{\circ}-{ }^{3} P^{\circ}$ & $\begin{array}{l}{\left[21.97 \times 10^{4}\right]} \\
{\left[10.06 \times 10^{4}\right]}\end{array}$ & $\begin{array}{l}111251 \\
111706\end{array}$ & $\begin{array}{l}111706 \\
112700\end{array}$ & $\begin{array}{l}1 \\
3\end{array}$ & $\begin{array}{l}3 \\
5\end{array}$ & $\begin{array}{l}\mathrm{m} \\
\mathrm{m}\end{array}$ & $\begin{array}{l}0.00170 \\
0.0132\end{array}$ & $\begin{array}{l}2.00 \\
2.50\end{array}$ & $\begin{array}{l}B \\
B\end{array}$ & $\begin{array}{l}1 \\
1\end{array}$ \\
\hline 2 & & ${ }^{3} \mathrm{P}^{\circ}-{ }^{1} \mathrm{P}^{\circ}$ & $\begin{array}{l}{[964.31]} \\
{[968.56]} \\
{[977.98]}\end{array}$ & $\begin{array}{l}111251 \\
111706 \\
112700\end{array}$ & $\begin{array}{l}214952 \\
214952 \\
214952\end{array}$ & $\begin{array}{l}1 \\
3 \\
5\end{array}$ & $\begin{array}{l}3 \\
3 \\
3\end{array}$ & $\begin{array}{l}\mathrm{m} \\
\mathrm{m} \\
\mathrm{m}\end{array}$ & $\begin{array}{c}0.91 \\
100 \\
1.10\end{array}$ & $\begin{array}{l}9.1 \times 10^{-5} \\
0.0101 \\
1.14 \times 10^{-4}\end{array}$ & $\begin{array}{l}\mathrm{C} \\
\mathrm{C} \\
\mathrm{C}\end{array}$ & $\begin{array}{l}1 \\
1 \\
1\end{array}$ \\
\hline
\end{tabular}

\section{Ne VIII.}

Ground State

Ionization Potential
$1 s^{2} 2 s^{2} \mathrm{~S}_{1 / 2}$

$239 \mathrm{eV}=1928000 \mathrm{~cm}^{-1}(?)$

\section{Allowed Transitions}

The extensive self-consistent field calculations including exchange by Weiss [1] are used for this ion. Values have been calculated in both the dipole length and velocity approximations and agree quite well. The dipole length values are chosen. For the $2 s-2 p$ transition an experimental result from the lifetime measurement of Berkner et al. [2] is available and agrees very well with Weiss' value.

\section{References}

[1] Weiss, A. W., Astrophys. J. 138, 1262-1276 (1963).

[2] Berkner, K. H., Cooper, W. L., Kaplan, S. N., and Pyle, R. V., Phys. Letters 16, 35 (1965).

Ne VIII. Allowed Transitions

\begin{tabular}{|c|c|c|c|c|c|c|c|c|c|c|c|c|c|}
\hline No. & $\begin{array}{c}\text { Transition } \\
\text { Array }\end{array}$ & $\begin{array}{l}\text { Multi- } \\
\text { plet }\end{array}$ & $\lambda(\AA)$ & $E_{i}\left(\mathrm{~cm}^{-1}\right)$ & $E_{k}\left(\mathrm{~cm}^{-1}\right)$ & $g_{i}$ & $g_{k}$ & $\begin{array}{c}A_{k i}\left(10^{8}\right. \\
\left.\sec ^{-1}\right)\end{array}$ & $f_{i k}$ & $S$ (at.u.) & $\log g f$ & $\begin{array}{l}\text { Accu- } \\
\text { racy }\end{array}$ & Source \\
\hline 1 & $2 s-2 p$ & ${ }^{2} \mathrm{~S}-{ }^{2} \mathrm{P}^{\circ}$ & 773.69 & .0 & 129251 & 2 & 6 & 5.64 & 0.152 & 0.774 & -0.517 & $\mathrm{~B}+$ & 1. 2 \\
\hline & & & $\begin{array}{l}770.402 \\
780.324\end{array}$ & $\begin{array}{l}0 \\
0\end{array}$ & $\begin{array}{l}129801 \\
128152\end{array}$ & $\begin{array}{l}2 \\
2\end{array}$ & $\begin{array}{l}4 \\
2\end{array}$ & $\begin{array}{l}5.72 \\
5.50\end{array}$ & $\begin{array}{l}0.102 \\
0.0502\end{array}$ & $\begin{array}{l}0.516 \\
0.258\end{array}$ & $\begin{array}{l}-0.692 \\
-0.998\end{array}$ & $\begin{array}{l}\mathrm{B}+ \\
\mathrm{B}+\end{array}$ & Is \\
\hline 2 & $2 s-3 p$ & ${ }^{2} \mathrm{~S}-{ }^{2} \mathrm{P}^{\circ}$ & [88.134] & 0 & [1134634] & 2 & 6 & 853 & 0.298 & 0.173 & -0.225 & B & 1 \\
\hline 3 & $2 p-3 s$ & ${ }^{2} \mathrm{P}^{\circ}-{ }^{2} \mathrm{~S}$ & [103.05] & 129251 & [1099681] & 6 & 2 & 462 & 0.0245 & 0.0499 & -0.833 & B & l \\
\hline 4 & $2 p-3 d$ & ${ }^{2} \mathrm{P}^{\circ}-{ }^{2} \mathrm{D}$ & [98.308] & 129251 & [1146459] & 6 & 10 & 2760 & 0.667 & 1.30 & 0.602 & B & 1 \\
\hline 5 & $3 s-3 p$ & ${ }^{2} \mathrm{~S}-{ }^{2} \mathrm{P}^{0}$ & [2860.1] & [1099681] & [1134634] & 2 & 6 & 0.696 & 0.256 & 4.82 & -0.291 & B & 1 \\
\hline 6 & $3 p-3 d$ & ${ }^{2} \mathrm{P}^{\circ}-{ }^{2} \mathrm{D}$ & [8454.3] & [1134634] & [1146459] & 6 & 10 & 0.0214 & 0.0382 & 6.38 & -0.640 & B & l \\
\hline
\end{tabular}


Ne IX.

Ground State

$1 s^{2}{ }^{1} \mathrm{~S}_{n}$

Ionization Potential

$1195 \mathrm{eV}=9641000 \mathrm{~cm}^{-1}(?)$

\section{Allowed Transitions}

The results of extensive non-relativistic variational calculations by Weiss [1] are chosen. Values have been calculated in both the dipole length and dipole velocity approximations and agree to within $1 \%$, except for the $3 p^{1} \mathrm{P}^{\circ}-3 d^{1} \mathrm{D}$ transition where agreement is not as good. The average of the two approximations is adopted $\left.{ }^{1}\right]$.

\section{Reference}

['] Weiss, A. W., private communication (1964).

Ne IX. Allowed Transitions

\begin{tabular}{|c|c|c|c|c|c|c|c|c|c|c|c|c|c|}
\hline No. & $\begin{array}{c}\text { Transition } \\
\text { Array }\end{array}$ & $\begin{array}{l}\text { Multi- } \\
\text { plet }\end{array}$ & $\lambda(\AA)$ & $E_{i}\left(\mathrm{~cm}^{-1}\right)$ & $E_{k}\left(\mathrm{~cm}^{-1}\right)$ & $g_{i}$ & $g_{k}$ & $A_{k i}\left(10^{8} \mathrm{sec}^{-1}\right)$ & $f_{i k}$ & $S$ (at.u.) & $\log g f$ & $\begin{array}{l}\text { Accu- } \\
\text { racy }\end{array}$ & Source \\
\hline 1 & $\begin{array}{c}1 s^{2}- \\
\quad l s 2 p\end{array}$ & ${ }^{1} \mathrm{~S}-{ }^{1} \mathrm{P}^{\circ}$ & {$[13.460]$} & 0 & [7429270] & 1 & 3 & $8.87 \times 10^{4}$ & 0.723 & 0.0320 & -0.141 & A & 1 \\
\hline 2 & $\frac{1 s^{2}-}{1 s 3 p}$ & ${ }^{1} \mathrm{~S}-{ }^{1} \mathrm{P}^{\circ}$ & [11.558] & 0 & [8652380] & 1 & 3 & $2.48 \times 10^{4}$ & 0.149 & 0.00567 & -0.827 & A & 1 \\
\hline 3 & $\begin{array}{r}1 s 2 s- \\
\quad 1 s 2 p\end{array}$ & ${ }^{1} \mathrm{~S}-{ }^{1} \mathrm{P}^{\circ}$ & [1901.5] & [7376680] & [7429270] & 1 & 3 & 0.329 & 0.0535 & 0.335 & -1.272 & $A$ & 1 \\
\hline 4 & $\begin{array}{r}l s 2 s- \\
\quad l s 3 p\end{array}$ & ${ }^{1} \mathrm{~S}-{ }^{1} \mathrm{P}^{\circ}$ & [78.388] & [7376680] & [8652380] & 1 & 3 & 1400 & 0.386 & 0.0996 & -0.413 & A & 1 \\
\hline 5 & $\begin{array}{r}1 s 2 p- \\
\quad l s 3 d\end{array}$ & ${ }^{1} \mathrm{P}^{\circ}-{ }^{1} \mathrm{D}$ & {$[82.010]$} & [7429270] & {$[8648630]$} & 3 & 5 & 4180 & 0.703 & 0.569 & 0.324 & A & 1 \\
\hline 6 & $\begin{array}{r}1 s 3 d- \\
\quad 1 s 3 p\end{array}$ & ${ }^{1} \mathrm{D}-{ }^{1} \mathrm{P}^{\circ}$ & {$[26660] ?$} & [8648630] & [8652380] & 5 & 3 & $9.99 \times 10^{-4}$ & 0.00639 & 2.80 & -1.496 & $\mathrm{C}+$ & 1 \\
\hline 7 & $\begin{array}{l}1 s 2 s- \\
\quad 1 s 2 p\end{array}$ & ${ }^{3} \mathrm{~S}-{ }^{3} \mathrm{P}^{0}$ & [1297.5] & [7294740] & [7371810] & 3 & 9 & 0.980 & 0.0742 & 0.951 & -0.653 & $A$ & 1 \\
\hline 8 & $\begin{array}{l}1 s 2 s- \\
\quad l s 3 p\end{array}$ & ${ }^{3} \mathrm{~S}-{ }^{3} \mathrm{P}^{\circ}$ & [74.527] & [7294740] & [8636540] & 3 & 9 & 1460 & 0.365 & 0.269 & 0.039 & A & 1 \\
\hline 9 & $\begin{array}{r}1 s 2 p- \\
1 s 3 d\end{array}$ & ${ }^{3} \mathrm{P}^{\circ}-{ }^{3} \mathrm{D}$ & [78.356] & [7371810] & [8648030] & 9 & 15 & 4380 & 0.672 & 1.56 & 0.782 & A & 1 \\
\hline 10 & $\begin{array}{r}1 s 3 p- \\
1 s 3 d\end{array}$ & ${ }^{3} \mathrm{P}^{\circ}-{ }^{3} \mathrm{D}$ & [8700.8] & [8636540] & [8648030] & 9 & 15 & 0.0155 & 0.0294 & 7.58 & -0.577 & A & 1 \\
\hline
\end{tabular}


List of Recent Additional Material

(New material which would have been employed if received before eut-off date)

\begin{tabular}{|c|c|c|c|}
\hline Spectrum & Referenees & Spectrum & References \\
\hline He I & $\mathrm{D}, \mathrm{H}, \mathrm{K}$ & $\mathrm{N}_{\text {II }}$ & \\
\hline & & N III & C, L \\
\hline Li I & $A, B$ & $\begin{array}{l}\text { N IV } \\
\text { N V }\end{array}$ & B, E \\
\hline Be I & I & & \\
\hline Be II & B & $\begin{array}{l}\mathrm{O}_{\text {I }} \\
\mathrm{O}_{\text {II }}\end{array}$ & $\begin{array}{l}G, J \\
F\end{array}$ \\
\hline B I & $\mathrm{C}, \mathrm{L}$ & O III & $\mathrm{F}$ \\
\hline B II & $\mathrm{L}$ & $\mathrm{O}$ IV & C \\
\hline B III & B & $\begin{array}{l}\mathrm{O} V \\
\mathrm{O} \mathrm{VI}\end{array}$ & $\begin{array}{l}\mathrm{I} \\
\mathrm{B}, \mathrm{E}\end{array}$ \\
\hline C I & $\mathrm{L}$ & & \\
\hline C II & C & $\mathrm{Fv}$ & C \\
\hline $\begin{array}{l}\mathrm{C} \text { III } \\
\mathrm{C} \text { IV }\end{array}$ & $\mathrm{B}, \mathrm{E}$ & $\mathrm{Ne} I$ & D \\
\hline $\mathrm{N}_{I}$ & $\mathrm{G}, \mathrm{J}, \mathrm{L}$ & $\begin{array}{l}\text { Ne II } \\
\text { Ne VI }\end{array}$ & $\begin{array}{l}\mathrm{F} \\
\mathrm{C}\end{array}$ \\
\hline
\end{tabular}

References and Comments

A. Anderson, E. M., and Zilitis, V. A., Optics and Spectroscopy (U.S.S.R.) 16, 211-214 (1963).

Li I.

Semi-empirical calculations. Extensive tabulations, in fair agreement with our adopted values except for some of the high-lying transitions, especially those of the resonance series where these values are as much as $50 \%$ lower.

B. Flannery, M. R., and Stewart, A. L., Monthly Notices Roy. Astron. Soc. 126, 387-392 (1963).

Li I, Be II, B III, C IV, N v, O VI.

Variational calculations. Good agreement with tabulated values, usually within 10-25\%, except for cases where cancellation occurs. Some transitions are covered for which we have no values. Has been used for F VII.

C. Nikitin, A. A., and Yakubovskii, O. A., Soviet Phys. -Doklady 9, 409-411 (1964).

B I, C II, N III, O IV, F v, Ne VI.

Quantum mechanical calculations for forbidden transitions. Values are presented for quadrupole transitions in $\mathrm{sp}^{2}$ configurations. We have no values for these transitions.

D. Bennett, Jr., W. R., Kindlmann, P. J., and Mercer, G. N., Applied Optics Supplement 2 of Chemical Lasers pp. 3457, (1965).

$\mathrm{He} \mathbf{I}, \mathrm{Ne} \mathbf{I}$.

Lifetime determinations. The results for Ne agree within $25 \%$ with the adopted values and with the experimental results of Klose. The He results have been incorporated from an earlier paper which was referenced in this tabulation.

E. Berkner, K., Cooper III, W. S., Kaplan, S. N., and Pyle, R. V., Physics Letters 16, 35-36 (1965).

C IV, N V, O VI.

Lifetime determinations using the accelerator technique. Agrees with Weiss' extensive calculations within the stated experimental and theoretical error limits. Has been used for F vil and Ne VIII.

F. Froese, C., Phys. Rev. 137, Al644-A1648 (1965).

O II, O III, Ne II.

Self-consistent field calculations, Excellent agreement with the adopted values of Kelly, Mastrup, and Wiese, and the Coulomb approximation. Tends to be a few percent high in all cases except one and should be used in preference to the Coulomb approximation.

G. Morse, F. A., and Kaufman, F., J. Chem. Phys. 42, 1785-1790 (1965).

$\mathrm{N}_{1}, \mathrm{O}_{\mathrm{I}}$.

Absorption of resonance radiation. The lower limit given for $\mathrm{N}_{\mathbf{I}}$ is considerably lower than the values from the arc experiment of Labuhn and the lifetime determination of Lawrence and Savage (See ref. L), but is closer to the value of Prag, Fairchild, and Clark (See ref. J). For Or the values are in excellent agreement with the tabulated values.

H. Pendleton, W. R., and Hughes, R. H., Phys. Rev. 138, A683-A687 (1965).

He I.

Lifetime determination. Supports the theory quite well and usually agrees with other referenced lifetime experiments within the stated error limits.

I. Pfennig, H., Steele, R., and Trefftz, E., J. Quant. Spectrosc. Radiat. Transfer 5, 335-357 (1965).

Be I, C III, N IV, O v.

Self-consistent field calculations. Good agreement with tabulated values of Weiss; fair agreement with Kelly and the Coulomb approximation, with better agreement for the visible lines where Kelly and the Coulomb approximation have been averaged. Large divergences may occur where cancellation is significant..

J. Prag, A. B., Fairchild, C. E., and Clark, K. C. Phys. Rev. 137, A1358-A1363 (1965).

$\mathrm{N}$ I, O I.

Absorption of resonance radiation. For NI, disagrees by as much as a factor of $3-4$ (low) with the adopted values (arc experiment by Labuhn) and with the lifetime experiment of Lawrence and Savage (See ref. L). Agrees well for $\mathrm{O}$ I but this is to be expected because of the choice of "best" values.

K. Green, L. C., Kolchin, E. K., and Johnson, N. C., Submitted for publication in the Transactions of the International Astronomical Union Symposium, \#26 (1965).

$\mathrm{He}$.

Extensive variational calculations. Excellent agreement, within the assigned error limits, except for l'S -7 , 8 ip. where the disagreement is $10-15 \%$ low. These new values should be used for l'S $-7,8$ 'P.

L. Lawrence, G. M., and Savage, B. D., (To be published in Phys. Rev.)

$\mathrm{B}$ I, II, C I, $\mathrm{N}$ I, II, III.

Lifetime experiment using the phase shift method. Supports the adopted results of Weiss and Bototin and the are experiments of Boldt and Labuhn. The lifetimes tend to be somewhat longer than the adopted values. 


\section{National Standard Reference Data Series- National Bureau of Standards}

National Standard Reference Data System, Plan of Operation NSRDS-NBS $1-15 \phi^{*}$

Thermal Properties Of Aqueous Uni-univalent Electrolytes NSRDS-NBS 2-45 $\phi^{*}$

Selected Tables of Atomic Spectra, Atomic Energy Levels and Multiplet TablesSi II, Si III, Si IV NSRDS - NBS 3 Section $1-35 \phi^{*}$

Atomic Transition Probabilities, Hydrogen Through Neon NSRDS-NBS 4 Volume I$\$ 2.50^{*}$

\footnotetext{
*Send orders with remittance to: Superintendent of Documents, U.S. Government Printing Office, Washington, D.C., 20402. Remittances from foreign countries should include an additional one-fourth of the purchase price for postage.
} 


\section{THE NATIONAL BUREAU OF STANDARDS}

The National Bureau of Standards is a principal focal point in the Federal Government for assuring maximum application of the physical and engineering sciences to the advancement of technology in industry and commerce. Its responsibilities include development and maintenance of the national standards of measurement, and the provisions of means for making measurements consistent with those standards; determination of physical constants and properties of materials; development of methods for testing materials, mechanisms, and structures, and making such tests as may be necessary, particularly for government agencies; cooperation in the establishment of standard practices for incorporation in codes and specifications; advisory service to government agencies on scientific and technical problems; invention and development of devices to serve special needs of the Government; assistance to industry, business, and consumers in the development and acceptance of commercial standards and simplified trade practice recommendations; administration of programs in cooperation with United States business groups and standards organizations for the development of international standards of practice; and maintenance of a clearinghouse for the collection and dissemination of scientific, technical, and engineering information. The scope of the Bureau's activities is suggested in the following listing of its three Institutes and their organizational units.

Institute for Basic Standards. Applied Mathematics. Electricity. Metrology. Mechanics. Heat. Atomic Physics. Physical Chemistry. Laboratory Astrophysics.* Radiation Physics. Radio Standards Laboratory:* Radio Standards Physics; Radio Standards Engineering. Office of Standard Reference Data.

Institute for Materials Research. Analytical Chemistry. Polymers. Metallurgy. Inorganic Materials. Reactor Radiations. Cryogenics.* Materials Evaluation Laboratory. Office of Standard Reference Materials.

Institute for Applied Technology. Building Research. Information Technology. Performance Test Development. Electronic Instrumentation. Textile and Apparel Technology Center. Technical Analysis. Office of Weights and Measures. Office of Engineering Standards. Office of Invention and Innovation. Office of Technical Resources. Clearinghouse for Federal Scientific and Technical Information. **

*Located at Boulder, Colorado, 80301

* Located at 5285 Port Royal Road, Springfield, Virginia, 22171. 


\section{Announcement of New Publications on Standard Reference Data}

Superintendent of Documents, Government Printing Office,

Washington, D.C. 20402

Dear Sir:

Please add my name to the announcement list of new publications to be issued in the series: National Standard Reference Data Series - National Bureau of Standards.

Name

Company

Address

City State Zip Code

(Notification Key N337) 


